\title{
Tris(butadiene) Metal Complexes of the First Row Transition Metals Versus Coupling of Butadiene to Eight and Twelve-Carbon Hydrocarbon Chains
}

\author{
Qunchao Fan, ${ }^{a^{*}}$ Huidong $\mathrm{Li}^{,}{ }^{\mathrm{a}}$ Jia Fu, ${ }^{\mathrm{a}}$ Zhixiang Fan, ${ }^{\mathrm{a}}$ Yonggen $\mathrm{Xu},{ }^{\mathrm{a}} \mathrm{Hao}$ Feng, ${ }^{\mathrm{a}}$ \\ Yaoming Xie, ${ }^{b}$ R. Bruce King, ${ }^{\text {b* }}$ and Henry F. Schaefer III \\ aSchool of Science, Key Laboratory of High Performance Scientific \\ Computation, Xihua University, Chengdu, China 610039 \\ ${ }^{b}$ Department of Chemistry and Center for Computational Quantum Chemistry \\ University of Georgia, Athens, Georgia 30602, USA \\ rbking@chem.uga.edu; fanqunchao@mail.xhu.edu.cn
}

\section{Supporting Information}

Tables S1 to S47: Atomic coordinates of the optimized structures for the $\left(\mathrm{C}_{4} \mathrm{H}_{6}\right)_{3} \mathrm{M}$ $(\mathrm{M}=\mathrm{Ti}, \mathrm{V}, \mathrm{Cr}, \mathrm{Mn}, \mathrm{Fe}, \mathrm{Co}, \mathrm{Ni})$ complexes.

Tables S48 to S94: Harmonic vibrational frequencies (in $\mathrm{cm}^{-1}$ ) and infrared intensities (in parentheses in $\mathrm{km} / \mathrm{mol})$ for the $\left(\mathrm{C}_{4} \mathrm{H}_{6}\right)_{3} \mathrm{M}(\mathrm{M}=\mathrm{Ti}, \mathrm{V}, \mathrm{Cr}, \mathrm{Mn}, \mathrm{Fe}, \mathrm{Co}$, $\mathrm{Ni)}$ complexes.

Tables S95 to S101: The distances (in $\AA$ ) of $\mathrm{M}-\mathrm{C}$ bonds for the $\left(\mathrm{C}_{4} \mathrm{H}_{6}\right)_{3} \mathrm{M}(\mathrm{M}=\mathrm{Ti}, \mathrm{V}$, $\mathrm{Cr}, \mathrm{Mn}, \mathrm{Fe}, \mathrm{Co}, \mathrm{Ni}$ ) complexes;

Tables S102 to S108: Total energies ( $\mathrm{E}$ in hartree), relative energies $(\Delta \mathrm{E}$ in $\mathrm{kcal} / \mathrm{mol})$, and the spin expectation $\left\langle\mathrm{S}^{2}\right\rangle$ values for the $\left(\mathrm{C}_{4} \mathrm{H}_{6}\right)_{3} \mathrm{M}(\mathrm{M}=\mathrm{Ti}, \mathrm{V}, \mathrm{Cr}, \mathrm{Mn}, \mathrm{Fe}, \mathrm{Co}, \mathrm{Ni})$ complexes by all four methods.

Table S109. The theoretical (B3LYP*) Ni-C and C-C distances (in $\AA$ ) of the $\mathrm{Ni}\left(\mathrm{C}_{12} \mathrm{H}_{19}\right)^{+}$ cation. The experimental results of those in $\left[\mathrm{Ni}\left(\mathrm{C}_{12} \mathrm{H}_{19}\right)\right]^{+} \mathrm{X}^{-}\left(\mathrm{X}=\mathrm{BF}_{4}, \mathrm{~F}_{3} \mathrm{CSO}_{3}\right)$ are listed for comparison.

Table S110. The Mulliken spin density on the metal center. It shows that the spin states are mainly metal-centered, and the contribution from the ligand-based spin density are less than \pm 1.0 , except for the high-lying Ni-1T.

Complete Gaussian09 reference (Reference 39). 
Table S1. Optimized coordinates for the $\left(\mathrm{C}_{4} \mathbf{H}_{6}\right)_{3}$ Ti structure Ti-1S.

\begin{tabular}{|c|c|c|c|c|c|c|}
\hline & \multicolumn{3}{|c|}{ B3LYP } & \multicolumn{3}{|c|}{ B3LYP* } \\
\hline & $\mathrm{x}$ & $\mathrm{y}$ & $\mathrm{Z}$ & $\mathrm{x}$ & $\mathrm{y}$ & $\mathrm{Z}$ \\
\hline 6 & 0.174776 & -1.285607 & -1.739588 & 0.171099 & -1.286774 & -1.740284 \\
\hline 6 & 2.602424 & -1.049431 & 0.315028 & 2.601049 & -1.050308 & 0.310213 \\
\hline 6 & 1.216559 & -1.679074 & 0.491510 & 1.213052 & -1.674876 & 0.493235 \\
\hline 6 & 0.339179 & -2.029604 & -0.539673 & 0.332965 & -2.029107 & -0.537932 \\
\hline 1 & -0.439806 & -2.752200 & -0.298699 & -0.448494 & -2.751382 & -0.295017 \\
\hline 1 & 1.078161 & -2.221450 & 1.427191 & 1.074102 & -2.214277 & 1.432889 \\
\hline 1 & 3.114022 & -1.113210 & 1.284329 & 3.118128 & -1.115111 & 1.278761 \\
\hline 1 & 3.203137 & -1.641884 & -0.393273 & 3.198115 & -1.645032 & -0.402357 \\
\hline 1 & 1.038502 & -0.790689 & -2.188690 & 1.036533 & -0.790073 & -2.189076 \\
\hline 1 & -0.578203 & -1.607514 & -2.455535 & -0.585027 & -1.605495 & -2.457200 \\
\hline 6 & -2.427937 & 0.690351 & -0.793382 & -2.425637 & 0.691537 & -0.794333 \\
\hline 6 & -1.425408 & 0.131150 & 1.871799 & -1.418522 & 0.130741 & 1.873570 \\
\hline 6 & -2.070965 & -0.881115 & 1.085167 & -2.067413 & -0.880881 & 1.087086 \\
\hline 6 & -2.544705 & -0.612804 & -0.211479 & -2.542534 & -0.611794 & -0.211069 \\
\hline 1 & -2.810591 & -1.461802 & -0.841604 & -2.809539 & -1.462425 & -0.842051 \\
\hline 1 & -2.016313 & -1.920462 & 1.406889 & -2.013775 & -1.922156 & 1.409486 \\
\hline 1 & -0.958920 & -0.174702 & 2.806257 & -0.950540 & -0.174571 & 2.809607 \\
\hline 1 & -1.855332 & 1.137048 & 1.902964 & -1.843103 & 1.141288 & 1.901069 \\
\hline 1 & -2.670866 & 1.566666 & -0.190284 & -2.662169 & 1.570699 & -0.189273 \\
\hline 1 & -2.673715 & 0.803776 & -1.847548 & -2.673177 & 0.806610 & -1.849832 \\
\hline 6 & 2.586791 & 0.428846 & -0.116362 & 2.584545 & 0.428849 & -0.120761 \\
\hline 6 & 0.116494 & 2.223508 & -0.994239 & 0.111506 & 2.223209 & -0.993650 \\
\hline 6 & 0.742025 & 2.211605 & 0.273081 & 0.738836 & 2.211705 & 0.274011 \\
\hline 6 & 1.611125 & 1.219611 & 0.742138 & 1.610249 & 1.218018 & 0.741670 \\
\hline 1 & 1.842204 & 1.225762 & 1.807618 & 1.843983 & 1.222647 & 1.808623 \\
\hline 1 & 0.272516 & 2.831496 & 1.041842 & 0.269232 & 2.832663 & 1.044772 \\
\hline 1 & -0.610360 & 2.995685 & -1.226073 & -0.617797 & 2.995335 & -1.226730 \\
\hline 1 & 0.616272 & 1.794384 & -1.866343 & 0.608510 & 1.788798 & -1.867315 \\
\hline 1 & 2.341062 & 0.520906 & -1.180955 & 2.334300 & 0.521468 & -1.186491 \\
\hline 1 & 3.598015 & 0.856855 & -0.017609 & 3.597553 & 0.859013 & -0.026005 \\
\hline \multirow[t]{3}{*}{22} & -0.364179 & 0.170306 & -0.067022 & -0.360545 & 0.169095 & -0.065655 \\
\hline & \multicolumn{3}{|c|}{ BP86 } & \multicolumn{3}{|c|}{ M06L } \\
\hline & $\mathrm{x}$ & $\mathrm{y}$ & $\mathrm{Z}$ & $\mathrm{X}$ & $\mathrm{y}$ & $\mathrm{Z}$ \\
\hline 6 & 0.151261 & -1.294621 & -1.742668 & 0.139510 & -1.322289 & -1.712046 \\
\hline 6 & 2.599832 & -1.054428 & 0.292557 & 2.536442 & -1.062662 & 0.301232 \\
\hline 6 & 1.201832 & -1.658291 & 0.501272 & 1.154239 & -1.659018 & 0.525311 \\
\hline 6 & 0.310347 & -2.030034 & -0.528884 & 0.276030 & -2.035056 & -0.494044 \\
\hline 1 & -0.476972 & -2.752313 & -0.275113 & -0.533212 & -2.712989 & -0.224978 \\
\hline 1 & 1.063156 & -2.187044 & 1.454618 & 1.011409 & -2.163001 & 1.481169 \\
\hline 1 & 3.136622 & -1.124797 & 1.257418 & 3.080094 & -1.114626 & 1.251744 \\
\hline 1 & 3.181037 & -1.658982 & -0.434833 & 3.108739 & -1.673616 & -0.412313 \\
\hline 1 & 1.023274 & -0.798500 & -2.197180 & 1.029088 & -0.893712 & -2.180877 \\
\hline 1 & -0.614652 & -1.609907 & -2.460327 & -0.626088 & -1.634942 & -2.417800 \\
\hline 6 & -2.417674 & 0.694895 & -0.799751 & -2.386257 & 0.682265 & -0.787464 \\
\hline 6 & -1.394845 & 0.136115 & 1.882050 & -1.362068 & 0.186116 & 1.851850 \\
\hline 6 & -2.054232 & -0.877802 & 1.097852 & -2.000798 & -0.849400 & 1.101339 \\
\hline 6 & -2.534355 & -0.610162 & -0.208161 & -2.486637 & -0.612162 & -0.192264 \\
\hline 1 & -2.803823 & -1.468533 & -0.839060 & -2.732636 & -1.475554 & -0.809968 \\
\hline 1 & -2.003618 & -1.924495 & 1.425403 & -1.916220 & -1.881152 & 1.439169 \\
\hline 1 & -0.923438 & -0.166336 & 2.824456 & -0.891777 & -0.079752 & 2.794947 \\
\hline 1 & -1.806846 & 1.159881 & 1.898306 & -1.800728 & 1.189736 & 1.843994 \\
\hline 1 & -2.644358 & 1.583301 & -0.191958 & -2.637859 & 1.558393 & -0.186238 \\
\hline 1 & -2.671907 & 0.809454 & -1.860152 & -2.650011 & 0.786922 & -1.836962 \\
\hline 6 & 2.581103 & 0.428970 & -0.136903 & 2.510855 & 0.396780 & -0.150737 \\
\hline 6 & 0.093166 & 2.221483 & -0.995467 & 0.129076 & 2.218043 & -0.998569 \\
\hline 6 & 0.729451 & 2.212948 & 0.274896 & 0.741807 & 2.210474 & 0.269026 \\
\hline 6 & 1.609057 & 1.212135 & 0.739827 & 1.591863 & 1.204199 & 0.735047 \\
\hline 1 & 1.851289 & 1.214725 & 1.811441 & 1.838923 & 1.196679 & 1.795627 \\
\hline 1 & 0.258896 & 2.836265 & 1.052761 & 0.268347 & 2.827294 & 1.036775 \\
\hline 1 & -0.639986 & 2.997847 & -1.231805 & -0.594372 & 2.985406 & -1.248907 \\
\hline 1 & 0.585341 & 1.775986 & -1.875618 & 0.641545 & 1.780684 & -1.861051 \\
\hline 1 & 2.316010 & 0.524149 & -1.206862 & 2.200597 & 0.470142 & -1.202014 \\
\hline 1 & 3.599124 & 0.867964 & -0.055128 & 3.525633 & 0.823377 & -0.138565 \\
\hline 22 & -0.349037 & 0.165185 & -0.061640 & -0.335720 & 0.175772 & -0.082538 \\
\hline
\end{tabular}


Table S2. Optimized coordinates for the $\left(\mathrm{C}_{4} \mathbf{H}_{6}\right)_{3} \mathbf{T i}$ structure $\mathbf{T i - 2 S}$.

\begin{tabular}{|c|c|c|c|c|c|c|}
\hline & \multicolumn{3}{|c|}{ B3LYP } & \multicolumn{3}{|c|}{ B3LYP* } \\
\hline & $\mathrm{x}$ & $\mathrm{y}$ & $\mathrm{Z}$ & $\mathrm{x}$ & $\mathrm{y}$ & $\mathrm{Z}$ \\
\hline 6 & -0.718425 & 1.491536 & 1.631129 & -0.660804 & 1.520936 & 1.629872 \\
\hline 6 & 0.000643 & 2.139993 & -1.059552 & 0.042767 & 2.130730 & -1.071278 \\
\hline 6 & -1.336937 & 1.794487 & -0.730400 & -1.299779 & 1.815099 & -0.728361 \\
\hline 6 & -1.701879 & 1.493823 & 0.602627 & -1.656595 & 1.532022 & 0.612740 \\
\hline 1 & -2.704981 & 1.114707 & 0.792036 & -2.667043 & 1.174844 & 0.817154 \\
\hline 1 & -2.060373 & 1.601627 & -1.523172 & -2.036914 & 1.634595 & -1.514087 \\
\hline 1 & 0.255092 & 2.223565 & -2.115459 & 0.292624 & 2.199845 & -2.131277 \\
\hline 1 & 0.577491 & 2.787382 & -0.400762 & 0.641750 & 2.767829 & -0.419179 \\
\hline 1 & 0.036443 & 2.275409 & 1.661827 & 0.111601 & 2.290716 & 1.644070 \\
\hline 1 & -1.005730 & 1.105718 & 2.608948 & -0.941182 & 1.144829 & 2.615611 \\
\hline 6 & -0.353971 & -2.048933 & 1.184004 & -0.409669 & -2.030689 & 1.194417 \\
\hline 6 & -0.843423 & -1.499001 & -1.702844 & -0.871338 & -1.485176 & -1.698317 \\
\hline 6 & -1.813269 & -1.305102 & -0.705752 & -1.843135 & -1.260440 & -0.706734 \\
\hline 6 & -1.576957 & -1.574149 & 0.675566 & -1.620626 & -1.529149 & 0.678149 \\
\hline 1 & -2.334595 & -1.245129 & 1.384761 & -2.374058 & -1.177128 & 1.383918 \\
\hline 1 & -2.734746 & -0.782834 & -0.956839 & -2.751120 & -0.714526 & -0.965618 \\
\hline 1 & -1.052593 & -1.156343 & -2.713999 & -1.064878 & -1.140736 & -2.714035 \\
\hline 1 & -0.130890 & -2.314009 & -1.632265 & -0.181315 & -2.321361 & -1.619849 \\
\hline 1 & 0.285944 & -2.681256 & 0.572502 & 0.218336 & -2.681684 & 0.586419 \\
\hline 1 & -0.244968 & -2.180752 & 2.256206 & -0.306973 & -2.161776 & 2.269292 \\
\hline 6 & 1.839274 & -0.557217 & 1.521128 & 1.818993 & -0.608126 & 1.513843 \\
\hline 6 & 1.936908 & -0.810494 & -1.359405 & 1.901234 & -0.844344 & -1.363832 \\
\hline 6 & 2.254382 & 0.383536 & -0.719817 & 2.256635 & 0.341377 & -0.720012 \\
\hline 6 & 2.153342 & 0.534349 & 0.702304 & 2.164179 & 0.484715 & 0.702979 \\
\hline 1 & 2.237826 & 1.533206 & 1.124498 & 2.278129 & 1.478971 & 1.134419 \\
\hline 1 & 2.478002 & 1.266402 & -1.313123 & 2.503605 & 1.222348 & -1.310722 \\
\hline 1 & 1.935710 & -0.861881 & -2.444739 & 1.894740 & -0.891819 & -2.451276 \\
\hline 1 & 1.993795 & -1.762680 & -0.839537 & 1.949428 & -1.801896 & -0.848136 \\
\hline 1 & 2.163766 & -1.557534 & 1.250752 & 2.128253 & -1.614479 & 1.238434 \\
\hline 1 & 1.671342 & -0.391400 & 2.582347 & 1.656606 & -0.445008 & 2.578376 \\
\hline \multirow[t]{3}{*}{22} & -0.018394 & 0.034947 & -0.023996 & -0.012853 & 0.028850 & -0.025196 \\
\hline & \multicolumn{3}{|c|}{ BP86 } & \multicolumn{3}{|c|}{ M06L } \\
\hline & $\mathrm{x}$ & $\mathrm{y}$ & $\mathrm{Z}$ & $\mathrm{x}$ & $\mathrm{y}$ & $\mathrm{Z}$ \\
\hline 6 & -0.364268 & 1.642567 & 1.623151 & -0.615347 & 1.501241 & 1.591515 \\
\hline 6 & 0.313860 & 2.080745 & -1.105221 & 0.227231 & 2.083241 & -1.027127 \\
\hline 6 & -1.051472 & 1.932106 & -0.727943 & -1.148566 & 1.849599 & -0.765382 \\
\hline 6 & -1.394324 & 1.731078 & 0.641794 & -1.575637 & 1.586879 & 0.552492 \\
\hline 1 & -2.439006 & 1.511261 & 0.896282 & -2.605670 & 1.273222 & 0.714404 \\
\hline 1 & -1.831702 & 1.832869 & -1.494363 & -1.851318 & 1.703535 & -1.585634 \\
\hline 1 & 0.556682 & 2.086183 & -2.175526 & 0.535994 & 2.149932 & -2.069048 \\
\hline 1 & 1.006606 & 2.644072 & -0.468047 & 0.810202 & 2.704606 & -0.346301 \\
\hline 1 & 0.506784 & 2.306025 & 1.568755 & 0.199459 & 2.224153 & 1.632731 \\
\hline 1 & -0.638472 & 1.323833 & 2.637359 & -0.943975 & 1.138405 & 2.564542 \\
\hline 6 & -0.754100 & -1.914707 & 1.229140 & -0.528450 & -1.951409 & 1.182023 \\
\hline 6 & -1.024184 & -1.373360 & -1.686495 & -0.959560 & -1.384739 & -1.663234 \\
\hline 6 & -1.999056 & -0.979715 & -0.737873 & -1.932015 & -1.110068 & -0.685182 \\
\hline 6 & -1.871715 & -1.254097 & 0.663186 & -1.719895 & -1.395875 & 0.688813 \\
\hline 1 & -2.597099 & -0.784754 & 1.339709 & -2.435427 & -0.993668 & 1.403348 \\
\hline 1 & -2.811153 & -0.307845 & -1.044169 & -2.793427 & -0.494719 & -0.938096 \\
\hline 1 & -1.124026 & -1.029600 & -2.722505 & -1.125141 & -1.040666 & -2.681139 \\
\hline 1 & -0.483205 & -2.317302 & -1.565387 & -0.352940 & -2.283140 & -1.582680 \\
\hline 1 & -0.211114 & -2.668456 & 0.645131 & 0.062072 & -2.618142 & 0.552021 \\
\hline 1 & -0.702853 & -2.045546 & 2.314343 & -0.408328 & -2.103945 & 2.248666 \\
\hline 6 & 1.682426 & -0.895790 & 1.487464 & 1.764661 & -0.605181 & 1.537085 \\
\hline 6 & 1.739447 & -1.025491 & -1.379874 & 1.845703 & -1.025228 & -1.281664 \\
\hline 6 & 2.277631 & 0.087973 & -0.699782 & 2.281522 & 0.168936 & -0.725750 \\
\hline 6 & 2.208131 & 0.181348 & 0.729256 & 2.182797 & 0.412086 & 0.678800 \\
\hline 1 & 2.485737 & 1.126436 & 1.211066 & 2.321544 & 1.427341 & 1.043671 \\
\hline 1 & 2.657993 & 0.947797 & -1.262825 & 2.580220 & 0.989629 & -1.371290 \\
\hline 1 & 1.726658 & -1.036954 & -2.474879 & 1.849564 & -1.171095 & -2.356477 \\
\hline 1 & 1.722243 & -2.012604 & -0.901024 & 1.784222 & -1.930008 & -0.679658 \\
\hline 1 & 1.876570 & -1.927505 & 1.170564 & 2.008024 & -1.640208 & 1.312170 \\
\hline 1 & 1.552547 & -0.761959 & 2.567074 & 1.581635 & -0.384682 & 2.584531 \\
\hline 22 & 0.007843 & -0.007359 & -0.021017 & -0.006880 & 0.012389 & -0.042731 \\
\hline
\end{tabular}


Table S3. Optimized coordinates for the $\left(\mathbf{C}_{4} \mathbf{H}_{6}\right)_{3} \mathbf{T i}$ structure Ti-1T.

\begin{tabular}{|c|c|c|c|c|c|c|}
\hline & \multicolumn{3}{|c|}{ B3LYP } & \multicolumn{3}{|c|}{ B3LYP* } \\
\hline & $\mathrm{x}$ & $\mathrm{y}$ & $\mathrm{Z}$ & $\mathrm{x}$ & $\mathrm{y}$ & $\mathrm{z}$ \\
\hline 6 & 0.357237 & -1.486921 & -1.705975 & 0.387657 & -1.467640 & -1.729686 \\
\hline 6 & 2.666816 & -0.849048 & 0.476265 & 2.656976 & -0.823011 & 0.508149 \\
\hline 6 & 1.357797 & -1.631805 & 0.608654 & 1.350957 & -1.617084 & 0.607415 \\
\hline 6 & 0.562910 & -2.102213 & -0.441896 & 0.579659 & -2.088894 & -0.465972 \\
\hline 1 & -0.172237 & -2.863668 & -0.164035 & -0.158417 & -2.855918 & -0.203384 \\
\hline 1 & 1.233186 & -2.154309 & 1.557590 & 1.211312 & -2.151406 & 1.549897 \\
\hline 1 & 3.127250 & -0.810760 & 1.472405 & 3.094334 & -0.783018 & 1.516538 \\
\hline 1 & 3.374375 & -1.390924 & -0.172702 & 3.385953 & -1.355187 & -0.128184 \\
\hline 1 & 1.171554 & -0.923289 & -2.168547 & 1.201591 & -0.890964 & -2.180609 \\
\hline 1 & -0.317255 & -1.962771 & -2.415323 & -0.279975 & -1.939758 & -2.450555 \\
\hline 6 & -2.665749 & 0.450334 & -0.904078 & -2.651609 & 0.431252 & -0.899068 \\
\hline 6 & -1.694406 & 0.308115 & 1.930336 & -1.695760 & 0.312674 & 1.932170 \\
\hline 6 & -2.076684 & -0.795945 & 1.183905 & -2.050852 & -0.806033 & 1.190092 \\
\hline 6 & -2.534990 & -0.726951 & -0.184392 & -2.510617 & -0.746993 & -0.179337 \\
\hline 1 & -2.675201 & -1.673411 & -0.705471 & -2.635942 & -1.696816 & -0.702118 \\
\hline 1 & -1.913082 & -1.788561 & 1.602208 & -1.866149 & -1.795186 & 1.612758 \\
\hline 1 & -1.255621 & 0.175432 & 2.915581 & -1.244858 & 0.195209 & 2.915964 \\
\hline 1 & -1.995251 & 1.319022 & 1.664435 & -2.024102 & 1.315887 & 1.663407 \\
\hline 1 & -2.717682 & 1.422903 & -0.423096 & -2.716216 & 1.404257 & -0.416073 \\
\hline 1 & -2.911320 & 0.420855 & -1.962117 & -2.888616 & 0.400800 & -1.960818 \\
\hline 6 & 2.517722 & 0.598329 & -0.034761 & 2.498613 & 0.625276 & -0.002180 \\
\hline 6 & 0.089469 & 2.162238 & -1.186590 & 0.083533 & 2.129826 & -1.223859 \\
\hline 6 & 0.569456 & 2.282105 & 0.136579 & 0.516040 & 2.275195 & 0.115163 \\
\hline 6 & 1.454876 & 1.371675 & 0.741171 & 1.403352 & 1.378617 & 0.751198 \\
\hline 1 & 1.638318 & 1.472367 & 1.811102 & 1.567211 & 1.492843 & 1.824925 \\
\hline 1 & 0.001752 & 2.929077 & 0.810191 & -0.082989 & 2.919155 & 0.766743 \\
\hline 1 & -0.694509 & 2.833248 & -1.527496 & -0.715132 & 2.771518 & -1.592594 \\
\hline 1 & 0.727371 & 1.771175 & -1.980312 & 0.760598 & 1.762627 & -1.998147 \\
\hline 1 & 2.292889 & 0.594664 & -1.109045 & 2.295453 & 0.619418 & -1.082992 \\
\hline 1 & 3.485594 & 1.120686 & 0.043392 & 3.457007 & 1.164992 & 0.095684 \\
\hline \multirow[t]{3}{*}{22} & -0.274190 & 0.092253 & -0.135034 & -0.262216 & 0.081929 & -0.129771 \\
\hline & \multicolumn{3}{|c|}{ BP86 } & \multicolumn{3}{|c|}{ M06L } \\
\hline & $\mathrm{x}$ & $\mathrm{y}$ & $\mathrm{Z}$ & $\mathrm{x}$ & $\mathrm{y}$ & $\mathrm{Z}$ \\
\hline 6 & -0.525089 & -1.579368 & 1.560331 & 0.502210 & -1.377006 & -1.786291 \\
\hline 6 & -2.619992 & -0.780524 & -0.807695 & 2.586307 & -0.765122 & 0.610621 \\
\hline 6 & -1.252643 & -1.478177 & -0.874556 & 1.302047 & -1.579065 & 0.603893 \\
\hline 6 & -0.568840 & -2.071181 & 0.222410 & 0.622569 & -2.033796 & -0.536411 \\
\hline 1 & 0.214647 & -2.797463 & -0.047698 & -0.140540 & -2.792630 & -0.334429 \\
\hline 1 & -1.015472 & -1.923748 & -1.851522 & 1.113121 & -2.152359 & 1.511669 \\
\hline 1 & -2.941312 & -0.584638 & -1.848754 & 2.943805 & -0.715607 & 1.646065 \\
\hline 1 & -3.384635 & -1.454082 & -0.364786 & 3.375594 & -1.275855 & 0.037819 \\
\hline 1 & -1.396367 & -1.072277 & 1.997812 & 1.347355 & -0.812241 & -2.187604 \\
\hline 1 & 0.117738 & -2.088676 & 2.287203 & -0.127217 & -1.829154 & -2.549213 \\
\hline 6 & 2.616042 & -0.396467 & 1.003544 & -2.624105 & 0.385345 & -0.848267 \\
\hline 6 & 1.883075 & 1.255268 & -1.356113 & -1.635190 & 0.311092 & 1.921788 \\
\hline 6 & 2.051260 & -0.139186 & -1.427776 & -1.959728 & -0.829445 & 1.209165 \\
\hline 6 & 2.450668 & -0.930908 & -0.280507 & -2.450719 & -0.792220 & -0.141755 \\
\hline 1 & 2.505845 & -2.019226 & -0.411208 & -2.549532 & -1.741028 & -0.666870 \\
\hline 1 & 1.776074 & -0.672493 & -2.347133 & -1.714805 & -1.803377 & 1.629419 \\
\hline 1 & 1.475494 & 1.799599 & -2.214411 & -1.148405 & 0.234508 & 2.888511 \\
\hline 1 & 2.384213 & 1.859010 & -0.589526 & -2.014146 & 1.292213 & 1.645151 \\
\hline 1 & 2.722381 & 0.679691 & 1.185787 & -2.701577 & 1.350901 & -0.353550 \\
\hline 1 & 2.806325 & -1.053275 & 1.857572 & -2.872549 & 0.364585 & -1.903907 \\
\hline 6 & -2.592761 & 0.568202 & -0.052600 & 2.419478 & 0.666201 & 0.093138 \\
\hline 6 & -0.175537 & 1.743285 & 1.625917 & 0.092924 & 2.037968 & -1.290338 \\
\hline 6 & -0.562626 & 2.166081 & 0.324840 & 0.400552 & 2.238311 & 0.074658 \\
\hline 6 & -1.420967 & 1.421787 & -0.535018 & 1.277889 & 1.385981 & 0.783343 \\
\hline 1 & -1.495680 & 1.761472 & -1.578766 & 1.374508 & 1.513638 & 1.860794 \\
\hline 1 & 0.043921 & 2.952645 & -0.146827 & -0.282814 & 2.848933 & 0.668378 \\
\hline 1 & 0.595395 & 2.316782 & 2.155119 & -0.712396 & 2.617896 & -1.733055 \\
\hline 1 & -0.871543 & 1.202616 & 2.280133 & 0.871481 & 1.749583 & -1.997185 \\
\hline 1 & -2.529490 & 0.400616 & 1.038415 & 2.254893 & 0.650544 & -0.993062 \\
\hline 1 & -3.550398 & 1.110614 & -0.209000 & 3.358238 & 1.228131 & 0.210364 \\
\hline 22 & 0.311242 & 0.041362 & 0.108678 & -0.253655 & 0.062812 & -0.160935 \\
\hline
\end{tabular}


Table S4. Optimized coordinates for the $\left(\mathbf{C}_{4} \mathbf{H}_{6}\right)_{3} \mathbf{V}$ structure V-1D.

\begin{tabular}{|c|c|c|c|c|c|c|}
\hline & \multicolumn{3}{|c|}{$\overline{\text { B3LYP }}$} & \multicolumn{3}{|c|}{ B3LYP* } \\
\hline & $\mathrm{x}$ & $\mathrm{y}$ & $\mathrm{Z}$ & $\mathrm{x}$ & $\mathrm{y}$ & $\mathrm{Z}$ \\
\hline 6 & -0.830041 & -1.292386 & 1.533106 & -0.811508 & -1.285262 & 1.536215 \\
\hline 6 & -2.999361 & 0.604313 & 0.086347 & -3.000750 & 0.586202 & 0.089206 \\
\hline 6 & -2.433324 & -0.750530 & -0.342855 & -2.415247 & -0.759650 & -0.344693 \\
\hline 6 & -1.711497 & -1.642532 & 0.453399 & -1.686983 & -1.648480 & 0.454737 \\
\hline 1 & -1.612151 & -2.661431 & 0.059347 & -1.576462 & -2.668797 & 0.061521 \\
\hline 1 & -2.879026 & -1.184017 & -1.239497 & -2.853316 & -1.196967 & -1.245722 \\
\hline 1 & -3.699901 & 0.935916 & -0.690945 & -3.709580 & 0.910610 & -0.686313 \\
\hline 1 & -3.584584 & 0.508735 & 1.013318 & -3.582085 & 0.481321 & 1.019903 \\
\hline 1 & -1.066892 & -0.429409 & 2.159728 & -1.056495 & -0.419108 & 2.158783 \\
\hline 1 & -0.354971 & -2.109069 & 2.075207 & -0.324680 & -2.094798 & 2.082328 \\
\hline 6 & 1.750031 & -1.342807 & -1.408816 & 1.754837 & -1.344127 & -1.407227 \\
\hline 6 & 2.633663 & 1.092570 & 0.569472 & 2.620772 & 1.100132 & 0.570872 \\
\hline 6 & 2.225344 & -0.337794 & 0.870995 & 2.201777 & -0.327498 & 0.873260 \\
\hline 6 & 2.089544 & -1.399780 & -0.009680 & 2.084024 & -1.397827 & -0.006391 \\
\hline 1 & 3.183604 & 1.135357 & -0.381604 & 3.174955 & 1.137827 & -0.380320 \\
\hline 1 & 3.317633 & 1.447988 & 1.353834 & 3.304974 & 1.455002 & 1.358196 \\
\hline 6 & -1.883231 & 1.658433 & 0.240927 & -1.893871 & 1.651571 & 0.239155 \\
\hline 6 & 1.396003 & 2.009508 & 0.464339 & 1.385688 & 2.021015 & 0.457753 \\
\hline 6 & 0.563877 & 1.491071 & -0.692446 & 0.555032 & 1.492288 & -0.695845 \\
\hline 6 & -0.841223 & 1.377861 & -0.830648 & -0.852569 & 1.371328 & -0.833978 \\
\hline 1 & -1.237150 & 1.396997 & -1.850187 & -1.250492 & 1.388983 & -1.854939 \\
\hline 1 & 1.110205 & 1.526192 & -1.641052 & 1.101873 & 1.524954 & -1.647029 \\
\hline 1 & 1.701455 & 3.057215 & 0.299334 & 1.694113 & 3.068863 & 0.285379 \\
\hline 1 & 0.837127 & 1.987228 & 1.409392 & 0.823901 & 2.006647 & 1.403580 \\
\hline 1 & -1.435475 & 1.591246 & 1.240377 & -1.442157 & 1.589988 & 1.239536 \\
\hline 1 & -2.285232 & 2.681787 & 0.158090 & -2.303366 & 2.674071 & 0.154452 \\
\hline 1 & 2.201909 & -0.599093 & 1.930234 & 2.166683 & -0.585366 & 1.935045 \\
\hline 1 & 2.135989 & -0.526115 & -2.021158 & 2.131857 & -0.520684 & -2.019423 \\
\hline 1 & 1.697742 & -2.297820 & -1.934502 & 1.700133 & -2.299270 & -1.935765 \\
\hline 1 & 1.951752 & -2.378075 & 0.462547 & 1.944730 & -2.377183 & 0.467866 \\
\hline \multirow[t]{3}{*}{23} & 0.011272 & -0.560487 & -0.348143 & 0.017748 & -0.558011 & -0.347629 \\
\hline & \multicolumn{3}{|c|}{ BP86 } & \multicolumn{3}{|c|}{ M06L } \\
\hline & $\mathrm{x}$ & $\mathrm{y}$ & $\mathrm{Z}$ & $\mathrm{X}$ & $\mathrm{y}$ & $\mathrm{Z}$ \\
\hline 6 & -0.769775 & -1.285988 & 1.540741 & -0.774424 & -1.247991 & 1.535531 \\
\hline 6 & -3.002060 & 0.544015 & 0.104991 & -2.966091 & 0.543982 & 0.091994 \\
\hline 6 & -2.357360 & -0.768680 & -0.362189 & -2.349526 & -0.770362 & -0.357194 \\
\hline 6 & -1.627271 & -1.670101 & 0.444104 & -1.627382 & -1.647504 & 0.457564 \\
\hline 1 & -1.486360 & -2.689098 & 0.037888 & -1.469767 & -2.656490 & 0.057729 \\
\hline 1 & -2.769454 & -1.200971 & -1.286061 & -2.772234 & -1.213700 & -1.259095 \\
\hline 1 & -3.744129 & 0.850948 & -0.655562 & -3.684261 & 0.864025 & -0.671709 \\
\hline 1 & -3.561601 & 0.400833 & 1.051173 & -3.542129 & 0.415558 & 1.018926 \\
\hline 1 & -1.042082 & -0.418016 & 2.161960 & -1.057812 & -0.385550 & 2.144942 \\
\hline 1 & -0.260848 & -2.085152 & 2.094640 & -0.266539 & -2.030529 & 2.095774 \\
\hline 6 & 1.753223 & -1.361530 & -1.398781 & 1.753038 & -1.308138 & -1.399955 \\
\hline 6 & 2.594510 & 1.120732 & 0.565110 & 2.565378 & 1.095910 & 0.576557 \\
\hline 6 & 2.133826 & -0.296291 & 0.877024 & 2.139032 & -0.316662 & 0.877506 \\
\hline 6 & 2.067278 & -1.400329 & 0.008613 & 2.058994 & -1.384898 & -0.002951 \\
\hline 1 & 3.155463 & 1.137238 & -0.390600 & 3.113644 & 1.126586 & -0.376414 \\
\hline 1 & 3.287855 & 1.470843 & 1.356294 & 3.256058 & 1.448333 & 1.353721 \\
\hline 6 & -1.920960 & 1.642675 & 0.240931 & -1.879972 & 1.610683 & 0.241128 \\
\hline 6 & 1.369733 & 2.059595 & 0.435711 & 1.338570 & 2.005821 & 0.458226 \\
\hline 6 & 0.533922 & 1.497903 & -0.702165 & 0.540820 & 1.482095 & -0.704443 \\
\hline 6 & -0.883056 & 1.360605 & -0.839968 & -0.864992 & 1.348071 & -0.844954 \\
\hline 1 & -1.286753 & 1.373557 & -1.865847 & -1.271721 & 1.371317 & -1.859320 \\
\hline 1 & 1.080412 & 1.509241 & -1.663461 & 1.096271 & 1.514694 & -1.648561 \\
\hline 1 & 1.687946 & 3.106688 & 0.234120 & 1.632691 & 3.059255 & 0.319340 \\
\hline 1 & 0.805736 & 2.073584 & 1.388127 & 0.755119 & 1.964412 & 1.389556 \\
\hline 1 & -1.455147 & 1.595554 & 1.243870 & -1.404086 & 1.530899 & 1.228685 \\
\hline 1 & -2.349021 & 2.664627 & 0.151621 & -2.290569 & 2.631887 & 0.195800 \\
\hline 1 & 2.073359 & -0.539314 & 1.947893 & 2.070430 & -0.570086 & 1.935877 \\
\hline 1 & 2.114336 & -0.527719 & -2.019193 & 2.128576 & -0.460652 & -1.977277 \\
\hline 1 & 1.688889 & -2.322097 & -1.928613 & 1.719903 & -2.243730 & -1.956378 \\
\hline 1 & 1.927307 & -2.380055 & 0.496587 & 1.892759 & -2.362141 & 0.461457 \\
\hline 23 & 0.034001 & -0.551144 & -0.340851 & 0.021435 & -0.542180 & -0.349005 \\
\hline
\end{tabular}


Table S5. Optimized coordinates for the $\left(\mathbf{C}_{4} \mathbf{H}_{6}\right)_{3} \mathbf{V}$ structure V-2D.

\begin{tabular}{|c|c|c|c|c|c|c|}
\hline & \multicolumn{3}{|c|}{ B3LYP } & \multicolumn{3}{|c|}{ B3LYP* } \\
\hline & $\mathrm{x}$ & $\mathrm{y}$ & $\mathrm{Z}$ & $\mathrm{x}$ & $\mathrm{y}$ & $\mathrm{Z}$ \\
\hline 6 & -0.765588 & 1.460239 & 1.628592 & -0.733180 & 1.471022 & 1.630455 \\
\hline 6 & -0.142200 & 2.091290 & -1.121724 & -0.136669 & 2.079907 & -1.128890 \\
\hline 6 & -1.425501 & 1.659804 & -0.740762 & -1.421839 & 1.660339 & -0.732890 \\
\hline 6 & -1.743121 & 1.368115 & 0.614007 & -1.725281 & 1.377549 & 0.627947 \\
\hline 1 & -2.713820 & 0.923268 & 0.828092 & -2.697289 & 0.937312 & 0.856473 \\
\hline 1 & -2.151463 & 1.392159 & -1.508242 & -2.160581 & 1.396823 & -1.492463 \\
\hline 1 & 0.081933 & 2.164779 & -2.184442 & 0.078210 & 2.146484 & -2.196008 \\
\hline 1 & 0.437418 & 2.749099 & -0.478019 & 0.453164 & 2.740161 & -0.493350 \\
\hline 1 & -0.034733 & 2.265775 & 1.618955 & -0.000412 & 2.277301 & 1.608304 \\
\hline 1 & -1.014566 & 1.082558 & 2.619540 & -0.970202 & 1.096849 & 2.627856 \\
\hline 6 & -0.148118 & -2.075686 & 1.139233 & -0.158477 & -2.070381 & 1.138753 \\
\hline 6 & -0.798028 & -1.500442 & -1.677880 & -0.799166 & -1.493943 & -1.673610 \\
\hline 6 & -1.729585 & -1.352253 & -0.643887 & -1.736316 & -1.335219 & -0.642537 \\
\hline 6 & -1.412074 & -1.636261 & 0.720740 & -1.422551 & -1.620858 & 0.723299 \\
\hline 1 & -2.145015 & -1.364344 & 1.478689 & -2.154464 & -1.344478 & 1.483594 \\
\hline 1 & -2.688269 & -0.879045 & -0.847452 & -2.692830 & -0.854586 & -0.850098 \\
\hline 1 & -1.050369 & -1.137718 & -2.672377 & -1.043785 & -1.128339 & -2.671174 \\
\hline 1 & -0.055109 & -2.288850 & -1.646701 & -0.068771 & -2.296891 & -1.640539 \\
\hline 1 & 0.482032 & -2.670740 & 0.483200 & 0.464261 & -2.673689 & 0.479621 \\
\hline 1 & 0.028803 & -2.211754 & 2.202143 & 0.019989 & -2.210286 & 2.202937 \\
\hline 6 & 1.818525 & -0.362237 & 1.512876 & 1.808499 & -0.389049 & 1.502179 \\
\hline 6 & 1.929901 & -0.782152 & -1.321766 & 1.902848 & -0.782644 & -1.328499 \\
\hline 6 & 2.169795 & 0.471098 & -0.770011 & 2.162890 & 0.468460 & -0.771134 \\
\hline 6 & 2.060964 & 0.707934 & 0.638047 & 2.061873 & 0.693164 & 0.638855 \\
\hline 1 & 2.096278 & 1.731968 & 1.001976 & 2.110824 & 1.714428 & 1.014987 \\
\hline 1 & 2.325280 & 1.323289 & -1.426485 & 2.326692 & 1.324677 & -1.423772 \\
\hline 1 & 1.910999 & -0.896296 & -2.402143 & 1.877313 & -0.891175 & -2.411250 \\
\hline 1 & 2.064805 & -1.693502 & -0.748329 & 2.046137 & -1.699099 & -0.761023 \\
\hline 1 & 2.244425 & -1.338880 & 1.303072 & 2.239724 & -1.364189 & 1.283656 \\
\hline 1 & 1.629448 & -0.153885 & 2.563040 & 1.623966 & -0.189625 & 2.556917 \\
\hline \multirow[t]{3}{*}{23} & -0.014692 & 0.030671 & -0.002144 & -0.011640 & 0.029054 & -0.003401 \\
\hline & \multicolumn{3}{|c|}{ BP86 } & \multicolumn{3}{|c|}{ M06L } \\
\hline & $\mathrm{x}$ & $\mathrm{y}$ & $\mathrm{Z}$ & $\mathrm{X}$ & $\mathrm{y}$ & $\mathrm{Z}$ \\
\hline 6 & -0.646000 & 1.491077 & 1.642600 & -0.617550 & 1.478191 & 1.602772 \\
\hline 6 & -0.147603 & 2.047884 & -1.146642 & 0.056357 & 2.029619 & -1.110741 \\
\hline 6 & -1.433493 & 1.649513 & -0.700875 & -1.267915 & 1.725247 & -0.747865 \\
\hline 6 & -1.685943 & 1.386022 & 0.679701 & -1.611027 & 1.473518 & 0.602546 \\
\hline 1 & -2.656135 & 0.949721 & 0.954026 & -2.610707 & 1.098623 & 0.818088 \\
\hline 1 & -2.210526 & 1.393343 & -1.433935 & -2.006513 & 1.510196 & -1.519651 \\
\hline 1 & 0.031583 & 2.101800 & -2.227926 & 0.298283 & 2.092143 & -2.169589 \\
\hline 1 & 0.463819 & 2.725463 & -0.537162 & 0.670703 & 2.652479 & -0.461106 \\
\hline 1 & 0.078815 & 2.312176 & 1.582540 & 0.168843 & 2.232143 & 1.580493 \\
\hline 1 & -0.842522 & 1.126096 & 2.659324 & -0.885386 & 1.130666 & 2.599506 \\
\hline 6 & -0.151308 & -2.061071 & 1.123277 & -0.312200 & -2.037950 & 1.096364 \\
\hline 6 & -0.794926 & -1.475069 & -1.661591 & -0.921237 & -1.367047 & -1.642661 \\
\hline 6 & -1.748015 & -1.299882 & -0.632461 & -1.850660 & -1.150472 & -0.617916 \\
\hline 6 & -1.429353 & -1.592331 & 0.735439 & -1.543645 & -1.483625 & 0.731174 \\
\hline 1 & -2.155393 & -1.319012 & 1.512104 & -2.223054 & -1.145550 & 1.512013 \\
\hline 1 & -2.707001 & -0.810017 & -0.843348 & -2.754258 & -0.573980 & -0.806240 \\
\hline 1 & -1.027897 & -1.100038 & -2.665855 & -1.124540 & -0.974630 & -2.636554 \\
\hline 1 & -0.099843 & -2.319321 & -1.632444 & -0.281946 & -2.243841 & -1.616751 \\
\hline 1 & 0.440595 & -2.689141 & 0.446122 & 0.238694 & -2.673724 & 0.405195 \\
\hline 1 & 0.039727 & -2.222586 & 2.189208 & -0.104685 & -2.209613 & 2.147180 \\
\hline 6 & 1.789767 & -0.460688 & 1.462728 & 1.752472 & -0.442562 & 1.502367 \\
\hline 6 & 1.822974 & -0.753769 & -1.362317 & 1.827650 & -0.969329 & -1.262944 \\
\hline 6 & 2.135797 & 0.489593 & -0.779660 & 2.184047 & 0.282262 & -0.781259 \\
\hline 6 & 2.061913 & 0.666716 & 0.639987 & 2.095681 & 0.582259 & 0.610404 \\
\hline 1 & 2.147340 & 1.675903 & 1.059458 & 2.186493 & 1.615851 & 0.937062 \\
\hline 1 & 2.315849 & 1.363997 & -1.415022 & 2.399546 & 1.088361 & -1.476373 \\
\hline 1 & 1.779791 & -0.835099 & -2.453460 & 1.794638 & -1.146514 & -2.332874 \\
\hline 1 & 2.008191 & -1.690067 & -0.824380 & 1.886191 & -1.854184 & -0.635173 \\
\hline 1 & 2.251159 & -1.423456 & 1.210604 & 2.111132 & -1.451815 & 1.314241 \\
\hline 1 & 1.626547 & -0.296074 & 2.533793 & 1.562815 & -0.205571 & 2.545456 \\
\hline 23 & -0.005520 & 0.022971 & -0.004989 & -0.003395 & 0.014708 & -0.004277 \\
\hline
\end{tabular}


Table S6. Optimized coordinates for the $\left(\mathbf{C}_{4} \mathbf{H}_{6}\right)_{3} \mathbf{V}$ structure V-3D.

\begin{tabular}{|c|c|c|c|c|c|c|}
\hline & \multicolumn{3}{|c|}{$\overline{B 3 L Y P}$} & \multicolumn{3}{|c|}{ B3LYP* } \\
\hline & $\mathrm{x}$ & $\mathrm{y}$ & $\mathrm{Z}$ & $\mathrm{x}$ & $\mathrm{y}$ & $\mathrm{Z}$ \\
\hline 6 & -1.513229 & -1.430585 & -0.874307 & -1.497593 & -1.488758 & -0.776909 \\
\hline 6 & -2.670018 & 1.153724 & 0.861799 & -2.675523 & 1.080498 & 0.916334 \\
\hline 6 & -3.394549 & 0.074841 & 0.080982 & -3.384208 & 0.095691 & 0.006559 \\
\hline 6 & -2.929936 & -0.985920 & -0.612137 & -2.901400 & -0.955986 & -0.693733 \\
\hline 1 & -3.711383 & -1.619340 & -1.049733 & -3.651841 & -1.492191 & -1.290102 \\
\hline 1 & -4.482062 & 0.185823 & 0.123040 & -4.461194 & 0.279728 & -0.071489 \\
\hline 1 & -3.045800 & 2.134099 & 0.529449 & -3.077246 & 2.086131 & 0.709610 \\
\hline 1 & -2.975619 & 1.066433 & 1.916601 & -2.955172 & 0.860254 & 1.961165 \\
\hline 1 & -1.419963 & -2.504823 & -0.607919 & -1.377613 & -2.351236 & -0.077577 \\
\hline 1 & -1.323749 & -1.406346 & -1.970339 & -1.315052 & -1.909153 & -1.785206 \\
\hline 6 & 1.259094 & -1.172498 & 1.675633 & 1.311027 & -1.209653 & 1.635116 \\
\hline 6 & 3.063287 & 0.690768 & -0.247534 & 3.040031 & 0.718041 & -0.239356 \\
\hline 6 & 2.497376 & -0.716831 & -0.485466 & 2.486671 & -0.685912 & -0.538851 \\
\hline 6 & 1.984472 & -1.582379 & 0.499305 & 2.000152 & -1.594810 & 0.428119 \\
\hline 1 & 1.886028 & -2.634435 & 0.210300 & 1.897381 & -2.640030 & 0.112011 \\
\hline 1 & 2.850235 & -1.208847 & -1.392916 & 2.831476 & -1.134697 & -1.473621 \\
\hline 1 & 3.634305 & 0.973693 & -1.141531 & 3.644465 & 1.031075 & -1.102965 \\
\hline 1 & 3.775956 & 0.692009 & 0.591614 & 3.720936 & 0.693991 & 0.627415 \\
\hline 1 & 1.587102 & -0.285006 & 2.222441 & 1.661656 & -0.329102 & 2.183225 \\
\hline 1 & 0.879142 & -1.961561 & 2.324180 & 0.938701 & -2.005028 & 2.282842 \\
\hline 6 & -1.138263 & 1.172398 & 0.793530 & -1.144493 & 1.141327 & 0.820291 \\
\hline 6 & 1.947997 & 1.734552 & -0.019469 & 1.913121 & 1.753249 & -0.014506 \\
\hline 6 & 0.777245 & 1.395085 & -0.936535 & 0.742011 & 1.413247 & -0.934257 \\
\hline 6 & -0.588026 & 1.343261 & -0.580770 & -0.620918 & 1.358285 & -0.554021 \\
\hline 1 & -1.321761 & 1.333406 & -1.386135 & -1.368588 & 1.324086 & -1.348769 \\
\hline 1 & 0.985717 & 1.507978 & -2.001762 & 0.936763 & 1.548036 & -2.001140 \\
\hline 1 & 2.318450 & 2.755439 & -0.210125 & 2.278397 & 2.778670 & -0.204429 \\
\hline 1 & 1.628716 & 1.723048 & 1.031032 & 1.587154 & 1.740050 & 1.035012 \\
\hline 1 & -0.791237 & 0.168826 & 1.282012 & -0.765439 & 0.119811 & 1.290779 \\
\hline 1 & -0.726073 & 1.881943 & 1.525438 & -0.733483 & 1.830270 & 1.575597 \\
\hline \multirow[t]{3}{*}{23} & 0.194752 & -0.559166 & -0.127210 & 0.199801 & -0.529652 & -0.119612 \\
\hline & \multicolumn{3}{|c|}{ BP86 } & \multicolumn{3}{|c|}{ M06L } \\
\hline & $\mathrm{x}$ & $\mathrm{y}$ & $\underline{Z}$ & $\mathrm{X}$ & $\mathrm{y}$ & $\mathrm{Z}$ \\
\hline 6 & -1.513354 & -1.479473 & -0.743629 & -1.554752 & -1.507665 & -0.661444 \\
\hline 6 & -2.658611 & 0.984085 & 0.995445 & -2.641318 & 0.989063 & 0.983283 \\
\hline 6 & -3.371893 & 0.155752 & -0.061685 & -3.352711 & 0.188473 & -0.073631 \\
\hline 6 & -2.880111 & -0.882465 & -0.797645 & -2.879892 & -0.868130 & -0.769845 \\
\hline 1 & -3.574073 & -1.308375 & -1.542587 & -3.555892 & -1.270430 & -1.529007 \\
\hline 1 & -4.419782 & 0.442470 & -0.238073 & -4.370563 & 0.512140 & -0.289598 \\
\hline 1 & -3.084457 & 2.006977 & 0.977900 & -3.047010 & 2.008708 & 0.975793 \\
\hline 1 & -2.891355 & 0.584203 & 2.005724 & -2.876790 & 0.594894 & 1.983503 \\
\hline 1 & -1.376645 & -2.108100 & 0.182778 & -1.437320 & -2.042783 & 0.310595 \\
\hline 1 & -1.319794 & -2.148050 & -1.608669 & -1.395631 & -2.260082 & -1.446969 \\
\hline 6 & 1.295775 & -1.231835 & 1.619762 & 1.326982 & -1.181279 & 1.597116 \\
\hline 6 & 3.039382 & 0.718877 & -0.267878 & 3.005105 & 0.752536 & -0.267935 \\
\hline 6 & 2.436136 & -0.666728 & -0.571659 & 2.441359 & -0.630512 & -0.579888 \\
\hline 6 & 1.980903 & -1.605404 & 0.397553 & 2.009168 & -1.562062 & 0.387905 \\
\hline 1 & 1.875400 & -2.651478 & 0.063339 & 1.911443 & -2.602094 & 0.063490 \\
\hline 1 & 2.741193 & -1.108148 & -1.532043 & 2.763453 & -1.064353 & -1.527134 \\
\hline 1 & 3.628077 & 1.032147 & -1.150596 & 3.575259 & 1.092979 & -1.140198 \\
\hline 1 & 3.749677 & 0.666991 & 0.582250 & 3.718106 & 0.711690 & 0.567619 \\
\hline 1 & 1.654931 & -0.359640 & 2.190038 & 1.685380 & -0.304151 & 2.144479 \\
\hline 1 & 0.924758 & -2.043257 & 2.260070 & 0.988276 & -1.981138 & 2.253401 \\
\hline 6 & -1.121921 & 1.100863 & 0.859192 & -1.117587 & 1.085034 & 0.845750 \\
\hline 6 & 1.926057 & 1.764493 & -0.004163 & 1.872500 & 1.744122 & 0.008962 \\
\hline 6 & 0.741494 & 1.418597 & -0.908793 & 0.725106 & 1.392263 & -0.914115 \\
\hline 6 & -0.631270 & 1.367855 & -0.516498 & -0.634243 & 1.314087 & -0.533030 \\
\hline 1 & -1.398171 & 1.351997 & -1.303128 & -1.398064 & 1.284625 & -1.309490 \\
\hline 1 & 0.925315 & 1.560160 & -1.984576 & 0.922071 & 1.540121 & -1.976529 \\
\hline 1 & 2.288909 & 2.800355 & -0.181962 & 2.200217 & 2.788661 & -0.115079 \\
\hline 1 & 1.617865 & 1.732530 & 1.059534 & 1.547168 & 1.662698 & 1.056977 \\
\hline 1 & -0.676905 & 0.072655 & 1.326048 & -0.683384 & 0.102447 & 1.317090 \\
\hline 1 & -0.712531 & 1.779087 & 1.636007 & -0.707313 & 1.789548 & 1.584941 \\
\hline 23 & 0.199655 & -0.529140 & -0.119220 & 0.215751 & -0.559090 & -0.133159 \\
\hline
\end{tabular}


Table S7. Optimized coordinates for the $\left(\mathbf{C}_{4} \mathbf{H}_{6}\right)_{3} \mathbf{V}$ structure V-4D.

\begin{tabular}{|c|c|c|c|c|c|c|}
\hline & \multicolumn{3}{|c|}{$\overline{\text { B3LYP }}$} & \multicolumn{3}{|c|}{ B3LYP* } \\
\hline & $\mathrm{x}$ & $\mathrm{y}$ & $\mathrm{Z}$ & $\mathrm{x}$ & $\mathrm{y}$ & $\mathrm{Z}$ \\
\hline 6 & 0.252778 & -1.793968 & 0.755732 & 0.184905 & -1.876344 & 0.702561 \\
\hline 6 & -2.547151 & -0.446549 & 1.077328 & -2.544237 & -0.409195 & 1.124690 \\
\hline 6 & -2.070606 & -1.392783 & -0.015219 & -2.128212 & -1.326987 & -0.016176 \\
\hline 6 & -0.930018 & -2.208140 & 0.051736 & -1.026695 & -2.198646 & -0.002193 \\
\hline 1 & -0.843012 & -3.029627 & -0.667260 & -0.989901 & -2.988970 & -0.763024 \\
\hline 1 & -2.831697 & -1.668546 & -0.749842 & -2.910056 & -1.533370 & -0.754941 \\
\hline 1 & -3.442165 & -0.846456 & 1.580847 & -3.463314 & -0.777506 & 1.612905 \\
\hline 1 & -1.777894 & -0.348683 & 1.852515 & -1.765738 & -0.399779 & 1.899905 \\
\hline 1 & 0.177301 & -1.280005 & 1.714777 & 0.152296 & -1.396735 & 1.683180 \\
\hline 1 & 1.152528 & -2.398956 & 0.658933 & 1.047108 & -2.532056 & 0.574226 \\
\hline 6 & 3.852927 & -0.641200 & 0.645963 & 3.875328 & -0.636311 & 0.697061 \\
\hline 6 & 1.728603 & 1.599241 & 0.550459 & 1.810411 & 1.638090 & 0.394378 \\
\hline 6 & 1.820804 & 0.447985 & -0.471512 & 1.784795 & 0.328844 & -0.410370 \\
\hline 6 & 2.985760 & -0.458736 & -0.376476 & 2.926609 & -0.593878 & -0.269868 \\
\hline 1 & 3.150066 & -1.083292 & -1.263650 & 2.992450 & -1.369344 & -1.045381 \\
\hline 1 & 1.816575 & 0.862176 & -1.507933 & 1.665930 & 0.544604 & -1.517941 \\
\hline 1 & 2.375538 & 2.442441 & 0.261317 & 2.406000 & 2.413048 & -0.118773 \\
\hline 1 & 2.097349 & 1.259031 & 1.525217 & 2.299563 & 1.474731 & 1.363928 \\
\hline 1 & 3.800400 & -0.065463 & 1.567969 & 3.918423 & 0.090416 & 1.508090 \\
\hline 1 & 4.648461 & -1.380424 & 0.575631 & 4.636236 & -1.416644 & 0.696564 \\
\hline 6 & -2.813371 & 0.920847 & 0.424069 & -2.721778 & 1.006882 & 0.539665 \\
\hline 6 & 0.253307 & 2.072028 & 0.700450 & 0.358426 & 2.132554 & 0.613562 \\
\hline 6 & -0.501340 & 1.697886 & -0.558170 & -0.455790 & 1.721416 & -0.593094 \\
\hline 6 & -1.794520 & 1.157674 & -0.697374 & -1.758640 & 1.182624 & -0.640841 \\
\hline 1 & -2.230193 & 1.249874 & -1.696429 & -2.246914 & 1.232404 & -1.621335 \\
\hline 1 & -0.079250 & 2.130863 & -1.474806 & -0.077381 & 2.121835 & -1.546959 \\
\hline 1 & 0.197363 & 3.156348 & 0.888367 & 0.325280 & 3.224812 & 0.774345 \\
\hline 1 & -0.209979 & 1.586906 & 1.570105 & -0.058461 & 1.668375 & 1.520001 \\
\hline 1 & -2.795400 & 1.732370 & 1.165476 & -2.568524 & 1.781267 & 1.306479 \\
\hline 1 & -3.817501 & 0.927428 & -0.020744 & -3.749774 & 1.134356 & 0.168746 \\
\hline \multirow[t]{3}{*}{23} & -0.122240 & -0.390074 & -0.804453 & -0.149737 & -0.395032 & -0.807663 \\
\hline & \multicolumn{3}{|c|}{ BP86 } & \multicolumn{3}{|c|}{ M06L } \\
\hline & $\mathrm{x}$ & $\mathrm{y}$ & $\mathrm{Z}$ & $\mathrm{X}$ & $\mathrm{y}$ & $\mathrm{Z}$ \\
\hline 6 & 0.163386 & -1.893945 & 0.676067 & 0.313124 & -1.838657 & 0.657742 \\
\hline 6 & -2.563246 & -0.405291 & 1.144743 & -2.376969 & -0.406534 & 1.180141 \\
\hline 6 & -2.151381 & -1.289584 & -0.027533 & -2.025763 & -1.352629 & 0.058403 \\
\hline 6 & -1.058874 & -2.185776 & -0.037400 & -0.918839 & -2.211432 & 0.033591 \\
\hline 1 & -1.030749 & -2.953756 & -0.831019 & -0.904244 & -3.003860 & -0.720644 \\
\hline 1 & -2.934140 & -1.459378 & -0.784485 & -2.846640 & -1.579159 & -0.625985 \\
\hline 1 & -3.506387 & -0.767138 & 1.606021 & -3.179443 & -0.805162 & 1.819417 \\
\hline 1 & -1.792447 & -0.447811 & 1.936468 & -1.506963 & -0.263579 & 1.835244 \\
\hline 1 & 0.138306 & -1.429336 & 1.671668 & 0.322140 & -1.342583 & 1.629973 \\
\hline 1 & 1.019021 & -2.567476 & 0.537922 & 1.192013 & -2.458040 & 0.488681 \\
\hline 6 & 3.869801 & -0.663212 & 0.728152 & 3.805939 & -0.555136 & 0.700101 \\
\hline 6 & 1.854514 & 1.650011 & 0.323441 & 1.719450 & 1.657231 & 0.409582 \\
\hline 6 & 1.771762 & 0.286833 & -0.382579 & 1.754245 & 0.390726 & -0.435947 \\
\hline 6 & 2.895067 & -0.654063 & -0.226787 & 2.902836 & -0.500147 & -0.302165 \\
\hline 1 & 2.922744 & -1.478585 & -0.961395 & 3.009650 & -1.246855 & -1.097230 \\
\hline 1 & 1.587671 & 0.412531 & -1.513770 & 1.611159 & 0.624087 & -1.530988 \\
\hline 1 & 2.420375 & 2.382699 & -0.290086 & 2.280870 & 2.475726 & -0.066989 \\
\hline 1 & 2.410060 & 1.557360 & 1.274198 & 2.220444 & 1.477501 & 1.367830 \\
\hline 1 & 3.945814 & 0.112577 & 1.499499 & 3.794104 & 0.146043 & 1.532283 \\
\hline 1 & 4.613134 & -1.468304 & 0.761469 & 4.580979 & -1.316005 & 0.715100 \\
\hline 6 & -2.682480 & 1.038362 & 0.597360 & -2.742412 & 0.915550 & 0.512269 \\
\hline 6 & 0.413156 & 2.163532 & 0.578718 & 0.258147 & 2.070831 & 0.638915 \\
\hline 6 & -0.427108 & 1.723995 & -0.602564 & -0.515021 & 1.684336 & -0.587077 \\
\hline 6 & -1.740241 & 1.169134 & -0.610180 & -1.804030 & 1.115855 & -0.668845 \\
\hline 1 & -2.253859 & 1.209598 & -1.587190 & -2.287670 & 1.195994 & -1.647147 \\
\hline 1 & -0.073259 & 2.115553 & -1.578559 & -0.120839 & 2.098579 & -1.525840 \\
\hline 1 & 0.393263 & 3.265757 & 0.720557 & 0.166858 & 3.143481 & 0.871935 \\
\hline 1 & 0.018124 & 1.713862 & 1.510468 & -0.148051 & 1.534050 & 1.509449 \\
\hline 1 & -2.455010 & 1.790121 & 1.376892 & -2.703861 & 1.759377 & 1.214490 \\
\hline 1 & -3.718210 & 1.238892 & 0.262151 & -3.773575 & 0.869274 & 0.141797 \\
\hline 23 & -0.163938 & -0.385528 & -0.807801 & -0.170920 & -0.396906 & -0.830071 \\
\hline
\end{tabular}


Table S8. Optimized coordinates for the $\left(\mathrm{C}_{4} \mathrm{H}_{6}\right)_{3} \mathbf{V}$ structure $\mathbf{V - 1 Q}$.

\begin{tabular}{|c|c|c|c|c|c|c|}
\hline & \multicolumn{3}{|c|}{ B3LYP } & \multicolumn{3}{|c|}{ B3LYP* } \\
\hline & $\mathrm{x}$ & $\mathrm{y}$ & $\mathrm{Z}$ & $\mathrm{x}$ & $\mathrm{y}$ & $\mathrm{Z}$ \\
\hline 6 & 1.605593 & -1.607594 & -1.190594 & 1.564845 & -1.602520 & -1.198936 \\
\hline 6 & 2.563430 & 1.207434 & -0.206864 & 2.572766 & 1.188879 & -0.211280 \\
\hline 6 & 2.430582 & -0.082851 & 0.610741 & 2.426060 & -0.101332 & 0.605145 \\
\hline 6 & 2.237009 & -1.357054 & 0.051928 & 2.207045 & -1.372648 & 0.044510 \\
\hline 1 & 2.348947 & -2.205439 & 0.732176 & 2.305123 & -2.227105 & 0.722378 \\
\hline 1 & 2.815736 & -0.046915 & 1.628757 & 2.813451 & -0.073188 & 1.624723 \\
\hline 1 & 3.153621 & 1.929879 & 0.371288 & 3.167479 & 1.909026 & 0.368760 \\
\hline 1 & 3.111543 & 1.029665 & -1.143440 & 3.121226 & 1.007328 & -1.149274 \\
\hline 1 & 1.720285 & -0.894121 & -2.011813 & 1.697088 & -0.889630 & -2.020479 \\
\hline 1 & 1.449205 & -2.637572 & -1.504982 & 1.379373 & -2.628937 & -1.516275 \\
\hline 6 & -1.236961 & -1.725409 & 1.380169 & -1.223467 & -1.699185 & 1.391242 \\
\hline 6 & -2.816813 & 0.367918 & -0.451395 & -2.811945 & 0.369267 & -0.458700 \\
\hline 6 & -1.951575 & -0.826549 & -0.855306 & -1.946437 & -0.828345 & -0.853994 \\
\hline 6 & -1.574213 & -1.872789 & 0.009883 & -1.562272 & -1.865876 & 0.021165 \\
\hline 1 & -3.258007 & 0.192993 & 0.540764 & -3.260986 & 0.195620 & 0.532403 \\
\hline 1 & -3.658722 & 0.459403 & -1.153995 & -3.649656 & 0.463498 & -1.169128 \\
\hline 6 & 1.189573 & 1.852702 & -0.536547 & 1.199141 & 1.835873 & -0.537708 \\
\hline 6 & -2.051956 & 1.712995 & -0.419151 & -2.040307 & 1.711254 & -0.419235 \\
\hline 6 & -1.064118 & 1.745612 & 0.725715 & -1.054271 & 1.731614 & 0.728300 \\
\hline 6 & 0.298503 & 1.844571 & 0.694214 & 0.314139 & 1.828418 & 0.697990 \\
\hline 1 & 0.818842 & 1.973703 & 1.643773 & 0.837130 & 1.963832 & 1.647362 \\
\hline 1 & -1.524442 & 1.750102 & 1.717262 & -1.516531 & 1.737421 & 1.721066 \\
\hline 1 & -2.764706 & 2.542678 & -0.277738 & -2.751183 & 2.545509 & -0.277474 \\
\hline 1 & -1.554203 & 1.879825 & -1.382666 & -1.538240 & 1.879034 & -1.382724 \\
\hline 1 & 0.701805 & 1.296897 & -1.363465 & 0.706656 & 1.271443 & -1.360736 \\
\hline 1 & 1.308848 & 2.870534 & -0.938429 & 1.312758 & 2.851966 & -0.951541 \\
\hline 1 & -1.985934 & -1.077792 & -1.917052 & -1.975967 & -1.087224 & -1.916072 \\
\hline 1 & -1.738487 & -0.970263 & 1.989003 & -1.734725 & -0.943247 & 1.994235 \\
\hline 1 & -0.901520 & -2.605029 & 1.927292 & -0.876684 & -2.570977 & 1.947789 \\
\hline 1 & -1.240803 & -2.796346 & -0.469869 & -1.222022 & -2.793386 & -0.450448 \\
\hline \multirow[t]{3}{*}{23} & 0.148855 & -0.445483 & 0.118973 & 0.144084 & -0.425364 & 0.121063 \\
\hline & \multicolumn{3}{|c|}{ BP86 } & \multicolumn{3}{|c|}{ M06L } \\
\hline & $\mathrm{x}$ & $\mathrm{y}$ & $\mathrm{Z}$ & $\mathrm{X}$ & $\mathrm{y}$ & $\mathrm{Z}$ \\
\hline 6 & 1.440246 & -1.579235 & -1.245190 & 1.509907 & -1.570487 & -1.194667 \\
\hline 6 & 2.606715 & 1.117057 & -0.207825 & 2.556624 & 1.135858 & -0.220362 \\
\hline 6 & 2.395160 & -0.178347 & 0.594206 & 2.385417 & -0.125271 & 0.616234 \\
\hline 6 & 2.107515 & -1.435424 & 0.008670 & 2.142833 & -1.387330 & 0.056722 \\
\hline 1 & 2.159517 & -2.315896 & 0.668196 & 2.189427 & -2.242626 & 0.733891 \\
\hline 1 & 2.783078 & -0.186519 & 1.621284 & 2.766729 & -0.093732 & 1.635015 \\
\hline 1 & 3.222871 & 1.815259 & 0.389023 & 3.162608 & 1.862982 & 0.332419 \\
\hline 1 & 3.160756 & 0.924059 & -1.148076 & 3.095828 & 0.924100 & -1.154076 \\
\hline 1 & 1.636952 & -0.857532 & -2.055324 & 1.711050 & -0.854210 & -1.997788 \\
\hline 1 & 1.174509 & -2.585133 & -1.592586 & 1.299109 & -2.580265 & -1.539090 \\
\hline 6 & -1.188188 & -1.601627 & 1.444163 & -1.200955 & -1.622296 & 1.402945 \\
\hline 6 & -2.791427 & 0.379007 & -0.488409 & -2.768128 & 0.357643 & -0.461214 \\
\hline 6 & -1.933826 & -0.840795 & -0.843336 & -1.918172 & -0.837777 & -0.852590 \\
\hline 6 & -1.540742 & -1.845504 & 0.079017 & -1.530471 & -1.848279 & 0.043417 \\
\hline 1 & -3.269784 & 0.223975 & 0.499630 & -3.202183 & 0.189745 & 0.535802 \\
\hline 1 & -3.613059 & 0.478779 & -1.226725 & -3.614805 & 0.453793 & -1.154359 \\
\hline 6 & 1.247047 & 1.799468 & -0.530605 & 1.192569 & 1.767460 & -0.542978 \\
\hline 6 & -1.992126 & 1.710981 & -0.440344 & -1.988479 & 1.681559 & -0.428412 \\
\hline 6 & -1.021600 & 1.707624 & 0.725280 & -1.029466 & 1.707068 & 0.727323 \\
\hline 6 & 0.366293 & 1.800297 & 0.712032 & 0.337696 & 1.809941 & 0.699647 \\
\hline 1 & 0.886752 & 1.953179 & 1.667440 & 0.870492 & 1.947560 & 1.639268 \\
\hline 1 & -1.499076 & 1.724220 & 1.717846 & -1.499818 & 1.716302 & 1.713095 \\
\hline 1 & -2.695342 & 2.563851 & -0.313819 & -2.685699 & 2.527399 & -0.323549 \\
\hline 1 & -1.469928 & 1.866940 & -1.403079 & -1.463904 & 1.826698 & -1.382293 \\
\hline 1 & 0.734004 & 1.231219 & -1.354091 & 0.680845 & 1.153408 & -1.324872 \\
\hline 1 & 1.369970 & 2.814359 & -0.963790 & 1.277182 & 2.746665 & -1.034583 \\
\hline 1 & -1.958961 & -1.141403 & -1.901087 & -1.950573 & -1.112678 & -1.907380 \\
\hline 1 & -1.721981 & -0.832080 & 2.022225 & -1.752768 & -0.862844 & 1.964043 \\
\hline 1 & -0.814260 & -2.445053 & 2.039326 & -0.860728 & -2.466465 & 1.999287 \\
\hline 1 & -1.195522 & -2.797024 & -0.354977 & -1.162927 & -2.775261 & -0.402223 \\
\hline 23 & 0.127787 & -0.375487 & 0.123593 & 0.130604 & -0.381265 & 0.112653 \\
\hline
\end{tabular}


Table S9. Optimized coordinates for the $\left(\mathbf{C}_{4} \mathbf{H}_{6}\right)_{3} \mathbf{V}$ structure $\mathbf{V - 2 Q}$.

\begin{tabular}{|c|c|c|c|c|c|c|}
\hline & \multicolumn{3}{|c|}{ B3LYP } & \multicolumn{3}{|c|}{ B3LYP* } \\
\hline & $\mathrm{x}$ & $\mathrm{y}$ & Z & $\mathrm{x}$ & $\mathrm{y}$ & Z \\
\hline 6 & -0.751211 & -1.281522 & 1.575663 & -0.722614 & -1.226992 & 1.576416 \\
\hline 6 & -2.992559 & 0.061133 & -0.219175 & -3.000290 & 0.055586 & -0.240347 \\
\hline 6 & -2.100029 & -1.147014 & -0.528454 & -2.097036 & -1.152003 & -0.518845 \\
\hline 6 & -1.384885 & -1.861702 & 0.451242 & -1.368687 & -1.839503 & 0.472346 \\
\hline 1 & -1.075652 & -2.876573 & 0.187159 & -1.054006 & -2.859820 & 0.228254 \\
\hline 1 & -2.418224 & -1.731618 & -1.393430 & -2.409171 & -1.759805 & -1.372724 \\
\hline 1 & -3.551266 & 0.305954 & -1.133516 & -3.538937 & 0.291848 & -1.171210 \\
\hline 1 & -3.746644 & -0.201674 & 0.540541 & -3.773000 & -0.203197 & 0.505031 \\
\hline 1 & -1.188517 & -0.413420 & 2.067151 & -1.160998 & -0.350668 & 2.056828 \\
\hline 1 & -0.171981 & -1.928809 & 2.231612 & -0.131726 & -1.857916 & 2.241357 \\
\hline 6 & 1.959178 & -1.988966 & 0.011211 & 1.935089 & -1.989791 & -0.002365 \\
\hline 6 & 2.132225 & 1.126017 & 0.892203 & 2.155571 & 1.122448 & 0.893116 \\
\hline 6 & 2.366125 & 0.461671 & -0.462160 & 2.362370 & 0.460194 & -0.467776 \\
\hline 6 & 2.472903 & -0.910242 & -0.741692 & 2.452433 & -0.913028 & -0.757759 \\
\hline 1 & 2.754487 & -1.138518 & -1.775154 & 2.720590 & -1.139304 & -1.797353 \\
\hline 1 & 2.720088 & 1.128362 & -1.248805 & 2.712619 & 1.127886 & -1.258005 \\
\hline 1 & 3.043287 & 1.641184 & 1.233843 & 3.065172 & 1.665558 & 1.199690 \\
\hline 1 & 1.908174 & 0.367736 & 1.651726 & 1.979170 & 0.359476 & 1.662751 \\
\hline 1 & 1.928673 & -1.944125 & 1.097988 & 1.915774 & -1.949064 & 1.086736 \\
\hline 1 & 2.036034 & -2.989296 & -0.410637 & 1.992944 & -2.990827 & -0.430629 \\
\hline 6 & -2.249706 & 1.339964 & 0.229869 & -2.265414 & 1.337319 & 0.212610 \\
\hline 6 & 0.936647 & 2.144722 & 0.820171 & 0.927478 & 2.106665 & 0.855232 \\
\hline 6 & 0.177926 & 1.973410 & -0.486085 & 0.184730 & 1.953447 & -0.462960 \\
\hline 6 & -1.120210 & 1.626997 & -0.726726 & -1.116141 & 1.612885 & -0.725022 \\
\hline 1 & -1.429645 & 1.646438 & -1.776203 & -1.412062 & 1.648793 & -1.780258 \\
\hline 1 & 0.750672 & 2.263892 & -1.366993 & 0.765919 & 2.265167 & -1.333489 \\
\hline 1 & 1.315580 & 3.176581 & 0.860383 & 1.270119 & 3.150667 & 0.943143 \\
\hline 1 & 0.275370 & 2.018835 & 1.684181 & 0.263516 & 1.918888 & 1.707996 \\
\hline 1 & -1.887222 & 1.257055 & 1.258209 & -1.925170 & 1.263985 & 1.251318 \\
\hline 1 & -2.951684 & 2.190146 & 0.215919 & -2.966396 & 2.190559 & 0.176761 \\
\hline \multirow[t]{3}{*}{23} & 0.217828 & -0.523433 & -0.383495 & 0.213914 & -0.506268 & -0.394293 \\
\hline & \multicolumn{3}{|c|}{ BP86 } & \multicolumn{3}{|c|}{ M06L } \\
\hline & $\mathrm{x}$ & $\mathrm{y}$ & $\mathrm{z}$ & $\mathrm{x}$ & $\mathrm{y}$ & $\mathrm{Z}$ \\
\hline 6 & -1.244964 & -1.742484 & 1.122214 & -0.631524 & -1.115628 & 1.606280 \\
\hline 6 & -2.912229 & 0.638490 & 0.067021 & -2.958559 & -0.033627 & -0.243904 \\
\hline 6 & -2.249951 & -0.477401 & -0.764726 & -2.021067 & -1.212597 & -0.464400 \\
\hline 6 & -1.807574 & -1.694919 & -0.179341 & -1.275575 & -1.820162 & 0.562783 \\
\hline 1 & -1.675037 & -2.571978 & -0.833708 & -0.915568 & -2.832839 & 0.365934 \\
\hline 1 & -2.549141 & -0.531978 & -1.821023 & -2.332232 & -1.885584 & -1.264957 \\
\hline 1 & -3.614897 & 1.190281 & -0.586059 & -3.484632 & 0.157901 & -1.188348 \\
\hline 1 & -3.516241 & 0.213928 & 0.893282 & -3.740271 & -0.289850 & 0.487905 \\
\hline 1 & -1.541193 & -0.994397 & 1.873093 & -1.113178 & -0.250352 & 2.062333 \\
\hline 1 & -0.898830 & -2.696113 & 1.531472 & -0.000467 & -1.682362 & 2.289516 \\
\hline 6 & 1.572016 & -1.968140 & 0.623967 & 1.906352 & -1.948848 & -0.023242 \\
\hline 6 & 2.698118 & 0.946985 & 0.414261 & 2.094701 & 1.116360 & 0.885638 \\
\hline 6 & 2.316601 & -0.011215 & -0.728834 & 2.331501 & 0.488614 & -0.473775 \\
\hline 6 & 2.097641 & -1.406154 & -0.563211 & 2.427437 & -0.874853 & -0.774605 \\
\hline 1 & 2.108024 & -2.032383 & -1.470004 & 2.689042 & -1.092011 & -1.814306 \\
\hline 1 & 2.611384 & 0.306623 & -1.738400 & 2.669591 & 1.170796 & -1.252733 \\
\hline 1 & 3.522172 & 1.602391 & 0.072569 & 2.984658 & 1.666154 & 1.224315 \\
\hline 1 & 3.086223 & 0.387626 & 1.286148 & 1.923577 & 0.330304 & 1.631876 \\
\hline 1 & 1.746213 & -1.489566 & 1.598577 & 1.908616 & -1.904558 & 1.065844 \\
\hline 1 & 1.351751 & -3.040555 & 0.658875 & 1.984378 & -2.949004 & -0.442471 \\
\hline 6 & -1.852131 & 1.630722 & 0.608452 & -2.270616 & 1.270052 & 0.172866 \\
\hline 6 & 1.468112 & 1.816700 & 0.818742 & 0.853142 & 2.061863 & 0.848945 \\
\hline 6 & 0.563483 & 1.877499 & -0.402052 & 0.148412 & 1.932307 & -0.479612 \\
\hline 6 & -0.816165 & 1.778970 & -0.488533 & -1.136951 & 1.563816 & -0.759541 \\
\hline 1 & -1.248560 & 1.981537 & -1.480714 & -1.422928 & 1.588930 & -1.815028 \\
\hline 1 & 1.079795 & 2.175394 & -1.325954 & 0.741038 & 2.253700 & -1.335012 \\
\hline 1 & 1.765008 & 2.837732 & 1.142675 & 1.157128 & 3.108178 & 0.992612 \\
\hline 1 & 0.936821 & 1.363557 & 1.678643 & 0.171516 & 1.824512 & 1.673973 \\
\hline 1 & -1.393823 & 1.251817 & 1.542033 & -1.924403 & 1.237893 & 1.210539 \\
\hline 1 & -2.298651 & 2.617683 & 0.861390 & -2.995136 & 2.097475 & 0.126136 \\
\hline 23 & 0.066575 & -0.474170 & -0.306550 & 0.212859 & -0.483177 & -0.398380 \\
\hline
\end{tabular}


Table S10. Optimized coordinates for the $\left(\mathbf{C}_{4} \mathbf{H}_{6}\right)_{3} \mathbf{V}$ structure V-3Q.

\begin{tabular}{|c|c|c|c|c|c|c|}
\hline & \multicolumn{3}{|c|}{ B3LYP } & \multicolumn{3}{|c|}{ B3LYP* } \\
\hline & $\mathrm{x}$ & $\mathrm{y}$ & $\mathrm{Z}$ & $\mathrm{x}$ & $\mathrm{y}$ & $\mathrm{Z}$ \\
\hline 6 & -1.561975 & -2.183261 & -0.259391 & -1.569071 & -2.163846 & -0.297549 \\
\hline 6 & -1.585450 & 0.482842 & 1.537155 & -1.563641 & 0.457482 & 1.566182 \\
\hline 6 & -2.409456 & 0.118965 & 0.306257 & -2.394513 & 0.130156 & 0.329712 \\
\hline 6 & -2.517118 & -1.146320 & -0.304318 & -2.517614 & -1.119418 & -0.315312 \\
\hline 1 & -3.265693 & -1.210198 & -1.101112 & -3.269793 & -1.156058 & -1.113148 \\
\hline 1 & -3.175562 & 0.848539 & 0.037212 & -3.154870 & 0.875455 & 0.079301 \\
\hline 1 & -2.207569 & 0.500125 & 2.446672 & -2.186504 & 0.476945 & 2.477633 \\
\hline 1 & -0.805512 & -0.267863 & 1.724382 & -0.798822 & -0.313954 & 1.741728 \\
\hline 1 & -0.974830 & -2.355423 & 0.639912 & -0.981200 & -2.363642 & 0.597389 \\
\hline 1 & -1.712498 & -3.055688 & -0.892174 & -1.720290 & -3.017032 & -0.959153 \\
\hline 6 & 2.302590 & -1.485729 & 1.740301 & 2.321894 & -1.482181 & 1.732301 \\
\hline 6 & 2.523838 & 0.241999 & -0.756716 & 2.499140 & 0.202422 & -0.794576 \\
\hline 6 & 1.534850 & -0.932432 & -0.637282 & 1.489822 & -0.949178 & -0.627161 \\
\hline 6 & 1.685510 & -1.776602 & 0.568585 & 1.654238 & -1.776483 & 0.587342 \\
\hline 1 & 1.223912 & -2.765552 & 0.496596 & 1.161171 & -2.754178 & 0.550130 \\
\hline 1 & 1.594138 & -1.586708 & -1.528375 & 1.499844 & -1.618087 & -1.512802 \\
\hline 1 & 2.700672 & 0.469493 & -1.819061 & 2.648526 & 0.412560 & -1.866514 \\
\hline 1 & 3.505357 & -0.012744 & -0.323924 & 3.489604 & -0.068381 & -0.387161 \\
\hline 1 & 2.814074 & -0.541129 & 1.916674 & 2.868712 & -0.548994 & 1.870961 \\
\hline 1 & 2.323792 & -2.209539 & 2.552573 & 2.348712 & -2.191096 & 2.559867 \\
\hline 6 & -0.909990 & 1.868670 & 1.322567 & -0.857736 & 1.831793 & 1.362680 \\
\hline 6 & 1.993468 & 1.527442 & -0.078775 & 2.011465 & 1.506498 & -0.117632 \\
\hline 6 & 0.699777 & 1.959076 & -0.723200 & 0.703949 & 1.949832 & -0.726467 \\
\hline 6 & -0.532776 & 2.083122 & -0.141708 & -0.518538 & 2.062891 & -0.109375 \\
\hline 1 & -1.324118 & 2.508278 & -0.763286 & -1.321969 & 2.512629 & -0.701427 \\
\hline 1 & 0.785729 & 2.268879 & -1.770577 & 0.765985 & 2.286096 & -1.769360 \\
\hline 1 & 2.729357 & 2.340684 & -0.181783 & 2.759248 & 2.306478 & -0.252968 \\
\hline 1 & 1.849857 & 1.350637 & 0.992573 & 1.898517 & 1.343216 & 0.961708 \\
\hline 1 & -0.036616 & 1.967589 & 1.976874 & 0.038234 & 1.893800 & 1.993715 \\
\hline 1 & -1.611122 & 2.666784 & 1.602721 & -1.529792 & 2.643641 & 1.680307 \\
\hline \multirow[t]{3}{*}{23} & -0.510999 & -0.237513 & -0.932467 & -0.524856 & -0.200835 & -0.934394 \\
\hline & \multicolumn{3}{|c|}{ BP86 } & \multicolumn{3}{|c|}{ M06L } \\
\hline & $\mathrm{x}$ & $\mathrm{y}$ & $\mathrm{Z}$ & $\mathrm{x}$ & $\mathrm{y}$ & $\mathrm{Z}$ \\
\hline 6 & -1.469995 & -2.181187 & -0.361030 & -1.947869 & -1.842688 & -0.445332 \\
\hline 6 & -1.568036 & 0.347378 & 1.614707 & -1.348321 & 0.481716 & 1.647069 \\
\hline 6 & -2.377439 & 0.056033 & 0.352136 & -2.287221 & 0.470696 & 0.460995 \\
\hline 6 & -2.462958 & -1.171375 & -0.360772 & -2.681638 & -0.652207 & -0.289327 \\
\hline 1 & -3.204468 & -1.194505 & -1.177610 & -3.453464 & -0.456356 & -1.039387 \\
\hline 1 & -3.156745 & 0.798476 & 0.122163 & -2.890167 & 1.370368 & 0.330705 \\
\hline 1 & -2.207117 & 0.335408 & 2.523330 & -1.895202 & 0.611862 & 2.592287 \\
\hline 1 & -0.794726 & -0.429061 & 1.773303 & -0.837089 & -0.487299 & 1.733146 \\
\hline 1 & -0.901063 & -2.403770 & 0.548598 & -1.376333 & -2.249038 & 0.388711 \\
\hline 1 & -1.556085 & -3.005302 & -1.079970 & -2.303318 & -2.579191 & -1.161233 \\
\hline 6 & 2.460211 & -1.450611 & 1.674951 & 2.136443 & -1.431191 & 1.763195 \\
\hline 6 & 2.438315 & 0.248204 & -0.849098 & 2.360369 & -0.288090 & -1.007504 \\
\hline 6 & 1.446601 & -0.910444 & -0.610238 & 1.197488 & -1.177079 & -0.579373 \\
\hline 6 & 1.691008 & -1.743548 & 0.584925 & 1.281943 & -1.749164 & 0.763756 \\
\hline 1 & 1.179818 & -2.719633 & 0.590977 & 0.543950 & -2.526328 & 0.986886 \\
\hline 1 & 1.353654 & -1.571556 & -1.509711 & 0.991049 & -1.977263 & -1.313213 \\
\hline 1 & 2.532292 & 0.446332 & -1.935625 & 2.375766 & -0.192718 & -2.103287 \\
\hline 1 & 3.455680 & -0.015610 & -0.487015 & 3.332978 & -0.726815 & -0.724698 \\
\hline 1 & 3.033721 & -0.518859 & 1.758889 & 2.942044 & -0.709784 & 1.637662 \\
\hline 1 & 2.540032 & -2.155479 & 2.510891 & 2.072439 & -1.922035 & 2.730262 \\
\hline 6 & -0.865852 & 1.734914 & 1.442774 & -0.278563 & 1.594995 & 1.481959 \\
\hline 6 & 1.963920 & 1.556347 & -0.160340 & 2.247414 & 1.119024 & -0.400862 \\
\hline 6 & 0.619495 & 1.972805 & -0.715938 & 0.961383 & 1.790811 & -0.783128 \\
\hline 6 & -0.597498 & 2.011598 & -0.040394 & -0.125708 & 1.998843 & 0.031290 \\
\hline 1 & -1.434018 & 2.480673 & -0.583471 & -0.917121 & 2.641009 & -0.361432 \\
\hline 1 & 0.632409 & 2.370641 & -1.745500 & 0.929759 & 2.246387 & -1.777433 \\
\hline 1 & 2.700869 & 2.368071 & -0.331362 & 3.089311 & 1.742263 & -0.728743 \\
\hline 1 & 1.898438 & 1.394291 & 0.930790 & 2.310836 & 1.047159 & 0.690083 \\
\hline 1 & 0.065501 & 1.769004 & 2.035328 & 0.675837 & 1.241207 & 1.890008 \\
\hline 1 & -1.521058 & 2.540510 & 1.827970 & -0.561625 & 2.483404 & 2.061051 \\
\hline 23 & -0.534077 & -0.143927 & -0.921830 & -0.614085 & -0.063080 & -0.942948 \\
\hline
\end{tabular}


Table S11. Optimized coordinates for the $\left(\mathrm{C}_{4} \mathrm{H}_{6}\right)_{3} \mathrm{~V}$ structure $\mathrm{V}-4 \mathrm{Q}$.

\begin{tabular}{|c|c|c|c|c|c|c|}
\hline & \multicolumn{3}{|c|}{$\overline{\text { B3LYP }}$} & \multicolumn{3}{|c|}{ B3LYP* } \\
\hline & $\mathrm{x}$ & $\mathrm{y}$ & $\mathrm{Z}$ & $\mathrm{x}$ & $\mathrm{y}$ & $\mathrm{Z}$ \\
\hline 6 & 1.480182 & -1.724537 & 1.205006 & 1.495187 & -1.722144 & 1.184741 \\
\hline 6 & 0.369443 & -1.605034 & -1.533111 & 0.417407 & -1.570852 & -1.567068 \\
\hline 6 & 1.705320 & -1.247870 & -1.233833 & 1.740982 & -1.191474 & -1.242910 \\
\hline 6 & 2.235448 & -1.310687 & 0.087796 & 2.255516 & -1.267429 & 0.086586 \\
\hline 1 & 3.213630 & -0.859621 & 0.255154 & 3.223373 & -0.798564 & 0.276481 \\
\hline 1 & 2.313552 & -0.756001 & -1.992694 & 2.349255 & -0.671149 & -1.985440 \\
\hline 1 & -0.003828 & -1.417934 & -2.538046 & 0.048774 & -1.371194 & -2.573238 \\
\hline 1 & -0.100199 & -2.443423 & -1.019339 & -0.049630 & -2.420554 & -1.066041 \\
\hline 1 & 0.712933 & -2.492316 & 1.104519 & 0.738344 & -2.498959 & 1.057992 \\
\hline 1 & 1.922339 & -1.638727 & 2.195137 & 1.918786 & -1.643382 & 2.185545 \\
\hline 6 & 1.190303 & 1.786609 & 1.366348 & 1.145593 & 1.767657 & 1.388271 \\
\hline 6 & -0.451213 & 1.808772 & -1.121867 & -0.498168 & 1.793845 & -1.090643 \\
\hline 6 & 0.947119 & 1.823253 & -1.117345 & 0.903141 & 1.843649 & -1.094303 \\
\hline 6 & 1.742109 & 1.806765 & 0.077281 & 1.700658 & 1.825579 & 0.098009 \\
\hline 1 & 2.814460 & 1.658857 & -0.044584 & 2.778009 & 1.702615 & -0.026368 \\
\hline 1 & 1.472281 & 1.685213 & -2.062131 & 1.428211 & 1.732365 & -2.045142 \\
\hline 1 & -0.985820 & 1.698160 & -2.061027 & -1.035821 & 1.682528 & -2.030308 \\
\hline 1 & -1.033100 & 2.200784 & -0.290093 & -1.085175 & 2.174258 & -0.254115 \\
\hline 1 & 0.233890 & 2.258992 & 1.579054 & 0.183067 & 2.228094 & 1.609661 \\
\hline 1 & 1.846916 & 1.651420 & 2.222638 & 1.805292 & 1.627659 & 2.244044 \\
\hline 6 & -1.477987 & -0.020689 & 1.683028 & -1.454484 & -0.096698 & 1.688831 \\
\hline 6 & -3.819504 & 0.289189 & -0.342145 & -3.800507 & 0.279894 & -0.329087 \\
\hline 6 & -2.856135 & -0.656467 & -0.357788 & -2.837027 & -0.669022 & -0.373065 \\
\hline 6 & -1.879829 & -0.911121 & 0.712142 & -1.854929 & -0.954358 & 0.681738 \\
\hline 1 & -1.550365 & -1.946979 & 0.804617 & -1.516517 & -1.992567 & 0.734363 \\
\hline 1 & -2.799937 & -1.331260 & -1.214084 & -2.787225 & -1.322324 & -1.249106 \\
\hline 1 & -4.507510 & 0.401854 & -1.177304 & -4.492818 & 0.415947 & -1.159617 \\
\hline 1 & -3.953244 & 0.960458 & 0.504339 & -3.929074 & 0.930538 & 0.536568 \\
\hline 1 & -1.872961 & 0.993078 & 1.710614 & -1.863034 & 0.911724 & 1.759237 \\
\hline 1 & -0.931362 & -0.358223 & 2.559649 & -0.909173 & -0.465976 & 2.555805 \\
\hline \multirow[t]{3}{*}{23} & 0.352034 & -0.021453 & 0.126544 & 0.344136 & -0.019694 & 0.123612 \\
\hline & \multicolumn{3}{|c|}{ BP86 } & \multicolumn{3}{|c|}{ M06L } \\
\hline & $\mathrm{x}$ & $\mathrm{y}$ & $\mathrm{Z}$ & $\mathrm{X}$ & $\mathrm{y}$ & $\mathrm{Z}$ \\
\hline 6 & 1.486398 & -1.742303 & 1.135932 & 1.461083 & -1.742569 & 1.088661 \\
\hline 6 & 0.513850 & -1.463194 & -1.650447 & 0.438948 & -1.444665 & -1.630139 \\
\hline 6 & 1.819370 & -1.067721 & -1.254099 & 1.748834 & -1.059963 & -1.277725 \\
\hline 6 & 2.283376 & -1.199487 & 0.096620 & 2.238981 & -1.203689 & 0.049038 \\
\hline 1 & 3.232993 & -0.711310 & 0.355877 & 3.181357 & -0.714820 & 0.294096 \\
\hline 1 & 2.444511 & -0.488344 & -1.947175 & 2.349009 & -0.473932 & -1.972785 \\
\hline 1 & 0.171804 & -1.215135 & -2.662192 & 0.070783 & -1.207741 & -2.625590 \\
\hline 1 & 0.042533 & -2.349187 & -1.204622 & -0.005539 & -2.332051 & -1.176692 \\
\hline 1 & 0.757903 & -2.538868 & 0.929604 & 0.733446 & -2.529006 & 0.881410 \\
\hline 1 & 1.859600 & -1.700581 & 2.165759 & 1.852429 & -1.728124 & 2.102250 \\
\hline 6 & 1.060962 & 1.704209 & 1.450934 & 1.129128 & 1.670937 & 1.420354 \\
\hline 6 & -0.559358 & 1.773636 & -1.033796 & -0.578660 & 1.806040 & -0.949203 \\
\hline 6 & 0.849947 & 1.877903 & -1.034712 & 0.816019 & 1.860454 & -1.026964 \\
\hline 6 & 1.640574 & 1.836970 & 0.165557 & 1.655097 & 1.792795 & 0.126079 \\
\hline 1 & 2.729115 & 1.748906 & 0.048391 & 2.723716 & 1.664476 & -0.039384 \\
\hline 1 & 1.384350 & 1.823226 & -1.993186 & 1.292531 & 1.775081 & -2.002705 \\
\hline 1 & -1.093480 & 1.672123 & -1.984280 & -1.170852 & 1.737067 & -1.856595 \\
\hline 1 & -1.164553 & 2.124116 & -0.187648 & -1.111309 & 2.162165 & -0.066290 \\
\hline 1 & 0.091238 & 2.162068 & 1.679720 & 0.178746 & 2.135618 & 1.678138 \\
\hline 1 & 1.715654 & 1.537382 & 2.314456 & 1.804316 & 1.501793 & 2.254957 \\
\hline 6 & -1.417840 & -0.212902 & 1.687095 & -1.417214 & -0.186142 & 1.710929 \\
\hline 6 & -3.777821 & 0.270707 & -0.318587 & -3.630379 & 0.305596 & -0.379380 \\
\hline 6 & -2.813144 & -0.689628 & -0.406682 & -2.739078 & -0.707911 & -0.384193 \\
\hline 6 & -1.822192 & -1.022564 & 0.623739 & -1.802239 & -1.023898 & 0.683376 \\
\hline 1 & -1.468415 & -2.064916 & 0.615045 & -1.446986 & -2.055960 & 0.710960 \\
\hline 1 & -2.780863 & -1.315478 & -1.311715 & -2.718510 & -1.380720 & -1.242899 \\
\hline 1 & -4.480425 & 0.442517 & -1.142098 & -4.294152 & 0.477341 & -1.221180 \\
\hline 1 & -3.895244 & 0.892909 & 0.577388 & -3.718054 & 0.986470 & 0.465650 \\
\hline 1 & -1.853091 & 0.785325 & 1.822528 & -1.842876 & 0.813081 & 1.801443 \\
\hline 1 & -0.882713 & -0.634220 & 2.544727 & -0.887518 & -0.568457 & 2.578472 \\
\hline 23 & 0.330624 & -0.024534 & 0.113483 & 0.308102 & -0.028878 & 0.123989 \\
\hline
\end{tabular}


Table S12. Optimized coordinates for the $\left(\mathbf{C}_{4} \mathbf{H}_{6}\right)_{3} \mathbf{V}$ structure V-5Q.

\begin{tabular}{|c|c|c|c|c|c|c|}
\hline & \multicolumn{3}{|c|}{ B3LYP } & \multicolumn{3}{|c|}{ B3LYP* } \\
\hline & $\mathrm{x}$ & $\mathrm{y}$ & $\mathrm{Z}$ & $\mathrm{x}$ & $\mathrm{y}$ & $\mathrm{Z}$ \\
\hline 6 & 1.199496 & -0.856445 & -1.573201 & 1.185985 & -0.865741 & -1.566784 \\
\hline 6 & 3.740221 & -0.161962 & 0.118168 & 3.738469 & -0.146009 & 0.101562 \\
\hline 6 & 2.708389 & -0.960652 & 0.487679 & 2.710123 & -0.946166 & 0.486542 \\
\hline 6 & 1.591033 & -1.395212 & -0.349712 & 1.588557 & -1.391231 & -0.338283 \\
\hline 1 & 1.115931 & -2.324881 & -0.032251 & 1.113469 & -2.318270 & -0.005908 \\
\hline 1 & 2.709514 & -1.359976 & 1.504556 & 2.719925 & -1.336627 & 1.509038 \\
\hline 1 & 4.528683 & 0.094313 & 0.822321 & 4.532785 & 0.119951 & 0.798389 \\
\hline 1 & 3.832856 & 0.229399 & -0.893440 & 3.822318 & 0.235581 & -0.916634 \\
\hline 1 & 1.746645 & -0.027944 & -2.017990 & 1.729917 & -0.039187 & -2.024057 \\
\hline 1 & 0.564394 & -1.428460 & -2.246262 & 0.549223 & -1.448651 & -2.232040 \\
\hline 6 & -2.350983 & -0.799175 & -1.113649 & -2.343121 & -0.780257 & -1.110984 \\
\hline 6 & -1.843776 & -0.424033 & 1.774939 & -1.843001 & -0.427097 & 1.774168 \\
\hline 6 & -1.504392 & -1.594809 & 1.084895 & -1.503403 & -1.598581 & 1.081583 \\
\hline 6 & -1.732712 & -1.775758 & -0.317088 & -1.728002 & -1.770483 & -0.322846 \\
\hline 1 & -1.265108 & -2.636998 & -0.795896 & -1.262559 & -2.631717 & -0.808417 \\
\hline 1 & -0.894463 & -2.340619 & 1.596242 & -0.895292 & -2.348724 & 1.593140 \\
\hline 1 & -1.542538 & -0.314388 & 2.812975 & -1.543071 & -0.318895 & 2.814743 \\
\hline 1 & -2.663206 & 0.211565 & 1.449081 & -2.662716 & 0.211005 & 1.446968 \\
\hline 1 & -3.119975 & -0.151506 & -0.705343 & -3.120470 & -0.142387 & -0.697026 \\
\hline 1 & -2.377135 & -0.932518 & -2.193291 & -2.367302 & -0.902329 & -2.194010 \\
\hline 6 & -1.519046 & 2.010843 & -0.742778 & -1.508532 & 2.005126 & -0.747703 \\
\hline 6 & 0.348486 & 1.622925 & 1.521263 & 0.333150 & 1.606502 & 1.525178 \\
\hline 6 & 0.776329 & 1.947714 & 0.225483 & 0.780934 & 1.933202 & 0.232819 \\
\hline 6 & -0.128809 & 2.148799 & -0.864452 & -0.115014 & 2.139755 & -0.864344 \\
\hline 1 & 0.299634 & 2.246594 & -1.862292 & 0.320004 & 2.236121 & -1.861702 \\
\hline 1 & 1.836512 & 1.864900 & -0.009400 & 1.845172 & 1.844392 & 0.008635 \\
\hline 1 & 1.091707 & 1.343238 & 2.264893 & 1.067220 & 1.319020 & 2.277864 \\
\hline 1 & -0.594679 & 1.990670 & 1.913657 & -0.609110 & 1.990743 & 1.910219 \\
\hline 1 & -2.029897 & 2.205926 & 0.196876 & -2.022918 & 2.207993 & 0.190873 \\
\hline 1 & -2.135373 & 2.071168 & -1.635315 & -2.122540 & 2.065846 & -1.644164 \\
\hline \multirow[t]{3}{*}{23} & -0.382996 & 0.029831 & -0.072974 & -0.385459 & 0.029666 & -0.069123 \\
\hline & \multicolumn{3}{|c|}{ BP86 } & \multicolumn{3}{|c|}{ M06L } \\
\hline & $\mathrm{X}$ & $\mathrm{y}$ & $\mathrm{z}$ & $\mathrm{x}$ & $\mathrm{y}$ & $\mathrm{Z}$ \\
\hline 6 & 1.157543 & -0.889012 & -1.551325 & 1.152103 & -0.930128 & -1.536689 \\
\hline 6 & 3.737285 & -0.135725 & 0.090486 & 3.625312 & -0.126343 & 0.143454 \\
\hline 6 & 2.703520 & -0.933582 & 0.500990 & 2.620517 & -0.942701 & 0.534555 \\
\hline 6 & 1.576940 & -1.394081 & -0.308610 & 1.535679 & -1.425887 & -0.298726 \\
\hline 1 & 1.093197 & -2.314777 & 0.051609 & 1.042907 & -2.332948 & 0.055084 \\
\hline 1 & 2.720826 & -1.307196 & 1.536714 & 2.619120 & -1.302386 & 1.564853 \\
\hline 1 & 4.540843 & 0.145643 & 0.780568 & 4.397552 & 0.191103 & 0.837482 \\
\hline 1 & 3.815347 & 0.224299 & -0.943056 & 3.707556 & 0.227218 & -0.883281 \\
\hline 1 & 1.704870 & -0.068538 & -2.031862 & 1.705699 & -0.116818 & -2.004925 \\
\hline 1 & 0.522633 & -1.496049 & -2.207824 & 0.517727 & -1.521193 & -2.192848 \\
\hline 6 & -2.312138 & -0.721258 & -1.127784 & -2.238512 & -0.852839 & -1.122933 \\
\hline 6 & -1.858988 & -0.402342 & 1.748264 & -1.900455 & -0.302675 & 1.696234 \\
\hline 6 & -1.515290 & -1.589511 & 1.065252 & -1.489726 & -1.515061 & 1.133180 \\
\hline 6 & -1.719290 & -1.749516 & -0.348408 & -1.653017 & -1.790919 & -0.256654 \\
\hline 1 & -1.259168 & -2.618007 & -0.841215 & -1.142455 & -2.662542 & -0.667617 \\
\hline 1 & -0.920490 & -2.349829 & 1.591525 & -0.867442 & -2.191122 & 1.720231 \\
\hline 1 & -1.575510 & -0.291499 & 2.799890 & -1.648498 & -0.080414 & 2.728230 \\
\hline 1 & -2.684770 & 0.232935 & 1.406808 & -2.740496 & 0.249334 & 1.282612 \\
\hline 1 & -3.119809 & -0.108683 & -0.713003 & -3.049873 & -0.217451 & -0.778663 \\
\hline 1 & -2.318561 & -0.821261 & -2.220061 & -2.219426 & -1.037927 & -2.194579 \\
\hline 6 & -1.468892 & 1.988195 & -0.778384 & -1.536565 & 2.018151 & -0.571782 \\
\hline 6 & 0.274188 & 1.568704 & 1.540141 & 0.522995 & 1.574722 & 1.387992 \\
\hline 6 & 0.796832 & 1.896359 & 0.261897 & 0.838980 & 1.895624 & 0.058760 \\
\hline 6 & -0.061993 & 2.113943 & -0.868391 & -0.181620 & 2.110186 & -0.913727 \\
\hline 1 & 0.402700 & 2.205085 & -1.859983 & 0.108283 & 2.165228 & -1.962334 \\
\hline 1 & 1.874880 & 1.786483 & 0.080481 & 1.866717 & 1.778710 & -0.286381 \\
\hline 1 & 0.972529 & 1.261528 & 2.328263 & 1.324185 & 1.279847 & 2.061921 \\
\hline 1 & -0.664289 & 2.010683 & 1.893056 & -0.366745 & 1.979809 & 1.862268 \\
\hline 1 & -2.003335 & 2.228739 & 0.148840 & -1.873912 & 2.276356 & 0.429470 \\
\hline 1 & -2.065044 & 2.049780 & -1.694709 & -2.289096 & 2.072815 & -1.351924 \\
\hline 23 & -0.386746 & 0.031201 & -0.063079 & -0.385476 & 0.042156 & -0.075721 \\
\hline
\end{tabular}


Table S13. Optimized coordinates for the $\left(\mathrm{C}_{4} \mathbf{H}_{6}\right)_{3} \mathbf{C r}$ structure $\mathrm{Cr}-\mathbf{1 S}$.

\begin{tabular}{|c|c|c|c|c|c|c|}
\hline & \multicolumn{3}{|c|}{ B3LYP } & \multicolumn{3}{|c|}{ B3LYP* } \\
\hline & $\mathrm{x}$ & $\mathrm{y}$ & $\mathrm{Z}$ & $\mathrm{x}$ & $\mathrm{y}$ & $\mathrm{Z}$ \\
\hline 6 & 0.809067 & -0.830901 & -1.468781 & 0.799294 & -0.867949 & -1.444344 \\
\hline 6 & 3.661001 & -0.561666 & -0.225519 & 3.651059 & -0.574001 & -0.203635 \\
\hline 6 & 2.556884 & -1.024296 & 0.397314 & 2.547394 & -1.033015 & 0.428097 \\
\hline 6 & 1.239566 & -1.300904 & -0.210592 & 1.226493 & -1.310907 & -0.169471 \\
\hline 1 & 0.818904 & -2.242765 & 0.138026 & 0.800054 & -2.244961 & 0.199584 \\
\hline 1 & 2.639467 & -1.273034 & 1.459843 & 2.635611 & -1.275248 & 1.493845 \\
\hline 1 & 4.593107 & -0.416414 & 0.317474 & 4.587846 & -0.427021 & 0.334540 \\
\hline 1 & 3.666759 & -0.335534 & -1.290457 & 3.651547 & -0.354245 & -1.271900 \\
\hline 1 & 1.428015 & -0.118995 & -2.008506 & 1.429695 & -0.177346 & -2.002474 \\
\hline 1 & 0.208362 & -1.466344 & -2.114356 & 0.203585 & -1.522775 & -2.078753 \\
\hline 6 & -2.401849 & -0.050362 & -1.008671 & -2.389398 & -0.041026 & -1.022665 \\
\hline 6 & -1.618276 & -0.738785 & 1.679524 & -1.630147 & -0.698247 & 1.676484 \\
\hline 6 & -1.592575 & -1.693327 & 0.642611 & -1.609381 & -1.673010 & 0.654661 \\
\hline 6 & -1.972207 & -1.354994 & -0.680098 & -1.979633 & -1.349765 & -0.674940 \\
\hline 1 & -1.754236 & -2.071903 & -1.469155 & -1.768372 & -2.081833 & -1.454639 \\
\hline 1 & -1.105966 & -2.653055 & 0.804486 & -1.134453 & -2.637796 & 0.834368 \\
\hline 1 & -1.192639 & -1.007811 & 2.642680 & -1.209159 & -0.955263 & 2.647145 \\
\hline 1 & -2.420220 & -0.000748 & 1.734747 & -2.431199 & 0.044942 & 1.718260 \\
\hline 1 & -3.036986 & 0.512518 & -0.322118 & -3.023090 & 0.539119 & -0.345565 \\
\hline 1 & -2.557888 & 0.191875 & -2.056762 & -2.537908 & 0.190067 & -2.076301 \\
\hline 6 & -0.735125 & 2.076808 & -0.952594 & -0.692048 & 2.053084 & -1.003082 \\
\hline 6 & 0.032017 & 1.391267 & 1.732778 & 0.023550 & 1.421286 & 1.704483 \\
\hline 6 & 1.000262 & 1.613875 & 0.734522 & 1.014721 & 1.609816 & 0.718842 \\
\hline 6 & 0.622413 & 1.948065 & -0.590372 & 0.662029 & 1.915240 & -0.620386 \\
\hline 1 & 1.393395 & 1.927238 & -1.357136 & 1.445852 & 1.867100 & -1.375723 \\
\hline 1 & 2.035279 & 1.337918 & 0.908967 & 2.045094 & 1.325329 & 0.918520 \\
\hline 1 & 0.359860 & 1.012966 & 2.697627 & 0.328029 & 1.061732 & 2.686145 \\
\hline 1 & -0.876465 & 1.995017 & 1.775688 & -0.879634 & 2.038353 & 1.714853 \\
\hline 1 & -1.450654 & 2.538151 & -0.269598 & -1.408083 & 2.549937 & -0.342252 \\
\hline 1 & -0.980737 & 2.200206 & -2.004235 & -0.922683 & 2.151670 & -2.062778 \\
\hline \multirow[t]{3}{*}{24} & -0.483808 & 0.132008 & 0.013924 & -0.481514 & 0.133300 & 0.008286 \\
\hline & \multicolumn{3}{|c|}{ BP86 } & \multicolumn{3}{|c|}{ M06L } \\
\hline & $\mathrm{X}$ & $\mathrm{y}$ & $\mathrm{Z}$ & $\mathrm{X}$ & $\mathrm{y}$ & $\mathrm{Z}$ \\
\hline 6 & 0.762207 & -0.847091 & -1.448465 & 0.692877 & -1.093028 & -1.287628 \\
\hline 6 & 3.634482 & -0.579864 & -0.215653 & 3.523326 & -0.625500 & -0.229618 \\
\hline 6 & 2.530547 & -1.062714 & 0.419560 & 2.479295 & -1.038648 & 0.514912 \\
\hline 6 & 1.197913 & -1.308896 & -0.164172 & 1.136779 & -1.363763 & 0.033675 \\
\hline 1 & 0.760747 & -2.248973 & 0.198533 & 0.702420 & -2.222665 & 0.545133 \\
\hline 1 & 2.637146 & -1.350878 & 1.479149 & 2.641223 & -1.175176 & 1.587788 \\
\hline 1 & 4.587421 & -0.463033 & 0.314453 & 4.491119 & -0.417164 & 0.218009 \\
\hline 1 & 3.617355 & -0.313687 & -1.280103 & 3.443174 & -0.508942 & -1.309498 \\
\hline 1 & 1.412206 & -0.164669 & -2.008501 & 1.347612 & -0.534740 & -1.955476 \\
\hline 1 & 0.179795 & -1.512691 & -2.096726 & 0.081960 & -1.826649 & -1.806818 \\
\hline 6 & -2.372375 & -0.008398 & -1.025782 & -2.349899 & 0.110803 & -1.041261 \\
\hline 6 & -1.630817 & -0.683843 & 1.670517 & -1.686557 & -0.526615 & 1.646569 \\
\hline 6 & -1.618244 & -1.669844 & 0.645866 & -1.717962 & -1.535065 & 0.659464 \\
\hline 6 & -1.979049 & -1.335894 & -0.688515 & -2.071540 & -1.217001 & -0.668457 \\
\hline 1 & -1.776275 & -2.071852 & -1.476293 & -1.922380 & -1.973050 & -1.434288 \\
\hline 1 & -1.155232 & -2.647683 & 0.828769 & -1.302723 & -2.520832 & 0.856158 \\
\hline 1 & -1.212611 & -0.945270 & 2.648390 & -1.283370 & -0.772287 & 2.624819 \\
\hline 1 & -2.439674 & 0.062408 & 1.712864 & -2.468088 & 0.236498 & 1.670371 \\
\hline 1 & -3.021626 & 0.566917 & -0.346591 & -2.886264 & 0.772350 & -0.355211 \\
\hline 1 & -2.513315 & 0.233560 & -2.084706 & -2.495348 & 0.346638 & -2.090266 \\
\hline 6 & -0.668256 & 2.048387 & -0.990144 & -0.365877 & 1.894616 & -1.214056 \\
\hline 6 & 0.033737 & 1.402516 & 1.710857 & -0.042415 & 1.570994 & 1.586419 \\
\hline 6 & 1.044428 & 1.581329 & 0.729767 & 1.072136 & 1.558492 & 0.737748 \\
\hline 6 & 0.696895 & 1.894225 & -0.613797 & 0.916219 & 1.678045 & -0.660237 \\
\hline 1 & 1.485497 & 1.844133 & -1.373821 & 1.772955 & 1.426192 & -1.282490 \\
\hline 1 & 2.076513 & 1.277906 & 0.933905 & 2.035349 & 1.226612 & 1.113054 \\
\hline 1 & 0.325766 & 1.033397 & 2.699878 & 0.082198 & 1.348974 & 2.640993 \\
\hline 1 & -0.858630 & 2.048579 & 1.718310 & -0.907400 & 2.199763 & 1.356825 \\
\hline 1 & -1.371602 & 2.576083 & -0.326504 & -1.058270 & 2.589745 & -0.732554 \\
\hline 1 & -0.901646 & 2.150715 & -2.055598 & -0.474277 & 1.846314 & -2.293948 \\
\hline 24 & -0.484193 & 0.139398 & 0.013932 & -0.471591 & 0.144935 & 0.007592 \\
\hline
\end{tabular}


Table S14. Optimized coordinates for the $\left(\mathrm{C}_{4} \mathrm{H}_{6}\right)_{3} \mathrm{Cr}$ structure $\mathrm{Cr}-2 \mathrm{~S}$.

\begin{tabular}{|c|c|c|c|c|c|c|}
\hline & \multicolumn{3}{|c|}{ B3LYP } & \multicolumn{3}{|c|}{ B3LYP* } \\
\hline & $\mathrm{x}$ & $\mathrm{y}$ & $\mathrm{Z}$ & $\mathrm{x}$ & $\mathrm{y}$ & $\mathrm{Z}$ \\
\hline 6 & -0.725782 & 1.493125 & 1.612240 & -0.664343 & 1.511057 & 1.614479 \\
\hline 6 & -0.039662 & 2.036665 & -1.209895 & 0.019760 & 2.003044 & -1.232287 \\
\hline 6 & -1.308818 & 1.649650 & -0.795559 & -1.262202 & 1.674880 & -0.794528 \\
\hline 6 & -1.651087 & 1.409392 & 0.572488 & -1.599770 & 1.461212 & 0.578255 \\
\hline 1 & -2.637614 & 0.992729 & 0.772175 & -2.600115 & 1.076942 & 0.787338 \\
\hline 1 & -2.042551 & 1.371317 & -1.551589 & -2.017704 & 1.419002 & -1.540097 \\
\hline 1 & 0.173391 & 2.066341 & -2.276609 & 0.215368 & 2.013572 & -2.304867 \\
\hline 1 & 0.597814 & 2.649911 & -0.580600 & 0.685997 & 2.608816 & -0.622266 \\
\hline 1 & 0.078280 & 2.221882 & 1.596556 & 0.165368 & 2.213087 & 1.594439 \\
\hline 1 & -1.022927 & 1.147119 & 2.601031 & -0.972047 & 1.178411 & 2.606775 \\
\hline 6 & -0.185566 & -2.082923 & 1.074803 & -0.237097 & -2.077040 & 1.057173 \\
\hline 6 & -0.831701 & -1.430401 & -1.643490 & -0.870491 & -1.394509 & -1.630736 \\
\hline 6 & -1.758634 & -1.227580 & -0.615818 & -1.798534 & -1.162532 & -0.604580 \\
\hline 6 & -1.432101 & -1.545449 & 0.738865 & -1.474245 & -1.494969 & 0.746712 \\
\hline 1 & -2.126829 & -1.258258 & 1.527406 & -2.154469 & -1.194485 & 1.545997 \\
\hline 1 & -2.696823 & -0.712404 & -0.813757 & -2.723121 & -0.618429 & -0.800978 \\
\hline 1 & -1.045490 & -1.035309 & -2.635511 & -1.066049 & -0.987098 & -2.623815 \\
\hline 1 & -0.158682 & -2.279445 & -1.621062 & -0.239296 & -2.278085 & -1.612593 \\
\hline 1 & 0.358216 & -2.728454 & 0.390940 & 0.263642 & -2.749210 & 0.362294 \\
\hline 1 & 0.040364 & -2.231403 & 2.127381 & 0.002115 & -2.238706 & 2.106936 \\
\hline 6 & 1.663900 & -0.281536 & 1.549900 & 1.640220 & -0.320072 & 1.549127 \\
\hline 6 & 1.896682 & -0.933742 & -1.206537 & 1.852604 & -0.985206 & -1.201443 \\
\hline 6 & 2.145043 & 0.358431 & -0.760705 & 2.149025 & 0.302610 & -0.761059 \\
\hline 6 & 1.983593 & 0.724336 & 0.612988 & 1.998685 & 0.677553 & 0.610631 \\
\hline 1 & 2.073655 & 1.770662 & 0.896167 & 2.125688 & 1.723069 & 0.891562 \\
\hline 1 & 2.337240 & 1.142575 & -1.488784 & 2.369193 & 1.077873 & -1.493849 \\
\hline 1 & 1.895324 & -1.130450 & -2.275189 & 1.840757 & -1.184902 & -2.271435 \\
\hline 1 & 2.008920 & -1.796760 & -0.561554 & 1.952430 & -1.850763 & -0.554191 \\
\hline 1 & 2.118279 & -1.262435 & 1.441809 & 2.078077 & -1.311900 & 1.448056 \\
\hline 1 & 1.444659 & 0.002159 & 2.576490 & 1.427616 & -0.027222 & 2.576641 \\
\hline \multirow[t]{3}{*}{24} & 0.002899 & 0.002101 & 0.012459 & 0.005203 & -0.001922 & 0.013066 \\
\hline & \multicolumn{3}{|c|}{ BP86 } & \multicolumn{3}{|c|}{ M06L } \\
\hline & $\mathrm{X}$ & $\mathrm{y}$ & $\mathrm{z}$ & $\mathrm{x}$ & $\mathrm{y}$ & $\mathrm{Z}$ \\
\hline 6 & -0.452398 & 1.550453 & 1.626862 & -0.692755 & 1.444448 & 1.597640 \\
\hline 6 & 0.190526 & 1.877799 & -1.305052 & -0.009150 & 1.889586 & -1.259350 \\
\hline 6 & -1.113280 & 1.754817 & -0.783566 & -1.291861 & 1.588173 & -0.804189 \\
\hline 6 & -1.419256 & 1.622655 & 0.606907 & -1.625850 & 1.397839 & 0.563491 \\
\hline 1 & -2.456966 & 1.354675 & 0.853398 & -2.612214 & 0.981741 & 0.769167 \\
\hline 1 & -1.938271 & 1.579310 & -1.487854 & -2.043180 & 1.294396 & -1.536441 \\
\hline 1 & 0.310485 & 1.834339 & -2.395336 & 0.170071 & 1.883596 & -2.332603 \\
\hline 1 & 0.949022 & 2.461236 & -0.771857 & 0.646272 & 2.533946 & -0.678713 \\
\hline 1 & 0.455269 & 2.158630 & 1.586019 & 0.124713 & 2.158464 & 1.576874 \\
\hline 1 & -0.786128 & 1.266609 & 2.633034 & -1.004414 & 1.120644 & 2.588665 \\
\hline 6 & -0.390554 & -2.061574 & 0.981764 & -0.132725 & -2.108453 & 0.908032 \\
\hline 6 & -1.021187 & -1.260390 & -1.585437 & -0.852550 & -1.310718 & -1.600128 \\
\hline 6 & -1.928936 & -0.942250 & -0.542952 & -1.787658 & -1.152333 & -0.564030 \\
\hline 6 & -1.589267 & -1.328569 & 0.791474 & -1.399516 & -1.521817 & 0.752101 \\
\hline 1 & -2.209196 & -1.001186 & 1.637788 & -2.037195 & -1.279080 & 1.601576 \\
\hline 1 & -2.810163 & -0.311303 & -0.716820 & -2.723799 & -0.618470 & -0.717904 \\
\hline 1 & -1.177012 & -0.807732 & -2.574238 & -1.060339 & -0.860206 & -2.570385 \\
\hline 1 & -0.531564 & -2.239450 & -1.604499 & -0.225661 & -2.196730 & -1.637320 \\
\hline 1 & -0.074452 & -2.810653 & 0.243880 & 0.252416 & -2.789855 & 0.149758 \\
\hline 1 & -0.092128 & -2.287544 & 2.011541 & 0.209808 & -2.322599 & 1.916269 \\
\hline 6 & 1.572586 & -0.443113 & 1.540967 & 1.574830 & -0.206033 & 1.575677 \\
\hline 6 & 1.696184 & -1.130735 & -1.203546 & 1.895522 & -0.929645 & -1.107471 \\
\hline 6 & 2.158035 & 0.126472 & -0.772524 & 2.148318 & 0.375941 & -0.712073 \\
\hline 6 & 2.058242 & 0.519226 & 0.597397 & 1.941818 & 0.786828 & 0.633196 \\
\hline 1 & 2.311906 & 1.551520 & 0.873166 & 2.019281 & 1.844524 & 0.881074 \\
\hline 1 & 2.464278 & 0.872041 & -1.515853 & 2.342832 & 1.134046 & -1.466257 \\
\hline 1 & 1.641899 & -1.324077 & -2.280239 & 1.896983 & -1.173866 & -2.164991 \\
\hline 1 & 1.766133 & -2.013766 & -0.562594 & 2.008144 & -1.760091 & -0.420407 \\
\hline 1 & 1.950882 & -1.470146 & 1.458227 & 2.072268 & -1.172536 & 1.511470 \\
\hline 1 & 1.395850 & -0.122289 & 2.574138 & 1.330243 & 0.093401 & 2.591717 \\
\hline 24 & 0.011083 & -0.016623 & 0.013514 & 0.019342 & -0.023295 & 0.022596 \\
\hline
\end{tabular}


Table S15. Optimized coordinates for the $\left(\mathrm{C}_{4} \mathbf{H}_{6}\right)_{3} \mathrm{Cr}$ structure $\mathrm{Cr}-\mathbf{1 T}$.

\begin{tabular}{|c|c|c|c|c|c|c|}
\hline & \multicolumn{3}{|c|}{ B3LYP } & \multicolumn{3}{|c|}{ B3LYP* } \\
\hline & $\mathrm{x}$ & $\mathrm{y}$ & Z & $\mathrm{x}$ & $\mathrm{y}$ & $\mathrm{Z}$ \\
\hline 6 & -2.384460 & 0.981929 & -0.075421 & -2.381254 & 0.983464 & -0.072935 \\
\hline 6 & 0.260542 & 1.714242 & 1.476917 & 0.265584 & 1.699467 & 1.480172 \\
\hline 6 & -0.063010 & 2.132626 & 0.165485 & -0.052488 & 2.124203 & 0.167529 \\
\hline 6 & -1.079272 & 1.552624 & -0.608105 & -1.073352 & 1.547731 & -0.606925 \\
\hline 1 & -1.092640 & 1.806927 & -1.670059 & -1.085501 & 1.801127 & -1.671276 \\
\hline 1 & 0.685866 & 2.717421 & -0.370012 & 0.700008 & 2.709009 & -0.366811 \\
\hline 1 & 1.159278 & 2.116945 & 1.939318 & 1.168667 & 2.094328 & 1.945566 \\
\hline 1 & -0.516376 & 1.424896 & 2.178470 & -0.517030 & 1.418960 & 2.182078 \\
\hline 1 & -2.370098 & 0.940440 & 1.018086 & -2.366622 & 0.944741 & 1.022824 \\
\hline 1 & -3.215170 & 1.656343 & -0.339665 & -3.212165 & 1.660060 & -0.339720 \\
\hline 6 & -2.660481 & -0.411679 & -0.673825 & -2.656111 & -0.412633 & -0.668140 \\
\hline 6 & -0.702510 & -1.231660 & 1.756473 & -0.692903 & -1.224374 & 1.758587 \\
\hline 6 & -0.866843 & -1.904851 & 0.529688 & -0.853335 & -1.901565 & 0.531944 \\
\hline 6 & -1.481522 & -1.383781 & -0.619282 & -1.468496 & -1.376087 & -0.618437 \\
\hline 1 & -1.359901 & -1.987283 & -1.517737 & -1.347031 & -1.980066 & -1.519059 \\
\hline 1 & -0.224099 & -2.773747 & 0.365287 & -0.207908 & -2.771180 & 0.368038 \\
\hline 1 & -0.101095 & -1.695362 & 2.533670 & -0.087470 & -1.682446 & 2.538731 \\
\hline 1 & -1.463593 & -0.542311 & 2.112094 & -1.459985 & -0.537686 & 2.112899 \\
\hline 1 & -3.542652 & -0.860973 & -0.188530 & -3.534668 & -0.867073 & -0.176673 \\
\hline 1 & -2.924521 & -0.281284 & -1.731914 & -2.926848 & -0.284555 & -1.726870 \\
\hline 6 & 2.297712 & -0.909824 & 0.786748 & 2.288729 & -0.907340 & 0.782786 \\
\hline 6 & 1.167414 & -0.670345 & -1.865657 & 1.151895 & -0.671791 & -1.865430 \\
\hline 6 & 2.009994 & 0.340498 & -1.328332 & 1.995824 & 0.342471 & -1.333730 \\
\hline 6 & 2.565429 & 0.216822 & -0.030595 & 2.553953 & 0.221285 & -0.035012 \\
\hline 1 & 3.030497 & 1.098410 & 0.409733 & 3.019311 & 1.105374 & 0.405049 \\
\hline 1 & 2.086680 & 1.304615 & -1.830213 & 2.069632 & 1.307654 & -1.838421 \\
\hline 1 & 0.649059 & -0.463284 & -2.799489 & 0.627705 & -0.467497 & -2.798884 \\
\hline 1 & 1.403993 & -1.723236 & -1.707296 & 1.391764 & -1.725931 & -1.705997 \\
\hline 1 & 2.256845 & -1.909065 & 0.352046 & 2.249948 & -1.908487 & 0.347284 \\
\hline 1 & 2.610082 & -0.877587 & 1.827957 & 2.600274 & -0.874406 & 1.826226 \\
\hline \multirow[t]{3}{*}{24} & 0.356245 & -0.104645 & 0.097237 & 0.352068 & -0.103788 & 0.094690 \\
\hline & \multicolumn{3}{|c|}{ BP86 } & \multicolumn{3}{|c|}{ M06L } \\
\hline & $\mathrm{x}$ & $\mathrm{y}$ & $\mathrm{Z}$ & $\mathrm{x}$ & $\mathrm{y}$ & $\mathrm{Z}$ \\
\hline 6 & -2.381198 & 0.986626 & -0.069523 & -2.324028 & 1.003932 & -0.058961 \\
\hline 6 & 0.270999 & 1.670244 & 1.483557 & 0.279486 & 1.652107 & 1.484023 \\
\hline 6 & -0.028373 & 2.101421 & 0.159851 & -0.002057 & 2.097757 & 0.174649 \\
\hline 6 & -1.063966 & 1.525820 & -0.615007 & -1.026182 & 1.546401 & -0.606786 \\
\hline 1 & -1.072256 & 1.769935 & -1.688585 & -1.031256 & 1.795375 & -1.669803 \\
\hline 1 & 0.733100 & 2.686689 & -0.374790 & 0.775861 & 2.654314 & -0.348553 \\
\hline 1 & 1.184678 & 2.049397 & 1.957254 & 1.179258 & 2.017282 & 1.973817 \\
\hline 1 & -0.530565 & 1.420560 & 2.187026 & -0.530471 & 1.398222 & 2.164149 \\
\hline 1 & -2.362046 & 0.963891 & 1.034204 & -2.278319 & 0.953763 & 1.034890 \\
\hline 1 & -3.212087 & 1.671046 & -0.345788 & -3.149807 & 1.694842 & -0.286989 \\
\hline 6 & -2.656688 & -0.421839 & -0.645167 & -2.628699 & -0.371873 & -0.654640 \\
\hline 6 & -0.661452 & -1.201041 & 1.768081 & -0.701080 & -1.223671 & 1.734413 \\
\hline 6 & -0.814002 & -1.894835 & 0.540519 & -0.855124 & -1.892314 & 0.507379 \\
\hline 6 & -1.440554 & -1.357996 & -0.613343 & -1.457324 & -1.338654 & -0.633322 \\
\hline 1 & -1.321645 & -1.964921 & -1.520534 & -1.340391 & -1.925320 & -1.542697 \\
\hline 1 & -0.160964 & -2.767233 & 0.375383 & -0.200057 & -2.749266 & 0.328492 \\
\hline 1 & -0.042885 & -1.642872 & 2.556557 & -0.102584 & -1.682046 & 2.515912 \\
\hline 1 & -1.451087 & -0.527440 & 2.121447 & -1.475285 & -0.544307 & 2.086749 \\
\hline 1 & -3.519774 & -0.890524 & -0.125886 & -3.502443 & -0.819885 & -0.157181 \\
\hline 1 & -2.955525 & -0.309075 & -1.704891 & -2.917989 & -0.232274 & -1.703487 \\
\hline 6 & 2.271741 & -0.891821 & 0.784088 & 2.241422 & -0.916634 & 0.771800 \\
\hline 6 & 1.122800 & -0.689796 & -1.866073 & 1.119389 & -0.669304 & -1.850239 \\
\hline 6 & 1.964882 & 0.342729 & -1.351187 & 1.960424 & 0.344187 & -1.323812 \\
\hline 6 & 2.527998 & 0.239977 & -0.044593 & 2.513781 & 0.218405 & -0.030666 \\
\hline 1 & 2.991141 & 1.136863 & 0.388632 & 2.964708 & 1.102326 & 0.418967 \\
\hline 1 & 2.030255 & 1.307923 & -1.871559 & 2.019535 & 1.312515 & -1.819164 \\
\hline 1 & 0.586965 & -0.501439 & -2.803940 & 0.595813 & -0.475172 & -2.783038 \\
\hline 1 & 1.373603 & -1.746461 & -1.691891 & 1.366359 & -1.720162 & -1.684579 \\
\hline 1 & 2.246729 & -1.902786 & 0.352591 & 2.217514 & -1.909127 & 0.316899 \\
\hline 1 & 2.581353 & -0.847195 & 1.834475 & 2.556601 & -0.902338 & 1.811525 \\
\hline 24 & 0.342828 & -0.098470 & 0.088878 & 0.338871 & -0.111282 & 0.094211 \\
\hline
\end{tabular}


Table S16. Optimized coordinates for the $\left(\mathrm{C}_{4} \mathbf{H}_{6}\right)_{3} \mathrm{Cr}$ structure $\mathrm{Cr}-2 \mathrm{~T}$.

\begin{tabular}{|c|c|c|c|c|c|c|}
\hline & \multicolumn{3}{|c|}{ B3LYP } & \multicolumn{3}{|c|}{ B3LYP* } \\
\hline & $\mathrm{x}$ & $\mathrm{y}$ & $\mathrm{Z}$ & $\mathrm{x}$ & $\mathrm{y}$ & $\mathrm{Z}$ \\
\hline 6 & -1.764147 & -1.989989 & -0.018314 & -1.750337 & -1.993202 & -0.014145 \\
\hline 6 & -2.129237 & 1.156200 & 0.865544 & -2.132180 & 1.153072 & 0.864253 \\
\hline 6 & -2.329201 & 0.434559 & -0.467670 & -2.324176 & 0.429826 & -0.469905 \\
\hline 6 & -2.363387 & -0.937936 & -0.755758 & -2.351618 & -0.945252 & -0.758006 \\
\hline 1 & -2.606638 & -1.164583 & -1.798951 & -2.589316 & -1.174846 & -1.803920 \\
\hline 1 & -2.680510 & 1.079701 & -1.272791 & -2.675919 & 1.074192 & -1.278138 \\
\hline 1 & -3.035206 & 1.722080 & 1.126762 & -3.042781 & 1.716705 & 1.123201 \\
\hline 1 & -1.966669 & 0.439594 & 1.677886 & -1.968197 & 0.436125 & 1.678681 \\
\hline 1 & -1.786995 & -1.981407 & 1.069516 & -1.778496 & -1.983302 & 1.075564 \\
\hline 1 & -1.762989 & -2.981655 & -0.467834 & -1.737740 & -2.987108 & -0.463203 \\
\hline 6 & 0.573079 & -0.961800 & 1.586137 & 0.566558 & -0.946449 & 1.586289 \\
\hline 6 & 2.997630 & -0.091224 & -0.171852 & 2.999047 & -0.091161 & -0.168275 \\
\hline 6 & 1.941515 & -1.175983 & -0.444970 & 1.938442 & -1.172779 & -0.440733 \\
\hline 6 & 1.140449 & -1.784228 & 0.548391 & 1.135040 & -1.779115 & 0.555130 \\
\hline 1 & 0.823316 & -2.818812 & 0.432095 & 0.819996 & -2.817346 & 0.446892 \\
\hline 1 & 2.167891 & -1.812259 & -1.305153 & 2.161420 & -1.811407 & -1.302784 \\
\hline 1 & 3.605102 & 0.019301 & -1.080744 & 3.609392 & 0.016060 & -1.077958 \\
\hline 1 & 3.688556 & -0.402873 & 0.626832 & 3.688785 & -0.403542 & 0.633804 \\
\hline 1 & 1.180861 & -0.170962 & 2.025711 & 1.175201 & -0.150390 & 2.020352 \\
\hline 1 & -0.089739 & -1.442005 & 2.306670 & -0.101603 & -1.418674 & 2.310303 \\
\hline 6 & -0.886834 & 2.115197 & 0.742317 & -0.891186 & 2.114819 & 0.739230 \\
\hline 6 & 2.349362 & 1.269112 & 0.145500 & 2.349060 & 1.270390 & 0.143349 \\
\hline 6 & 1.162402 & 1.391783 & -0.782618 & 1.159329 & 1.379815 & -0.784265 \\
\hline 6 & -0.151999 & 1.788482 & -0.557371 & -0.158236 & 1.781191 & -0.560882 \\
\hline 1 & -0.692411 & 2.104771 & -1.450403 & -0.696579 & 2.102612 & -1.456004 \\
\hline 1 & 1.452228 & 1.366282 & -1.837294 & 1.450100 & 1.352837 & -1.840922 \\
\hline 1 & 3.058197 & 2.094961 & -0.031219 & 3.055827 & 2.099603 & -0.038517 \\
\hline 1 & 2.050431 & 1.339894 & 1.195313 & 2.050612 & 1.345632 & 1.195295 \\
\hline 1 & -0.234016 & 2.002498 & 1.613439 & -0.236241 & 2.004704 & 1.611613 \\
\hline 1 & -1.209400 & 3.166052 & 0.722412 & -1.215486 & 3.167251 & 0.717154 \\
\hline \multirow[t]{3}{*}{24} & -0.216658 & -0.410234 & -0.320345 & -0.216976 & -0.407334 & -0.320985 \\
\hline & \multicolumn{3}{|c|}{ BP86 } & \multicolumn{3}{|c|}{ M06L } \\
\hline & $\mathrm{X}$ & $\mathrm{y}$ & $\mathrm{z}$ & $\mathrm{x}$ & $\mathrm{y}$ & $\mathrm{Z}$ \\
\hline 6 & -1.702284 & -2.007911 & 0.000240 & -1.741106 & -1.948497 & -0.000068 \\
\hline 6 & -2.148040 & 1.139629 & 0.858820 & -2.075858 & 1.132507 & 0.866215 \\
\hline 6 & -2.307997 & 0.412752 & -0.481600 & -2.293356 & 0.455149 & -0.474688 \\
\hline 6 & -2.310174 & -0.973013 & -0.769780 & -2.333643 & -0.914431 & -0.762058 \\
\hline 1 & -2.523556 & -1.211748 & -1.825859 & -2.562639 & -1.148289 & -1.805039 \\
\hline 1 & -2.661517 & 1.052604 & -1.301501 & -2.632208 & 1.114059 & -1.271962 \\
\hline 1 & -3.077901 & 1.688073 & 1.112936 & -2.976167 & 1.680151 & 1.175323 \\
\hline 1 & -1.974619 & 0.419606 & 1.678104 & -1.899221 & 0.381819 & 1.646447 \\
\hline 1 & -1.762753 & -1.995655 & 1.096121 & -1.780343 & -1.912483 & 1.088430 \\
\hline 1 & -1.654778 & -3.009037 & -0.446687 & -1.739946 & -2.951780 & -0.419810 \\
\hline 6 & 0.556502 & -0.903993 & 1.589918 & 0.550332 & -0.925386 & 1.567973 \\
\hline 6 & 3.003978 & -0.084021 & -0.160697 & 2.957064 & -0.127811 & -0.155988 \\
\hline 6 & 1.926409 & -1.154819 & -0.435569 & 1.881764 & -1.173297 & -0.453268 \\
\hline 6 & 1.124978 & -1.766711 & 0.573355 & 1.084434 & -1.783117 & 0.544918 \\
\hline 1 & 0.822393 & -2.817919 & 0.489385 & 0.749695 & -2.812822 & 0.441377 \\
\hline 1 & 2.136580 & -1.795469 & -1.308512 & 2.095663 & -1.804880 & -1.319791 \\
\hline 1 & 3.624365 & 0.014148 & -1.072456 & 3.587232 & -0.029579 & -1.048953 \\
\hline 1 & 3.689328 & -0.401895 & 0.651628 & 3.625527 & -0.459373 & 0.651900 \\
\hline 1 & 1.173185 & -0.094991 & 2.007713 & 1.194297 & -0.158171 & 2.002060 \\
\hline 1 & -0.123676 & -1.355163 & 2.327081 & -0.139541 & -1.362147 & 2.291777 \\
\hline 6 & -0.916014 & 2.117956 & 0.731828 & -0.839395 & 2.077862 & 0.751941 \\
\hline 6 & 2.348000 & 1.282305 & 0.136470 & 2.330257 & 1.232895 & 0.142758 \\
\hline 6 & 1.144881 & 1.347217 & -0.785874 & 1.161344 & 1.354721 & -0.790462 \\
\hline 6 & -0.183499 & 1.766491 & -0.569084 & -0.142970 & 1.784697 & -0.562423 \\
\hline 1 & -0.713654 & 2.100219 & -1.473377 & -0.689160 & 2.121210 & -1.443409 \\
\hline 1 & 1.435261 & 1.313309 & -1.850292 & 1.452731 & 1.317491 & -1.843926 \\
\hline 1 & 3.044944 & 2.123888 & -0.065987 & 3.047112 & 2.052899 & -0.019715 \\
\hline 1 & 2.054757 & 1.372316 & 1.196760 & 2.013447 & 1.317134 & 1.187606 \\
\hline 1 & -0.255676 & 2.019444 & 1.610222 & -0.162051 & 1.919136 & 1.598666 \\
\hline 1 & -1.250387 & 3.174385 & 0.701513 & -1.141550 & 3.132673 & 0.793720 \\
\hline 24 & -0.216781 & -0.402142 & -0.318957 & -0.219837 & -0.391200 & -0.323075 \\
\hline
\end{tabular}


Table S17. Optimized coordinates for the $\left(\mathbf{C}_{4} \mathbf{H}_{6}\right)_{3} \mathbf{C r}$ structure $\mathrm{Cr}-3 \mathrm{~T}$.

\begin{tabular}{|c|c|c|c|c|c|c|}
\hline & \multicolumn{3}{|c|}{$\overline{\text { B3LYP }}$} & \multicolumn{3}{|c|}{ B3LYP* } \\
\hline & $\mathrm{x}$ & $\mathrm{y}$ & $\mathrm{Z}$ & $\mathrm{x}$ & $\mathrm{y}$ & $\mathrm{Z}$ \\
\hline 6 & 1.532403 & -1.464384 & 1.350411 & 1.536731 & -1.445196 & 1.351414 \\
\hline 6 & -0.123734 & -1.901921 & -0.979802 & -0.111734 & -1.902825 & -0.978674 \\
\hline 6 & 1.259658 & -1.599832 & -1.101640 & 1.269248 & -1.585951 & -1.102910 \\
\hline 6 & 2.077607 & -1.433733 & 0.049953 & 2.085493 & -1.409007 & 0.050462 \\
\hline 1 & 3.094274 & -1.069059 & -0.095027 & 3.100404 & -1.033169 & -0.093359 \\
\hline 1 & 1.685797 & -1.349407 & -2.072317 & 1.693385 & -1.331709 & -2.075756 \\
\hline 1 & -0.723750 & -1.875152 & -1.887259 & -0.715856 & -1.879490 & -1.885827 \\
\hline 1 & -0.454866 & -2.631981 & -0.243737 & -0.435525 & -2.637725 & -0.241301 \\
\hline 1 & 0.722929 & -2.148218 & 1.600039 & 0.734344 & -2.140583 & 1.601045 \\
\hline 1 & 2.159943 & -1.163756 & 2.187097 & 2.159025 & -1.133481 & 2.190540 \\
\hline 6 & 0.969173 & 1.915678 & 1.115927 & 0.951482 & 1.918191 & 1.109796 \\
\hline 6 & -0.139981 & 1.510643 & -1.523826 & -0.168235 & 1.496355 & -1.519258 \\
\hline 6 & 1.249938 & 1.511953 & -1.308673 & 1.225308 & 1.507807 & -1.315128 \\
\hline 6 & 1.804508 & 1.659033 & -0.004354 & 1.786668 & 1.664028 & -0.012911 \\
\hline 1 & 2.859908 & 1.427107 & 0.132377 & 2.846405 & 1.440356 & 0.120175 \\
\hline 1 & 1.912423 & 1.179783 & -2.107947 & 1.885916 & 1.177826 & -2.119565 \\
\hline 1 & -0.524754 & 1.212539 & -2.496362 & -0.560000 & 1.188455 & -2.488137 \\
\hline 1 & -0.805575 & 2.133633 & -0.931128 & -0.834757 & 2.119266 & -0.923752 \\
\hline 1 & 0.152263 & 2.628052 & 1.028317 & 0.129647 & 2.627962 & 1.022800 \\
\hline 1 & 1.423271 & 1.873267 & 2.104565 & 1.408185 & 1.878903 & 2.099460 \\
\hline 6 & -1.311830 & 0.532287 & 1.572247 & -1.291250 & 0.516979 & 1.577602 \\
\hline 6 & -3.643144 & 0.371340 & -0.451830 & -3.632036 & 0.365700 & -0.440204 \\
\hline 6 & -2.801418 & -0.630677 & -0.129376 & -2.786971 & -0.639454 & -0.127303 \\
\hline 6 & -1.754110 & -0.606211 & 0.912501 & -1.731739 & -0.621174 & 0.906760 \\
\hline 1 & -1.538437 & -1.571647 & 1.368520 & -1.516533 & -1.590928 & 1.359163 \\
\hline 1 & -2.911473 & -1.581125 & -0.654656 & -2.901009 & -1.588936 & -0.658149 \\
\hline 1 & -4.392215 & 0.242282 & -1.230231 & -4.387691 & 0.240712 & -1.215542 \\
\hline 1 & -3.615221 & 1.338146 & 0.047690 & -3.599979 & 1.331589 & 0.065129 \\
\hline 1 & -1.734916 & 1.505850 & 1.340829 & -1.728896 & 1.489801 & 1.360399 \\
\hline 1 & -0.833090 & 0.446068 & 2.543828 & -0.817396 & 0.423702 & 2.553339 \\
\hline \multirow[t]{3}{*}{24} & 0.367044 & 0.009107 & 0.098174 & 0.364272 & 0.009364 & 0.097144 \\
\hline & \multicolumn{3}{|c|}{ BP86 } & \multicolumn{3}{|c|}{ M06L } \\
\hline & $\mathrm{X}$ & $\mathrm{y}$ & Z & $\mathrm{X}$ & $\mathrm{y}$ & Z \\
\hline 6 & 1.543520 & -1.396033 & 1.359518 & 1.513265 & -1.451516 & 1.284788 \\
\hline 6 & -0.064985 & -1.901604 & -0.984853 & -0.120441 & -1.867575 & -0.996815 \\
\hline 6 & 1.313087 & -1.543320 & -1.108233 & 1.247914 & -1.527515 & -1.153430 \\
\hline 6 & 2.115511 & -1.336599 & 0.059147 & 2.068764 & -1.362802 & -0.009019 \\
\hline 1 & 3.126822 & -0.928670 & -0.072314 & 3.068420 & -0.952901 & -0.144749 \\
\hline 1 & 1.738772 & -1.276368 & -2.084617 & 1.643174 & -1.232027 & -2.124049 \\
\hline 1 & -0.673881 & -1.886844 & -1.897055 & -0.752934 & -1.842408 & -1.881624 \\
\hline 1 & -0.368075 & -2.659022 & -0.251003 & -0.402607 & -2.625813 & -0.266181 \\
\hline 1 & 0.767169 & -2.134754 & 1.599611 & 0.739460 & -2.189769 & 1.497731 \\
\hline 1 & 2.143493 & -1.055462 & 2.212330 & 2.123451 & -1.163518 & 2.137407 \\
\hline 6 & 0.901982 & 1.923117 & 1.098350 & 0.967039 & 1.871334 & 1.117829 \\
\hline 6 & -0.250043 & 1.459868 & -1.500868 & -0.287740 & 1.519924 & -1.398446 \\
\hline 6 & 1.157094 & 1.501438 & -1.332440 & 1.116119 & 1.516442 & -1.305111 \\
\hline 6 & 1.740324 & 1.684312 & -0.035082 & 1.752997 & 1.651522 & -0.042802 \\
\hline 1 & 2.814231 & 1.489899 & 0.085218 & 2.814311 & 1.420378 & 0.030883 \\
\hline 1 & 1.812136 & 1.183026 & -2.154858 & 1.715830 & 1.188399 & -2.153160 \\
\hline 1 & -0.664005 & 1.125391 & -2.459068 & -0.762939 & 1.241447 & -2.335770 \\
\hline 1 & -0.916649 & 2.085543 & -0.895032 & -0.882225 & 2.163533 & -0.750630 \\
\hline 1 & 0.067815 & 2.630083 & 1.014909 & 0.145337 & 2.584515 & 1.079391 \\
\hline 1 & 1.368706 & 1.892750 & 2.090904 & 1.462428 & 1.815858 & 2.085111 \\
\hline 6 & -1.245146 & 0.468696 & 1.594090 & -1.256098 & 0.466280 & 1.632527 \\
\hline 6 & -3.621299 & 0.344804 & -0.405930 & -3.441606 & 0.384920 & -0.499238 \\
\hline 6 & -2.755399 & -0.665580 & -0.131461 & -2.709244 & -0.667065 & -0.092262 \\
\hline 6 & -1.683022 & -0.666713 & 0.888017 & -1.694597 & -0.664738 & 0.962335 \\
\hline 1 & -1.465280 & -1.651417 & 1.324536 & -1.447999 & -1.638132 & 1.385674 \\
\hline 1 & -2.869777 & -1.606988 & -0.690436 & -2.886249 & -1.634052 & -0.564852 \\
\hline 1 & -4.391262 & 0.232826 & -1.178229 & -4.180884 & 0.284499 & -1.287597 \\
\hline 1 & -3.591319 & 1.301875 & 0.129959 & -3.322925 & 1.374836 & -0.061503 \\
\hline 1 & -1.717359 & 1.441082 & 1.413195 & -1.702467 & 1.437241 & 1.427819 \\
\hline 1 & -0.784856 & 0.351563 & 2.581628 & -0.752425 & 0.373532 & 2.589786 \\
\hline 24 & 0.362232 & 0.009632 & 0.092867 & 0.351792 & 0.007463 & 0.097257 \\
\hline
\end{tabular}


Table S18. Optimized coordinates for the $\left(\mathrm{C}_{4} \mathrm{H}_{6}\right)_{3} \mathrm{Cr}$ structure $\mathrm{Cr}-\mathbf{1 P}$.

\begin{tabular}{|c|c|c|c|c|c|c|}
\hline & \multicolumn{3}{|c|}{ B3LYP } & \multicolumn{3}{|c|}{ B3LYP* } \\
\hline & $\mathrm{x}$ & $\mathrm{y}$ & $\mathrm{Z}$ & $\mathrm{x}$ & $\mathrm{y}$ & $\mathrm{Z}$ \\
\hline 6 & -1.179281 & -1.264721 & 1.678809 & -1.129604 & -1.240779 & 1.680688 \\
\hline 6 & -2.846377 & 0.558598 & -0.321197 & -2.869856 & 0.522619 & -0.295836 \\
\hline 6 & -2.255199 & -0.838702 & -0.546203 & -2.254941 & -0.863677 & -0.527655 \\
\hline 6 & -1.826793 & -1.686537 & 0.493615 & -1.780926 & -1.695430 & 0.506927 \\
\hline 1 & -1.745794 & -2.750448 & 0.246119 & -1.672567 & -2.759925 & 0.264438 \\
\hline 1 & -2.614963 & -1.340636 & -1.444894 & -2.615344 & -1.377526 & -1.421753 \\
\hline 1 & -3.284108 & 0.888088 & -1.273609 & -3.333305 & 0.840864 & -1.242095 \\
\hline 1 & -3.682566 & 0.491473 & 0.393497 & -3.690670 & 0.446757 & 0.438590 \\
\hline 1 & -1.425085 & -0.305533 & 2.132586 & -1.408676 & -0.288323 & 2.134357 \\
\hline 1 & -0.810205 & -2.019600 & 2.370074 & -0.722806 & -1.978061 & 2.372848 \\
\hline 6 & 1.772920 & -0.999794 & -1.726004 & 1.791676 & -1.025258 & -1.707618 \\
\hline 6 & 2.514690 & 0.908799 & 0.752368 & 2.509953 & 0.928651 & 0.741807 \\
\hline 6 & 2.152559 & -0.582109 & 0.727280 & 2.134618 & -0.559076 & 0.744400 \\
\hline 6 & 2.171645 & -1.394732 & -0.433721 & 2.160048 & -1.400709 & -0.398645 \\
\hline 1 & 3.121563 & 1.154228 & -0.132726 & 3.115776 & 1.155131 & -0.151418 \\
\hline 1 & 3.158454 & 1.085820 & 1.624865 & 3.156946 & 1.122021 & 1.611105 \\
\hline 6 & -1.899694 & 1.689112 & 0.163181 & -1.922367 & 1.665830 & 0.158846 \\
\hline 6 & 1.335383 & 1.918600 & 0.818424 & 1.328304 & 1.937570 & 0.786869 \\
\hline 6 & 0.591139 & 1.971954 & -0.494665 & 0.571342 & 1.927734 & -0.520533 \\
\hline 6 & -0.728739 & 1.866814 & -0.768724 & -0.753433 & 1.803540 & -0.782823 \\
\hline 1 & -1.009894 & 1.942343 & -1.823341 & -1.039164 & 1.835487 & -1.840521 \\
\hline 1 & 1.245831 & 2.137886 & -1.354926 & 1.218982 & 2.063230 & -1.393849 \\
\hline 1 & 1.748713 & 2.920944 & 1.018500 & 1.738174 & 2.951618 & 0.942054 \\
\hline 1 & 0.672650 & 1.672463 & 1.656785 & 0.674860 & 1.719386 & 1.642640 \\
\hline 1 & -1.566213 & 1.524350 & 1.192593 & -1.585877 & 1.527682 & 1.193389 \\
\hline 1 & -2.479444 & 2.626366 & 0.175986 & -2.497519 & 2.608462 & 0.145981 \\
\hline 24 & 0.069363 & -0.734021 & -0.153046 & 0.073746 & -0.689059 & -0.165460 \\
\hline 1 & 2.332401 & -1.107367 & 1.665450 & 2.288046 & -1.064927 & 1.700033 \\
\hline 1 & 1.890371 & 0.031447 & -2.056412 & 1.941734 & -0.003552 & -2.060556 \\
\hline 1 & 1.737487 & -1.745188 & -2.517490 & 1.751918 & -1.788776 & -2.484153 \\
\hline \multirow[t]{3}{*}{1} & 2.232563 & -2.473826 & -0.258930 & 2.200702 & -2.478228 & -0.198610 \\
\hline & \multicolumn{3}{|c|}{ BP86 } & \multicolumn{3}{|c|}{ M06L } \\
\hline & $\mathrm{x}$ & $\mathrm{y}$ & $\mathrm{z}$ & $\mathrm{x}$ & $\mathrm{y}$ & $\mathrm{Z}$ \\
\hline 6 & -1.010830 & -1.186045 & 1.685500 & -1.093616 & -1.220997 & 1.666050 \\
\hline 6 & -2.899449 & 0.457785 & -0.223344 & -2.834596 & 0.493492 & -0.278538 \\
\hline 6 & -2.227709 & -0.901636 & -0.486668 & -2.203801 & -0.869111 & -0.533775 \\
\hline 6 & -1.665887 & -1.711875 & 0.533538 & -1.730642 & -1.698504 & 0.499657 \\
\hline 1 & -1.498969 & -2.775634 & 0.293597 & -1.589022 & -2.754858 & 0.252368 \\
\hline 1 & -2.589262 & -1.433963 & -1.377858 & -2.567767 & -1.382013 & -1.423390 \\
\hline 1 & -3.429306 & 0.751682 & -1.149764 & -3.314403 & 0.819337 & -1.209852 \\
\hline 1 & -3.678838 & 0.355432 & 0.560989 & -3.644828 & 0.398020 & 0.460104 \\
\hline 1 & -1.368833 & -0.257288 & 2.150381 & -1.419239 & -0.283578 & 2.118339 \\
\hline 1 & -0.513599 & -1.881760 & 2.372152 & -0.675181 & -1.940015 & 2.365672 \\
\hline 6 & 1.774268 & -1.109641 & -1.666025 & 1.739017 & -0.963372 & -1.697296 \\
\hline 6 & 2.515777 & 0.932972 & 0.706821 & 2.477386 & 0.906163 & 0.735304 \\
\hline 6 & 2.068259 & -0.535487 & 0.777237 & 2.119288 & -0.572655 & 0.738181 \\
\hline 6 & 2.078391 & -1.450039 & -0.322685 & 2.128041 & -1.390646 & -0.412130 \\
\hline 1 & 3.115817 & 1.098617 & -0.211353 & 3.057018 & 1.141187 & -0.171076 \\
\hline 1 & 3.185668 & 1.146706 & 1.563014 & 3.143941 & 1.106154 & 1.582797 \\
\hline 6 & -1.947704 & 1.629007 & 0.162105 & -1.899882 & 1.630452 & 0.172055 \\
\hline 6 & 1.349201 & 1.965340 & 0.713957 & 1.288987 & 1.885931 & 0.802061 \\
\hline 6 & 0.561826 & 1.836745 & -0.573664 & 0.561725 & 1.919339 & -0.508893 \\
\hline 6 & -0.784011 & 1.673155 & -0.802470 & -0.755794 & 1.798668 & -0.777100 \\
\hline 1 & -1.085260 & 1.635056 & -1.862583 & -1.043708 & 1.841271 & -1.830542 \\
\hline 1 & 1.188028 & 1.923089 & -1.476899 & 1.218615 & 2.067087 & -1.370339 \\
\hline 1 & 1.768768 & 2.993053 & 0.778854 & 1.667070 & 2.895583 & 1.021560 \\
\hline 1 & 0.710474 & 1.812908 & 1.603194 & 0.618675 & 1.612719 & 1.628756 \\
\hline 1 & -1.598258 & 1.545999 & 1.206001 & -1.536437 & 1.480352 & 1.195207 \\
\hline 1 & -2.512598 & 2.584268 & 0.096781 & -2.480999 & 2.564057 & 0.197573 \\
\hline 24 & 0.062646 & -0.564787 & -0.200109 & 0.072225 & -0.662591 & -0.174517 \\
\hline 1 & 2.156715 & -0.994237 & 1.771498 & 2.271855 & -1.084492 & 1.687384 \\
\hline 1 & 2.028795 & -0.118242 & -2.065435 & 1.931872 & 0.066354 & -2.005329 \\
\hline 1 & 1.696759 & -1.910580 & -2.410934 & 1.706694 & -1.687512 & -2.506898 \\
\hline 1 & 2.047614 & -2.521909 & -0.064828 & 2.145746 & -2.470026 & -0.237386 \\
\hline
\end{tabular}


Table S19. Optimized coordinates for the $\left(\mathrm{C}_{4} \mathbf{H}_{6}\right)_{3} \mathrm{Cr}$ structure $\mathrm{Cr}-\mathbf{2 P}$.

\begin{tabular}{|c|c|c|c|c|c|c|}
\hline & \multicolumn{3}{|c|}{ B3LYP } & \multicolumn{3}{|c|}{ B3LYP* } \\
\hline & $\mathrm{x}$ & $\mathrm{y}$ & Z & $\mathrm{x}$ & $\mathrm{y}$ & Z \\
\hline 6 & 1.838762 & -2.025506 & -0.785760 & 1.824557 & -2.022871 & -0.787256 \\
\hline 6 & 2.589435 & 0.932156 & 0.014130 & 2.587010 & 0.930001 & 0.015810 \\
\hline 6 & 2.037752 & -0.185780 & 0.899765 & 2.031200 & -0.185819 & 0.902139 \\
\hline 6 & 2.083251 & -1.540785 & 0.524770 & 2.073321 & -1.543276 & 0.525972 \\
\hline 1 & 2.024998 & -2.278015 & 1.333528 & 2.015279 & -2.283634 & 1.334573 \\
\hline 1 & 2.040267 & 0.016957 & 1.970937 & 2.033468 & 0.017066 & 1.975315 \\
\hline 1 & 3.237724 & 1.562074 & 0.641336 & 3.237585 & 1.560471 & 0.643633 \\
\hline 1 & 3.242727 & 0.488221 & -0.750810 & 3.240676 & 0.482113 & -0.749466 \\
\hline 1 & 2.081023 & -1.399566 & -1.648786 & 2.065944 & -1.393069 & -1.650234 \\
\hline 1 & 1.862721 & -3.097368 & -0.968748 & 1.841452 & -3.095884 & -0.975282 \\
\hline 6 & -1.838602 & -2.025626 & -0.785773 & -1.824434 & -2.023028 & -0.787206 \\
\hline 6 & -2.589511 & 0.931981 & 0.014134 & -2.587068 & 0.929813 & 0.015778 \\
\hline 6 & -2.037758 & -0.185924 & 0.899764 & -2.031178 & -0.185944 & 0.902137 \\
\hline 6 & -2.083141 & -1.540927 & 0.524758 & -2.073221 & -1.543416 & 0.526011 \\
\hline 1 & -2.024836 & -2.278156 & 1.333512 & -2.015134 & -2.283748 & 1.334633 \\
\hline 1 & -2.040286 & 0.016806 & 1.970937 & -2.033455 & 0.016974 & 1.975306 \\
\hline 1 & -3.237848 & 1.561850 & 0.641340 & -3.237710 & 1.560236 & 0.643578 \\
\hline 1 & -3.242768 & 0.488005 & -0.750812 & -3.240680 & 0.481858 & -0.749505 \\
\hline 1 & -2.080895 & -1.399698 & -1.648799 & -2.065865 & -1.393261 & -1.650196 \\
\hline 1 & -1.862489 & -3.097490 & -0.968765 & -1.841261 & -3.096047 & -0.975207 \\
\hline 6 & 1.585535 & 1.863543 & -0.710365 & 1.584935 & 1.862999 & -0.711046 \\
\hline 6 & -1.585676 & 1.863443 & -0.710356 & -1.585054 & 1.862883 & -0.711069 \\
\hline 6 & -0.672802 & 2.617180 & 0.222542 & -0.673836 & 2.620730 & 0.220388 \\
\hline 6 & 0.672619 & 2.617224 & 0.222537 & 0.673647 & 2.620779 & 0.220399 \\
\hline 1 & 1.182108 & 3.223635 & 0.977228 & 1.184832 & 3.229859 & 0.974771 \\
\hline 1 & -1.182324 & 3.223559 & 0.977238 & -1.185078 & 3.229773 & 0.974751 \\
\hline 1 & -2.170244 & 2.588995 & -1.299584 & -2.172257 & 2.586377 & -1.304089 \\
\hline 1 & -1.005609 & 1.283886 & -1.440629 & -1.001734 & 1.281132 & -1.440248 \\
\hline 1 & 1.005502 & 1.283940 & -1.440629 & 1.001667 & 1.281207 & -1.440234 \\
\hline 1 & 2.170050 & 2.589129 & -1.299604 & 2.172092 & 2.586538 & -1.304055 \\
\hline \multirow[t]{3}{*}{24} & 0.000041 & -1.029277 & 0.016260 & 0.000028 & -1.022346 & 0.025195 \\
\hline & \multicolumn{3}{|c|}{ BP86 } & \multicolumn{3}{|c|}{ M06L } \\
\hline & $\mathrm{x}$ & $\mathrm{y}$ & $\mathrm{Z}$ & $\mathrm{x}$ & $\mathrm{y}$ & $\mathrm{Z}$ \\
\hline 6 & -1.756168 & -2.022855 & 0.816191 & 1.758665 & -1.914627 & -0.839914 \\
\hline 6 & -2.589244 & 0.916029 & 0.000336 & 2.593756 & 0.884524 & 0.068852 \\
\hline 6 & -2.026771 & -0.197674 & -0.890103 & 2.037131 & -0.221245 & 0.947034 \\
\hline 6 & -2.048231 & -1.564102 & -0.503743 & 2.019040 & -1.553076 & 0.502954 \\
\hline 1 & -1.999897 & -2.316239 & -1.311117 & 1.906248 & -2.333732 & 1.261469 \\
\hline 1 & -2.048357 & -0.001459 & -1.971181 & 2.082313 & -0.060986 & 2.023135 \\
\hline 1 & -3.259563 & 1.539599 & -0.625338 & 3.188015 & 1.564295 & 0.693441 \\
\hline 1 & -3.231481 & 0.458703 & 0.779483 & 3.294977 & 0.442388 & -0.653114 \\
\hline 1 & -1.986084 & -1.381214 & 1.682457 & 2.068505 & -1.235593 & -1.639787 \\
\hline 1 & -1.745544 & -3.099192 & 1.021425 & 1.726133 & -2.964357 & -1.116312 \\
\hline 6 & 1.756279 & -2.022842 & 0.816075 & -1.758776 & -1.914542 & -0.839915 \\
\hline 6 & 2.589193 & 0.916142 & 0.000418 & -2.593700 & 0.884662 & 0.068851 \\
\hline 6 & 2.026777 & -0.197527 & -0.890092 & -2.037139 & -0.221142 & 0.947031 \\
\hline 6 & 2.048302 & -1.563981 & -0.503831 & -2.019136 & -1.552976 & 0.502949 \\
\hline 1 & 1.999986 & -2.316060 & -1.311261 & -1.906417 & -2.333642 & 1.261463 \\
\hline 1 & 2.048331 & -0.001232 & -1.971156 & -2.082336 & -0.060887 & 2.023133 \\
\hline 1 & 3.259535 & 1.539746 & -0.625198 & -3.187911 & 1.564473 & 0.693442 \\
\hline 1 & 3.231397 & 0.458798 & 0.779581 & -3.294955 & 0.442573 & -0.653112 \\
\hline 1 & 1.986195 & -1.381258 & 1.682384 & -2.068539 & -1.235472 & -1.639788 \\
\hline 1 & 1.745707 & -3.099195 & 1.021232 & -1.726307 & -2.964270 & -1.116324 \\
\hline 6 & -1.585004 & 1.864455 & 0.714075 & 1.577117 & 1.729343 & -0.714454 \\
\hline 6 & 1.584893 & 1.864523 & 0.714134 & -1.577008 & 1.729415 & -0.714457 \\
\hline 6 & 0.677994 & 2.615564 & -0.231180 & -0.670485 & 2.518997 & 0.175267 \\
\hline 6 & -0.678102 & 2.615533 & -0.231207 & 0.670631 & 2.518969 & 0.175267 \\
\hline 1 & -1.193086 & 3.218445 & -0.997459 & 1.180329 & 3.150506 & 0.906916 \\
\hline 1 & 1.192982 & 3.218500 & -0.997410 & -1.180158 & 3.150556 & 0.906914 \\
\hline 1 & 2.174431 & 2.594814 & 1.309045 & -2.128901 & 2.415580 & -1.373212 \\
\hline 1 & 0.989874 & 1.285963 & 1.447911 & -0.997767 & 1.080932 & -1.393205 \\
\hline 1 & -0.989987 & 1.285928 & 1.447878 & 0.997846 & 1.080902 & -1.393214 \\
\hline 1 & -2.174595 & 2.594726 & 1.308959 & 2.129058 & 2.415483 & -1.373195 \\
\hline 24 & 0.000027 & -0.997457 & -0.064028 & -0.000030 & -0.893690 & 0.037690 \\
\hline
\end{tabular}


Table S20. Optimized coordinates for the $\left(\mathrm{C}_{4} \mathbf{H}_{6}\right)_{3} \mathbf{M n}$ structure Mn-1D.

\begin{tabular}{|c|c|c|c|c|c|c|}
\hline & \multicolumn{3}{|c|}{ B3LYP } & \multicolumn{3}{|c|}{ B3LYP* } \\
\hline & $\mathrm{x}$ & $\mathrm{y}$ & $\mathrm{Z}$ & $\mathrm{x}$ & $\mathrm{y}$ & $\mathrm{Z}$ \\
\hline 6 & 1.391602 & -1.482051 & 1.296947 & 1.385865 & -1.472594 & 1.297489 \\
\hline 6 & 0.335010 & -1.679342 & -1.337306 & 0.337419 & -1.674395 & -1.335748 \\
\hline 6 & 1.660956 & -1.216826 & -1.139260 & 1.663606 & -1.207280 & -1.138651 \\
\hline 6 & 2.193721 & -1.130912 & 0.180849 & 2.194077 & -1.118924 & 0.184061 \\
\hline 1 & 3.148591 & -0.627382 & 0.323028 & 3.149183 & -0.612269 & 0.329394 \\
\hline 1 & 2.221037 & -0.761511 & -1.954639 & 2.223725 & -0.749108 & -1.955195 \\
\hline 1 & -0.110785 & -1.537444 & -2.320733 & -0.111875 & -1.530552 & -2.319515 \\
\hline 1 & -0.035319 & -2.545726 & -0.795714 & -0.029585 & -2.545625 & -0.795623 \\
\hline 1 & 0.745096 & -2.357806 & 1.268347 & 0.745101 & -2.355240 & 1.267771 \\
\hline 1 & 1.763441 & -1.225669 & 2.287522 & 1.751667 & -1.211709 & 2.291313 \\
\hline 6 & 0.923636 & 1.825246 & 1.213116 & 0.917717 & 1.821321 & 1.208793 \\
\hline 6 & -0.639141 & 1.497688 & -1.130653 & -0.652490 & 1.484269 & -1.123039 \\
\hline 6 & 0.763527 & 1.661420 & -1.233976 & 0.750551 & 1.654664 & -1.238272 \\
\hline 6 & 1.555331 & 1.805234 & -0.054968 & 1.548332 & 1.803072 & -0.061907 \\
\hline 1 & 2.638030 & 1.729131 & -0.142149 & 2.633063 & 1.730614 & -0.152849 \\
\hline 1 & 1.270823 & 1.493575 & -2.182781 & 1.253612 & 1.487915 & -2.191893 \\
\hline 1 & -1.190380 & 1.199392 & -2.018914 & -1.210220 & 1.178211 & -2.006982 \\
\hline 1 & -1.224313 & 2.059658 & -0.406926 & -1.236606 & 2.049974 & -0.398147 \\
\hline 1 & 0.008717 & 2.390834 & 1.362207 & 0.002333 & 2.389366 & 1.360475 \\
\hline 1 & 1.547439 & 1.727367 & 2.100246 & 1.543955 & 1.722171 & 2.096533 \\
\hline 6 & -1.175755 & 0.030226 & 1.603324 & -1.158470 & 0.027816 & 1.601147 \\
\hline 6 & -3.753352 & 0.161201 & -0.152255 & -3.746394 & 0.161153 & -0.144114 \\
\hline 6 & -2.707267 & -0.669894 & -0.320817 & -2.701409 & -0.673607 & -0.318518 \\
\hline 6 & -1.587710 & -0.872135 & 0.623311 & -1.572781 & -0.874365 & 0.615076 \\
\hline 1 & -1.325923 & -1.919156 & 0.769594 & -1.313720 & -1.924328 & 0.762532 \\
\hline 1 & -2.687554 & -1.301018 & -1.212963 & -2.691075 & -1.309043 & -1.210570 \\
\hline 1 & -4.543117 & 0.228548 & -0.898256 & -4.544097 & 0.227225 & -0.884531 \\
\hline 1 & -3.854023 & 0.785969 & 0.733580 & -3.838023 & 0.790111 & 0.742134 \\
\hline 1 & -1.646847 & 1.008238 & 1.663577 & -1.637576 & 1.004057 & 1.667199 \\
\hline 1 & -0.720667 & -0.321781 & 2.524251 & -0.713174 & -0.330029 & 2.527138 \\
\hline \multirow[t]{3}{*}{25} & 0.409297 & 0.015826 & 0.064434 & 0.409088 & 0.016059 & 0.063717 \\
\hline & \multicolumn{3}{|c|}{ BP86 } & \multicolumn{3}{|c|}{ M06L } \\
\hline & $\mathrm{x}$ & $\mathrm{y}$ & $\mathrm{Z}$ & $\mathrm{x}$ & $\mathrm{y}$ & $\mathrm{Z}$ \\
\hline 6 & 1.376666 & -1.451280 & 1.300814 & 0.565573 & -1.470170 & 1.827321 \\
\hline 6 & 0.364939 & -1.661482 & -1.338508 & 0.511537 & -1.859166 & -0.932636 \\
\hline 6 & 1.691439 & -1.173052 & -1.136186 & 1.755636 & -1.599040 & -0.306001 \\
\hline 6 & 2.209068 & -1.079754 & 0.198817 & 1.785757 & -1.413191 & 1.104986 \\
\hline 1 & 3.162955 & -0.561209 & 0.360762 & 2.700162 & -1.049107 & 1.569206 \\
\hline 1 & 2.254042 & -0.701481 & -1.952668 & 2.642142 & -1.349617 & -0.885980 \\
\hline 1 & -0.088455 & -1.515567 & -2.327460 & 0.459446 & -1.782569 & -2.017535 \\
\hline 1 & 0.013990 & -2.553730 & -0.807293 & -0.180060 & -2.580211 & -0.500695 \\
\hline 1 & 0.759773 & -2.359890 & 1.259201 & -0.169759 & -2.245705 & 1.608235 \\
\hline 1 & 1.720102 & -1.182662 & 2.307827 & 0.573564 & -1.138995 & 2.863769 \\
\hline 6 & 0.883744 & 1.814481 & 1.207935 & 0.891994 & 1.758272 & 1.347522 \\
\hline 6 & -0.687062 & 1.448632 & -1.107779 & 0.178129 & 1.362094 & -1.313432 \\
\hline 6 & 0.720270 & 1.646842 & -1.245535 & 1.545823 & 1.271488 & -0.951996 \\
\hline 6 & 1.524950 & 1.809308 & -0.067417 & 1.910381 & 1.448931 & 0.413277 \\
\hline 1 & 2.617565 & 1.754933 & -0.159670 & 2.911521 & 1.164737 & 0.732114 \\
\hline 1 & 1.219844 & 1.492108 & -2.210797 & 2.284136 & 0.876772 & -1.647263 \\
\hline 1 & -1.253881 & 1.124320 & -1.987746 & -0.116553 & 1.034451 & -2.307269 \\
\hline 1 & -1.276971 & 2.021689 & -0.381549 & -0.466812 & 2.133534 & -0.891190 \\
\hline 1 & -0.037454 & 2.387455 & 1.358865 & 0.136664 & 2.500899 & 1.101722 \\
\hline 1 & 1.510572 & 1.716107 & 2.103580 & 1.123044 & 1.682179 & 2.408452 \\
\hline 6 & -1.123732 & 0.002942 & 1.595392 & -1.493550 & 0.454223 & 1.081350 \\
\hline 6 & -3.744354 & 0.157139 & -0.122118 & -3.101009 & 0.808564 & -1.529214 \\
\hline 6 & -2.697893 & -0.683837 & -0.324170 & -2.359489 & -0.263447 & -1.201910 \\
\hline 6 & -1.545266 & -0.890222 & 0.581575 & -1.725482 & -0.508905 & 0.094773 \\
\hline 1 & -1.287816 & -1.950154 & 0.718448 & -1.747577 & -1.551196 & 0.413616 \\
\hline 1 & -2.711496 & -1.320480 & -1.223960 & -2.226753 & -1.051238 & -1.947550 \\
\hline 1 & -4.563304 & 0.227521 & -0.848325 & -3.538342 & 0.915187 & -2.517750 \\
\hline 1 & -3.815138 & 0.785731 & 0.774522 & -3.291313 & 1.608946 & -0.815829 \\
\hline 1 & -1.624208 & 0.975470 & 1.683106 & -1.744244 & 1.494396 & 0.872905 \\
\hline 1 & -0.708601 & -0.381609 & 2.533056 & -1.501874 & 0.183638 & 2.132821 \\
\hline 25 & 0.410874 & 0.016126 & 0.061727 & 0.452231 & -0.049843 & 0.247826 \\
\hline
\end{tabular}


Table S21. Optimized coordinates for the $\left(\mathbf{C}_{4} \mathbf{H}_{6}\right)_{3} \mathbf{M n}$ structure $M n-2 D$.

\begin{tabular}{|c|c|c|c|c|c|c|}
\hline & \multicolumn{3}{|c|}{ B3LYP } & \multicolumn{3}{|c|}{ B3LYP* } \\
\hline & $\mathrm{x}$ & $\mathrm{y}$ & $\mathrm{z}$ & $\mathrm{x}$ & $\mathrm{y}$ & $\mathrm{Z}$ \\
\hline 6 & -1.679967 & -1.994153 & -0.034916 & -1.665370 & -1.991403 & -0.034125 \\
\hline 6 & -2.142550 & 1.200757 & 0.871023 & -2.147397 & 1.201260 & 0.878127 \\
\hline 6 & -2.244446 & 0.445711 & -0.447682 & -2.239915 & 0.448016 & -0.442890 \\
\hline 6 & -2.244433 & -0.923690 & -0.772120 & -2.230899 & -0.921801 & -0.776228 \\
\hline 1 & -2.449176 & -1.124002 & -1.830224 & -2.433475 & -1.120178 & -1.837367 \\
\hline 1 & -2.559444 & 1.079143 & -1.278875 & -2.554419 & 1.084306 & -1.274917 \\
\hline 1 & -3.051279 & 1.795943 & 1.048631 & -3.059188 & 1.796719 & 1.052667 \\
\hline 1 & -2.035723 & 0.510402 & 1.712196 & -2.042297 & 0.509815 & 1.721260 \\
\hline 1 & -1.777721 & -2.050052 & 1.045948 & -1.776037 & -2.052923 & 1.047520 \\
\hline 1 & -1.638933 & -2.961168 & -0.537305 & -1.612027 & -2.958502 & -0.539778 \\
\hline 6 & 0.425429 & -0.859931 & 1.558110 & 0.417342 & -0.847286 & 1.550046 \\
\hline 6 & 2.983012 & -0.105241 & -0.168440 & 2.986289 & -0.108904 & -0.171627 \\
\hline 6 & 1.930826 & -1.201663 & -0.378307 & 1.926900 & -1.199047 & -0.382520 \\
\hline 6 & 1.090662 & -1.724888 & 0.625633 & 1.084527 & -1.721255 & 0.623550 \\
\hline 1 & 0.782220 & -2.767392 & 0.558467 & 0.779197 & -2.767245 & 0.564811 \\
\hline 1 & 2.197906 & -1.915970 & -1.162595 & 2.188376 & -1.915020 & -1.170258 \\
\hline 1 & 3.539333 & 0.009954 & -1.109359 & 3.541334 & 0.008584 & -1.115274 \\
\hline 1 & 3.720617 & -0.409828 & 0.590975 & 3.725711 & -0.420410 & 0.585983 \\
\hline 1 & 0.966409 & -0.012438 & 1.974360 & 0.958159 & 0.005036 & 1.961834 \\
\hline 1 & -0.259066 & -1.317543 & 2.273512 & -0.272523 & -1.298357 & 2.267574 \\
\hline 6 & -0.878823 & 2.132692 & 0.776093 & -0.882530 & 2.132103 & 0.781094 \\
\hline 6 & 2.349284 & 1.251147 & 0.180567 & 2.351097 & 1.246225 & 0.182113 \\
\hline 6 & 1.166041 & 1.408124 & -0.741732 & 1.161639 & 1.388771 & -0.735812 \\
\hline 6 & -0.134942 & 1.809805 & -0.518211 & -0.142620 & 1.797474 & -0.513988 \\
\hline 1 & -0.670220 & 2.126379 & -1.413454 & -0.673018 & 2.125364 & -1.410725 \\
\hline 1 & 1.455307 & 1.376365 & -1.796885 & 1.450998 & 1.356884 & -1.793229 \\
\hline 1 & 3.065101 & 2.071576 & 0.008407 & 3.062193 & 2.072523 & 0.005878 \\
\hline 1 & 2.061175 & 1.310677 & 1.232948 & 2.068093 & 1.304343 & 1.238234 \\
\hline 1 & -0.236626 & 1.995455 & 1.650340 & -0.238583 & 1.997027 & 1.657084 \\
\hline 1 & -1.172637 & 3.192543 & 0.758754 & -1.174225 & 3.194602 & 0.760314 \\
\hline \multirow[t]{3}{*}{25} & -0.212423 & -0.453548 & -0.398965 & -0.212418 & -0.451556 & -0.402457 \\
\hline & \multicolumn{3}{|c|}{ BP86 } & \multicolumn{3}{|c|}{ M06L } \\
\hline & $\mathrm{x}$ & $\mathrm{y}$ & $\mathrm{Z}$ & $\mathrm{x}$ & $\mathrm{y}$ & $\mathrm{z}$ \\
\hline 6 & -1.558824 & -2.038848 & 0.069541 & -1.601024 & -1.976928 & 0.058559 \\
\hline 6 & -2.231706 & 1.162394 & 0.798550 & -2.125184 & 1.186677 & 0.826366 \\
\hline 6 & -2.229646 & 0.348301 & -0.493522 & -2.211696 & 0.406702 & -0.465004 \\
\hline 6 & -2.144132 & -1.042658 & -0.771126 & -2.182214 & -0.968869 & -0.753196 \\
\hline 1 & -2.312545 & -1.308387 & -1.831047 & -2.384385 & -1.217817 & -1.800168 \\
\hline 1 & -2.548058 & 0.933635 & -1.369697 & -2.517280 & 1.015431 & -1.317238 \\
\hline 1 & -3.179227 & 1.730050 & 0.910716 & -3.041464 & 1.769739 & 1.002976 \\
\hline 1 & -2.131633 & 0.509381 & 1.681093 & -2.005739 & 0.512610 & 1.679976 \\
\hline 1 & -1.734769 & -2.049127 & 1.152663 & -1.752374 & -1.985475 & 1.137237 \\
\hline 1 & -1.427408 & -3.035732 & -0.376117 & -1.521170 & -2.970022 & -0.384510 \\
\hline 6 & 0.404637 & -0.717595 & 1.592015 & 0.356683 & -0.718752 & 1.568437 \\
\hline 6 & 2.993171 & -0.023269 & -0.152374 & 2.946235 & -0.099965 & -0.124907 \\
\hline 6 & 1.945645 & -1.139123 & -0.312707 & 1.890980 & -1.184778 & -0.301539 \\
\hline 6 & 1.119798 & -1.640168 & 0.735304 & 1.048474 & -1.658134 & 0.729767 \\
\hline 1 & 0.861254 & -2.707156 & 0.754568 & 0.751120 & -2.705325 & 0.738872 \\
\hline 1 & 2.220243 & -1.894751 & -1.068920 & 2.173413 & -1.949391 & -1.031501 \\
\hline 1 & 3.540670 & 0.072804 & -1.110468 & 3.513851 & -0.024894 & -1.061745 \\
\hline 1 & 3.748703 & -0.293108 & 0.614479 & 3.676542 & -0.378018 & 0.649221 \\
\hline 1 & 0.914731 & 0.183885 & 1.956812 & 0.895906 & 0.149679 & 1.947746 \\
\hline 1 & -0.292099 & -1.140674 & 2.331033 & -0.352923 & -1.117353 & 2.296664 \\
\hline 6 & -0.993422 & 2.131788 & 0.690946 & -0.881038 & 2.115948 & 0.695534 \\
\hline 6 & 2.309539 & 1.325110 & 0.150624 & 2.307347 & 1.256838 & 0.148917 \\
\hline 6 & 1.103933 & 1.348903 & -0.764824 & 1.140656 & 1.344356 & -0.786083 \\
\hline 6 & -0.227264 & 1.743911 & -0.579814 & -0.160561 & 1.762312 & -0.590160 \\
\hline 1 & -0.743225 & 2.040899 & -1.504116 & -0.695083 & 2.051850 & -1.494148 \\
\hline 1 & 1.400753 & 1.275682 & -1.826339 & 1.439343 & 1.257505 & -1.835472 \\
\hline 1 & 2.981154 & 2.181042 & -0.073400 & 3.012351 & 2.078128 & -0.049632 \\
\hline 1 & 2.032412 & 1.419971 & 1.213587 & 2.003760 & 1.372503 & 1.193300 \\
\hline 1 & -0.357507 & 2.049350 & 1.587707 & -0.218351 & 1.996032 & 1.558234 \\
\hline 1 & -1.311764 & 3.191596 & 0.619441 & -1.170169 & 3.175455 & 0.672135 \\
\hline 25 & -0.184482 & -0.476473 & -0.377507 & -0.199172 & -0.472923 & -0.397683 \\
\hline
\end{tabular}


Table S22. Optimized coordinates for the $\left(\mathbf{C}_{4} \mathbf{H}_{6}\right)_{3} \mathbf{M n}$ structure $M n-3 D$.

\begin{tabular}{|c|c|c|c|c|c|c|}
\hline & \multicolumn{3}{|c|}{ B3LYP } & \multicolumn{3}{|c|}{ B3LYP* } \\
\hline & $\mathrm{x}$ & $\mathrm{y}$ & $\mathrm{Z}$ & $\mathrm{x}$ & $\mathrm{y}$ & $\mathrm{Z}$ \\
\hline 6 & 0.988608 & -0.640260 & -1.507831 & 0.978613 & -0.644437 & -1.503980 \\
\hline 6 & 3.675208 & -0.464128 & 0.138330 & 3.675413 & -0.452073 & 0.125482 \\
\hline 6 & 2.525068 & -1.057412 & 0.522847 & 2.528357 & -1.048891 & 0.521695 \\
\hline 6 & 1.307585 & -1.271514 & -0.282723 & 1.304136 & -1.266445 & -0.271906 \\
\hline 1 & 0.871775 & -2.255585 & -0.108065 & 0.868612 & -2.251171 & -0.089107 \\
\hline 1 & 2.482926 & -1.468864 & 1.536112 & 2.496458 & -1.458750 & 1.538273 \\
\hline 1 & 4.517988 & -0.378231 & 0.821480 & 4.525571 & -0.362461 & 0.801943 \\
\hline 1 & 3.812199 & -0.074483 & -0.869229 & 3.802762 & -0.064543 & -0.886293 \\
\hline 1 & 1.645250 & 0.140009 & -1.888707 & 1.635706 & 0.135836 & -1.889709 \\
\hline 1 & 0.466506 & -1.194353 & -2.286967 & 0.464988 & -1.209861 & -2.283609 \\
\hline 6 & -2.226918 & -0.447449 & -1.191971 & -2.230664 & -0.426394 & -1.177475 \\
\hline 6 & -1.780509 & -0.482995 & 1.616580 & -1.767450 & -0.491181 & 1.621146 \\
\hline 6 & -1.459953 & -1.601706 & 0.833030 & -1.451381 & -1.607613 & 0.827486 \\
\hline 6 & -1.648103 & -1.577473 & -0.582056 & -1.647418 & -1.569423 & -0.586782 \\
\hline 1 & -1.207397 & -2.374520 & -1.178693 & -1.214676 & -2.364576 & -1.195536 \\
\hline 1 & -0.892501 & -2.421295 & 1.270475 & -0.879889 & -2.431485 & 1.256481 \\
\hline 1 & -1.490223 & -0.473867 & 2.663621 & -1.470148 & -0.489160 & 2.668239 \\
\hline 1 & -2.613655 & 0.167628 & 1.360325 & -2.607721 & 0.158224 & 1.376861 \\
\hline 1 & -3.054656 & 0.078839 & -0.722377 & -3.065749 & 0.084860 & -0.698948 \\
\hline 1 & -2.196439 & -0.373711 & -2.277519 & -2.205442 & -0.339000 & -2.264099 \\
\hline 6 & -1.266222 & 2.073007 & -0.668165 & -1.251915 & 2.065320 & -0.685854 \\
\hline 6 & 0.298342 & 1.344094 & 1.592764 & 0.266998 & 1.339825 & 1.594338 \\
\hline 6 & 0.924488 & 1.692493 & 0.381740 & 0.923092 & 1.677760 & 0.391718 \\
\hline 6 & 0.135414 & 2.088064 & -0.739221 & 0.153404 & 2.073001 & -0.743372 \\
\hline 1 & 0.625950 & 2.215418 & -1.702316 & 0.657533 & 2.196221 & -1.702129 \\
\hline 1 & 1.984394 & 1.506068 & 0.236987 & 1.986568 & 1.484651 & 0.267114 \\
\hline 1 & 0.907242 & 0.881242 & 2.367347 & 0.858622 & 0.873175 & 2.382829 \\
\hline 1 & -0.572417 & 1.885968 & 1.954806 & -0.599431 & 1.902310 & 1.942667 \\
\hline 1 & -1.786418 & 2.303209 & 0.259009 & -1.778685 & 2.313560 & 0.235515 \\
\hline 1 & -1.841133 & 2.236581 & -1.575388 & -1.818267 & 2.227211 & -1.601087 \\
\hline \multirow[t]{3}{*}{25} & -0.426618 & 0.095984 & -0.065464 & -0.432215 & 0.096268 & -0.063140 \\
\hline & \multicolumn{3}{|c|}{ BP86 } & \multicolumn{3}{|c|}{ M06L } \\
\hline & $\mathrm{x}$ & $\mathrm{y}$ & $\mathrm{Z}$ & $\mathrm{X}$ & $\mathrm{y}$ & $\mathrm{Z}$ \\
\hline 6 & 0.926437 & -0.564014 & -1.542186 & 0.921529 & -0.508593 & -1.550278 \\
\hline 6 & 3.708423 & -0.213439 & -0.066344 & 3.635522 & -0.189600 & -0.029273 \\
\hline 6 & 2.624528 & -0.899480 & 0.390743 & 2.575279 & -0.915404 & 0.375791 \\
\hline 6 & 1.363621 & -1.176594 & -0.324961 & 1.342384 & -1.170471 & -0.366720 \\
\hline 1 & 0.996767 & -2.194488 & -0.129304 & 0.969972 & -2.185351 & -0.219867 \\
\hline 1 & 2.689991 & -1.337655 & 1.401198 & 2.635122 & -1.399615 & 1.354473 \\
\hline 1 & 4.600696 & -0.084199 & 0.557791 & 4.511711 & -0.066463 & 0.600872 \\
\hline 1 & 3.741370 & 0.207149 & -1.079676 & 3.662427 & 0.282469 & -1.010931 \\
\hline 1 & 1.523327 & 0.261177 & -1.952535 & 1.520561 & 0.326946 & -1.915694 \\
\hline 1 & 0.431410 & -1.168037 & -2.314835 & 0.429235 & -1.072073 & -2.343015 \\
\hline 6 & -2.244752 & -0.476433 & -1.073595 & -2.203951 & -0.531631 & -1.067659 \\
\hline 6 & -1.649342 & -0.655143 & 1.677825 & -1.651212 & -0.658494 & 1.659861 \\
\hline 6 & -1.309272 & -1.731636 & 0.822142 & -1.265934 & -1.723455 & 0.835677 \\
\hline 6 & -1.571501 & -1.634029 & -0.583216 & -1.507366 & -1.652240 & -0.565633 \\
\hline 1 & -1.137924 & -2.384268 & -1.257376 & -1.025993 & -2.376039 & -1.221134 \\
\hline 1 & -0.679681 & -2.550225 & 1.193239 & -0.602892 & -2.496265 & 1.218772 \\
\hline 1 & -1.306698 & -0.681301 & 2.717538 & -1.327445 & -0.642800 & 2.695307 \\
\hline 1 & -2.550871 & -0.054755 & 1.502090 & -2.545087 & -0.075939 & 1.443129 \\
\hline 1 & -3.109234 & -0.065409 & -0.535449 & -3.066570 & -0.134362 & -0.533610 \\
\hline 1 & -2.272918 & -0.325014 & -2.159865 & -2.229667 & -0.379535 & -2.144629 \\
\hline 6 & -1.363921 & 1.982898 & -0.593676 & -1.422367 & 1.990320 & -0.460508 \\
\hline 6 & 0.187936 & 1.285716 & 1.646940 & 0.310485 & 1.246967 & 1.592627 \\
\hline 6 & 0.846546 & 1.679351 & 0.447466 & 0.851188 & 1.677937 & 0.362769 \\
\hline 6 & 0.049311 & 2.063100 & -0.678494 & -0.040269 & 2.083143 & -0.667975 \\
\hline 1 & 0.536793 & 2.239405 & -1.645001 & 0.353908 & 2.256543 & -1.666711 \\
\hline 1 & 1.926328 & 1.540786 & 0.320924 & 1.905152 & 1.544599 & 0.131498 \\
\hline 1 & 0.800502 & 0.823624 & 2.431340 & 0.984313 & 0.773775 & 2.305113 \\
\hline 1 & -0.689881 & 1.833866 & 2.015390 & -0.535920 & 1.764860 & 2.043660 \\
\hline 1 & -1.885556 & 2.215514 & 0.343854 & -1.849075 & 2.154077 & 0.527706 \\
\hline 1 & -1.955730 & 2.137613 & -1.501942 & -2.100774 & 2.151078 & -1.291943 \\
\hline 25 & -0.442671 & 0.064977 & -0.025730 & -0.438428 & 0.067329 & -0.027403 \\
\hline
\end{tabular}


Table S23. Optimized coordinates for the $\left(\mathbf{C}_{4} \mathbf{H}_{6}\right)_{3} \mathbf{M n}$ structure $M n-1 Q$.

\begin{tabular}{|c|c|c|c|c|c|c|}
\hline & \multicolumn{3}{|c|}{ B3LYP } & \multicolumn{3}{|c|}{ B3LYP* } \\
\hline & $\mathrm{x}$ & $\mathrm{y}$ & $\mathrm{Z}$ & $\mathrm{x}$ & $\mathrm{y}$ & $\mathrm{Z}$ \\
\hline 6 & -0.619074 & 1.180641 & -1.692306 & -0.592313 & 1.183556 & -1.690448 \\
\hline 6 & -2.578322 & 2.273533 & 0.466993 & -2.592267 & 2.250832 & 0.444489 \\
\hline 6 & -1.236086 & 2.436379 & 0.449512 & -1.248085 & 2.414143 & 0.455954 \\
\hline 6 & -0.294496 & 1.993058 & -0.591088 & -0.287452 & 1.989614 & -0.575393 \\
\hline 1 & 0.655994 & 2.524628 & -0.611384 & 0.659161 & 2.532702 & -0.579415 \\
\hline 1 & -0.778456 & 2.958949 & 1.293594 & -0.805749 & 2.921963 & 1.319770 \\
\hline 1 & -3.172739 & 2.634112 & 1.303539 & -3.204601 & 2.595020 & 1.277491 \\
\hline 1 & -3.114898 & 1.788478 & -0.346108 & -3.112558 & 1.781572 & -0.390881 \\
\hline 1 & -1.650825 & 0.909826 & -1.901583 & -1.622030 & 0.912078 & -1.918735 \\
\hline 1 & 0.049974 & 1.155996 & -2.552767 & 0.089006 & 1.172750 & -2.544059 \\
\hline 6 & 3.436503 & -0.305048 & -0.463466 & 3.410885 & -0.325912 & -0.478291 \\
\hline 6 & 1.270281 & -0.543224 & 1.710049 & 1.260096 & -0.540850 & 1.710417 \\
\hline 6 & 1.763998 & 0.660060 & 1.168150 & 1.759538 & 0.660789 & 1.164286 \\
\hline 6 & 2.803978 & 0.735164 & 0.138823 & 2.789922 & 0.723958 & 0.124148 \\
\hline 1 & 3.053275 & 1.743366 & -0.200787 & 3.040442 & 1.730073 & -0.227279 \\
\hline 1 & 1.464571 & 1.604207 & 1.619492 & 1.469680 & 1.609971 & 1.616373 \\
\hline 1 & 0.606234 & -0.493072 & 2.572124 & 0.596873 & -0.484961 & 2.575159 \\
\hline 1 & 1.817336 & -1.475614 & 1.598863 & 1.806061 & -1.476742 & 1.603392 \\
\hline 1 & 3.268851 & -1.337744 & -0.166972 & 3.241729 & -1.356565 & -0.169031 \\
\hline 1 & 4.164052 & -0.129743 & -1.252826 & 4.129857 & -0.160916 & -1.280254 \\
\hline 6 & 0.237152 & -2.253685 & -0.655146 & 0.246703 & -2.234179 & -0.652493 \\
\hline 6 & -2.251059 & -2.088490 & 1.145642 & -2.235162 & -2.082972 & 1.158518 \\
\hline 6 & -2.180952 & -1.658740 & -0.138578 & -2.168143 & -1.633915 & -0.120790 \\
\hline 6 & -1.018184 & -1.729482 & -1.031807 & -1.010194 & -1.703701 & -1.022404 \\
\hline 1 & -1.207950 & -1.521044 & -2.083024 & -1.206996 & -1.495848 & -2.074543 \\
\hline 1 & -3.066030 & -1.178389 & -0.562245 & -3.052200 & -1.137154 & -0.532780 \\
\hline 1 & -3.158502 & -1.950311 & 1.729139 & -3.138525 & -1.943375 & 1.751473 \\
\hline 1 & -1.421296 & -2.593457 & 1.635235 & -1.405699 & -2.604969 & 1.634970 \\
\hline 1 & 0.346385 & -2.850485 & 0.246708 & 0.357745 & -2.834588 & 0.249092 \\
\hline 1 & 0.972392 & -2.453133 & -1.434264 & 0.978557 & -2.435533 & -1.436907 \\
\hline \multirow[t]{3}{*}{25} & 0.206768 & -0.141503 & -0.157096 & 0.214594 & -0.131607 & -0.155415 \\
\hline & \multicolumn{3}{|c|}{ BP86 } & \multicolumn{3}{|c|}{ M06L } \\
\hline & $\mathrm{x}$ & $\mathrm{y}$ & $\mathrm{Z}$ & $\mathrm{x}$ & $\mathrm{y}$ & $\mathrm{Z}$ \\
\hline 6 & 0.563250 & 1.751408 & -1.334754 & 0.828357 & -0.915966 & -1.684288 \\
\hline 6 & -2.053144 & 2.609301 & 0.185221 & 3.198901 & -1.247917 & 0.255071 \\
\hline 6 & -0.816078 & 2.416387 & 0.733050 & 1.983145 & -1.816208 & 0.396212 \\
\hline 6 & 0.439922 & 2.135565 & 0.027230 & 0.872476 & -1.750007 & -0.548276 \\
\hline 1 & 1.353929 & 2.425195 & 0.563348 & 0.144839 & -2.557205 & -0.475473 \\
\hline 1 & -0.725594 & 2.492288 & 1.828414 & 1.790295 & -2.393082 & 1.303644 \\
\hline 1 & -2.924665 & 2.808356 & 0.819400 & 3.957641 & -1.341002 & 1.025674 \\
\hline 1 & -2.216733 & 2.598865 & -0.899404 & 3.468589 & -0.686055 & -0.637829 \\
\hline 1 & -0.303877 & 1.780290 & -2.007664 & 1.713155 & -0.361326 & -1.993871 \\
\hline 1 & 1.538057 & 1.847616 & -1.829934 & 0.128295 & -1.148603 & -2.485680 \\
\hline 6 & 2.337936 & -1.257342 & -0.658709 & -3.173002 & -0.474475 & -0.559512 \\
\hline 6 & 0.678625 & -1.075604 & 1.756872 & -1.348356 & 0.132876 & 1.772744 \\
\hline 6 & 1.760483 & -0.190936 & 1.535775 & -1.481836 & -1.125600 & 1.165232 \\
\hline 6 & 2.542258 & -0.258864 & 0.321037 & -2.359028 & -1.382019 & 0.035273 \\
\hline 1 & 3.183943 & 0.602019 & 0.084921 & -2.293204 & -2.379585 & -0.402363 \\
\hline 1 & 1.904871 & 0.674425 & 2.195178 & -0.945756 & -1.976959 & 1.579687 \\
\hline 1 & -0.002826 & -0.892252 & 2.595045 & -0.745670 & 0.218920 & 2.672830 \\
\hline 1 & 0.694683 & -2.106695 & 1.381876 & -2.113913 & 0.895721 & 1.648090 \\
\hline 1 & 2.044916 & -2.275480 & -0.380851 & -3.303612 & 0.527389 & -0.156423 \\
\hline 1 & 2.845346 & -1.159028 & -1.625217 & -3.746489 & -0.730144 & -1.444537 \\
\hline 6 & -0.392661 & -1.587645 & -1.469735 & -0.941116 & 2.120364 & -0.665870 \\
\hline 6 & -2.604965 & -2.303981 & 0.644453 & 1.317947 & 2.045687 & 1.282601 \\
\hline 6 & -2.363654 & -1.076039 & 0.108892 & 1.474827 & 1.906908 & -0.062896 \\
\hline 6 & -1.435771 & -0.754596 & -0.993991 & 0.403777 & 1.965783 & -1.038074 \\
\hline 1 & -1.756964 & 0.082448 & -1.631550 & 0.670209 & 1.858968 & -2.086358 \\
\hline 1 & -2.923901 & -0.213747 & 0.502155 & 2.457368 & 1.622121 & -0.441514 \\
\hline 1 & -3.325856 & -2.431039 & 1.460558 & 2.147885 & 1.873190 & 1.960613 \\
\hline 1 & -2.100196 & -3.207244 & 0.278956 & 0.381393 & 2.382203 & 1.719896 \\
\hline 1 & -0.240955 & -2.585906 & -1.040345 & -1.204530 & 2.592628 & 0.278917 \\
\hline 1 & -0.004493 & -1.453334 & -2.486928 & -1.698104 & 2.181838 & -1.443439 \\
\hline 25 & 0.440925 & -0.057308 & -0.197600 & -0.218598 & 0.106577 & -0.108447 \\
\hline
\end{tabular}


Table S24. Optimized coordinates for the $\left(\mathbf{C}_{4} \mathbf{H}_{6}\right)_{3} \mathbf{M n}$ structure $M n-2 Q$.

\begin{tabular}{|c|c|c|c|c|c|c|}
\hline & \multicolumn{3}{|c|}{ B3LYP } & \multicolumn{3}{|c|}{ B3LYP* } \\
\hline & $\mathrm{x}$ & $\mathrm{y}$ & $\mathrm{Z}$ & $\mathrm{x}$ & $\mathrm{y}$ & $\mathrm{Z}$ \\
\hline 6 & 1.411743 & -0.770114 & 1.392210 & 1.377932 & -0.767260 & 1.396617 \\
\hline 6 & 2.390443 & 0.472590 & -0.966018 & 2.396230 & 0.485707 & -0.928580 \\
\hline 6 & 3.186069 & -0.626788 & -0.308667 & 3.166594 & -0.641356 & -0.289286 \\
\hline 6 & 2.711801 & -1.228220 & 0.801643 & 2.672897 & -1.247615 & 0.813376 \\
\hline 1 & 3.257925 & -2.051680 & 1.272206 & 3.197009 & -2.092275 & 1.275646 \\
\hline 1 & 4.143459 & -0.939723 & -0.738723 & 4.117231 & -0.973076 & -0.725022 \\
\hline 1 & 2.857564 & 1.456953 & -0.811554 & 2.854300 & 1.464846 & -0.709150 \\
\hline 1 & 2.304322 & 0.330951 & -2.054937 & 2.349171 & 0.396284 & -2.026763 \\
\hline 1 & 0.802986 & -1.561677 & 1.841279 & 0.758437 & -1.546952 & 1.856514 \\
\hline 1 & 1.541641 & 0.044040 & 2.131051 & 1.517520 & 0.056105 & 2.127289 \\
\hline 6 & -0.191364 & -1.289010 & -1.065238 & -0.176735 & -1.254637 & -1.101909 \\
\hline 6 & -3.221401 & -0.562840 & -0.096345 & -3.206692 & -0.577362 & -0.061481 \\
\hline 6 & -2.413096 & -1.829237 & 0.093998 & -2.382783 & -1.840393 & 0.077450 \\
\hline 6 & -1.148441 & -2.115212 & -0.300953 & -1.120646 & -2.105892 & -0.345535 \\
\hline 1 & -0.762244 & -3.088218 & 0.014153 & -0.722378 & -3.085345 & -0.059433 \\
\hline 1 & -2.939173 & -2.616057 & 0.639311 & -2.893863 & -2.648512 & 0.610057 \\
\hline 1 & -3.881258 & -0.463023 & 0.778846 & -3.833495 & -0.495290 & 0.842218 \\
\hline 1 & -3.902235 & -0.694533 & -0.954052 & -3.922516 & -0.703272 & -0.894002 \\
\hline 1 & -0.639387 & -0.728639 & -1.900230 & -0.637922 & -0.682491 & -1.924530 \\
\hline 1 & 0.677051 & -1.852797 & -1.413700 & 0.694146 & -1.805927 & -1.470187 \\
\hline 6 & 0.088162 & 2.559481 & -0.418556 & 0.084867 & 2.547628 & -0.452372 \\
\hline 6 & -2.466214 & 0.769163 & -0.281733 & -2.469199 & 0.762608 & -0.258114 \\
\hline 6 & -1.493971 & 1.104277 & 0.840954 & -1.479493 & 1.107142 & 0.847346 \\
\hline 6 & -0.557984 & 2.145337 & 0.769422 & -0.545125 & 2.151585 & 0.751674 \\
\hline 1 & -0.092340 & 2.448886 & 1.711462 & -0.067818 & 2.470489 & 1.685050 \\
\hline 1 & -1.789472 & 0.763322 & 1.834233 & -1.763623 & 0.777521 & 1.850089 \\
\hline 1 & -3.220964 & 1.572306 & -0.330904 & -3.233670 & 1.560408 & -0.291119 \\
\hline 1 & -1.974552 & 0.790336 & -1.262085 & -1.995517 & 0.790146 & -1.249005 \\
\hline 1 & -0.422418 & 2.499297 & -1.379766 & -0.439359 & 2.470811 & -1.407223 \\
\hline 1 & 0.887724 & 3.292681 & -0.362590 & 0.886937 & 3.282325 & -0.420008 \\
\hline \multirow[t]{3}{*}{25} & 0.535075 & 0.360840 & -0.071132 & 0.529133 & 0.361731 & -0.070622 \\
\hline & \multicolumn{3}{|c|}{ BP86 } & \multicolumn{3}{|c|}{ M06L } \\
\hline & $\mathrm{x}$ & $\mathrm{y}$ & $\mathrm{Z}$ & $\mathrm{x}$ & $\mathrm{y}$ & $\mathrm{Z}$ \\
\hline 6 & 1.281986 & -0.731545 & 1.477549 & 1.669475 & -0.111745 & 1.829149 \\
\hline 6 & 2.461301 & 0.547245 & -0.724957 & 2.573871 & 0.036830 & -0.866083 \\
\hline 6 & 3.046931 & -0.734068 & -0.237725 & 2.208955 & -1.216842 & -0.292170 \\
\hline 6 & 2.470840 & -1.357411 & 0.842485 & 1.764258 & -1.283639 & 1.055214 \\
\hline 1 & 2.821421 & -2.348346 & 1.171116 & 1.289156 & -2.201614 & 1.396912 \\
\hline 1 & 3.869771 & -1.218255 & -0.787589 & 2.085958 & -2.097278 & -0.918713 \\
\hline 1 & 2.707838 & 1.409123 & -0.061356 & 3.150620 & 0.755143 & -0.282448 \\
\hline 1 & 2.718486 & 0.821631 & -1.761237 & 2.757375 & 0.074856 & -1.936464 \\
\hline 1 & 0.660640 & -1.396892 & 2.097064 & 1.160302 & -0.150079 & 2.788138 \\
\hline 1 & 1.521286 & 0.198512 & 2.051548 & 2.409040 & 0.681849 & 1.721571 \\
\hline 6 & -0.183392 & -1.097867 & -1.322253 & -0.499712 & -1.033686 & -1.465407 \\
\hline 6 & -3.131026 & -0.642262 & 0.095456 & -3.077873 & -0.311473 & 0.261297 \\
\hline 6 & -2.246900 & -1.872486 & 0.031261 & -2.249129 & -1.562929 & 0.302838 \\
\hline 6 & -1.017993 & -2.043245 & -0.544087 & -1.162119 & -1.856588 & -0.463664 \\
\hline 1 & -0.549329 & -3.024643 & -0.364092 & -0.680075 & -2.816047 & -0.251143 \\
\hline 1 & -2.664235 & -2.749628 & 0.549875 & -2.562624 & -2.311815 & 1.029435 \\
\hline 1 & -3.622293 & -0.640147 & 1.091038 & -3.562354 & -0.195473 & 1.240561 \\
\hline 1 & -3.966487 & -0.753498 & -0.630461 & -3.909949 & -0.431771 & -0.449934 \\
\hline 1 & -0.738928 & -0.489619 & -2.062563 & -1.151106 & -0.357898 & -2.027385 \\
\hline 1 & 0.680353 & -1.583203 & -1.804619 & 0.139193 & -1.595876 & -2.150223 \\
\hline 6 & 0.059999 & 2.481884 & -0.624256 & 0.462029 & 2.210546 & -0.923645 \\
\hline 6 & -2.483327 & 0.742265 & -0.126018 & -2.359333 & 1.006439 & -0.069736 \\
\hline 6 & -1.394216 & 1.118420 & 0.877381 & -1.119083 & 1.257293 & 0.746831 \\
\hline 6 & -0.478857 & 2.181042 & 0.656383 & -0.076422 & 2.139905 & 0.367675 \\
\hline 1 & 0.058959 & 2.573004 & 1.534828 & 0.538577 & 2.545660 & 1.173293 \\
\hline 1 & -1.606751 & 0.848485 & 1.922510 & -1.262843 & 1.144400 & 1.823840 \\
\hline 1 & -3.288634 & 1.505079 & -0.037030 & -3.069135 & 1.824153 & 0.133447 \\
\hline 1 & -2.121232 & 0.833270 & -1.165542 & -2.140973 & 1.082212 & -1.140036 \\
\hline 1 & -0.533742 & 2.328226 & -1.535188 & -0.138973 & 1.959594 & -1.797491 \\
\hline 1 & 0.867926 & 3.216079 & -0.711134 & 1.342476 & 2.815257 & -1.112800 \\
\hline 25 & 0.514915 & 0.356760 & -0.076179 & 0.591833 & 0.145202 & -0.085374 \\
\hline
\end{tabular}


Table S25. Optimized coordinates for the $\left(\mathbf{C}_{4} \mathbf{H}_{6}\right)_{3} \mathbf{M n}$ structure $M \mathbf{M n}-\mathbf{H}$.

\begin{tabular}{|c|c|c|c|c|c|c|}
\hline & \multicolumn{3}{|c|}{ B3LYP } & \multicolumn{3}{|c|}{ B3LYP* } \\
\hline & $\mathrm{x}$ & $\mathrm{y}$ & $\mathrm{Z}$ & $\mathrm{x}$ & $\mathrm{y}$ & Z \\
\hline 6 & -1.429126 & -1.589116 & 0.948811 & -1.406332 & -1.592522 & 0.955180 \\
\hline 6 & -3.129647 & 1.103652 & 0.303531 & -3.114610 & 1.088574 & 0.317342 \\
\hline 6 & -3.285683 & -0.290333 & -0.280274 & -3.255090 & -0.297965 & -0.287758 \\
\hline 6 & -2.573189 & -1.409363 & 0.024423 & -2.534795 & -1.417029 & 0.015982 \\
\hline 1 & -2.855373 & -2.306511 & -0.536975 & -2.798198 & -2.311896 & -0.561871 \\
\hline 1 & -4.081611 & -0.397353 & -1.018999 & -4.038182 & -0.402083 & -1.043087 \\
\hline 1 & -3.691090 & 1.798670 & -0.336655 & -3.692514 & 1.787293 & -0.306938 \\
\hline 1 & -3.607074 & 1.162690 & 1.295038 & -3.582480 & 1.125782 & 1.316654 \\
\hline 1 & -1.478755 & -0.945676 & 1.837420 & -1.464603 & -0.951043 & 1.846841 \\
\hline 1 & -1.334007 & -2.631941 & 1.275502 & -1.288373 & -2.637958 & 1.270133 \\
\hline 6 & 2.167689 & -1.546330 & -0.964681 & 2.129937 & -1.561979 & -0.971231 \\
\hline 6 & 2.785227 & 1.341710 & 0.261398 & 2.789717 & 1.308064 & 0.269278 \\
\hline 6 & 2.671426 & 0.023239 & 0.997231 & 2.643272 & -0.009241 & 1.001621 \\
\hline 6 & 2.520846 & -1.214720 & 0.416847 & 2.473439 & -1.245315 & 0.413380 \\
\hline 1 & 3.197357 & 1.178712 & -0.745263 & 3.207064 & 1.138351 & -0.736422 \\
\hline 1 & 3.506809 & 1.977285 & 0.793524 & 3.519270 & 1.931911 & 0.808542 \\
\hline 6 & -1.684062 & 1.644547 & 0.445034 & -1.672159 & 1.644944 & 0.452099 \\
\hline 6 & 1.458411 & 2.136345 & 0.131187 & 1.475887 & 2.124031 & 0.129988 \\
\hline 6 & 0.511551 & 1.561612 & -0.897708 & 0.523494 & 1.556868 & -0.898558 \\
\hline 6 & -0.826447 & 1.371279 & -0.773608 & -0.818525 & 1.372476 & -0.769994 \\
\hline 1 & -1.370467 & 1.035425 & -1.660385 & -1.369086 & 1.046925 & -1.659129 \\
\hline 1 & 0.955883 & 1.374894 & -1.879717 & 0.963446 & 1.375840 & -1.885877 \\
\hline 1 & 1.695915 & 3.164572 & -0.189339 & 1.729695 & 3.148984 & -0.195749 \\
\hline 1 & 0.969633 & 2.217157 & 1.109923 & 0.984240 & 2.216934 & 1.108503 \\
\hline 1 & -1.207018 & 1.227991 & 1.340278 & -1.186964 & 1.235457 & 1.348950 \\
\hline 1 & -1.730081 & 2.732079 & 0.612413 & -1.726555 & 2.734758 & 0.616198 \\
\hline 25 & 0.228643 & -0.998457 & -0.231167 & 0.214278 & -0.949186 & -0.235436 \\
\hline 1 & 2.797896 & 0.061595 & 2.079889 & 2.756888 & 0.023479 & 2.087794 \\
\hline 1 & 2.497022 & -0.838838 & -1.733168 & 2.474533 & -0.857119 & -1.737881 \\
\hline 1 & 2.359641 & -2.582691 & -1.248802 & 2.285396 & -2.604663 & -1.260327 \\
\hline \multirow[t]{3}{*}{1} & 2.537262 & -2.061776 & 1.111333 & 2.464058 & -2.096724 & 1.105591 \\
\hline & \multicolumn{3}{|c|}{ BP86 } & \multicolumn{3}{|c|}{ M06L } \\
\hline & $\mathrm{X}$ & $\mathrm{y}$ & $\mathrm{Z}$ & $\mathrm{x}$ & $\mathrm{y}$ & $\mathrm{Z}$ \\
\hline 6 & -1.344127 & -1.643143 & 0.830592 & 1.348970 & -1.559542 & -1.089628 \\
\hline 6 & -3.025687 & 1.087836 & 0.472339 & 3.082610 & 0.934620 & -0.258179 \\
\hline 6 & -3.221040 & -0.242679 & -0.237802 & 3.035762 & -0.405353 & 0.427562 \\
\hline 6 & -2.485810 & -1.395591 & -0.061331 & 2.291773 & -1.476462 & 0.020873 \\
\hline 1 & -2.762103 & -2.238509 & -0.717766 & 2.369121 & -2.371345 & 0.645478 \\
\hline 1 & -4.027629 & -0.274484 & -0.983722 & 3.649207 & -0.523521 & 1.318966 \\
\hline 1 & -3.659825 & 1.837598 & -0.037505 & 3.729770 & 1.602802 & 0.321803 \\
\hline 1 & -3.403035 & 1.036111 & 1.515619 & 3.552672 & 0.852967 & -1.249242 \\
\hline 1 & -1.352289 & -1.065010 & 1.774861 & 1.566020 & -0.891388 & -1.931077 \\
\hline 1 & -1.154162 & -2.711548 & 1.028223 & 1.172061 & -2.578187 & -1.443617 \\
\hline 6 & 1.988275 & -1.699378 & -0.847699 & -1.998389 & -1.464825 & 1.150666 \\
\hline 6 & 2.835641 & 1.163743 & 0.253543 & -2.718691 & 1.235446 & -0.322658 \\
\hline 6 & 2.528263 & -0.079248 & 1.064853 & -2.553842 & -0.129920 & -0.934682 \\
\hline 6 & 2.252814 & -1.338888 & 0.541375 & -2.398673 & -1.304887 & -0.227952 \\
\hline 1 & 3.266052 & 0.885683 & -0.729215 & -3.128682 & 1.142209 & 0.694579 \\
\hline 1 & 3.605031 & 1.762702 & 0.779385 & -3.460761 & 1.798466 & -0.901055 \\
\hline 6 & -1.564169 & 1.639168 & 0.521464 & 1.712200 & 1.617412 & -0.432267 \\
\hline 6 & 1.588532 & 2.066002 & 0.020036 & -1.425129 & 2.064697 & -0.247215 \\
\hline 6 & 0.584180 & 1.474487 & -0.954193 & -0.507088 & 1.599026 & 0.844822 \\
\hline 6 & -0.778221 & 1.317388 & -0.740285 & 0.836426 & 1.441145 & 0.776279 \\
\hline 1 & -1.395009 & 1.049822 & -1.613700 & 1.358556 & 1.174666 & 1.698085 \\
\hline 1 & 0.954287 & 1.327513 & -1.982484 & -0.978982 & 1.467942 & 1.822159 \\
\hline 1 & 1.918829 & 3.042871 & -0.395323 & -1.674455 & 3.116359 & -0.039547 \\
\hline 1 & 1.103682 & 2.281665 & 0.990271 & -0.910644 & 2.058409 & -1.218037 \\
\hline 1 & -1.037763 & 1.249061 & 1.413485 & 1.197084 & 1.227951 & -1.322564 \\
\hline 1 & -1.604512 & 2.740440 & 0.662697 & 1.851300 & 2.690011 & -0.630920 \\
\hline 25 & 0.171774 & -0.761540 & -0.304725 & -0.201271 & -0.820902 & 0.136679 \\
\hline 1 & 2.562039 & 0.022668 & 2.158855 & -2.693992 & -0.203656 & -2.011855 \\
\hline 1 & 2.466295 & -1.091485 & -1.634091 & -2.293054 & -0.670230 & 1.845185 \\
\hline 1 & 1.981571 & -2.775286 & -1.071705 & -2.114728 & -2.461971 & 1.570839 \\
\hline 1 & 2.092295 & -2.139496 & 1.282877 & -2.394298 & -2.217090 & -0.831881 \\
\hline
\end{tabular}


Table S26. Optimized coordinates for the $\left(\mathbf{C}_{4} \mathbf{H}_{6}\right)_{3} \mathbf{M n}$ structure $M n-2 H$.

\begin{tabular}{|c|c|c|c|c|c|c|}
\hline & \multicolumn{3}{|c|}{ B3LYP } & \multicolumn{3}{|c|}{ B3LYP* } \\
\hline & $\mathrm{x}$ & $\mathrm{y}$ & $\mathrm{Z}$ & $\mathrm{x}$ & $\mathrm{y}$ & $\mathrm{Z}$ \\
\hline 6 & -1.959085 & -1.826142 & 0.378173 & -1.923438 & -1.814940 & 0.377136 \\
\hline 6 & -2.668242 & 1.172013 & 0.796303 & -2.673925 & 1.168051 & 0.797753 \\
\hline 6 & -3.380639 & 0.229784 & -0.146476 & -3.377509 & 0.218851 & -0.144692 \\
\hline 6 & -3.034964 & -1.070035 & -0.322840 & -3.010791 & -1.078264 & -0.322702 \\
\hline 1 & -3.586434 & -1.622231 & -1.090497 & -3.552966 & -1.639539 & -1.093282 \\
\hline 1 & -4.183899 & 0.650026 & -0.754629 & -4.188137 & 0.628914 & -0.753298 \\
\hline 1 & -3.389729 & 1.826177 & 1.306557 & -3.400363 & 1.825315 & 1.301062 \\
\hline 1 & -2.158015 & 0.600331 & 1.582435 & -2.166456 & 0.600051 & 1.591339 \\
\hline 1 & -1.981445 & -1.647691 & 1.467517 & -1.935190 & -1.629515 & 1.467457 \\
\hline 1 & -2.067769 & -2.907915 & 0.227750 & -2.005429 & -2.900842 & 0.228819 \\
\hline 6 & 1.959084 & -1.826143 & 0.378172 & 1.923435 & -1.814939 & 0.377137 \\
\hline 6 & 2.668243 & 1.172012 & 0.796303 & 2.673927 & 1.168051 & 0.797753 \\
\hline 6 & 3.380639 & 0.229783 & -0.146476 & 3.377508 & 0.218850 & -0.144692 \\
\hline 6 & 3.034963 & -1.070036 & -0.322841 & 3.010789 & -1.078265 & -0.322702 \\
\hline 1 & 3.586433 & -1.622231 & -1.090498 & 3.552964 & -1.639541 & -1.093282 \\
\hline 1 & 4.183899 & 0.650025 & -0.754629 & 4.188136 & 0.628911 & -0.753299 \\
\hline 1 & 3.389730 & 1.826175 & 1.306558 & 3.400366 & 1.825316 & 1.301060 \\
\hline 1 & 2.158016 & 0.600329 & 1.582436 & 2.166458 & 0.600053 & 1.591340 \\
\hline 1 & 1.981444 & -1.647693 & 1.467516 & 1.935187 & -1.629514 & 1.467458 \\
\hline 1 & 2.067767 & -2.907916 & 0.227748 & 2.005426 & -2.900842 & 0.228821 \\
\hline 6 & -1.623720 & 2.083352 & 0.083412 & -1.623854 & 2.077573 & 0.086993 \\
\hline 6 & 1.623722 & 2.083352 & 0.083413 & 1.623856 & 2.077574 & 0.086993 \\
\hline 6 & 0.679421 & 1.336062 & -0.827830 & 0.681695 & 1.332375 & -0.829218 \\
\hline 6 & -0.679419 & 1.336062 & -0.827830 & -0.681692 & 1.332375 & -0.829218 \\
\hline 1 & -1.182557 & 0.800146 & -1.638133 & -1.186568 & 0.809444 & -1.649838 \\
\hline 1 & 1.182559 & 0.800146 & -1.638132 & 1.186571 & 0.809446 & -1.649838 \\
\hline 1 & 2.169970 & 2.804117 & -0.545282 & 2.168181 & 2.804684 & -0.539591 \\
\hline 1 & 1.074221 & 2.668715 & 0.830684 & 1.070820 & 2.658965 & 0.837836 \\
\hline 1 & -1.074220 & 2.668716 & 0.830682 & -1.070818 & 2.658964 & 0.837836 \\
\hline 1 & -2.169969 & 2.804117 & -0.545284 & -2.168179 & 2.804683 & -0.539591 \\
\hline \multirow[t]{3}{*}{25} & -0.000001 & -1.177749 & -0.092068 & -0.000001 & -1.106587 & -0.109336 \\
\hline & \multicolumn{3}{|c|}{ BP86 } & \multicolumn{3}{|c|}{ M06L } \\
\hline & $\mathrm{x}$ & $\mathrm{y}$ & $\mathrm{Z}$ & $\mathrm{X}$ & $\mathrm{y}$ & $\mathrm{Z}$ \\
\hline 6 & -1.746846 & -1.833134 & 0.406963 & -1.912646 & -1.819806 & 0.343322 \\
\hline 6 & -2.695342 & 1.080520 & 0.810116 & -2.602103 & 1.124152 & 0.809924 \\
\hline 6 & -3.351224 & 0.084515 & -0.120266 & -3.352402 & 0.210604 & -0.112610 \\
\hline 6 & -2.881736 & -1.192704 & -0.300218 & -3.005404 & -1.081695 & -0.318882 \\
\hline 1 & -3.371648 & -1.797229 & -1.082131 & -3.567767 & -1.625355 & -1.082375 \\
\hline 1 & -4.191417 & 0.435778 & -0.736432 & -4.166995 & 0.644237 & -0.692468 \\
\hline 1 & -3.450659 & 1.743332 & 1.275885 & -3.284589 & 1.766247 & 1.380019 \\
\hline 1 & -2.195183 & 0.543475 & 1.640174 & -2.048773 & 0.532060 & 1.554294 \\
\hline 1 & -1.730491 & -1.617302 & 1.498151 & -1.914103 & -1.650087 & 1.435159 \\
\hline 1 & -1.699792 & -2.926310 & 0.261079 & -1.987683 & -2.901300 & 0.188666 \\
\hline 6 & 1.746845 & -1.833134 & 0.406962 & 1.912652 & -1.819803 & 0.343320 \\
\hline 6 & 2.695343 & 1.080519 & 0.810117 & 2.602098 & 1.124154 & 0.809925 \\
\hline 6 & 3.351224 & 0.084514 & -0.120266 & 3.352402 & 0.210610 & -0.112609 \\
\hline 6 & 2.881735 & -1.192705 & -0.300218 & 3.005407 & -1.081689 & -0.318883 \\
\hline 1 & 3.371648 & -1.797230 & -1.082131 & 3.567772 & -1.625346 & -1.082378 \\
\hline 1 & 4.191417 & 0.435777 & -0.736432 & 4.166994 & 0.644248 & -0.692466 \\
\hline 1 & 3.450660 & 1.743331 & 1.275886 & 3.284581 & 1.766250 & 1.380023 \\
\hline 1 & 2.195183 & 0.543475 & 1.640174 & 2.048769 & 0.532059 & 1.554293 \\
\hline 1 & 1.730490 & -1.617303 & 1.498151 & 1.914110 & -1.650085 & 1.435157 \\
\hline 1 & 1.699791 & -2.926310 & 0.261078 & 1.987691 & -2.901297 & 0.188662 \\
\hline 6 & -1.632050 & 1.983532 & 0.095102 & -1.597651 & 2.028384 & 0.062039 \\
\hline 6 & 1.632052 & 1.983532 & 0.095103 & 1.597645 & 2.028385 & 0.062041 \\
\hline 6 & 0.698104 & 1.245895 & -0.848733 & 0.679679 & 1.269531 & -0.846417 \\
\hline 6 & -0.698103 & 1.245895 & -0.848733 & -0.679684 & 1.269531 & -0.846417 \\
\hline 1 & -1.197326 & 0.835379 & -1.742735 & -1.190628 & 0.714156 & -1.640422 \\
\hline 1 & 1.197327 & 0.835379 & -1.742735 & 1.190624 & 0.714156 & -1.640420 \\
\hline 1 & 2.171231 & 2.740131 & -0.512468 & 2.164036 & 2.736430 & -0.559698 \\
\hline 1 & 1.061142 & 2.544979 & 0.858365 & 1.027184 & 2.631393 & 0.778330 \\
\hline 1 & -1.061140 & 2.544979 & 0.858364 & -1.027192 & 2.631393 & 0.778328 \\
\hline 1 & -2.171229 & 2.740132 & -0.512469 & -2.164043 & 2.736428 & -0.559701 \\
\hline 25 & -0.000001 & -0.857117 & -0.137414 & 0.000002 & -1.058789 & -0.078861 \\
\hline
\end{tabular}


Table S27. Optimized coordinates for the $\left(\mathrm{C}_{4} \mathrm{H}_{6}\right)_{3} \mathbf{F e}$ structure Fe-1S.

\begin{tabular}{|c|c|c|c|c|c|c|}
\hline & \multicolumn{3}{|c|}{ B3LYP } & \multicolumn{3}{|c|}{ B3LYP* } \\
\hline & $\mathrm{x}$ & $\mathrm{y}$ & $\mathrm{Z}$ & $\mathrm{x}$ & $\mathrm{y}$ & Z \\
\hline 6 & 1.366613 & -1.440371 & -1.222219 & 1.343343 & -1.427657 & -1.233223 \\
\hline 6 & 2.474530 & 1.256450 & -0.247463 & 2.479467 & 1.244223 & -0.254833 \\
\hline 6 & 2.266865 & -0.017707 & 0.583561 & 2.264791 & -0.028434 & 0.578533 \\
\hline 6 & 2.005269 & -1.284713 & 0.037291 & 1.983751 & -1.293265 & 0.030349 \\
\hline 1 & 2.059087 & -2.144392 & 0.706957 & 2.029619 & -2.159561 & 0.695431 \\
\hline 1 & 2.604525 & 0.012930 & 1.618802 & 2.604431 & -0.001910 & 1.615409 \\
\hline 1 & 3.053206 & 1.992518 & 0.324557 & 3.060860 & 1.983043 & 0.314582 \\
\hline 1 & 3.034033 & 1.054080 & -1.170642 & 3.037183 & 1.037499 & -1.180447 \\
\hline 1 & 1.604737 & -0.757530 & -2.041225 & 1.600779 & -0.746874 & -2.050476 \\
\hline 1 & 1.107970 & -2.444301 & -1.554103 & 1.060980 & -2.425452 & -1.570984 \\
\hline 6 & -0.806927 & -1.541688 & 1.427195 & -0.796098 & -1.513507 & 1.438017 \\
\hline 6 & -2.816617 & 0.187846 & -0.448431 & -2.817131 & 0.188479 & -0.459246 \\
\hline 6 & -1.747695 & -0.852196 & -0.790058 & -1.744484 & -0.852741 & -0.788611 \\
\hline 6 & -1.210932 & -1.827141 & 0.091390 & -1.199912 & -1.817503 & 0.102805 \\
\hline 1 & -3.291755 & -0.061830 & 0.511638 & -3.304906 & -0.063427 & 0.496189 \\
\hline 1 & -3.613245 & 0.186719 & -1.207001 & -3.605280 & 0.191093 & -1.229357 \\
\hline 6 & 1.068513 & 1.808967 & -0.558918 & 1.068801 & 1.787669 & -0.558192 \\
\hline 6 & -2.160743 & 1.570794 & -0.334380 & -2.154108 & 1.567613 & -0.330963 \\
\hline 6 & -1.110211 & 1.466193 & 0.750652 & -1.102343 & 1.434648 & 0.751863 \\
\hline 6 & 0.248029 & 1.695626 & 0.708551 & 0.261203 & 1.677842 & 0.716965 \\
\hline 1 & 0.781478 & 1.855111 & 1.644001 & 0.795715 & 1.853036 & 1.651371 \\
\hline 1 & -1.533519 & 1.366355 & 1.753437 & -1.528981 & 1.331871 & 1.755169 \\
\hline 1 & -2.894064 & 2.349910 & -0.065392 & -2.883482 & 2.350486 & -0.053391 \\
\hline 1 & -1.718762 & 1.855660 & -1.297417 & -1.709281 & 1.859808 & -1.292866 \\
\hline 1 & 0.591134 & 1.156762 & -1.336900 & 0.583352 & 1.115772 & -1.323788 \\
\hline 1 & 1.049418 & 2.806688 & -1.023628 & 1.030369 & 2.775928 & -1.046789 \\
\hline 1 & -1.736585 & -1.145359 & -1.843230 & -1.726702 & -1.154676 & -1.841391 \\
\hline 1 & -1.397314 & -0.891114 & 2.069991 & -1.397466 & -0.869932 & 2.080695 \\
\hline 1 & -0.267090 & -2.323980 & 1.962006 & -0.241218 & -2.285830 & 1.976559 \\
\hline 1 & -0.822705 & -2.744207 & -0.352857 & -0.807341 & -2.739658 & -0.332401 \\
\hline \multirow[t]{3}{*}{26} & 0.151127 & -0.317553 & 0.050691 & 0.149142 & -0.302131 & 0.052912 \\
\hline & \multicolumn{3}{|c|}{ BP86 } & \multicolumn{3}{|c|}{ M06L } \\
\hline & $\mathrm{x}$ & $\mathrm{y}$ & Z & $\mathrm{x}$ & $\mathrm{y}$ & Z \\
\hline 6 & 1.303064 & -1.386179 & -1.265261 & 1.315317 & -1.376298 & -1.240447 \\
\hline 6 & 2.480801 & 1.227930 & -0.271987 & 2.441759 & 1.211610 & -0.270761 \\
\hline 6 & 2.253549 & -0.041214 & 0.572272 & 2.215032 & -0.030204 & 0.586302 \\
\hline 6 & 1.938615 & -1.307029 & 0.016181 & 1.931184 & -1.292692 & 0.035197 \\
\hline 1 & 1.973857 & -2.193015 & 0.667204 & 1.942721 & -2.164268 & 0.690253 \\
\hline 1 & 2.597093 & -0.020982 & 1.615319 & 2.545318 & 0.006909 & 1.623049 \\
\hline 1 & 3.066318 & 1.980161 & 0.288692 & 3.041749 & 1.959370 & 0.260388 \\
\hline 1 & 3.036461 & 1.010395 & -1.204188 & 2.977980 & 0.973340 & -1.198270 \\
\hline 1 & 1.616298 & -0.710656 & -2.076648 & 1.625963 & -0.687478 & -2.030721 \\
\hline 1 & 0.965328 & -2.367885 & -1.622399 & 0.998377 & -2.349563 & -1.611194 \\
\hline 6 & -0.759034 & -1.447271 & 1.472942 & -0.761133 & -1.452461 & 1.447703 \\
\hline 6 & -2.813952 & 0.166136 & -0.488429 & -2.778484 & 0.155383 & -0.468703 \\
\hline 6 & -1.718231 & -0.867100 & -0.786639 & -1.695117 & -0.861478 & -0.788041 \\
\hline 6 & -1.156219 & -1.808201 & 0.137085 & -1.143251 & -1.803699 & 0.121665 \\
\hline 1 & -3.329464 & -0.095135 & 0.457677 & -3.256702 & -0.098600 & 0.489085 \\
\hline 1 & -3.583266 & 0.165035 & -1.286318 & -3.570607 & 0.143068 & -1.228958 \\
\hline 6 & 1.054841 & 1.741690 & -0.557997 & 1.036694 & 1.739187 & -0.551927 \\
\hline 6 & -2.154140 & 1.549384 & -0.331684 & -2.127718 & 1.526803 & -0.339432 \\
\hline 6 & -1.102800 & 1.373292 & 0.753079 & -1.099236 & 1.382795 & 0.750032 \\
\hline 6 & 0.275908 & 1.654988 & 0.736341 & 0.260901 & 1.662590 & 0.731378 \\
\hline 1 & 0.811268 & 1.873505 & 1.669292 & 0.801208 & 1.851412 & 1.656301 \\
\hline 1 & -1.539173 & 1.268659 & 1.759614 & -1.536833 & 1.264097 & 1.744317 \\
\hline 1 & -2.888406 & 2.332715 & -0.040682 & -2.853451 & 2.316295 & -0.085968 \\
\hline 1 & -1.701522 & 1.860908 & -1.291863 & -1.661678 & 1.810380 & -1.292797 \\
\hline 1 & 0.548663 & 1.011098 & -1.292144 & 0.527759 & 1.031601 & -1.281979 \\
\hline 1 & 0.960978 & 2.697136 & -1.116491 & 0.959540 & 2.688707 & -1.102418 \\
\hline 1 & -1.686022 & -1.197246 & -1.837917 & -1.669077 & -1.179986 & -1.833445 \\
\hline 1 & -1.399406 & -0.826766 & 2.112774 & -1.401249 & -0.824630 & 2.067205 \\
\hline 1 & -0.162202 & -2.187839 & 2.026877 & -0.190067 & -2.192044 & 2.011409 \\
\hline 1 & -0.746123 & -2.743522 & -0.272708 & -0.712685 & -2.716554 & -0.293805 \\
\hline 26 & 0.147881 & -0.269043 & 0.058788 & 0.148309 & -0.269280 & 0.051529 \\
\hline
\end{tabular}


Table S28. Optimized coordinates for the $\left(\mathrm{C}_{4} \mathrm{H}_{6}\right)_{3} \mathbf{F e}$ structure Fe-2S.

\begin{tabular}{|c|c|c|c|c|c|c|}
\hline & \multicolumn{3}{|c|}{ B3LYP } & \multicolumn{3}{|c|}{ B3LYP* } \\
\hline & $\mathrm{x}$ & $\mathrm{y}$ & $\mathrm{Z}$ & $\mathrm{x}$ & $\mathrm{y}$ & $\mathrm{Z}$ \\
\hline 6 & 0.990923 & -0.726440 & -1.452067 & 0.982280 & -0.730203 & -1.445406 \\
\hline 6 & 3.694276 & -0.136762 & 0.039304 & 3.693520 & -0.115021 & 0.031993 \\
\hline 6 & 2.623168 & -0.789189 & 0.536083 & 2.629483 & -0.777692 & 0.536747 \\
\hline 6 & 1.404211 & -1.196311 & -0.189343 & 1.405357 & -1.188198 & -0.176503 \\
\hline 1 & 1.065339 & -2.185196 & 0.117629 & 1.068860 & -2.177504 & 0.140065 \\
\hline 1 & 2.659060 & -1.100567 & 1.583776 & 2.678468 & -1.093769 & 1.584717 \\
\hline 1 & 4.553584 & 0.090449 & 0.667423 & 4.559338 & 0.114616 & 0.653502 \\
\hline 1 & 3.754496 & 0.160874 & -1.006419 & 3.742180 & 0.186362 & -1.015290 \\
\hline 1 & 1.564058 & 0.047300 & -1.958615 & 1.553531 & 0.042763 & -1.960224 \\
\hline 1 & 0.457490 & -1.390711 & -2.128243 & 0.459905 & -1.409224 & -2.119379 \\
\hline 6 & -2.044440 & -0.986654 & -1.146198 & -2.026603 & -0.959434 & -1.155477 \\
\hline 6 & -1.886526 & -0.198665 & 1.573394 & -1.896870 & -0.207214 & 1.563763 \\
\hline 6 & -1.308338 & -1.415476 & 1.175627 & -1.310964 & -1.422652 & 1.165629 \\
\hline 6 & -1.427204 & -1.808877 & -0.196740 & -1.413953 & -1.801713 & -0.212751 \\
\hline 1 & -0.848620 & -2.667278 & -0.535045 & -0.833386 & -2.659272 & -0.556616 \\
\hline 1 & -0.668840 & -1.990785 & 1.842169 & -0.675702 & -2.002065 & 1.836124 \\
\hline 1 & -1.665043 & 0.186157 & 2.566267 & -1.682915 & 0.176786 & 2.560731 \\
\hline 1 & -2.836385 & 0.122181 & 1.156737 & -2.846375 & 0.112478 & 1.139921 \\
\hline 1 & -2.911316 & -0.385763 & -0.898378 & -2.911138 & -0.381064 & -0.905500 \\
\hline 1 & -1.937804 & -1.237199 & -2.199744 & -1.909182 & -1.192457 & -2.214000 \\
\hline 6 & -1.612676 & 1.834842 & -0.414338 & -1.609724 & 1.833347 & -0.407884 \\
\hline 6 & 0.589782 & 1.472663 & 1.301806 & 0.570718 & 1.446033 & 1.311392 \\
\hline 6 & 0.787015 & 1.775843 & -0.051880 & 0.787303 & 1.758572 & -0.042314 \\
\hline 6 & -0.314864 & 1.928137 & -0.949731 & -0.309717 & 1.924426 & -0.944870 \\
\hline 1 & -0.134230 & 1.978005 & -2.021344 & -0.126389 & 1.980375 & -2.017842 \\
\hline 1 & 1.787759 & 1.686437 & -0.465745 & 1.793385 & 1.668260 & -0.448353 \\
\hline 1 & 1.459238 & 1.189760 & 1.889972 & 1.433937 & 1.149486 & 1.905969 \\
\hline 1 & -0.244443 & 1.877528 & 1.863168 & -0.258406 & 1.870463 & 1.870545 \\
\hline 1 & -1.835674 & 2.256913 & 0.561268 & -1.831032 & 2.257121 & 0.569787 \\
\hline 1 & -2.458057 & 1.794757 & -1.097350 & -2.457418 & 1.795794 & -1.091229 \\
\hline \multirow[t]{3}{*}{26} & -0.412791 & 0.040326 & -0.049732 & -0.413948 & 0.038437 & -0.049186 \\
\hline & \multicolumn{3}{|c|}{ BP86 } & \multicolumn{3}{|c|}{ M06L } \\
\hline & $\mathrm{x}$ & $\mathrm{y}$ & $\mathrm{Z}$ & $\mathrm{X}$ & $\mathrm{y}$ & $\mathrm{Z}$ \\
\hline 6 & 0.962453 & -0.745314 & -1.425050 & 0.953369 & -0.764357 & -1.415484 \\
\hline 6 & 3.703870 & -0.068608 & 0.010141 & 3.621805 & -0.060200 & 0.026214 \\
\hline 6 & 2.649968 & -0.741825 & 0.546212 & 2.596424 & -0.755940 & 0.551085 \\
\hline 6 & 1.412272 & -1.165853 & -0.135647 & 1.383406 & -1.190520 & -0.138196 \\
\hline 1 & 1.078995 & -2.154150 & 0.213203 & 1.035038 & -2.162925 & 0.210614 \\
\hline 1 & 2.728359 & -1.053772 & 1.600729 & 2.671408 & -1.065520 & 1.596206 \\
\hline 1 & 4.587051 & 0.172995 & 0.613646 & 4.490131 & 0.205891 & 0.621696 \\
\hline 1 & 3.727703 & 0.223661 & -1.047636 & 3.631252 & 0.240403 & -1.020801 \\
\hline 1 & 1.527088 & 0.027006 & -1.964391 & 1.528710 & -0.006898 & -1.948866 \\
\hline 1 & 0.473630 & -1.468513 & -2.091151 & 0.435637 & -1.462139 & -2.071782 \\
\hline 6 & -1.986915 & -0.911511 & -1.173078 & -1.978990 & -0.907136 & -1.146516 \\
\hline 6 & -1.928929 & -0.208693 & 1.527234 & -1.897436 & -0.175188 & 1.507508 \\
\hline 6 & -1.312028 & -1.428012 & 1.152629 & -1.290759 & -1.393795 & 1.159275 \\
\hline 6 & -1.379651 & -1.794364 & -0.237917 & -1.375990 & -1.773504 & -0.216413 \\
\hline 1 & -0.794714 & -2.656676 & -0.585762 & -0.782992 & -2.619826 & -0.561841 \\
\hline 1 & -0.688600 & -2.007566 & 1.844939 & -0.658357 & -1.955794 & 1.842756 \\
\hline 1 & -1.740311 & 0.191663 & 2.530159 & -1.704663 & 0.253799 & 2.487522 \\
\hline 1 & -2.886810 & 0.084979 & 1.083651 & -2.853201 & 0.100879 & 1.067646 \\
\hline 1 & -2.916550 & -0.388930 & -0.925550 & -2.884357 & -0.363225 & -0.891957 \\
\hline 1 & -1.845278 & -1.115433 & -2.241820 & -1.858119 & -1.126725 & -2.205374 \\
\hline 6 & -1.602950 & 1.824065 & -0.404039 & -1.574709 & 1.808551 & -0.347428 \\
\hline 6 & 0.513517 & 1.393764 & 1.332345 & 0.573622 & 1.387238 & 1.273027 \\
\hline 6 & 0.790376 & 1.716485 & -0.025282 & 0.802830 & 1.692237 & -0.083757 \\
\hline 6 & -0.294258 & 1.904010 & -0.949357 & -0.307200 & 1.873594 & -0.961166 \\
\hline 1 & -0.101365 & 1.968222 & -2.027063 & -0.162996 & 1.915410 & -2.037720 \\
\hline 1 & 1.814215 & 1.620799 & -0.404989 & 1.800299 & 1.568281 & -0.500983 \\
\hline 1 & 1.356938 & 1.064898 & 1.951434 & 1.432221 & 1.078821 & 1.866452 \\
\hline 1 & -0.301435 & 1.879639 & 1.878983 & -0.223289 & 1.871385 & 1.831445 \\
\hline 1 & -1.819009 & 2.272603 & 0.572483 & -1.728353 & 2.279048 & 0.621613 \\
\hline 1 & -2.457889 & 1.785901 & -1.089006 & -2.464236 & 1.761926 & -0.970591 \\
\hline 26 & -0.419553 & 0.034915 & -0.046962 & -0.413168 & 0.040051 & -0.045574 \\
\hline
\end{tabular}


Table S29. Optimized coordinates for the $\left(\mathrm{C}_{4} \mathrm{H}_{6}\right)_{3} \mathbf{F e}$ structure Fe-1T.

\begin{tabular}{|c|c|c|c|c|c|c|}
\hline & \multicolumn{3}{|c|}{ B3LYP } & \multicolumn{3}{|c|}{ B3LYP* } \\
\hline & $\mathrm{x}$ & $\mathrm{y}$ & $\mathrm{Z}$ & $\mathrm{x}$ & $\mathrm{y}$ & $\mathrm{Z}$ \\
\hline 6 & -0.893268 & -1.003197 & 1.740627 & -0.860229 & -0.982403 & 1.737354 \\
\hline 6 & -2.892005 & 0.434941 & -0.227642 & -2.902788 & 0.435989 & -0.192620 \\
\hline 6 & -2.144445 & -0.890201 & -0.423380 & -2.149148 & -0.885012 & -0.403475 \\
\hline 6 & -1.536832 & -1.613731 & 0.627842 & -1.511364 & -1.605426 & 0.635005 \\
\hline 1 & -1.343325 & -2.676960 & 0.454575 & -1.304741 & -2.666862 & 0.456316 \\
\hline 1 & -2.482014 & -1.487757 & -1.270302 & -2.490105 & -1.483865 & -1.250720 \\
\hline 1 & -3.417752 & 0.662271 & -1.164888 & -3.452406 & 0.658967 & -1.119244 \\
\hline 1 & -3.670429 & 0.328214 & 0.543909 & -3.662768 & 0.328747 & 0.599497 \\
\hline 1 & -1.272698 & -0.074092 & 2.161326 & -1.248398 & -0.056280 & 2.162462 \\
\hline 1 & -0.373516 & -1.642806 & 2.451165 & -0.315875 & -1.612026 & 2.441036 \\
\hline 6 & 1.537232 & -1.084603 & -1.705180 & 1.522749 & -1.151542 & -1.668971 \\
\hline 6 & 2.527726 & 0.963720 & 0.649165 & 2.547534 & 0.955692 & 0.624544 \\
\hline 6 & 2.041441 & -0.488237 & 0.702560 & 2.014392 & -0.477435 & 0.721343 \\
\hline 6 & 1.950506 & -1.406512 & -0.387873 & 1.908095 & -1.439686 & -0.332876 \\
\hline 1 & 3.125131 & 1.126196 & -0.260213 & 3.138197 & 1.076837 & -0.297792 \\
\hline 1 & 3.193745 & 1.158900 & 1.501046 & 3.228249 & 1.159286 & 1.465591 \\
\hline 6 & -1.976542 & 1.638100 & 0.105167 & -1.970066 & 1.634864 & 0.109140 \\
\hline 6 & 1.359557 & 1.977834 & 0.651364 & 1.392006 & 1.983085 & 0.604067 \\
\hline 6 & 0.548300 & 1.728786 & -0.599454 & 0.562741 & 1.669978 & -0.622584 \\
\hline 6 & -0.788065 & 1.569968 & -0.818705 & -0.787089 & 1.506625 & -0.819632 \\
\hline 1 & -1.071834 & 1.449907 & -1.867520 & -1.083995 & 1.370908 & -1.864888 \\
\hline 1 & 1.162709 & 1.746321 & -1.501800 & 1.162266 & 1.670656 & -1.537624 \\
\hline 1 & 1.751302 & 3.008487 & 0.635412 & 1.787540 & 3.012572 & 0.537196 \\
\hline 1 & 0.759516 & 1.867067 & 1.562285 & 0.803703 & 1.913726 & 1.529326 \\
\hline 1 & -1.670873 & 1.642551 & 1.155829 & -1.659200 & 1.657934 & 1.160477 \\
\hline 1 & -2.524749 & 2.578643 & -0.065696 & -2.499766 & 2.584614 & -0.081489 \\
\hline 1 & 2.175703 & -0.963937 & 1.674378 & 2.111790 & -0.918397 & 1.715835 \\
\hline 1 & 1.806854 & -0.139081 & -2.168243 & 1.837003 & -0.234805 & -2.165653 \\
\hline 1 & 1.371856 & -1.904830 & -2.401468 & 1.332220 & -1.992338 & -2.336901 \\
\hline 1 & 1.923736 & -2.466401 & -0.116624 & 1.842242 & -2.488320 & -0.021131 \\
\hline \multirow[t]{3}{*}{26} & 0.082885 & -0.583612 & -0.123466 & 0.072040 & -0.532681 & -0.143848 \\
\hline & \multicolumn{3}{|c|}{ BP86 } & \multicolumn{3}{|c|}{ M06L } \\
\hline & $\mathrm{x}$ & $\mathrm{y}$ & $\mathrm{Z}$ & $\mathrm{X}$ & $\mathrm{y}$ & $\mathrm{Z}$ \\
\hline 6 & -0.767205 & -0.956163 & 1.728545 & -0.804355 & -0.960692 & 1.702758 \\
\hline 6 & -2.910367 & 0.421403 & -0.117614 & -2.870044 & 0.409709 & -0.148168 \\
\hline 6 & -2.118719 & -0.877740 & -0.370586 & -2.099354 & -0.882006 & -0.405220 \\
\hline 6 & -1.439791 & -1.610970 & 0.649955 & -1.456329 & -1.615605 & 0.621668 \\
\hline 1 & -1.221236 & -2.674734 & 0.465375 & -1.231419 & -2.668359 & 0.437833 \\
\hline 1 & -2.461334 & -1.475111 & -1.227594 & -2.448190 & -1.470769 & -1.253131 \\
\hline 1 & -3.510674 & 0.631947 & -1.022953 & -3.456075 & 0.636200 & -1.046977 \\
\hline 1 & -3.633599 & 0.289987 & 0.712806 & -3.600203 & 0.276192 & 0.662849 \\
\hline 1 & -1.173538 & -0.033726 & 2.164856 & -1.234113 & -0.056587 & 2.134606 \\
\hline 1 & -0.175583 & -1.566400 & 2.420644 & -0.234261 & -1.564079 & 2.404984 \\
\hline 6 & 1.466252 & -1.236015 & -1.611290 & 1.512382 & -1.100684 & -1.635232 \\
\hline 6 & 2.572996 & 0.943177 & 0.578994 & 2.510326 & 0.937322 & 0.618941 \\
\hline 6 & 1.966958 & -0.457293 & 0.745506 & 1.962289 & -0.474562 & 0.740864 \\
\hline 6 & 1.831894 & -1.489228 & -0.250549 & 1.864966 & -1.444166 & -0.302140 \\
\hline 1 & 3.141896 & 1.001633 & -0.370543 & 3.075427 & 1.037438 & -0.319619 \\
\hline 1 & 3.289692 & 1.152563 & 1.397790 & 3.215706 & 1.146298 & 1.433260 \\
\hline 6 & -1.971901 & 1.631373 & 0.136119 & -1.947617 & 1.604737 & 0.129837 \\
\hline 6 & 1.437972 & 1.995962 & 0.540364 & 1.363339 & 1.953368 & 0.600797 \\
\hline 6 & 0.575775 & 1.603502 & -0.647048 & 0.556187 & 1.644890 & -0.629192 \\
\hline 6 & -0.804062 & 1.439913 & -0.812501 & -0.794567 & 1.479957 & -0.819352 \\
\hline 1 & -1.126453 & 1.316277 & -1.859328 & -1.103333 & 1.344990 & -1.858443 \\
\hline 1 & 1.148308 & 1.599295 & -1.588495 & 1.156933 & 1.651413 & -1.540409 \\
\hline 1 & 1.843308 & 3.022944 & 0.408670 & 1.742714 & 2.986365 & 0.564514 \\
\hline 1 & 0.869892 & 1.979211 & 1.488496 & 0.760192 & 1.860931 & 1.514050 \\
\hline 1 & -1.637952 & 1.677437 & 1.187596 & -1.604348 & 1.626920 & 1.170332 \\
\hline 1 & -2.495347 & 2.589442 & -0.069320 & -2.482324 & 2.552872 & -0.031917 \\
\hline 1 & 2.017170 & -0.837191 & 1.775189 & 2.025219 & -0.894998 & 1.744047 \\
\hline 1 & 1.856410 & -0.365951 & -2.153536 & 1.905899 & -0.193585 & -2.091615 \\
\hline 1 & 1.222442 & -2.099163 & -2.244192 & 1.324495 & -1.911048 & -2.336597 \\
\hline 1 & 1.718450 & -2.520913 & 0.118397 & 1.773251 & -2.490168 & -0.000787 \\
\hline 26 & 0.049590 & -0.466734 & -0.193201 & 0.062734 & -0.507063 & -0.170783 \\
\hline
\end{tabular}


Table S30. Optimized coordinates for the $\left(\mathrm{C}_{4} \mathrm{H}_{6}\right)_{3} \mathbf{F e}$ structure Fe-2T.

\begin{tabular}{|c|c|c|c|c|c|c|}
\hline & \multicolumn{3}{|c|}{ B3LYP } & \multicolumn{3}{|c|}{ B3LYP* } \\
\hline & $\mathrm{x}$ & $\mathrm{y}$ & $\mathrm{Z}$ & $\mathrm{x}$ & $\mathrm{y}$ & $\mathrm{Z}$ \\
\hline 6 & -2.034642 & 1.694564 & 0.792162 & -2.034590 & 1.685338 & 0.794450 \\
\hline 6 & 0.922901 & 2.454739 & -0.001623 & 0.920777 & 2.449348 & 0.001267 \\
\hline 6 & -0.159981 & 1.773214 & -0.844974 & -0.158644 & 1.761640 & -0.842243 \\
\hline 6 & -1.544204 & 1.906607 & -0.525759 & -1.546786 & 1.899009 & -0.527074 \\
\hline 1 & -2.273230 & 1.884776 & -1.343506 & -2.277539 & 1.881997 & -1.346123 \\
\hline 1 & 0.068712 & 1.720408 & -1.910678 & 0.073650 & 1.704599 & -1.909123 \\
\hline 1 & 1.453723 & 3.160346 & -0.659037 & 1.447042 & 3.161303 & -0.656414 \\
\hline 1 & 0.438973 & 3.068592 & 0.770682 & 0.432447 & 3.059276 & 0.776770 \\
\hline 1 & -1.416395 & 1.953652 & 1.654901 & -1.413231 & 1.946970 & 1.656824 \\
\hline 1 & -3.107449 & 1.716731 & 0.970498 & -3.108801 & 1.706411 & 0.976301 \\
\hline 6 & -2.034704 & -1.694515 & 0.792131 & -2.034507 & -1.685416 & 0.794471 \\
\hline 6 & 0.922832 & -2.454758 & -0.001599 & 0.920881 & -2.449316 & 0.001251 \\
\hline 6 & -0.160013 & -1.773206 & -0.844976 & -0.158579 & -1.761647 & -0.842243 \\
\hline 6 & -1.544244 & -1.906561 & -0.525786 & -1.546711 & -1.899071 & -0.527058 \\
\hline 1 & -2.273251 & -1.884700 & -1.343548 & -2.277478 & -1.882101 & -1.346095 \\
\hline 1 & 0.068707 & -1.720398 & -1.910673 & 0.073698 & -1.704601 & -1.909126 \\
\hline 1 & 1.453634 & -3.160399 & -0.658994 & 1.447174 & -3.161237 & -0.656444 \\
\hline 1 & 0.438872 & -3.068576 & 0.770712 & 0.432585 & -3.059277 & 0.776750 \\
\hline 1 & -1.416498 & -1.953650 & 1.654884 & -1.413116 & -1.947006 & 1.656835 \\
\hline 1 & -3.107518 & -1.716653 & 0.970433 & -3.108712 & -1.706536 & 0.976342 \\
\hline 6 & 1.979603 & 1.567743 & 0.699224 & 1.984408 & 1.566718 & 0.699133 \\
\hline 6 & 1.979558 & -1.567785 & 0.699239 & 1.984479 & -1.566648 & 0.699122 \\
\hline 6 & 2.748340 & -0.672596 & -0.237699 & 2.753179 & -0.673560 & -0.240191 \\
\hline 6 & 2.748361 & 0.672523 & -0.237705 & 2.753147 & 0.673671 & -0.240187 \\
\hline 1 & 3.349553 & 1.187330 & -0.992869 & 3.353329 & 1.190275 & -0.997857 \\
\hline 1 & 3.349515 & -1.187430 & -0.992859 & 3.353386 & -1.190131 & -0.997864 \\
\hline 1 & 2.686055 & -2.237998 & 1.215488 & 2.690802 & -2.240945 & 1.214629 \\
\hline 1 & 1.491184 & -0.975928 & 1.482401 & 1.499362 & -0.971203 & 1.484498 \\
\hline 1 & 1.491215 & 0.975906 & 1.482392 & 1.499314 & 0.971247 & 1.484502 \\
\hline 1 & 2.686118 & 2.237943 & 1.215464 & 2.690703 & 2.241038 & 1.214649 \\
\hline \multirow[t]{3}{*}{26} & -1.089414 & 0.000009 & -0.036596 & -1.092959 & -0.000018 & -0.039356 \\
\hline & \multicolumn{3}{|c|}{ BP86 } & \multicolumn{3}{|c|}{ M06L } \\
\hline & $\mathrm{X}$ & $\mathrm{y}$ & $\mathrm{Z}$ & $\mathrm{x}$ & $\mathrm{y}$ & $\mathrm{Z}$ \\
\hline 6 & -2.085572 & 1.658670 & 0.771253 & 1.928673 & -1.648402 & 0.838734 \\
\hline 6 & 0.918626 & 2.416130 & 0.095468 & -0.894399 & -2.446101 & -0.059588 \\
\hline 6 & -0.129416 & 1.715774 & -0.780500 & 0.172609 & -1.740561 & -0.882822 \\
\hline 6 & -1.541314 & 1.895303 & -0.535894 & 1.549834 & -1.859014 & -0.517934 \\
\hline 1 & -2.240665 & 1.920559 & -1.390702 & 2.322251 & -1.817403 & -1.291363 \\
\hline 1 & 0.154675 & 1.649576 & -1.842137 & -0.030084 & -1.689360 & -1.953488 \\
\hline 1 & 1.367592 & 3.222491 & -0.521916 & -1.463115 & -3.107287 & -0.728457 \\
\hline 1 & 0.408893 & 2.928833 & 0.934377 & -0.400984 & -3.107709 & 0.664859 \\
\hline 1 & -1.488694 & 1.896177 & 1.666558 & 1.249836 & -1.961466 & 1.637138 \\
\hline 1 & -3.171655 & 1.697967 & 0.916045 & 2.981037 & -1.652265 & 1.109285 \\
\hline 6 & -2.085481 & -1.658758 & 0.771269 & 1.928800 & 1.648262 & 0.838720 \\
\hline 6 & 0.918738 & -2.416088 & 0.095455 & -0.894224 & 2.446152 & -0.059570 \\
\hline 6 & -0.129341 & -1.715759 & -0.780498 & 0.172731 & 1.740546 & -0.882821 \\
\hline 6 & -1.541232 & -1.895373 & -0.535885 & 1.549967 & 1.858909 & -0.517946 \\
\hline 1 & -2.240585 & -1.920699 & -1.390687 & 2.322376 & 1.817290 & -1.291381 \\
\hline 1 & 0.154748 & -1.649552 & -1.842135 & -0.029983 & 1.689377 & -1.953485 \\
\hline 1 & 1.367719 & -3.222428 & -0.521944 & -1.462877 & 3.107402 & -0.728428 \\
\hline 1 & 0.409032 & -2.928816 & 0.934365 & -0.400754 & 3.107701 & 0.664895 \\
\hline 1 & -1.488537 & -1.896181 & 1.666552 & 1.249996 & 1.961374 & 1.637134 \\
\hline 1 & -3.171554 & -1.698142 & 0.916110 & 2.981169 & 1.652062 & 1.109254 \\
\hline 6 & 2.071140 & 1.560365 & 0.690671 & -1.886696 & -1.560861 & 0.702724 \\
\hline 6 & 2.071235 & -1.560296 & 0.690655 & -1.886598 & 1.560985 & 0.702727 \\
\hline 6 & 2.775446 & -0.678126 & -0.312050 & -2.683393 & 0.670553 & -0.194821 \\
\hline 6 & 2.775403 & 0.678248 & -0.312044 & -2.683427 & -0.670370 & -0.194823 \\
\hline 1 & 3.319793 & 1.199714 & -1.116365 & -3.304615 & -1.183805 & -0.932823 \\
\hline 1 & 3.319868 & -1.199551 & -1.116376 & -3.304551 & 1.184024 & -0.932820 \\
\hline 1 & 2.806104 & -2.257066 & 1.146506 & -2.565813 & 2.211982 & 1.271872 \\
\hline 1 & 1.671473 & -0.947832 & 1.519113 & -1.339402 & 0.970221 & 1.451183 \\
\hline 1 & 1.671382 & 0.947863 & 1.519103 & -1.339451 & -0.970142 & 1.451178 \\
\hline 1 & 2.805974 & 2.257148 & 1.146558 & -2.565967 & -2.211800 & 1.271869 \\
\hline 26 & -1.144806 & -0.000023 & -0.068093 & 1.032987 & -0.000030 & -0.041996 \\
\hline
\end{tabular}


Table S31. Optimized coordinates for the $\left(\mathrm{C}_{4} \mathrm{H}_{6}\right)_{3} \mathbf{F e}$ structure Fe-3T.

\begin{tabular}{|c|c|c|c|c|c|c|}
\hline & \multicolumn{3}{|c|}{ B3LYP } & \multicolumn{3}{|c|}{ B3LYP* } \\
\hline & $\mathrm{x}$ & $\mathrm{y}$ & $\mathrm{Z}$ & $\mathrm{x}$ & $\mathrm{y}$ & $\mathrm{Z}$ \\
\hline 6 & -1.911097 & 0.597036 & -1.246160 & -1.883128 & 0.474074 & -1.318522 \\
\hline 6 & -3.095064 & -1.867799 & 0.258065 & -3.003310 & -1.935618 & 0.317078 \\
\hline 6 & -2.707098 & -0.698609 & 0.812687 & -2.684767 & -0.713429 & 0.802577 \\
\hline 6 & -2.227875 & 0.507582 & 0.114940 & -2.234601 & 0.464185 & 0.039517 \\
\hline 1 & -2.355274 & 1.436299 & 0.669832 & -2.413977 & 1.423311 & 0.527617 \\
\hline 1 & -2.757862 & -0.609220 & 1.900622 & -2.774684 & -0.554239 & 1.882108 \\
\hline 1 & -3.431420 & -2.695512 & 0.878781 & -3.323454 & -2.736363 & 0.983361 \\
\hline 1 & -3.103548 & -2.025054 & -0.819044 & -2.969540 & -2.164227 & -0.748700 \\
\hline 1 & -2.073531 & -0.239190 & -1.920169 & -2.014846 & -0.407441 & -1.942993 \\
\hline 1 & -1.870157 & 1.575229 & -1.721716 & -1.856439 & 1.422789 & -1.856066 \\
\hline 6 & 3.254007 & -1.731273 & -0.015976 & 3.336605 & -1.571612 & -0.013490 \\
\hline 6 & 0.427349 & -1.396396 & -1.290428 & 0.488597 & -1.393298 & -1.275297 \\
\hline 6 & 0.723382 & -1.663864 & 0.063156 & 0.805070 & -1.624095 & 0.083140 \\
\hline 6 & 2.075414 & -1.759526 & 0.642386 & 2.163946 & -1.654131 & 0.654453 \\
\hline 1 & 2.100232 & -1.884958 & 1.727703 & 2.202540 & -1.775745 & 1.741894 \\
\hline 1 & -0.063546 & -2.127627 & 0.661663 & 0.042765 & -2.117290 & 0.694165 \\
\hline 1 & -0.489689 & -1.792064 & -1.720781 & -0.406102 & -1.850886 & -1.695765 \\
\hline 1 & 1.224722 & -1.204194 & -2.005280 & 1.276339 & -1.177250 & -1.997542 \\
\hline 1 & 3.310570 & -1.641183 & -1.099365 & 3.379615 & -1.481259 & -1.099420 \\
\hline 1 & 4.195831 & -1.818966 & 0.521453 & 4.287488 & -1.613367 & 0.517300 \\
\hline 6 & 0.917971 & 0.974037 & 1.770515 & 0.804482 & 0.961576 & 1.783365 \\
\hline 6 & 1.573724 & 1.633157 & -1.022215 & 1.508481 & 1.666386 & -0.975671 \\
\hline 6 & 0.663424 & 2.386367 & -0.266093 & 0.548177 & 2.387380 & -0.241816 \\
\hline 6 & 0.328140 & 2.050661 & 1.093764 & 0.189968 & 2.027424 & 1.105699 \\
\hline 1 & -0.512406 & 2.576426 & 1.544837 & -0.683354 & 2.515494 & 1.541273 \\
\hline 1 & 0.052853 & 3.140568 & -0.762062 & -0.080552 & 3.119178 & -0.753131 \\
\hline 1 & 1.669251 & 1.835585 & -2.086438 & 1.620715 & 1.876158 & -2.039092 \\
\hline 1 & 2.407345 & 1.102802 & -0.563308 & 2.364316 & 1.188137 & -0.495318 \\
\hline 1 & 1.934906 & 0.658904 & 1.556165 & 1.846156 & 0.703677 & 1.603996 \\
\hline 1 & 0.528354 & 0.683329 & 2.743735 & 0.396879 & 0.638477 & 2.741241 \\
\hline \multirow[t]{3}{*}{26} & -0.034627 & 0.340022 & -0.192095 & -0.043499 & 0.325300 & -0.206581 \\
\hline & \multicolumn{3}{|c|}{ BP86 } & \multicolumn{3}{|c|}{ M06L } \\
\hline & $\mathrm{x}$ & $\mathrm{y}$ & $\mathrm{Z}$ & $\mathrm{x}$ & $\mathrm{y}$ & $\mathrm{Z}$ \\
\hline 6 & -1.932375 & -1.282011 & 0.220742 & -1.886529 & 0.626422 & -1.224359 \\
\hline 6 & -3.418936 & 1.472746 & 0.404866 & -2.890981 & -1.873118 & 0.243421 \\
\hline 6 & -2.656277 & 1.040995 & -0.636076 & -2.599182 & -0.690771 & 0.822910 \\
\hline 6 & -1.999132 & -0.271736 & -0.772726 & -2.180963 & 0.531985 & 0.145481 \\
\hline 1 & -1.819154 & -0.588001 & -1.814640 & -2.319917 & 1.453238 & 0.708059 \\
\hline 1 & -2.517869 & 1.725166 & -1.488847 & -2.675317 & -0.619299 & 1.909345 \\
\hline 1 & -3.856732 & 2.477533 & 0.405003 & -3.182930 & -2.734286 & 0.836008 \\
\hline 1 & -3.639300 & 0.832117 & 1.268093 & -2.855502 & -2.009346 & -0.836740 \\
\hline 1 & -2.309940 & -1.083194 & 1.233410 & -2.083647 & -0.210099 & -1.892060 \\
\hline 1 & -1.922754 & -2.338001 & -0.072383 & -1.883639 & 1.605085 & -1.700260 \\
\hline 6 & 2.968183 & 2.170876 & 0.189717 & 3.165808 & -1.596561 & 0.025953 \\
\hline 6 & 0.149588 & 1.267003 & 1.183285 & 0.415225 & -1.361679 & -1.315348 \\
\hline 6 & 0.491147 & 1.689973 & -0.129983 & 0.655760 & -1.644092 & 0.050030 \\
\hline 6 & 1.850382 & 2.045299 & -0.577442 & 1.982634 & -1.727620 & 0.656392 \\
\hline 1 & 1.946456 & 2.240572 & -1.657590 & 1.990677 & -1.912739 & 1.732138 \\
\hline 1 & -0.317823 & 2.074458 & -0.766264 & -0.155268 & -2.103470 & 0.618372 \\
\hline 1 & -0.889708 & 1.400181 & 1.521106 & -0.490764 & -1.743593 & -1.782414 \\
\hline 1 & 0.899350 & 1.242755 & 1.981584 & 1.251113 & -1.211272 & -1.997148 \\
\hline 1 & 2.949267 & 2.022179 & 1.276918 & 3.228519 & -1.427377 & -1.048043 \\
\hline 1 & 3.928987 & 2.452417 & -0.256594 & 4.104121 & -1.673064 & 0.566805 \\
\hline 6 & 1.573881 & -0.957028 & -1.372918 & 0.800968 & 0.886387 & 1.759712 \\
\hline 6 & 1.259896 & -1.384264 & 1.505211 & 1.603737 & 1.523281 & -0.922111 \\
\hline 6 & 0.783576 & -2.327124 & 0.563043 & 0.679322 & 2.334065 & -0.234198 \\
\hline 6 & 0.926456 & -2.110685 & -0.858367 & 0.270249 & 1.998757 & 1.096274 \\
\hline 1 & 0.342507 & -2.754845 & -1.531512 & -0.582233 & 2.531404 & 1.513523 \\
\hline 1 & 0.128958 & -3.143104 & 0.894937 & 0.127882 & 3.108268 & -0.764009 \\
\hline 1 & 0.983144 & -1.496705 & 2.559578 & 1.781913 & 1.708435 & -1.977763 \\
\hline 1 & 2.175610 & -0.806597 & 1.317006 & 2.410540 & 1.008992 & -0.395119 \\
\hline 1 & 2.439989 & -0.505312 & -0.872164 & 1.829902 & 0.575968 & 1.589044 \\
\hline 1 & 1.506070 & -0.756414 & -2.449289 & 0.366226 & 0.572281 & 2.705324 \\
\hline 26 & -0.000207 & -0.427672 & 0.005213 & -0.036845 & 0.347636 & -0.246539 \\
\hline
\end{tabular}


Table S32. Optimized coordinates for the $\left(\mathrm{C}_{4} \mathrm{H}_{6}\right)_{3} \mathbf{F e}$ structure Fe-1P.

\begin{tabular}{|c|c|c|c|c|c|c|}
\hline & \multicolumn{3}{|c|}{ B3LYP } & \multicolumn{3}{|c|}{ B3LYP* } \\
\hline & $\mathrm{x}$ & $\mathrm{y}$ & $\mathrm{Z}$ & $\mathrm{x}$ & $\mathrm{y}$ & $\mathrm{Z}$ \\
\hline 6 & -1.041611 & -1.319632 & 1.348170 & -1.003933 & -1.303862 & 1.368366 \\
\hline 6 & -3.097821 & 0.834410 & 0.137040 & -3.089955 & 0.807525 & 0.149605 \\
\hline 6 & -2.895696 & -0.614458 & -0.267707 & -2.853939 & -0.634076 & -0.263815 \\
\hline 6 & -2.051766 & -1.533737 & 0.320951 & -1.992377 & -1.541117 & 0.331212 \\
\hline 1 & -2.099201 & -2.545439 & -0.094385 & -2.015912 & -2.555671 & -0.084439 \\
\hline 1 & -3.531250 & -0.976793 & -1.075988 & -3.472262 & -1.007337 & -1.082930 \\
\hline 1 & -3.719396 & 1.314422 & -0.631072 & -3.729036 & 1.276578 & -0.613401 \\
\hline 1 & -3.676002 & 0.895676 & 1.073603 & -3.663386 & 0.850754 & 1.092537 \\
\hline 1 & -1.221307 & -0.518246 & 2.068665 & -1.192267 & -0.492654 & 2.077603 \\
\hline 1 & -0.658582 & -2.226000 & 1.820480 & -0.583815 & -2.195149 & 1.840336 \\
\hline 6 & 1.784731 & -1.422504 & -1.244709 & 1.743036 & -1.430932 & -1.247912 \\
\hline 6 & 2.775225 & 1.166811 & 0.417224 & 2.781938 & 1.146367 & 0.413217 \\
\hline 6 & 2.598304 & -0.259126 & 0.896680 & 2.590666 & -0.278127 & 0.889487 \\
\hline 6 & 2.250031 & -1.356871 & 0.138861 & 2.206752 & -1.370299 & 0.133910 \\
\hline 1 & 3.214270 & 1.179340 & -0.591975 & 3.217047 & 1.157817 & -0.599920 \\
\hline 1 & 3.488916 & 1.679173 & 1.076474 & 3.503034 & 1.652561 & 1.072450 \\
\hline 6 & -1.805670 & 1.674107 & 0.307351 & -1.810664 & 1.670903 & 0.309810 \\
\hline 6 & 1.456435 & 1.985434 & 0.381431 & 1.464763 & 1.970320 & 0.382306 \\
\hline 6 & 0.559762 & 1.513153 & -0.743467 & 0.565104 & 1.489293 & -0.738194 \\
\hline 6 & -0.810192 & 1.398440 & -0.799552 & -0.815295 & 1.382256 & -0.795386 \\
\hline 1 & -1.249513 & 1.247321 & -1.788906 & -1.255724 & 1.259691 & -1.790557 \\
\hline 1 & 1.081440 & 1.424538 & -1.700106 & 1.083369 & 1.420713 & -1.700915 \\
\hline 1 & 1.695204 & 3.048027 & 0.203945 & 1.705094 & 3.034517 & 0.203599 \\
\hline 1 & 0.950057 & 1.932087 & 1.353361 & 0.962428 & 1.918191 & 1.358896 \\
\hline 1 & -1.347851 & 1.491929 & 1.285856 & -1.349296 & 1.506385 & 1.292324 \\
\hline 1 & -2.071876 & 2.743546 & 0.293778 & -2.090815 & 2.738796 & 0.283616 \\
\hline 1 & 2.772246 & -0.430458 & 1.959735 & 2.762868 & -0.454456 & 1.954107 \\
\hline 1 & 2.185079 & -0.684521 & -1.945439 & 2.150023 & -0.701213 & -1.955564 \\
\hline 1 & 1.763906 & -2.428173 & -1.668979 & 1.684699 & -2.438462 & -1.668808 \\
\hline 1 & 2.195263 & -2.299559 & 0.691511 & 2.133464 & -2.311877 & 0.689888 \\
\hline \multirow[t]{3}{*}{26} & 0.073008 & -0.663194 & -0.295546 & 0.055151 & -0.619565 & -0.306325 \\
\hline & \multicolumn{3}{|c|}{ BP86 } & \multicolumn{3}{|c|}{ M06L } \\
\hline & $\mathrm{x}$ & $\mathrm{y}$ & $\mathrm{Z}$ & $\mathrm{x}$ & $\mathrm{y}$ & $\mathrm{Z}$ \\
\hline 6 & -0.979133 & -1.301724 & 1.354335 & -0.972226 & -1.270971 & 1.403160 \\
\hline 6 & -3.090667 & 0.804879 & 0.188869 & -3.035921 & 0.747216 & 0.159917 \\
\hline 6 & -2.850039 & -0.625814 & -0.262787 & -2.732819 & -0.655160 & -0.304207 \\
\hline 6 & -1.943990 & -1.533958 & 0.293457 & -1.886820 & -1.550353 & 0.325839 \\
\hline 1 & -1.945967 & -2.544231 & -0.149635 & -1.847593 & -2.556182 & -0.101181 \\
\hline 1 & -3.463539 & -0.988050 & -1.098906 & -3.288399 & -1.020319 & -1.166134 \\
\hline 1 & -3.761338 & 1.285957 & -0.547916 & -3.714217 & 1.208230 & -0.567174 \\
\hline 1 & -3.638140 & 0.823530 & 1.155757 & -3.587798 & 0.727964 & 1.111909 \\
\hline 1 & -1.176196 & -0.489751 & 2.070332 & -1.220281 & -0.452585 & 2.082692 \\
\hline 1 & -0.525483 & -2.192152 & 1.810357 & -0.525603 & -2.129288 & 1.902530 \\
\hline 6 & 1.694744 & -1.464136 & -1.230930 & 1.701262 & -1.404544 & -1.268731 \\
\hline 6 & 2.807960 & 1.116982 & 0.393029 & 2.739570 & 1.106299 & 0.424117 \\
\hline 6 & 2.568282 & -0.291357 & 0.897112 & 2.513397 & -0.304625 & 0.886104 \\
\hline 6 & 2.124123 & -1.391240 & 0.158583 & 2.133497 & -1.379358 & 0.110887 \\
\hline 1 & 3.241929 & 1.096481 & -0.627666 & 3.167544 & 1.110524 & -0.590053 \\
\hline 1 & 3.546894 & 1.620019 & 1.045902 & 3.474524 & 1.592210 & 1.076054 \\
\hline 6 & -1.801800 & 1.670825 & 0.322110 & -1.795788 & 1.646481 & 0.307119 \\
\hline 6 & 1.498744 & 1.961639 & 0.346143 & 1.441952 & 1.935404 & 0.394325 \\
\hline 6 & 0.588164 & 1.435124 & -0.751683 & 0.570631 & 1.463436 & -0.737350 \\
\hline 6 & -0.818148 & 1.329753 & -0.784988 & -0.817118 & 1.360180 & -0.798922 \\
\hline 1 & -1.272890 & 1.229574 & -1.784485 & -1.258522 & 1.266732 & -1.793805 \\
\hline 1 & 1.086268 & 1.392411 & -1.734933 & 1.094106 & 1.420775 & -1.696490 \\
\hline 1 & 1.747910 & 3.025535 & 0.136715 & 1.676606 & 3.002924 & 0.256200 \\
\hline 1 & 1.001641 & 1.939406 & 1.334542 & 0.920448 & 1.856042 & 1.359075 \\
\hline 1 & -1.332543 & 1.525781 & 1.312651 & -1.314899 & 1.495407 & 1.282191 \\
\hline 1 & -2.076897 & 2.746603 & 0.274083 & -2.096098 & 2.705329 & 0.293100 \\
\hline 1 & 2.713821 & -0.453029 & 1.974843 & 2.638243 & -0.489976 & 1.952743 \\
\hline 1 & 2.119127 & -0.753205 & -1.955687 & 2.115099 & -0.649852 & -1.942910 \\
\hline 1 & 1.565404 & -2.478507 & -1.635344 & 1.639868 & -2.393275 & -1.722464 \\
\hline 1 & 2.009192 & -2.322514 & 0.736576 & 2.017578 & -2.320714 & 0.654294 \\
\hline 26 & 0.052745 & -0.566527 & -0.302180 & 0.036603 & -0.559153 & -0.300159 \\
\hline
\end{tabular}


Table S33. Optimized coordinates for the $\left(\mathrm{C}_{4} \mathbf{H}_{6}\right)_{3} \mathbf{F e}$ structure Fe-2P.

\begin{tabular}{|c|c|c|c|c|c|c|}
\hline & \multicolumn{3}{|c|}{ B3LYP } & \multicolumn{3}{|c|}{ B3LYP* } \\
\hline & $\mathrm{x}$ & $\mathrm{y}$ & $\mathrm{z}$ & $\mathrm{x}$ & $\mathrm{y}$ & $\mathrm{z}$ \\
\hline 6 & 1.993346 & -1.897726 & -0.574088 & 2.003008 & -1.911025 & -0.505666 \\
\hline 6 & 2.717753 & 1.127786 & 0.029806 & 2.708926 & 1.134303 & 0.039106 \\
\hline 6 & 2.260564 & 0.088055 & 1.024918 & 2.226774 & 0.119572 & 1.046285 \\
\hline 6 & 2.057618 & -1.246351 & 0.734943 & 2.017606 & -1.220876 & 0.786036 \\
\hline 1 & 1.885205 & -1.898170 & 1.601030 & 1.791129 & -1.844995 & 1.661378 \\
\hline 1 & 2.140127 & 0.404914 & 2.060901 & 2.063096 & 0.466899 & 2.068668 \\
\hline 1 & 3.317986 & 1.880483 & 0.561620 & 3.315224 & 1.890827 & 0.562742 \\
\hline 1 & 3.384127 & 0.656559 & -0.706916 & 3.374489 & 0.641765 & -0.687296 \\
\hline 1 & 2.447934 & -1.365255 & -1.414297 & 2.468823 & -1.389439 & -1.349246 \\
\hline 1 & 2.236395 & -2.963888 & -0.573947 & 2.242571 & -2.978780 & -0.479096 \\
\hline 6 & -1.993582 & -1.897556 & -0.574018 & -2.002758 & -1.911247 & -0.505627 \\
\hline 6 & -2.717611 & 1.128071 & 0.029762 & -2.709074 & 1.133992 & 0.039046 \\
\hline 6 & -2.260540 & 0.088322 & 1.024910 & -2.226841 & 0.119349 & 1.046275 \\
\hline 6 & -2.057755 & -1.246120 & 0.734986 & -2.017480 & -1.221074 & 0.786064 \\
\hline 1 & -1.885401 & -1.897923 & 1.601096 & -1.790947 & -1.845144 & 1.661425 \\
\hline 1 & -2.140053 & 0.405208 & 2.060878 & -2.063253 & 0.466725 & 2.068656 \\
\hline 1 & -3.317786 & 1.880836 & 0.561545 & -3.315484 & 1.890462 & 0.562633 \\
\hline 1 & -3.384011 & 0.656891 & -0.706966 & -3.374547 & 0.641354 & -0.687370 \\
\hline 1 & -2.448132 & -1.365073 & -1.414240 & -2.468632 & -1.389736 & -1.349222 \\
\hline 1 & -2.236741 & -2.963692 & -0.573828 & -2.242196 & -2.979030 & -0.479039 \\
\hline 6 & 1.596641 & 1.869104 & -0.747462 & 1.594842 & 1.871935 & -0.755258 \\
\hline 6 & -1.596409 & 1.869268 & -0.747491 & -1.595040 & 1.871728 & -0.755292 \\
\hline 6 & -0.672466 & 2.664059 & 0.140649 & -0.673911 & 2.689976 & 0.115413 \\
\hline 6 & 0.672764 & 2.663988 & 0.140662 & 0.673588 & 2.690062 & 0.115428 \\
\hline 1 & 1.178071 & 3.323347 & 0.852831 & 1.180552 & 3.367316 & 0.812793 \\
\hline 1 & -1.177718 & 3.323472 & 0.852808 & -1.180977 & 3.367167 & 0.812764 \\
\hline 1 & -2.082462 & 2.556768 & -1.459049 & -2.090004 & 2.542393 & -1.479728 \\
\hline 1 & -1.036090 & 1.151165 & -1.360017 & -1.027626 & 1.143127 & -1.351735 \\
\hline 1 & 1.036259 & 1.151061 & -1.360000 & 1.027534 & 1.143289 & -1.351747 \\
\hline 1 & 2.082779 & 2.556555 & -1.459008 & 2.089763 & 2.542674 & -1.479655 \\
\hline \multirow[t]{3}{*}{26} & -0.000093 & -1.490718 & -0.247304 & 0.000102 & -1.533733 & -0.316453 \\
\hline & \multicolumn{3}{|c|}{ BP86 } & \multicolumn{3}{|c|}{ M06L } \\
\hline & $\mathrm{x}$ & $\mathrm{y}$ & $\mathrm{z}$ & $\mathrm{x}$ & $\mathrm{y}$ & $\mathrm{z}$ \\
\hline 6 & 1.982064 & -1.902094 & -0.561519 & 1.965097 & -1.823021 & -0.606787 \\
\hline 6 & 2.677138 & 1.115271 & 0.080349 & 2.688984 & 1.068930 & 0.105239 \\
\hline 6 & 2.149366 & 0.088943 & 1.055034 & 2.190669 & 0.049336 & 1.083497 \\
\hline 6 & 1.952416 & -1.261112 & 0.750899 & 1.974124 & -1.268090 & 0.734495 \\
\hline 1 & 1.745014 & -1.921272 & 1.614548 & 1.742963 & -1.959497 & 1.553798 \\
\hline 1 & 1.945410 & 0.416923 & 2.083197 & 2.030220 & 0.350851 & 2.117054 \\
\hline 1 & 3.271528 & 1.865434 & 0.639526 & 3.229441 & 1.861597 & 0.638351 \\
\hline 1 & 3.371453 & 0.623399 & -0.629625 & 3.414546 & 0.595397 & -0.570719 \\
\hline 1 & 2.463142 & -1.358307 & -1.390593 & 2.479451 & -1.253048 & -1.385632 \\
\hline 1 & 2.203496 & -2.981732 & -0.567738 & 2.146761 & -2.896757 & -0.678393 \\
\hline 6 & -1.982056 & -1.902113 & -0.561503 & -1.965557 & -1.822689 & -0.606668 \\
\hline 6 & -2.677143 & 1.115257 & 0.080340 & -2.688709 & 1.069491 & 0.105154 \\
\hline 6 & -2.149364 & 0.088939 & 1.055032 & -2.190650 & 0.049844 & 1.083486 \\
\hline 6 & -1.952407 & -1.261118 & 0.750909 & -1.974425 & -1.267658 & 0.734572 \\
\hline 1 & -1.744999 & -1.921268 & 1.614562 & -1.743384 & -1.959058 & 1.553915 \\
\hline 1 & -1.945406 & 0.416928 & 2.083191 & -2.030102 & 0.351400 & 2.117015 \\
\hline 1 & -3.271543 & 1.865415 & 0.639511 & -3.229025 & 1.862299 & 0.638198 \\
\hline 1 & -3.371450 & 0.623374 & -0.629635 & -3.414336 & 0.596079 & -0.570818 \\
\hline 1 & -2.463142 & -1.358336 & -1.390580 & -2.479819 & -1.252669 & -1.385540 \\
\hline 1 & -2.203482 & -2.981751 & -0.567712 & -2.147442 & -2.896393 & -0.678190 \\
\hline 6 & 1.598745 & 1.875668 & -0.751586 & 1.584764 & 1.720616 & -0.743634 \\
\hline 6 & -1.598754 & 1.875661 & -0.751592 & -1.584301 & 1.720903 & -0.743686 \\
\hline 6 & -0.677706 & 2.722021 & 0.094362 & -0.670122 & 2.578049 & 0.072286 \\
\hline 6 & 0.677690 & 2.722024 & 0.094365 & 0.670717 & 2.577922 & 0.072313 \\
\hline 1 & 1.188409 & 3.419019 & 0.779867 & 1.177106 & 3.280678 & 0.738941 \\
\hline 1 & -1.188431 & 3.419015 & 0.779862 & -1.176406 & 3.280901 & 0.738891 \\
\hline 1 & -2.129920 & 2.531527 & -1.474071 & -2.052246 & 2.330045 & -1.530827 \\
\hline 1 & -1.026291 & 1.149522 & -1.360869 & -1.023935 & 0.934282 & -1.276820 \\
\hline 1 & 1.026287 & 1.149523 & -1.360861 & 1.024263 & 0.934136 & -1.276830 \\
\hline 1 & 2.129907 & 2.531535 & -1.474066 & 2.052888 & 2.329692 & -1.530720 \\
\hline 26 & 0.000003 & -1.505886 & -0.284579 & -0.000173 & -1.323528 & -0.267433 \\
\hline
\end{tabular}


Table S34. Optimized coordinates for the $\left(\mathrm{C}_{4} \mathrm{H}_{6}\right)_{3} \mathrm{Co}$ structure $\mathrm{Co-1D}$.

\begin{tabular}{|c|c|c|c|c|c|c|}
\hline & \multicolumn{3}{|c|}{ B3LYP } & \multicolumn{3}{|c|}{ B3LYP* } \\
\hline & $\mathrm{x}$ & $\mathrm{y}$ & $\mathrm{z}$ & $\mathrm{x}$ & $\mathrm{y}$ & $\mathrm{z}$ \\
\hline 6 & 0.904283 & -1.122279 & -1.790006 & 0.887673 & -1.115171 & -1.793147 \\
\hline 6 & 2.589620 & 0.624585 & 0.463849 & 2.587490 & 0.623826 & 0.459256 \\
\hline 6 & 2.028346 & -0.801226 & 0.452465 & 2.022999 & -0.800966 & 0.447492 \\
\hline 6 & 1.690639 & -1.570363 & -0.693890 & 1.681018 & -1.569776 & -0.701453 \\
\hline 1 & 1.749556 & -2.657801 & -0.565478 & 1.741819 & -2.659783 & -0.577399 \\
\hline 1 & 2.426619 & -1.395445 & 1.276842 & 2.423503 & -1.398514 & 1.271142 \\
\hline 1 & 2.759465 & 0.904277 & 1.513382 & 2.758809 & 0.903991 & 1.510549 \\
\hline 1 & 3.589131 & 0.582174 & 0.000531 & 3.588539 & 0.579278 & -0.005457 \\
\hline 1 & 0.996636 & -0.114656 & -2.187787 & 0.980867 & -0.104711 & -2.189255 \\
\hline 1 & 0.580081 & -1.862500 & -2.519053 & 0.557636 & -1.853352 & -2.524603 \\
\hline 6 & -1.071101 & -0.724032 & 1.890374 & -1.050356 & -0.718624 & 1.892746 \\
\hline 6 & -2.476631 & 0.772267 & -0.645982 & -2.477501 & 0.768624 & -0.640876 \\
\hline 6 & -2.085397 & -0.695355 & -0.410088 & -2.080072 & -0.697518 & -0.403852 \\
\hline 6 & -1.856227 & -1.297470 & 0.859401 & -1.843440 & -1.298673 & 0.867931 \\
\hline 1 & -3.055563 & 1.128692 & 0.219800 & -3.056374 & 1.124406 & 0.227779 \\
\hline 1 & -3.169468 & 0.780991 & -1.497831 & -3.173608 & 0.774758 & -1.492641 \\
\hline 6 & 1.812698 & 1.788058 & -0.207777 & 1.810859 & 1.788719 & -0.212180 \\
\hline 6 & -1.369296 & 1.823673 & -0.942345 & -1.373164 & 1.823817 & -0.939814 \\
\hline 6 & -0.663887 & 2.299540 & 0.302591 & -0.666116 & 2.300419 & 0.303913 \\
\hline 6 & 0.645582 & 2.274945 & 0.611787 & 0.646103 & 2.276305 & 0.610482 \\
\hline 1 & 0.924299 & 2.657198 & 1.598748 & 0.927901 & 2.658407 & 1.598945 \\
\hline 1 & -1.338155 & 2.710319 & 1.060936 & -1.340498 & 2.710939 & 1.065323 \\
\hline 1 & -1.863802 & 2.694533 & -1.402187 & -1.872001 & 2.694882 & -1.399527 \\
\hline 1 & -0.668834 & 1.429262 & -1.685461 & -0.672027 & 1.430099 & -1.685667 \\
\hline 1 & 1.509317 & 1.529535 & -1.225426 & 1.503988 & 1.528777 & -1.230683 \\
\hline 1 & 2.521718 & 2.625130 & -0.306813 & 2.522109 & 2.626269 & -0.314042 \\
\hline 1 & -2.501570 & -1.374219 & -1.155097 & -2.498397 & -1.380535 & -1.146909 \\
\hline 1 & -1.046150 & 0.352125 & 2.044838 & -1.027532 & 0.360189 & 2.043666 \\
\hline 1 & -0.838845 & -1.329047 & 2.764282 & -0.809678 & -1.321004 & 2.768617 \\
\hline 1 & -2.019195 & -2.379923 & 0.916776 & -2.006668 & -2.382961 & 0.930403 \\
\hline \multirow[t]{3}{*}{27} & -0.053593 & -0.982026 & 0.066916 & -0.052642 & -0.984335 & 0.066918 \\
\hline & \multicolumn{3}{|c|}{ BP86 } & \multicolumn{3}{|c|}{ M06L } \\
\hline & $\mathrm{x}$ & $\mathrm{y}$ & $\mathrm{Z}$ & $\mathrm{x}$ & $\mathrm{y}$ & $\mathrm{Z}$ \\
\hline 6 & 0.839242 & -1.098802 & -1.802729 & 0.804529 & -1.068057 & -1.780223 \\
\hline 6 & 2.595665 & 0.616884 & 0.436735 & 2.632650 & 0.535827 & 0.401741 \\
\hline 6 & 2.011021 & -0.802758 & 0.435045 & 1.994838 & -0.841961 & 0.429331 \\
\hline 6 & 1.651337 & -1.575357 & -0.720469 & 1.589350 & -1.584709 & -0.713195 \\
\hline 1 & 1.705109 & -2.673544 & -0.600795 & 1.565639 & -2.673104 & -0.586672 \\
\hline 1 & 2.415885 & -1.409495 & 1.258911 & 2.382567 & -1.462116 & 1.238036 \\
\hline 1 & 2.787713 & 0.898493 & 1.491042 & 2.884620 & 0.809980 & 1.434807 \\
\hline 1 & 3.594672 & 0.560899 & -0.046971 & 3.596742 & 0.455015 & -0.123135 \\
\hline 1 & 0.949971 & -0.083535 & -2.203270 & 0.977965 & -0.072181 & -2.184946 \\
\hline 1 & 0.486224 & -1.833365 & -2.537298 & 0.415582 & -1.774506 & -2.509730 \\
\hline 6 & -1.000270 & -0.712935 & 1.901573 & -1.070620 & -0.689772 & 1.882468 \\
\hline 6 & -2.485560 & 0.764812 & -0.628305 & -2.444338 & 0.819266 & -0.657627 \\
\hline 6 & -2.066208 & -0.698792 & -0.394045 & -2.066928 & -0.638279 & -0.425027 \\
\hline 6 & -1.813760 & -1.306172 & 0.885085 & -1.853053 & -1.252719 & 0.841593 \\
\hline 1 & -3.066691 & 1.114554 & 0.249766 & -3.024550 & 1.182232 & 0.204265 \\
\hline 1 & -3.190086 & 0.766249 & -1.481718 & -3.128845 & 0.851135 & -1.512747 \\
\hline 6 & 1.813338 & 1.788982 & -0.224867 & 1.847775 & 1.709121 & -0.205703 \\
\hline 6 & -1.386492 & 1.831181 & -0.929258 & -1.307424 & 1.825600 & -0.915463 \\
\hline 6 & -0.669518 & 2.292712 & 0.316667 & -0.603006 & 2.190188 & 0.351675 \\
\hline 6 & 0.653683 & 2.265675 & 0.614551 & 0.702094 & 2.126271 & 0.655709 \\
\hline 1 & 0.943338 & 2.634846 & 1.613114 & 0.983652 & 2.401287 & 1.675906 \\
\hline 1 & -1.341469 & 2.694591 & 1.094540 & -1.274987 & 2.526696 & 1.147052 \\
\hline 1 & -1.895504 & 2.710537 & -1.378034 & -1.749102 & 2.735745 & -1.346701 \\
\hline 1 & -0.686382 & 1.443273 & -1.689584 & -0.613302 & 1.426100 & -1.665171 \\
\hline 1 & 1.494874 & 1.531704 & -1.248469 & 1.518157 & 1.490746 & -1.226167 \\
\hline 1 & 2.527088 & 2.632946 & -0.329356 & 2.541064 & 2.557783 & -0.293080 \\
\hline 1 & -2.486011 & -1.391839 & -1.137202 & -2.467724 & -1.315506 & -1.178860 \\
\hline 1 & -0.992449 & 0.373029 & 2.056379 & -1.084574 & 0.381559 & 2.075620 \\
\hline 1 & -0.738432 & -1.316969 & 2.778597 & -0.848728 & -1.308101 & 2.748469 \\
\hline 1 & -1.971528 & -2.398359 & 0.950501 & -1.990489 & -2.339061 & 0.872880 \\
\hline 27 & -0.051526 & -0.979503 & 0.067406 & -0.075514 & -0.913273 & 0.075500 \\
\hline
\end{tabular}


Table S35. Optimized coordinates for the $\left(\mathrm{C}_{4} \mathbf{H}_{6}\right)_{3} \mathrm{Co}$ structure $\mathrm{Co-2D}$.

\begin{tabular}{|c|c|c|c|c|c|c|}
\hline & \multicolumn{3}{|c|}{$\overline{\text { B3LYP }}$} & \multicolumn{3}{|c|}{ B3LYP* } \\
\hline & $\mathrm{x}$ & $\mathrm{y}$ & $\mathrm{Z}$ & $\mathrm{x}$ & $\mathrm{y}$ & $\mathrm{Z}$ \\
\hline 6 & -0.874360 & -2.182707 & -0.214445 & -0.885586 & -2.167158 & -0.222322 \\
\hline 6 & -2.147261 & 0.232024 & 1.408423 & -2.159146 & 0.240978 & 1.410389 \\
\hline 6 & -2.456098 & -0.269755 & -0.001098 & -2.456801 & -0.245634 & -0.008159 \\
\hline 6 & -2.041207 & -1.464650 & -0.605345 & -2.045374 & -1.440260 & -0.621762 \\
\hline 1 & -2.471619 & -1.684381 & -1.585918 & -2.471191 & -1.652261 & -1.608231 \\
\hline 1 & -3.242257 & 0.278760 & -0.521956 & -3.235565 & 0.314257 & -0.532221 \\
\hline 1 & -3.078715 & 0.368478 & 1.976031 & -3.099188 & 0.408900 & 1.958740 \\
\hline 1 & -1.557499 & -0.510363 & 1.958782 & -1.606140 & -0.524091 & 1.971515 \\
\hline 1 & -0.624267 & -2.280425 & 0.842169 & -0.645643 & -2.268212 & 0.838545 \\
\hline 1 & -0.554461 & -3.020646 & -0.830811 & -0.561566 & -3.005624 & -0.839294 \\
\hline 6 & 2.602262 & -1.099574 & 1.704863 & 2.583398 & -1.094337 & 1.716905 \\
\hline 6 & 2.478647 & 0.674639 & -0.733123 & 2.486487 & 0.642812 & -0.747313 \\
\hline 6 & 1.667394 & -0.633171 & -0.638534 & 1.656654 & -0.652294 & -0.635230 \\
\hline 6 & 1.987460 & -1.463511 & 0.554739 & 1.960365 & -1.468351 & 0.572062 \\
\hline 1 & 1.681055 & -2.510264 & 0.482618 & 1.635441 & -2.512648 & 0.518102 \\
\hline 1 & 1.808401 & -1.252575 & -1.537032 & 1.783576 & -1.288197 & -1.526527 \\
\hline 1 & 2.599390 & 0.955874 & -1.789943 & 2.602155 & 0.914836 & -1.809021 \\
\hline 1 & 3.491379 & 0.566499 & -0.314022 & 3.502606 & 0.522387 & -0.334219 \\
\hline 1 & 2.964555 & -0.089076 & 1.884001 & 2.964385 & -0.085543 & 1.877096 \\
\hline 1 & 2.773769 & -1.824800 & 2.498382 & 2.743874 & -1.809454 & 2.524367 \\
\hline 6 & -1.345692 & 1.577055 & 1.308829 & -1.309261 & 1.559320 & 1.326764 \\
\hline 6 & 1.698068 & 1.794908 & -0.029132 & 1.721806 & 1.775310 & -0.045247 \\
\hline 6 & 0.340949 & 1.830260 & -0.691538 & 0.357709 & 1.810042 & -0.695633 \\
\hline 6 & -0.929771 & 1.783025 & -0.146098 & -0.912450 & 1.774155 & -0.133106 \\
\hline 1 & -1.742624 & 2.095929 & -0.802672 & -1.729888 & 2.114848 & -0.773646 \\
\hline 1 & 0.382939 & 2.107599 & -1.750371 & 0.389173 & 2.098563 & -1.754219 \\
\hline 1 & 2.191802 & 2.774137 & -0.137377 & 2.221745 & 2.752183 & -0.165353 \\
\hline 1 & 1.610126 & 1.588155 & 1.043712 & 1.641970 & 1.577322 & 1.031962 \\
\hline 1 & -0.472046 & 1.548250 & 1.968523 & -0.422083 & 1.480264 & 1.967593 \\
\hline 1 & -1.961532 & 2.427876 & 1.631933 & -1.885178 & 2.427228 & 1.683498 \\
\hline \multirow[t]{3}{*}{27} & -0.358546 & -0.230011 & -0.611900 & -0.363529 & -0.217491 & -0.612325 \\
\hline & \multicolumn{3}{|c|}{ BP86 } & \multicolumn{3}{|c|}{ M06L } \\
\hline & $\mathrm{x}$ & $\mathrm{y}$ & $\mathrm{Z}$ & $\mathrm{x}$ & $\mathrm{y}$ & $\mathrm{Z}$ \\
\hline 6 & -0.907188 & -2.145814 & -0.255535 & -0.997298 & -2.123420 & -0.304841 \\
\hline 6 & -2.145440 & 0.251077 & 1.428421 & -1.877430 & 0.281558 & 1.468064 \\
\hline 6 & -2.446705 & -0.195173 & -0.007196 & -2.404176 & -0.129152 & 0.108508 \\
\hline 6 & -2.052329 & -1.386967 & -0.661897 & -2.126691 & -1.309217 & -0.594299 \\
\hline 1 & -2.480186 & -1.569568 & -1.660751 & -2.636065 & -1.435714 & -1.551327 \\
\hline 1 & -3.216348 & 0.397222 & -0.523082 & -3.181210 & 0.513196 & -0.305396 \\
\hline 1 & -3.088155 & 0.440561 & 1.978858 & -2.672879 & 0.320055 & 2.224719 \\
\hline 1 & -1.614634 & -0.545181 & 1.980885 & -1.153522 & -0.461233 & 1.831441 \\
\hline 1 & -0.693362 & -2.277750 & 0.815049 & -0.730631 & -2.311008 & 0.735679 \\
\hline 1 & -0.580344 & -2.978636 & -0.890582 & -0.758611 & -2.941771 & -0.980171 \\
\hline 6 & 2.519436 & -1.076705 & 1.758628 & 2.352403 & -1.073592 & 1.778352 \\
\hline 6 & 2.507808 & 0.577512 & -0.772742 & 2.504544 & 0.472273 & -0.765345 \\
\hline 6 & 1.637185 & -0.691292 & -0.627779 & 1.605351 & -0.756478 & -0.634280 \\
\hline 6 & 1.894469 & -1.475291 & 0.612465 & 1.782348 & -1.497014 & 0.628535 \\
\hline 1 & 1.536496 & -2.516778 & 0.590879 & 1.398736 & -2.519736 & 0.626045 \\
\hline 1 & 1.733600 & -1.362019 & -1.505293 & 1.713231 & -1.447952 & -1.480357 \\
\hline 1 & 2.622539 & 0.826755 & -1.846644 & 2.656113 & 0.711542 & -1.827103 \\
\hline 1 & 3.529091 & 0.431095 & -0.361899 & 3.503153 & 0.302402 & -0.331973 \\
\hline 1 & 2.933411 & -0.069095 & 1.886891 & 2.790753 & -0.083222 & 1.887351 \\
\hline 1 & 2.648077 & -1.770812 & 2.598278 & 2.418485 & -1.727950 & 2.643513 \\
\hline 6 & -1.249775 & 1.545945 & 1.353625 & -1.165962 & 1.649253 & 1.292930 \\
\hline 6 & 1.767729 & 1.738569 & -0.085237 & 1.789912 & 1.645339 & -0.108876 \\
\hline 6 & 0.389916 & 1.756291 & -0.718510 & 0.443005 & 1.714617 & -0.766466 \\
\hline 6 & -0.885809 & 1.757488 & -0.121325 & -0.817238 & 1.794083 & -0.180019 \\
\hline 1 & -1.707642 & 2.152195 & -0.737202 & -1.626705 & 2.163223 & -0.810160 \\
\hline 1 & 0.402475 & 2.053896 & -1.782995 & 0.481708 & 1.961608 & -1.832966 \\
\hline 1 & 2.275880 & 2.714392 & -0.235077 & 2.330092 & 2.595915 & -0.228323 \\
\hline 1 & 1.699700 & 1.566373 & 1.004344 & 1.678183 & 1.471012 & 0.968654 \\
\hline 1 & -0.342948 & 1.418905 & 1.970954 & -0.271029 & 1.701180 & 1.923005 \\
\hline 1 & -1.785016 & 2.432918 & 1.746034 & -1.816551 & 2.476573 & 1.601553 \\
\hline 27 & -0.372163 & -0.195493 & -0.609117 & -0.394661 & -0.196208 & -0.615843 \\
\hline
\end{tabular}


Table S36. Optimized coordinates for the $\left(\mathbf{C}_{4} \mathbf{H}_{6}\right)_{3} \mathbf{C o}$ structure $\mathbf{C o - 3 D}$.

\begin{tabular}{|c|c|c|c|c|c|c|}
\hline & \multicolumn{3}{|c|}{$\overline{B 3 L Y P}$} & \multicolumn{3}{|c|}{ B3LYP* } \\
\hline & $\mathrm{x}$ & $\mathrm{y}$ & $\mathrm{Z}$ & $\mathrm{x}$ & $\mathrm{y}$ & $\mathrm{Z}$ \\
\hline 6 & 1.001479 & -1.679158 & 0.628640 & 0.993794 & -1.676246 & 0.623700 \\
\hline 6 & 3.728575 & -0.201466 & 0.960304 & 3.713240 & -0.188847 & 0.965899 \\
\hline 6 & 3.101872 & -0.467789 & -0.206531 & 3.093187 & -0.457987 & -0.206200 \\
\hline 6 & 1.843026 & -1.210118 & -0.403209 & 1.836025 & -1.201854 & -0.408549 \\
\hline 1 & 1.738333 & -1.657756 & -1.393399 & 1.733209 & -1.648245 & -1.401613 \\
\hline 1 & 3.568028 & -0.108086 & -1.127602 & 3.565002 & -0.097510 & -1.126545 \\
\hline 1 & 4.661815 & 0.357437 & 0.977420 & 4.647405 & 0.371955 & 0.988648 \\
\hline 1 & 3.344142 & -0.548743 & 1.917681 & 3.322799 & -0.537662 & 1.922525 \\
\hline 1 & 1.274294 & -1.530368 & 1.671490 & 1.270007 & -1.532827 & 1.668601 \\
\hline 1 & 0.376121 & -2.552006 & 0.445535 & 0.370587 & -2.552417 & 0.437880 \\
\hline 6 & 0.911853 & 1.724884 & -1.224332 & 0.917074 & 1.711795 & -1.209327 \\
\hline 6 & 0.177382 & 1.199860 & 1.612525 & 0.158289 & 1.172361 & 1.616511 \\
\hline 6 & -0.712770 & 1.744278 & 0.683534 & -0.722522 & 1.736261 & 0.686059 \\
\hline 6 & -0.369956 & 1.956036 & -0.701010 & -0.369110 & 1.954090 & -0.695624 \\
\hline 1 & -1.194020 & 2.156211 & -1.386959 & -1.188552 & 2.163161 & -1.387695 \\
\hline 1 & -1.766424 & 1.808594 & 0.948376 & -1.779329 & 1.805917 & 0.945506 \\
\hline 1 & -0.195337 & 0.907639 & 2.591813 & -0.223669 & 0.874213 & 2.592555 \\
\hline 1 & 1.255354 & 1.355385 & 1.546529 & 1.240169 & 1.321784 & 1.559545 \\
\hline 1 & 1.807043 & 1.857012 & -0.620614 & 1.809936 & 1.842852 & -0.598039 \\
\hline 1 & 1.059996 & 1.779321 & -2.300574 & 1.074412 & 1.765709 & -2.286319 \\
\hline 6 & -1.235973 & -1.060889 & -1.487367 & -1.202972 & -1.038304 & -1.497333 \\
\hline 6 & -3.960718 & -0.432999 & 1.009383 & -3.946737 & -0.444731 & 0.998130 \\
\hline 6 & -3.113665 & -0.265374 & -0.027985 & -3.089257 & -0.262406 & -0.031093 \\
\hline 6 & -1.947443 & -1.104372 & -0.297851 & -1.915463 & -1.091074 & -0.300855 \\
\hline 1 & -1.788625 & -1.933081 & 0.391725 & -1.767036 & -1.935851 & 0.374735 \\
\hline 1 & -3.321040 & 0.522800 & -0.757306 & -3.295293 & 0.533884 & -0.755393 \\
\hline 1 & -4.837511 & 0.198494 & 1.133728 & -4.829606 & 0.181835 & 1.120730 \\
\hline 1 & -3.801019 & -1.212919 & 1.753160 & -3.789692 & -1.233446 & 1.736025 \\
\hline 1 & -1.544221 & -0.384809 & -2.283573 & -1.522197 & -0.360959 & -2.291146 \\
\hline 1 & -0.619497 & -1.902860 & -1.793687 & -0.599925 & -1.887730 & -1.817845 \\
\hline \multirow[t]{3}{*}{27} & 0.127430 & -0.012208 & -0.184828 & 0.117351 & -0.013075 & -0.182595 \\
\hline & \multicolumn{3}{|c|}{ BP86 } & \multicolumn{3}{|c|}{ M06L } \\
\hline & $\mathrm{x}$ & $\mathrm{y}$ & $\mathrm{Z}$ & $\mathrm{X}$ & $\mathrm{y}$ & $\mathrm{Z}$ \\
\hline 6 & 1.018795 & -1.562689 & 0.848041 & 1.007447 & -1.602810 & 0.794322 \\
\hline 6 & 3.726311 & -0.014668 & 0.816104 & 3.584491 & 0.049092 & 0.905228 \\
\hline 6 & 3.045600 & -0.452817 & -0.279367 & 3.018935 & -0.430966 & -0.220183 \\
\hline 6 & 1.792320 & -1.233088 & -0.305280 & 1.802528 & -1.237994 & -0.316372 \\
\hline 1 & 1.652537 & -1.832615 & -1.215710 & 1.688886 & -1.796479 & -1.245452 \\
\hline 1 & 3.463934 & -0.215885 & -1.270829 & 3.492015 & -0.189404 & -1.174226 \\
\hline 1 & 4.654214 & 0.559444 & 0.710149 & 4.486040 & 0.652760 & 0.869192 \\
\hline 1 & 3.393402 & -0.238202 & 1.837484 & 3.175692 & -0.165061 & 1.891743 \\
\hline 1 & 1.376625 & -1.289904 & 1.848835 & 1.339636 & -1.365062 & 1.804130 \\
\hline 1 & 0.395450 & -2.466841 & 0.824535 & 0.380484 & -2.490885 & 0.725015 \\
\hline 6 & 0.814368 & 1.539775 & -1.347024 & 0.837103 & 1.519443 & -1.359956 \\
\hline 6 & 0.171096 & 1.299291 & 1.562903 & 0.174782 & 1.284353 & 1.511073 \\
\hline 6 & -0.753044 & 1.773068 & 0.609004 & -0.738084 & 1.746985 & 0.559403 \\
\hline 6 & -0.455860 & 1.857429 & -0.804638 & -0.433018 & 1.820884 & -0.841589 \\
\hline 1 & -1.308514 & 2.000139 & -1.483615 & -1.277090 & 1.931916 & -1.523201 \\
\hline 1 & -1.809289 & 1.848582 & 0.899676 & -1.787901 & 1.818508 & 0.840108 \\
\hline 1 & -0.170911 & 1.092737 & 2.583168 & -0.158777 & 1.098011 & 2.527633 \\
\hline 1 & 1.255989 & 1.450474 & 1.446692 & 1.252986 & 1.431378 & 1.390357 \\
\hline 1 & 1.741501 & 1.758660 & -0.800971 & 1.740037 & 1.747471 & -0.792284 \\
\hline 1 & 0.919261 & 1.478192 & -2.436853 & 0.969083 & 1.465530 & -2.437234 \\
\hline 6 & -1.041270 & -1.174548 & -1.402565 & -1.084543 & -1.214264 & -1.368387 \\
\hline 6 & -3.947883 & -0.387943 & 0.891421 & -3.882151 & -0.290765 & 0.943358 \\
\hline 6 & -3.019036 & -0.282831 & -0.098213 & -2.991628 & -0.253033 & -0.065727 \\
\hline 6 & -1.809630 & -1.100619 & -0.214032 & -1.828503 & -1.112511 & -0.191243 \\
\hline 1 & -1.711862 & -1.900441 & 0.534167 & -1.713694 & -1.875242 & 0.579069 \\
\hline 1 & -3.198194 & 0.438488 & -0.913301 & -3.152056 & 0.455891 & -0.884398 \\
\hline 1 & -4.855755 & 0.226111 & 0.890965 & -4.751995 & 0.358506 & 0.959821 \\
\hline 1 & -3.826215 & -1.101959 & 1.716744 & -3.766647 & -0.985446 & 1.773318 \\
\hline 1 & -1.353521 & -0.597765 & -2.284105 & -1.389440 & -0.649613 & -2.249372 \\
\hline 1 & -0.467408 & -2.083069 & -1.623729 & -0.490623 & -2.104209 & -1.565553 \\
\hline 27 & 0.096228 & -0.025493 & -0.108201 & 0.117008 & -0.037372 & -0.132897 \\
\hline
\end{tabular}


Table S37. Optimized coordinates for the $\left(\mathrm{C}_{4} \mathbf{H}_{6}\right)_{3} \mathrm{Co}$ structure $\mathrm{Co}-4 \mathrm{D}$.

\begin{tabular}{|c|c|c|c|c|c|c|}
\hline & \multicolumn{3}{|c|}{ B3LYP } & \multicolumn{3}{|c|}{ B3LYP* } \\
\hline & $\mathrm{x}$ & $\mathrm{y}$ & $\mathrm{Z}$ & $\mathrm{x}$ & $\mathrm{y}$ & $\mathrm{Z}$ \\
\hline 6 & 1.052361 & -1.698178 & 1.523306 & 1.286897 & -1.564872 & 1.510806 \\
\hline 6 & -0.378177 & -2.367982 & -0.975635 & -0.081420 & -2.336862 & -0.971162 \\
\hline 6 & 0.966169 & -2.046419 & -0.939778 & 1.232469 & -1.880824 & -0.954946 \\
\hline 6 & 1.693705 & -1.733599 & 0.272200 & 1.927524 & -1.512533 & 0.258241 \\
\hline 1 & 2.735235 & -1.427658 & 0.177212 & 2.919456 & -1.065557 & 0.165015 \\
\hline 1 & 1.486461 & -1.887885 & -1.885302 & 1.721410 & -1.648391 & -1.904094 \\
\hline 1 & -0.889099 & -2.444364 & -1.932664 & -0.599284 & -2.440695 & -1.924247 \\
\hline 1 & -0.904062 & -2.763265 & -0.113202 & -0.524965 & -2.857383 & -0.125725 \\
\hline 1 & 0.210443 & -2.350819 & 1.747817 & 0.534095 & -2.321816 & 1.734833 \\
\hline 1 & 1.618877 & -1.361137 & 2.390110 & 1.803637 & -1.154294 & 2.379156 \\
\hline 6 & 1.756951 & 2.950174 & 0.443123 & 1.295105 & 3.168545 & 0.444411 \\
\hline 6 & -0.035946 & 1.180681 & -1.398910 & -0.211184 & 1.134628 & -1.389414 \\
\hline 6 & 1.329599 & 1.026613 & -1.153126 & 1.162721 & 1.190005 & -1.137108 \\
\hline 6 & 2.132210 & 1.828588 & -0.210243 & 1.830141 & 2.094924 & -0.182454 \\
\hline 1 & 3.145797 & 1.457282 & -0.038922 & 2.880747 & 1.859955 & 0.018528 \\
\hline 1 & 1.913218 & 0.414836 & -1.841246 & 1.836869 & 0.685492 & -1.833485 \\
\hline 1 & -0.480768 & 0.716898 & -2.276894 & -0.575712 & 0.628048 & -2.283220 \\
\hline 1 & -0.606002 & 2.003996 & -0.977257 & -0.905834 & 1.849749 & -0.952720 \\
\hline 1 & 0.777505 & 3.402908 & 0.300914 & 0.268489 & 3.489490 & 0.265926 \\
\hline 1 & 2.436935 & 3.450678 & 1.129244 & 1.885372 & 3.761511 & 1.142847 \\
\hline 6 & -1.012700 & 0.699408 & 1.488721 & -1.046118 & 0.509165 & 1.507568 \\
\hline 6 & -3.486333 & 1.186285 & -0.346795 & -3.578587 & 0.820868 & -0.296866 \\
\hline 6 & -2.858862 & 0.011638 & -0.140960 & -2.831247 & -0.293083 & -0.146770 \\
\hline 6 & -1.816479 & -0.254316 & 0.869091 & -1.755623 & -0.494880 & 0.842212 \\
\hline 1 & -1.878390 & -1.238788 & 1.334685 & -1.722425 & -1.499433 & 1.272079 \\
\hline 1 & -3.159404 & -0.850673 & -0.740024 & -3.050892 & -1.156270 & -0.782689 \\
\hline 1 & -4.254996 & 1.285297 & -1.110777 & -4.362750 & 0.874507 & -1.052353 \\
\hline 1 & -3.262293 & 2.069870 & 0.248312 & -3.439937 & 1.696373 & 0.338469 \\
\hline 1 & -1.091087 & 1.749865 & 1.217812 & -1.222404 & 1.558987 & 1.272651 \\
\hline 1 & -0.569691 & 0.493223 & 2.460600 & -0.604726 & 0.312903 & 2.484197 \\
\hline \multirow[t]{3}{*}{27} & 0.248753 & -0.274726 & 0.123097 & 0.287955 & -0.280876 & 0.106582 \\
\hline & \multicolumn{3}{|c|}{ BP86 } & \multicolumn{3}{|c|}{ M06L } \\
\hline & $\mathrm{x}$ & $\mathrm{y}$ & $\mathrm{Z}$ & $\mathrm{x}$ & $\mathrm{y}$ & $\mathrm{Z}$ \\
\hline 6 & 1.039604 & -1.719423 & 1.536753 & 1.241735 & -1.527246 & 1.478649 \\
\hline 6 & -0.358849 & -2.217480 & -0.937604 & -0.146032 & -2.216332 & -0.925115 \\
\hline 6 & 1.041448 & -2.024802 & -0.929503 & 1.190035 & -1.827579 & -0.962591 \\
\hline 6 & 1.746517 & -1.783078 & 0.309841 & 1.907057 & -1.491115 & 0.237654 \\
\hline 1 & 2.798579 & -1.469009 & 0.258047 & 2.901764 & -1.056862 & 0.145127 \\
\hline 1 & 1.583337 & -1.881443 & -1.875016 & 1.658031 & -1.599337 & -1.920007 \\
\hline 1 & -0.885556 & -2.207689 & -1.899821 & -0.707494 & -2.290423 & -1.853076 \\
\hline 1 & -0.864830 & -2.763015 & -0.132514 & -0.556598 & -2.762608 & -0.079636 \\
\hline 1 & 0.182235 & -2.376162 & 1.734622 & 0.494706 & -2.293473 & 1.689353 \\
\hline 1 & 1.568946 & -1.361344 & 2.428582 & 1.751970 & -1.134684 & 2.355827 \\
\hline 6 & 1.562797 & 2.967055 & 0.385080 & 1.277404 & 2.986987 & 0.517516 \\
\hline 6 & -0.089513 & 1.014398 & -1.447626 & -0.279475 & 1.115988 & -1.367523 \\
\hline 6 & 1.293683 & 0.962777 & -1.160375 & 1.107905 & 1.159401 & -1.200433 \\
\hline 6 & 2.003751 & 1.807244 & -0.186642 & 1.810226 & 2.000495 & -0.234478 \\
\hline 1 & 3.018804 & 1.465973 & 0.073474 & 2.875859 & 1.790495 & -0.122278 \\
\hline 1 & 1.945401 & 0.384273 & -1.831545 & 1.740323 & 0.653794 & -1.930585 \\
\hline 1 & -0.467311 & 0.538274 & -2.361534 & -0.702418 & 0.624676 & -2.241814 \\
\hline 1 & -0.728338 & 1.812808 & -1.052101 & -0.931621 & 1.854225 & -0.901482 \\
\hline 1 & 0.586666 & 3.400949 & 0.135802 & 0.228767 & 3.267959 & 0.434252 \\
\hline 1 & 2.182941 & 3.511416 & 1.106742 & 1.880767 & 3.542390 & 1.228815 \\
\hline 6 & -0.884438 & 0.630122 & 1.477429 & -0.985801 & 0.561956 & 1.494589 \\
\hline 6 & -3.397459 & 1.319907 & -0.269940 & -3.463029 & 0.795678 & -0.320639 \\
\hline 6 & -2.815337 & 0.098689 & -0.145434 & -2.763785 & -0.329503 & -0.092762 \\
\hline 6 & -1.753078 & -0.265243 & 0.813210 & -1.686295 & -0.483347 & 0.884587 \\
\hline 1 & -1.874889 & -1.265629 & 1.256938 & -1.622161 & -1.468334 & 1.349766 \\
\hline 1 & -3.178016 & -0.721775 & -0.784359 & -3.020476 & -1.227834 & -0.657190 \\
\hline 1 & -4.187964 & 1.499119 & -1.008656 & -4.249204 & 0.832517 & -1.069052 \\
\hline 1 & -3.112858 & 2.160894 & 0.374868 & -3.272919 & 1.709122 & 0.241017 \\
\hline 1 & -0.907549 & 1.700053 & 1.234648 & -1.213312 & 1.594934 & 1.229347 \\
\hline 1 & -0.494798 & 0.383375 & 2.471617 & -0.529444 & 0.420445 & 2.470634 \\
\hline 27 & 0.266003 & -0.372802 & 0.069526 & 0.296807 & -0.256641 & 0.095343 \\
\hline
\end{tabular}


Table S38. Optimized coordinates for the $\left(\mathbf{C}_{4} \mathbf{H}_{6}\right)_{3} \mathbf{C o}$ structure $\mathbf{C o - 5 D}$.

\begin{tabular}{|c|c|c|c|c|c|c|}
\hline & \multicolumn{3}{|c|}{ B3LYP } & \multicolumn{3}{|c|}{ B3LYP* } \\
\hline & $\mathrm{x}$ & $\mathrm{y}$ & $\mathrm{z}$ & $\mathrm{x}$ & y & $\mathrm{Z}$ \\
\hline 6 & -2.895576 & 0.650349 & 1.019991 & -2.836137 & 0.709105 & 1.019480 \\
\hline 6 & -0.130418 & 1.807582 & 0.087692 & -0.080473 & 1.774010 & -0.050281 \\
\hline 6 & -1.389404 & 1.938193 & -0.612228 & -1.372562 & 1.877072 & -0.730808 \\
\hline 6 & -2.606392 & 1.447756 & -0.228597 & -2.578859 & 1.430983 & -0.284324 \\
\hline 1 & -3.446477 & 1.629539 & -0.900155 & -3.441252 & 1.585682 & -0.937630 \\
\hline 1 & -1.350907 & 2.459590 & -1.571949 & -1.354088 & 2.343295 & -1.721684 \\
\hline 1 & 0.631910 & 2.533486 & -0.200351 & 0.639192 & 2.532107 & -0.378603 \\
\hline 1 & -0.182944 & 1.642115 & 1.168356 & -0.142124 & 1.734293 & 1.046500 \\
\hline 1 & -2.675226 & 1.262824 & 1.909558 & -2.590484 & 1.370811 & 1.868723 \\
\hline 1 & -3.969396 & 0.426351 & 1.057050 & -3.910772 & 0.489846 & 1.099827 \\
\hline 6 & -2.097215 & -0.680349 & 1.154799 & -2.035801 & -0.613920 & 1.203169 \\
\hline 6 & -0.292040 & -1.013595 & -1.509497 & -0.290876 & -1.046867 & -1.470692 \\
\hline 6 & -1.486854 & -1.809781 & -1.058115 & -1.476149 & -1.831124 & -0.974762 \\
\hline 6 & -2.279186 & -1.662667 & 0.025894 & -2.244055 & -1.644116 & 0.122395 \\
\hline 1 & -3.135406 & -2.336487 & 0.113325 & -3.102304 & -2.311631 & 0.253070 \\
\hline 1 & -1.774432 & -2.622998 & -1.736374 & -1.779143 & -2.663599 & -1.625445 \\
\hline 1 & -0.620872 & -0.224503 & -2.204966 & -0.631793 & -0.294207 & -2.202257 \\
\hline 1 & 0.376442 & -1.675094 & -2.087889 & 0.386378 & -1.730737 & -2.017479 \\
\hline 1 & -1.031092 & -0.447424 & 1.257590 & -0.965480 & -0.372709 & 1.263024 \\
\hline 1 & -2.407337 & -1.152838 & 2.098839 & -2.318352 & -1.043938 & 2.178248 \\
\hline 27 & 0.912706 & -0.037349 & -0.264208 & 0.909686 & -0.018946 & -0.284014 \\
\hline 6 & 1.863707 & -1.513995 & 1.053888 & 1.840435 & -1.605817 & 0.885776 \\
\hline 6 & 2.894660 & 0.503322 & -0.890449 & 2.813018 & 0.647298 & -0.772603 \\
\hline 6 & 2.789041 & 0.735995 & 0.494983 & 2.695038 & 0.723631 & 0.639349 \\
\hline 6 & 2.311336 & -0.242731 & 1.429044 & 2.244996 & -0.380720 & 1.432349 \\
\hline 1 & 2.069055 & 0.115271 & 2.430717 & 1.964379 & -0.159700 & 2.465476 \\
\hline 1 & 2.880175 & 1.759132 & 0.859337 & 2.736010 & 1.701626 & 1.121999 \\
\hline 1 & 3.123775 & 1.339899 & -1.547061 & 2.997064 & 1.567717 & -1.327754 \\
\hline 1 & 3.146756 & -0.478663 & -1.291958 & 3.168126 & -0.259455 & -1.270527 \\
\hline 1 & 2.280828 & -2.041279 & 0.195600 & 2.290908 & -2.003622 & -0.026930 \\
\hline \multirow[t]{3}{*}{1} & 1.352126 & -2.140973 & 1.779523 & 1.326817 & -2.335687 & 1.508797 \\
\hline & \multicolumn{3}{|c|}{ BP86 } & \multicolumn{3}{|c|}{ M06L } \\
\hline & $\mathrm{x}$ & $\mathrm{y}$ & $\mathrm{Z}$ & $\mathrm{x}$ & $\mathrm{y}$ & $\mathrm{Z}$ \\
\hline 6 & -2.542663 & 0.851315 & 0.936225 & 2.534963 & -0.803794 & 0.973343 \\
\hline 6 & 0.179829 & 1.997499 & 0.110934 & -0.107815 & -1.960759 & 0.079906 \\
\hline 6 & -0.973768 & 1.893130 & -0.777582 & 1.073305 & -1.889328 & -0.761907 \\
\hline 6 & -2.217175 & 1.404823 & -0.432964 & 2.273818 & -1.373146 & -0.388515 \\
\hline 1 & -2.986502 & 1.334818 & -1.213040 & 3.067709 & -1.308586 & -1.129915 \\
\hline 1 & -0.819265 & 2.189910 & -1.826426 & 0.959101 & -2.207864 & -1.799513 \\
\hline 1 & 0.957657 & 2.701190 & -0.222566 & -0.858481 & -2.686784 & -0.238840 \\
\hline 1 & -0.043815 & 2.108292 & 1.187121 & 0.091803 & -2.034791 & 1.156866 \\
\hline 1 & -2.237951 & 1.561727 & 1.732438 & 2.206538 & -1.506045 & 1.754026 \\
\hline 1 & -3.634402 & 0.704320 & 1.035513 & 3.610484 & -0.653775 & 1.119596 \\
\hline 6 & -1.826148 & -0.509710 & 1.182171 & 1.811119 & 0.539812 & 1.186911 \\
\hline 6 & -0.182230 & -1.213427 & -1.407957 & 0.209228 & 1.188316 & -1.430221 \\
\hline 6 & -1.444073 & -1.887473 & -0.934411 & 1.443845 & 1.867693 & -0.941347 \\
\hline 6 & -2.170023 & -1.583024 & 0.172580 & 2.147443 & 1.590189 & 0.171417 \\
\hline 1 & -3.100147 & -2.140917 & 0.363915 & 3.061467 & 2.155176 & 0.361063 \\
\hline 1 & -1.832416 & -2.707897 & -1.566803 & 1.837893 & 2.676095 & -1.568220 \\
\hline 1 & -0.427553 & -0.522772 & -2.245563 & 0.474248 & 0.483302 & -2.237320 \\
\hline 1 & 0.515747 & -1.964463 & -1.824609 & -0.475474 & 1.918698 & -1.881342 \\
\hline 1 & -0.714504 & -0.322321 & 1.194918 & 0.717212 & 0.343931 & 1.200664 \\
\hline 1 & -2.047226 & -0.864898 & 2.210755 & 2.020573 & 0.910793 & 2.200412 \\
\hline 27 & 0.670333 & 0.042638 & -0.114873 & -0.701598 & -0.030318 & -0.134189 \\
\hline 6 & 1.577922 & -1.627039 & 0.745442 & -1.650403 & 1.607004 & 0.728641 \\
\hline 6 & 2.423629 & 0.376149 & -0.964828 & -2.455178 & -0.442772 & -0.933696 \\
\hline 6 & 2.556694 & 0.595191 & 0.474658 & -2.541954 & -0.639330 & 0.495532 \\
\hline 6 & 2.122695 & -0.441699 & 1.348361 & -2.136859 & 0.420768 & 1.337949 \\
\hline 1 & 1.984915 & -0.219855 & 2.416174 & -1.959722 & 0.215176 & 2.392961 \\
\hline 1 & 2.821196 & 1.579254 & 0.884439 & -2.732509 & -1.622204 & 0.921956 \\
\hline 1 & 2.531893 & 1.257779 & -1.613395 & -2.559149 & -1.324785 & -1.565120 \\
\hline 1 & 2.799711 & -0.552711 & -1.419662 & -2.849134 & 0.470670 & -1.383842 \\
\hline 1 & 2.111262 & -2.113904 & -0.080367 & -2.174125 & 2.034801 & -0.123599 \\
\hline 1 & 0.994262 & -2.313202 & 1.372958 & -1.104351 & 2.326850 & 1.335184 \\
\hline
\end{tabular}


Table S39. Optimized coordinates for the $\left(\mathrm{C}_{4} \mathbf{H}_{6}\right)_{3}$ Co structure $\mathbf{C o - 1 Q}$.

\begin{tabular}{|c|c|c|c|c|c|c|}
\hline & \multicolumn{3}{|c|}{$\overline{B 3 L Y P}$} & \multicolumn{3}{|c|}{ B3LYP* } \\
\hline & $\mathrm{x}$ & $\mathrm{y}$ & $\mathrm{Z}$ & $\mathrm{x}$ & $\mathrm{y}$ & $\mathrm{Z}$ \\
\hline 6 & -1.066016 & -1.402900 & 1.255703 & -0.997339 & -1.352762 & 1.326025 \\
\hline 6 & -3.048606 & 0.954449 & 0.288704 & -3.046003 & 0.878446 & 0.252581 \\
\hline 6 & -2.949274 & -0.473265 & -0.212732 & -2.858175 & -0.546645 & -0.231662 \\
\hline 6 & -2.126694 & -1.473680 & 0.271408 & -2.005315 & -1.503542 & 0.305604 \\
\hline 1 & -2.246910 & -2.446474 & -0.217246 & -2.063308 & -2.493893 & -0.162925 \\
\hline 1 & -3.632785 & -0.747318 & -1.016478 & -3.493348 & -0.865255 & -1.060604 \\
\hline 1 & -3.697966 & 1.508879 & -0.402060 & -3.712774 & 1.390907 & -0.456498 \\
\hline 1 & -3.551799 & 0.986639 & 1.268525 & -3.567245 & 0.893452 & 1.225717 \\
\hline 1 & -1.120278 & -0.632270 & 2.026999 & -1.098865 & -0.552123 & 2.062420 \\
\hline 1 & -0.709564 & -2.361422 & 1.635306 & -0.577093 & -2.273445 & 1.735200 \\
\hline 6 & 1.756954 & -1.459071 & -1.067872 & 1.714901 & -1.419963 & -1.151400 \\
\hline 6 & 2.843972 & 1.163939 & 0.337892 & 2.819955 & 1.132241 & 0.385003 \\
\hline 6 & 2.699926 & -0.210499 & 0.950445 & 2.628696 & -0.262055 & 0.934459 \\
\hline 6 & 2.271553 & -1.336215 & 0.301394 & 2.190619 & -1.357947 & 0.225783 \\
\hline 1 & 3.244222 & 1.090085 & -0.684812 & 3.249758 & 1.091813 & -0.629814 \\
\hline 1 & 3.574295 & 1.742987 & 0.918992 & 3.542770 & 1.674824 & 1.012553 \\
\hline 6 & -1.701362 & 1.713843 & 0.408297 & -1.737101 & 1.703963 & 0.379709 \\
\hline 6 & 1.516752 & 1.964661 & 0.286768 & 1.503516 & 1.951366 & 0.323735 \\
\hline 6 & 0.577727 & 1.487413 & -0.798734 & 0.581553 & 1.471879 & -0.776203 \\
\hline 6 & -0.790032 & 1.419261 & -0.764532 & -0.795189 & 1.401784 & -0.767373 \\
\hline 1 & -1.299574 & 1.265335 & -1.719687 & -1.283855 & 1.268599 & -1.739106 \\
\hline 1 & 1.047596 & 1.380188 & -1.780027 & 1.064724 & 1.394407 & -1.756127 \\
\hline 1 & 1.747462 & 3.020701 & 0.064310 & 1.748272 & 3.008491 & 0.111555 \\
\hline 1 & 1.029703 & 1.948964 & 1.269276 & 1.002515 & 1.931448 & 1.301466 \\
\hline 1 & -1.199104 & 1.466354 & 1.349357 & -1.243631 & 1.506913 & 1.339565 \\
\hline 1 & -1.903661 & 2.796476 & 0.446496 & -1.986007 & 2.779766 & 0.378799 \\
\hline 1 & 2.945093 & -0.297688 & 2.009783 & 2.831765 & -0.399140 & 1.999311 \\
\hline 1 & 2.201518 & -0.788025 & -1.810281 & 2.157051 & -0.719725 & -1.867876 \\
\hline 1 & 1.717550 & -2.488817 & -1.435593 & 1.625042 & -2.431494 & -1.561298 \\
\hline 1 & 2.209243 & -2.240851 & 0.912575 & 2.097621 & -2.284997 & 0.801732 \\
\hline \multirow[t]{3}{*}{27} & -0.009794 & -0.714495 & -0.384292 & -0.010893 & -0.648190 & -0.369320 \\
\hline & \multicolumn{3}{|c|}{ BP86 } & \multicolumn{3}{|c|}{ M06L } \\
\hline & $\mathrm{x}$ & $\mathrm{y}$ & $\underline{Z}$ & $\mathrm{X}$ & $\mathrm{y}$ & $\mathrm{Z}$ \\
\hline 6 & -0.934716 & -1.294960 & 1.352405 & -0.748609 & -0.978348 & 1.824590 \\
\hline 6 & -3.063852 & 0.823382 & 0.245869 & -2.914036 & 0.440641 & 0.061504 \\
\hline 6 & -2.830175 & -0.599446 & -0.227029 & -2.206723 & -0.873220 & -0.227603 \\
\hline 6 & -1.900563 & -1.509338 & 0.302865 & -1.425408 & -1.554132 & 0.753005 \\
\hline 1 & -1.906320 & -2.512687 & -0.156555 & -1.215120 & -2.609568 & 0.551008 \\
\hline 1 & -3.448258 & -0.953992 & -1.062810 & -2.703705 & -1.525416 & -0.946008 \\
\hline 1 & -3.757013 & 1.310556 & -0.465310 & -3.575226 & 0.665641 & -0.784361 \\
\hline 1 & -3.579905 & 0.831339 & 1.229798 & -3.566097 & 0.362944 & 0.945030 \\
\hline 1 & -1.079709 & -0.471967 & 2.065158 & -0.999516 & 0.006000 & 2.208161 \\
\hline 1 & -0.456379 & -2.184133 & 1.782726 & -0.085242 & -1.581849 & 2.436275 \\
\hline 6 & 1.721224 & -1.404317 & -1.191602 & 1.682620 & -1.175439 & -1.422551 \\
\hline 6 & 2.792525 & 1.111962 & 0.430580 & 2.599652 & 1.062585 & 0.603824 \\
\hline 6 & 2.514898 & -0.274447 & 0.964632 & 2.325780 & -0.379519 & 0.898830 \\
\hline 6 & 2.078975 & -1.367970 & 0.211071 & 1.962169 & -1.344899 & -0.029306 \\
\hline 1 & 3.273241 & 1.054372 & -0.567739 & 3.113496 & 1.162879 & -0.364869 \\
\hline 1 & 3.503127 & 1.634108 & 1.099699 & 3.277285 & 1.472371 & 1.361198 \\
\hline 6 & -1.766517 & 1.682127 & 0.347326 & -1.928666 & 1.605869 & 0.222025 \\
\hline 6 & 1.493946 & 1.958285 & 0.300869 & 1.313847 & 1.910024 & 0.548192 \\
\hline 6 & 0.590363 & 1.409489 & -0.788699 & 0.516328 & 1.555375 & -0.671713 \\
\hline 6 & -0.810459 & 1.317353 & -0.774596 & -0.852784 & 1.436700 & -0.809991 \\
\hline 1 & -1.294582 & 1.182603 & -1.757520 & -1.226687 & 1.362590 & -1.836330 \\
\hline 1 & 1.067324 & 1.368610 & -1.782452 & 1.092471 & 1.598650 & -1.599754 \\
\hline 1 & 1.759497 & 3.007944 & 0.044396 & 1.580539 & 2.977947 & 0.511520 \\
\hline 1 & 0.969715 & 1.987514 & 1.274055 & 0.723401 & 1.758706 & 1.462179 \\
\hline 1 & -1.278780 & 1.542181 & 1.328204 & -1.489574 & 1.632034 & 1.225396 \\
\hline 1 & -2.033327 & 2.759257 & 0.287946 & -2.438660 & 2.571605 & 0.090680 \\
\hline 1 & 2.616513 & -0.420917 & 2.049068 & 2.360101 & -0.684843 & 1.943797 \\
\hline 1 & 2.181588 & -0.681408 & -1.880326 & 2.137833 & -0.334921 & -1.948683 \\
\hline 1 & 1.569876 & -2.400795 & -1.633081 & 1.603730 & -2.082223 & -2.021370 \\
\hline 1 & 1.928407 & -2.302723 & 0.774341 & 1.789979 & -2.345548 & 0.376967 \\
\hline 27 & 0.024115 & -0.587503 & -0.358213 & -0.086076 & -0.542253 & -0.522803 \\
\hline
\end{tabular}


Table S40. Optimized coordinates for the $\left(\mathbf{C}_{4} \mathbf{H}_{6}\right)_{3}$ Co structure $\mathbf{C o - 2 Q}$.

\begin{tabular}{|c|c|c|c|c|c|c|}
\hline & \multicolumn{3}{|c|}{$\overline{B 3 L Y P}$} & \multicolumn{3}{|c|}{ B3LYP* } \\
\hline & $\mathrm{x}$ & $\mathrm{y}$ & $\mathrm{Z}$ & $\mathrm{x}$ & $\mathrm{y}$ & $\mathrm{Z}$ \\
\hline 6 & -1.715704 & -1.769174 & 0.480776 & -1.684447 & -1.755042 & 0.489201 \\
\hline 6 & -2.665766 & 1.140006 & 0.816756 & -2.687218 & 1.126945 & 0.811966 \\
\hline 6 & -3.317827 & 0.119661 & -0.084623 & -3.324415 & 0.094773 & -0.085890 \\
\hline 6 & -2.854491 & -1.152835 & -0.229279 & -2.831290 & -1.170543 & -0.227884 \\
\hline 1 & -3.348379 & -1.775317 & -0.982067 & -3.306371 & -1.807294 & -0.983627 \\
\hline 1 & -4.157858 & 0.450390 & -0.697680 & -4.170434 & 0.407726 & -0.703215 \\
\hline 1 & -3.421197 & 1.804226 & 1.259818 & -3.449303 & 1.802374 & 1.230552 \\
\hline 1 & -2.164176 & 0.633456 & 1.650780 & -2.203489 & 0.629929 & 1.664954 \\
\hline 1 & -1.662996 & -1.506011 & 1.547552 & -1.626285 & -1.475712 & 1.553356 \\
\hline 1 & -1.684689 & -2.859459 & 0.373525 & -1.615078 & -2.845599 & 0.385631 \\
\hline 6 & 1.715706 & -1.769174 & 0.480771 & 1.684452 & -1.755044 & 0.489189 \\
\hline 6 & 2.665765 & 1.140005 & 0.816758 & 2.687212 & 1.126945 & 0.811973 \\
\hline 6 & 3.317828 & 0.119663 & -0.084622 & 3.324415 & 0.094780 & -0.085888 \\
\hline 6 & 2.854493 & -1.152832 & -0.229281 & 2.831293 & -1.170536 & -0.227892 \\
\hline 1 & 3.348382 & -1.775312 & -0.982071 & 3.306377 & -1.807281 & -0.983638 \\
\hline 1 & 4.157859 & 0.450394 & -0.697677 & 4.170433 & 0.407740 & -0.703210 \\
\hline 1 & 3.421194 & 1.804225 & 1.259822 & 3.449294 & 1.802374 & 1.230565 \\
\hline 1 & 2.164175 & 0.633453 & 1.650781 & 2.203483 & 0.629922 & 1.664957 \\
\hline 1 & 1.662997 & -1.506015 & 1.547548 & 1.626289 & -1.475719 & 1.553346 \\
\hline 1 & 1.684692 & -2.859459 & 0.373517 & 1.615087 & -2.845600 & 0.385614 \\
\hline 6 & -1.619561 & 2.032235 & 0.080875 & -1.619024 & 2.001916 & 0.082945 \\
\hline 6 & 1.619559 & 2.032235 & 0.080879 & 1.619018 & 2.001916 & 0.082953 \\
\hline 6 & 0.683302 & 1.285738 & -0.843817 & 0.687517 & 1.246533 & -0.841635 \\
\hline 6 & -0.683302 & 1.285738 & -0.843819 & -0.687518 & 1.246532 & -0.841638 \\
\hline 1 & -1.182630 & 0.817815 & -1.696915 & -1.186412 & 0.807038 & -1.712829 \\
\hline 1 & 1.182632 & 0.817815 & -1.696913 & 1.186415 & 0.807038 & -1.712823 \\
\hline 1 & 2.162580 & 2.756637 & -0.546484 & 2.146193 & 2.741549 & -0.543712 \\
\hline 1 & 1.059593 & 2.616266 & 0.821141 & 1.052582 & 2.573496 & 0.831142 \\
\hline 1 & -1.059597 & 2.616269 & 0.821136 & -1.052591 & 2.573500 & 0.831133 \\
\hline 1 & -2.162582 & 2.756635 & -0.546490 & -2.146199 & 2.741546 & -0.543724 \\
\hline \multirow[t]{3}{*}{27} & 0.000000 & -0.953467 & -0.226206 & 0.000001 & -0.896373 & -0.229217 \\
\hline & \multicolumn{3}{|c|}{ BP86 } & \multicolumn{3}{|c|}{ M06L } \\
\hline & $\mathrm{x}$ & $\mathrm{y}$ & $\mathrm{Z}$ & $\mathrm{X}$ & $\mathrm{y}$ & $\mathrm{z}$ \\
\hline 6 & -1.496388 & -1.616545 & 0.985834 & -1.661436 & -1.744098 & 0.489270 \\
\hline 6 & -2.849854 & 1.106910 & 0.557031 & -2.641384 & 1.096815 & 0.819733 \\
\hline 6 & -3.197831 & -0.144307 & -0.208311 & -3.288892 & 0.087569 & -0.076218 \\
\hline 6 & -2.517128 & -1.343248 & -0.016183 & -2.791484 & -1.171459 & -0.230249 \\
\hline 1 & -2.764302 & -2.172680 & -0.699692 & -3.250630 & -1.798509 & -0.998464 \\
\hline 1 & -3.922253 & -0.073623 & -1.031517 & -4.122948 & 0.410562 & -0.698343 \\
\hline 1 & -3.742808 & 1.745204 & 0.700125 & -3.384116 & 1.762488 & 1.275007 \\
\hline 1 & -2.487666 & 0.830054 & 1.564822 & -2.135571 & 0.582870 & 1.647701 \\
\hline 1 & -1.518866 & -1.033028 & 1.920666 & -1.594179 & -1.450196 & 1.546619 \\
\hline 1 & -1.253375 & -2.678911 & 1.147719 & -1.565287 & -2.828983 & 0.389198 \\
\hline 6 & 1.680308 & -1.661366 & 0.036604 & 1.661459 & -1.744096 & 0.489159 \\
\hline 6 & 2.683449 & 1.023069 & 0.970216 & 2.641375 & 1.096800 & 0.819779 \\
\hline 6 & 3.409656 & 0.169070 & -0.045254 & 3.288916 & 0.087604 & -0.076206 \\
\hline 6 & 2.916393 & -1.043115 & -0.462399 & 2.791526 & -1.171421 & -0.230306 \\
\hline 1 & 3.464687 & -1.576730 & -1.255842 & 3.250702 & -1.798436 & -0.998531 \\
\hline 1 & 4.335515 & 0.556524 & -0.492654 & 4.122989 & 0.410633 & -0.698289 \\
\hline 1 & 3.381964 & 1.726139 & 1.461610 & 3.384086 & 1.762478 & 1.275079 \\
\hline 1 & 2.272162 & 0.375606 & 1.770040 & 2.135576 & 0.582811 & 1.647729 \\
\hline 1 & 1.549460 & -1.529270 & 1.139347 & 1.594191 & -1.450240 & 1.546522 \\
\hline 1 & 1.570958 & -2.731306 & -0.220135 & 1.565325 & -2.828979 & 0.389048 \\
\hline 6 & -1.732582 & 1.953807 & -0.151012 & -1.595742 & 1.961911 & 0.079885 \\
\hline 6 & 1.496053 & 1.851178 & 0.362272 & 1.595706 & 1.961895 & 0.079965 \\
\hline 6 & 0.705116 & 1.119855 & -0.702440 & 0.688765 & 1.199832 & -0.844627 \\
\hline 6 & -0.686877 & 1.152773 & -0.921127 & -0.688762 & 1.199835 & -0.844662 \\
\hline 1 & -1.019269 & 0.822789 & -1.922243 & -1.187179 & 0.772778 & -1.721093 \\
\hline 1 & 1.329637 & 0.780303 & -1.545328 & 1.187226 & 0.772769 & -1.721029 \\
\hline 1 & 1.918939 & 2.759141 & -0.117119 & 2.123906 & 2.707599 & -0.532120 \\
\hline 1 & 0.841596 & 2.204126 & 1.182012 & 1.009949 & 2.529411 & 0.812810 \\
\hline 1 & -1.245345 & 2.601491 & 0.601331 & -1.010011 & 2.529483 & 0.812706 \\
\hline 1 & -2.215680 & 2.635517 & -0.880457 & -2.123963 & 2.707566 & -0.532240 \\
\hline 27 & -0.109528 & -0.764809 & -0.213113 & -0.000013 & -0.834934 & -0.233165 \\
\hline
\end{tabular}


Table S41. Optimized coordinates for the $\left(\mathrm{C}_{4} \mathbf{H}_{6}\right)_{3} \mathbf{N i}$ structure Ni-1S.

\begin{tabular}{|c|c|c|c|c|c|c|}
\hline & \multicolumn{3}{|c|}{ B3LYP } & \multicolumn{3}{|c|}{ B3LYP* } \\
\hline & $\mathrm{x}$ & $\mathrm{y}$ & $\mathrm{Z}$ & $\mathrm{x}$ & $\mathrm{y}$ & $\mathrm{Z}$ \\
\hline 6 & 0.867801 & -1.139400 & -1.771832 & 0.858747 & -1.134220 & -1.774886 \\
\hline 6 & 2.551024 & 0.623053 & 0.464852 & 2.548221 & 0.628646 & 0.461903 \\
\hline 6 & 1.993278 & -0.804244 & 0.448541 & 1.992073 & -0.799302 & 0.445470 \\
\hline 6 & 1.665358 & -1.592250 & -0.687427 & 1.661633 & -1.589022 & -0.692108 \\
\hline 1 & 1.735457 & -2.676223 & -0.556759 & 1.737366 & -2.675089 & -0.564661 \\
\hline 1 & 2.376315 & -1.389734 & 1.286817 & 2.378022 & -1.386523 & 1.283931 \\
\hline 1 & 2.700124 & 0.905402 & 1.516668 & 2.697244 & 0.912643 & 1.515399 \\
\hline 1 & 3.560813 & 0.571359 & 0.025305 & 3.560064 & 0.577613 & 0.021746 \\
\hline 1 & 0.958714 & -0.131525 & -2.169397 & 0.946725 & -0.123402 & -2.171342 \\
\hline 1 & 0.516423 & -1.877763 & -2.490083 & 0.505217 & -1.872850 & -2.494774 \\
\hline 6 & -1.015929 & -0.726604 & 1.870321 & -0.999124 & -0.721034 & 1.873017 \\
\hline 6 & -2.462181 & 0.757964 & -0.591203 & -2.464868 & 0.751559 & -0.588024 \\
\hline 6 & -2.056494 & -0.711232 & -0.390038 & -2.053220 & -0.715857 & -0.384768 \\
\hline 6 & -1.813945 & -1.328956 & 0.868463 & -1.801550 & -1.331322 & 0.876025 \\
\hline 1 & -2.997712 & 1.101709 & 0.306353 & -3.000890 & 1.095150 & 0.311797 \\
\hline 1 & -3.198162 & 0.769507 & -1.406183 & -3.203446 & 0.759848 & -1.403370 \\
\hline 6 & 1.798797 & 1.789941 & -0.230052 & 1.793285 & 1.794096 & -0.234363 \\
\hline 6 & -1.376742 & 1.820373 & -0.933910 & -1.382636 & 1.817936 & -0.932882 \\
\hline 6 & -0.662365 & 2.354639 & 0.282333 & -0.668979 & 2.355605 & 0.282333 \\
\hline 6 & 0.649511 & 2.334021 & 0.579837 & 0.645468 & 2.338062 & 0.577910 \\
\hline 1 & 0.944610 & 2.766516 & 1.540986 & 0.942657 & 2.772503 & 1.539902 \\
\hline 1 & -1.327374 & 2.811133 & 1.022556 & -1.335495 & 2.811990 & 1.024343 \\
\hline 1 & -1.893906 & 2.665770 & -1.415685 & -1.903913 & 2.662295 & -1.416757 \\
\hline 1 & -0.678399 & 1.415022 & -1.672282 & -0.682070 & 1.412599 & -1.672192 \\
\hline 1 & 1.481564 & 1.513030 & -1.237999 & 1.472506 & 1.513102 & -1.242322 \\
\hline 1 & 2.530177 & 2.603922 & -0.355435 & 2.524952 & 2.609832 & -0.364636 \\
\hline 28 & -0.048703 & -0.948580 & 0.057708 & -0.046203 & -0.953300 & 0.057913 \\
\hline 1 & -2.461874 & -1.380865 & -1.149401 & -2.459647 & -1.390591 & -1.142010 \\
\hline 1 & -1.003900 & 0.353141 & 2.003890 & -0.988445 & 0.361449 & 2.001369 \\
\hline 1 & -0.734986 & -1.314986 & 2.741291 & -0.710914 & -1.306012 & 2.746329 \\
\hline \multirow[t]{3}{*}{1} & -1.972869 & -2.409003 & 0.934205 & -1.960543 & -2.413048 & 0.947912 \\
\hline & \multicolumn{3}{|c|}{ BP86 } & \multicolumn{3}{|c|}{ M06L } \\
\hline & $\mathrm{x}$ & $\mathrm{y}$ & $\mathrm{Z}$ & $\mathrm{x}$ & $\mathrm{y}$ & $\mathrm{Z}$ \\
\hline 6 & 0.833617 & -1.127530 & -1.782218 & 0.776675 & -1.144066 & -1.752261 \\
\hline 6 & 2.548545 & 0.645622 & 0.445385 & 2.574367 & 0.508557 & 0.435724 \\
\hline 6 & 1.990104 & -0.784012 & 0.440178 & 1.931912 & -0.867375 & 0.452386 \\
\hline 6 & 1.652292 & -1.587343 & -0.700100 & 1.555311 & -1.646690 & -0.676153 \\
\hline 1 & 1.737151 & -2.679768 & -0.572788 & 1.553889 & -2.730872 & -0.536667 \\
\hline 1 & 2.382407 & -1.372579 & 1.283653 & 2.283659 & -1.468633 & 1.291922 \\
\hline 1 & 2.710989 & 0.936568 & 1.501917 & 2.768197 & 0.798960 & 1.476872 \\
\hline 1 & 3.561369 & 0.592521 & -0.009319 & 3.568282 & 0.398423 & -0.024173 \\
\hline 1 & 0.926387 & -0.112001 & -2.186725 & 0.944707 & -0.150921 & -2.166145 \\
\hline 1 & 0.469676 & -1.870800 & -2.502337 & 0.373738 & -1.858169 & -2.466418 \\
\hline 6 & -0.953907 & -0.710260 & 1.881809 & -1.032969 & -0.643725 & 1.851662 \\
\hline 6 & -2.478397 & 0.730957 & -0.579163 & -2.410582 & 0.831159 & -0.579005 \\
\hline 6 & -2.042383 & -0.731948 & -0.374475 & -2.057654 & -0.639753 & -0.397590 \\
\hline 6 & -1.767244 & -1.345625 & 0.894790 & -1.833098 & -1.262627 & 0.860756 \\
\hline 1 & -3.017997 & 1.069995 & 0.328494 & -2.902513 & 1.193091 & 0.335942 \\
\hline 1 & -3.224619 & 0.729439 & -1.396488 & -3.168786 & 0.888496 & -1.368272 \\
\hline 6 & 1.778819 & 1.807810 & -0.249759 & 1.858118 & 1.686199 & -0.247234 \\
\hline 6 & -1.405173 & 1.811464 & -0.928860 & -1.279152 & 1.823275 & -0.928387 \\
\hline 6 & -0.690835 & 2.353884 & 0.286386 & -0.559034 & 2.318296 & 0.286281 \\
\hline 6 & 0.634036 & 2.345232 & 0.575060 & 0.748251 & 2.251763 & 0.579224 \\
\hline 1 & 0.936750 & 2.782360 & 1.541769 & 1.060902 & 2.645790 & 1.549955 \\
\hline 1 & -1.359938 & 2.808044 & 1.037391 & -1.212270 & 2.770720 & 1.038451 \\
\hline 1 & -1.938582 & 2.655665 & -1.415089 & -1.734657 & 2.686606 & -1.434742 \\
\hline 1 & -0.698667 & 1.407579 & -1.674042 & -0.590914 & 1.365962 & -1.649010 \\
\hline 1 & 1.445443 & 1.516758 & -1.258831 & 1.507185 & 1.415447 & -1.247147 \\
\hline 1 & 2.508172 & 2.632350 & -0.393734 & 2.606756 & 2.476346 & -0.400086 \\
\hline 28 & -0.038093 & -0.956527 & 0.058925 & -0.086421 & -0.904130 & 0.064632 \\
\hline 1 & -2.447262 & -1.421199 & -1.129312 & -2.468710 & -1.295463 & -1.164871 \\
\hline 1 & -0.959114 & 0.380689 & 2.002466 & -1.047313 & 0.439723 & 1.973587 \\
\hline 1 & -0.642879 & -1.287936 & 2.760424 & -0.759630 & -1.209411 & 2.738211 \\
\hline 1 & -1.919514 & -2.434432 & 0.978461 & -1.995603 & -2.340542 & 0.930480 \\
\hline
\end{tabular}


Table S42. Optimized coordinates for the $\left(\mathrm{C}_{4} \mathbf{H}_{6}\right)_{3} \mathbf{N i}$ structure $\mathrm{Ni}-2 \mathrm{~S}$.

\begin{tabular}{|c|c|c|c|c|c|c|}
\hline & \multicolumn{3}{|c|}{ B3LYP } & \multicolumn{3}{|c|}{ B3LYP* } \\
\hline & $\mathrm{x}$ & $\mathrm{y}$ & $\mathrm{Z}$ & $\mathrm{x}$ & $\mathrm{y}$ & Z \\
\hline 6 & -0.542761 & -1.968880 & 1.046541 & -0.549150 & -1.947846 & 1.066948 \\
\hline 6 & -2.850374 & 0.079605 & 0.361145 & -2.857213 & 0.088660 & 0.349333 \\
\hline 6 & -2.174365 & -0.961384 & -0.526863 & -2.172291 & -0.956652 & -0.528018 \\
\hline 6 & -1.350526 & -2.023864 & -0.125702 & -1.345877 & -2.015808 & -0.113918 \\
\hline 1 & -1.100522 & -2.769733 & -0.883053 & -1.089855 & -2.770717 & -0.863113 \\
\hline 1 & -2.555839 & -1.015411 & -1.548223 & -2.546535 & -1.018093 & -1.553807 \\
\hline 1 & -3.941536 & 0.027817 & 0.232071 & -3.948494 & 0.042578 & 0.203006 \\
\hline 1 & -2.651204 & -0.137649 & 1.416085 & -2.674690 & -0.129499 & 1.409199 \\
\hline 1 & -0.917052 & -1.505021 & 1.960018 & -0.932412 & -1.473513 & 1.973751 \\
\hline 1 & 0.185376 & -2.761544 & 1.206362 & 0.182198 & -2.737367 & 1.241173 \\
\hline 6 & 3.388701 & -0.770303 & -1.049978 & 3.361425 & -0.779050 & -1.068175 \\
\hline 6 & 2.082982 & 1.332804 & 0.836988 & 2.091764 & 1.322876 & 0.849814 \\
\hline 6 & 1.667318 & -0.153312 & 0.752675 & 1.667952 & -0.161004 & 0.760326 \\
\hline 6 & 2.511047 & -1.062749 & -0.060387 & 2.490755 & -1.072858 & -0.069739 \\
\hline 1 & 2.843173 & 1.559677 & 0.078603 & 2.863250 & 1.544033 & 0.098169 \\
\hline 1 & 2.539640 & 1.567600 & 1.809428 & 2.540001 & 1.554400 & 1.829008 \\
\hline 6 & -2.308465 & 1.499671 & 0.002576 & -2.298278 & 1.504128 & -0.005010 \\
\hline 6 & 0.856273 & 2.221433 & 0.573415 & 0.870712 & 2.215965 & 0.571940 \\
\hline 6 & 0.211725 & 1.662921 & -0.676652 & 0.230363 & 1.644336 & -0.675651 \\
\hline 6 & -1.108752 & 1.358175 & -0.930162 & -1.095813 & 1.344050 & -0.933137 \\
\hline 1 & -1.363350 & 1.147304 & -1.970104 & -1.349976 & 1.138539 & -1.976580 \\
\hline 1 & 0.885016 & 1.620943 & -1.537820 & 0.906361 & 1.599393 & -1.537210 \\
\hline 1 & 1.138571 & 3.276238 & 0.420514 & 1.158382 & 3.270258 & 0.410998 \\
\hline 1 & 0.163915 & 2.186759 & 1.423643 & 0.172808 & 2.191423 & 1.420641 \\
\hline 1 & -2.034411 & 2.045100 & 0.911628 & -2.018708 & 2.045338 & 0.907329 \\
\hline 1 & -3.077579 & 2.098696 & -0.505476 & -3.059607 & 2.115304 & -0.514795 \\
\hline 28 & -0.137933 & -0.379111 & -0.115063 & -0.140302 & -0.372836 & -0.114609 \\
\hline 1 & 1.552342 & -0.569877 & 1.760603 & 1.550691 & -0.585110 & 1.766562 \\
\hline 1 & 3.613314 & 0.249121 & -1.358483 & 3.591420 & 0.243337 & -1.369664 \\
\hline 1 & 3.913873 & -1.564133 & -1.577431 & 3.874484 & -1.573766 & -1.609787 \\
\hline \multirow[t]{3}{*}{1} & 2.371583 & -2.125494 & 0.161834 & 2.343058 & -2.137919 & 0.145897 \\
\hline & \multicolumn{3}{|c|}{ BP86 } & \multicolumn{3}{|c|}{ M06L } \\
\hline & $\mathrm{X}$ & $\mathrm{y}$ & $\mathrm{Z}$ & $\mathrm{x}$ & $\mathrm{y}$ & $\mathrm{Z}$ \\
\hline 6 & -0.600591 & -1.879978 & 1.146052 & -0.672027 & -1.891842 & 1.113065 \\
\hline 6 & -2.871449 & 0.146644 & 0.294573 & -2.762094 & 0.264718 & 0.323990 \\
\hline 6 & -2.161228 & -0.922214 & -0.541013 & -2.165067 & -0.829820 & -0.535251 \\
\hline 6 & -1.351686 & -1.986144 & -0.070120 & -1.428339 & -1.939359 & -0.089732 \\
\hline 1 & -1.081300 & -2.776915 & -0.786305 & -1.200147 & -2.717392 & -0.818967 \\
\hline 1 & -2.502944 & -1.007047 & -1.583662 & -2.520172 & -0.866998 & -1.565803 \\
\hline 1 & -3.961094 & 0.122994 & 0.091144 & -3.857739 & 0.272712 & 0.239554 \\
\hline 1 & -2.746907 & -0.068782 & 1.370411 & -2.539772 & 0.070132 & 1.379362 \\
\hline 1 & -1.015302 & -1.365354 & 2.025305 & -1.058670 & -1.380397 & 1.996150 \\
\hline 1 & 0.124536 & -2.670533 & 1.377339 & 0.013487 & -2.709123 & 1.327904 \\
\hline 6 & 3.223616 & -0.804094 & -1.171849 & 3.074482 & -0.744290 & -1.241963 \\
\hline 6 & 2.130297 & 1.267338 & 0.915382 & 2.122701 & 1.158688 & 0.997942 \\
\hline 6 & 1.671714 & -0.207298 & 0.793118 & 1.652557 & -0.288200 & 0.814862 \\
\hline 6 & 2.402143 & -1.114301 & -0.124482 & 2.320382 & -1.127814 & -0.185376 \\
\hline 1 & 2.950964 & 1.468023 & 0.200067 & 2.983468 & 1.356803 & 0.343627 \\
\hline 1 & 2.536914 & 1.479061 & 1.923570 & 2.471768 & 1.341085 & 2.021525 \\
\hline 6 & -2.250865 & 1.543970 & -0.047377 & -2.140570 & 1.616347 & -0.101323 \\
\hline 6 & 0.939399 & 2.189787 & 0.583402 & 0.982778 & 2.102045 & 0.624036 \\
\hline 6 & 0.313469 & 1.591593 & -0.665199 & 0.401432 & 1.548537 & -0.647346 \\
\hline 6 & -1.031238 & 1.318085 & -0.947762 & -0.927657 & 1.338093 & -0.970899 \\
\hline 1 & -1.271821 & 1.123755 & -2.004235 & -1.146566 & 1.123903 & -2.017425 \\
\hline 1 & 1.004282 & 1.525142 & -1.522335 & 1.122370 & 1.427987 & -1.460647 \\
\hline 1 & 1.257887 & 3.239154 & 0.403174 & 1.321030 & 3.140815 & 0.486507 \\
\hline 1 & 0.216719 & 2.202777 & 1.420532 & 0.222795 & 2.113211 & 1.416849 \\
\hline 1 & -1.964648 & 2.076494 & 0.876274 & -1.862975 & 2.208369 & 0.776957 \\
\hline 1 & -2.978143 & 2.192110 & -0.575702 & -2.856411 & 2.224861 & -0.668377 \\
\hline 28 & -0.142708 & -0.363369 & -0.101028 & -0.155505 & -0.380644 & -0.085553 \\
\hline 1 & 1.565676 & -0.673479 & 1.787854 & 1.566364 & -0.815105 & 1.769635 \\
\hline 1 & 3.467770 & 0.229833 & -1.446481 & 3.308688 & 0.300739 & -1.441607 \\
\hline 1 & 3.678003 & -1.594856 & -1.780152 & 3.485467 & -1.473687 & -1.933169 \\
\hline 1 & 2.233747 & -2.188360 & 0.063622 & 2.149698 & -2.202507 & -0.068621 \\
\hline
\end{tabular}


Table S43. Optimized coordinates for the $\left(\mathrm{C}_{4} \mathbf{H}_{6}\right)_{3} \mathbf{N i}$ structure $\mathrm{Ni}-3 \mathrm{~S}$.

\begin{tabular}{|c|c|c|c|c|c|c|}
\hline & \multicolumn{3}{|c|}{ B3LYP } & \multicolumn{3}{|c|}{ B3LYP* } \\
\hline & $\mathrm{x}$ & $\mathrm{y}$ & $\mathrm{Z}$ & $\mathrm{x}$ & $\mathrm{y}$ & $\mathrm{Z}$ \\
\hline 6 & -0.814261 & -2.150736 & -0.192186 & -0.810986 & -2.144917 & -0.199434 \\
\hline 6 & -2.194859 & 0.211920 & 1.403318 & -2.196774 & 0.208008 & 1.409043 \\
\hline 6 & -2.454691 & -0.299527 & -0.011689 & -2.453745 & -0.294010 & -0.010653 \\
\hline 6 & -2.000076 & -1.478191 & -0.610423 & -1.994779 & -1.468827 & -0.620524 \\
\hline 1 & -2.405162 & -1.711724 & -1.597435 & -2.398765 & -1.697450 & -1.611321 \\
\hline 1 & -3.234883 & 0.234631 & -0.556779 & -3.234406 & 0.244754 & -0.554617 \\
\hline 1 & -3.153223 & 0.390377 & 1.911730 & -3.157391 & 0.391799 & 1.915724 \\
\hline 1 & -1.661268 & -0.541212 & 1.992086 & -1.669158 & -0.552692 & 1.997261 \\
\hline 1 & -0.583683 & -2.239486 & 0.868981 & -0.583135 & -2.236637 & 0.864076 \\
\hline 1 & -0.446649 & -2.970896 & -0.806332 & -0.437495 & -2.961973 & -0.817624 \\
\hline 6 & 2.702557 & -1.024071 & 1.682661 & 2.696701 & -1.020215 & 1.687404 \\
\hline 6 & 2.425583 & 0.684424 & -0.775947 & 2.431741 & 0.674768 & -0.779409 \\
\hline 6 & 1.639006 & -0.631692 & -0.617655 & 1.637067 & -0.636300 & -0.616336 \\
\hline 6 & 2.015550 & -1.421978 & 0.587103 & 2.004368 & -1.421775 & 0.594019 \\
\hline 1 & 1.701409 & -2.468647 & 0.570266 & 1.681114 & -2.467802 & 0.585226 \\
\hline 1 & 1.756956 & -1.271422 & -1.504606 & 1.744817 & -1.282347 & -1.502253 \\
\hline 1 & 2.495205 & 0.944993 & -1.842229 & 2.500713 & 0.933386 & -1.848089 \\
\hline 1 & 3.457156 & 0.593742 & -0.401314 & 3.465032 & 0.578021 & -0.404969 \\
\hline 1 & 3.078998 & -0.010464 & 1.806042 & 3.081775 & -0.006784 & 1.801772 \\
\hline 1 & 2.916926 & -1.723155 & 2.489116 & 2.907018 & -1.715706 & 2.500534 \\
\hline 6 & -1.346115 & 1.529790 & 1.335682 & -1.337421 & 1.520180 & 1.343597 \\
\hline 6 & 1.667673 & 1.811152 & -0.057667 & 1.675097 & 1.802727 & -0.061704 \\
\hline 6 & 0.295972 & 1.853761 & -0.685392 & 0.301387 & 1.834712 & -0.688019 \\
\hline 6 & -0.953974 & 1.775336 & -0.115354 & -0.951823 & 1.760884 & -0.111404 \\
\hline 1 & -1.785420 & 2.055480 & -0.763482 & -1.784974 & 2.056110 & -0.754287 \\
\hline 1 & 0.309855 & 2.121393 & -1.746583 & 0.312036 & 2.107506 & -1.750159 \\
\hline 1 & 2.161464 & 2.787121 & -0.190606 & 2.166649 & 2.781698 & -0.197338 \\
\hline 1 & 1.608177 & 1.613424 & 1.018193 & 1.617945 & 1.606205 & 1.016671 \\
\hline 1 & -0.458914 & 1.440418 & 1.970215 & -0.445220 & 1.420055 & 1.973109 \\
\hline 1 & -1.922587 & 2.390413 & 1.704831 & -1.904741 & 2.386358 & 1.720057 \\
\hline \multirow[t]{3}{*}{28} & -0.347448 & -0.242719 & -0.592028 & -0.352386 & -0.231283 & -0.593331 \\
\hline & \multicolumn{3}{|c|}{ BP86 } & \multicolumn{3}{|c|}{ M06L } \\
\hline & $\mathrm{x}$ & $\mathrm{y}$ & $\mathrm{Z}$ & $\mathrm{x}$ & $\mathrm{y}$ & $\mathrm{Z}$ \\
\hline 6 & -0.811268 & -2.137888 & -0.216777 & -0.987700 & -2.077196 & -0.261030 \\
\hline 6 & -2.199714 & 0.201878 & 1.425834 & -2.010603 & 0.304088 & 1.456806 \\
\hline 6 & -2.448345 & -0.271373 & -0.009718 & -2.420950 & -0.100719 & 0.055737 \\
\hline 6 & -1.986740 & -1.443872 & -0.649899 & -2.110563 & -1.284970 & -0.622424 \\
\hline 1 & -2.388393 & -1.656224 & -1.652614 & -2.570308 & -1.429571 & -1.600557 \\
\hline 1 & -3.227924 & 0.283943 & -0.552262 & -3.167663 & 0.538028 & -0.416364 \\
\hline 1 & -3.167280 & 0.396517 & 1.930190 & -2.896837 & 0.510636 & 2.071768 \\
\hline 1 & -1.685571 & -0.580116 & 2.010856 & -1.476615 & -0.515997 & 1.949318 \\
\hline 1 & -0.597376 & -2.242393 & 0.855802 & -0.755672 & -2.225682 & 0.794149 \\
\hline 1 & -0.425426 & -2.949440 & -0.846341 & -0.694611 & -2.899908 & -0.909287 \\
\hline 6 & 2.691302 & -1.002767 & 1.702871 & 2.482677 & -1.008557 & 1.752323 \\
\hline 6 & 2.449153 & 0.645748 & -0.798376 & 2.461620 & 0.427139 & -0.839232 \\
\hline 6 & 1.631913 & -0.652479 & -0.613231 & 1.559615 & -0.782689 & -0.605826 \\
\hline 6 & 1.977772 & -1.418356 & 0.618006 & 1.788248 & -1.458670 & 0.685175 \\
\hline 1 & 1.627899 & -2.462612 & 0.637736 & 1.350097 & -2.454811 & 0.777445 \\
\hline 1 & 1.713615 & -1.321374 & -1.492930 & 1.619869 & -1.515128 & -1.421368 \\
\hline 1 & 2.511570 & 0.894395 & -1.876300 & 2.540446 & 0.632689 & -1.915332 \\
\hline 1 & 3.490270 & 0.533481 & -0.429101 & 3.485670 & 0.251950 & -0.471278 \\
\hline 1 & 3.104248 & 0.009801 & 1.787967 & 2.986317 & -0.043390 & 1.760252 \\
\hline 1 & 2.891201 & -1.686078 & 2.537409 & 2.586699 & -1.615646 & 2.647722 \\
\hline 6 & -1.313278 & 1.501669 & 1.367492 & -1.082196 & 1.549794 & 1.357389 \\
\hline 6 & 1.698738 & 1.781808 & -0.082259 & 1.812053 & 1.628134 & -0.166077 \\
\hline 6 & 0.315702 & 1.785448 & -0.701027 & 0.436978 & 1.735203 & -0.753719 \\
\hline 6 & -0.948055 & 1.732079 & -0.100493 & -0.786305 & 1.784703 & -0.108909 \\
\hline 1 & -1.785533 & 2.070182 & -0.728955 & -1.626651 & 2.155516 & -0.695640 \\
\hline 1 & 0.312900 & 2.069335 & -1.767938 & 0.422011 & 1.999732 & -1.814933 \\
\hline 1 & 2.183953 & 2.769547 & -0.229753 & 2.367871 & 2.561206 & -0.336626 \\
\hline 1 & 1.650164 & 1.592207 & 1.005080 & 1.756427 & 1.479289 & 0.918915 \\
\hline 1 & -0.406585 & 1.372850 & 1.983209 & -0.156004 & 1.374573 & 1.915752 \\
\hline 1 & -1.856975 & 2.382078 & 1.765284 & -1.553639 & 2.439053 & 1.796452 \\
\hline 28 & -0.367423 & -0.207409 & -0.592567 & -0.395523 & -0.197861 & -0.598274 \\
\hline
\end{tabular}


Table S44. Optimized coordinates for the $\left(\mathrm{C}_{4} \mathrm{H}_{6}\right)_{3} \mathrm{Ni}$ structure Ni-4S.

\begin{tabular}{|c|c|c|c|c|c|c|}
\hline & \multicolumn{3}{|c|}{ B3LYP } & \multicolumn{3}{|c|}{ B3LYP* } \\
\hline & $\mathrm{x}$ & $\mathrm{y}$ & $\mathrm{Z}$ & $\mathrm{x}$ & $\mathrm{y}$ & $\mathrm{Z}$ \\
\hline 6 & -1.621293 & -1.874154 & 0.774955 & -1.599529 & -1.874940 & 0.784486 \\
\hline 6 & -2.476100 & 1.183846 & 0.644310 & -2.493481 & 1.173221 & 0.640806 \\
\hline 6 & -2.546044 & 0.096260 & -0.418415 & -2.544317 & 0.081457 & -0.419865 \\
\hline 6 & -2.401922 & -1.288092 & -0.260773 & -2.382833 & -1.303763 & -0.258678 \\
\hline 1 & -2.646383 & -1.909431 & -1.125048 & -2.619657 & -1.933358 & -1.121619 \\
\hline 1 & -2.957864 & 0.421733 & -1.375827 & -2.956729 & 0.399697 & -1.381745 \\
\hline 1 & -3.454417 & 1.674986 & 0.759852 & -3.477121 & 1.661319 & 0.742660 \\
\hline 1 & -2.224844 & 0.750129 & 1.618275 & -2.251236 & 0.742912 & 1.620792 \\
\hline 1 & -1.618796 & -1.454996 & 1.782436 & -1.601406 & -1.446925 & 1.790443 \\
\hline 1 & -1.435821 & -2.944792 & 0.730819 & -1.399694 & -2.945281 & 0.747586 \\
\hline 6 & 1.135131 & -1.534906 & 0.476935 & 1.142926 & -1.526031 & 0.477607 \\
\hline 6 & 3.175233 & 0.889513 & 0.433482 & 3.170956 & 0.900626 & 0.434593 \\
\hline 6 & 3.297606 & -0.426131 & -0.310009 & 3.302534 & -0.413887 & -0.309290 \\
\hline 6 & 2.394383 & -1.433938 & -0.307471 & 2.400340 & -1.425480 & -0.308586 \\
\hline 1 & 2.616044 & -2.285087 & -0.958812 & 2.622999 & -2.278168 & -0.960815 \\
\hline 1 & 4.193101 & -0.541128 & -0.924026 & 4.201515 & -0.526291 & -0.922233 \\
\hline 1 & 3.786581 & 1.630114 & -0.101796 & 3.781031 & 1.646144 & -0.099185 \\
\hline 1 & 3.621870 & 0.811101 & 1.437970 & 3.616403 & 0.823873 & 1.441991 \\
\hline 1 & 1.246441 & -1.102074 & 1.481509 & 1.250761 & -1.085765 & 1.481165 \\
\hline 1 & 0.874593 & -2.590887 & 0.598200 & 0.880580 & -2.582950 & 0.601630 \\
\hline 6 & -1.374389 & 2.198494 & 0.217833 & -1.388044 & 2.186925 & 0.218678 \\
\hline 6 & 1.737490 & 1.460439 & 0.580046 & 1.727968 & 1.463141 & 0.576799 \\
\hline 6 & 0.856758 & 1.154353 & -0.618461 & 0.846161 & 1.135762 & -0.616189 \\
\hline 6 & -0.455601 & 1.524900 & -0.801291 & -0.472443 & 1.505777 & -0.801077 \\
\hline 1 & -0.828236 & 1.514352 & -1.826085 & -0.840761 & 1.507434 & -1.829855 \\
\hline 1 & 1.396792 & 0.823501 & -1.506003 & 1.390384 & 0.812894 & -1.506811 \\
\hline 1 & 1.816710 & 2.553624 & 0.686187 & 1.800302 & 2.560056 & 0.669802 \\
\hline 1 & 1.272690 & 1.102012 & 1.504767 & 1.267070 & 1.112795 & 1.509205 \\
\hline 1 & -0.809605 & 2.547784 & 1.089246 & -0.820413 & 2.531330 & 1.092670 \\
\hline 1 & -1.821266 & 3.087659 & -0.249062 & -1.830787 & 3.081482 & -0.246533 \\
\hline \multirow[t]{3}{*}{28} & -0.476968 & -0.564004 & -0.217480 & -0.474095 & -0.553502 & -0.219459 \\
\hline & \multicolumn{3}{|c|}{ BP86 } & \multicolumn{3}{|c|}{ M06L } \\
\hline & $\mathrm{x}$ & $\mathrm{y}$ & $\mathrm{Z}$ & $\mathrm{x}$ & $\mathrm{y}$ & $\mathrm{Z}$ \\
\hline 6 & -1.556108 & -1.878583 & 0.808811 & -1.548877 & -1.870707 & 0.790001 \\
\hline 6 & -2.531839 & 1.149383 & 0.633014 & -2.405537 & 1.137272 & 0.694058 \\
\hline 6 & -2.534705 & 0.051206 & -0.428448 & -2.513235 & 0.075015 & -0.374768 \\
\hline 6 & -2.339687 & -1.341152 & -0.259680 & -2.355810 & -1.311541 & -0.232256 \\
\hline 1 & -2.556996 & -1.989632 & -1.122341 & -2.610817 & -1.933375 & -1.090794 \\
\hline 1 & -2.945399 & 0.355444 & -1.403217 & -2.947366 & 0.413833 & -1.316401 \\
\hline 1 & -3.531515 & 1.624645 & 0.710588 & -3.382972 & 1.590651 & 0.914362 \\
\hline 1 & -2.302175 & 0.723618 & 1.625638 & -2.051479 & 0.695204 & 1.633052 \\
\hline 1 & -1.578505 & -1.430608 & 1.813603 & -1.528452 & -1.433248 & 1.790086 \\
\hline 1 & -1.324881 & -2.950186 & 0.790501 & -1.329805 & -2.934434 & 0.757514 \\
\hline 6 & 1.161329 & -1.507274 & 0.483052 & 1.157666 & -1.504387 & 0.451018 \\
\hline 6 & 3.167338 & 0.915941 & 0.445734 & 3.105781 & 0.889969 & 0.493017 \\
\hline 6 & 3.313323 & -0.388425 & -0.316690 & 3.284502 & -0.376050 & -0.297364 \\
\hline 6 & 2.407422 & -1.408611 & -0.321613 & 2.398968 & -1.395089 & -0.330417 \\
\hline 1 & 2.621669 & -2.260333 & -0.988392 & 2.624350 & -2.226444 & -1.002482 \\
\hline 1 & 4.215091 & -0.490423 & -0.938797 & 4.185325 & -0.448548 & -0.905913 \\
\hline 1 & 3.789379 & 1.675472 & -0.066886 & 3.744178 & 1.662247 & 0.046145 \\
\hline 1 & 3.598607 & 0.823401 & 1.465272 & 3.487824 & 0.764928 & 1.517526 \\
\hline 1 & 1.270895 & -1.045706 & 1.483924 & 1.254679 & -1.044006 & 1.445539 \\
\hline 1 & 0.895581 & -2.568367 & 0.619062 & 0.893141 & -2.555988 & 0.589672 \\
\hline 6 & -1.422950 & 2.168728 & 0.218581 & -1.379955 & 2.166847 & 0.180266 \\
\hline 6 & 1.713406 & 1.472448 & 0.570298 & 1.662621 & 1.434678 & 0.567245 \\
\hline 6 & 0.828194 & 1.097746 & -0.610980 & 0.824993 & 1.084795 & -0.636843 \\
\hline 6 & -0.509610 & 1.469293 & -0.800988 & -0.489870 & 1.468905 & -0.838225 \\
\hline 1 & -0.864548 & 1.499335 & -1.842046 & -0.851892 & 1.474642 & -1.866453 \\
\hline 1 & 1.380503 & 0.793054 & -1.512459 & 1.386473 & 0.764901 & -1.515806 \\
\hline 1 & 1.778346 & 2.579007 & 0.626376 & 1.713835 & 2.530147 & 0.651718 \\
\hline 1 & 1.253478 & 1.149962 & 1.522018 & 1.164575 & 1.099541 & 1.486671 \\
\hline 1 & -0.851917 & 2.511464 & 1.099349 & -0.790152 & 2.586688 & 1.002768 \\
\hline 1 & -1.862256 & 3.071886 & -0.249008 & -1.882513 & 3.013067 & -0.304538 \\
\hline 28 & -0.470072 & -0.531294 & -0.219990 & -0.483086 & -0.529216 & -0.236681 \\
\hline
\end{tabular}


Table S45. Optimized coordinates for the $\left(\mathrm{C}_{4} \mathrm{H}_{6}\right)_{3} \mathbf{N i}$ structure Ni-5S.

\begin{tabular}{|c|c|c|c|c|c|c|}
\hline & \multicolumn{3}{|c|}{ B3LYP } & \multicolumn{3}{|c|}{ B3LYP* } \\
\hline & $\mathrm{x}$ & $\mathrm{y}$ & $\mathrm{Z}$ & $\mathrm{x}$ & $\mathrm{y}$ & $\mathrm{Z}$ \\
\hline 6 & -3.014999 & -0.744075 & -0.203564 & -3.004471 & -0.750048 & -0.200195 \\
\hline 6 & -0.081553 & -1.626675 & -0.954485 & -0.073198 & -1.627375 & -0.964994 \\
\hline 6 & -0.811228 & -1.935422 & 0.298625 & -0.792167 & -1.933058 & 0.293734 \\
\hline 6 & -2.064512 & -1.548703 & 0.646169 & -2.047665 & -1.549375 & 0.647405 \\
\hline 1 & -2.438067 & -1.844036 & 1.630165 & -2.415685 & -1.843369 & 1.636063 \\
\hline 1 & -0.256149 & -2.517091 & 1.041241 & -0.229832 & -2.512149 & 1.035742 \\
\hline 1 & 0.654549 & -2.397502 & -1.207842 & 0.667650 & -2.393794 & -1.224059 \\
\hline 1 & -0.722550 & -1.435150 & -1.815435 & -0.719080 & -1.430993 & -1.823540 \\
\hline 1 & -2.633403 & -0.657341 & -1.224204 & -2.625849 & -0.662883 & -1.224161 \\
\hline 1 & -3.973511 & -1.283535 & -0.277386 & -3.963086 & -1.293908 & -0.270263 \\
\hline 6 & -3.329311 & 0.655769 & 0.373371 & -3.321591 & 0.650273 & 0.376732 \\
\hline 6 & -0.418461 & 1.124487 & -1.208276 & -0.417857 & 1.121440 & -1.217672 \\
\hline 6 & -1.016253 & 1.822803 & -0.045132 & -1.010487 & 1.820823 & -0.053813 \\
\hline 6 & -2.183572 & 1.621198 & 0.619750 & -2.177665 & 1.619324 & 0.616564 \\
\hline 1 & -2.373945 & 2.312674 & 1.445153 & -2.366683 & 2.314628 & 1.441706 \\
\hline 1 & -0.403356 & 2.637210 & 0.355307 & -0.396093 & 2.638615 & 0.342761 \\
\hline 1 & 0.160114 & 1.812981 & -1.836957 & 0.162882 & 1.805558 & -1.851697 \\
\hline 1 & -1.137194 & 0.590277 & -1.827144 & -1.137342 & 0.579205 & -1.832080 \\
\hline 1 & -4.066475 & 1.144641 & -0.287503 & -4.064672 & 1.136566 & -0.282873 \\
\hline 1 & -3.850545 & 0.517222 & 1.332498 & -3.839705 & 0.511189 & 1.339735 \\
\hline 6 & 2.403747 & 1.616168 & -0.184472 & 2.379892 & 1.612005 & -0.191834 \\
\hline 6 & 2.757151 & -1.286261 & 0.140055 & 2.736772 & -1.280135 & 0.135837 \\
\hline 6 & 2.351393 & -0.466303 & 1.171148 & 2.337779 & -0.461107 & 1.176729 \\
\hline 6 & 2.179023 & 0.970387 & 1.014997 & 2.163870 & 0.974256 & 1.019264 \\
\hline 1 & 1.693592 & 1.506790 & 1.829002 & 1.679131 & 1.515100 & 1.833445 \\
\hline 1 & 1.982710 & -0.915430 & 2.092898 & 1.971680 & -0.913067 & 2.100569 \\
\hline 1 & 2.709240 & -2.365727 & 0.259353 & 2.686864 & -2.361827 & 0.252449 \\
\hline 1 & 3.297324 & -0.924515 & -0.730936 & 3.289407 & -0.917163 & -0.729339 \\
\hline 1 & 2.107392 & 2.655688 & -0.300837 & 2.078850 & 2.651622 & -0.314456 \\
\hline 1 & 3.049314 & 1.215513 & -0.961905 & 3.039533 & 1.214307 & -0.961894 \\
\hline \multirow[t]{3}{*}{28} & 0.913300 & -0.045461 & -0.340164 & 0.912242 & -0.043563 & -0.331952 \\
\hline & \multicolumn{3}{|c|}{ BP86 } & \multicolumn{3}{|c|}{ M06L } \\
\hline & $\mathrm{x}$ & $\mathrm{y}$ & $\mathrm{Z}$ & $\mathrm{x}$ & $\mathrm{y}$ & $\mathrm{Z}$ \\
\hline 6 & -2.975690 & -0.767380 & -0.187232 & -2.913047 & -0.725315 & -0.170156 \\
\hline 6 & -0.048060 & -1.629296 & -1.004203 & -0.066800 & -1.641803 & -1.037633 \\
\hline 6 & -0.732770 & -1.924823 & 0.274810 & -0.706095 & -1.899862 & 0.254799 \\
\hline 6 & -1.996537 & -1.551216 & 0.651824 & -1.933703 & -1.487794 & 0.662114 \\
\hline 1 & -2.343021 & -1.841604 & 1.656342 & -2.249434 & -1.732368 & 1.678064 \\
\hline 1 & -0.148593 & -2.499679 & 1.012524 & -0.109926 & -2.459682 & 0.981427 \\
\hline 1 & 0.703645 & -2.386033 & -1.282708 & 0.671131 & -2.394953 & -1.324639 \\
\hline 1 & -0.710799 & -1.418174 & -1.855266 & -0.744600 & -1.427313 & -1.865031 \\
\hline 1 & -2.609871 & -0.681239 & -1.223913 & -2.550048 & -0.643810 & -1.199105 \\
\hline 1 & -3.935436 & -1.324071 & -0.241336 & -3.855698 & -1.289331 & -0.228427 \\
\hline 6 & -3.299015 & 0.638052 & 0.388528 & -3.245812 & 0.662088 & 0.394955 \\
\hline 6 & -0.414012 & 1.117430 & -1.249153 & -0.428836 & 1.124181 & -1.251616 \\
\hline 6 & -0.988645 & 1.815853 & -0.076765 & -0.963659 & 1.809004 & -0.072039 \\
\hline 6 & -2.156834 & 1.614579 & 0.612653 & -2.107154 & 1.614873 & 0.631985 \\
\hline 1 & -2.336108 & 2.316095 & 1.443297 & -2.258206 & 2.290627 & 1.475244 \\
\hline 1 & -0.368778 & 2.642244 & 0.311218 & -0.320673 & 2.604391 & 0.318997 \\
\hline 1 & 0.170898 & 1.792299 & -1.898513 & 0.157487 & 1.787686 & -1.895049 \\
\hline 1 & -1.138254 & 0.554140 & -1.851922 & -1.162079 & 0.580451 & -1.846842 \\
\hline 1 & -4.055108 & 1.118387 & -0.271944 & -3.986904 & 1.140859 & -0.264739 \\
\hline 1 & -3.813357 & 0.501161 & 1.361343 & -3.770361 & 0.524455 & 1.350523 \\
\hline 6 & 2.320698 & 1.590730 & -0.226968 & 2.265240 & 1.554129 & -0.176702 \\
\hline 6 & 2.669567 & -1.269504 & 0.129633 & 2.618115 & -1.270176 & 0.159651 \\
\hline 6 & 2.299584 & -0.444339 & 1.198898 & 2.197796 & -0.472957 & 1.212794 \\
\hline 6 & 2.131441 & 0.991140 & 1.027676 & 2.025835 & 0.952882 & 1.054021 \\
\hline 1 & 1.660505 & 1.556750 & 1.841799 & 1.506289 & 1.498072 & 1.838751 \\
\hline 1 & 1.943571 & -0.895570 & 2.134816 & 1.794663 & -0.935845 & 2.111643 \\
\hline 1 & 2.611430 & -2.357517 & 0.247665 & 2.561130 & -2.351112 & 0.248907 \\
\hline 1 & 3.262708 & -0.912834 & -0.720399 & 3.232325 & -0.893370 & -0.654221 \\
\hline 1 & 2.017156 & 2.633873 & -0.374042 & 1.958800 & 2.584087 & -0.340219 \\
\hline 1 & 3.016333 & 1.191652 & -0.975006 & 2.994017 & 1.164275 & -0.883572 \\
\hline 28 & 0.900526 & -0.038473 & -0.305435 & 0.873974 & -0.055792 & -0.320394 \\
\hline
\end{tabular}


Table S46. Optimized coordinates for the $\left(\mathrm{C}_{4} \mathrm{H}_{6}\right)_{3} \mathbf{N i}$ structure Ni-1T.

\begin{tabular}{|c|c|c|c|c|c|c|}
\hline & \multicolumn{3}{|c|}{ B3LYP } & \multicolumn{3}{|c|}{ B3LYP* } \\
\hline & $\mathrm{x}$ & $\mathrm{y}$ & $\mathrm{Z}$ & $\mathrm{x}$ & $\mathrm{y}$ & $\mathrm{Z}$ \\
\hline 6 & 1.147187 & -1.582879 & -1.100642 & 1.109910 & -1.562383 & -1.120407 \\
\hline 6 & 2.952550 & 0.908607 & -0.289461 & 2.956406 & 0.898201 & -0.299112 \\
\hline 6 & 2.822758 & -0.422850 & 0.423380 & 2.820522 & -0.441655 & 0.395339 \\
\hline 6 & 2.034203 & -1.493531 & 0.028893 & 1.998237 & -1.495264 & 0.003310 \\
\hline 1 & 2.054394 & -2.362702 & 0.692041 & 2.011145 & -2.372961 & 0.658525 \\
\hline 1 & 3.381771 & -0.535111 & 1.351862 & 3.388151 & -0.575040 & 1.318048 \\
\hline 1 & 3.666833 & 1.525411 & 0.270580 & 3.683538 & 1.500584 & 0.263477 \\
\hline 1 & 3.384176 & 0.774205 & -1.293476 & 3.377383 & 0.774708 & -1.311348 \\
\hline 1 & 1.336548 & -0.927825 & -1.956324 & 1.281211 & -0.888189 & -1.966874 \\
\hline 1 & 0.808090 & -2.579158 & -1.383739 & 0.737786 & -2.547023 & -1.407924 \\
\hline 6 & -1.636980 & -1.566848 & 1.234512 & -1.611693 & -1.551144 & 1.236441 \\
\hline 6 & -2.727528 & 0.973597 & -0.392664 & -2.741931 & 0.956286 & -0.410076 \\
\hline 6 & -2.199312 & -0.354048 & -0.911321 & -2.202833 & -0.369060 & -0.921851 \\
\hline 6 & -1.955257 & -1.510932 & -0.150954 & -1.915897 & -1.513136 & -0.154878 \\
\hline 1 & -3.203548 & 0.833332 & 0.588872 & -3.233235 & 0.812871 & 0.565762 \\
\hline 1 & -3.510309 & 1.344479 & -1.070279 & -3.513985 & 1.332048 & -1.100387 \\
\hline 6 & 1.622679 & 1.709483 & -0.440213 & 1.627995 & 1.709946 & -0.421055 \\
\hline 6 & -1.623368 & 2.048679 & -0.259637 & -1.629857 & 2.020224 & -0.253597 \\
\hline 6 & -0.641631 & 1.653520 & 0.821370 & -0.654225 & 1.579263 & 0.818535 \\
\hline 6 & 0.715251 & 1.525367 & 0.761799 & 0.714159 & 1.467949 & 0.767510 \\
\hline 1 & 1.232548 & 1.349011 & 1.706610 & 1.229201 & 1.298500 & 1.717193 \\
\hline 1 & -1.090605 & 1.547669 & 1.812816 & -1.106593 & 1.466135 & 1.809687 \\
\hline 1 & -2.072649 & 3.017260 & 0.017163 & -2.068551 & 2.990507 & 0.042872 \\
\hline 1 & -1.119518 & 2.189794 & -1.223482 & -1.122375 & 2.175103 & -1.215849 \\
\hline 1 & 1.100340 & 1.414672 & -1.359340 & 1.109347 & 1.457878 & -1.357253 \\
\hline 1 & 1.856881 & 2.779084 & -0.561045 & 1.865102 & 2.785764 & -0.492388 \\
\hline 28 & -0.121402 & -0.551235 & 0.113142 & -0.110670 & -0.505233 & 0.133978 \\
\hline 1 & -2.229745 & -0.486094 & -1.993472 & -2.218318 & -0.505063 & -2.006021 \\
\hline 1 & -2.006147 & -0.805222 & 1.920971 & -2.014879 & -0.800603 & 1.917967 \\
\hline 1 & -1.450604 & -2.541692 & 1.680355 & -1.390428 & -2.518457 & 1.688098 \\
\hline \multirow[t]{3}{*}{1} & -1.802525 & -2.431514 & -0.718467 & -1.740497 & -2.435612 & -0.715931 \\
\hline & \multicolumn{3}{|c|}{ BP86 } & \multicolumn{3}{|c|}{ M06L } \\
\hline & $\mathrm{x}$ & $\mathrm{y}$ & Z & $\mathrm{X}$ & $\mathrm{y}$ & Z \\
\hline 6 & 1.033880 & -1.514202 & -1.165188 & 1.128810 & -1.548621 & -1.140024 \\
\hline 6 & 2.994244 & 0.839659 & -0.295452 & 2.885307 & 0.883780 & -0.291117 \\
\hline 6 & 2.819758 & -0.513797 & 0.363802 & 2.723566 & -0.413757 & 0.451110 \\
\hline 6 & 1.898422 & -1.508446 & -0.022911 & 1.922692 & -1.474915 & 0.039705 \\
\hline 1 & 1.868820 & -2.406041 & 0.615194 & 1.879067 & -2.332723 & 0.713421 \\
\hline 1 & 3.400093 & -0.704002 & 1.276281 & 3.214637 & -0.503593 & 1.418162 \\
\hline 1 & 3.747666 & 1.407625 & 0.280296 & 3.617524 & 1.501137 & 0.240133 \\
\hline 1 & 3.407923 & 0.731044 & -1.320153 & 3.303444 & 0.714476 & -1.293935 \\
\hline 1 & 1.221779 & -0.807677 & -1.989177 & 1.360887 & -0.874982 & -1.970117 \\
\hline 1 & 0.600541 & -2.472156 & -1.478704 & 0.750751 & -2.520911 & -1.448051 \\
\hline 6 & -1.620907 & -1.514813 & 1.255666 & -1.528563 & -1.510467 & 1.276438 \\
\hline 6 & -2.725215 & 0.964931 & -0.447643 & -2.707505 & 0.875920 & -0.419764 \\
\hline 6 & -2.158963 & -0.361489 & -0.938211 & -2.104966 & -0.420549 & -0.906393 \\
\hline 6 & -1.878046 & -1.510188 & -0.151342 & -1.828881 & -1.546058 & -0.107963 \\
\hline 1 & -3.249961 & 0.818613 & 0.517829 & -3.186583 & 0.720831 & 0.558531 \\
\hline 1 & -3.477951 & 1.343654 & -1.167886 & -3.499506 & 1.207453 & -1.103842 \\
\hline 6 & 1.678200 & 1.686970 & -0.380098 & 1.568293 & 1.683524 & -0.431265 \\
\hline 6 & -1.600272 & 2.014026 & -0.248013 & -1.642710 & 1.966981 & -0.272617 \\
\hline 6 & -0.647514 & 1.493320 & 0.818559 & -0.685372 & 1.560831 & 0.812838 \\
\hline 6 & 0.746153 & 1.372633 & 0.783158 & 0.682555 & 1.475886 & 0.771003 \\
\hline 1 & 1.251912 & 1.214893 & 1.748378 & 1.204021 & 1.327134 & 1.717348 \\
\hline 1 & -1.107957 & 1.401343 & 1.816120 & -1.146357 & 1.444903 & 1.796772 \\
\hline 1 & -2.025310 & 2.991172 & 0.071211 & -2.098496 & 2.935309 & -0.012089 \\
\hline 1 & -1.074849 & 2.184191 & -1.206015 & -1.117570 & 2.108697 & -1.226385 \\
\hline 1 & 1.163273 & 1.505334 & -1.342633 & 1.029623 & 1.380483 & -1.342794 \\
\hline 1 & 1.939320 & 2.767082 & -0.378705 & 1.785630 & 2.753075 & -0.570393 \\
\hline 28 & -0.130285 & -0.446048 & 0.149854 & -0.092344 & -0.457793 & 0.098465 \\
\hline 1 & -2.138489 & -0.505590 & -2.028311 & -2.117883 & -0.582749 & -1.984211 \\
\hline 1 & -2.054757 & -0.754669 & 1.916865 & -1.954482 & -0.732453 & 1.910766 \\
\hline 1 & -1.366095 & -2.468148 & 1.735239 & -1.296525 & -2.444365 & 1.782658 \\
\hline 1 & -1.696419 & -2.448965 & -0.695713 & -1.621900 & -2.478862 & -0.634683 \\
\hline
\end{tabular}


Table S47. Optimized coordinates for the $\left(\mathrm{C}_{4} \mathrm{H}_{6}\right)_{3} \mathbf{N i}$ structure Ni-2T.

\begin{tabular}{|c|c|c|c|c|c|c|}
\hline & \multicolumn{3}{|c|}{$\overline{B 3 L Y P}$} & \multicolumn{3}{|c|}{ B3LYP* } \\
\hline & $\mathrm{x}$ & $\mathrm{y}$ & $\mathrm{Z}$ & $\mathrm{x}$ & $\mathrm{y}$ & $\mathrm{Z}$ \\
\hline 6 & -1.296460 & -1.822423 & -0.203532 & -1.288921 & -1.779702 & -0.246924 \\
\hline 6 & -2.249396 & 0.540616 & 1.456524 & -2.297432 & 0.505861 & 1.459289 \\
\hline 6 & -3.081858 & -0.143904 & 0.400754 & -3.111499 & -0.156639 & 0.376339 \\
\hline 6 & -2.610226 & -1.179823 & -0.351896 & -2.609058 & -1.162584 & -0.403156 \\
\hline 1 & -3.249037 & -1.540732 & -1.163447 & -3.227324 & -1.511240 & -1.238153 \\
\hline 1 & -4.071043 & 0.262718 & 0.184219 & -4.105786 & 0.241198 & 0.158299 \\
\hline 1 & -2.879729 & 0.879963 & 2.290020 & -2.946881 & 0.863247 & 2.272525 \\
\hline 1 & -1.524587 & -0.169046 & 1.871871 & -1.605952 & -0.225439 & 1.899701 \\
\hline 1 & -0.959156 & -1.944243 & 0.831270 & -0.953238 & -1.905940 & 0.789669 \\
\hline 1 & -1.207701 & -2.769750 & -0.750413 & -1.158836 & -2.709820 & -0.819290 \\
\hline 6 & 2.263787 & -0.849549 & 2.172879 & 2.233397 & -0.931494 & 2.154705 \\
\hline 6 & 2.654916 & 0.683231 & -0.442449 & 2.676390 & 0.683173 & -0.404709 \\
\hline 6 & 2.027437 & -0.717850 & -0.366339 & 2.030724 & -0.711398 & -0.379453 \\
\hline 6 & 2.007910 & -1.381758 & 0.952675 & 1.985088 & -1.420795 & 0.911751 \\
\hline 1 & 1.737168 & -2.442119 & 0.926554 & 1.700134 & -2.477804 & 0.846305 \\
\hline 1 & 2.449906 & -1.397948 & -1.122119 & 2.427789 & -1.372884 & -1.167811 \\
\hline 1 & 2.978450 & 0.877123 & -1.475497 & 3.016412 & 0.905178 & -1.428594 \\
\hline 1 & 3.556118 & 0.754459 & 0.188061 & 3.570070 & 0.727284 & 0.242281 \\
\hline 1 & 2.561652 & 0.188339 & 2.307889 & 2.545031 & 0.098994 & 2.326009 \\
\hline 1 & 2.198376 & -1.459186 & 3.071880 & 2.148465 & -1.569906 & 3.034078 \\
\hline 6 & -1.458619 & 1.775395 & 0.916910 & -1.450738 & 1.718521 & 0.947456 \\
\hline 6 & 1.657722 & 1.797182 & -0.036149 & 1.673652 & 1.785979 & 0.018640 \\
\hline 6 & 0.409518 & 1.675481 & -0.878723 & 0.434265 & 1.663369 & -0.838476 \\
\hline 6 & -0.895630 & 1.617985 & -0.478162 & -0.879445 & 1.574528 & -0.446185 \\
\hline 1 & -1.648690 & 1.576292 & -1.267757 & -1.628752 & 1.557479 & -1.243229 \\
\hline 1 & 0.588566 & 1.713917 & -1.960629 & 0.620186 & 1.744656 & -1.919044 \\
\hline 1 & 2.111649 & 2.787623 & -0.196594 & 2.121980 & 2.785004 & -0.116002 \\
\hline 1 & 1.410716 & 1.719208 & 1.027487 & 1.417521 & 1.681673 & 1.080172 \\
\hline 1 & -0.669748 & 2.035841 & 1.632010 & -0.658064 & 1.933891 & 1.676345 \\
\hline 1 & -2.143103 & 2.636698 & 0.871617 & -2.102002 & 2.608474 & 0.915792 \\
\hline \multirow[t]{3}{*}{28} & 0.078057 & -0.559881 & -0.932906 & 0.087168 & -0.499534 & -0.935882 \\
\hline & \multicolumn{3}{|c|}{ BP86 } & \multicolumn{3}{|c|}{ M06L } \\
\hline & $\mathrm{x}$ & $\mathrm{y}$ & $\mathrm{Z}$ & $\mathrm{X}$ & $\mathrm{y}$ & $\mathrm{Z}$ \\
\hline 6 & -1.178681 & -1.728015 & -0.260655 & -1.348473 & -1.698786 & -0.215004 \\
\hline 6 & -2.500446 & 0.364848 & 1.418662 & -2.257106 & 0.603447 & 1.408991 \\
\hline 6 & -3.186055 & -0.308038 & 0.256540 & -3.099765 & -0.017500 & 0.340848 \\
\hline 6 & -2.524367 & -1.236273 & -0.525179 & -2.631917 & -1.050450 & -0.416622 \\
\hline 1 & -3.030800 & -1.601126 & -1.433352 & -3.243452 & -1.386378 & -1.256820 \\
\hline 1 & -4.196425 & 0.015871 & -0.029061 & -4.066909 & 0.425819 & 0.107372 \\
\hline 1 & -3.239782 & 0.731501 & 2.154986 & -2.874752 & 1.029696 & 2.206971 \\
\hline 1 & -1.863419 & -0.372552 & 1.942590 & -1.632904 & -0.169547 & 1.875735 \\
\hline 1 & -0.885408 & -1.809054 & 0.799417 & -1.034720 & -1.788336 & 0.831004 \\
\hline 1 & -0.878136 & -2.617790 & -0.846948 & -1.224326 & -2.650755 & -0.745719 \\
\hline 6 & 2.304315 & -1.279045 & 1.957312 & 1.950281 & -0.843678 & 2.214050 \\
\hline 6 & 2.699583 & 0.844544 & -0.230917 & 2.731056 & 0.538339 & -0.351788 \\
\hline 6 & 2.092721 & -0.549322 & -0.475432 & 2.003456 & -0.799050 & -0.313933 \\
\hline 6 & 2.071611 & -1.516700 & 0.626103 & 1.781209 & -1.407793 & 0.993691 \\
\hline 1 & 1.830136 & -2.554009 & 0.339503 & 1.409934 & -2.436400 & 0.970712 \\
\hline 1 & 2.411657 & -1.017798 & -1.430191 & 2.389000 & -1.529757 & -1.038378 \\
\hline 1 & 3.102400 & 1.236880 & -1.184992 & 3.130121 & 0.708053 & -1.360601 \\
\hline 1 & 3.548269 & 0.800817 & 0.482816 & 3.595128 & 0.552171 & 0.332300 \\
\hline 1 & 2.573974 & -0.284906 & 2.334417 & 2.347089 & 0.162253 & 2.339583 \\
\hline 1 & 2.244529 & -2.089156 & 2.693260 & 1.724115 & -1.394110 & 3.122643 \\
\hline 6 & -1.583139 & 1.571486 & 0.994389 & -1.312692 & 1.712301 & 0.876368 \\
\hline 6 & 1.605861 & 1.821284 & 0.275069 & 1.770009 & 1.685424 & -0.012886 \\
\hline 6 & 0.429443 & 1.686167 & -0.674334 & 0.558530 & 1.554541 & -0.887644 \\
\hline 6 & -0.924099 & 1.474690 & -0.369196 & -0.763118 & 1.492881 & -0.502859 \\
\hline 1 & -1.625670 & 1.529993 & -1.217503 & -1.510762 & 1.483202 & -1.299995 \\
\hline 1 & 0.667987 & 1.916262 & -1.730396 & 0.759485 & 1.595788 & -1.965706 \\
\hline 1 & 1.979528 & 2.866516 & 0.298806 & 2.248380 & 2.662373 & -0.166917 \\
\hline 1 & 1.304452 & 1.554307 & 1.304239 & 1.475101 & 1.638359 & 1.041514 \\
\hline 1 & -0.826318 & 1.739101 & 1.782330 & -0.500145 & 1.867836 & 1.595441 \\
\hline 1 & -2.212362 & 2.485342 & 0.966338 & -1.872205 & 2.658186 & 0.836451 \\
\hline 28 & 0.116246 & -0.335855 & -0.899302 & 0.092607 & -0.501661 & -0.936602 \\
\hline
\end{tabular}


Table S48. Harmonic vibrational frequencies (in $\mathrm{cm}^{-1}$ ) and infrared intensities (in parentheses in $\mathrm{km} / \mathrm{mol})$ for the $\left(\mathbf{C}_{4} \mathbf{H}_{6}\right)_{3} \mathbf{T i}$ structure Ti-1S.

\begin{tabular}{|c|c|c|c|c|c|c|c|}
\hline \multicolumn{2}{|c|}{ B3LYP } & \multicolumn{2}{|c|}{ B3LYP* } & \multicolumn{2}{|c|}{ BP86 } & \multicolumn{2}{|c|}{ M06L } \\
\hline 62( & 0) & 60( & 0) & 57( & 0) & 54( & 0) \\
\hline 94( & 1) & 97( & 1) & 97( & 2) & 97( & 1) \\
\hline 119( & 1) & 119 & 1) & 109( & 1) & 111( & 1) \\
\hline 152( & 2) & 155( & 3) & 149( & 1) & 160( & 0) \\
\hline 158( & 1) & 159 & 0) & 161( & 1) & 172( & 2) \\
\hline 178( & 3) & 178( & 2) & 174( & 2) & 179( & 1) \\
\hline 191( & 1) & 194( & 1) & 201( & 1) & 213( & 1) \\
\hline 233( & 2) & 232( & 2) & 225( & 1) & 237( & 1) \\
\hline 256( & 3) & 255( & 3) & 248( & 4) & 262( & 3) \\
\hline 268( & 3) & 268( & 3) & 262( & 2) & 281( & 3) \\
\hline 286( & 7) & 288( & 7) & 290( & 5) & 305( & 4) \\
\hline 323( & 3) & 321( & 3) & 315( & 3) & 335( & 4) \\
\hline 327( & 5) & 325( & 5) & 327( & 5) & 346( & 7) \\
\hline 349 ( & 2) & 346( & 2) & 338( & 1) & 361( & 2) \\
\hline 373( & 5) & 372( & 5) & 361( & 5) & 386( & 4) \\
\hline 405( & 3) & 402( & 3) & 396( & 3) & 425( & 3) \\
\hline 429 ( & 2) & 427( & 2) & 413( & 1) & 434( & 3) \\
\hline 458( & 3) & 457( & 2) & 441( & 2) & 455( & 4) \\
\hline 486( & 7) & 484( & 7) & 468( & 7) & 489 & 8) \\
\hline 510( & 4) & 512( & 3) & 497( & 4) & 523( & 4) \\
\hline 559( & 2) & 556( & 2) & 537( & 1) & 554( & 1) \\
\hline 607( & 9) & 602( & 7) & 573( & 9) & 577( & 11) \\
\hline 628( & 18) & 622 & 19) & 5961 & 13) & 605( & 12) \\
\hline 638( & 5) & 633 & 5) & 610( & 2) & 628( & 2) \\
\hline 674( & 1) & 668( & 1) & 644( & 1) & 671( & 9) \\
\hline 680( & 15) & 676( & 14) & 6531 & 14) & 675( & 14) \\
\hline 705( & 4) & 703 ( & 4) & 675( & 6) & 697( & 4) \\
\hline 777( & 13) & 769 & 14) & 747( & 13) & 762( & 2) \\
\hline 792( & 24) & 784( & 14) & 758( & 13) & 773( & 36) \\
\hline 796( & 14) & 786( & 26) & 762( & 30) & 784( & 16) \\
\hline 810 & 30) & 806( & 27) & 770( & 23) & 787( & 16) \\
\hline 823( & 48) & 816( & 46) & 791( & 25) & 814( & 12) \\
\hline 835( & 1) & 830( & 1) & 800( & 13) & 828( & 15) \\
\hline 848( & 4) & 839 & 4) & 810( & 7) & 835( & 15) \\
\hline 861( & 6) & 852( & 6) & 821( & 3) & 851( & 5) \\
\hline 871( & 1) & 864( & 1) & 843( & 4) & 876( & 4) \\
\hline 932( & 5) & 924( & 4) & 897( & 4) & 927( & 3) \\
\hline 946( & 9) & 939 & 8) & 905( & 4) & 939( & 2) \\
\hline 956( & 0) & 943( & 0) & 906( & 2) & 943( & 9) \\
\hline 966( & 3) & 960 & 4) & 912( & 5) & 952( & 3) \\
\hline 999( & 6) & 991( & 10) & 957( & 16) & 989 & 14) \\
\hline 1003 ( & 7) & 995( & 4) & 965( & 1) & 998( & 2) \\
\hline 1017( & 4) & 1007( & 3) & 976( & 1) & 1010( & 1) \\
\hline 1050( & 2) & 1043( & 2) & 1016( & 2) & 1052( & 2) \\
\hline 1064( & 5) & 1057 & 12) & 1030( & 13) & 1065( & 11) \\
\hline 1065( & 12) & 1057( & 5) & 1032( & 4) & 1066( & 8) \\
\hline
\end{tabular}




\begin{tabular}{|c|c|c|c|c|c|c|c|}
\hline 1069 ( & 11) & 1062( & 11) & 1037( & 10) & 1070( & 9) \\
\hline 1143( & 5) & 1135( & 5) & 1105( & 4) & 1140( & 5) \\
\hline 1165( & 7) & 1156( & 6) & 1127( & 6) & 1165( & 6) \\
\hline 1189( & 9) & 1182( & 9) & 1153( & 9) & 1190( & 10) \\
\hline 1212( & 2) & 1203( & 2) & 1170( & 3) & 1214( & 2) \\
\hline 1236( & 7) & 1226( & 7) & 1193( & 6) & 1240( & 11) \\
\hline 1247( & 13) & 1239 & 12) & 1214( & 9) & 1249 & 7) \\
\hline 1269( & 5) & 1260( & 5) & 1227( & 3) & 1263( & 5) \\
\hline 1275( & 6) & 1266 & 6) & 1235( & 5) & 1269 & 4) \\
\hline 1323( & 2) & 1311( & 2) & 1269 & 2) & 1316( & 1) \\
\hline 1356( & 6) & 1343( & 6) & 1302( & 5) & 1353( & 7) \\
\hline 1396( & 0) & 1385( & 0) & 1344( & 0) & 1378( & 0) \\
\hline 1404( & 2) & 1391( & 2) & 1349 & 2) & 1394( & 1) \\
\hline 1417( & 0) & 1405( & 0) & 1364( & 1) & 1412( & 0) \\
\hline 1475( & 6) & 1463( & 6) & 1419 & 7) & 1448( & 8) \\
\hline 1480( & 0) & 1467( & 0) & 1426( & 0) & 1464( & 1) \\
\hline 1484( & 3) & 1471( & 3) & 1428( & 4) & 1469 & 3) \\
\hline 1496( & 3) & 1483( & 3) & 1441( & 3) & 1481( & 17) \\
\hline 1498( & 8) & 1486( & 8) & 1446( & 6) & 1484( & 1) \\
\hline 1505( & 16) & 1491( & 17) & 1448( & 20) & 1486( & 12) \\
\hline 1529( & 20) & 1519 ( & 18) & 1488( & 14) & 1537( & 16) \\
\hline 1561( & 21) & 1551( & 20) & 1509 & 20) & 1555( & 22) \\
\hline 1576( & 38) & 1565( & 37) & 1528( & 29) & 1574( & 37) \\
\hline 3012( & 22) & 2991( & 22) & 2933( & 29) & 3031( & 26) \\
\hline 3026( & 84) & 3006( & 81) & 2952( & 82) & 3042( & 145) \\
\hline 3074( & 3) & 3055( & 2) & 2994( & 1) & 3089 & 11) \\
\hline 3094( & 27) & 3075( & 26) & 30160 & 28) & 3091( & 16) \\
\hline 3097( & 7) & 3077( & 7) & 3017 & 8) & 3106( & 19) \\
\hline 3123( & 2) & 3102( & 2) & 3042( & 4) & 3110( & 21) \\
\hline 3124( & 11) & 3106( & 10) & 30460 & 7) & 3112( & 10) \\
\hline 3128( & 8) & 3107( & 8) & 3050 & 11) & 3121( & 32) \\
\hline 3134( & 7) & 3113( & 7) & 3054( & 8) & 3145( & 16) \\
\hline 3161( & 3) & 3141( & 4) & 3085( & 4) & 3157( & 13) \\
\hline 3164( & 1) & 3143( & 2) & 3088( & 2) & 3158( & 6) \\
\hline 3174( & 10) & 3154( & 10) & 3101( & 9) & 3179 & 50) \\
\hline 3179 & 5) & 3159 & 5) & 3103( & 10) & 3179 & 4) \\
\hline 3184( & 18) & 3163( & 18) & 3107( & 19) & 3182( & 26) \\
\hline 3210( & 7) & 3192( & 6) & 3140 & 6) & 3213( & 20) \\
\hline 3217 ( & 4) & 3198( & 4) & 3146( & 3) & 3215( & 10) \\
\hline 3222( & 10) & 3203( & 9) & 3149 & 10) & 3220 & 16) \\
\hline 3241( & 6) & 3221( & 6) & 3168( & 6) & 3244( & 15) \\
\hline
\end{tabular}


Table S49. Harmonic vibrational frequencies (in $\mathrm{cm}^{-1}$ ) and infrared intensities (in parentheses in $\mathrm{km} / \mathrm{mol})$ for the $\left(\mathbf{C}_{4} \mathbf{H}_{6}\right)_{3} \mathbf{T i}$ structure Ti-2S.

\begin{tabular}{|c|c|c|c|c|c|c|c|}
\hline \multicolumn{2}{|c|}{ B3LYP } & \multicolumn{2}{|c|}{ B3LYP* } & \multicolumn{2}{|c|}{ BP86 } & \multicolumn{2}{|c|}{ M06L } \\
\hline 70( & 2) & 65( & 1) & 49( & 1) & 79( & 2) \\
\hline 95( & 2) & 95( & 2) & 86( & 1) & 101( & 1) \\
\hline 118( & 1) & 117( & 1) & 109 & 1) & 134( & 1) \\
\hline 145( & 4) & 144( & 5) & 146( & 8) & 162( & 5) \\
\hline 171( & 5) & 173( & 5) & 174( & 7) & 178( & 5) \\
\hline 188( & 0) & 194( & 0) & 198( & 1) & 204( & 1) \\
\hline 209( & 1) & 212( & 0) & 222( & 4) & 229 & 0) \\
\hline 235( & 4) & 234( & 6) & 238( & 1) & 260( & 6) \\
\hline 240( & 7) & 242( & 3) & 252( & 0) & 269 & 3) \\
\hline 256( & 1) & 257( & 1) & 264( & 1) & 280( & 1) \\
\hline 263( & 5) & 266( & 5) & 282( & 3) & 289 & 4) \\
\hline 277( & 7) & 274( & 6) & 287( & 9) & 305( & 6) \\
\hline 306( & 2) & 305( & 2) & 303( & 2) & 319 ( & 2) \\
\hline 337( & 1) & 335( & 1) & 332( & 3) & 364( & 1) \\
\hline 343( & 4) & 341( & 3) & 341( & 1) & 369 & 6) \\
\hline 364( & 4) & 362( & 4) & 358( & 1) & 395( & 0) \\
\hline 385( & 2) & 382( & 3) & 369( & 6) & 403( & 4) \\
\hline 445( & 23) & 446( & 24) & 446( & 25) & 453( & 25) \\
\hline 485( & 3) & 483( & 4) & 470( & 3) & 494( & 3) \\
\hline 494( & 2) & 493( & 1) & 486( & 1) & 502( & 2) \\
\hline 502( & 2) & 500 & 1) & 495( & 1) & 515( & 1) \\
\hline 576( & 1) & 570( & 1) & 548( & 1) & 556( & 2) \\
\hline 596( & 1) & 594( & 1) & 585( & 1) & 595( & 2) \\
\hline 620( & 5) & 615( & 4) & 599 & 2) & 615( & 4) \\
\hline 674( & 21) & 660( & 20) & 645( & 21) & 657( & 17) \\
\hline 686( & 10) & 680 & 12) & 662( & 9) & 677( & 10) \\
\hline 695( & 3) & 684( & 1) & 674( & 1) & 688( & 3) \\
\hline 714( & 4) & 706( & 4) & 689 & 2) & 702( & 7) \\
\hline 720( & 4) & 715( & 4) & 6961 & 5) & 710( & 3) \\
\hline 743( & 21) & 736( & 20) & 718( & 17) & 732( & 15) \\
\hline 799( & 15) & 790 & 14) & 769 & 8) & 766( & 8) \\
\hline 824( & 8) & 807( & 16) & 779 ( & 10) & 799( & 8) \\
\hline 828( & 7) & 812( & 2) & 787 & 10) & 804( & 1) \\
\hline 844( & 6) & 838( & 6) & 819 & 4) & 821( & 3) \\
\hline 864( & 11) & 856( & 12) & 826( & 3) & 829 & 12) \\
\hline 872( & 1) & 860( & 1) & 831( & 10) & 849 & 4) \\
\hline 892( & 6) & 882( & 8) & 855( & 8) & 866( & 2) \\
\hline 899( & 1) & 885 & 2) & 858( & 16) & 879) & 13) \\
\hline 900( & 8) & 890( & 6) & 864( & 1) & 900( & 6) \\
\hline 937( & 4) & 931( & 1) & 890( & 3) & 928( & 3) \\
\hline 941( & 1) & 932( & 7) & 899 & 3) & 940( & 6) \\
\hline 947( & 2) & 934( & 1) & 910( & 2) & 941( & 2) \\
\hline 951( & 2) & 944 ( & 1) & 921( & 2) & 950( & 3) \\
\hline 958( & 3) & 948( & 5) & 9231 & 2) & 961( & 4) \\
\hline 963( & 3) & 950 & 0) & 927( & 0) & 964( & 2) \\
\hline 1072( & 1) & 1064( & 2) & 1040 & 2) & 1071( & 2) \\
\hline
\end{tabular}




\begin{tabular}{|c|c|c|c|c|c|c|c|}
\hline 1073( & 2) & 1065( & 2) & 1041( & 1) & 1072( & 4) \\
\hline 1073( & 2) & 1067( & 3) & 1042( & 6) & 1075( & 3) \\
\hline 1080( & 9) & 1072( & 7) & 1047( & 2) & 1077( & 6) \\
\hline 1088( & 1) & 1079( & 1) & 1048( & 1) & 1085( & 1) \\
\hline 1093( & 7) & 1084( & 7) & 1055( & 7) & 1088( & 6) \\
\hline 1202( & 14) & 1192( & 13) & 1167( & 11) & 1199( & 13) \\
\hline 1227( & 2) & 1216( & 2) & 1177( & 3) & 1221( & 3) \\
\hline 1232( & 10) & 1221( & 10) & 1188( & 9) & 1228( & 10) \\
\hline 1252( & 24) & 1243( & 22) & 1216( & 23) & 1251( & 20) \\
\hline 1277( & 36) & 1266( & 32) & 1227( & 18) & 1274( & 39) \\
\hline 1287( & 13) & 1276( & 11) & 1244( & 10) & 1283( & 9) \\
\hline 1400( & 0) & 1387( & 0) & 1348( & 0) & 1383( & 1) \\
\hline 1409 & 1) & 1396( & 0) & 1351( & 0) & 1391( & 0) \\
\hline 1413( & 0) & 1400 & 0) & 1359 & 0) & 1392( & 0) \\
\hline 1467( & 34) & 1455( & 28) & 1418( & 14) & 1457( & 17) \\
\hline 1479( & 1) & 1467( & 2) & 1430( & 4) & 1468( & 9) \\
\hline 1483( & 5) & 1471( & 5) & 1433( & 6) & 1471( & 2) \\
\hline 1517( & 13) & 1504( & 13) & 1469 & 12) & 1503( & 13) \\
\hline 1529 ( & 32) & 1517( & 30) & 1479 & 14) & 1525( & 14) \\
\hline 1537( & 40) & 1524( & 42) & 1484( & 25) & 1530( & 24) \\
\hline 1543( & 19) & 1528( & 13) & 1486( & 15) & 1536( & 58) \\
\hline 1552( & 10) & 1537( & 8) & 1490( & 23) & 1549( & 7) \\
\hline 1564( & 20) & 1550 & 21) & 1506( & 14) & 1566( & 30) \\
\hline 3132( & 14) & 3113( & 14) & 3062( & 14) & 3112( & 37) \\
\hline 3135( & 7) & 3115( & 6) & 3067( & 7) & 3121( & 20) \\
\hline 3158( & 3) & 3137( & 1) & 3071( & 15) & 3138( & 17) \\
\hline 3163( & 2) & 3142( & 16) & 3075( & 10) & 3149( & 19) \\
\hline 3166r & 4) & 3142( & 1) & 3080 & 5) & 3152( & 9) \\
\hline 3167( & 12) & 3143( & 3) & 3090( & 7) & 3159 ( & 10) \\
\hline 3168( & 5) & 3147( & 6) & 3093( & 1) & 3160( & 22) \\
\hline 3180( & 3) & 3158( & 3) & 3101( & 3) & 3171( & 6) \\
\hline 3184( & 6) & 3162( & 6) & 3109 & 5) & 3184( & 9) \\
\hline 3193( & 2) & 3169 & 2) & 3110( & 3) & 3186( & 44) \\
\hline 3196( & 11) & 3174( & 11) & 3116( & 17) & 3188( & 4) \\
\hline 3211( & 5) & 3188( & 6) & 3127( & 11) & 3208( & 18) \\
\hline 3217 ( & 12) & 3197( & 10) & 3151( & 10) & 3211( & 29) \\
\hline 3219 ( & 8) & 3200 & 7) & 3152( & 6) & 3213( & 9) \\
\hline 3253( & 1) & 3230( & 3) & 3165( & 5) & 3241( & 11) \\
\hline 3256( & 2) & 3233( & 2) & 3169 & 4) & 3251( & 15) \\
\hline 3257 ( & 10) & 3235( & 8) & 3175( & 7) & 3257( & 7) \\
\hline 3259 & 4) & 3237( & 3) & 3178( & 5) & 32580 & 14) \\
\hline
\end{tabular}


Table S50. Harmonic vibrational frequencies (in $\mathrm{cm}^{-1}$ ) and infrared intensities (in parentheses in $\mathrm{km} / \mathrm{mol})$ for the $\left(\mathbf{C}_{4} \mathbf{H}_{6}\right)_{3} \mathbf{T i}$ structure Ti-1T.

\begin{tabular}{|c|c|c|c|c|c|c|c|}
\hline \multicolumn{2}{|c|}{ B3LYP } & \multicolumn{2}{|c|}{ B3LYP* } & \multicolumn{2}{|r|}{ BP86 } & \multicolumn{2}{|c|}{ M06L } \\
\hline 45( & 1) & 41( & 1) & 45( & 0) & 49 & 0) \\
\hline 54( & 0) & 45( & 0) & 62( & 0) & 62( & 0) \\
\hline 111( & 0) & 106( & 0) & 126( & 2) & 105( & 1) \\
\hline 117( & 0) & 121( & 0) & 130( & 1) & 124( & 0) \\
\hline 139 & 0) & 143( & 0) & 161( & 0) & 150( & 1) \\
\hline 164( & 1) & 164( & 1) & 168( & 0) & 181( & 0) \\
\hline 182( & 2) & 184( & 2) & 188( & 1) & 208( & 1) \\
\hline 202( & 1) & 193( & 1) & 196( & 0) & 214( & 1) \\
\hline 217( & 1) & 221( & 0) & 218( & 1) & 252( & 1) \\
\hline 2360 & 1) & 237( & 1) & 228( & 0) & 267( & 1) \\
\hline 249 & 3) & 250( & 2) & 255( & 1) & 272( & 0) \\
\hline 284( & 3) & 275( & 2) & 295( & 2) & 297( & 1) \\
\hline 297( & 5) & 299 & 5) & 318( & 2) & 335( & 4) \\
\hline 326( & 2) & 326( & 2) & 320( & 2) & 353( & 8) \\
\hline 332 & 13) & 329 & 11) & 326( & 4) & 355( & 5) \\
\hline 354( & 3) & 353( & 1) & 360( & 1) & 377( & 0) \\
\hline 361( & 4) & 357( & 3) & 364( & 2) & 3866 & 2) \\
\hline 426( & 5) & 421( & 7) & 423( & 3) & 430( & 2) \\
\hline 448( & 7) & 439 & 6) & 445( & 7) & 450( & 9) \\
\hline 462( & 7) & 459 & 6) & 460( & 5) & 474( & 6) \\
\hline 566( & 2) & 560( & 2) & 545( & 1) & 565( & 3) \\
\hline 582( & 1) & 577( & 1) & 548( & 0) & 582( & 1) \\
\hline 607( & 15) & 601( & 11) & 579 & 3) & 608( & 10) \\
\hline 627( & 4) & 620( & 4) & 609 & 2) & 624( & 4) \\
\hline 663 & 8) & 654( & 7) & 625( & 7) & 659 & 5) \\
\hline 672( & 3) & 664( & 2) & 640( & 3) & 670 & 3) \\
\hline 700( & 4) & 687( & 4) & 659 & 4) & 689 & 2) \\
\hline 754( & 5) & 747( & 6) & 720( & 16) & 742( & 0) \\
\hline 768( & 25) & 757( & 22) & 731( & 38) & 750( & 22) \\
\hline 769 & 26) & 763( & 31) & 740( & 24) & 759 & 25) \\
\hline 793( & 75) & 783( & 72) & 757( & 29) & 773( & 40) \\
\hline 808( & 17) & 800( & 10) & 762( & 13) & 783( & 27) \\
\hline 817( & 22) & 809 & 20) & 789 & 14) & 804( & 15) \\
\hline 828( & 10) & 818( & 14) & 799 & 16) & 827( & 16) \\
\hline 857( & 4) & 851( & 5) & 835( & 2) & 8681 & 5) \\
\hline 915 & 1) & 910( & 2) & 848( & 8) & 898( & 14) \\
\hline 919 & 17) & 911( & 18) & 883( & 5) & 912( & 7) \\
\hline 924( & 5) & 912( & 3) & 888( & 2) & 918( & 6) \\
\hline 946( & 0) & 937( & 0) & 891( & 11) & 923( & 2) \\
\hline 983( & 6) & 954( & 3) & 913( & 4) & 929 & 1) \\
\hline 991( & 5) & 979 & 8) & 9161 & 4) & 973( & 8) \\
\hline 1001( & 1) & 989 & 1) & 964( & 3) & 993( & 1) \\
\hline 1003( & 2) & 992( & 3) & 967( & 1) & 1001( & 4) \\
\hline 1053( & 4) & 1042( & 3) & 1014( & 3) & 1052 & 5) \\
\hline 1060 & 12) & 1050 & 13) & 1025( & 16) & 1056( & 18) \\
\hline 1064( & 1) & 1056( & 1) & 1028( & 3) & 1062( & 3) \\
\hline
\end{tabular}




\begin{tabular}{|c|c|c|c|c|c|c|c|}
\hline 1089 & 4) & 1081( & 4) & 1049 & 5) & 1086( & 4) \\
\hline 1138( & 7) & 1127( & 6) & 1104( & 4) & 1131( & 5) \\
\hline 1161( & 14) & 1150( & 15) & 1119 & 9) & 1154( & 17) \\
\hline 1205( & 1) & 1192( & 2) & 1164( & 3) & 1207( & 3) \\
\hline 1234( & 10) & 1221( & 8) & 1186( & 6) & 1229 & 16) \\
\hline 1239 & 5) & 1229( & 6) & 1192( & 7) & 12350 & 3) \\
\hline 1258( & 6) & 1245( & 6) & 1226( & 6) & 1248( & 2) \\
\hline 1276( & 11) & 1268( & 10) & 1234( & 7) & 1276( & 7) \\
\hline 1296( & 31) & 12866 & 24) & 12460 & 15) & 1292( & 17) \\
\hline 1323( & 1) & 1309 & 1) & 1268( & 2) & 1315( & 0) \\
\hline 1356( & 6) & 1341( & 5) & 1301( & 6) & 1357( & 5) \\
\hline 1404( & 1) & 1390( & 1) & 1347( & 1) & 1394( & 1) \\
\hline 1413( & 0) & 1399( & 1) & 1353( & 2) & 1395( & 0) \\
\hline 1414( & 1) & 1401( & 1) & 1357( & 2) & 1406( & 2) \\
\hline 1462( & 31) & 1452( & 23) & 1413( & 13) & 1457( & 8) \\
\hline 1477( & 1) & 1462( & 1) & 1420 & 0) & 1461( & 2) \\
\hline 1482( & 3) & 1468( & 3) & 1427( & 2) & 14660 & 4) \\
\hline 1489 ( & 1) & 1474( & 0) & 1433( & 2) & 1472( & 1) \\
\hline 1499( & 14) & 1486( & 15) & 1448( & 12) & 1481( & 12) \\
\hline 1551( & 23) & 1534( & 27) & 1496( & 21) & 1528( & 34) \\
\hline 1559 ( & 91) & 1547( & 73) & 1498( & 14) & 1558( & 55) \\
\hline 1570( & 69) & 1555( & 64) & 1502( & 19) & 1561( & 45) \\
\hline 1573( & 8) & 1559( & 5) & 1506( & 64) & 1564( & 11) \\
\hline 3008( & 15) & 2991( & 18) & 2931( & 24) & 3018( & 76) \\
\hline 3018( & 94) & 3000( & 87) & 2942( & 108) & 3035( & 105) \\
\hline 3068( & 1) & 3048( & 1) & 29960 & 4) & 3085( & 10) \\
\hline 3087( & 31) & 3068( & 30) & 3019 & 26) & 3102( & 31) \\
\hline 31160 & 4) & 3097( & 4) & 3054( & 4) & 3111( & 19) \\
\hline 3118( & 11) & 3100( & 10) & 3064( & 9) & 3124( & 18) \\
\hline 3138( & 9) & 3120( & 7) & 3069 & 5) & 3138( & 23) \\
\hline 3139 & 1) & 3123( & 3) & 3073( & 4) & 3146( & 14) \\
\hline 3161( & 1) & 3144( & 12) & 3083( & 1) & 3159 & 32) \\
\hline 3164( & 19) & 3145( & 7) & 3085( & 16) & 3163( & 10) \\
\hline 3173( & 5) & 3153( & 9) & 3089 & 11) & 3169 & 9) \\
\hline 3174( & 10) & 3154( & 5) & 3092( & 3) & 3181( & 1) \\
\hline 3177( & 1) & 3158( & 1) & 3106( & 1) & 3181( & 28) \\
\hline 3192( & 5) & 3172( & 5) & 3119 & 5) & 3202( & 15) \\
\hline 3211( & 14) & 3194( & 13) & 3152( & 10) & 3217( & 21) \\
\hline 3232( & 9) & 3212( & 9) & 3157( & 9) & 32300 & 20) \\
\hline 3256( & 3) & 3238( & 3) & 3179 & 4) & 3264( & 10) \\
\hline 32660 & 3) & 32476 & 3) & 31880 & 3) & 32690 & 9) \\
\hline
\end{tabular}


Table S51 Harmonic vibrational frequencies (in $\mathrm{cm}^{-1}$ ) and infrared intensities (in parentheses in $\mathrm{km} / \mathrm{mol})$ for the $\left(\mathbf{C}_{4} \mathbf{H}_{6}\right)_{3} \mathbf{V}$ structure V-1D.

\begin{tabular}{|c|c|c|c|c|c|c|c|}
\hline \multicolumn{2}{|c|}{ B3LYP } & \multicolumn{2}{|c|}{ B3LYP* } & \multicolumn{2}{|c|}{ BP86 } & \multicolumn{2}{|c|}{ M06L } \\
\hline 62( & 1) & 631 & 1) & 581 & 1) & 48( & 1) \\
\hline 107( & 2) & 114( & 1) & 112( & 0) & 117( & 2) \\
\hline 137( & 1) & 140( & 3) & 137( & 4) & 144( & 3) \\
\hline 147( & 5) & 148( & 2) & 153( & 1) & 165( & 2) \\
\hline 165( & 0) & 165( & 0) & 173( & 0) & 182( & 0) \\
\hline 206( & 3) & 205( & 3) & 201( & 0) & 210( & 1) \\
\hline 215( & 2) & 215 ( & 2) & 213( & 4) & 224( & 4) \\
\hline 222( & 7) & 227( & 8) & 224( & 0) & 247 & 6) \\
\hline 238( & 0) & 236( & 0) & 242( & 8) & 254( & 2) \\
\hline 251( & 0) & 252( & 0) & 252( & 3) & 265( & 1) \\
\hline 284( & 0) & 283( & 0) & 277( & 1) & 291( & 0) \\
\hline 348( & 0) & 349 & 0) & 350( & 1) & 363( & 1) \\
\hline 387( & 1) & 384( & 2) & 384( & 3) & 402( & 2) \\
\hline 419( & 9) & 417( & 5) & 410( & 1) & 430( & 2) \\
\hline 433( & 4) & 431( & 5) & 424( & 7) & 440( & 6) \\
\hline 455( & 5) & 452( & 6) & 440( & 3) & 473 & 1) \\
\hline 475( & 8) & 469 & 6) & 461( & 1) & 483( & 5) \\
\hline 482( & 10) & 476( & 10) & 471( & 8) & 495( & 10) \\
\hline 519( & 5) & 513( & 5) & 495( & 6) & 523( & 6) \\
\hline 571( & 4) & 564( & 3) & 539 & 2) & 559 & 3) \\
\hline 588( & 2) & 581( & 1) & 562( & 1) & 591( & 1) \\
\hline 651( & 3) & 6431 & 2) & 6281 & 2) & 659 & 4) \\
\hline 671( & 4) & 6661 & 4) & 642( & 2) & 669 & 5) \\
\hline 695( & 3) & 682( & 3) & 670( & 3) & 705( & 3) \\
\hline 771( & 14) & 7631 & 13) & 7431 & 13) & 752( & 11) \\
\hline 788( & 22) & 781( & 14) & 761( & 7) & 775( & 2) \\
\hline 791( & 8) & 787( & 14) & 765( & 13) & 783( & 16) \\
\hline 798( & 9) & 791( & 9) & 7731 & 6) & 800 & 6) \\
\hline 819 & 14) & 812( & 15) & 795( & 23) & 824( & 23) \\
\hline 848( & 50) & 837( & 45) & 815( & 15) & 841( & 21) \\
\hline 855 & 8) & 845( & 7) & 834( & 7) & 858( & 7) \\
\hline 862( & 6) & 856( & 3) & 836( & 6) & 861( & 6) \\
\hline 885 & 0) & 872( & 1) & 847( & 3) & 876( & 5) \\
\hline 926( & 5) & 916( & 3) & 879 & 3) & 915( & 2) \\
\hline 936( & 13) & 925( & 13) & 892( & 2) & 926( & 3) \\
\hline 950( & 1) & 935( & 2) & 902( & 11) & 935( & 14) \\
\hline 962( & 1) & 952( & 1) & 927( & 1) & 962( & 2) \\
\hline 992( & 1) & 968( & 0) & 932( & 1) & 973( & 1) \\
\hline 998( & 4) & 989 & 4) & 9631 & 4) & 998( & 4) \\
\hline 1004( & 4) & 995( & 4) & 966( & 3) & 1004( & 4) \\
\hline 1024( & 10) & 1017( & 10) & 991( & 10) & 1027 & 11) \\
\hline 1037( & 2) & 1028 & 2) & 999( & 2) & 1037 & 2) \\
\hline 1050( & 8) & 1042( & 7) & 1014( & 7) & 1052( & 5) \\
\hline 1091( & 4) & 1083 & 5) & 1056 & 4) & 1094( & 4) \\
\hline 1127( & 8) & 1118( & 8) & 1090( & 8) & 1125( & 9) \\
\hline 1152( & 7) & 1143( & 7) & 1114( & 6) & 1155 & 8) \\
\hline 1181( & 4) & 1172( & 4) & 1142( & 5) & 1184( & 5) \\
\hline 1200( & 1) & 1189 & 1) & 1156( & 2) & 1201( & 3) \\
\hline
\end{tabular}




\begin{tabular}{|c|c|c|c|c|c|c|c|}
\hline 1222( & 3) & 1211( & 3) & 1176( & 1) & 1226( & 1) \\
\hline 1232( & 2) & 1220 & 2) & 1189 & 2) & 1239 & 6) \\
\hline 1259( & 16) & 1248( & 16) & 1216( & 8) & 1250 & 12) \\
\hline 1266( & 6) & 1255( & 5) & 1220( & 7) & 1259 & 4) \\
\hline 1288( & 7) & 1276( & 6) & 1244( & 5) & 1283 & 6) \\
\hline 1310( & 1) & 12960 & 0) & 1257( & 0) & 1304( & 2) \\
\hline 1324( & 4) & 1311( & 5) & 1269 & 6) & 1320 & 3) \\
\hline 1325( & 3) & 1315( & 4) & 1270 & 4) & 1321( & 3) \\
\hline 1343( & 5) & 1327( & 4) & 1285( & 4) & 1344( & 5) \\
\hline 1394( & 1) & 1380 & 1) & 1339 & 1) & 1386( & 2) \\
\hline 1403( & 3) & 1389 & 3) & 1348( & 4) & 1397( & 4) \\
\hline 1414( & 2) & 1400( & 2) & 1356( & 3) & 1403 & 3) \\
\hline 1477( & 0) & 1465( & 1) & 1420 & 3) & 1459 & 1) \\
\hline 1480( & 5) & 1466( & 5) & 1422( & 4) & 1465( & 7) \\
\hline 1483( & 15) & 1470( & 6) & 1427( & 16) & 1469 & 17) \\
\hline 1484( & 4) & 1471( & 13) & 1430 & 4) & 1471( & 3) \\
\hline 1490( & 3) & 1477( & 3) & 1436( & 3) & 1477( & 2) \\
\hline 1502( & 11) & 1486( & 12) & 1446( & 11) & 1499 & 8) \\
\hline 1517 ( & 16) & 1503( & 15) & 1462( & 11) & 1516( & 15) \\
\hline 1560( & 27) & 1546( & 23) & 1501( & 15) & 1555 & 21) \\
\hline 1586( & 28) & 1569 & 27) & 1517( & 22) & 1586( & 24) \\
\hline 2998( & 60) & 2977( & 60) & 2919 & 67) & 3010 & 68) \\
\hline 3010 ( & 58) & 2989 & 59) & 2932 & 67) & 3020 & 117) \\
\hline 30331 & 72) & 3013( & 70) & 2955 & 73) & 3032( & 96) \\
\hline 3037( & 65) & 3017( & 64) & 2966( & 68) & 3053 & 119) \\
\hline 3067( & 16) & 3047( & 13) & 2990 & 14) & 3067( & 57) \\
\hline 3074( & 5) & 3053( & 5) & 2995( & 2) & 3083 & 10) \\
\hline 3082( & 13) & 3061( & 9) & 3004 & 8) & 3089 & 11) \\
\hline 3093( & 27) & 3072( & 4) & 3012( & 32) & 3092( & 20) \\
\hline 3095( & 12) & 3072( & 31) & 3015( & 7) & 3093 & 51) \\
\hline 3098( & 13) & 3074( & 24) & 3021( & 28) & 3103( & 16) \\
\hline 3112( & 5) & 3090( & 6) & 3032( & 9) & 3111( & 17) \\
\hline 3113( & 9) & 3093( & 8) & 3041( & 6) & 3114 & 41) \\
\hline 3116( & 5) & 3097( & 1) & 3044( & 1) & 3116( & 13) \\
\hline 3123( & 28) & 3101( & 28) & 3045( & 26) & 3125 & 47) \\
\hline 3160 & 12) & 3139 & 12) & 3080 & 13) & 3160 & 31) \\
\hline 3162( & 14) & 3142( & 14) & 3086( & 15) & 3169 & 30) \\
\hline 3194( & 9) & 3178( & 7) & 3128( & 4) & 3205( & 3) \\
\hline 3202( & 12) & 3183( & 12) & 3130( & 13) & 3206( & 33) \\
\hline
\end{tabular}


Table S52. Harmonic vibrational frequencies (in $\mathrm{cm}^{-1}$ ) and infrared intensities (in parentheses in $\mathrm{km} / \mathrm{mol}$ ) for the $\left(\mathbf{C}_{4} \mathbf{H}_{6}\right)_{3} \mathbf{V}$ structure $\mathrm{V}-2 \mathrm{D}$.

\begin{tabular}{|c|c|c|c|c|c|c|c|}
\hline \multicolumn{2}{|c|}{ B3LYP } & \multicolumn{2}{|c|}{ B3LYP* } & \multicolumn{2}{|c|}{ BP86 } & \multicolumn{2}{|c|}{ M06L } \\
\hline 83( & 1) & 84( & 1) & 80( & 1) & 96( & 1) \\
\hline 116( & 1) & 117( & 2) & 117( & 2) & 115( & 2) \\
\hline 125( & 2) & 119 & 1) & 123( & 1) & 135( & 2) \\
\hline 181( & 5) & 180( & 6) & 178( & 9) & 194( & 7) \\
\hline 181( & 1) & 188( & 4) & 195( & 5) & 203( & 5) \\
\hline 194( & 2) & 203( & 1) & 216( & 1) & 212( & 1) \\
\hline 218( & 2) & 222( & 1) & 240( & 1) & 234( & 1) \\
\hline 231( & 5) & 233( & 3) & 247( & 1) & 260( & 3) \\
\hline 240( & 2) & 245( & 3) & 260( & 1) & 264( & 1) \\
\hline 254( & 4) & 254( & 4) & 268( & 3) & 278( & 2) \\
\hline 268( & 11) & 273( & 7) & 287( & 4) & 289 ( & 15) \\
\hline 285( & 9) & 287( & 10) & 298( & 11) & 309( & 5) \\
\hline 304( & 2) & 306( & 4) & 309 & 3) & 318( & 5) \\
\hline 329 & 2) & 330 & 2) & 334( & 2) & 354( & 4) \\
\hline 338( & 3) & 341( & 4) & 348( & 6) & 364( & 7) \\
\hline 3581 & 3) & 363( & 3) & 3600 & 2) & 384( & 1) \\
\hline 393( & 7) & 3931 & 7) & 389 & 8) & 408( & 8) \\
\hline 447( & 25) & 449 & 25) & 451( & 29) & 442( & 24) \\
\hline 481( & 2) & 477( & 3) & 469 & 2) & 487( & 4) \\
\hline 498( & 3) & 494( & 4) & 489 & 3) & 502( & 3) \\
\hline 504( & 0) & 501( & 0) & 496( & 1) & 508( & 1) \\
\hline 593( & 1) & 588( & 1) & 577( & 0) & 584( & 1) \\
\hline 604( & 1) & 602( & 1) & 595( & 1) & 598( & 1) \\
\hline 620 & 3) & 613( & 3) & 599( & 2) & 616( & 4) \\
\hline 694( & 16) & 6861 & 12) & 671( & 8) & 650( & 2) \\
\hline 710( & 1) & 6981 & 3) & 685( & 2) & 680( & 11) \\
\hline 713( & 11) & 711( & 6) & 698( & 6) & 706( & 3) \\
\hline 728( & 2) & 718( & 2) & 705( & 2) & 708( & 2) \\
\hline 739 & 10) & 731( & 8) & 726( & 15) & 724( & 12) \\
\hline 754( & 9) & 751( & 16) & 742( & 10) & 744( & 11) \\
\hline 824( & 15) & 812( & 12) & 798( & 8) & 793( & 10) \\
\hline 827( & 16) & 823( & 18) & 808( & 22) & 801( & 7) \\
\hline 847( & 5) & 8331 & 7) & 826( & 5) & 821( & 4) \\
\hline 861( & 6) & 850 & 5) & 840( & 8) & 8331 & 2) \\
\hline 8831 & 9) & 880 & 7) & 849 & 4) & 852( & 5) \\
\hline 890( & 3) & 883( & 9) & 857( & 11) & 868( & 2) \\
\hline 895( & 0) & 891( & 1) & 866( & 1) & 871( & 2) \\
\hline 906( & 3) & 902( & 3) & 877( & 8) & 877( & 7) \\
\hline 910( & 4) & 907( & 5) & 892( & 1) & 893( & 9) \\
\hline 943( & 3) & 936( & 3) & 908( & 6) & 929 & 3) \\
\hline 950( & 2) & 942( & 1) & 911( & 1) & 940( & 3) \\
\hline 952( & 0) & 944( & 3) & 918( & 4) & 942( & 2) \\
\hline 959 & 1) & 947( & 1) & 926( & 1) & 949( & 1) \\
\hline 964( & 1) & 952( & 3) & 929( & 2) & 954( & 1) \\
\hline 968( & 6) & 955( & 1) & 936( & 0) & 959) & 3) \\
\hline 1075( & 1) & 1066( & 1) & 1042( & 3) & 1071( & 1) \\
\hline
\end{tabular}




\begin{tabular}{|c|c|c|c|c|c|c|c|}
\hline 1076( & 1) & 1068( & 2) & 1045( & 0) & 1074( & 1) \\
\hline 1076( & 1) & 1069( & 1) & 1046( & 1) & 1077( & 3) \\
\hline 1087( & 8) & 1078( & 7) & 1052( & 5) & 1084( & 3) \\
\hline 1091( & 3) & 1082( & 3) & 1054( & 2) & 1085( & 7) \\
\hline 1095( & 4) & 1085( & 3) & 1058( & 3) & 1086( & 1) \\
\hline 1217( & 13) & 1206( & 13) & 1176( & 2) & 1213( & 16) \\
\hline 1228( & 5) & 1215( & 4) & 1179( & 13) & 1218( & 3) \\
\hline 1235( & 5) & 1223( & 5) & 1188( & 4) & 1225( & 3) \\
\hline 1268( & 32) & 1257( & 28) & 1225( & 18) & 1264( & 28) \\
\hline 1278( & 24) & 1266( & 21) & 1230( & 18) & 1269 & 27) \\
\hline 1287( & 12) & 1275( & 9) & 1240( & 5) & 1278( & 6) \\
\hline 1409 & 0) & 1395( & 1) & 1353( & 1) & 1385( & 1) \\
\hline 1409 & 1) & 13960 & 1) & 1357( & 0) & 1390( & 1) \\
\hline 1416( & 0) & 1403( & 0) & 1362( & 0) & 1394( & 0) \\
\hline 1470( & 20) & 1461( & 19) & 1424( & 8) & 1459( & 11) \\
\hline 1482( & 1) & 1470( & 1) & 1435( & 2) & 1472( & 1) \\
\hline 1485( & 11) & 1472( & 8) & 14390 & 8) & 1473( & 5) \\
\hline 1536( & 21) & 1522( & 20) & 1476( & 7) & 1521( & 17) \\
\hline 1540( & 27) & 1528( & 19) & 1482( & 7) & 1529 ( & 22) \\
\hline 1545( & 16) & 1532( & 11) & 1489 & 3) & 1534( & 3) \\
\hline 1549( & 34) & 1535( & 35) & 1497( & 10) & 1544( & 26) \\
\hline 1559 ( & 9) & 1543( & 9) & 1499( & 36) & 1546( & 32) \\
\hline 1566( & 15) & 1551( & 15) & 1507( & 5) & 1557( & 16) \\
\hline 3140( & 12) & 3118( & 13) & 3058( & 18) & 3121( & 41) \\
\hline 3143( & 9) & 3120( & 8) & 3064( & 8) & 3127( & 24) \\
\hline 3158( & 7) & 3136 & 7) & 3065( & 20) & 3143( & 44) \\
\hline 3165( & 13) & 3141( & 14) & 3076( & 21) & 3146( & 17) \\
\hline 3166( & 1) & 3144( & 1) & 3084( & 1) & 3154( & 18) \\
\hline 3167( & 5) & 3145( & 5) & 3084( & 3) & 3160( & 10) \\
\hline 3172( & 5) & 3149 & 5) & 3085( & 8) & 3166( & 6) \\
\hline 3179 & 4) & 3157( & 3) & 3097( & 5) & 3177( & 9) \\
\hline 3183( & 8) & 3160( & 9) & 30999 & 16) & 3181( & 30) \\
\hline 3192( & 2) & 3171( & 3) & 3110( & 18) & 3184( & 19) \\
\hline 3194( & 15) & 3171( & 14) & 3112( & 3) & 3191( & 26) \\
\hline 3211( & 7) & 3188( & 7) & 3128( & 12) & 3211( & 21) \\
\hline 3225( & 11) & 3203( & 11) & 3146( & 10) & 3216( & 20) \\
\hline 3232( & 7) & 3209 & 7) & 3149 & 8) & 3223( & 18) \\
\hline 3249 & 4) & 3227( & 4) & 3160( & 5) & 3245( & 13) \\
\hline 3256( & 6) & 3233( & 6) & 3170( & 5) & 3246( & 11) \\
\hline 3263( & 6) & 3239 & 3) & 3173( & 5) & 3248( & 15) \\
\hline 3264( & 5) & 32400 & 7) & 31750 & 10) & 3258( & 14) \\
\hline
\end{tabular}


Table S53. Harmonic vibrational frequencies (in $\mathrm{cm}^{-1}$ ) and infrared intensities (in parentheses in $\mathrm{km} / \mathrm{mol}$ ) for the $\left(\mathbf{C}_{4} \mathbf{H}_{6}\right)_{3} \mathbf{V}$ structure V-3D.

\begin{tabular}{|c|c|c|c|c|c|c|c|}
\hline \multicolumn{2}{|c|}{ B3LYP } & \multicolumn{2}{|c|}{ B3LYP* } & \multicolumn{2}{|r|}{ BP86 } & \multicolumn{2}{|r|}{ M06L } \\
\hline 72( & 0) & 24( & 0) & 70( & 0) & 62( & 1) \\
\hline 102( & 0) & 99( & 0) & 97( & 2) & 96( & 2) \\
\hline 114( & 2) & 103( & 1) & 99 & 0) & 113( & 2) \\
\hline 141( & 1) & 134( & 0) & 137( & 1) & 137( & 2) \\
\hline 146( & 2) & 142( & 3) & 153( & 2) & 158( & 2) \\
\hline 203( & 0) & 203( & 1) & 200( & 1) & 199( & 1) \\
\hline 223( & 1) & 223( & 1) & 215( & 0) & 219 & 3) \\
\hline 233( & 8) & 234( & 8) & 245( & 6) & 234( & 5) \\
\hline 264( & 4) & 261( & 4) & 2531 & 1) & 256( & 1) \\
\hline 284( & 1) & 274( & 3) & 280 & 15) & 286( & 14) \\
\hline 316( & 1) & 313( & 2) & 327( & 4) & 354( & 7) \\
\hline 340( & 2) & 355( & 2) & 361( & 2) & 370( & 2) \\
\hline 360( & 3) & 3600 & 2) & 371( & 1) & 378( & 2) \\
\hline 412( & 4) & 419) & 5) & 418( & 14) & 422( & 10) \\
\hline 440( & 2) & 429 & 13) & 426( & 4) & 443( & 4) \\
\hline 461( & 14) & 447( & 6) & 448( & 3) & 464( & 2) \\
\hline 466( & 21) & 464( & 7) & 456( & 5) & 474( & 13) \\
\hline 470( & 19) & 474( & 34) & 470 & 18) & 492( & 31) \\
\hline 493( & 10) & 490( & 8) & 483( & 14) & 498( & 6) \\
\hline 510 & 4) & 509 & 3) & 510 & 6) & 525( & 5) \\
\hline 590( & 1) & 583( & 1) & 566( & 0) & 589 & 0) \\
\hline 646( & 1) & 640 & 1) & 621( & 1) & 643( & 0) \\
\hline 689( & 7) & 672( & 32) & 649 & 34) & 667( & 22) \\
\hline 690( & 29) & 685( & 5) & 668( & 6) & 687( & 9) \\
\hline 730( & 4) & 728( & 4) & 715( & 5) & 728( & 9) \\
\hline 765( & 9) & 757( & 11) & 739 & 11) & 747 ( & 5) \\
\hline 791( & 8) & 781( & 7) & 7531 & 3) & 766( & 3) \\
\hline 806( & 9) & 797( & 9) & 775( & 8) & 798( & 3) \\
\hline 822( & 3) & 827( & 3) & 809 & 7) & 834( & 9) \\
\hline 848( & 6) & 836( & 5) & 818( & 5) & 846( & 9) \\
\hline 860( & 20) & 848( & 18) & 8331 & 11) & 860( & 9) \\
\hline 885( & 2) & 8731 & 2) & 852( & 3) & 873( & 5) \\
\hline 899( & 6) & 888( & 2) & 860 & 1) & 888( & 1) \\
\hline 925( & 9) & 915( & 2) & 870 & 1) & 903( & 2) \\
\hline 930( & 15) & 919 & 15) & 897( & 15) & 927( & 21) \\
\hline 935( & 4) & 930( & 11) & 906( & 0) & 942( & 0) \\
\hline 984( & 3) & 967( & 1) & 928( & 5) & 961( & 4) \\
\hline 991( & 1) & 981( & 4) & 959 & 2) & 994( & 3) \\
\hline 1006( & 3) & 998( & 3) & 971( & 6) & 1012( & 5) \\
\hline 1020( & 7) & 1011( & 4) & 978( & 0) & 1014( & 1) \\
\hline 1035( & 3) & 1024( & 3) & 990( & 4) & 1028( & 6) \\
\hline 1070( & 10) & 1053( & 9) & 1005( & 3) & 1041( & 3) \\
\hline 1098( & 3) & 1081( & 4) & 1043( & 8) & 1082( & 13) \\
\hline 1111( & 2) & 1095( & 1) & 1067( & 6) & 1107( & 3) \\
\hline 1136( & 1) & 1122( & 2) & 1086( & 7) & 1127( & 9) \\
\hline 1145( & 9) & 11350 & 9) & 1107( & 10) & 1144( & 16) \\
\hline 1185( & 2) & 1175( & 2) & 1139 & 2) & 1175( & 4) \\
\hline 1222( & 7) & 1208( & 9) & 1169 & 10) & 1215( & 13) \\
\hline
\end{tabular}




\begin{tabular}{|c|c|c|c|c|c|c|c|}
\hline 1234( & 2) & 1224( & 5) & 1183( & 2) & 1233( & 4) \\
\hline 1241( & 13) & 1228( & 9) & 1194( & 9) & 1235( & 6) \\
\hline 1259( & 1) & 1242( & 3) & 1208( & 3) & 1251( & 2) \\
\hline 1261( & 5) & 1246( & 3) & 1213 & 2) & 1257( & 3) \\
\hline 1289 & 4) & 1273( & 1) & 1226( & 0) & 1271( & 1) \\
\hline 1314( & 2) & 1300( & 2) & 1253( & 1) & 1300( & 3) \\
\hline 1336( & 1) & 1317( & 1) & 1265( & 2) & 1322( & 2) \\
\hline 1342( & 6) & 1325( & 6) & 1285 & 6) & 1339 & 6) \\
\hline 1378( & 1) & 1362( & 0) & 1311( & 0) & 1360 & 1) \\
\hline 1390( & 2) & 1374( & 2) & 1335( & 3) & 1379 & 3) \\
\hline 1402( & 4) & 1388( & 1) & 1345( & 2) & 1392( & 3) \\
\hline 1404( & 3) & 1390 & 7) & 1353( & 7) & 1409 & 6) \\
\hline 1418( & 7) & 1406( & 6) & 1373 & 3) & 1431( & 6) \\
\hline 1471( & 4) & 1456( & 4) & 1414( & 5) & 1454( & 5) \\
\hline 1475( & 9) & 1462( & 9) & 1422( & 8) & 1463( & 5) \\
\hline 1480( & 1) & 1466( & 1) & 1426( & 1) & 1466( & 6) \\
\hline 1495( & 11) & 1481( & 12) & 1438( & 11) & 1475 & 6) \\
\hline 1507( & 16) & 1490( & 16) & 1449 & 12) & 1500( & 22) \\
\hline 1530( & 22) & 1510 & 19) & 1469 & 12) & 1519 & 13) \\
\hline 1663( & 21) & 1679 & 15) & 1618( & 13) & 1694( & 20) \\
\hline 1699 & 17) & 1695( & 22) & 1711( & 11) & 1757( & 14) \\
\hline 2280( & 4) & 2119 & 12) & 1928( & 23) & 2267( & 10) \\
\hline 2913( & 34) & 2878( & 31) & 2788( & 39) & 2901( & 45) \\
\hline 2947( & 22) & 2976( & 20) & 2927( & 57) & 3019 & 24) \\
\hline 3009 & 44) & 2986( & 47) & 2939 & 31) & 3021( & 75) \\
\hline 3020 & 32) & 3000 & 31) & 2958( & 36) & 3038( & 108) \\
\hline 3032( & 75) & 3015( & 67) & 2961( & 73) & 3045( & 121) \\
\hline 3048( & 26) & 3027( & 33) & 2967( & 13) & 3058( & 21) \\
\hline 3050( & 24) & 3034( & 16) & 2985( & 9) & 3071( & 29) \\
\hline 3067( & 2) & 3054( & 1) & 2987( & 20) & 3087( & 30) \\
\hline 3087( & 32) & 3074( & 34) & 3017( & 29) & 3098 & 23) \\
\hline 30899 & 35) & 3074( & 29) & 3030 & 11) & 3106( & 42) \\
\hline 3110( & 6) & 3091( & 9) & 3038( & 27) & 3119 & 50) \\
\hline 3111( & 9) & 3098 & 4) & 3044( & 3) & 3127( & 10) \\
\hline 3140( & 60) & 3126( & 54) & 3069 & 11) & 3148( & 28) \\
\hline 3151( & 8) & 3134( & 7) & 3079 & 15) & 3165 & 35) \\
\hline 3161( & 13) & 3143( & 12) & 3084( & 44) & 3176( & 71) \\
\hline 3174( & 6) & 3153( & 6) & 3089 & 8) & 3184( & 11) \\
\hline 3198( & 7) & 3183( & 6) & 3123 & 7) & 3198( & 16) \\
\hline
\end{tabular}


Table S54. Harmonic vibrational frequencies (in $\mathrm{cm}^{-1}$ ) and infrared intensities (in parentheses in $\mathrm{km} / \mathrm{mol})$ for the $\left(\mathbf{C}_{4} \mathbf{H}_{6}\right)_{3} \mathbf{V}$ structure V-4D.

\begin{tabular}{|c|c|c|c|c|c|c|c|}
\hline \multicolumn{2}{|c|}{ B3LYP } & \multicolumn{2}{|c|}{ B3LYP* } & \multicolumn{2}{|c|}{ BP86 } & \multicolumn{2}{|c|}{ M06L } \\
\hline 32( & 1) & 47( & 1) & 44( & 0) & 50( & 1) \\
\hline 72( & 0) & 62( & 1) & 62( & 0) & 72( & 2) \\
\hline 88( & 0) & 83( & 0) & 79 & 0) & 112( & 0) \\
\hline 108( & 1) & 128( & 0) & 128( & 0) & 144( & 1) \\
\hline 143( & 11) & 163( & 5) & 162( & 0) & 170( & 2) \\
\hline 165( & 1) & 168( & 7) & 178( & 5) & 188( & 10) \\
\hline 202( & 8) & 211( & 10) & 216( & 5) & 225( & 5) \\
\hline 228( & 3) & 235( & 2) & 238( & 5) & 254( & 5) \\
\hline 245( & 3) & 248( & 2) & 244( & 2) & 261( & 3) \\
\hline 251( & 4) & 273 & 9) & 265( & 2) & 282( & 8) \\
\hline 277( & 2) & 276( & 6) & 277( & 5) & 290( & 3) \\
\hline 282( & 3) & 298( & 2) & 297( & 3) & 300( & 2) \\
\hline 318( & 20) & 315( & 36) & 348( & 25) & 332( & 45) \\
\hline 343( & 6) & 3631 & 4) & 374( & 2) & 380( & 1) \\
\hline 393( & 3) & 385( & 3) & 386( & 2) & 401( & 3) \\
\hline 431( & 16) & 429 & 17) & 427( & 15) & 447( & 15) \\
\hline 459( & 0) & 465( & 0) & 4631 & 0) & 472( & 3) \\
\hline 497( & 2) & 492( & 3) & 479 ( & 3) & 498( & 2) \\
\hline 549( & 1) & 547( & 2) & 529 & 1) & 543( & 3) \\
\hline 559( & 1) & 5631 & 1) & 551( & 2) & 565( & 2) \\
\hline 581( & 2) & 578( & 1) & 559 & 0) & 583( & 2) \\
\hline 607( & 1) & 590( & 2) & 564( & 2) & 606( & 2) \\
\hline 633( & 7) & 629 & 15) & 627 & 8) & 633 & 13) \\
\hline 6731 & 1) & 6631 & 1) & 637 & 8) & 657 & 1) \\
\hline 765( & 3) & 764( & 7) & 742( & 6) & 745( & 1) \\
\hline 773( & 2) & 769 & 0) & 750( & 1) & 770 & 8) \\
\hline 779 & 3) & 779 ( & 12) & 766( & 10) & 782( & 5) \\
\hline 826( & 29) & 825( & 17) & 806( & 13) & 822( & 55) \\
\hline 852( & 49) & 852( & 75) & 821( & 62) & 830( & 12) \\
\hline 857( & 34) & 854( & 8) & 835( & 4) & 835( & 1) \\
\hline 867( & 6) & 864( & 6) & 847( & 13) & 849 & 8) \\
\hline 877( & 4) & 869 & 5) & 852( & 1) & 880( & 12) \\
\hline 900( & 7) & 892( & 9) & 874( & 14) & 890( & 20) \\
\hline 921( & 5) & 915( & 1) & 8831 & 9) & 901( & 0) \\
\hline 938( & 1) & 930( & 1) & 906( & 4) & 928( & 5) \\
\hline 947( & 2) & 940( & 7) & 9231 & 15) & 951( & 9) \\
\hline 978( & 3) & 973( & 3) & 947( & 3) & 982( & 0) \\
\hline 982( & 13) & 978( & 7) & 952( & 0) & 987 & 1) \\
\hline 999( & 3) & 994( & 4) & 971( & 5) & 1002( & 1) \\
\hline 1015( & 7) & 1006( & 6) & 977( & 6) & 1015( & 7) \\
\hline 1026( & 6) & 1020 & 7) & 997( & 8) & 1031( & 8) \\
\hline 1036( & 2) & 1031( & 1) & 1010( & 8) & 1038 & 3) \\
\hline 1047( & 9) & 1038 & 7) & 1013( & 3) & 1048 & 13) \\
\hline 1089( & 1) & 1081( & 4) & 1061( & 12) & 1092( & 4) \\
\hline 1121( & 9) & 1109 & 10) & 1075( & 12) & 1120 & 11) \\
\hline 1145( & 18) & 1132( & 7) & 1098( & 6) & 1148 & 5) \\
\hline 1182( & 7) & 1171( & 3) & 1137( & 4) & 1179 & 2) \\
\hline 1203( & 8) & 1193( & 8) & 1161( & 8) & 1198( & 9) \\
\hline
\end{tabular}




\begin{tabular}{|c|c|c|c|c|c|c|c|}
\hline 1225( & 5) & 1216( & 3) & 1180( & 1) & 1228( & 6) \\
\hline 1232( & 1) & 1221( & 2) & 1188( & 2) & 1234( & 1) \\
\hline 1243( & 1) & 1235( & 1) & 1205( & 3) & 1243 & 1) \\
\hline 1262( & 20) & 1250( & 17) & 1216( & 10) & 1257( & 13) \\
\hline 1297( & 2) & 1275( & 5) & 1230( & 4) & 1290 & 2) \\
\hline 1308( & 0) & 1300( & 1) & 1258( & 1) & 1304( & 2) \\
\hline 1316( & 3) & 1306( & 2) & 1266( & 2) & 1316( & 3) \\
\hline 1323( & 3) & 1314( & 6) & 1271( & 4) & 1322( & 5) \\
\hline 1334( & 6) & 1316( & 2) & 1279 ( & 3) & 1335( & 5) \\
\hline 1354( & 5) & 1338( & 5) & 1294( & 6) & 1361( & 6) \\
\hline 1393( & 2) & 1381( & 2) & 1339 ( & 2) & 1390 & 2) \\
\hline 1403( & 1) & 1390( & 2) & 1351( & 2) & 1396( & 2) \\
\hline 1445( & 6) & 1435( & 3) & 1394( & 1) & 1431( & 2) \\
\hline 1478( & 3) & 1461( & 3) & 1418( & 3) & 1455( & 2) \\
\hline 1481( & 1) & 1465( & 1) & 1423( & 4) & 1466( & 0) \\
\hline 1488( & 3) & 1472( & 2) & 1429 ( & 3) & 1468( & 3) \\
\hline 1495( & 3) & 1477( & 7) & 1434( & 7) & 1477( & 11) \\
\hline 1500( & 10) & 1486( & 9) & 1447( & 9) & 1485( & 3) \\
\hline 1522( & 28) & 1512( & 24) & 1465( & 16) & 1521( & 29) \\
\hline 1537( & 8) & 1530( & 5) & 1497( & 3) & 1547( & 4) \\
\hline 1654( & 90) & 1639 & 74) & 1591( & 48) & 1663( & 68) \\
\hline 2859 & 58) & 2677 ( & 115) & 25200 & 115) & 2757( & 103) \\
\hline 3012( & 21) & 2989 & 24) & 2933( & 39) & 3016( & 39) \\
\hline 3019) & 49) & 2999( & 82) & 2944( & 68) & 3025( & 139) \\
\hline 3024( & 78) & 3001( & 44) & 2947( & 55) & 3033( & 62) \\
\hline 3043( & 76) & 3027( & 70) & 2962( & 7) & 3049 & 146) \\
\hline 3061( & 7) & 3030 & 5) & 2973( & 71) & 3049 & 8) \\
\hline 3072( & 21) & 3052( & 29) & 2998( & 25) & 3083( & 45) \\
\hline 3077( & 5) & 3059 & 2) & 3004( & 4) & 3094( & 11) \\
\hline 3090( & 30) & 3076( & 23) & 3016( & 9) & 3094( & 41) \\
\hline 3096( & 22) & 3077 & 14) & 3024( & 10) & 3107( & 32) \\
\hline 3098( & 23) & 3080( & 20) & 3025( & 19) & 3109 & 18) \\
\hline 3111( & 6) & 3084( & 7) & 3029( & 26) & 3117( & 55) \\
\hline 3120( & 23) & 3092( & 26) & 3030( & 25) & 3127( & 5) \\
\hline 3131( & 8) & 3114( & 9) & 3054( & 6) & 3131( & 27) \\
\hline 3142( & 19) & 3120 & 17) & 3064( & 19) & 3149 & 40) \\
\hline 3154( & 5) & 3137( & 5) & 3080( & 6) & 3154( & 17) \\
\hline 3219x & 4) & 31964 & 5) & 3137( & 4) & 3221( & 11) \\
\hline 3241( & 20) & 3226( & 16) & 3170( & 15) & 3250 & 30) \\
\hline
\end{tabular}


Table S55. Harmonic vibrational frequencies (in $\mathrm{cm}^{-1}$ ) and infrared intensities (in parentheses in $\mathrm{km} / \mathrm{mol})$ for the $\left(\mathbf{C}_{4} \mathbf{H}_{6}\right)_{3} \mathbf{V}$ structure V-1Q.

\begin{tabular}{|c|c|c|c|c|c|c|c|}
\hline \multicolumn{2}{|c|}{ B3LYP } & \multicolumn{2}{|c|}{ B3LYP* } & \multicolumn{2}{|c|}{ BP86 } & \multicolumn{2}{|c|}{ M06L } \\
\hline 67( & 1) & 651 & 0) & 681 & 0) & 75( & 0) \\
\hline 96( & 1) & 102( & 1) & 97( & 1) & 106( & 1) \\
\hline 126( & 0) & 129 & 0) & 134( & 0) & 134( & 0) \\
\hline 138( & 1) & 143( & 1) & 154( & 0) & 158( & 0) \\
\hline 150( & 0) & 152( & 0) & 162( & 0) & 195( & 1) \\
\hline 169 & 1) & 176( & 1) & 190( & 1) & 211( & 1) \\
\hline 210( & 2) & 212( & 1) & 208( & 1) & 235( & 0) \\
\hline 214( & 3) & 218( & 3) & 231( & 0) & 239 & 0) \\
\hline 232( & 1) & 234( & 1) & 237 & 2) & 246( & 3) \\
\hline 273( & 2) & 272( & 1) & 264( & 0) & 282( & 1) \\
\hline 278( & 3) & 280( & 3) & 286( & 2) & 312( & 3) \\
\hline 305( & 0) & 308( & 1) & 3231 & 0) & 335( & 2) \\
\hline 325( & 3) & 328( & 3) & 334( & 1) & 342( & 1) \\
\hline 357( & 17) & 358( & 15) & 361( & 9) & 379 & 9) \\
\hline 405( & 3) & 407( & 3) & 4081 & 5) & 419 & 4) \\
\hline 428( & 6) & 4331 & 6) & 440( & 6) & 444( & 6) \\
\hline 457 & 10) & 455( & 7) & 445( & 6) & 469 & 7) \\
\hline 468( & 7) & 468( & 6) & 456( & 3) & 484( & 4) \\
\hline 510( & 4) & 505( & 5) & 494( & 3) & 523( & 6) \\
\hline 569( & 2) & 564( & 2) & 551( & 1) & 572( & 2) \\
\hline 586( & 2) & 582( & 2) & 5681 & 1) & 597( & 3) \\
\hline 626( & 5) & 626( & 6) & 614( & 6) & 636( & 6) \\
\hline 682( & 6) & 6831 & 3) & 652( & 3) & 687( & 0) \\
\hline 6931 & 8) & 6861 & 7) & 6631 & 6) & 691( & 8) \\
\hline 728( & 38) & 723( & 29) & 695( & 21) & 709 & 18) \\
\hline 746( & 8) & 747( & 12) & 7331 & 10) & 737( & 12) \\
\hline 770 & 35) & 768( & 27) & 748( & 24) & 762( & 27) \\
\hline 780 & 101) & 778( & 67) & 764( & 34) & 778( & 75) \\
\hline 786( & 11) & 786( & 37) & 7681 & 20) & 789 & 5) \\
\hline 794( & 6) & 792( & 6) & 778( & 15) & 797( & 2) \\
\hline 820 & 6) & 815( & 4) & 796( & 2) & 821( & 2) \\
\hline 859 & 2) & 855( & 3) & 839 & 5) & 873 & 6) \\
\hline 909 & 4) & 902( & 4) & 867( & 2) & 901( & 2) \\
\hline 934( & 2) & 9231 & 3) & 889 & 4) & 921( & 4) \\
\hline 952( & 2) & 9431 & 3) & 8961 & 1) & 939 & 3) \\
\hline 970( & 2) & 952( & 2) & 904( & 3) & 944( & 2) \\
\hline 972( & 4) & 959( & 5) & 917( & 2) & 955( & 2) \\
\hline 978( & 3) & 964( & 2) & 920( & 5) & 9631 & 3) \\
\hline 998( & 7) & 989 & 7) & 960( & 5) & 995( & 4) \\
\hline 1001( & 3) & 993( & 2) & 969 & 2) & 1008( & 3) \\
\hline 1021( & 5) & 1014( & 5) & 990( & 6) & 1029 & 5) \\
\hline 1039( & 5) & 1031( & 5) & 1004( & 7) & 1041( & 9) \\
\hline 1054( & 7) & 1045( & 7) & 1016( & 5) & 1057 & 4) \\
\hline 1091( & 3) & 1082( & 3) & 1054( & 3) & 1091( & 3) \\
\hline 1140( & 4) & 1130 & 4) & 1098( & 4) & 1134( & 4) \\
\hline 1163( & 3) & 1151( & 4) & 1119( & 5) & 1159 & 4) \\
\hline 1180( & 0) & 1169 & 0) & 1137( & 1) & 1183( & 1) \\
\hline 1194( & 2) & 1182( & 2) & 1148( & 2) & 1193( & 2) \\
\hline
\end{tabular}




\begin{tabular}{|c|c|c|c|c|c|c|c|}
\hline 1223( & 3) & 1209 & 3) & 1173( & 2) & 1224( & 2) \\
\hline 1231( & 1) & 1218( & 1) & 1187( & 1) & 1235 & 4) \\
\hline 1256( & 16) & 1246( & 12) & 1210( & 6) & 1246( & 9) \\
\hline 1264( & 21) & 1253( & 18) & 1220( & 11) & 1253( & 12) \\
\hline 1282( & 10) & 1268( & 12) & 1229 & 12) & 1267( & 13) \\
\hline 1314( & 0) & 1301( & 0) & 1260( & 1) & 1307( & 1) \\
\hline 1331( & 4) & 1317( & 4) & 1277( & 2) & 1327( & 0) \\
\hline 1333( & 2) & 1319 & 3) & 1280 & 5) & 1329 & 5) \\
\hline 1336( & 2) & 1323( & 1) & 1287( & 1) & 1340( & 2) \\
\hline 1398( & 0) & 1384( & 0) & 1342( & 0) & 1389 & 0) \\
\hline 1401( & 1) & 1388( & 1) & 1347( & 2) & 1394( & 3) \\
\hline 1420( & 2) & 1406( & 2) & 1360 & 2) & 1410 & 2) \\
\hline 1474( & 7) & 1460 & 8) & 1420 & 3) & 1456( & 2) \\
\hline 1476( & 1) & 1463( & 0) & 1422( & 0) & 1460( & 10) \\
\hline 1484( & 9) & 1470( & 4) & 1428( & 8) & 1467( & 3) \\
\hline 1485( & 3) & 1473( & 11) & 1432( & 10) & 1474( & 4) \\
\hline 1487( & 11) & 1473( & 6) & 1433( & 8) & 1476( & 11) \\
\hline 1503( & 14) & 1491( & 15) & 1455( & 14) & 1527( & 11) \\
\hline 1549 & 62) & 1537( & 56) & 1495( & 40) & 1545( & 50) \\
\hline 1557( & 61) & 1545( & $50)$ & 1502( & 25) & 1556( & 37) \\
\hline 1628( & 15) & 1603( & 21) & 1533( & 23) & 1611( & 29) \\
\hline 29350 & 8) & 2902( & 8) & 2797( & 7) & 28620 & 8) \\
\hline 3011( & 32) & 2988( & 36) & 2923 & 49) & 3019 & 40) \\
\hline 3026( & 88) & 3005( & 84) & 2949 & 80) & 3025 & 145) \\
\hline 3036( & 17) & 3015( & 14) & 2952( & 25) & 3042( & 58) \\
\hline 3044( & 63) & 3023( & 66) & 2967( & 69) & 3056 & 91) \\
\hline 3067( & 18) & 3047( & 18) & 2994( & 16) & 3082( & 55) \\
\hline 3089 & 15) & 3069 & 18) & 3014 & 20) & 3096( & 20) \\
\hline 3090 & 23) & 3071( & 19) & 3019 & 17) & 3097( & 29) \\
\hline 3104( & 7) & 3085( & 6) & 3028( & 7) & 3106( & 18) \\
\hline 3117( & 6) & 3098( & 6) & 3040 & 7) & 3111( & 29) \\
\hline 3135( & 3) & 3115( & 3) & 3061( & 4) & 3143( & 11) \\
\hline 3140( & 8) & 3119 & 7) & 3064 & 11) & 3146( & 11) \\
\hline 3140( & 7) & 3120 & 8) & 3067( & 6) & 3160 & 13) \\
\hline 3155( & 17) & 3135( & 17) & 3082( & 19) & 3163 & 41) \\
\hline 3176( & 2) & 31560 & 1) & 3100 & 1) & 3174( & 32) \\
\hline 3181( & 15) & 3160( & 15) & 3103( & 18) & 3183 & 19) \\
\hline 3210 ( & 3) & 3190 & 3) & 3130 & 13) & 3208( & 24) \\
\hline 3211( & 23) & 3191( & 22) & 3135( & 14) & 3211( & 30) \\
\hline
\end{tabular}


Table S56. Harmonic vibrational frequencies (in $\mathrm{cm}^{-1}$ ) and infrared intensities (in parentheses in $\mathrm{km} / \mathrm{mol}$ ) for the $\left(\mathbf{C}_{4} \mathbf{H}_{6}\right)_{3} \mathbf{V}$ structure $\mathrm{V}-2 \mathrm{Q}$.

\begin{tabular}{|c|c|c|c|c|c|c|c|}
\hline \multicolumn{2}{|c|}{ B3LYP } & \multicolumn{2}{|c|}{ B3LYP* } & \multicolumn{2}{|c|}{ BP86 } & \multicolumn{2}{|c|}{ M06L } \\
\hline 24( & 0) & 53( & 0) & 29( & 0) & 60( & 1) \\
\hline 63( & 0) & 81( & 0) & 83( & 0) & 111( & 0) \\
\hline 123( & 3) & 130( & 3) & 132( & 1) & 151( & 1) \\
\hline 157( & 1) & 165( & 2) & 155( & 1) & 167( & 2) \\
\hline 165( & 2) & 174( & 1) & 173( & 3) & 186( & 2) \\
\hline 172( & 1) & 177( & 0) & 187( & 4) & 198( & 0) \\
\hline 178( & 1) & 182( & 1) & 210( & 0) & 203( & 0) \\
\hline 217( & 0) & 218( & 0) & 225( & 1) & 228( & 2) \\
\hline 236( & 3) & 234( & 3) & 237( & 2) & 237( & 1) \\
\hline 245( & 1) & 250( & 1) & 258( & 1) & 257( & 0) \\
\hline 265( & 1) & 279 & 3) & 260( & 1) & 284( & 2) \\
\hline 277( & 6) & 282( & 4) & 2999 & 9) & 298( & 5) \\
\hline 307( & 0) & 310( & 0) & 313( & 3) & 333( & 1) \\
\hline 327( & 12) & 332( & 10) & 327( & 0) & 349( & 10) \\
\hline 379 & 1) & 382( & 1) & 409) & 4) & 390( & 1) \\
\hline 419 & 1) & 420( & 1) & 428( & 3) & 419( & 1) \\
\hline 449 & 14) & 451( & 14) & 436( & 4) & 449 & 14) \\
\hline 467( & 9) & 467( & 7) & 465( & 2) & 476( & 6) \\
\hline 5231 & 3) & 522( & 3) & 491( & 2) & 538( & 4) \\
\hline 561( & 2) & 555( & 3) & 546( & 2) & 568( & 3) \\
\hline 591( & 0) & 590( & 0) & 568( & 1) & 599( & 1) \\
\hline 6531 & 4) & 650( & 3) & 635( & 0) & 653( & 3) \\
\hline 678( & 16) & 674( & 13) & 675( & 5) & 686( & 8) \\
\hline 700( & 8) & 696( & 6) & 684( & 9) & 688( & 8) \\
\hline 744( & 24) & 741( & 25) & 720( & 15) & 745( & 19) \\
\hline 756( & 99) & 754( & 87) & 757( & 10) & 7531 & 75) \\
\hline 778( & 27) & 776( & 21) & 7666 & 3) & 774( & 14) \\
\hline 783( & 0) & 780( & 2) & 773( & 9) & 787( & 4) \\
\hline 804( & 27) & 803( & 23) & 781( & 13) & 796( & 24) \\
\hline 817( & 9) & 815( & 9) & 794( & 23) & 819( & 8) \\
\hline 837( & 14) & 834( & 15) & 817( & 2) & 838( & 7) \\
\hline 854( & 1) & 850( & 2) & 840( & 2) & 863( & 1) \\
\hline 889 & 0) & 884( & 0) & 880( & 1) & 901( & 2) \\
\hline 942( & 1) & 932( & 1) & 8921 & 1) & 934( & 1) \\
\hline 946( & 10) & 9381 & 6) & 897( & 0) & 940( & 2) \\
\hline 967( & 4) & 9521 & 4) & 913( & 4) & 950( & 5) \\
\hline 986( & 3) & 975( & 3) & 916( & 3) & 971( & 1) \\
\hline 993( & 1) & 980( & 2) & 932( & 1) & 980( & 2) \\
\hline 1000( & 1) & 987( & 0) & 957( & 1) & 993( & 2) \\
\hline 1014( & 3) & 1005( & 2) & 981( & 5) & 1018( & 1) \\
\hline 1024( & 8) & 1015( & 8) & 984( & 1) & 1022( & 6) \\
\hline 1036( & 5) & 1029 & 6) & 997( & 6) & 1041( & 12) \\
\hline 1050 & 5) & 1042( & 4) & 1019 & 4) & 1051( & 4) \\
\hline 1100( & 7) & 1094( & 7) & 1064( & 3) & 1103( & 6) \\
\hline 1147( & 4) & 1138( & 5) & 1109 & 3) & 1145( & 6) \\
\hline 1159 & 6) & 1151( & 6) & 1127( & 1) & 1158( & 5) \\
\hline
\end{tabular}




\begin{tabular}{|c|c|c|c|c|c|c|c|}
\hline 1195( & 2) & 1184( & 2) & 1143( & 1) & 1198( & 4) \\
\hline 1212( & 5) & 1202( & 5) & 1158( & 6) & 1220( & 5) \\
\hline 1229 & 6) & 1220 & 5) & 1183( & 5) & 1239 & 5) \\
\hline 1242( & 1) & 1232( & 1) & 1192( & 0) & 1253( & 2) \\
\hline 1260( & 17) & 1254( & 15) & 1208( & 5) & 1258( & 11) \\
\hline 1279 ( & 17) & 1267( & 14) & 1219 & 5) & 1269 & 15) \\
\hline 1295( & 0) & 1281( & 1) & 1251( & 12) & 1286( & 0) \\
\hline 1309 & 7) & 1297( & 6) & 1255( & 2) & 1304( & 11) \\
\hline 1315( & 4) & 1302( & 6) & 1264( & 5) & 1308( & 1) \\
\hline 1333( & 0) & 1319 & 0) & 1270( & 2) & 1332( & 0) \\
\hline 1355( & 9) & 1343( & 8) & 1284( & 3) & 1361( & 8) \\
\hline 1398( & 0) & 1387( & 0) & 1343( & 2) & 1389 & 1) \\
\hline 1418( & 1) & 1405( & 1) & 1348( & 2) & 1406( & 1) \\
\hline 1436( & 3) & 1422( & 3) & 1376( & 1) & 1434( & 4) \\
\hline 1472( & 3) & 1462( & 3) & 1422( & 2) & 1457( & 2) \\
\hline 1476( & 3) & 1464( & 3) & 1426( & 4) & 1464( & 11) \\
\hline 1479 ( & 1) & 1467( & 1) & 1426( & 2) & 14660 & 0) \\
\hline 1490( & 9) & 1477( & 8) & 1429 & 5) & 1478( & 3) \\
\hline 1495( & 7) & 1483( & 11) & 1443( & 15) & 1480( & 7) \\
\hline 1498( & 16) & 1488( & 14) & 1444( & 13) & 1492( & 14) \\
\hline 1557( & 66) & 1545( & 59) & 1504( & 12) & 1548( & 55) \\
\hline 1571( & 71) & 1559 & 60) & 1513( & 34) & 1570( & 49) \\
\hline 1650( & 5) & 1627( & 8) & 1564( & 12) & 1648( & 14) \\
\hline 3011( & 29) & 2989 & 24) & 2924( & 47) & 3027( & 60) \\
\hline 3021( & 68) & 2999 & 97) & 2931( & 68) & 3029 & 75) \\
\hline 3021( & 62) & 3002( & 33) & 2967( & 72) & 3038( & 151) \\
\hline 3040( & 60) & 3018( & 69) & 2972( & 52) & 3050 & 113) \\
\hline 3066( & 19) & 30460 & 18) & 2987( & 23) & 3083( & 27) \\
\hline 3087( & 5) & 3072( & 4) & 2996( & 4) & 3088( & 21) \\
\hline 3108( & 14) & 3092( & 9) & 3019 & 23) & 3115( & 10) \\
\hline 3116( & 10) & 3094( & 9) & 3022( & 24) & 3121( & 15) \\
\hline 3119 & 2) & 3098( & 4) & 3053( & 4) & 3122( & 22) \\
\hline 3129 & 9) & 3109 & 13) & 3057( & 7) & 3125( & 15) \\
\hline 3132( & 2) & 3111( & 2) & 3058( & 4) & 3126( & 7) \\
\hline 3140( & 22) & 3122( & 19) & 3059 & 5) & 3139 & 4) \\
\hline 3145( & 6) & 3125( & 6) & 3063( & 6) & 3140( & 57) \\
\hline 3156( & 15) & 3135( & 14) & 3087( & 4) & 3159 & 33) \\
\hline 3161( & 4) & 3140 & 3) & 3090( & 15) & 3165( & 9) \\
\hline 3174( & 24) & 3153( & 25) & 3094( & 25) & 31760 & 53) \\
\hline 3220( & 15) & 3201( & 15) & 3159 & 6) & 3211( & 23) \\
\hline 3231( & 6) & 3212( & 6) & 3167( & 12) & 3221( & 17) \\
\hline
\end{tabular}


Table S57. Harmonic vibrational frequencies (in $\mathrm{cm}^{-1}$ ) and infrared intensities (in parentheses in $\mathrm{km} / \mathrm{mol}$ ) for the $\left(\mathbf{C}_{4} \mathbf{H}_{6}\right)_{3} \mathbf{V}$ structure V-3Q.

\begin{tabular}{|c|c|c|c|c|c|c|c|}
\hline \multicolumn{2}{|c|}{ B3LYP } & \multicolumn{2}{|c|}{ B3LYP* } & \multicolumn{2}{|c|}{ BP86 } & \multicolumn{2}{|c|}{ M06L } \\
\hline 49( & 0) & 51( & 0) & 50( & 0) & 64( & 0) \\
\hline 73( & 1) & 76( & 0) & 80( & 0) & 89 ( & 1) \\
\hline 89( & 1) & 95( & 1) & 90( & 1) & 95( & 1) \\
\hline 99( & 5) & 97( & 2) & 98( & 0) & 98( & 1) \\
\hline 119 & 0) & 124( & 0) & 126( & 0) & 122( & 0) \\
\hline 127( & 0) & 134( & 0) & 137( & 0) & 135( & 0) \\
\hline 149 & 1) & 145( & 0) & 148( & 0) & 166( & 0) \\
\hline 169 & 10) & 177( & 3) & 186( & 1) & 187( & 2) \\
\hline 208( & 45) & 224( & 17) & 219 ( & 1) & 240( & 1) \\
\hline 231( & 8) & 237( & 30) & 245( & 4) & 255( & 25) \\
\hline 235( & 18) & 243( & 5) & 252( & 1) & 263( & 24) \\
\hline 246( & 5) & 252( & 1) & 262( & 1) & 278( & 1) \\
\hline 270( & 13) & 273 ( & 21) & 274( & 13) & 281( & 10) \\
\hline 281( & 6) & 285( & 5) & 286( & 20) & 290( & 23) \\
\hline 295( & 13) & 292( & 12) & 296( & 9) & 321( & 10) \\
\hline 326( & 3) & 329 & 2) & 3331 & 1) & 350( & 4) \\
\hline 336( & 6) & 335( & 4) & 3390 & 3) & 358( & 5) \\
\hline 356( & 4) & 358( & 4) & 3531 & 7) & 376( & 0) \\
\hline 412( & 26) & 414( & 25) & 414( & 19) & 435( & 19) \\
\hline 424( & 3) & 435( & 3) & 426( & 4) & 443( & 7) \\
\hline 463( & 5) & 454( & 4) & 439) & 3) & 458( & 3) \\
\hline 533( & 24) & 531( & 23) & 523( & 22) & 542( & 22) \\
\hline 588( & 3) & 587( & 10) & 564( & 10) & 581( & 11) \\
\hline 592( & 13) & 591( & 4) & 574( & 3) & 595( & 7) \\
\hline 603( & 3) & 600( & 3) & 584( & 1) & 602( & 1) \\
\hline 636( & 10) & 646( & 8) & 627( & 4) & 635( & 5) \\
\hline 657( & 44) & 657( & 38) & 639) & 22) & 651( & 29) \\
\hline 676( & 3) & 675( & 4) & 647( & 4) & 674( & 7) \\
\hline 689 & 1) & 682( & 1) & 660( & 2) & 684( & 1) \\
\hline 722( & 6) & 715( & 6) & 687( & 4) & 705( & 2) \\
\hline 750( & 4) & 752( & 2) & 7371 & 4) & 727( & 4) \\
\hline 790( & 7) & 790( & 6) & 763( & 7) & 764( & 0) \\
\hline 802( & 17) & 797( & 13) & 765( & 9) & 770( & 19) \\
\hline 810( & 16) & 805( & 18) & 7799 & 15) & 780( & 12) \\
\hline 855( & 3) & 841( & 4) & 797( & 9) & 808( & 9) \\
\hline 885( & 1) & 877( & 2) & 850( & 2) & 856( & 41) \\
\hline 8851 & 4) & 880( & 3) & 855( & 48) & 869) & 1) \\
\hline 899 & 8) & 894( & 9) & 859( & 9) & 877( & 11) \\
\hline 917 & 1) & 906( & 48) & 866( & 9) & 889 ( & 1) \\
\hline 923( & 49) & 915( & 2) & 888( & 0) & 922( & 0) \\
\hline 932( & 3) & 9231 & 3) & 896( & 4) & 926( & 1) \\
\hline 950( & 4) & 935( & 7) & 903( & 6) & 926( & 5) \\
\hline 952( & 5) & 944( & 2) & 911( & 0) & 932( & 5) \\
\hline 956( & 2) & 947( & 1) & 913( & 1) & 939( & 1) \\
\hline 1010 & 9) & 994( & 9) & 963( & 10) & 995( & 7) \\
\hline 1063( & 3) & 1056( & 3) & 10300 & 2) & 1057 & 1) \\
\hline
\end{tabular}




\begin{tabular}{|c|c|c|c|c|c|c|c|}
\hline 1065( & 1) & 1056( & 2) & 1032( & 3) & 1064( & 6) \\
\hline 1068( & 3) & 1059 & 2) & 1035( & 3) & 1067( & 3) \\
\hline 1079( & 1) & 1071( & 1) & 1041( & 1) & 1074( & 3) \\
\hline 1083( & 13) & 1074( & 11) & 1044( & 8) & 1077( & 7) \\
\hline 1097( & 7) & 1087( & 8) & 1059 & 9) & 1092( & 7) \\
\hline 1208( & 13) & 1200( & 12) & 1171( & 10) & 1204( & 14) \\
\hline 1221( & 16) & 1210 & 16) & 1177( & 14) & 1219 ( & 14) \\
\hline 1259 & 113) & 1251( & 97) & 1220( & 12) & 1256( & 88) \\
\hline 1268( & 35) & 12560 & 28) & 1224( & 60) & 1262( & 19) \\
\hline 1280( & 15) & 1269 & 10) & 1237( & 4) & 1275( & 15) \\
\hline 1325( & 8) & 1312( & 7) & 1277( & 5) & 1315( & 8) \\
\hline 1406( & 1) & 1394( & 0) & 1354( & 0) & 1386( & 1) \\
\hline 1408( & 0) & 13960 & 0) & 1355( & 0) & 1390( & 0) \\
\hline 1420( & 9) & 1407( & 7) & 1366( & 3) & 1402( & 2) \\
\hline 1454( & 16) & 1442( & 14) & 1405( & 8) & 1442( & 7) \\
\hline 1463( & 56) & 1455( & 47) & 1420( & 9) & 1456( & 18) \\
\hline 1474( & 25) & 1462( & 17) & 1423( & 10) & 1460( & 9) \\
\hline 1523( & 191) & 1514( & 162) & 1477( & 72) & 1516( & 86) \\
\hline 1530( & 29) & 1520( & 40) & 1482( & 73) & 1520( & 128) \\
\hline 1538( & 21) & 1528( & 13) & 1486( & 15) & 1535( & 14) \\
\hline 1548( & 10) & 1533( & 11) & 1492( & 3) & 1538( & 16) \\
\hline 1584( & 88) & 1567( & 81) & 1520( & 53) & 1575( & 77) \\
\hline 1656( & 24) & 1638( & 27) & 1585( & 28) & 1652( & 36) \\
\hline 3135( & 5) & 3117( & 1) & 3057( & 7) & 3117( & 19) \\
\hline 3136( & 6) & 3117( & 9) & 3061( & 6) & 3122( & 14) \\
\hline 3152( & 6) & 3133( & 3) & 3072( & 7) & 3136( & 16) \\
\hline 3153( & 1) & 3133( & 8) & 3074( & 4) & 3141( & 6) \\
\hline 3156( & 5) & 3134( & 4) & 3076( & 3) & 3143( & 12) \\
\hline 3157( & 4) & 3136 & 4) & 3077 ( & 5) & 3148( & 9) \\
\hline 3161( & 6) & 31380 & 5) & 3080( & 9) & 3153( & 20) \\
\hline 3164( & 0) & 3144( & 0) & 3086( & 0) & 3165( & 10) \\
\hline 3173( & 1) & 3149 & 3) & 3089x & 4) & 3167( & 4) \\
\hline 3174( & 3) & 3150 & 1) & 3090( & 3) & 3171( & 4) \\
\hline 3181( & 9) & 3160 & 7) & 3102( & 2) & 3183( & 54) \\
\hline 3188( & 18) & 31660 & 19) & 3104( & 31) & 3187( & 20) \\
\hline 3225( & 9) & 3208( & 8) & 31531 & 9) & 3221( & 20) \\
\hline 3229 & 6) & 3211( & 5) & 3154( & 5) & 3225( & 16) \\
\hline 3243( & 7) & 3222( & 8) & 3162( & 10) & 3237( & 17) \\
\hline 3250( & 7) & 3230 & 3) & 3171( & 4) & 3244( & 9) \\
\hline 3251( & 2) & 3231( & 7) & 3175( & 5) & 3250( & 5) \\
\hline 3253 ( & 5) & 3234( & 3) & 31771 & 7) & 32550 & 19) \\
\hline
\end{tabular}


Table S58. Harmonic vibrational frequencies (in $\mathrm{cm}^{-1}$ ) and infrared intensities (in parentheses in $\mathrm{km} / \mathrm{mol}$ ) for the $\left(\mathbf{C}_{4} \mathbf{H}_{6}\right)_{3} \mathbf{V}$ structure V-4Q.

\begin{tabular}{|c|c|c|c|c|c|c|c|}
\hline \multicolumn{2}{|c|}{ B3LYP } & \multicolumn{2}{|c|}{ B3LYP* } & \multicolumn{2}{|c|}{ BP86 } & \multicolumn{2}{|c|}{ M06L } \\
\hline 38( & 0) & 37( & 0) & 30( & 0) & 72( & 1) \\
\hline 75( & 0) & 73( & 0) & 74( & 0) & 98( & 0) \\
\hline 110( & 0) & 101( & 0) & 100( & 0) & 132( & 0) \\
\hline 134( & 1) & 140( & 1) & 134( & 0) & 142( & 0) \\
\hline 148( & 0) & 151( & 0) & 148( & 1) & 175( & 2) \\
\hline 162( & 3) & 166( & 3) & 174( & 1) & 178( & 0) \\
\hline 187( & 1) & 193( & 0) & 205( & 0) & 216( & 0) \\
\hline 204( & 0) & 207( & 0) & 214( & 0) & 227( & 1) \\
\hline 2350 & 2) & 239 & 3) & 242( & 0) & 255( & 4) \\
\hline 249 & 1) & 250( & 1) & 250( & 2) & 265( & 1) \\
\hline 268( & 3) & 270( & 3) & 268( & 2) & 285( & 2) \\
\hline 278( & 1) & 274( & 1) & 277( & 2) & 292( & 0) \\
\hline 305( & 2) & 304( & 2) & 300( & 4) & 315( & 2) \\
\hline 321( & 15) & 318( & 13) & 317( & 8) & 339 & 9) \\
\hline 339 & 4) & 342( & 4) & 346( & 5) & 360( & 4) \\
\hline 402( & 11) & 402( & 9) & 397( & 5) & 417( & 7) \\
\hline 441( & 6) & 438( & 3) & 429 & 1) & 453( & 0) \\
\hline 477( & 11) & 474( & 11) & 466( & 9) & 494( & 12) \\
\hline 551( & 5) & 547( & 4) & 528( & 2) & 544( & 1) \\
\hline 564( & 1) & 5531 & 1) & 534( & 3) & 562( & 6) \\
\hline 576( & 2) & 570( & 1) & 553( & 1) & 568( & 3) \\
\hline 603( & 3) & 597( & 3) & 578( & 2) & 612( & 3) \\
\hline 647( & 9) & 638( & 8) & 611( & 7) & 639 & 8) \\
\hline 696( & 5) & 690( & 5) & 665( & 3) & 6960 & 6) \\
\hline 753( & 9) & 742( & 7) & 715( & 7) & 726( & 5) \\
\hline 775( & 45) & 7666 & 37) & 745( & 32) & 765( & 35) \\
\hline 781( & 18) & 780( & 20) & 762( & 10) & 780 & 3) \\
\hline 812( & 38) & 808( & 28) & 7899 & 12) & 800( & 54) \\
\hline 825( & 42) & 820( & 47) & 795( & 76) & 815( & 29) \\
\hline 836( & 58) & 828( & 55) & 800( & 14) & 821( & 24) \\
\hline 858( & 1) & 851( & 1) & 832( & 1) & 858( & 1) \\
\hline 882( & 15) & 872( & 14) & 841( & 1) & 877( & 3) \\
\hline 891( & 11) & 882( & 4) & 861( & 6) & 892( & 5) \\
\hline 946( & 7) & 939x & 6) & 898( & 1) & 929 & 1) \\
\hline 957( & 11) & 946( & 6) & 915( & 2) & 945( & 1) \\
\hline 979( & 2) & 958( & 4) & 920( & 4) & 949 & 3) \\
\hline 987( & 3) & 975( & 1) & 923( & 0) & 974( & 3) \\
\hline 991( & 8) & 978( & 6) & 951( & 1) & 988( & 3) \\
\hline 1015( & 2) & 1006( & 2) & 975( & 9) & 1007( & 7) \\
\hline 1021( & 7) & 1012( & 7) & 982( & 5) & 1017( & 3) \\
\hline 1026( & 10) & 1017 ( & 10) & 988( & 10) & 1028( & 9) \\
\hline 1038( & 4) & 1029( & 5) & 1002( & 5) & 1041( & 8) \\
\hline 1048( & 5) & 1039 & 4) & 1012( & 4) & 1050( & 5) \\
\hline 1093( & 5) & 1084( & 4) & 1058( & 2) & 1094( & 4) \\
\hline 1128( & 9) & 1117( & 9) & 1089 & 8) & 1124( & 8) \\
\hline 1151( & 7) & 1140( & 7) & 1111( & 6) & 1152( & 6) \\
\hline 1192( & 2) & 1183( & 3) & 1151( & 3) & 1197( & 4) \\
\hline 1204( & 2) & 1192( & 2) & 1162( & 2) & 1217( & 3) \\
\hline
\end{tabular}




\begin{tabular}{|c|c|c|c|c|c|c|c|}
\hline 1221( & 5) & 1208( & 5) & 1171( & 3) & 1224( & 3) \\
\hline 1238( & 1) & 1224( & 1) & 1193 & 1) & 1246( & 4) \\
\hline 1275( & 7) & 1264( & 2) & 1229 & 0) & 1265 & 2) \\
\hline 1283( & 18) & 1271( & 22) & 1237( & 20) & 1276( & 18) \\
\hline 1314( & 0) & 1299 & 0) & 1256( & 0) & 1308( & 2) \\
\hline 1315( & 2) & 1303( & 2) & 1268( & 5) & 1309 & 0) \\
\hline 1325( & 6) & 1309 & 1) & 1271( & 0) & 1317( & 1) \\
\hline 1328( & 1) & 1311( & 5) & 1274( & 1) & 1328( & 2) \\
\hline 1335( & 2) & 1321( & 3) & 1284( & 2) & 1345( & 4) \\
\hline 1347( & 2) & 1334( & 2) & 1293 & 2) & 1356( & 2) \\
\hline 1417( & 3) & 1403( & 3) & 1361( & 3) & 1407( & 3) \\
\hline 1421( & 1) & 1407( & 1) & 1368( & 1) & 1415( & 3) \\
\hline 1443( & 4) & 1431( & 4) & 1392( & 2) & 1436( & 2) \\
\hline 1479 & 5) & 1465( & 3) & 1424( & 3) & 1463( & 4) \\
\hline 1485( & 5) & 1472( & 7) & 1432( & 4) & 1466( & 10) \\
\hline 1488( & 5) & 1474( & 5) & 1433( & 9) & 1474( & 6) \\
\hline 1491( & 6) & 1477( & 6) & 1437( & 5) & 1476( & 7) \\
\hline 1505( & 9) & 1489 & 9) & 1446( & 11) & 1491( & 4) \\
\hline 1562( & 63) & 1551( & 54) & 1514( & 34) & 1563( & 49) \\
\hline 1629 & 9) & 1604( & 14) & 1537( & 19) & 1607( & 21) \\
\hline 1645( & 91) & 1632( & 84) & 1590 & 60) & 1659 & 77) \\
\hline 2962( & 17) & 29350 & 16) & 2851( & 18) & 29920 & 30) \\
\hline 3004( & 65) & 2984( & 64) & 2928( & 67) & 3005 & 114) \\
\hline 3013( & 39) & 2993( & 40) & 2937( & $50)$ & 3032( & 49) \\
\hline 3029 & 54) & 3011( & 52) & 2959 & 55) & 3041( & 85) \\
\hline 3051( & 26) & 3032( & 30) & 2974( & 40) & 3057( & 64) \\
\hline 3053( & 25) & 30331 & 22) & 2986( & 18) & 3062 & 61) \\
\hline 3070( & 25) & 3052( & 20) & 3000 & 17) & 3094 & 20) \\
\hline 3104( & 4) & 3083( & 4) & 3026( & 6) & 3110 & 11) \\
\hline 3116( & 10) & 3094( & 9) & 3035( & 9) & 3121( & 19) \\
\hline 3117( & 5) & 3097( & 5) & 3041( & 12) & 3128( & 1) \\
\hline 3119 & 21) & 3098( & 19) & 3044( & 6) & 3133 & 29) \\
\hline 3132( & 17) & 3113( & 16) & 3061( & 13) & 3139 & 15) \\
\hline 3148( & 4) & 3125( & 6) & 3063 & 16) & 3144( & 14) \\
\hline 3150 & 7) & 3129 & 15) & 3072( & 18) & 3150 & 6) \\
\hline 3153( & 6) & 3133( & 9) & 3077( & 1) & 3154( & 32) \\
\hline 3155( & 15) & 3134( & 2) & 3077( & 6) & 3163 & 34) \\
\hline 3238 ( & 16) & 3219 & 20) & 31660 & 13) & 3237( & 12) \\
\hline 3238( & 10) & 3220( & 4) & 31660 & 9) & 3242( & 28) \\
\hline
\end{tabular}


Table S59. Harmonic vibrational frequencies (in $\mathrm{cm}^{-1}$ ) and infrared intensities (in parentheses in $\mathrm{km} / \mathrm{mol}$ ) for the $\left(\mathbf{C}_{4} \mathbf{H}_{6}\right)_{3} \mathbf{V}$ structure $\mathrm{V}-5 \mathrm{Q}$.

\begin{tabular}{|c|c|c|c|c|c|c|c|}
\hline \multicolumn{2}{|c|}{ B3LYP } & \multicolumn{2}{|c|}{ B3LYP* } & \multicolumn{2}{|c|}{ BP86 } & \multicolumn{2}{|c|}{ M06L } \\
\hline 56( & 0) & 50( & 0) & 51( & 0) & 57( & 0) \\
\hline 67( & 1) & 61( & 1) & 68( & 1) & 71( & 1) \\
\hline 87( & 0) & 82( & 0) & 89 & 0) & 88( & 0) \\
\hline 99 & 0) & 98( & 0) & 100( & 0) & 122( & 2) \\
\hline 129( & 1) & 131( & 1) & 130( & 0) & 125( & 0) \\
\hline 143( & 2) & 140( & 1) & 145( & 2) & 160( & 1) \\
\hline 164( & 3) & 161( & 2) & 167( & 2) & 178( & 3) \\
\hline 178( & 2) & 173( & 2) & 179( & 3) & 191( & 5) \\
\hline 215( & 19) & 218( & 11) & 235( & 3) & 219 & 22) \\
\hline 235( & 5) & 237( & 4) & 251( & 0) & 255( & 6) \\
\hline 250( & 2) & 251( & 2) & 261( & 3) & 266( & 1) \\
\hline 274( & 2) & 275( & 1) & 271( & 0) & 275( & 0) \\
\hline 285( & 2) & 285( & 1) & 289 & 1) & 289 & 25) \\
\hline 292( & 19) & 294( & 8) & 304( & 6) & 305( & 4) \\
\hline 298( & 9) & 297( & 13) & 311( & 2) & 309 & 12) \\
\hline 303( & 9) & 305( & 15) & 321( & 15) & 312( & 6) \\
\hline 346( & 4) & 340( & 5) & 346( & 8) & 356( & 4) \\
\hline 365( & 4) & 366( & 3) & 372( & 1) & 398( & 3) \\
\hline 418( & 14) & 413( & 15) & 417( & 16) & 428( & 10) \\
\hline 464( & 1) & 459 & 2) & 459( & 3) & 463( & 3) \\
\hline 466( & 2) & 464( & 2) & 463( & 2) & 476( & 1) \\
\hline 552( & 11) & 545( & 12) & 534( & 10) & 550 & 12) \\
\hline 589 & 8) & 582( & 9) & 571( & 9) & 582( & 10) \\
\hline 593( & 3) & 589 & 1) & 575( & 4) & 5881 & 2) \\
\hline 5981 & 0) & 594( & 0) & 586( & 0) & 601( & 2) \\
\hline 670( & 40) & 664( & 32) & 654( & 19) & 650 & 33) \\
\hline 684( & 1) & 680 & 0) & 665( & 2) & 682( & 0) \\
\hline 705( & 7) & 699 & 6) & 677( & 6) & 690( & 5) \\
\hline 711( & 7) & 702( & 10) & 686( & 5) & 700( & 5) \\
\hline 718( & 2) & 711( & 1) & 691( & 2) & 712( & 5) \\
\hline 778 ( & 4) & 777 & 5) & 770 & 14) & 738( & 5) \\
\hline 807( & 6) & 794( & 7) & 778( & 8) & 777 & 3) \\
\hline 824( & 0) & 819 & 0) & 797( & 2) & 789 & 3) \\
\hline 840( & 2) & 8331 & 1) & 812( & 1) & 809 & 4) \\
\hline 861( & 9) & 852( & 11) & 829 & 11) & 831( & 3) \\
\hline 884( & 4) & 875( & 3) & 837( & 35) & 855( & 30) \\
\hline 891( & 11) & 879 & 34) & 842( & 9) & 876( & 4) \\
\hline 894( & 30) & 881( & 8) & 848( & 5) & 877( & 3) \\
\hline 898( & 3) & 884( & 4) & 860 & 6) & 889 & 5) \\
\hline 915( & 13) & 901( & 15) & 879 & 9) & 909 & 3) \\
\hline 934( & 1) & 927( & 0) & 895( & 4) & 928( & 1) \\
\hline 937( & 7) & 930( & 7) & 904( & 4) & 931( & 1) \\
\hline 950( & 3) & 935( & 2) & 909 & 1) & 935( & 5) \\
\hline 954( & 1) & 938( & 4) & 913( & 6) & 939 & 7) \\
\hline 1002( & 7) & 990( & 8) & 958( & 9) & 996( & 8) \\
\hline 1065 & 3) & 1055 & 2) & 1031( & 3) & 1057 & 2) \\
\hline
\end{tabular}




\begin{tabular}{|c|c|c|c|c|c|c|c|}
\hline 1067( & 1) & 1060 & 1) & 1034( & 0) & 1064( & 5) \\
\hline 1070( & 3) & 1062( & 4) & 1038( & 4) & 10666 & 3) \\
\hline 1085( & 8) & 1076( & 7) & 1045( & 6) & 1076( & 5) \\
\hline 1089( & 0) & 1079( & 1) & 1050 & 1) & 1082( & 1) \\
\hline 1097( & 6) & 1087( & 7) & 1058( & 8) & 1091( & 6) \\
\hline 1224( & 11) & 1213( & 11) & 1179 & 10) & 1214( & 12) \\
\hline 1228( & 8) & 1216( & 7) & 1182( & 5) & 1220( & 8) \\
\hline 1258( & 32) & 1246( & 23) & 1214( & 7) & 12566 & 20) \\
\hline 1274( & 95) & 1263( & 78) & 1229 & 47) & 1261( & 75) \\
\hline 1283( & 8) & 1270 & 6) & 1235( & 4) & 1272( & 15) \\
\hline 1320( & 18) & 1307( & 14) & 1277( & 8) & 1314( & 10) \\
\hline 1410( & 1) & 1397( & 1) & 1354( & 1) & 1386( & 0) \\
\hline 1413( & 0) & 1398( & 0) & 1356 & 0) & 1394( & 1) \\
\hline 1420( & 7) & 1406( & 5) & 1366( & 2) & 1401( & 2) \\
\hline 1452( & 28) & 1441( & 22) & 1407( & 11) & 1444( & 9) \\
\hline 1468( & 75) & 1457( & 56) & 1424( & 25) & 1458( & 33) \\
\hline 1478( & 3) & 1466( & 4) & 1431( & 8) & 1466( & 12) \\
\hline 1536( & 105) & 1523( & 90) & 1480( & 31) & 1523( & 103) \\
\hline 1547( & 12) & 1531( & 11) & 1483( & 1) & 1530( & 3) \\
\hline 1547 ( & 31) & 1533( & 4) & 1488( & 27) & 1533( & 25) \\
\hline 1551( & 40) & 1538( & 42) & 1497( & 14) & 1546( & 45) \\
\hline 1554( & 92) & 1543( & 94) & 1506( & 67) & 1561( & 75) \\
\hline 1626( & 41) & 1611( & 41) & 1566( & 39) & 1638( & 45) \\
\hline 3147( & 11) & 3125( & 11) & 3069 & 9) & 3137( & 14) \\
\hline 3151( & 3) & 3130 & 3) & 3070( & 4) & 3140( & 20) \\
\hline 3157( & 3) & 3136( & 3) & 3078( & 9) & 3143( & 20) \\
\hline 3158( & 1) & 3136( & 1) & 3080 & 9) & 3148( & 14) \\
\hline 3164( & 13) & 3141( & 5) & 3080 & 6) & 3154( & 10) \\
\hline 3164( & 7) & 3141( & 10) & 3082( & 8) & 3156( & 16) \\
\hline 3165( & 3) & 3142( & 6) & 3083( & 5) & 3156( & 5) \\
\hline 3166r & 3) & 3144( & 5) & 3086( & 3) & 3167( & 27) \\
\hline 3169 & 1) & 3146( & 5) & 3088( & 4) & 3171( & 5) \\
\hline 3170( & 5) & 3147( & 2) & 3089 & 2) & 3172( & 29) \\
\hline 3177( & 7) & 3154( & 7) & 3097( & 11) & 3175( & 4) \\
\hline 3189 ( & 4) & 3166( & 4) & 3103( & 9) & 3185( & 22) \\
\hline 3243( & 3) & 3222( & 3) & 3162( & 3) & 3236( & 13) \\
\hline 3251( & 9) & 3230 & 5) & 3166( & 6) & 3238( & 6) \\
\hline 3254( & 11) & 3231( & 7) & 3167( & 5) & 3240( & 9) \\
\hline 3255( & 4) & 3231( & 9) & 3174( & 6) & 3252( & 19) \\
\hline 3256( & 2) & 3234( & 3) & 3178( & 9) & 32566 & 16) \\
\hline 3257 ( & 2) & 3236( & 3) & 3178( & 7) & 32666 & 9) \\
\hline
\end{tabular}


Table S60. Harmonic vibrational frequencies (in $\mathrm{cm}^{-1}$ ) and infrared intensities (in parentheses in $\mathrm{km} / \mathrm{mol})$ for the $\left(\mathbf{C}_{4} \mathbf{H}_{6}\right)_{3} \mathbf{C r}$ structure $\mathbf{C r}-\mathbf{1 S}$.

\begin{tabular}{|c|c|c|c|c|c|c|c|}
\hline \multicolumn{2}{|c|}{ B3LYP } & \multicolumn{2}{|c|}{ B3LYP* } & \multicolumn{2}{|r|}{ BP86 } & \multicolumn{2}{|c|}{ M06L } \\
\hline 36( & 0) & 36( & 0) & 47( & 0) & 68( & 0) \\
\hline 92( & 1) & 86( & 1) & 87( & 1) & 100( & 1) \\
\hline 96( & 1) & 101( & 1) & 100( & 1) & 119( & 1) \\
\hline 125( & 1) & 124( & 1) & 123( & 1) & 144( & 1) \\
\hline 189( & 2) & 190( & 2) & 195( & 1) & 205( & 1) \\
\hline 202( & 3) & 200( & 3) & 210( & 3) & 218( & 4) \\
\hline 220( & 0) & 218( & 0) & 220( & 1) & 226( & 1) \\
\hline 244( & 2) & 247( & 2) & 244( & 2) & 263( & 2) \\
\hline 261( & 3) & 258( & 2) & 249 ( & 2) & 273( & 1) \\
\hline 281( & 0) & 281( & 1) & 288( & 1) & 300( & 2) \\
\hline 286( & 7) & 289 & 5) & 297( & 2) & 315( & 4) \\
\hline 315( & 1) & 315( & 0) & 316( & 0) & 327( & 0) \\
\hline 322( & 1) & 321( & 1) & 321( & 1) & 352( & 1) \\
\hline 349 & 7) & 354( & 7) & 367( & 4) & 382( & 4) \\
\hline 369( & 7) & 371( & 7) & 379( & 5) & 392( & 5) \\
\hline 389r & 1) & 391( & 0) & 392( & 2) & 433( & 3) \\
\hline 425( & 6) & 428( & 4) & 433( & 1) & 464( & 14) \\
\hline 444( & 10) & 446( & 11) & 449( & 11) & 468( & 3) \\
\hline 467( & 20) & 465( & 18) & 465( & 13) & 483( & 8) \\
\hline 490( & 1) & 485( & 1) & 472( & 3) & 504( & 2) \\
\hline 496( & 3) & 491( & 3) & 482( & 5) & 516( & 5) \\
\hline 562( & 6) & 558( & 6) & 546( & 4) & 563( & 4) \\
\hline 613( & 3) & 608( & 3) & 591( & 3) & 613( & 7) \\
\hline 625( & 0) & 621( & 0) & 603( & 0) & 630( & 1) \\
\hline 686( & 25) & 683( & 25) & 663( & 21) & 689( & 19) \\
\hline 721( & 19) & 713( & 17) & 681( & 17) & 707( & 16) \\
\hline 737( & 5) & 729 & 5) & 711( & 11) & 731( & 8) \\
\hline 748( & 12) & 736( & 12) & 717( & 6) & 740( & 9) \\
\hline 763( & 1) & 753( & 1) & 729) & 1) & 751( & 1) \\
\hline 781( & 8) & 772( & 6) & 763( & 2) & 781( & 3) \\
\hline 872( & 19) & 866( & 18) & 826( & 7) & 846( & 17) \\
\hline 878( & 7) & 871( & 14) & 843( & 64) & 857( & 27) \\
\hline 888( & 0) & 874( & 2) & 845( & 28) & 861( & 4) \\
\hline 898( & 1) & 887( & 3) & 856( & 1) & 869) & 2) \\
\hline 900( & 12) & 890( & 12) & 860( & 1) & 890( & 2) \\
\hline 907( & 44) & 893( & 39) & 864( & 2) & 898( & 2) \\
\hline 916( & 2) & 907( & 1) & 876( & 1) & 899 & 2) \\
\hline 921( & 7) & 908( & 8) & 886( & 2) & 914( & 15) \\
\hline 936( & 4) & 925( & 3) & 905( & 1) & 916( & 5) \\
\hline 947( & 2) & 937( & 2) & 910( & 3) & 937( & 2) \\
\hline 950( & 3) & 940( & 3) & 918( & 4) & 950( & 4) \\
\hline 966( & 0) & 957( & 0) & 923( & 1) & 965( & 1) \\
\hline 972( & 0) & 961( & 1) & 926( & 2) & 973( & 1) \\
\hline 977( & 1) & 961( & 0) & 940( & 0) & 978( & 1) \\
\hline 1025( & 10) & 1015( & 10) & 987( & 11) & 1018( & 8) \\
\hline 1067( & 5) & 1056( & 5) & 1028( & 5) & 1059 ( & 3) \\
\hline
\end{tabular}




\begin{tabular}{|c|c|c|c|c|c|c|c|}
\hline 1080( & 4) & 1071( & 2) & 1044( & 1) & 1079 & 0) \\
\hline 1081( & 2) & 1072( & 3) & 1048( & 3) & 1085( & 8) \\
\hline 1085( & 1) & 1077( & 2) & 1053( & 2) & 1086( & 1) \\
\hline 1094( & 8) & 1085( & 7) & 1059( & 7) & 1091( & 6) \\
\hline 1109 & 1) & 1102( & 1) & 1072( & 2) & 1117( & 1) \\
\hline 1209 ( & 0) & 1197( & 0) & 1162( & 0) & 1202( & 2) \\
\hline 1215( & 8) & 1205( & 8) & 1170( & 7) & 1211( & 8) \\
\hline 1241( & 39) & 1229 & 33) & 1190( & 21) & 1229 & 29) \\
\hline 1263( & 29) & 1251( & 24) & 1220( & 15) & 1261( & 22) \\
\hline 1267( & 12) & 1257( & 13) & 1225( & 7) & 1264( & 11) \\
\hline 1320( & 3) & 1308( & 3) & 1277( & 2) & 1316( & 3) \\
\hline 1410( & 0) & 1396( & 0) & 1354( & 0) & 1390( & 0) \\
\hline 1414( & 9) & 1402( & 1) & 1360( & 1) & 1396( & 0) \\
\hline 1415( & 5) & 1403( & 11) & 1360( & 7) & 1400( & 5) \\
\hline 1451( & 13) & 1439( & 13) & 1401( & 10) & 1444( & 11) \\
\hline 1483( & 7) & 1470( & 4) & 1432( & 1) & 1469( & 2) \\
\hline 1492( & 7) & 1480( & 9) & 1443( & 12) & 1482( & 7) \\
\hline 1536( & 7) & 1520( & 2) & 1473( & 11) & 1519( & 17) \\
\hline 1540( & 9) & 1525( & 13) & 1476( & 7) & 1522( & 3) \\
\hline 1541( & 15) & 1526( & 11) & 1480( & 5) & 1525( & 10) \\
\hline 1543( & 18) & 1531( & 20) & 1498( & 8) & 1548( & 16) \\
\hline 1551( & 28) & 1539 & 20) & 1506( & 9) & 1554( & 11) \\
\hline 1664( & 54) & 1649 ( & 55) & 1600( & 50) & 1669( & 67) \\
\hline 3118( & 1) & 3095( & 0) & 3032( & 1) & 31066 & 4) \\
\hline 3122( & 0) & 3098( & 0) & 3034( & 0) & 3109 & 19) \\
\hline 3124( & 18) & 3101( & 2) & 3038( & 5) & 3114( & 24) \\
\hline 3125( & 4) & 3102( & 20) & 3043( & 32) & 3122( & 40) \\
\hline 3130( & 27) & 3107( & 26) & 3047( & 19) & 3131( & 37) \\
\hline 3152( & 3) & 3132( & 3) & 3070( & 17) & 3142( & 28) \\
\hline 3157( & 11) & 3134( & 11) & 3076( & 6) & 3153( & 16) \\
\hline 3174( & 3) & 3151( & 4) & 3088( & 8) & 3168( & 17) \\
\hline 3183( & 4) & 3161( & 4) & 30999 & 4) & 3177( & 9) \\
\hline 3194( & 6) & 3172( & 5) & 3113( & 10) & 3185( & 5) \\
\hline 3196( & 11) & 3174( & 12) & 3114( & 11) & 3199( & 31) \\
\hline 3216( & 1) & 3195( & 1) & 3132( & 1) & 3215( & 3) \\
\hline 3223( & 0) & 3201( & 0) & 3144( & 0) & 3222( & 14) \\
\hline 3228( & 13) & 3206( & 12) & 3147( & 7) & 3228( & 24) \\
\hline 3229 & 13) & 3207( & 13) & 3148( & 16) & 3228( & 20) \\
\hline 3235( & 3) & 3213( & 16) & 3150( & 25) & 3231( & 41) \\
\hline 3237( & 17) & 3213( & 4) & 3155( & 3) & 3234( & 8) \\
\hline 3244( & 12) & 32240 & 12) & 31690 & 14) & 32480 & 28) \\
\hline
\end{tabular}


Table S61. Harmonic vibrational frequencies (in $\mathrm{cm}^{-1}$ ) and infrared intensities (in parentheses in $\mathrm{km} / \mathrm{mol})$ for the $\left(\mathbf{C}_{4} \mathbf{H}_{6}\right)_{3} \mathbf{C r}$ structure $\mathrm{Cr}-2 \mathrm{~S}$.

\begin{tabular}{|c|c|c|c|c|c|c|c|}
\hline \multicolumn{2}{|c|}{ B3LYP } & \multicolumn{2}{|c|}{ B3LYP* } & \multicolumn{2}{|c|}{ BP86 } & \multicolumn{2}{|c|}{ M06L } \\
\hline 101( & 1) & 102( & 1) & 966 & 1) & 108( & 1) \\
\hline 126( & 4) & 124( & 2) & 111( & 1) & 136( & 1) \\
\hline 129( & 1) & 129 & 1) & 137( & 1) & 156( & 2) \\
\hline 181( & 0) & 192( & 0) & 193( & 2) & 197( & 1) \\
\hline 202( & 1) & 207( & 3) & 219 ( & 0) & 232( & 1) \\
\hline 209( & 8) & 213( & 2) & 234( & 2) & 2466 & 0) \\
\hline 213( & 3) & 221( & 6) & 246( & 2) & 257( & 2) \\
\hline 232( & 0) & 236( & 0) & 258( & 5) & 265( & 1) \\
\hline 245( & 2) & 250( & 3) & 260( & 1) & 277 ( & 11) \\
\hline 259( & 16) & 263( & 15) & 279 & 10) & 300( & 9) \\
\hline 291( & 12) & 295( & 10) & 309 & 2) & 318( & 8) \\
\hline 294( & 0) & 296( & 1) & 310( & 1) & 329 ( & 3) \\
\hline 308( & 8) & 306( & 7) & 323( & 7) & 342( & 6) \\
\hline 332( & 7) & 332( & 1) & 341( & 8) & 3681 & 25) \\
\hline 341( & 13) & 342( & 19) & 360( & 9) & 378( & 1) \\
\hline 374( & 3) & 371( & 2) & 375( & 9) & 410( & 6) \\
\hline 409 & 13) & 408( & 14) & 409 & 12) & 4331 & 12) \\
\hline 450( & 26) & 445( & 28) & 438( & 30) & 455( & 19) \\
\hline 471( & 2) & 468( & 1) & 458( & 1) & 4861 & 0) \\
\hline 490( & 4) & 489 & 6) & 475( & 2) & 506( & 2) \\
\hline 503( & 2) & 496( & 1) & 4891 & 3) & 513( & 2) \\
\hline 604( & 2) & 598( & 2) & 576( & 1) & 601( & 1) \\
\hline 606( & 1) & 604( & 1) & 597( & 1) & 612( & 2) \\
\hline 619 ( & 1) & 619 & 1) & 619x & 2) & 6399 & 3) \\
\hline 703( & 5) & 697( & 2) & 682( & 1) & 691( & 3) \\
\hline 717( & 7) & 714( & 6) & 7066 & 5) & 722( & 8) \\
\hline 737( & 6) & 735( & 1) & 722( & 1) & 745( & 1) \\
\hline 743( & 5) & 740( & 9) & 731( & 8) & 7531 & 4) \\
\hline 752( & 2) & 751( & 1) & 748( & 7) & 771( & 12) \\
\hline 775( & 15) & 769 & 13) & 7531 & 2) & 782( & 3) \\
\hline 838( & 9) & 833( & 8) & 817( & 11) & 821( & 8) \\
\hline 846( & 25) & 842( & 31) & 829( & 27) & 830( & 16) \\
\hline 868( & 3) & 861( & 3) & 849 & 1) & 855( & 2) \\
\hline 874( & 3) & 870( & 3) & 860( & 10) & 862( & 1) \\
\hline 889( & 5) & 889 & 10) & 861( & 7) & 888( & 5) \\
\hline 904( & 6) & 892( & 4) & 881( & 4) & 897( & 1) \\
\hline 927( & 1) & 918( & 0) & 900( & 3) & 921( & 0) \\
\hline 927( & 0) & 923( & 1) & 906( & 1) & 926( & 1) \\
\hline 938( & 3) & 933( & 4) & 916( & 5) & 931( & 7) \\
\hline 944( & 1) & 940( & 1) & 922( & 2) & 947( & 1) \\
\hline 953( & 1) & 948( & 2) & 930( & 3) & 955( & 1) \\
\hline 961( & 3) & 957( & 2) & 940( & 7) & 969) & 5) \\
\hline 965( & 2) & 961( & 4) & 944( & 3) & 975( & 1) \\
\hline 971( & 0) & 968( & 1) & 947( & 0) & 976( & 0) \\
\hline 980( & 2) & 972( & 2) & 950( & 1) & 991( & 8) \\
\hline 1076( & 1) & 1068( & 1) & 1043( & 3) & 1074( & 3) \\
\hline
\end{tabular}




\begin{tabular}{|c|c|c|c|c|c|c|c|}
\hline 1079 & 0) & 1071( & 0) & 1044( & 1) & 1075( & 2) \\
\hline 1080( & 1) & 1074( & 2) & 1048( & 3) & 1081( & 1) \\
\hline 1086( & 6) & 1077( & 4) & 1053( & 1) & 1085( & 1) \\
\hline 1092( & 1) & 1083( & 1) & 1058( & 0) & 1090( & 2) \\
\hline 1096( & 4) & 1087( & 4) & 1064( & 2) & 1095( & 2) \\
\hline 1225( & 8) & 1212( & 8) & 1171( & 8) & 1210( & 10) \\
\hline 1230( & 5) & 1217( & 5) & 1176( & 1) & 1216( & 3) \\
\hline 1244( & 5) & 1232( & 5) & 1200( & 6) & 1239 & 7) \\
\hline 1277( & 22) & 1263( & 20) & 1220( & 13) & 1263( & 19) \\
\hline 1280( & 19) & 1268( & 16) & 1231( & 10) & 1278( & 14) \\
\hline 1298( & 15) & 1288( & 13) & 1262( & 9) & 1297( & 12) \\
\hline 1409( & 1) & 1396( & 1) & 1357( & 1) & 1391( & 1) \\
\hline 1417( & 0) & 1403( & 0) & 1358( & 1) & 1394( & 0) \\
\hline 1422( & 0) & 1410( & 0) & 1370( & 0) & 1403( & 0) \\
\hline 1478( & 10) & 1466( & 7) & 1433( & 4) & 1463( & 2) \\
\hline 1482( & 8) & 1471( & 6) & 1440( & 5) & 1473( & 4) \\
\hline 1486( & 7) & 1475( & 6) & 1444( & 3) & 1483( & 3) \\
\hline 1540( & 8) & 1521( & 8) & 1473( & 3) & 1515( & 7) \\
\hline 1556( & 21) & 1542( & 13) & 1486( & 2) & 1530( & 3) \\
\hline 1560( & 22) & 1543( & 15) & 1497( & 10) & 1542( & 12) \\
\hline 1569( & 32) & 1555( & 15) & 1506( & 6) & 1551( & 7) \\
\hline 1571( & 9) & 1556( & 14) & 1514( & 7) & 1565( & 27) \\
\hline 1576( & 16) & 1561( & 26) & 1528( & 22) & 1566( & 19) \\
\hline 3151( & 9) & 3129 & 10) & 3056( & 21) & 3130( & 34) \\
\hline 3155( & 7) & 3131( & 11) & 3060 & 27) & 3132( & 40) \\
\hline 3156( & 11) & 3132( & 8) & 3065( & 10) & 3133( & 52) \\
\hline 3159( & 8) & 3135( & 9) & 3066( & 20) & 3139 & 39) \\
\hline 3160( & 15) & 31360 & 15) & 3070 & 8) & 3140( & 17) \\
\hline 3165( & 3) & 3140( & 5) & 3077( & 15) & 3157 ( & 36) \\
\hline 3170( & 10） & 3146( & 9) & 3082( & 9) & 3158( & 18) \\
\hline 3179 & 6) & 3154( & 8) & 3084( & 8) & 3162( & 9) \\
\hline 3179( & 5) & 3157 ( & 7) & 3091( & 17) & 3174( & 16) \\
\hline 3183( & 6) & 3157( & 5) & 3095( & 7) & 3178( & 35) \\
\hline 3186( & 28) & 3163( & 27) & 3099 & 30) & 3192( & 42) \\
\hline 3205( & 10) & 3182( & 10) & 3116( & 15) & 3206( & 28) \\
\hline 3242( & 7) & 3215( & 7) & 3147( & 17) & 3228( & 22) \\
\hline 3245( & 8) & 3223( & 7) & 3150 & 0) & 3229 ( & 21) \\
\hline 3248( & 8) & 3225( & 7) & 3154( & 10) & 3232( & 4) \\
\hline 3249 & 6) & 3225( & 8) & 3156( & 10) & 3234( & 20) \\
\hline 3257( & 7) & 3231( & 7) & 3171( & 7) & 3242( & 14) \\
\hline 3276( & 5) & 32531 & 4) & 3187( & 5) & 3278( & 9) \\
\hline
\end{tabular}


Table S62. Harmonic vibrational frequencies (in $\mathrm{cm}^{-1}$ ) and infrared intensities (in parentheses in $\mathrm{km} / \mathrm{mol})$ for the $\left(\mathbf{C}_{4} \mathbf{H}_{6}\right)_{3} \mathbf{C r s t r u c t u r e ~ C r - 1 T}$.

\begin{tabular}{|c|c|c|c|c|c|c|c|}
\hline \multicolumn{2}{|c|}{ B3LYP } & \multicolumn{2}{|c|}{ B3LYP* } & \multicolumn{2}{|c|}{ BP86 } & \multicolumn{2}{|c|}{ M06L } \\
\hline 83( & 0) & 83( & 0) & 82( & 0) & 79 ( & 0) \\
\hline 100( & 0) & 100( & 0) & 105( & 0) & 107( & 0) \\
\hline 151( & 2) & 154( & 1) & 148( & 1) & 158( & 1) \\
\hline 171( & 1) & 179 & 1) & 182( & 1) & 179( & 1) \\
\hline 176( & 1) & 183( & 2) & 191( & 2) & 188( & 2) \\
\hline 199 & 4) & 201( & 1) & 212( & 1) & 212( & 1) \\
\hline 208( & 2) & 210 & 3) & 218( & 1) & 217( & 1) \\
\hline 214( & 1) & 217( & 1) & 228( & 2) & 238( & 1) \\
\hline 244( & 5) & 247( & 4) & 256( & 2) & 267( & 2) \\
\hline 279 & 8) & 279 & 8) & 288( & 2) & 297( & 2) \\
\hline 286( & 8) & 294( & 11) & 299( & 19) & 305( & 18) \\
\hline 304( & 0) & 308( & 0) & 315( & 1) & 320( & 1) \\
\hline 314( & 0) & 316( & 0) & 316( & 0) & 327( & 0) \\
\hline 330( & 2) & 327( & 1) & 344( & 2) & 351( & 2) \\
\hline 373( & 4) & 377( & 6) & 383( & 7) & 388( & 5) \\
\hline 380( & 2) & 3831 & 2) & 3900 & 5) & 410( & 3) \\
\hline 442( & 3) & 443( & 3) & 438( & 4) & 440( & 2) \\
\hline 466( & 4) & 467( & 3) & 458( & 1) & 473( & 2) \\
\hline 470( & 1) & 470( & 3) & 468( & 8) & 479( & 4) \\
\hline 491( & 3) & 492( & 4) & 482( & 4) & 505( & 3) \\
\hline 571( & 2) & 567( & 2) & 556( & 3) & 573( & 3) \\
\hline 619 & 1) & 616( & 1) & 599( & 1) & 618( & 3) \\
\hline 653( & 5) & 646( & 4) & 628( & 4) & 651( & 6) \\
\hline 671( & 20) & 671( & 19) & 6531 & 14) & 663( & 16) \\
\hline 698( & 2) & 6961 & 2) & 677( & 4) & 690( & 3) \\
\hline 704( & 4) & 705( & 4) & 691( & 2) & 708( & 1) \\
\hline 732( & 2) & 727( & 1) & 713( & 2) & 726( & 2) \\
\hline 786( & 7) & 781( & 8) & 762( & 6) & 784( & 8) \\
\hline 807( & 15) & 805( & 16) & 788( & 16) & 7899 & 4) \\
\hline 825( & 17) & 821( & 20) & 800( & 15) & 811( & 7) \\
\hline 830( & 8) & 825( & 5) & 810( & 2) & 817( & 4) \\
\hline 837( & 14) & 832( & 14) & 824( & 20) & 838( & 9) \\
\hline 848( & 12) & 844( & 12) & 828( & 8) & 845( & 6) \\
\hline 861( & 7) & 858( & 7) & 838( & 6) & 849( & 16) \\
\hline 880( & 2) & 874( & 2) & 850( & 4) & 868( & 5) \\
\hline 8861 & 2) & 881( & 1) & 861( & 1) & 878( & 2) \\
\hline 928( & 4) & 921( & 4) & 8931 & 4) & 922( & 3) \\
\hline 947( & 5) & 939 & 5) & 914( & 0) & 941( & 0) \\
\hline 960( & 0) & 951( & 0) & 916 & 3) & 949( & 6) \\
\hline 990( & 4) & 979 & 4) & 936( & 5) & 976( & 5) \\
\hline 997( & 3) & 984( & 3) & 939x & 1) & 978( & 2) \\
\hline 1005( & 2) & 997( & 3) & 967( & 3) & 998( & 3) \\
\hline 1009 & 3) & 1000( & 1) & 975( & 1) & 1007( & 1) \\
\hline 1048( & 4) & 1041( & 3) & 1014( & 4) & 1048( & 4) \\
\hline 1070( & 11) & 1062( & 11) & 1033( & 11) & 1068( & 12) \\
\hline 1072( & 5) & 1064( & 5) & 1041( & 4) & 1072( & 6) \\
\hline
\end{tabular}




\begin{tabular}{|c|c|c|c|c|c|c|c|}
\hline 1079( & 8) & 1071( & 7) & 1044( & 6) & 1076( & 6) \\
\hline 1152( & 5) & 1143( & 5) & 1113( & 5) & 1150( & 6) \\
\hline 1169( & 7) & 1160 & 6) & 1131( & 4) & 1167( & 6) \\
\hline 1202( & 9) & 1195( & 8) & 1167( & 7) & 1199 & 8) \\
\hline 1217( & 2) & 1207( & 3) & 1175( & 4) & 1218( & 4) \\
\hline 1237( & 5) & 1227( & 5) & 1194( & 4) & 1242( & 9) \\
\hline 1255( & 18) & 1246( & 17) & 1219 ( & 11) & 1253( & 12) \\
\hline 1267( & 4) & 1257( & 3) & 1224( & 3) & 1261( & 2) \\
\hline 1283( & 5) & 1272( & 4) & 1237( & 3) & 1272( & 4) \\
\hline 1324( & 3) & 1310( & 3) & 1269 & 3) & 1318( & 2) \\
\hline 1363( & 4) & 1350 & 3) & 1308( & 3) & 1360( & 3) \\
\hline 1405( & 0) & 1392( & 0) & 1353( & 0) & 1388( & 0) \\
\hline 1413( & 1) & 1399 & 1) & 1358( & 1) & 1401( & 1) \\
\hline 1418( & 1) & 1405( & 1) & 1363( & 1) & 1410( & 1) \\
\hline 1478( & 2) & 1465( & 2) & 1425( & 2) & 1462( & 2) \\
\hline 1483( & 6) & 1471( & 6) & 1431( & 5) & 1470( & 5) \\
\hline $1489 x$ & 4) & 1474( & 3) & 1434( & 4) & 1473( & 3) \\
\hline 1501( & 0) & 1488( & 1) & 1445( & 3) & 1485( & 2) \\
\hline 1506( & 8) & 1494( & 10) & 1449 ( & 9) & 1488( & 7) \\
\hline 1522( & 6) & 1510 & 6) & 1470( & 5) & 1509 & 6) \\
\hline 1532( & 30) & 1522( & 28) & 1490( & 19) & 1535( & 24) \\
\hline 1561( & 14) & 1551( & 12) & 1509( & 10) & 1555( & 10) \\
\hline 1572( & 32) & 1561( & 30) & 1520( & 19) & 1563( & 27) \\
\hline 3012( & 21) & 2992( & 19) & 2936( & 25) & 3026( & 32) \\
\hline 3022( & 92) & 3002( & 92) & 2946( & 98) & 3037( & 167) \\
\hline 3072( & 14) & 3053( & 13) & 3000( & 9) & 3095( & 15) \\
\hline 3116( & 13) & 3095( & 13) & 3031( & 18) & 3113( & 27) \\
\hline 3129 ( & 8) & 3107( & 8) & 3050( & 15) & 3121( & 15) \\
\hline 3129 & 1) & 3110 & 0) & 3051( & 2) & 3124( & 20) \\
\hline 3133( & 12) & 3112( & 12) & 3055( & 8) & 3138( & 13) \\
\hline 3146( & 8) & 3125( & 8) & 3068( & 9) & 3143( & 9) \\
\hline 3157( & 4) & 3137( & 6) & 3073( & 12) & 3144( & 3) \\
\hline 3162( & 16) & 3142( & 14) & 3078( & 13) & 3146( & 58) \\
\hline 3168( & 3) & 3147( & 3) & 30899 & 2) & 3166( & 33) \\
\hline 3172( & 8) & 3150( & 8) & 3092( & 13) & 3168( & 7) \\
\hline 3182( & 13) & 3159 & 13) & 3100( & 16) & 3184( & 29) \\
\hline 3186( & 14) & 3164( & 14) & 3105( & 18) & 3190( & 34) \\
\hline 3221( & 8) & 3200( & 8) & 3144( & 8) & 3213( & 20) \\
\hline 3228( & 6) & 3208( & 6) & 3152( & 7) & 32321 & 18) \\
\hline 3245( & 7) & 3223( & 7) & 3162( & 8) & 3236( & 15) \\
\hline 3251( & 10) & 32310 & 9) & 31660 & 11) & 32430 & 17) \\
\hline
\end{tabular}


Table S63. Harmonic vibrational frequencies (in $\mathrm{cm}^{-1}$ ) and infrared intensities (in parentheses in $\mathrm{km} / \mathrm{mol})$ for the $\left(\mathbf{C}_{4} \mathbf{H}_{6}\right)_{3} \mathbf{C r}$ structure $\mathrm{Cr}-2 \mathrm{~T}$.

\begin{tabular}{|c|c|c|c|c|c|c|c|}
\hline \multicolumn{2}{|c|}{ B3LYP } & \multicolumn{2}{|c|}{ B3LYP* } & \multicolumn{2}{|c|}{ BP86 } & \multicolumn{2}{|c|}{ M06L } \\
\hline 95( & 1) & 980 & 0) & 966 & 0) & 99 & 1) \\
\hline 105( & 1) & 105( & 1) & 104( & 1) & 108( & 0) \\
\hline 146( & 4) & 148( & 4) & 148( & 1) & 153( & 2) \\
\hline 173( & 2) & 174( & 2) & 167( & 1) & 171( & 2) \\
\hline 193( & 1) & 195( & 0) & 197( & 1) & 197( & 0) \\
\hline 207( & 8) & 210( & 1) & 201( & 1) & 209( & 1) \\
\hline 209 ( & 5) & 213( & 11) & 222( & 5) & 213( & 14) \\
\hline 251( & 5) & 2521 & 6) & 2476 & 4) & 247( & 6) \\
\hline 255( & 2) & 255( & 2) & 2566 & 5) & 262( & 3) \\
\hline 263( & 5) & 265( & 5) & 276( & 5) & 294( & 5) \\
\hline 307( & 0) & 312( & 0) & 305( & 0) & 317( & 0) \\
\hline 330( & 2) & 334( & 1) & 3360 & 1) & 341( & 1) \\
\hline 346( & 2) & 346( & 2) & 354( & 3) & 361( & 2) \\
\hline 379 & 4) & 381( & 4) & 384( & 3) & 399( & 3) \\
\hline 407( & 6) & 409 & 7) & 413( & 8) & 418( & 8) \\
\hline 455( & 1) & 4531 & 1) & 441( & 1) & 455( & 0) \\
\hline 481( & 4) & 480( & 4) & 474( & 2) & 493( & 2) \\
\hline 528( & 5) & 530( & 4) & 512( & 3) & 530( & 5) \\
\hline 543( & 8) & 5400 & 8) & 5321 & 9) & 550( & 8) \\
\hline 554( & 3) & 551( & 3) & 538( & 2) & 559( & 2) \\
\hline 600( & 1) & 596( & 1) & 583( & 0) & 610( & 1) \\
\hline 658( & 4) & 654( & 4) & 636( & 2) & 665( & 3) \\
\hline 690( & 10) & 684( & 10) & 670( & 7) & 683( & 9) \\
\hline 717( & 2) & 716( & 2) & 698( & 1) & 717( & 2) \\
\hline 760( & 22) & 757( & 22) & 737( & 21) & 757( & 14) \\
\hline 780( & 4) & 775( & 3) & 755( & 5) & 771( & 6) \\
\hline 804( & 9) & 799) & 9) & 777 ( & 3) & 792( & 3) \\
\hline 819 ( & 9) & 815( & 9) & 7931 & 10) & 815( & 12) \\
\hline 838( & 31) & 832( & 29) & 817( & 29) & 824( & 10) \\
\hline 845( & 11) & 841( & 9) & 8221 & 2) & 844( & 12) \\
\hline 861( & 4) & 859 & 5) & 838( & 2) & 8666 & 2) \\
\hline 886( & 9) & 883( & 2) & 8531 & 3) & 875( & 9) \\
\hline 889 & 2) & 884( & 10) & 864( & 8) & 887( & 8) \\
\hline 907( & 2) & 900( & 2) & 8731 & 2) & 901( & 3) \\
\hline 931( & 5) & 923( & 4) & 8999 & 3) & 927( & 6) \\
\hline 941( & 2) & 935( & 2) & 909) & 1) & 942( & 1) \\
\hline 974( & 2) & 965( & 2) & 932( & 1) & 958( & 1) \\
\hline 983( & 1) & 976( & 2) & 938( & 2) & 976( & 4) \\
\hline 993( & 2) & 984( & 2) & 951( & 3) & 987( & 1) \\
\hline 1007( & 4) & 999( & 3) & 972( & 2) & 1010 & 1) \\
\hline 1014( & 4) & 1007( & 4) & 979 & 4) & 1016( & 5) \\
\hline 1029( & 6) & 1023( & 6) & 996( & 6) & 1035( & 11) \\
\hline 1045( & 2) & 1038( & 2) & 1008( & 1) & 1042( & 2) \\
\hline 1094( & 8) & $1087 x$ & 8) & 1058( & 7) & 1095( & 8) \\
\hline 1132( & 11) & 1125( & 10) & 1094( & 9) & 1130 & 11) \\
\hline 1151( & 4) & 1142( & 4) & 1112( & 3) & 1151( & 2) \\
\hline
\end{tabular}




\begin{tabular}{|c|c|c|c|c|c|c|c|}
\hline 1184( & 1) & 1175( & 1) & 1140( & 1) & 1182( & 2) \\
\hline 1205( & 3) & 1195( & 4) & 1161( & 5) & 1209 & 5) \\
\hline 1215( & 4) & 1205( & 4) & 1172( & 4) & 1212( & 6) \\
\hline 1232( & 2) & 1222( & 2) & 1185( & 1) & 1237( & 1) \\
\hline 1243( & 3) & 1233( & 2) & 1198( & 2) & 1249 ( & 3) \\
\hline 1281( & 10) & 1271( & 9) & 12331 & 6) & 1273( & 2) \\
\hline 1290( & 4) & 1280( & 4) & 1243( & 2) & 1281( & 19) \\
\hline 1302( & 10) & 1291( & 7) & 1250( & 4) & 1293( & 3) \\
\hline 1306( & 6) & 1295( & 8) & 1258( & 2) & 1299 & 0) \\
\hline 1317( & 4) & 1304( & 4) & 1260( & 12) & 1310( & 5) \\
\hline 1341( & 7) & 1328( & 7) & 1286( & 5) & 1340( & 6) \\
\hline 1387( & 1) & 1374( & 1) & 1333( & 2) & 1378( & 3) \\
\hline 1416( & 0) & 1405( & 0) & 1361( & 2) & 1404( & 1) \\
\hline 1422( & 5) & 1408( & 4) & 1364( & 3) & 1417 ( & 5) \\
\hline 1476( & 1) & 1463( & 1) & 1420 & 3) & 1453( & 3) \\
\hline 1477( & 3) & 1465( & 3) & 1421( & 7) & 1462( & 3) \\
\hline 1483( & 0) & 1470( & 0) & 14300 & 1) & 1467( & 12) \\
\hline 1488( & 9) & 1476( & 9) & 1433( & 6) & 1470( & 7) \\
\hline 1496( & 13) & 1483( & 14) & 1440( & 13) & 1477( & 4) \\
\hline 1504( & 7) & 1493( & 7) & 1451( & 6) & 1490( & 4) \\
\hline 1514( & 20) & 1503( & 19) & 1460( & 14) & 1502( & 14) \\
\hline 1567( & 14) & 1554( & 5) & 1505( & 8) & 1567( & 2) \\
\hline 1575( & 28) & 1559( & 32) & 1516( & 16) & 1573( & 31) \\
\hline 3014( & 36) & 2994( & 37) & 2939 & 46) & 3021( & 69) \\
\hline 3029 & 97) & 30099 & 93) & 2956( & 31) & 3039 & 67) \\
\hline 3036( & 18) & 3016 & 18) & 2958( & 88) & 3041( & 135) \\
\hline 3046( & 70) & 3025( & 69) & 2966( & 76) & 3050( & 106) \\
\hline 3070( & 15) & 3052( & 14) & 2999( & 8) & 3091( & 17) \\
\hline 3100( & 3) & 3080( & 3) & 3021( & 2) & 3095( & 20) \\
\hline 3108( & 1) & 3087( & 0) & 3024( & 3) & 3104( & 14) \\
\hline 3116( & 3) & 3096 & 11) & 3032( & 8) & 3116( & 8) \\
\hline 3117( & 13) & 3098( & 3) & 3035( & 36) & 3120( & 15) \\
\hline 3120( & 5) & 3100( & 5) & 3041( & 17) & 3125( & 20) \\
\hline 3125( & 4) & 3104( & 9) & 3042( & 0) & 3126( & 17) \\
\hline 3126( & 52) & 3104( & 47) & 3043( & 24) & 3130( & 21) \\
\hline 3142( & 7) & 3122( & 6) & 3062( & 7) & 3132( & 87) \\
\hline 3152( & 12) & 3128( & 16) & 3065( & 25) & 3162( & 21) \\
\hline 3170( & 27) & 3149 & 25) & 3092( & 25) & 3176( & 31) \\
\hline 3179 ( & 5) & 3159( & 6) & 3097( & 7) & 3176( & 42) \\
\hline 3198( & 23) & 3180( & 21) & 3115( & 23) & 3195( & 35) \\
\hline 3228( & 9) & 32080 & 8) & 31480 & 9) & 3222( & 18) \\
\hline
\end{tabular}


Table S64. Harmonic vibrational frequencies (in $\mathrm{cm}^{-1}$ ) and infrared intensities (in parentheses in $\mathrm{km} / \mathrm{mol})$ for the $\left(\mathbf{C}_{4} \mathbf{H}_{6}\right)_{3} \mathbf{C r}$ structure $\mathrm{Cr}-3 \mathrm{~T}$.

\begin{tabular}{|c|c|c|c|c|c|c|c|}
\hline \multicolumn{2}{|c|}{ B3LYP } & \multicolumn{2}{|c|}{ B3LYP* } & \multicolumn{2}{|c|}{ BP86 } & \multicolumn{2}{|c|}{ M06L } \\
\hline 59( & 0) & 63( & 0) & 700 & 0) & 82( & 0) \\
\hline 67( & 0) & 69 & 0) & 76( & 0) & 90( & 0) \\
\hline 86( & 0) & 91( & 0) & 95( & 0) & 99( & 0) \\
\hline 107( & 3) & 108( & 3) & 113( & 2) & 117( & 2) \\
\hline 154( & 1) & 150( & 2) & 154( & 1) & 154( & 0) \\
\hline 161( & 10) & 168( & 8) & 178( & 6) & 186( & 9) \\
\hline 185( & 1) & 189 & 1) & 190( & 3) & 199( & 1) \\
\hline 207( & 3) & 209 & 4) & 219 & 3) & 220( & 3) \\
\hline 237( & 8) & 243( & 6) & 247( & 0) & 272( & 0) \\
\hline 248( & 0) & 249 & 0) & 251( & 0) & 275( & 6) \\
\hline 253( & 3) & 260( & 3) & 269 & 2) & 281( & 1) \\
\hline 269 & 1) & 275( & 1) & 2860 & 2) & 299 & 0) \\
\hline 278( & 8) & 279 & 9) & 290( & 10) & 302( & 8) \\
\hline 297( & 4) & 301( & 4) & 313( & 2) & 309( & 7) \\
\hline 3300 & 7) & 334( & 9) & 344( & 10) & 354( & 12) \\
\hline 347( & 5) & 350 & 4) & 3571 & 2) & 366( & 3) \\
\hline 380( & 3) & 383( & 4) & 388( & 8) & 397( & 2) \\
\hline 411( & 4) & 417( & 3) & 424( & 2) & 434( & 3) \\
\hline $430 c$ & 22) & 435( & 26) & $446 c$ & 32) & 459 & 21) \\
\hline 468( & 1) & 467( & 0) & 460( & 3) & 477( & 2) \\
\hline 483( & 8) & 479 & 7) & 476( & 5) & 498( & 6) \\
\hline 561( & 3) & 560( & 3) & 542( & 2) & 561( & 2) \\
\hline 611( & 7) & 607( & 5) & 5931 & 5) & 616( & 8) \\
\hline 613( & 1) & 611( & 1) & 602( & 3) & 624( & 3) \\
\hline 644( & 20) & 644( & 22) & 632( & 23) & 641( & 17) \\
\hline 686( & 10) & 6861 & 9) & 6631 & 9) & 6861 & 10) \\
\hline 695( & 5) & 690 & 5) & 673( & 3) & 699( & 5) \\
\hline 727( & 10) & 718( & 9) & 707( & 11) & 718( & 12) \\
\hline 734( & 14) & 729 & 16) & 715( & 11) & 723( & 8) \\
\hline 745( & 1) & 742( & 0) & 726( & 0) & 736( & 1) \\
\hline 817( & 18) & 811( & 17) & 787( & 18) & 7999 & 12) \\
\hline 825( & 18) & 820( & 18) & 797( & 13) & 8066 & 10) \\
\hline 856( & 6) & 852( & 6) & 822( & 7) & 838( & 5) \\
\hline 8621 & 8) & 859 & 9) & 8361 & 15) & 848( & 3) \\
\hline 882( & 2) & 876( & 2) & 852( & 38) & 8666 & 2) \\
\hline 893( & 3) & 8831 & 0) & 854( & 10) & 873( & 39) \\
\hline 895( & 0) & 887( & 3) & 8631 & 2) & 8799 & 2) \\
\hline 911( & 46) & 900( & 50) & 867( & 1) & 885( & 2) \\
\hline 915( & 5) & 910( & 1) & 882( & 3) & 890( & 3) \\
\hline 937( & 1) & 929 & 1) & 9066 & 0) & 931( & 3) \\
\hline 946( & 1) & 938( & 1) & 911( & 2) & 945( & 1) \\
\hline 951( & 3) & 947( & 3) & 913( & 3) & 947( & 4) \\
\hline 958( & 2) & 951( & 1) & 918( & 1) & 950( & 2) \\
\hline 964( & 1) & 956( & 2) & 922( & 1) & 955( & 2) \\
\hline 1016( & 11) & 1006( & 11) & 974( & 12) & 1015( & 9) \\
\hline 1065( & 3) & 1058 & 3) & 10288 & 4) & 1058( & 2) \\
\hline
\end{tabular}




\begin{tabular}{|c|c|c|c|c|c|c|c|}
\hline 1066( & 5) & 1059 & 4) & 1033( & 3) & 1069( & 5) \\
\hline 1068( & 0) & 1062( & 1) & 1035( & 1) & 1070( & 0) \\
\hline 1081( & 1) & 1072( & 1) & 1042( & 1) & 1075( & 1) \\
\hline 1084( & 9) & 1075( & 9) & 1046( & 7) & 1077( & 9) \\
\hline 1106( & 5) & 1096( & 5) & 10699 & 5) & 1102( & 4) \\
\hline 1204( & 4) & 11960 & 3) & 1163( & 4) & 12000 & 5) \\
\hline 1211( & 19) & 1202( & 18) & 1172( & 15) & 1210( & 17) \\
\hline 1251( & 43) & 1243( & 38) & 1212( & 26) & 1248( & 37) \\
\hline 1259 ( & 33) & 1251( & 29) & 1214( & 24) & 12560 & 30) \\
\hline 1268( & 3) & 1259 & 3) & 1226( & 1) & 1265( & 1) \\
\hline 1330( & 5) & 1319 & 5) & 1284( & 4) & 1325( & 6) \\
\hline 1404( & 0) & 1392( & 0) & 1350( & 0) & 1388( & 1) \\
\hline 1406( & 0) & 1395( & 0) & 1354( & 0) & 1392( & 1) \\
\hline 1416( & 6) & 1404( & 6) & 1361( & 5) & 1401( & 3) \\
\hline 1455( & 9) & 1443( & 9) & 1405( & 7) & 1448( & 7) \\
\hline 1475( & 4) & 1464( & 3) & 1425( & 1) & 1469 & 1) \\
\hline 1481( & 13) & 1470( & 11) & 1431( & 8) & 1473( & 7) \\
\hline 1518( & 13) & 1507( & 5) & 1466( & 4) & 1509 & 3) \\
\hline 1523( & 17) & 1509 & 18) & 1471( & 5) & 1513( & 9) \\
\hline 1533( & 52) & 1522( & 52) & 1481( & 42) & 1526( & 57) \\
\hline 1540( & 4) & 1529 & 2) & 1489x & 1) & 1534( & 3) \\
\hline 1565( & 36) & 1549 & 33) & 1500( & 26) & 1562( & 38) \\
\hline 1672( & 33) & 1659 & 34) & 1615( & 36) & 1679 & 39) \\
\hline 3138( & 10) & 3119 & 10) & 3058( & 14) & 3122( & 34) \\
\hline 3142( & 10) & 3122( & 8) & 3063( & 9) & 3129 & 39) \\
\hline 3146( & 5) & 3124( & 6) & 3064( & 11) & 31300 & 13) \\
\hline 3153( & 4) & 3132( & 7) & 3072( & 7) & 3140( & 12) \\
\hline 3155( & 3) & 31360 & 3) & 3077( & 9) & 3149 & 23) \\
\hline 3159 & 12) & 3137( & 10) & 3078( & 13) & 3152( & 9) \\
\hline 3162( & 7) & 3140 & 8) & 30799 & 9) & 3164( & 13) \\
\hline 3166r & 0) & 3145( & 0) & 3088( & 8) & 3167( & 2) \\
\hline 3173( & 1) & 3151( & 1) & 3088( & 0) & 3172( & 5) \\
\hline 3176( & 9) & 3154( & 9) & 3094( & 3) & 3180 & 21) \\
\hline 3183( & 2) & 3162( & 0) & 3104( & 2) & 3184( & 40) \\
\hline 3189 & 24) & 3167( & 25) & 3108( & 32) & 3188( & 32) \\
\hline 3228( & 4) & 3208( & 4) & 3149 & 4) & 3221( & 15) \\
\hline 3229 & 8) & 3209 & 7) & 3151( & 6) & 3225( & 17) \\
\hline 3234( & 9) & 3212( & 9) & 3153( & 11) & 3227( & 18) \\
\hline 3244( & 5) & 3225( & 4) & 3166( & 6) & 3236( & 12) \\
\hline 3247( & 13) & 3226( & 13) & 3168( & 6) & 3249 & 23) \\
\hline 3253 ( & 4) & 32300 & 5) & 31710 & 14) & 32510 & 12) \\
\hline
\end{tabular}


Table S65. Harmonic vibrational frequencies (in $\mathrm{cm}^{-1}$ ) and infrared intensities (in parentheses in $\mathrm{km} / \mathrm{mol})$ for the $\left(\mathbf{C}_{4} \mathbf{H}_{6}\right)_{3} \mathbf{C r}$ structure $\mathrm{Cr}-1 \mathrm{P}$.

\begin{tabular}{|c|c|c|c|c|c|c|c|}
\hline \multicolumn{2}{|c|}{ B3LYP } & \multicolumn{2}{|c|}{ B3LYP* } & \multicolumn{2}{|c|}{ BP86 } & \multicolumn{2}{|c|}{ M06L } \\
\hline 58( & 2) & 60( & 1) & 58( & 0) & 66( & 2) \\
\hline 72( & 1) & 81( & 1) & 101( & 0) & 80( & 0) \\
\hline 106( & 1) & 106( & 1) & 109( & 0) & 117( & 1) \\
\hline 125( & 0) & 127( & 0) & 136( & 0) & 139( & 0) \\
\hline 135( & 1) & 138( & 1) & 160( & 1) & 153( & 1) \\
\hline 162( & 0) & 163( & 0) & 181( & 1) & 182( & 0) \\
\hline 197( & 3) & 196( & 2) & 197( & 1) & 213( & 1) \\
\hline 217( & 4) & 216( & 4) & 221( & 2) & 238( & 3) \\
\hline 240( & 4) & 239 & 3) & 239 ( & 2) & 264( & 1) \\
\hline 249( & 1) & 249( & 1) & 252( & 1) & 273( & 5) \\
\hline 255( & 2) & 254( & 2) & 254( & 2) & 285( & 3) \\
\hline 270( & 2) & 269 & 2) & 270( & 1) & 294( & 1) \\
\hline 311( & 4) & 310( & 4) & 310( & 6) & 328( & 4) \\
\hline 333( & 26) & 331( & 23) & 329 ( & 14) & 360( & 19) \\
\hline 399 & 6) & 394( & 5) & 385( & 3) & 411( & 4) \\
\hline 415( & 1) & 414( & 2) & 417( & 2) & 427( & 1) \\
\hline 439 & 11) & 438( & 10) & 438( & 4) & 452( & 9) \\
\hline 458( & 2) & 454( & 2) & 445( & 3) & 464( & 1) \\
\hline 492( & 2) & 490( & 2) & 482( & 2) & 498( & 2) \\
\hline 567( & 2) & 562( & 3) & 549( & 3) & 569( & 4) \\
\hline 601( & 0) & 596( & 0) & 580( & 0) & 610( & 0) \\
\hline 650( & 2) & 647( & 1) & 638( & 1) & 664( & 2) \\
\hline 703( & 10) & 6931 & 11) & 671( & 5) & 702( & 11) \\
\hline 709 & 11) & 703( & 8) & 682( & 7) & 705( & 5) \\
\hline 726( & 26) & 722( & 26) & 711( & 25) & 724( & 18) \\
\hline 742( & 7) & 737( & 4) & 728( & 7) & 730( & 14) \\
\hline 773( & 61) & 767( & 57) & 755( & 32) & 771( & 54) \\
\hline 787( & 10) & 7799 & 6) & 765( & 16) & 784( & 39) \\
\hline 793( & 77) & 784( & 71) & 7666 & 37) & 7899 & 3) \\
\hline 813( & 8) & 804( & 8) & 785( & 12) & 8099 & 3) \\
\hline 827( & 13) & 820( & 12) & 7999 & 4) & 8331 & 11) \\
\hline 846( & 1) & 842( & 1) & 828( & 4) & 857( & 3) \\
\hline 906( & 4) & 896( & 4) & 870( & 4) & 911( & 4) \\
\hline 946( & 5) & 939) & 4) & 907( & 1) & 949( & 4) \\
\hline 956( & 5) & 946( & 3) & 914( & 1) & 953( & 2) \\
\hline 986( & 9) & 969) & 7) & 919) & 6) & 962( & 4) \\
\hline 997( & 8) & 980( & 4) & 933( & 5) & 972( & 4) \\
\hline 1000( & 3) & 988( & 8) & 942( & 0) & 994( & 1) \\
\hline 1008( & 7) & 999( & 6) & 962( & 7) & 1002( & 5) \\
\hline 1020( & 0) & 1003( & 1) & 973( & 3) & 1016( & 5) \\
\hline 1033( & 8) & 1025( & 8) & 996( & 5) & 1040 & 9) \\
\hline 1040( & 6) & 1031( & 7) & 1004( & 10) & 1047( & 10) \\
\hline 1054( & 6) & 1045( & 6) & 1017( & 5) & 1057( & 4) \\
\hline 1108( & 1) & 1098( & 1) & 1068( & 1) & 1112( & 1) \\
\hline 1155( & 3) & 1144( & 3) & 1112( & 3) & 1155( & 4) \\
\hline 1168( & 4) & 1157( & 4) & 1125( & 5) & 1168( & 3) \\
\hline
\end{tabular}




\begin{tabular}{|c|c|c|c|c|c|c|c|}
\hline 1190( & 3) & 1179 & 2) & 1147( & 1) & 1195( & 3) \\
\hline 1214( & 3) & 1202( & 2) & 1165( & 1) & 1219 ( & 1) \\
\hline 1229 & 2) & 1215( & 2) & 1176( & 2) & 1232( & 1) \\
\hline 1237( & 2) & 1225( & 2) & 1190( & 1) & 1240( & 4) \\
\hline 1261( & 14) & 1249 & 12) & 1211( & 7) & 1252( & 5) \\
\hline 1268( & 26) & 1257( & 24) & 1223( & 16) & 1256( & 22) \\
\hline 1308( & 1) & 1296( & 2) & 1262( & 4) & 1303( & 3) \\
\hline 1326( & 1) & 1312( & 1) & 1269 & 1) & 1323( & 1) \\
\hline 1340( & 8) & 1324( & 7) & 12790 & 4) & 1337( & 6) \\
\hline 1342( & 0) & 1328( & 0) & 1283( & 1) & 1348( & 5) \\
\hline 1359 ( & 11) & 1345( & 9) & 1302( & 6) & 1367( & 7) \\
\hline 1400( & 1) & 1385( & 1) & 1345( & 1) & 1393( & 0) \\
\hline 1402( & 1) & 1388( & 1) & 1350( & 2) & 1394( & 1) \\
\hline 1434( & 2) & 1419 & 2) & 1376( & 2) & 1432( & 2) \\
\hline 1467( & 13) & 1454( & 12) & 1413( & 9) & 1455( & 8) \\
\hline 1475( & 1) & 1461( & 2) & 1420( & 4) & 1463( & 2) \\
\hline 1479( & 4) & 1464( & 5) & 1423( & 4) & 1469( & 7) \\
\hline 1481( & 5) & 1467( & 4) & 1425( & 2) & 1470( & 3) \\
\hline 1484( & 6) & 1469 & 6) & 1431( & 6) & 1474( & 6) \\
\hline 1496( & 13) & 1482( & 12) & 1445( & 10) & 1493( & 10) \\
\hline 1551( & 48) & 1539 & 49) & 1499( & 48) & 1546( & 32) \\
\hline 1556( & 88) & 1544( & 76) & 1505( & 42) & 1551( & 73) \\
\hline 1698( & 2) & 1678( & 1) & 1601( & 3) & 1714( & 1) \\
\hline 3014( & 27) & 2994( & 20) & 2933( & 31) & 3018 ( & 74) \\
\hline 3015( & 23) & 2994( & 27) & 2935( & 30) & 3022( & 46) \\
\hline 3026( & 84) & 3005( & 82) & 2949( & 99) & 3033( & 56) \\
\hline 3027( & 48) & 3007( & 54) & 2951( & 58) & 3035( & 120) \\
\hline 3070( & 15) & 3052( & 14) & 2999( & 24) & 3092( & 51) \\
\hline 3075( & 29) & 3055( & 28) & 3002( & 12) & 3096( & 35) \\
\hline 3094( & 13) & 3076( & 11) & 3023( & 12) & 30999 & 24) \\
\hline 3116( & 13) & 3094( & 13) & 3038( & 14) & 3126( & 11) \\
\hline 3117( & 5) & 3097( & 5) & 3047( & 3) & 3127( & 11) \\
\hline 3121( & 8) & 3101( & 8) & 3048( & 9) & 3128( & 26) \\
\hline 3124( & 10) & 3103( & 9) & 3049 & 11) & 3132( & 12) \\
\hline 3145( & 1) & 3124( & 2) & 3061( & 8) & 3137( & 10) \\
\hline 3146( & 4) & 3125( & 4) & 3063( & 5) & 3143( & 31) \\
\hline 3149 & 34) & 3128( & 31) & 3068( & 25) & 3161( & 49) \\
\hline 3166r & 17) & 3146( & 15) & 3090( & 17) & 3167( & 33) \\
\hline 3167( & 13) & 3147( & 13) & 3092( & 16) & 3172( & 36) \\
\hline 3231( & 12) & 3209 & 11) & 3149 & 13) & 3230( & 18) \\
\hline 3235( & 13) & 3214( & 12) & 31550 & 12) & 3231( & 26) \\
\hline
\end{tabular}


Table S66. Harmonic vibrational frequencies (in $\mathrm{cm}^{-1}$ ) and infrared intensities (in parentheses in $\mathrm{km} / \mathrm{mol})$ for the $\left(\mathbf{C}_{4} \mathbf{H}_{6}\right)_{3} \mathbf{C r}$ structure $\mathrm{Cr}-2 \mathrm{P}$.

\begin{tabular}{|c|c|c|c|c|c|c|c|}
\hline \multicolumn{2}{|c|}{ B3LYP } & \multicolumn{2}{|c|}{ B3LYP* } & \multicolumn{2}{|c|}{ BP86 } & \multicolumn{2}{|c|}{ M06L } \\
\hline 54( & 0) & 55( & 0) & 55( & 0) & 66( & 0) \\
\hline 60( & 0) & 65( & 0) & 57( & 0) & 80( & 0) \\
\hline 77( & 0) & 78( & 0) & 63( & 0) & 96( & 0) \\
\hline 96( & 0) & 93( & 0) & 95( & 0) & 126( & 0) \\
\hline 112( & 7) & 116( & 6) & 109( & 5) & 127( & 5) \\
\hline 149( & 9) & 157( & 8) & 157( & 4) & 169( & 4) \\
\hline 165( & 1) & 170( & 1) & 169( & 0) & 196( & 0) \\
\hline 204( & 0) & 207( & 0) & 212( & 0) & 226( & 0) \\
\hline 225( & 7) & 225( & 8) & 223( & 5) & 234( & 5) \\
\hline 266( & 4) & 262( & 3) & 2566 & 4) & 260( & 0) \\
\hline 272( & 0) & 2731 & 0) & 257 ( & 0) & 284( & 7) \\
\hline 300( & 3) & 299 & 3) & 305( & 2) & 321( & 2) \\
\hline 316( & 0) & 315( & 0) & 307( & 1) & 340( & 2) \\
\hline 343( & 21) & 342( & 19) & 340( & 14) & 361( & 16) \\
\hline 408( & 15) & 406( & 14) & 403( & 11) & 422( & 13) \\
\hline 412( & 1) & 412( & 1) & 410( & 1) & 426( & 1) \\
\hline 439( & 4) & 438( & 3) & 439) & 1) & 451( & 3) \\
\hline 476( & 17) & 474( & 16) & 461( & 14) & 490( & 13) \\
\hline 491( & 2) & 484( & 2) & 472( & 2) & 504( & 1) \\
\hline 566( & 1) & 560( & 1) & 545( & 1) & 579( & 1) \\
\hline 570( & 1) & 565( & 1) & 550( & 1) & 581( & 1) \\
\hline 613( & 1) & 609 & 1) & 595( & 1) & 625( & 1) \\
\hline 699( & 34) & 692( & 37) & 674( & 38) & 699( & 28) \\
\hline 711( & 5) & 706( & 5) & 691( & 4) & 721( & 7) \\
\hline 717( & 17) & 710( & 13) & 693( & 12) & 723( & 5) \\
\hline 741( & 14) & 739 & 13) & 724( & 7) & 733( & 10) \\
\hline 783( & 60) & 779 & 54) & 752( & 32) & 765( & 50) \\
\hline 804( & 82) & 797( & 75) & 772( & 56) & 789 ( & 43) \\
\hline 813( & 1) & 808( & 2) & 7899 & 3) & 807( & 12) \\
\hline 814( & 18) & 810( & 17) & 791( & 18) & 827( & 9) \\
\hline 821( & 1) & 816( & 1) & 796( & 3) & 831( & 4) \\
\hline 843( & 12) & 838( & 12) & 821( & 10) & 8561 & 9) \\
\hline 875( & 2) & 869 & 2) & 848( & 2) & 876( & 1) \\
\hline 969 & 12) & 960( & 11) & 926( & 7) & 962( & 3) \\
\hline 979 & 4) & 972( & 3) & 928( & 5) & 964( & 3) \\
\hline 983( & 2) & 974( & 2) & 935( & 5) & 978( & 6) \\
\hline 988( & 8) & 975( & 8) & 946( & 2) & 983( & 3) \\
\hline 1004( & 0) & 992( & 0) & 956( & 0) & 990( & 0) \\
\hline 1005( & 3) & 995( & 3) & 965( & 3) & 996( & 5) \\
\hline 1017( & 9) & 1008( & 9) & 982( & 10) & 1016( & 12) \\
\hline 1039 & 2) & 1030 & 2) & 1004( & 2) & 1045( & 2) \\
\hline 1054( & 5) & 1047( & 5) & 1023( & 7) & 1062( & 9) \\
\hline 1059 & 6) & 1052( & 6) & 1028( & 5) & 1065( & 4) \\
\hline 1099( & 2) & 1092( & 2) & 1065( & 1) & 1106( & 3) \\
\hline 1151( & 8) & 1143( & 7) & 1115( & 5) & 1149( & 3) \\
\hline 1170( & 0) & 1160 & 0) & 1133( & 1) & 1170( & 1) \\
\hline
\end{tabular}




\begin{tabular}{|c|c|c|c|c|c|c|c|}
\hline 1190( & 1) & 1180( & 1) & 1149 & 0) & 1197( & 0) \\
\hline 1215( & 0) & 1205( & 0) & 1173( & 0) & 1215( & 0) \\
\hline 1240( & 10) & 1229 & 9) & 1193( & 5) & 1238( & 15) \\
\hline 1245( & 0) & 1234( & 0) & 1198( & 1) & 1239 & 0) \\
\hline 1257( & 1) & 1247( & 1) & 1215( & 1) & 1247( & 14) \\
\hline 1258( & 36) & 1248( & 31) & 1216( & 22) & 1248( & 0) \\
\hline 1284( & 7) & 1272( & 8) & 1241( & 8) & 1288( & 8) \\
\hline 1325( & 1) & 1313( & 1) & 1273( & 1) & 1325( & 2) \\
\hline 1342( & 2) & 1329 & 2) & 1289 & 3) & 1343( & 5) \\
\hline 1350( & 17) & 1337( & 19) & 1296( & 12) & 1345( & 19) \\
\hline 1350( & 4) & 1340( & 1) & 1300( & 5) & 1363( & 1) \\
\hline 1400( & 0) & 1387( & 0) & 1346( & 0) & 1388( & 0) \\
\hline 1403( & 1) & 1390( & 2) & 1349 & 2) & 1392( & 1) \\
\hline 1430( & 0) & 1417( & 0) & 1379 & 1) & 1432( & 0) \\
\hline 1473( & 5) & 1459 & 4) & 1416( & 3) & 1455( & 3) \\
\hline 1475( & 4) & 1462( & 3) & 1420( & 3) & 1457( & 10) \\
\hline 1481( & 2) & 1466( & 0) & 1426( & 0) & 1470 & 4) \\
\hline 1483( & 2) & 1470( & 0) & 1428( & 3) & 1471( & 2) \\
\hline 1484( & 4) & 1471( & 7) & 1429 & 7) & 1480 & 0) \\
\hline 1492( & 21) & 1476( & 24) & 1434( & 24) & 1487( & 20) \\
\hline 1550( & 0) & 1538( & 0) & 1499 & 0) & 1550( & 1) \\
\hline 1553( & 143) & 1540( & 132) & 1500( & 96) & 1551( & 108) \\
\hline 1719( & 1) & 1706( & 1) & 1662( & 1) & 1749 & 2) \\
\hline 3014( & 0) & 2994( & 0) & 2937( & 1) & 3012( & 2) \\
\hline 3017( & 35) & 2997( & 36) & 2940( & 41) & 3024( & 144) \\
\hline 3027( & 85) & 3007( & 82) & 2950( & 86) & 3024( & 4) \\
\hline 3029) & 26) & 3009 & 22) & 2951( & 16) & 3035( & 56) \\
\hline 3057( & 46) & 3037( & 46) & 2980( & 48) & 3070( & 67) \\
\hline 3066( & 10) & 3047( & 9) & 2986( & 19) & 3078( & 47) \\
\hline 3074( & 1) & 3055( & 1) & 2999( & 1) & 3087( & 10) \\
\hline 3082( & 70) & 3062( & 68) & 3002( & 62) & 3090( & 101) \\
\hline 3111( & 1) & 3090( & 1) & 3034( & 1) & 3109 & 26) \\
\hline 3111( & 10) & 3091( & 10) & 3034( & 9) & 3109 & 1) \\
\hline 3113( & 16) & 3094( & 15) & 3038( & 17) & 3110( & 3) \\
\hline 3113( & 0) & 3094( & 0) & 3038( & 0) & 3111( & 31) \\
\hline 3122( & 8) & 3100( & 8) & 3046( & 8) & 3139 ( & 16) \\
\hline 3149( & 40) & 3127( & 39) & 3072( & 42) & 3165( & 63) \\
\hline 3169 & 26) & 3148( & 0) & 3091( & 0) & 3170( & 1) \\
\hline 3169 ( & 0) & 3148( & 24) & 3091( & 26) & 3171( & 57) \\
\hline 3223( & 0) & 3204( & 0) & 3152( & 0) & 3233 ( & 38) \\
\hline 3223( & 18) & 3204 ( & 16) & 31531 & 16) & 3234 ( & $0)$ \\
\hline
\end{tabular}


Table S67. Harmonic vibrational frequencies (in $\mathrm{cm}^{-1}$ ) and infrared intensities (in parentheses in $\mathrm{km} / \mathrm{mol})$ for the $\left(\mathbf{C}_{4} \mathbf{H}_{6}\right)_{3} \mathbf{M n}$ structure Mn-1D.

\begin{tabular}{|c|c|c|c|c|c|c|c|}
\hline \multicolumn{2}{|c|}{ B3LYP } & \multicolumn{2}{|c|}{ B3LYP* } & \multicolumn{2}{|c|}{ BP86 } & \multicolumn{2}{|c|}{ M06L } \\
\hline 74( & 0) & 76( & 0) & 82( & 0) & 78( & 0) \\
\hline 97( & 0) & 95( & 0) & 99( & 0) & 102( & 0) \\
\hline 114( & 2) & 116( & 2) & 118( & 2) & 122( & 3) \\
\hline 140( & 2) & 138( & 2) & 140( & 1) & 145( & 1) \\
\hline 168( & 0) & 170( & 0) & 166( & 1) & 178( & 1) \\
\hline 202( & 4) & 203( & 3) & 207( & 2) & 207( & 2) \\
\hline 210( & 6) & 216( & 6) & 220( & 8) & 223( & 8) \\
\hline 235( & 2) & 236( & 3) & 236( & 2) & 237( & 1) \\
\hline 255( & 3) & 249 & 3) & 246( & 2) & 262( & 3) \\
\hline 267( & 2) & 269 & 1) & 276( & 2) & 274( & 2) \\
\hline 272( & 3) & 275( & 2) & 282( & 1) & 293( & 4) \\
\hline 284( & 2) & 292( & 1) & 309 & 1) & 319 & 6) \\
\hline 298( & 6) & 304( & 8) & 315( & 6) & 321( & 1) \\
\hline 313( & 6) & 319 & 5) & 337( & 1) & 331( & 4) \\
\hline 334( & 3) & 341( & 3) & 357( & 1) & 367( & 3) \\
\hline 344( & 11) & 349 & 13) & 364( & 12) & 369 & 15) \\
\hline 401( & 5) & 404( & 7) & 409 & 9) & 421( & 5) \\
\hline 4331 & 2) & 434( & 2) & 443( & 2) & 452( & 3) \\
\hline 458( & 27) & 462( & 20) & 460( & 4) & 478( & 3) \\
\hline 474( & 4) & 476( & 13) & 471( & 11) & 491( & 14) \\
\hline 487( & 2) & 480( & 3) & 480( & 30) & 500( & 16) \\
\hline 567( & 6) & 565( & 5) & 556( & 4) & 574( & 5) \\
\hline 622( & 3) & 621( & 3) & 609 & 2) & 6331 & 4) \\
\hline 637( & 0) & 637( & 0) & 627( & 0) & 652( & 1) \\
\hline 667( & 23) & 664( & 24) & 652( & 25) & 6661 & 23) \\
\hline 712( & 6) & 705( & 2) & 692( & 3) & 705( & 4) \\
\hline 721( & 10) & 713( & 11) & 707( & 12) & 719 & 11) \\
\hline 729 & 3) & 733( & 4) & 712( & 5) & 7300 & 9) \\
\hline 740( & 3) & 741( & 4) & 722( & 2) & 741( & 2) \\
\hline 770 & 3) & 765( & 3) & 760( & 1) & 760 & 2) \\
\hline 855( & 22) & 852( & 26) & 831( & 26) & 845( & 13) \\
\hline 862( & 1) & 862( & 4) & 841( & 1) & 853( & 0) \\
\hline 874( & 5) & 870( & 8) & 849 & 27) & 857( & 0) \\
\hline 878( & 25) & 875( & 18) & 854( & 13) & 865( & 13) \\
\hline 884( & 1) & 878( & 1) & 857( & 5) & 873( & 36) \\
\hline 890( & 0) & 887( & 1) & 862( & 14) & 874( & 4) \\
\hline 904( & 1) & 898( & 1) & 864( & 18) & 883( & 2) \\
\hline 920( & 44) & 908( & 47) & 866( & 6) & 8881 & 2) \\
\hline 928( & 7) & 922( & 5) & 889 & 3) & 900( & 1) \\
\hline 942( & 1) & 934( & 0) & 895( & 2) & 921( & 1) \\
\hline 944( & 1) & 938( & 1) & 902( & 0) & 930( & 0) \\
\hline 948( & 1) & 940( & 0) & 916( & 1) & 939 & 1) \\
\hline 952( & 0) & 946( & 0) & 922( & 0) & 949 & 2) \\
\hline 968( & 0) & 957( & 0) & 943( & 0) & 955( & 0) \\
\hline 1021( & 10) & 1009 & 10) & 978( & 11) & 1008( & 8) \\
\hline 1064( & 4) & 1057( & 4) & 1032( & 5) & 1058( & 2) \\
\hline
\end{tabular}




\begin{tabular}{|c|c|c|c|c|c|c|c|}
\hline 1068( & 5) & 1060( & 4) & 1033( & 4) & 1064( & 7) \\
\hline 1069 & 3) & 1061( & 4) & 1035( & 2) & 1067( & 4) \\
\hline 1072( & 2) & 1063( & 1) & 1038( & 2) & 1069 & 4) \\
\hline 1075( & 9) & 1066( & 9) & 1039( & 6) & 1071( & 4) \\
\hline 1106( & 2) & 1098( & 2) & 1071( & 3) & 1098( & 4) \\
\hline 1199( & 2) & 1190( & 2) & 1158( & 2) & 1194( & 3) \\
\hline 1208( & 10) & 1198( & 9) & 1165( & 7) & 1203( & 8) \\
\hline 1242( & 32) & 1233( & 28) & 1202( & 18) & 1237( & 26) \\
\hline 1255( & 16) & 1244( & 20) & 1213( & 18) & 1247( & 25) \\
\hline 1263( & 13) & 1250( & 6) & 1218( & 2) & 1254( & 2) \\
\hline 1319( & 3) & 1310( & 2) & 1276( & 2) & 1315( & 3) \\
\hline 1401( & 0) & 1389 & 0) & 1349 ( & 0) & 1385( & 0) \\
\hline 1404( & 0) & 1391( & 0) & 1353( & 0) & 13880 & 0) \\
\hline 1418( & 4) & 1404( & 3) & 1367( & 2) & 13999 & 1) \\
\hline 1453( & 6) & 1442( & 6) & 1405( & 5) & 1441( & 5) \\
\hline 1471( & 3) & 1459 & 1) & 1426( & 1) & 1464( & 0) \\
\hline 1480( & 9) & 1469( & 9) & 1435( & 8) & 1472( & 5) \\
\hline 1520( & 30) & 1507( & 26) & 1466( & 1) & 1505( & 6) \\
\hline 1523( & 1) & 1509( & 1) & 1468( & 8) & 1509 & 9) \\
\hline 1526( & 8) & 1513( & 8) & 1472( & 12) & 1510( & 13) \\
\hline 1530( & 1) & 1518( & 0) & 1483( & 0) & 1524( & 0) \\
\hline 1563( & 19) & 1546( & 17) & 1498( & 11) & 1543( & 18) \\
\hline 1675( & 33) & 1662( & 34) & 1618( & 37) & 1682( & 47) \\
\hline 3137( & 10) & 3113( & 10) & 3051( & 16) & 3120( & 34) \\
\hline 3141( & 5) & 3118( & 10) & 3057( & 12) & 3127( & 32) \\
\hline 3144( & 14) & 3124( & 9) & 3064( & 11) & 3129 & 7) \\
\hline 3151( & 6) & 3129 ( & 4) & 3068( & 13) & 3132( & 57) \\
\hline 3154( & 5) & 3132( & 6) & 3070( & 2) & 3134( & 1) \\
\hline 3156( & 7) & 3135( & 8) & 3073( & 11) & 3142( & 22) \\
\hline 3158( & 11) & 3136( & 12) & 3075( & 20) & 3149 & 15) \\
\hline 3172( & 1) & 3146( & 10) & 3080( & 6) & 3170 & 3) \\
\hline 3173( & 9) & 3151( & 0) & 3092( & 0) & 3170( & 18) \\
\hline 3179 & 3) & 3157( & 3) & 3098( & 4) & 3177( & 9) \\
\hline 3188( & 1) & 3166( & 1) & 3107( & 1) & 3184( & 33) \\
\hline 3193( & 23) & 3171( & 23) & 3111( & 30) & 3194( & 36) \\
\hline 3224( & 8) & 3201( & 8) & 3141( & 9) & 3217( & 18) \\
\hline 3231( & 8) & 3211( & 7) & 3150( & 7) & 3222( & 14) \\
\hline 3241( & 4) & 3217( & 4) & 3157( & 4) & 3228( & 14) \\
\hline 3244( & 11) & 3223( & 3) & 3159( & 5) & 32344 & 10) \\
\hline 3245( & 10) & 3224( & 18) & 3162( & 15) & 3238( & 26) \\
\hline 3251( & 8) & 32271 & 7) & 31690 & 14) & 32460 & 24) \\
\hline
\end{tabular}


Table S68. Harmonic vibrational frequencies (in $\mathrm{cm}^{-1}$ ) and infrared intensities (in parentheses in $\mathrm{km} / \mathrm{mol}$ ) for the $\left(\mathbf{C}_{4} \mathbf{H}_{6}\right)_{3} \mathbf{M n}$ structure $\mathrm{Mn}-2 \mathrm{D}$.

\begin{tabular}{|c|c|c|c|c|c|c|c|}
\hline \multicolumn{2}{|c|}{ B3LYP } & \multicolumn{2}{|c|}{ B3LYP* } & \multicolumn{2}{|c|}{ BP86 } & \multicolumn{2}{|c|}{ M06L } \\
\hline 104( & 0) & 105( & 0) & 90( & 0) & -229 & 46) \\
\hline 117( & 0) & 117( & 0) & 112( & 0) & 104( & 0) \\
\hline 173( & 1) & 181( & 4) & 168( & 1) & 138( & 2) \\
\hline 179 & 5) & 187( & 2) & 193( & 0) & 187( & 4) \\
\hline 183( & 3) & 192( & 4) & 208( & 7) & 189( & 6) \\
\hline 213 ( & 3) & 214( & 3) & 217 ( & 2) & 219 & 8) \\
\hline 226( & 1) & 225( & 1) & 220( & 2) & 228( & 0) \\
\hline 246( & 5) & 241( & 5) & 2331 & 5) & 235( & 1) \\
\hline 263( & 5) & 265( & 5) & 2666 & 4) & 274( & 3) \\
\hline 278( & 4) & 281( & 5) & 291( & 8) & 293( & 4) \\
\hline 307( & 2) & 309( & 2) & 313( & 1) & 325( & 0) \\
\hline 3350 & 1) & 342( & 2) & 349 & 2) & 357( & 2) \\
\hline 353( & 2) & 361( & 3) & 371( & 5) & 388( & 5) \\
\hline 389 & 1) & 396( & 2) & 403( & 2) & 419( & 2) \\
\hline 415( & 6) & 420( & 7) & 425( & 8) & 433( & 5) \\
\hline 447( & 1) & 444( & 1) & 436( & 4) & 449 & 3) \\
\hline 492( & 3) & 490( & 3) & 479) & 2) & 500( & 1) \\
\hline 534( & 9) & 531( & 7) & 519x & 2) & 532( & 9) \\
\hline 542( & 3) & 540( & 3) & 526( & 5) & 543( & 3) \\
\hline 567( & 2) & 573( & 3) & 560( & 3) & 571( & 1) \\
\hline 605( & 0) & 601( & 0) & 583( & 1) & 601( & 3) \\
\hline 670( & 3) & 665( & 2) & 646( & 1) & 663( & 4) \\
\hline 732( & 2) & 725( & 2) & 706( & 1) & 720( & 7) \\
\hline 7531 & 3) & 746( & 1) & 731( & 3) & 757( & 3) \\
\hline 770( & 18) & 767( & 18) & 746( & 15) & 764( & 11) \\
\hline 793( & 6) & 787( & 5) & 768( & 6) & 792( & 7) \\
\hline 818( & 7) & 815( & 6) & 794( & 3) & 815( & 3) \\
\hline 837( & 8) & 832( & 8) & 811( & 8) & 841( & 6) \\
\hline 853( & 7) & 848( & 7) & 828( & 5) & 861( & 12) \\
\hline 871( & 26) & 870( & 21) & 847( & 8) & 874( & 2) \\
\hline 8866 & 6) & 883( & 8) & 866( & 5) & 886( & 24) \\
\hline 898( & 0) & 892( & 0) & 868( & 5) & 901( & 2) \\
\hline 915( & 9) & 912( & 7) & 883( & 5) & 910( & 18) \\
\hline 934( & 1) & 924( & 1) & 897( & 6) & 919) & 4) \\
\hline 943( & 3) & 934( & 3) & 911( & 5) & 936( & 5) \\
\hline 948( & 0) & 939( & 1) & 915( & 2) & 951( & 2) \\
\hline 982( & 1) & 972( & 0) & 9331 & 2) & 959( & 4) \\
\hline 986( & 4) & 978( & 5) & 951( & 3) & 972( & 1) \\
\hline 1004( & 1) & 994( & 1) & 958( & 3) & 994( & 6) \\
\hline 1013( & 3) & 1003( & 2) & 974( & 2) & 1010( & 1) \\
\hline 1021( & 7) & 1012( & 7) & 984( & 5) & 1022( & 6) \\
\hline 1037( & 6) & 1028( & 6) & 998( & 6) & 1039 & 11) \\
\hline 1050( & 1) & 1041( & 1) & 1009 & 0) & 1043( & 1) \\
\hline 1101( & 8) & 1092( & 8) & 1062( & 7) & 1100( & 8) \\
\hline 1141( & 4) & 1131( & 4) & 1099( & 4) & 1135( & 4) \\
\hline 1156( & 5) & 1146( & 5) & 1112( & 5) & 1154( & 4) \\
\hline
\end{tabular}




\begin{tabular}{|c|c|c|c|c|c|c|c|}
\hline 1190( & 3) & 1179 & 3) & 1144( & 3) & 1188( & 8) \\
\hline 1211( & 5) & 1201( & 6) & 1165( & 9) & 1213( & 9) \\
\hline 1227( & 3) & 1215( & 3) & 1180( & 2) & 1221( & 5) \\
\hline 1240( & 1) & 1230 & 1) & 1193( & 1) & 1243( & 0) \\
\hline 1250( & 5) & 1241( & 4) & 1204( & 2) & 1255( & 6) \\
\hline 1282( & 4) & 1272( & 3) & 1230( & 2) & 1277( & 2) \\
\hline 1300( & 1) & 1289 & 0) & 1246( & 1) & 1283( & 2) \\
\hline 1307( & 3) & 1295( & 3) & 1251( & 3) & 1297( & 6) \\
\hline 1314( & 12) & 1304( & 12) & 1261( & 4) & 1305( & 7) \\
\hline 1321( & 1) & 1308( & 1) & 1269 & 9) & 1315( & 2) \\
\hline 1350( & 7) & 1337( & 7) & 1294( & 5) & 1353( & 6) \\
\hline 1398( & 1) & 1385( & 1) & 1342( & 1) & 1388( & 3) \\
\hline 1424( & 1) & 1412( & 1) & 1369 & 2) & 1413( & 1) \\
\hline 1432( & 4) & 1418( & 4) & 1374( & 4) & 1426( & 4) \\
\hline 1477( & 2) & 1466( & 3) & 1419 & 3) & 1456( & 2) \\
\hline 1482( & 1) & 1466( & 1) & 1424( & 1) & 1461( & 4) \\
\hline 1486( & 0) & 1473( & 1) & 1432( & 1) & 1471( & 4) \\
\hline 1495( & 12) & 1481( & 10) & 1436( & 12) & 1474( & 10) \\
\hline 1498( & 7) & 1487( & 8) & 1441( & 7) & 1486( & 5) \\
\hline 1514( & 6) & 1500( & 7) & 1460 & 6) & 1499( & 3) \\
\hline 1537( & 24) & 1521( & 21) & 1479 & 14) & 1522( & 17) \\
\hline 1567( & 18) & 1553( & 13) & 1508( & 4) & 1556( & 8) \\
\hline 1611( & 7) & 1590( & 8) & 1531( & 6) & 1608( & 12) \\
\hline 3016( & 24) & 2997( & 23) & 2944( & 74) & 3033( & 39) \\
\hline 3026( & 123) & 3006( & 62) & 2944( & 18) & 3035( & 90) \\
\hline 3027( & 26) & 3007( & 87) & 2955( & 105) & 3045( & 163) \\
\hline 3044( & 69) & 3024( & 66) & 2967( & 69) & 3051( & 125) \\
\hline 3065( & 19) & 30460 & 17) & 2994( & 14) & 3088( & 40) \\
\hline 3096( & 10) & 3073( & 10) & 3009 & 13) & 3095( & 37) \\
\hline 3109 & 3) & 3086( & 2) & 3017( & 3) & 30999 & 21) \\
\hline 3118( & 4) & 3097( & 6) & 3032( & 31) & 3110( & 56) \\
\hline 3120( & 21) & 3097( & 17) & 3034( & 8) & 3117( & 6) \\
\hline 3124( & 1) & 3102( & 1) & 3039 & 3) & 3124( & 7) \\
\hline 3131( & 5) & 3109 & 12) & 3043( & 22) & 3125( & 28) \\
\hline 3134( & 17) & 3111( & 17) & 3045( & 37) & 3138( & 15) \\
\hline 3135( & 39) & 3113( & 32) & 3053( & 2) & 3142( & 46) \\
\hline 3143( & 11) & 3121( & 10) & 3059 & 9) & 3143( & 30) \\
\hline 3165( & 38) & 3141( & 42) & 3077( & 56) & 3165( & 69) \\
\hline 3169 ( & 14) & 3149 & 14) & 3084( & 16) & 3170( & 38) \\
\hline 3210( & 20) & 3187( & 19) & 3117( & 19) & 3196( & 27) \\
\hline 3226( & 8) & 3201( & 8) & 3129 & 11) & 32000 & 21) \\
\hline
\end{tabular}


Table S69. Harmonic vibrational frequencies (in $\mathrm{cm}^{-1}$ ) and infrared intensities (in parentheses in $\mathrm{km} / \mathrm{mol}$ ) for the $\left(\mathbf{C}_{4} \mathbf{H}_{6}\right)_{3} \mathbf{M n}$ structure Mn-3D.

\begin{tabular}{|c|c|c|c|c|c|c|c|}
\hline \multicolumn{2}{|c|}{ B3LYP } & \multicolumn{2}{|c|}{ B3LYP* } & \multicolumn{2}{|r|}{ BP86 } & \multicolumn{2}{|c|}{ M06L } \\
\hline 70( & 0) & 70( & 0) & 65( & 0) & 68( & 0) \\
\hline 931 & 1) & 92( & 1) & 93( & 1) & 94( & 0) \\
\hline 109 & 0) & 113( & 0) & 108( & 0) & 125( & 1) \\
\hline 126( & 1) & 129 & 1) & 121( & 1) & 130( & 1) \\
\hline 184( & 5) & 185( & 5) & 182( & 4) & 184( & 4) \\
\hline 205( & 0) & 206( & 0) & 203( & 1) & 200( & 0) \\
\hline 215( & 0) & 220( & 0) & 217( & 1) & 209( & 0) \\
\hline 222( & 7) & 226( & 7) & 2331 & 8) & 233( & 7) \\
\hline 261( & 12) & 268( & 9) & 270( & 1) & 276( & 3) \\
\hline 272( & 1) & 276( & 2) & 283( & 1) & 282( & 8) \\
\hline 285( & 1) & 291( & 1) & 2999 & 7) & 296( & 1) \\
\hline 294( & 0) & 302( & 0) & 302( & 0) & 306( & 2) \\
\hline 313( & 3) & 319 & 1) & 331( & 0) & 325( & 0) \\
\hline 318( & 9) & 325( & 10) & 338( & 7) & 333( & 6) \\
\hline 331( & 11) & 338( & 8) & 3431 & 4) & 343( & 5) \\
\hline 364( & 8) & 3731 & 7) & 399 & 3) & 390( & 7) \\
\hline 406( & 10) & 410( & 10) & 415( & 7) & 436( & 7) \\
\hline 437( & 7) & 437( & 7) & 437( & 7) & 449 & 15) \\
\hline 461( & 13) & 4631 & 14) & 4600 & 13) & 466( & 8) \\
\hline 478( & 7) & 480( & 8) & 473( & 8) & 478( & 5) \\
\hline 482( & 1) & 483( & 2) & 480( & 11) & 487( & 3) \\
\hline 566( & 6) & 563( & 5) & 550( & 4) & 568( & 4) \\
\hline 616( & 1) & 615( & 1) & 602( & 1) & 619 & 2) \\
\hline 632( & 1) & 632( & 1) & 621( & 1) & 637( & 2) \\
\hline 680 & 31) & 677( & 32) & 661( & 31) & 677( & 30) \\
\hline 714( & 5) & 713( & 5) & 691( & 3) & 698( & 4) \\
\hline 737( & 0) & 735( & 0) & 717( & 0) & 725( & 0) \\
\hline 757( & 3) & 753( & 5) & 7381 & 6) & 7361 & 7) \\
\hline 759 & 19) & 754( & 14) & 739 & 6) & 755( & 9) \\
\hline 781( & 5) & 7731 & 3) & 7566 & 2) & 773( & 2) \\
\hline 861( & 23) & 859 & 28) & 837( & 31) & 842( & 24) \\
\hline 876( & 1) & 870( & 1) & 847( & 28) & 851( & 29) \\
\hline 880( & 1) & 877( & 2) & 851( & 22) & 858( & 3) \\
\hline 8981 & 16) & 887( & 12) & 854( & 1) & 866( & 2) \\
\hline 902( & 30) & 894( & 43) & 868( & 11) & 878( & 8) \\
\hline 904( & 3) & 899( & 6) & 871( & 6) & 883( & 2) \\
\hline 910( & 14) & 902( & 3) & 880( & 3) & 8861 & 1) \\
\hline 921( & 4) & 917( & 3) & 896( & 2) & 895( & 2) \\
\hline 927( & 2) & 919) & 1) & 898( & 1) & 907( & 1) \\
\hline 938( & 2) & 933( & 3) & 909( & 4) & 913( & 5) \\
\hline 950 & 0) & 944( & 0) & 914( & 1) & 931( & 1) \\
\hline 956( & 2) & 949( & 0) & 921( & 1) & 938( & 1) \\
\hline 961( & 2) & 951( & 2) & 930( & 2) & 950( & 3) \\
\hline 967( & 1) & 958( & 1) & 9351 & 1) & 954( & 3) \\
\hline 1021( & 11) & 1012( & 11) & 975( & 12) & 1013( & 8) \\
\hline 1065( & 4) & 1056( & 5) & 10266 & 5) & 1047( & 3) \\
\hline
\end{tabular}




\begin{tabular}{|c|c|c|c|c|c|c|c|}
\hline 1078( & 1) & 1070 & 0) & 1043( & 0) & 1071( & 2) \\
\hline 1082( & 2) & 1075( & 1) & 1047( & 6) & 1075( & 4) \\
\hline 1084( & 6) & 1075( & 7) & 1048( & 1) & 1076( & 2) \\
\hline 1088( & 1) & 1079( & 2) & 1054( & 2) & 1080( & 4) \\
\hline 1113( & 2) & 1102( & 3) & 1074( & 5) & 1107( & 3) \\
\hline 1220( & 8) & 1208( & 7) & 1172( & 4) & 1207( & 6) \\
\hline 1225( & 9) & 1214( & 8) & 1179( & 7) & 1214( & 8) \\
\hline 1244( & 40) & 1233( & 32) & 1199( & 20) & 1237( & 27) \\
\hline 1267( & 22) & 1256( & 19) & 1222( & 14) & 1256( & 21) \\
\hline 1271( & 11) & 1259 & 10) & 1225( & 5) & 1259 & 5) \\
\hline 1323( & 4) & 1310( & 3) & 1276( & 3) & 1313( & 5) \\
\hline 1411( & 1) & 1398( & 2) & 1355( & 0) & 1388( & 0) \\
\hline 1411( & 1) & 1399 & 1) & 1356( & 2) & 1391( & 0) \\
\hline 1419( & 8) & 1405( & 7) & 1365( & 4) & 1402( & 3) \\
\hline 1452( & 14) & 1440( & 14) & 1403( & 11) & 1442( & 11) \\
\hline 1479( & 15) & 1469 & 10) & 1434( & 3) & 1465( & 5) \\
\hline 1483( & 4) & 1473( & 6) & 1439 & 9) & 1468( & 5) \\
\hline 1536( & 18) & 1522( & 13) & 1475( & 10) & 1515( & 2) \\
\hline 1540( & 12) & 1525( & 10) & 1477( & 2) & 1516( & 13) \\
\hline 1544( & 23) & 1529 & 7) & 1481( & 3) & 1522( & 14) \\
\hline 1548( & 8) & 1534( & 23) & 1492( & 4) & 1537( & 21) \\
\hline 1550( & 15) & 1535( & 8) & 1495( & 17) & 1538( & 9) \\
\hline 1662( & 61) & 1648( & 61) & 1604( & 62) & 1666( & 72) \\
\hline 3125( & 20) & 3103( & 20) & 3045( & 30) & 3111( & 39) \\
\hline 3131( & 9) & 3110 & 10) & 3047( & 6) & 3116( & 35) \\
\hline 3144( & 9) & 3121( & 10) & 3054 ( & 17) & 3128( & 37) \\
\hline 3146( & 8) & 3122( & 9) & 3056 & 15) & 3132( & 13) \\
\hline 3150( & 7) & 3131( & 6) & 3071( & 10) & 3144( & 40) \\
\hline 3156( & 2) & 3136( & 3) & 3072( & 14) & 3151( & 23) \\
\hline 3157( & 5) & 3137( & 7) & 3074( & 8) & 3151( & 5) \\
\hline 3158( & 14) & 3139 & 12) & 3078 ( & 5) & 3153( & 17) \\
\hline 3179( & 2) & 3156( & 2) & 3096( & 3) & 3175( & 6) \\
\hline 3189 & 4) & 3169 & 5) & 3110( & 2) & 3189x & 28) \\
\hline 3191( & 9) & 3170 & 8) & 3112( & 12) & 3192( & 7) \\
\hline 3212( & 13) & 3191( & 8) & 3120( & 5) & 3200( & 30) \\
\hline 3216( & 6) & 3192( & 12) & 3126( & 21) & 3205( & 10) \\
\hline 3232( & 5) & 3208( & 4) & 3142( & 3) & 3217 ( & 6) \\
\hline 3232( & 7) & 3209 & 7) & 3144( & 8) & 3221( & 17) \\
\hline 3243( & 13) & 3223( & 12) & 3163( & 11) & 3247( & 16) \\
\hline 3246( & 13) & 3226( & 14) & 3165( & 5) & 3248( & 28) \\
\hline 3247 ( & 1) & 3227 & 0) & $3168 d$ & 15) & 32521 & 15) \\
\hline
\end{tabular}


Table S70. Harmonic vibrational frequencies (in $\mathrm{cm}^{-1}$ ) and infrared intensities (in parentheses in $\mathrm{km} / \mathrm{mol}$ ) for the $\left(\mathbf{C}_{4} \mathbf{H}_{6}\right)_{3} \mathbf{M n}$ structure Mn-1Q.

\begin{tabular}{|c|c|c|c|c|c|c|c|}
\hline \multicolumn{2}{|c|}{ B3LYP } & \multicolumn{2}{|c|}{ B3LYP* } & \multicolumn{2}{|c|}{ BP86 } & \multicolumn{2}{|c|}{ M06L } \\
\hline 30( & 0) & 31( & 0) & 50( & 0) & -31( & 0) \\
\hline 38( & 0) & 41( & 0) & 54( & 1) & 39 & 0) \\
\hline 49( & 0) & 47( & 0) & 84( & 0) & 57( & 1) \\
\hline 60( & 0) & 61( & 0) & 89 & 0) & 74( & 1) \\
\hline 62( & 0) & 66( & 0) & 105( & 3) & 90( & 1) \\
\hline 77( & 0) & 79( & 0) & 126( & 3) & 103( & 0) \\
\hline 111( & 1) & 109 & 0) & 143( & 0) & 108( & 1) \\
\hline 1331 & 3) & 135( & 2) & 156( & 1) & 151( & 2) \\
\hline 144( & 4) & 148( & 3) & 180( & 4) & 154( & 9) \\
\hline 174( & 2) & 174( & 3) & 190( & 5) & 194( & 1) \\
\hline 179( & 10) & 181( & 9) & 219 & 2) & 213( & 2) \\
\hline 1966 & 7) & 202( & 6) & 249 & 7) & 2566 & 1) \\
\hline 278( & 2) & 281( & 1) & 272( & 0) & 274 ( & 2) \\
\hline 281( & 2) & 286( & 1) & 273( & 3) & 280 & 4) \\
\hline 291( & 1) & 290( & 1) & 294( & 11) & 301( & 4) \\
\hline 294( & 1) & 301( & 1) & 320( & 28) & 309 & 6) \\
\hline 307( & 40) & 316( & $35)$ & 346( & 7) & 323( & 12) \\
\hline 334( & 18) & 340( & 16) & 387( & 9) & 325( & 11) \\
\hline 413( & 6) & 418( & 4) & 417( & 3) & 432( & 4) \\
\hline 418( & 11) & 425( & 6) & 425( & 3) & 436( & 2) \\
\hline 426( & 2) & 430( & 4) & 454( & 3) & 446( & 2) \\
\hline 559( & 4) & 555( & 4) & 537( & 6) & 555( & 5) \\
\hline 566( & 2) & 560( & 3) & 542( & 6) & 567( & 4) \\
\hline 567( & 6) & 565( & 5) & 580( & 4) & 573( & 3) \\
\hline 633( & $35)$ & 631( & 37) & 609 & 21) & 618( & 28) \\
\hline 642( & 2) & 639 & 2) & 614( & 37) & 640( & 8) \\
\hline 652( & 37) & 649 & 34) & 667( & 11) & 651( & 26) \\
\hline 705( & 3) & 701( & 3) & 687( & 7) & 688( & 18) \\
\hline 713( & 1) & 707( & 1) & 691( & 6) & 700( & 1) \\
\hline 721( & 6) & 717( & 6) & 699 & 4) & 704( & 3) \\
\hline 824( & 1) & 818( & 3) & 798( & 19) & 780( & 6) \\
\hline 855( & 7) & 851( & 5) & 812( & 8) & 813( & 20) \\
\hline 872( & 15) & 864( & 12) & 818( & 5) & 821( & 5) \\
\hline 879 & 12) & 873( & 22) & 833( & 39) & 837( & 37) \\
\hline 881( & 33) & 876( & 47) & 846( & 12) & 849 & 9) \\
\hline 884( & 45) & 877( & 33) & 850( & 6) & 855( & 19) \\
\hline 886( & 13) & 880( & 14) & 851( & 42) & 860 & 25) \\
\hline 891( & 23) & 882( & 24) & 857( & 3) & 872( & 5) \\
\hline 894( & 13) & 888( & 3) & 860( & 6) & 880( & 4) \\
\hline 895( & 8) & 891( & 3) & 875( & 2) & 895( & 3) \\
\hline 9031 & 3) & 895( & 3) & 887( & 5) & 912( & 1) \\
\hline 908( & 1) & 901( & 0) & 903( & 0) & 913( & 2) \\
\hline 1005( & 15) & 995( & 16) & 908( & 0) & 987( & 12) \\
\hline 1009 & 9) & 1000( & 9) & 961( & 13) & 997( & 9) \\
\hline 1011( & 10) & 1001( & 10) & 979( & 9) & 1013( & 7) \\
\hline 1054( & 2) & $1047 x$ & 2) & 1025( & 2) & 1053 & 0) \\
\hline
\end{tabular}




\begin{tabular}{|c|c|c|c|c|c|c|c|}
\hline 1056( & 1) & 1048( & 1) & 1030( & 4) & 1053( & 0) \\
\hline 1057( & 5) & 1050( & 6) & 1030( & 1) & 1054( & 5) \\
\hline 1092( & 7) & 1083( & 7) & 1045( & 5) & 1087( & 6) \\
\hline 1095( & 6) & 1086( & 4) & 1055( & 2) & 1091( & 1) \\
\hline 1097( & 2) & 1088( & 2) & 1063( & 5) & 1094( & 3) \\
\hline 1237( & 62) & 1227( & 51) & 1181( & 17) & 1230( & 52) \\
\hline 1239 & 82) & 1229 & 66) & 1198( & 25) & 1233( & 36) \\
\hline 1251( & 9) & 1241( & 9) & 1202( & 42) & 1244( & 4) \\
\hline 1321( & 18) & 1310 & 10) & 12350 & 12) & 1306( & 13) \\
\hline 1322( & 5) & 1311( & 8) & 1275( & 8) & 1316( & 14) \\
\hline 1327( & 7) & 1315( & 5) & 1276( & 3) & 1318( & 7) \\
\hline 1410( & 27) & 1399 & 17) & 1356( & 2) & 1392( & 10) \\
\hline 1413( & 20) & 1400( & 20) & 1359( & 13) & 1396( & 3) \\
\hline 1415( & 3) & 1404( & 3) & 1364( & 8) & 1398( & 3) \\
\hline 1452( & 37) & 1440( & 35) & 1403( & 9) & 1445( & 18) \\
\hline 1453( & 44) & 1442( & 23) & 1408( & 13) & 1450( & 32) \\
\hline 1458( & 6) & 1447( & 13) & 1420( & 5) & 1455( & 1) \\
\hline 1528( & 100) & 1515( & 67) & 1480( & 45) & 1520( & 98) \\
\hline 1532( & 66) & 1520( & 68) & 1485( & 28) & 1529 ( & 44) \\
\hline 1538( & 16) & 1525( & 17) & 1486( & 20) & 1533( & 31) \\
\hline 1636( & 56) & 1624( & 48) & 1491( & 23) & 1616( & 51) \\
\hline 1640( & 39) & 1630 & 36) & 1584( & 31) & 1642( & 51) \\
\hline 1654( & 47) & 1642( & 45) & 1601( & 26) & 1656( & 68) \\
\hline 3139 & 6) & 3119 & 16) & 3060( & 8) & 3125( & 18) \\
\hline 3143( & 14) & 3120 & 4) & 3067( & 11) & 3144( & 19) \\
\hline 3143( & 7) & 3123( & 8) & 3068( & 8) & 3146( & 23) \\
\hline 3147( & 8) & 3127( & 15) & 3074( & 5) & 3147( & 17) \\
\hline 3149 ( & 9) & 3128( & 1) & 3078 ( & 2) & 3153( & 11) \\
\hline 3149 & 8) & 3128( & 8) & 3079) & 9) & 3156( & 18) \\
\hline 3159 & 3) & 3139 & 4) & 3082( & 4) & 3164( & 9) \\
\hline 3163( & 2) & 3143( & 2) & 3085( & 5) & 3166( & 9) \\
\hline 3165( & 2) & 3146( & 2) & 3088( & 6) & 3173( & 17) \\
\hline 3180( & 5) & 3157( & 6) & 3091( & 4) & 3178( & 8) \\
\hline 3188( & 7) & 31660 & 7) & 3098( & 9) & 3195( & 18) \\
\hline 3188( & 7) & 3167( & 6) & 3112( & 9) & 3202( & 17) \\
\hline 3231( & 6) & 3210 & 6) & 3148( & 7) & 3220( & 12) \\
\hline 3233( & 5) & 3213( & 4) & 3157( & 3) & 3242( & 15) \\
\hline 3238( & 4) & 3218( & 4) & 3169 & 3) & 32460 & 6) \\
\hline 3250( & 11) & 3231( & 11) & 3172( & 12) & 3251( & 24) \\
\hline 3254( & 8) & 3235( & 8) & 3174( & 5) & 3261( & 16) \\
\hline 3256( & 9) & 3237( & 8) & 31760 & 11) & 32671 & 10) \\
\hline
\end{tabular}


Table S71. Harmonic vibrational frequencies (in $\mathrm{cm}^{-1}$ ) and infrared intensities (in parentheses in $\mathrm{km} / \mathrm{mol})$ for the $\left(\mathbf{C}_{4} \mathbf{H}_{6}\right)_{3} \mathbf{M n}$ structure Mn-2Q.

\begin{tabular}{|c|c|c|c|c|c|c|c|}
\hline \multicolumn{2}{|c|}{ B3LYP } & \multicolumn{2}{|c|}{ B3LYP* } & \multicolumn{2}{|c|}{ BP86 } & \multicolumn{2}{|c|}{ M06L } \\
\hline 23( & 0) & 25( & 0) & 28( & 2) & 51( & 1) \\
\hline 78( & 0) & 78( & 0) & 78( & 0) & 78( & 2) \\
\hline 91( & 1) & 91( & 1) & 93( & 1) & 106( & 1) \\
\hline 110( & 0) & 112( & 0) & 100( & 0) & 138( & 1) \\
\hline 122( & 1) & 121( & 1) & 104( & 9) & 155( & 2) \\
\hline 138( & 1) & 134( & 1) & 126( & 2) & 168( & 0) \\
\hline 157( & 1) & 150( & 1) & 138( & 0) & 187( & 2) \\
\hline 185( & 0) & 186( & 0) & 190( & 0) & 235( & 7) \\
\hline 210 & 1) & 213( & 1) & 217 ( & 0) & 240( & 3) \\
\hline 2331 & 2) & 232( & 2) & 234( & 1) & 264( & 4) \\
\hline 255( & 0) & 254( & 0) & 247( & 0) & 288( & 2) \\
\hline 278( & 0) & 284( & 0) & 287( & 0) & 298( & 4) \\
\hline 310( & 1) & 311( & 1) & 312( & 2) & 336( & 4) \\
\hline 334( & 5) & 3331 & 6) & 3699 & 0) & 383( & 14) \\
\hline 390( & 4) & 3881 & 5) & 381( & 4) & 388( & 0) \\
\hline 397( & 7) & 401( & 5) & 3931 & 1) & 406( & 9) \\
\hline 419 & 4) & 420( & 5) & 418( & 9) & 422( & 3) \\
\hline 445( & 8) & 447( & 6) & 435( & 41) & 448( & 3) \\
\hline 469 & 9) & 471( & 10) & 436( & 5) & 464( & 5) \\
\hline 486( & 16) & 488( & 25) & 453( & 10) & 485( & 12) \\
\hline 518( & 31) & 508( & 21) & 489 & 14) & 559( & 3) \\
\hline 567( & 1) & 563( & 1) & 519( & 4) & 609 & 8) \\
\hline 581( & 4) & 574( & 4) & 548( & 1) & 661( & 18) \\
\hline 617( & 3) & 617( & 3) & 599( & 1) & 671( & 1) \\
\hline 689 & 13) & 678( & 19) & 659) & 4) & 683( & 4) \\
\hline 692( & 19) & 6861 & 13) & 665( & 16) & 687( & 33) \\
\hline 711( & 24) & 705( & 24) & 673( & 18) & 698( & 5) \\
\hline 721( & 0) & 715( & 1) & 687( & 12) & 7261 & 1) \\
\hline 727( & 1) & 722( & 1) & 695( & 3) & 779) & 6) \\
\hline 788( & 5) & 779 & 6) & 770( & 4) & 805( & 21) \\
\hline 809 & 45) & 804( & 43) & 788( & 27) & 816( & 6) \\
\hline 831( & 9) & 826( & 7) & 811( & 5) & 834( & 2) \\
\hline 855( & 4) & 850 & 4) & 829( & 6) & 852( & 6) \\
\hline 928( & 1) & 923( & 2) & 876( & 1) & 8566 & 8) \\
\hline 9331 & 4) & 926( & 1) & 895( & 3) & 859 & 2) \\
\hline 940( & 11) & 929 & 2) & 919) & 5) & 921( & 7) \\
\hline 956( & 31) & 955( & 21) & 924( & 17) & 9261 & 4) \\
\hline 967( & 22) & 9631 & 16) & 935( & 7) & 931( & 12) \\
\hline 990( & 5) & 981( & 7) & 942( & 7) & 940( & 5) \\
\hline 997( & 11) & 988( & 11) & 947( & 3) & 960( & 1) \\
\hline 1010 & 4) & 1000( & 2) & 960( & 4) & 977( & 13) \\
\hline 1019 & 3) & 1012( & 4) & 965( & 0) & 1002( & 2) \\
\hline 1030 & 12) & 1020( & 9) & 983( & 6) & 1017( & 2) \\
\hline 1043( & 5) & 1036( & 4) & 986( & 1) & 1046( & 2) \\
\hline 1054( & 2) & 1042( & 1) & 1010( & 3) & 1062( & 7) \\
\hline 1082( & 3) & 1066( & 2) & 1012( & 1) & 1071( & 3) \\
\hline
\end{tabular}




\begin{tabular}{|c|c|c|c|c|c|c|c|}
\hline 1085( & 1) & 1076( & 3) & 1048( & 2) & 1078( & 6) \\
\hline 1116( & 5) & 1109 & 4) & 1088( & 22) & 1136( & 23) \\
\hline 1123( & 17) & 1114( & 18) & 1099 & 6) & 1161( & 11) \\
\hline 1154( & 4) & 1144( & 5) & 1113( & 9) & 1198( & 6) \\
\hline 1196( & 16) & 1185( & 12) & 1156( & 10) & 1219 & 2) \\
\hline 1217( & 1) & 1206( & 1) & 1175( & 2) & 12350 & 4) \\
\hline 1235( & 9) & 1226( & 8) & 1194( & 4) & 1250 & 17) \\
\hline 1266( & 4) & 1256( & 4) & 1223( & 3) & 1270( & 4) \\
\hline 1291( & 1) & 1282( & 1) & 1251( & 1) & 1274( & 1) \\
\hline 1333( & 3) & 1321( & 3) & 1280( & 2) & 1324( & 4) \\
\hline 1368( & 5) & 1356( & 5) & 1326( & 3) & 1380 & 7) \\
\hline 1384( & 2) & 1373( & 2) & 1335( & 3) & 1385( & 1) \\
\hline 1416( & 1) & 1403( & 1) & 1363( & 1) & 1405( & 2) \\
\hline 1421( & 1) & 1407( & 1) & 1366( & 1) & 1415( & 2) \\
\hline 1426( & 4) & 1411( & 4) & 1379 & 6) & 1458( & 2) \\
\hline 1434( & 1) & 1420( & 1) & 1382( & 2) & 1462( & 6) \\
\hline 1463( & 1) & 1450( & 1) & 1408( & 4) & 14660 & 2) \\
\hline 1472( & 10) & 1459 & 10) & 1418( & 10) & 1476( & 4) \\
\hline 1487( & 12) & 1477( & 11) & 1435( & 13) & 1493( & 7) \\
\hline 1495( & 1) & 1485( & 3) & 1437( & 4) & 1502( & 8) \\
\hline 1559 ( & 29) & 1546( & 28) & 1501( & 22) & 1524( & 21) \\
\hline 1665( & 18) & 1648( & 15) & 1546( & 9) & 1548( & 25) \\
\hline 1666( & 6) & 1656( & 4) & 1611( & 2) & 1639 & 8) \\
\hline 2971( & 21) & 2946( & 20) & 2867( & 15) & 3013( & 30) \\
\hline 3004( & 10) & 2983( & 10) & 2907( & 13) & 3024( & 148) \\
\hline 3016( & 71) & 2995( & 68) & 2923( & 11) & 3061( & 28) \\
\hline 3023( & 19) & 3004( & 20) & 2935( & 76) & 3095( & 16) \\
\hline 3028( & 15) & 3009 ( & 14) & 2962( & 18) & 3117( & 20) \\
\hline 3045( & 11) & 3024( & 12) & 2976( & 8) & 3120 & 35) \\
\hline 3064( & 15) & 3045( & 13) & 3022( & 18) & 3126( & 31) \\
\hline 3080( & 26) & 3064( & 23) & 3047( & 3) & 3128( & 6) \\
\hline 3111( & 4) & 3091( & 3) & 3052( & 2) & 3144( & 27) \\
\hline 3119 & 17) & 3099( & 17) & 3060 & 4) & 3146( & 1) \\
\hline 3129 & 2) & 3107( & 2) & 3062( & 9) & 3164( & 31) \\
\hline 3134( & 5) & 3114( & 4) & 3072( & 13) & 3175( & 14) \\
\hline 3140( & 66) & 3121( & 62) & 3072( & 16) & 3180 & 60) \\
\hline 3147( & 4) & 3127( & 4) & 3073( & 8) & 3191( & 3) \\
\hline 3153( & 12) & 3132( & 13) & 3083( & 26) & 3209 & 17) \\
\hline 3164( & 33) & 3142( & 31) & 3086( & 48) & 3227( & 18) \\
\hline 3170( & 8) & 3147( & 8) & 3088( & 11) & 3234( & 12) \\
\hline 3245( & 7) & 32260 & 6) & 31680 & 6) & 3254( & 13) \\
\hline
\end{tabular}


Table S72. Harmonic vibrational frequencies (in $\mathrm{cm}^{-1}$ ) and infrared intensities (in parentheses in $\mathrm{km} / \mathrm{mol}$ ) for the $\left(\mathbf{C}_{4} \mathbf{H}_{6}\right)_{3} \mathbf{M n}$ structure $\mathrm{Mn}-1 \mathrm{H}$.

\begin{tabular}{|c|c|c|c|c|c|c|c|}
\hline \multicolumn{2}{|c|}{ B3LYP } & \multicolumn{2}{|c|}{ B3LYP* } & \multicolumn{2}{|c|}{ BP86 } & \multicolumn{2}{|c|}{ M06L } \\
\hline 371 & 0) & 37( & 1) & 43( & 0) & 46( & 1) \\
\hline 70( & 1) & 70( & 1) & 73( & 0) & 70( & 3) \\
\hline 75( & 2) & 731 & 1) & 79 ( & 0) & 80( & 0) \\
\hline 921 & 3) & 93( & 2) & 111( & 0) & 91( & 2) \\
\hline 110( & 2) & 110 & 2) & 124( & 1) & 118( & 2) \\
\hline 117( & 5) & 115( & 3) & 150( & 1) & 135( & 4) \\
\hline 147( & 2) & 145( & 2) & 153( & 1) & 162( & 2) \\
\hline 192( & 0) & 197( & 0) & 199 & 3) & 203( & 0) \\
\hline 216( & 0) & 213( & 1) & 213( & 0) & 217( & 2) \\
\hline 225( & 2) & 223( & 1) & 223( & 1) & 227( & 1) \\
\hline 239 & 3) & 2361 & 3) & 249 & 2) & 244( & 2) \\
\hline 291( & 1) & 292( & 1) & 2900 & 1) & 296( & 0) \\
\hline 330( & 1) & 328( & 1) & 328( & 2) & 341( & 1) \\
\hline 360( & 2) & 356( & 2) & 352( & 0) & 346( & 4) \\
\hline 394( & 6) & 390 & 9) & 382( & 12) & 387( & 12) \\
\hline 426( & 45) & 422( & 37) & 413( & 15) & 430( & 19) \\
\hline 446( & 5) & 443( & 6) & 441( & 7) & 446( & 6) \\
\hline 506( & 33) & 502( & 30) & 483( & 12) & 512( & 30) \\
\hline 5251 & 11) & 519 & 10) & 505( & 10) & 517( & 8) \\
\hline 560( & 2) & 556( & 2) & 542( & 5) & 554( & 1) \\
\hline 587( & 9) & 587( & 6) & 575( & 0) & 594( & 3) \\
\hline 617( & 3) & 614( & 2) & 605( & 1) & 626( & 2) \\
\hline 658( & 10) & 654 ( & 8) & 640( & 3) & 664( & 8) \\
\hline 679 & 27) & 673( & 23) & 658( & 25) & 677( & 25) \\
\hline 701( & 54) & 692( & 55) & 672( & 27) & 690( & 51) \\
\hline 734( & 24) & 724( & 28) & 6999 & 28) & 721( & 18) \\
\hline 744( & 41) & 738( & 35) & 719 & 41) & 735( & 31) \\
\hline 794( & 9) & 787( & 11) & 7699 & 18) & 795( & 33) \\
\hline 821( & 6) & 813( & 9) & 785( & 17) & 814( & 49) \\
\hline 851( & 47) & 837( & 95) & 804( & 48) & 831( & 25) \\
\hline 857( & 66) & 849 & 5) & 832( & 4) & 872( & 12) \\
\hline 909 & 6) & 902( & 5) & 869 ( & 12) & 8999 & 39) \\
\hline 946( & 66) & 9331 & 79) & 8799 & 22) & 915( & 27) \\
\hline 950 & 1) & 942( & 3) & 9031 & 12) & 946( & 1) \\
\hline 956( & 20) & 945( & 1) & 913( & 2) & 9566 & 3) \\
\hline 984( & 22) & 967( & 13) & 922( & 1) & 961( & 3) \\
\hline 988( & 2) & 974( & 3) & 926( & 1) & 980( & 8) \\
\hline 1005( & 2) & 991( & 4) & 940( & 5) & 986( & 3) \\
\hline 1009 & 18) & 1000 & 11) & 972( & 5) & 1008( & 6) \\
\hline 1015( & 5) & 1007( & 5) & 981( & 9) & 1023( & 6) \\
\hline 1018( & 6) & 1009 & 8) & 9860 & 2) & 1026( & 7) \\
\hline 1054( & 5) & 1047( & 5) & 1020( & 4) & 1062( & 1) \\
\hline 1066( & 4) & 1057( & 4) & 1028( & 5) & 1067( & 8) \\
\hline 1081( & 2) & 1074( & 1) & 1047( & 2) & 1089 & 2) \\
\hline 1133( & 20) & 1124( & 18) & 1098( & 9) & 1136( & 10) \\
\hline 1145( & 11) & 1136( & 10) & 1108( & 9) & 1152( & 8) \\
\hline
\end{tabular}




\begin{tabular}{|c|c|c|c|c|c|c|c|}
\hline 1190( & 6) & 1180( & 6) & 1148( & 4) & 1194( & 7) \\
\hline 1217 ( & 7) & 1206( & 6) & 1165( & 1) & 1218( & 8) \\
\hline 1232( & 3) & 1219 & 1) & 1178( & 2) & 1231( & 1) \\
\hline 1234( & 1) & 1222( & 2) & 1187( & 5) & 1234( & 6) \\
\hline 1270( & 13) & 1259 ( & 14) & 1225( & 14) & 1265( & 17) \\
\hline 1283( & 3) & 1272( & 5) & 1234( & 1) & 1268( & 5) \\
\hline 1284( & 5) & 1273( & 3) & 1236( & 10) & 1282( & 5) \\
\hline 1325( & 1) & 1312( & 1) & 1270( & 0) & 1319 & 1) \\
\hline 1339 & 3) & 13266 & 3) & 1282( & 1) & 1336( & 5) \\
\hline 1346( & 1) & 1333( & 0) & 1294( & 1) & 1346( & 1) \\
\hline 1371( & 6) & 1356( & 5) & 1311( & 2) & 1365( & 6) \\
\hline 1409( & 8) & 1396( & 8) & 1357( & 4) & 1402( & 6) \\
\hline 1411( & 3) & 1398( & 2) & 1359 & 1) & 1404( & 3) \\
\hline 1431( & 0) & 1417 ( & 0) & 1371( & 1) & 1432( & 1) \\
\hline 1460( & 3) & 1448( & 3) & 1413( & 1) & 1455( & 6) \\
\hline 1468( & 2) & 1456( & 2) & 1420( & 3) & 1457( & 6) \\
\hline 1473( & 20) & 1460( & 10) & 1421( & 7) & 1464( & 18) \\
\hline 1474( & 6) & 1462( & 17) & 1426( & 17) & 1465( & 3) \\
\hline 1484( & 2) & 1471( & 2) & 1430( & 6) & 1470 & 7) \\
\hline 1486( & 14) & 1473( & 14) & 1434( & 8) & 1473( & 8) \\
\hline 1601( & 41) & 1584( & 41) & 1534( & 29) & 1594( & 52) \\
\hline 1646( & 38) & 1625( & 34) & 1543( & 14) & 1626( & 44) \\
\hline 1666( & 4) & 1647( & 3) & 1561( & 18) & 1681( & 2) \\
\hline 3014( & 23) & 2993( & 23) & 2936( & 27) & 3023( & 34) \\
\hline 3017( & 42) & 2997( & 39) & 2938( & 20) & 3027( & 110) \\
\hline 3033( & 34) & 3011( & 36) & 2947( & 69) & 3028( & 39) \\
\hline 3035( & 53) & 3015 ( & 52) & 2960( & 61) & 3040 & 76) \\
\hline 3048( & 14) & 3032( & 13) & 2978( & 8) & 3072( & 20) \\
\hline 3060( & 12) & 3043( & 9) & 2988( & 2) & 3081( & 4) \\
\hline 3078( & 22) & 3058( & 22) & 3004( & 21) & 3092( & 20) \\
\hline 3084( & 11) & 3068 ( & 9) & 3010( & 38) & 3097( & 40) \\
\hline 3090( & 26) & 3069 & 29) & 3020 & 25) & 3102( & 53) \\
\hline 3094( & 22) & 3075( & 18) & 3022( & 2) & 3104( & 47) \\
\hline 3101( & 1) & 3085( & 2) & 3041( & 8) & 3112( & 16) \\
\hline 3115( & 6) & 3096( & 6) & 3051( & 3) & 3128( & 14) \\
\hline 3121( & 29) & 3104( & 28) & 3051( & 5) & 3131( & 11) \\
\hline 3130( & 3) & 3107( & 3) & 3066( & 23) & 3159 ( & 34) \\
\hline 3154( & 10) & 3132( & 9) & 3071( & 11) & 3161( & 19) \\
\hline 3164( & 36) & 3145( & 33) & 3095( & 30) & 3182( & 27) \\
\hline 3168( & 9) & 3149 & 10) & 3096( & 14) & 3189 ( & 44) \\
\hline 3174( & 23) & 3159 & 19) & 3115( & 13) & 3198( & 28) \\
\hline
\end{tabular}


Table S73. Harmonic vibrational frequencies (in $\mathrm{cm}^{-1}$ ) and infrared intensities (in parentheses in $\mathrm{km} / \mathrm{mol}$ ) for the $\left(\mathbf{C}_{4} \mathbf{H}_{6}\right)_{3} \mathbf{M n}$ structure $\mathrm{Mn}-2 \mathrm{H}$.

\begin{tabular}{|c|c|c|c|c|c|c|c|}
\hline \multicolumn{2}{|c|}{ B3LYP } & \multicolumn{2}{|c|}{ B3LYP* } & \multicolumn{2}{|r|}{ BP86 } & \multicolumn{2}{|c|}{ M06L } \\
\hline 34( & 0) & 35( & 0) & 466 & 0) & 31( & 0) \\
\hline 56( & 2) & 58( & 1) & 49( & 0) & 47( & 1) \\
\hline 81( & 6) & 72( & 4) & 90( & 0) & 73( & 3) \\
\hline 100( & 1) & 100( & 1) & 96( & 0) & 85( & 2) \\
\hline 108( & 2) & 105( & 2) & 129 & 2) & 104( & 3) \\
\hline 117( & 0) & 116( & 0) & 147( & 5) & 116( & 0) \\
\hline 118( & 1) & 124( & 1) & 165( & 1) & 129( & 1) \\
\hline 184( & 3) & 182( & 2) & $189 x$ & 1) & 187( & 2) \\
\hline 210( & 2) & 208( & 1) & 210( & 1) & 205( & 1) \\
\hline 241( & 1) & 244( & 0) & 259 ( & 1) & 245( & 0) \\
\hline 285( & 0) & 284( & 0) & 2788 & 0) & 287( & 0) \\
\hline 298( & 0) & 298( & 0) & 2966 & 1) & 316( & 1) \\
\hline 304( & 2) & 303( & 1) & 307( & 0) & 319 & 1) \\
\hline 343( & 2) & 344( & 2) & 359 ( & 1) & 349 & 3) \\
\hline 415( & 27) & 414( & 26) & 414( & 14) & 407( & 25) \\
\hline 426( & 36) & 423( & 35) & 420( & 19) & 417 ( & 22) \\
\hline 479( & 9) & 475( & 8) & 463( & 29) & 466( & 0) \\
\hline 506( & 38) & 502( & 38) & 4999 & 4) & 503( & 17) \\
\hline 517 ( & 20) & 512( & 18) & 510( & 1) & 508( & 72) \\
\hline 536( & 2) & 532( & 2) & 511( & 8) & 536( & 0) \\
\hline 560( & 3) & 556( & 2) & 543( & 1) & 557( & 1) \\
\hline 607( & 3) & 600( & 2) & 569 & 0) & 606( & 6) \\
\hline 615( & 15) & 609 & 18) & 602( & 19) & 610( & 1) \\
\hline 621( & 4) & 614( & 3) & 604( & 1) & 611( & 17) \\
\hline 717( & 7) & 709( & 8) & 682( & 9) & 705( & 5) \\
\hline 724( & 95) & 716( & 92) & 691( & 75) & 717( & 74) \\
\hline 765( & 50) & 759 & 50) & 724( & 31) & 750( & 54) \\
\hline 814( & 15) & 804( & 11) & 752( & 8) & 804( & 0) \\
\hline 843( & 0) & 836( & 0) & 810( & 1) & 845( & 0) \\
\hline 847( & 3) & 843( & 3) & 826( & 3) & 858( & 5) \\
\hline 867( & 3) & 862( & 1) & 848( & 1) & 880( & 0) \\
\hline 949 & 45) & 937( & 41) & 887( & 6) & 938( & 27) \\
\hline 952( & 5) & 945( & 5) & 8999 & 1) & 945( & 3) \\
\hline 9631 & 4) & 951( & 2) & 906( & 3) & 958( & 7) \\
\hline 972( & 1) & 963( & 3) & 917( & 4) & 965( & 2) \\
\hline 1001( & 6) & 992( & 6) & 931( & 2) & 983( & 7) \\
\hline 1004( & 34) & 995( & 36) & 940( & 0) & 995( & 16) \\
\hline 1013( & 51) & 997( & 25) & 949( & 14) & 1000 & 4) \\
\hline 1020 & 2) & 1004( & 17) & 972( & 3) & 1012( & 8) \\
\hline 1021( & 16) & 1005( & 3) & 975( & 1) & 1013( & 29) \\
\hline 1025( & 5) & 1017 ( & 3) & 982( & 1) & 1025( & 1) \\
\hline 1053( & 1) & 1045( & 1) & 1019x & 3) & 1058( & 1) \\
\hline 1057( & 2) & 1049( & 3) & 1023( & 0) & 1061( & 4) \\
\hline 1096 & 17) & $1087 x$ & 17) & 1054 ( & 8) & 1097 & 23) \\
\hline 1128( & 6) & 1116( & 6) & 1080( & 7) & 1117( & 7) \\
\hline 11380 & 9) & 1127( & 9) & 1093( & 6) & 1137( & 6) \\
\hline
\end{tabular}




\begin{tabular}{|c|c|c|c|c|c|c|c|}
\hline 1194( & 6) & 1183( & 5) & 1149 & 1) & 1188( & 3) \\
\hline 1205( & 4) & 1194( & 4) & 1162( & 1) & 1207( & 8) \\
\hline 1219 & 5) & 1207( & 4) & 1174( & 3) & 1215( & 5) \\
\hline 1244( & 0) & 1231( & 0) & 1195( & 1) & 1243( & 0) \\
\hline 1263( & 13) & 1252( & 12) & 1216( & 7) & 1260( & 6) \\
\hline 1263( & 9) & 1252( & 9) & 1217( & 5) & 1260( & 14) \\
\hline 1290( & 4) & 1278( & 6) & 1231( & 20) & 1272( & 11) \\
\hline 1320( & 0) & 1305( & 0) & 1267( & 1) & 1314( & 1) \\
\hline 1321( & 1) & 1306( & 1) & 1270( & 2) & 1321( & 3) \\
\hline 1326( & 0) & 1312( & 0) & 1271( & 1) & 1322( & 0) \\
\hline 1346( & 3) & 1331( & 2) & 1290( & 1) & 1337( & 3) \\
\hline 1404( & 22) & 1391( & 20) & 1348( & 9) & 1400( & 24) \\
\hline 1411( & 1) & 1397( & 1) & 1356( & 0) & 1407( & 1) \\
\hline 1433( & 0) & 1419( & 0) & 1368( & 2) & 1425( & 0) \\
\hline 1455( & 0) & 1440( & 0) & 1401( & 2) & 1444( & 1) \\
\hline 1457( & 9) & 1442( & 10) & 1405( & 7) & 1447( & 12) \\
\hline 1470( & 0) & 1458( & 0) & 1415( & 0) & 1456( & 0) \\
\hline 1480( & 13) & 1467( & 13) & 1423( & 5) & 1462( & 9) \\
\hline 1491( & 0) & 1478( & 0) & 1434( & 2) & 1483( & 0) \\
\hline 1492( & 17) & 1478( & 17) & 1435( & 20) & 1483( & 12) \\
\hline 1660( & 43) & 1641( & 39) & 1528( & 8) & 1673( & 4) \\
\hline 1663( & 24) & 1644( & 21) & 1577( & 7) & 1676( & 54) \\
\hline 1669( & 11) & 1646( & 10) & 1579 & 14) & 1679( & 26) \\
\hline 2998( & 0) & 2980( & 1) & 2935( & 1) & 3000( & 1) \\
\hline 2999( & 31) & 2981( & 29) & 2936( & 30) & 3001( & 48) \\
\hline 3027( & 0) & 3007( & 0) & 2948( & 1) & 3044( & 7) \\
\hline 3028( & 35) & 3008( & 34) & 2949 & 52) & 3044( & 81) \\
\hline 3043( & 17) & 3023( & 14) & 2962( & 9) & 3046( & 0) \\
\hline 3044( & 58) & 3023( & 56) & 2963( & 50) & 3047( & 82) \\
\hline 3082( & 11) & 3063( & 15) & 3006( & 21) & 3101( & 46) \\
\hline 3084( & 65) & 3065( & 53) & 3007( & 58) & 3102( & 87) \\
\hline 3085( & 2) & 3071( & 2) & 3014( & 21) & 3104( & 9) \\
\hline 3087( & 3) & 3072( & 11) & 3018( & 21) & 3113( & 1) \\
\hline 3094( & 22) & 3074( & 19) & 3038( & 6) & 3114( & 15) \\
\hline 3103( & 33) & 3082( & 31) & 3039 & 4) & 3117 ( & 16) \\
\hline 3123( & 12) & 3100( & 1) & 3039 & 4) & 3119 ( & 21) \\
\hline 3123( & 33) & 3103( & 15) & 3055( & 11) & 3130( & 57) \\
\hline 3123( & 4) & 3103( & 36) & 3056( & 53) & 31360 & 27) \\
\hline 3147( & 15) & 3124( & 15) & 3060 & 17) & 3136( & 76) \\
\hline 3162( & 53) & 3143( & 49) & 3094( & 46) & 3177( & 92) \\
\hline 3162( & 23) & 3143( & 21) & 3094( & 19) & 3177( & 30) \\
\hline
\end{tabular}


Table S74. Harmonic vibrational frequencies (in $\mathrm{cm}^{-1}$ ) and infrared intensities (in parentheses in $\mathrm{km} / \mathrm{mol}$ ) for the $\left(\mathbf{C}_{4} \mathbf{H}_{6}\right)_{3} \mathbf{F e}$ structure Fe-1S.

\begin{tabular}{|c|c|c|c|c|c|c|c|}
\hline \multicolumn{2}{|c|}{ B3LYP } & \multicolumn{2}{|c|}{ B3LYP* } & \multicolumn{2}{|c|}{ BP86 } & \multicolumn{2}{|c|}{ M06L } \\
\hline 74( & 2) & 86( & 1) & 108( & 0) & 117( & 0) \\
\hline 141( & 1) & 145( & 0) & 143( & 0) & 147( & 0) \\
\hline 142( & 1) & 151( & 0) & 181( & 0) & 205( & 2) \\
\hline 1850 & 5) & 199 & 2) & 208( & 3) & 219 & 1) \\
\hline 206( & 3) & 208( & 4) & 217( & 1) & 241( & 1) \\
\hline 216( & 3) & 227( & 3) & 238( & 1) & 248( & 4) \\
\hline 233( & 1) & 241( & 3) & 267( & 2) & 271( & 5) \\
\hline 266( & 0) & 272( & 2) & 271( & 3) & 284( & 2) \\
\hline 270 & 4) & 273( & 2) & 284( & 6) & 2980 & 1) \\
\hline 283( & 2) & 287( & 4) & 293( & 6) & 313( & 9) \\
\hline 317( & 3) & 323( & 4) & 331( & 6) & 346( & 4) \\
\hline 346( & 3) & 354( & 4) & 372( & 4) & 371( & 3) \\
\hline 377( & 1) & 382( & 2) & 394( & 5) & 412( & 5) \\
\hline 402( & 4) & 411( & 6) & 421( & 10) & 439 & 7) \\
\hline 460 & 8) & 463( & 7) & 470( & 4) & 482( & 4) \\
\hline 488( & 1) & 485( & 5) & 476( & 6) & 499 & 6) \\
\hline 492( & 8) & 496( & 5) & 491( & 1) & 506( & 1) \\
\hline 502( & 1) & 503( & 1) & 498( & 1) & 520 & 0) \\
\hline 525( & 3) & 525( & 4) & 525( & 8) & 560 & 7) \\
\hline 608( & 10) & 607( & 9) & 595( & 6) & 622( & 8) \\
\hline 610 & 5) & 609 & 5) & 600( & 8) & 630 & 7) \\
\hline 656( & 4) & 652( & 3) & 640( & 3) & 669 & 5) \\
\hline 7531 & 10) & 753( & 8) & 740 & 9) & 764( & 9) \\
\hline 763( & 4) & 757( & 5) & 751( & 4) & 775( & 3) \\
\hline 785( & 14) & 785( & 14) & 771( & 9) & 799 & 7) \\
\hline 807( & 6) & 805( & 5) & 791( & 1) & 819 & 3) \\
\hline 817 & 20) & 817( & 18) & 7966 & 14) & 822( & 11) \\
\hline 823( & 1) & 822( & 3) & 807( & 3) & 835( & 3) \\
\hline 839 & 5) & 835( & 5) & 818( & 5) & 842( & 4) \\
\hline 869 & 8) & 865( & 7) & 849 & 7) & 880 & 9) \\
\hline 900 & 18) & 8961 & 14) & 870( & 3) & 895( & 10) \\
\hline 906( & 6) & 902( & 3) & 880( & 10) & 905( & 5) \\
\hline 914( & 2) & 904( & 4) & 891( & 1) & 921( & 4) \\
\hline 936( & 1) & 930( & 1) & 902( & 4) & 9360 & 1) \\
\hline 958( & 1) & 949 & 1) & 914( & 1) & 943( & 1) \\
\hline 966( & 0) & 955( & 0) & 923( & 3) & 953( & 1) \\
\hline 975( & 2) & 959 & 2) & 927( & 1) & 961( & 2) \\
\hline 9831 & 1) & 977( & 1) & 929 & 0) & 968( & 1) \\
\hline 999 & 6) & 991( & 5) & 962( & 4) & 995( & 4) \\
\hline 1007( & 1) & 998( & 1) & 972( & 1) & 1007 & 2) \\
\hline 1018( & 3) & 1010 & 3) & 987( & 2) & 1022 & 2) \\
\hline 1038( & 3) & 1031( & 3) & 1000( & 3) & 1036( & 4) \\
\hline 1053( & 4) & 1045( & 3) & 1014( & 1) & 1052 & 2) \\
\hline 1094( & 2) & 1085( & 2) & 1053( & 2) & 1090 & 2) \\
\hline 1132( & 5) & 1121( & 5) & 1081( & 4) & 1116 & 5) \\
\hline 1159( & 1) & 1150( & 2) & 1116( & 1) & 1156( & 1) \\
\hline 1178( & 1) & 1169 & 1) & 1128( & 2) & 1169 & 3) \\
\hline 1189( & 2) & 1178( & 2) & 1141( & 2) & 1185 & 2) \\
\hline
\end{tabular}




\begin{tabular}{|c|c|c|c|c|c|c|c|}
\hline 1221( & 1) & 1209 & 1) & 1176( & 2) & 1227( & 2) \\
\hline 1224( & 0) & 1215( & 0) & 1180 & 0) & 1227( & 2) \\
\hline 1245( & 4) & 12331 & 3) & 1195 & 1) & 1232( & 2) \\
\hline 1259 & 5) & 1250( & 3) & 1212( & 5) & 1249 & 2) \\
\hline 1275( & 8) & 1259 & 8) & 12160 & 2) & 1254 & 6) \\
\hline 1304( & 0) & 1289 & 0) & 1239 & 1) & 1289 & 0) \\
\hline 1321( & 5) & 1306( & 4) & 1262( & 4) & 1313 & 2) \\
\hline 1324( & 3) & 1311( & 5) & 1269 & 7) & 1318( & 4) \\
\hline 1333( & 3) & 1321( & 2) & 1277( & 3) & 1327( & 5) \\
\hline 1400( & 1) & 13860 & 0) & 1343( & 0) & 1387( & 1) \\
\hline 1404( & 2) & 1391( & 2) & 1351( & 3) & 1397( & 4) \\
\hline 1417( & 3) & 1401( & 3) & 1356( & 4) & 1409 & 4) \\
\hline 1478( & 7) & 1466( & 6) & 1423( & 4) & 1459 & 6) \\
\hline 1485( & 1) & 1473( & 1) & 1429 & 1) & 1470 & 1) \\
\hline 1489 & 6) & 1476( & 6) & 1432( & 6) & 1472( & 5) \\
\hline 1490 & 2) & 1478( & 2) & 1434( & 2) & 1473 & 1) \\
\hline 1496( & 3) & 1486( & 3) & 1445( & 4) & 1481( & 4) \\
\hline 1521( & 11) & 1515( & 10) & 1472( & 10) & 1524( & 19) \\
\hline 1536( & 23) & 1521( & 20) & 1489 & 5) & 1539 & 6) \\
\hline 1555( & 14) & 1541( & 10) & 1494( & 5) & 1567( & 7) \\
\hline 1606( & 9) & 1579 & 9) & 1520 & 9) & 1619 & 9) \\
\hline 2806( & 11) & 2743( & 11) & 2528( & 7) & 2674( & 5) \\
\hline 3008( & 52) & 2987( & 56) & 2927( & 66) & 3024 & 92) \\
\hline 3028( & 84) & 3009 & 78) & 2948( & 31) & 3041( & 111) \\
\hline 3038( & 22) & 3014( & 25) & 2954( & 78) & 3044 & 47) \\
\hline 3054( & 61) & 3034( & 58) & 2979 & 60) & 3057( & 117) \\
\hline 3067( & 22) & 3048( & 21) & 2995( & 21) & 3091( & 45) \\
\hline 3089 & 22) & 3069 & 21) & 3011( & 22) & 3098( & 28) \\
\hline 3099 & 16) & 3080( & 15) & 3026( & 17) & 3104( & 48) \\
\hline 3110 & 11) & 3091( & 11) & 3030 & 13) & 3110 & 35) \\
\hline 3122( & 33) & 3101( & 31) & 3035( & 26) & 3115 & 26) \\
\hline 3124( & 1) & 3102( & 2) & 3042( & 20) & 3123 & 48) \\
\hline 3135( & 21) & 3111( & 25) & 3050 & 29) & 3128( & 43) \\
\hline 3155( & 6) & 3133( & 9) & 3062( & 35) & 3141( & 66) \\
\hline 3159 & 15) & 3134( & 15) & 3073( & 7) & 3147( & 14) \\
\hline 3178( & 13) & 3157( & 11) & 3095 & 7) & 3175 & 34) \\
\hline 3185( & 15) & 3162( & 18) & 3099 & 33) & 3182( & 39) \\
\hline 3203 ( & 13) & 3183( & 13) & 3119 & 15) & 31960 & 27) \\
\hline 3208( & 27) & 3187( & 24) & 3122( & 27) & 3199 & 37) \\
\hline
\end{tabular}


Table S75. Harmonic vibrational frequencies (in $\mathrm{cm}^{-1}$ ) and infrared intensities (in parentheses in $\mathrm{km} / \mathrm{mol})$ for the $\left(\mathbf{C}_{4} \mathbf{H}_{6}\right)_{3} \mathbf{F e}$ structure $\mathrm{Fe}-2 \mathrm{~S}$.

\begin{tabular}{|c|c|c|c|c|c|c|c|}
\hline \multicolumn{2}{|c|}{ B3LYP } & \multicolumn{2}{|c|}{ B3LYP* } & \multicolumn{2}{|c|}{ BP86 } & \multicolumn{2}{|c|}{ M06L } \\
\hline 83( & 1) & 831 & 1) & 80( & 0) & 93( & 0) \\
\hline 111( & 0) & 105( & 0) & 108( & 0) & 129 & 0) \\
\hline 118( & 0) & 114( & 1) & 118( & 0) & 138( & 0) \\
\hline 124( & 0) & 1266 & 0) & 137( & 0) & 155( & 0) \\
\hline 203 & 2) & 199 & 1) & 204( & 1) & 221( & 2) \\
\hline 207( & 2) & 213( & 2) & 218( & 1) & 223( & 1) \\
\hline 2260 & 2) & 229( & 1) & 238( & 1) & 239 & 2) \\
\hline 247( & 6) & 2600 & 2) & 2660 & 4) & 2850 & 7) \\
\hline 259 & 1) & 264( & 8) & 294( & 15) & 297( & 2) \\
\hline 272 & 38) & 284( & 27) & 300( & 0) & 304( & 17) \\
\hline 291( & 6) & 298( & 5) & 310 & 4) & $326 c$ & 13) \\
\hline 297( & 3) & 301( & 4) & 322( & 3) & 341( & 10) \\
\hline 318( & 22) & 321( & 15) & 336( & 2) & 3431 & 8) \\
\hline 325( & 8) & 332( & 14) & 349 & 17) & 351( & 20) \\
\hline 333( & 13) & 3381 & 18) & 352( & 21) & 362( & 13) \\
\hline 345 & 13) & 345( & 11) & 364( & 15) & 3931 & 10) \\
\hline 400 & 3) & 402( & 4) & 411( & 5) & 449 & 7) \\
\hline 4480 & 7) & 448( & 7) & 450( & 7) & 474( & 6) \\
\hline 474 & 20) & 474( & 20) & 464( & 4) & 4931 & 2) \\
\hline 481( & 1) & 478( & 1) & 471( & 12) & 499( & 9) \\
\hline 4860 & 4) & 484( & 9) & 4861 & 24) & 515( & 20) \\
\hline 559 & 7) & 559 & 6) & 547( & 4) & 561( & 5) \\
\hline 6260 & 3) & 624( & 4) & 618( & 4) & 6500 & 6) \\
\hline 635( & 0) & 6331 & 0) & 628( & 0) & 663( & 1) \\
\hline 680( & 31) & 676( & 33) & 662( & 35) & 687( & 32) \\
\hline 750( & 8) & 744( & 7) & 727( & 4) & 754( & 8) \\
\hline 753( & 4) & 752( & 4) & 743( & 4) & 765( & 3) \\
\hline 770( & 4) & 761( & 4) & 753( & 6) & 784( & 7) \\
\hline 778( & 9) & 771( & 7) & 760( & 3) & 791( & 1) \\
\hline 784( & 6) & 782( & 5) & 772( & 6) & 806( & 9) \\
\hline 865( & 19) & 858( & 21) & 845( & 18) & 860( & 1) \\
\hline 872( & 1) & 866( & 2) & 849 & 3) & 868( & 2) \\
\hline 875( & 3) & 871( & 3) & 853( & 2) & 879( & 4) \\
\hline 891( & 2) & 882( & 1) & 855( & 8) & 8800 & 36) \\
\hline 895( & 1) & 887( & 1) & 859 & 40) & 885( & 1) \\
\hline 909 ( & 20) & 896( & 37) & 873( & 2) & 8866 & 2) \\
\hline 914( & 34) & 904( & 15) & 876( & 4) & 896( & 3) \\
\hline 930( & 2) & 922( & 2) & 894( & 1) & 916( & 1) \\
\hline 936( & 1) & 929) & 0) & 911( & 0) & 927( & 0) \\
\hline 945( & 1) & 939 & 1) & 922( & 2) & 944( & 1) \\
\hline 954( & 0) & 9466 & 0) & 924( & 0) & 957( & 0) \\
\hline 959 ( & 0) & 953( & 0) & 931( & 0) & 960( & 1) \\
\hline 992( & 4) & 976( & 5) & 940( & 8) & 978( & 6) \\
\hline 1006( & 3) & 984( & 3) & 961( & 5) & 991( & 1) \\
\hline 1032( & 11) & 1016( & 11) & 987( & 12) & 1026( & 8) \\
\hline 1066( & 4) & 1057( & 4) & 1027( & 5) & 1057( & 2) \\
\hline
\end{tabular}




\begin{tabular}{|c|c|c|c|c|c|c|c|}
\hline 1075( & 3) & 1065( & 4) & 1033( & 5) & 1063( & 5) \\
\hline 1079( & 1) & 1070( & 0) & 1040( & 0) & 1070( & 0) \\
\hline 1087( & 4) & 1076( & 3) & 1049( & 2) & 1082( & 2) \\
\hline 1088( & 1) & 1078( & 1) & 1052( & 2) & 1088( & 5) \\
\hline 1115( & 2) & 1107( & 3) & 1079( & 5) & 1110( & 5) \\
\hline 1222( & 9) & 1209( & 8) & 1173( & 6) & 1209 ( & 7) \\
\hline 1230( & 18) & 1218( & 13) & 1181( & 7) & 1217( & 12) \\
\hline 1251( & 24) & 1240( & 23) & 1209 & 10) & 1247( & 16) \\
\hline 1272( & 8) & 1257( & 6) & 1217( & 4) & 1254( & 4) \\
\hline 1280( & 24) & 1264( & 21) & 1227( & 16) & 1264( & 28) \\
\hline 1322( & 3) & 1311( & 3) & 1277( & 2) & 1316( & 3) \\
\hline 1410( & 1) & 1395( & 1) & 1351( & 1) & 1389( & 0) \\
\hline 1415( & 4) & 1401( & 2) & 1360( & 1) & 1393( & 2) \\
\hline 1422( & 5) & 1411( & 5) & 1372( & 2) & 1410( & 1) \\
\hline 1454( & 11) & 1443( & 11) & 1407( & 9) & 1446( & 7) \\
\hline 1479( & 1) & 1467( & 2) & 1431( & 6) & 1467( & 2) \\
\hline 1481( & 15) & 1469( & 12) & 1434( & 4) & 1471( & 6) \\
\hline 1546( & 36) & 1532( & 26) & 1479( & 5) & 1524( & 7) \\
\hline 1547( & 14) & 1532( & 3) & 1487( & 10) & 1526( & 6) \\
\hline 1550( & 3) & 1535( & 19) & 1488( & 8) & 1529 ( & 26) \\
\hline 1555( & 17) & 1538( & 10) & 1494( & 10) & 1535( & 14) \\
\hline 1561( & 14) & 1542( & 12) & 1498( & 7) & 1537( & 8) \\
\hline 1668( & 45) & 1653( & 46) & 1608( & 44) & 1681( & 50) \\
\hline 3133( & 16) & 3110( & 16) & 3047( & 26) & 3118( & 41) \\
\hline 3142( & 10) & 3118( & 11) & 3052( & 8) & 3138( & 38) \\
\hline 3153( & 5) & 3131( & 6) & 3072( & 9) & 3140( & 38) \\
\hline 3157( & 14) & 3136( & 15) & 3073( & 29) & 3142( & 7) \\
\hline 3159 ( & 6) & 31380 & 4) & 3074( & 20) & 3147( & 29) \\
\hline 3164( & 4) & 3140( & 4) & 3075( & 3) & 3150( & 29) \\
\hline 3168( & 12) & 3142( & 14) & 3075( & 13) & 3152( & 23) \\
\hline 3172( & 7) & 3147( & 8) & 3079) & 6) & 3161( & 25) \\
\hline 3178( & 6) & 3154( & 6) & 3090( & 7) & 3173( & 14) \\
\hline 3194( & 3) & 3172( & 2) & 3112( & 1) & 3188( & 4) \\
\hline 3196( & 9) & 3173( & 9) & 3113( & 10) & 3195( & 24) \\
\hline 3212( & 4) & 3188( & 4) & 3123( & 7) & 3200( & 18) \\
\hline 3225( & 15) & 3200( & 16) & 3128( & 19) & 3211( & 24) \\
\hline 3243( & 11) & 3222( & 13) & 3158( & 16) & 3230( & 26) \\
\hline 3244( & 17) & 3223( & 13) & 3160( & 12) & 3235( & 14) \\
\hline 3248( & 2) & 3227( & 3) & 3161( & 0) & 3240( & 10) \\
\hline 3264( & 6) & 3236( & 6) & 3164( & 3) & 3244( & 12) \\
\hline 3264( & 3) & 32371 & 3) & 31690 & 14) & 32500 & 28) \\
\hline
\end{tabular}


Table S76. Harmonic vibrational frequencies (in $\mathrm{cm}^{-1}$ ) and infrared intensities (in parentheses in $\mathrm{km} / \mathrm{mol})$ for the $\left(\mathbf{C}_{4} \mathbf{H}_{6}\right)_{3} \mathbf{F e}$ structure $\mathrm{Fe}-1 \mathrm{~T}$.

\begin{tabular}{|c|c|c|c|c|c|c|c|}
\hline \multicolumn{2}{|c|}{ B3LYP } & \multicolumn{2}{|c|}{ B3LYP* } & \multicolumn{2}{|c|}{ BP86 } & \multicolumn{2}{|c|}{ M06L } \\
\hline 68( & 0) & 71( & 0) & 78( & 1) & -95( & 2) \\
\hline 104( & 1) & 124( & 1) & 126( & 1) & 87( & 1) \\
\hline 129 & 0) & 127( & 1) & 134( & 1) & 135( & 1) \\
\hline 162( & 1) & 165( & 1) & 170 & 1) & 157( & 3) \\
\hline 176( & 1) & 188( & 3) & 198( & 1) & 187( & 0) \\
\hline 190( & 0) & 194( & 0) & 207( & 0) & 197( & 1) \\
\hline 209 & 1) & 205( & 0) & 233( & 1) & 211( & 2) \\
\hline 233( & 1) & 233 ( & 1) & 249 & 0) & 226( & 2) \\
\hline 2560 & 0) & 2550 & 0) & 257( & 2) & 2670 & 0) \\
\hline 280 & 1) & 283( & 1) & 282( & 1) & 298 ( & 1) \\
\hline 285( & 0) & 286( & 1) & 309 & 2) & 312( & 1) \\
\hline 299 & 2) & 301( & 1) & 324( & 3) & 331( & 1) \\
\hline 331( & 1) & 337( & 0) & 351( & 1) & 355( & 0) \\
\hline 3560 & 3) & 3581 & 2) & 361( & 1) & 378( & 1) \\
\hline 407( & 3) & 412( & 3) & 418( & 4) & 423( & 4) \\
\hline 460( & 2) & 456( & 3) & 446( & 2) & 459 & 3) \\
\hline 471( & 4) & 468( & 3) & 462( & 3) & 473( & 2) \\
\hline 488( & 4) & 483( & 4) & 487( & 4) & 499( & 5) \\
\hline 510 & 2) & 507( & 2) & 4961 & 3) & 517( & 2) \\
\hline 580( & 6) & 574( & 5) & 561( & 4) & 575( & 7) \\
\hline 605( & 0) & 599 & 0) & 575( & 1) & 604( & 0) \\
\hline 667( & 2) & 661( & 2) & 640( & 1) & 666( & 2) \\
\hline 705( & 6) & 696( & 3) & 669 & 1) & 688( & 3) \\
\hline 722( & 2) & 714( & 2) & 706( & 3) & 716( & 2) \\
\hline 754( & 21) & 756( & 20) & 749 & 15) & 747( & 15) \\
\hline 786( & 3) & 781( & 1) & 767( & 4) & 787( & 1) \\
\hline 791( & 11) & 788( & 13) & 780 & 12) & 802( & 12) \\
\hline 818( & 15) & 811( & 12) & 797( & 10) & 822( & 10) \\
\hline 828( & 5) & 824( & 5) & 802( & 3) & 824( & 3) \\
\hline 844( & 24) & 838( & 21) & 823( & 15) & 842( & 19) \\
\hline 852( & 1) & 847( & 1) & 834( & 4) & 855( & 2) \\
\hline 871( & 5) & 863( & 7) & 847( & 7) & 870( & 10) \\
\hline 903( & 2) & 895( & 2) & 870( & 0) & 894( & 2) \\
\hline 941( & 1) & 929 & 0) & 8751 & 1) & 898( & 3) \\
\hline 949 & 0) & 933( & 1) & 888( & 6) & 908( & 2) \\
\hline 956( & 2) & 945( & 4) & 902( & 2) & 936( & 2) \\
\hline 970( & 3) & 946( & 1) & 923( & 3) & 955( & 2) \\
\hline 993( & 4) & 977( & 2) & 931( & 0) & 957( & 0) \\
\hline 1001( & 2) & 989 & 2) & 962( & 4) & 999( & 3) \\
\hline 1009( & 1) & 995( & 2) & 968( & 2) & 1000( & 2) \\
\hline 1025( & 7) & 1014( & 7) & 988( & 4) & 1026( & 2) \\
\hline 1035( & 7) & 1024( & 6) & 999( & 8) & 1035( & 17) \\
\hline 1052( & 8) & 1042( & 8) & 1013( & 7) & 1050( & 5) \\
\hline 1104( & 1) & 1093( & 1) & 1063( & 1) & 1103( & 1) \\
\hline 1144( & 6) & 1133( & 6) & 1102( & 7) & 1140( & 8) \\
\hline 1159 & 6) & 1148( & 5) & 1117 & 3) & 1157( & 6) \\
\hline 1182( & 1) & 1172( & 2) & 1145( & 3) & 1185( & 3) \\
\hline 1208( & 0) & 1193( & 0) & 1156( & 1) & 1209( & 1) \\
\hline
\end{tabular}




\begin{tabular}{|c|c|c|c|c|c|c|c|}
\hline 1223( & 1) & 1211( & 1) & 1177( & 0) & 1229 & 0) \\
\hline 1231( & 2) & 1218( & 2) & 1185( & 3) & 1232( & 5) \\
\hline 1248( & 5) & 1237( & 4) & 1204( & 2) & 1243 & 4) \\
\hline 1267( & 10) & 1254( & 9) & 1219 & 5) & 1247( & 8) \\
\hline 1318( & 0) & 1301( & 0) & 1256( & 0) & 1302( & 6) \\
\hline 1321( & 3) & 1305( & 4) & 1262( & 2) & 1312( & 0) \\
\hline 1331( & 2) & 1315( & 4) & 1271( & 8) & 1324( & 3) \\
\hline 1332( & 6) & 1317( & 5) & 1273 & 4) & 1330 & 5) \\
\hline 1350( & 6) & 13331 & 5) & 1289 & 3) & 1348( & 4) \\
\hline 1394( & 1) & 1382( & 2) & 1342( & 2) & 1385( & 2) \\
\hline 1406( & 3) & 1392( & 3) & 1354( & 4) & 1396( & 4) \\
\hline 1434( & 2) & 1416( & 2) & 1369 & 3) & 1427( & 3) \\
\hline 1467( & 5) & 1453( & 3) & 1414( & 1) & 1456( & 5) \\
\hline 1475( & 2) & 1464( & 1) & 1422( & 1) & 1461( & 3) \\
\hline 1479 & 2) & 1465( & 4) & 1426( & 3) & 1466( & 3) \\
\hline 1482( & 6) & 1468( & 6) & 1430 & 7) & 1468( & 5) \\
\hline 1487( & 2) & 1476( & 2) & 1437( & 1) & 1473( & 1) \\
\hline 1499 & 9) & 1488( & 9) & 1448( & 8) & 1491( & 7) \\
\hline 1532( & 29) & 1516( & 27) & 1469 & 20) & 1512( & 21) \\
\hline 1535( & 25) & 1525( & 21) & 1483( & 12) & 1523 & 14) \\
\hline 1666( & 2) & 1627( & 4) & 1543( & 5) & 1634( & 9) \\
\hline 3015 & 35) & 29931 & 40) & 2934( & 52) & 3024( & 61) \\
\hline 3021( & 21) & 3001( & 27) & 2943( & 46) & 3026( & 48) \\
\hline 3031( & 106) & 3012( & 108) & 2957( & 82) & 3037( & 200) \\
\hline 3033( & 53) & 3014( & 44) & 2962( & 72) & 3039 & 81) \\
\hline 3075( & 14) & 3056 & 17) & 3001( & 23) & 3087( & 19) \\
\hline 3077 ( & 28) & 3057( & 21) & 3007( & 6) & 3093 & 39) \\
\hline 3100( & 13) & 3077( & 13) & 3022 & 9) & 3104 & 36) \\
\hline 3117 ( & 16) & 3094( & 18) & 3030( & 30) & 3119 & 16) \\
\hline 3120 & 2) & 3103( & 3) & 3044( & 9) & 3131( & 15) \\
\hline 3123( & 12) & 3106( & 9) & 3057( & 3) & 3133 & 35) \\
\hline 31331 & 6) & 3111( & 6) & 3061( & 5) & 3136( & 15) \\
\hline 3148( & 9) & 31260 & 8) & 3062 & 8) & 3138( & 18) \\
\hline 3151( & 2) & 3129 & 1) & 3064( & 21) & 3143( & 18) \\
\hline 3158( & 34) & 3135( & 29) & 3067( & 17) & 3158( & 36) \\
\hline 3161( & 14) & 3141( & 17) & 3086( & 21) & 3166( & 41) \\
\hline 3170( & 17) & 3151( & 16) & 3089 & 18) & 3173 & 40) \\
\hline 3232( & 11) & 3212( & 10) & 3152( & 7) & 3224 & 20) \\
\hline 3239 & 12) & 3217( & 12) & 3153( & 15) & 3229 & 20) \\
\hline
\end{tabular}


Table S77. Harmonic vibrational frequencies (in $\mathrm{cm}^{-1}$ ) and infrared intensities (in parentheses in $\mathrm{km} / \mathrm{mol})$ for the $\left(\mathbf{C}_{4} \mathbf{H}_{6}\right)_{3} \mathbf{F e}$ structure $\mathrm{Fe}-2 \mathrm{~T}$.

\begin{tabular}{|c|c|c|c|c|c|c|c|}
\hline \multicolumn{2}{|c|}{ B3LYP } & \multicolumn{2}{|c|}{ B3LYP* } & \multicolumn{2}{|c|}{ BP86 } & \multicolumn{2}{|c|}{ M06L } \\
\hline 610 & 0) & 51( & 0) & 40( & 0) & -483( & 18) \\
\hline 73( & 0) & 69 & 0) & 55( & 0) & 67( & 0) \\
\hline 101( & 0) & 97( & 0) & 87( & 0) & 109 & 0) \\
\hline 120( & 1) & 120( & 1) & 117( & 2) & 116( & 1) \\
\hline 142( & 3) & 137( & 2) & 127( & 1) & 143( & 3) \\
\hline 181( & 0) & 176( & 0) & 165( & 0) & 187( & 0) \\
\hline 224( & 4) & 223( & 3) & 223( & 2) & 206( & 0) \\
\hline 253( & 1) & 255( & 1) & 248( & 0) & 2400 & 2) \\
\hline 268( & 1) & 270( & 1) & 261( & 1) & 277( & 0) \\
\hline 285( & 0) & 290( & 0) & 289( & 4) & 307( & 0) \\
\hline 295( & 8) & 291( & 7) & 3031 & 0) & 3131 & 5) \\
\hline 325( & 0) & 323( & 0) & 316( & 1) & 3431 & 1) \\
\hline 343( & 10) & 345( & 9) & 356( & 6) & 365( & 0) \\
\hline 370( & 6) & 372( & 6) & 371( & 2) & 400( & 3) \\
\hline 413( & 0) & 415( & 0) & 416( & 1) & 435( & 12) \\
\hline 459 & 14) & 454( & 3) & 4371 & 1) & 436( & 1) \\
\hline 460( & 3) & 460( & 14) & 473( & 5) & 483( & 5) \\
\hline 495( & 4) & 493( & 6) & 482( & 12) & 504( & 6) \\
\hline 502( & 7) & 4931 & 3) & 483( & 3) & 511( & 2) \\
\hline 559( & 4) & 554( & 4) & 533( & 5) & 542( & 1) \\
\hline 565( & 1) & 560( & 1) & 543( & 0) & 567( & 3) \\
\hline 610( & 1) & 605( & 1) & 593( & 0) & 619 & 1) \\
\hline 705( & 40) & 698( & 41) & 675( & 40) & 685( & 15) \\
\hline 730( & 13) & 723( & 12) & 698( & 17) & 712( & 21) \\
\hline 733( & 10) & 729 & 9) & 708( & 7) & 734( & 9) \\
\hline 796( & 4) & 791( & 4) & 775( & 5) & 805( & 4) \\
\hline 816( & 14) & 810 & 15) & 788( & 14) & 817( & 14) \\
\hline 834( & 5) & 829 & 3) & 809 & 5) & 827( & 0) \\
\hline 839 & 5) & 834( & 4) & 821( & 2) & 834( & 2) \\
\hline 848( & 9) & 838( & 10) & 825( & 3) & 837( & 2) \\
\hline 876( & 5) & 863( & 6) & 845( & 4) & 860( & 11) \\
\hline 877( & 2) & 871( & 2) & 852( & 12) & 876( & 9) \\
\hline 881( & 16) & 873( & 14) & 857( & 2) & 882( & 2) \\
\hline 962( & 4) & 946( & 3) & 8971 & 4) & 910( & 2) \\
\hline 966( & 7) & 955( & 4) & 909( & 1) & 918( & 2) \\
\hline 977( & 4) & 966( & 6) & 938( & 11) & 973( & 7) \\
\hline 986( & 1) & 978( & 1) & 9551 & 0) & 9866 & 1) \\
\hline 998( & 2) & 987( & 2) & 957( & 3) & 988( & 2) \\
\hline 1002( & 3) & 990( & 2) & 959( & 2) & 990( & 4) \\
\hline 1011( & 11) & 1002( & 12) & 973( & 11) & 1008( & 11) \\
\hline 1033( & 2) & 1025( & 2) & 9951 & 3) & 1035( & 3) \\
\hline 1054( & 5) & 1047( & 5) & 1022( & 5) & 1059 & 12) \\
\hline 1059 & 5) & 1052( & 5) & 1032( & 5) & 1062( & 4) \\
\hline 1097( & 1) & 1088( & 0) & 1062( & 0) & 1098( & 1) \\
\hline 1150( & 2) & 1141( & 2) & 1111( & 3) & 1142( & 2) \\
\hline 1166( & 6) & 1156 & 7) & 1127( & 9) & 1164( & 8) \\
\hline
\end{tabular}




\begin{tabular}{|c|c|c|c|c|c|c|c|}
\hline 1186( & 6) & 1176( & 5) & 1148( & 6) & 1189 & 4) \\
\hline 1221( & 1) & 1210( & 2) & 1181( & 1) & 1215( & 0) \\
\hline 1233( & 14) & 1221( & 12) & 1184( & 6) & 1216( & 10) \\
\hline 1234( & 2) & 1222( & 3) & 1185( & 8) & 1220( & 4) \\
\hline 1246( & 3) & 1236( & 2) & 1199 & 2) & 1249 ( & 0) \\
\hline 1248( & 2) & 12380 & 2) & 1200( & 3) & 1250( & 2) \\
\hline 1280( & 7) & 1268( & 7) & 1234( & 10) & 1284( & 10) \\
\hline 1326( & 1) & 1313( & 2) & 1273( & 1) & 1324( & 3) \\
\hline 1340( & 5) & 1328( & 4) & 1283( & 4) & 1339x & 5) \\
\hline 1349( & 19) & 1336( & 18) & 1292( & 17) & 1342( & 20) \\
\hline 1354( & 1) & 1340( & 1) & 1303( & 1) & 1360( & 3) \\
\hline 1393( & 0) & 1380( & 0) & 1338( & 0) & 1378( & 3) \\
\hline 1397( & 2) & 1384( & 2) & 1342( & 2) & 1379( & 1) \\
\hline 1429( & 1) & 1416( & 1) & 1376( & 2) & 1433( & 1) \\
\hline 1462( & 3) & 1448( & 3) & 1401( & 1) & 1448( & 0) \\
\hline 1463( & 0) & 1449 & 0) & 1402( & 0) & 1451( & 1) \\
\hline 1474( & 3) & 1459 & 2) & 1414( & 1) & 1467( & 5) \\
\hline 1480( & 1) & 1467( & 1) & 1424( & 1) & 1468( & 1) \\
\hline 1486( & 3) & 1473( & 4) & 1431( & 2) & 1478( & 2) \\
\hline 1496( & 17) & 1480( & 17) & 1437( & 16) & 1493( & 18) \\
\hline 1519( & 3) & 1505( & 3) & 1455( & 5) & 1495( & 23) \\
\hline 1521( & 53) & 1507( & 49) & 1456( & 36) & 1500( & 3) \\
\hline 1720( & 1) & 1706( & 1) & 1658( & 1) & 1749( & 2) \\
\hline 3018( & 3) & 2999 & 2) & 2940( & 52) & 3033( & 0) \\
\hline 3019) & 26) & 3000 & 26) & 2940( & 6) & 3037( & 17) \\
\hline 3027( & 107) & 3006( & 100) & 2951( & 58) & 3037 ( & 161) \\
\hline 3029 & 41) & 3009 & 39) & 2953( & 62) & 3045( & 81) \\
\hline 3069 ( & 21) & 3050 & 27) & 2997( & 21) & 3085( & 74) \\
\hline 3071( & 20) & 3052( & 16) & 2998( & 15) & 3095( & 25) \\
\hline 3086( & 11) & 3064( & 8) & 3014( & 8) & 30999 & 0) \\
\hline 3104( & 40) & 3079 & 42) & 3021( & 38) & 3106( & 4) \\
\hline 3108( & 1) & 3087( & 1) & 3026( & 2) & 3106( & 25) \\
\hline 3109 & 20) & 3088( & 19) & 3027( & 21) & 3112( & 66) \\
\hline 3118( & 1) & 3096( & 1) & 3036( & 1) & 3132( & 36) \\
\hline 3119 ( & 12) & 3097( & 12) & 3037( & 14) & 3132( & 2) \\
\hline 3122( & 10) & 3101( & 10) & 3048( & 10) & 3135( & 21) \\
\hline 3149 & 34) & 3128( & 31) & 3065( & 1) & 3151( & 89) \\
\hline 3153( & 1) & 3131( & 1) & 3065( & 32) & 3154( & 4) \\
\hline 3153( & 52) & 3131( & 52) & 3075( & 57) & 3162( & 89) \\
\hline 3222( & 0) & 3201( & 0) & 3144( & 1) & 3227( & 57) \\
\hline 3223( & 22) & 32020 & 21) & 3145( & 22) & 32280 & 1) \\
\hline
\end{tabular}


Table S78. Harmonic vibrational frequencies (in $\mathrm{cm}^{-1}$ ) and infrared intensities (in parentheses in $\mathrm{km} / \mathrm{mol})$ for the $\left(\mathbf{C}_{4} \mathbf{H}_{6}\right)_{3} \mathbf{F e}$ structure $\mathrm{Fe}-3 \mathrm{~T}$.

\begin{tabular}{|c|c|c|c|c|c|c|c|}
\hline \multicolumn{2}{|c|}{ B3LYP } & \multicolumn{2}{|c|}{ B3LYP* } & \multicolumn{2}{|c|}{ BP86 } & \multicolumn{2}{|c|}{ M06L } \\
\hline 29( & 0) & 30( & 0) & 381 & 0) & -47( & 1) \\
\hline 39( & 0) & 41( & 0) & 64( & 0) & 53( & 0) \\
\hline 77( & 0) & 75( & 0) & 85( & 0) & 98( & 0) \\
\hline 80( & 0) & 80( & 0) & 94( & 0) & 104( & 0) \\
\hline 98( & 1) & 99( & 1) & 112( & 1) & 113( & 0) \\
\hline 132( & 2) & 131( & 2) & 145( & 2) & 134( & 2) \\
\hline 153( & 0) & 154( & 0) & 157( & 3) & 152( & 0) \\
\hline 168( & 3) & 172( & 2) & 185( & 1) & 162( & 2) \\
\hline 174( & 1) & 180( & 1) & 193( & 2) & 177( & 1) \\
\hline 207( & 0) & 213( & 1) & 206( & 0) & 216( & 1) \\
\hline 219 ( & 2) & 225( & 2) & 245( & 1) & 227( & 1) \\
\hline 237( & 1) & 246( & 2) & 272( & 1) & 280( & 1) \\
\hline 275( & 1) & 273( & 1) & 278( & 2) & 287( & 1) \\
\hline 289 ( & 1) & 290( & 0) & 289 & 2) & 305( & 1) \\
\hline 294( & 0) & 298( & 0) & 312( & 1) & 308( & 2) \\
\hline 309 ( & 16) & 313( & 17) & 339 & 7) & 337( & 5) \\
\hline 359( & 4) & 362( & 5) & 365( & 2) & 383( & 5) \\
\hline 376( & 6) & 383( & 7) & 394( & 5) & 397( & 3) \\
\hline 405( & 1) & 410( & 1) & 404( & 10) & 429 & 3) \\
\hline 429( & 1) & 435( & 1) & 450( & 4) & 461( & 1) \\
\hline 451( & 1) & 455( & 1) & 467( & 2) & 471( & 1) \\
\hline 554( & 6) & 550( & 3) & 5371 & 5) & 550( & 2) \\
\hline 556( & 3) & 550( & 5) & 542( & 3) & 556( & 4) \\
\hline 607( & 5) & 603( & 5) & 588( & 4) & 612( & 6) \\
\hline 639 & 16) & 641( & 13) & 619 & 15) & 645( & 12) \\
\hline 654( & 25) & 651( & 27) & 639 & 17) & 650 & 24) \\
\hline 685( & 5) & 686( & 5) & 681( & 3) & 681( & 3) \\
\hline 714( & 0) & 711( & 1) & 700( & 2) & 706( & 12) \\
\hline 722( & 15) & 721( & 11) & 709 & 7) & 713( & 3) \\
\hline 742( & 2) & 737( & 3) & 718( & 6) & 730( & 1) \\
\hline 837( & 15) & 839 & 12) & 829 & 1) & 820( & 0) \\
\hline 848( & 11) & 844( & 16) & 8331 & 42) & 825( & 17) \\
\hline 870( & 0) & 864( & 0) & 837( & 9) & 843( & 5) \\
\hline 872( & 0) & 865( & 0) & 842( & 12) & 855( & 19) \\
\hline 877( & 5) & 873 & 3) & 851( & 12) & 860( & 13) \\
\hline 897( & 35) & 891( & 43) & 855( & 23) & 867( & 44) \\
\hline 903( & 8) & 895( & 46) & 856( & 28) & 873( & 4) \\
\hline 906( & 56) & 8981 & 8) & 862( & 5) & 880 & 2) \\
\hline 909( & 7) & 901( & 7) & 865( & 3) & 891( & 5) \\
\hline 910( & 4) & 908( & 5) & 883 & 7) & 898( & 7) \\
\hline 927( & 5) & 926( & 3) & 907( & 2) & 911( & 6) \\
\hline 935( & 2) & 933 & 5) & 912( & 1) & 935( & 1) \\
\hline 956( & 1) & 947( & 2) & 917( & 3) & 946( & 2) \\
\hline 1015 ( & 12) & 1005 & 12) & 983( & 15) & 1009 & 9) \\
\hline 1029 ( & 11) & 1019 & 11) & 984( & 9) & 1018( & 9) \\
\hline 1057 ( & 3) & 1050 & 1) & 1023( & 2) & 1047( & 2) \\
\hline
\end{tabular}




\begin{tabular}{|c|c|c|c|c|c|c|c|}
\hline 1059 & 2) & 1051( & 4) & 1027( & 2) & 1051( & 3) \\
\hline 1065( & 1) & 1057( & 1) & 1034( & 2) & 1062( & 3) \\
\hline 1083( & 8) & 1074( & 8) & 1044( & 7) & 1074( & 6) \\
\hline 1093( & 5) & 1085( & 5) & 1059( & 5) & 1087( & 4) \\
\hline 1096( & 1) & 1090( & 2) & 1063( & 6) & 1092( & 1) \\
\hline 1225( & 8) & 1214( & 7) & 1182( & 6) & 1214( & 8) \\
\hline 1239 & 60) & 1231( & 44) & 1198( & 21) & 1233( & 32) \\
\hline 1250( & 30) & 1242( & 28) & 1205( & 26) & 1238( & 17) \\
\hline 1276( & 23) & 1264( & 18) & 1233( & 9) & 1263( & 15) \\
\hline 1323( & 6) & 1313( & 4) & 1279 & 3) & 1317( & 4) \\
\hline 1325( & 4) & 1315( & 4) & 1280( & 3) & 1320( & 4) \\
\hline 1411( & 11) & 1399( & 6) & 1358( & 1) & 1392( & 1) \\
\hline 1412( & 4) & 1400( & 4) & 1359( & 8) & 1394( & 3) \\
\hline 1416( & 20) & 1406( & 14) & 1362( & 7) & 1399( & 11) \\
\hline 1451( & 20) & 1440( & 13) & 1405( & 5) & 1443( & 8) \\
\hline 1454( & 21) & 1444( & 17) & 1407( & 19) & 1448( & 15) \\
\hline 1465( & 17) & 1455( & 13) & 1423( & 7) & 1459( & 9) \\
\hline 1534( & 50) & 1521( & 41) & 1478( & 30) & 1518( & 7) \\
\hline 1539 ( & 24) & 1525( & 11) & 1483( & 6) & 1520( & 36) \\
\hline 1547( & 70) & 1536( & 5) & 1490( & 13) & 1531( & 6) \\
\hline 1550( & 4) & 1538( & 59) & 1492( & 30) & 1533( & 57) \\
\hline 1659 & 61) & 1647( & 48) & 1606( & 60) & 1669 & 71) \\
\hline 1662( & 41) & 1651( & 40) & 1608( & 27) & 1672( & 37) \\
\hline 3142( & 9) & 3118( & 1) & 3041( & 8) & 3113( & 8) \\
\hline 3143( & 2) & 3120( & 8) & 3048( & 5) & 3127( & 24) \\
\hline 31460 & 14) & 3124( & 14) & 3058( & 12) & 3138( & 10) \\
\hline 3148( & 6) & 3126( & 8) & 3062( & 5) & 3144( & 19) \\
\hline 3153( & 2) & 3131( & 2) & 3063( & 4) & 3147( & 17) \\
\hline 3154( & 9) & 3132( & 9) & 3065( & 7) & 3149 & 13) \\
\hline 3158( & 4) & 3137( & 5) & 3065( & 20) & 3152( & 12) \\
\hline 3159 & 2) & 3138( & 2) & 3080( & 4) & 3162( & 18) \\
\hline 3162( & 3) & 3140( & 2) & 3083( & 2) & 3168( & 13) \\
\hline 3174( & 2) & 3151( & 2) & 3087( & 4) & 3179 & 4) \\
\hline 3181( & 3) & 3162( & 2) & 3096( & 3) & 3186( & 17) \\
\hline 31900 & 13) & 3168( & 16) & 3111( & 11) & 3197( & 38) \\
\hline 3235( & 3) & 3213( & 3) & 3150( & 8) & 3222( & 12) \\
\hline 3239 & 5) & 3214( & 4) & 3151( & 2) & 3225( & 13) \\
\hline 3244( & 8) & 3221( & 8) & 31566 & 5) & 3233( & 15) \\
\hline 3248( & 11) & 3228( & 11) & 3159 & 6) & 3247( & 21) \\
\hline 3249 & 8) & 3229 & 8) & 3172( & 13) & 3251( & 22) \\
\hline 3252 ( & 3) & 32300 & 4) & 31750 & 10) & 3251( & 9) \\
\hline
\end{tabular}


Table S79. Harmonic vibrational frequencies (in $\mathrm{cm}^{-1}$ ) and infrared intensities (in parentheses in $\mathrm{km} / \mathrm{mol})$ for the $\left(\mathbf{C}_{4} \mathbf{H}_{6}\right)_{3} \mathbf{F e}$ structure $\mathrm{Fe}-1 \mathrm{P}$.

\begin{tabular}{|c|c|c|c|c|c|c|c|}
\hline \multicolumn{2}{|c|}{ B3LYP } & \multicolumn{2}{|c|}{ B3LYP* } & \multicolumn{2}{|c|}{ BP86 } & \multicolumn{2}{|c|}{ M06L } \\
\hline 54( & 1) & 55( & 1) & 631 & 0) & 33( & 0) \\
\hline 84( & 0) & 86( & 0) & 94( & 0) & 96( & 0) \\
\hline 102( & 0) & 100( & 0) & 108( & 0) & 108( & 0) \\
\hline 115( & 2) & 118( & 1) & 134( & 0) & 128( & 1) \\
\hline 140( & 0) & 142( & 0) & 151( & 0) & 153( & 0) \\
\hline 159( & 0) & 165( & 0) & 170( & 0) & 170( & 0) \\
\hline 175( & 1) & 182( & 1) & 200( & 0) & 202( & 0) \\
\hline 222( & 2) & 218( & 1) & 212( & 1) & 217( & 1) \\
\hline 231( & 1) & 231( & 1) & 233 ( & 1) & 233( & 0) \\
\hline 242( & 3) & 249 & 2) & 249 & 0) & 255( & 1) \\
\hline 261( & 1) & 272( & 3) & 281( & 0) & 287( & 0) \\
\hline 291( & 1) & 288( & 2) & 309 & 3) & 314( & 3) \\
\hline 358( & 1) & 359( & 1) & 366( & 1) & 373( & 2) \\
\hline 366( & 0) & 367( & 1) & 369 & 1) & 381( & 2) \\
\hline 415( & 6) & 409( & 13) & 396( & 6) & 412( & 9) \\
\hline 418( & 23) & 414( & 8) & 406( & 2) & 420( & 1) \\
\hline 457( & 8) & 451( & 7) & 441( & 3) & 450( & 6) \\
\hline 485( & 12) & 474( & 6) & 461( & 3) & 483( & 7) \\
\hline 522( & 4) & 513( & 2) & 494( & 1) & 524( & 4) \\
\hline 577( & 7) & 572( & 6) & 556( & 4) & 574( & 6) \\
\hline 605( & 0) & 599 & 0) & 579 & 0) & 597( & 0) \\
\hline 638( & 1) & 637( & 1) & 624( & 14) & 6351 & 1) \\
\hline 667( & 8) & 659 & 9) & 642( & 19) & 661( & 8) \\
\hline 682( & 15) & 675( & 12) & 660( & 6) & 681( & 12) \\
\hline 709 & 38) & 704( & 33) & 681( & 18) & 706( & 21) \\
\hline 737( & 49) & 723( & 58) & 704( & 57) & 720 & 53) \\
\hline 742( & 16) & 740( & 9) & 734( & 8) & 742( & 5) \\
\hline 797( & 7) & 790( & 8) & 773 ( & 8) & 797( & 5) \\
\hline 821( & 5) & 810( & 6) & 789 & 8) & 805( & 16) \\
\hline 844( & 28) & 824( & 26) & 803 ( & 22) & 818( & 21) \\
\hline 856( & 0) & 8481 & 37) & 821( & 18) & 847( & 25) \\
\hline 862( & 63) & 850( & 9) & 836( & 3) & 870( & 6) \\
\hline 906( & 4) & 895( & 3) & 865( & 2) & 897( & 2) \\
\hline 938( & 4) & 927( & 7) & 897( & 7) & 925( & 3) \\
\hline 944( & 4) & 9371 & 4) & 908( & 2) & 9381 & 5) \\
\hline 966( & 5) & 954( & 4) & 912( & 3) & 942( & 6) \\
\hline 981( & 1) & 960( & 2) & 919 & 5) & 954( & 4) \\
\hline 985( & 2) & 967( & 3) & 928( & 3) & 971( & 3) \\
\hline 1003( & 9) & 993( & 9) & 969 & 7) & 1005( & 2) \\
\hline 1010 & 6) & 1000 & 3) & 973( & 3) & 1010 & 7) \\
\hline 1017( & 7) & 1010 & 7) & 987( & 6) & 1024( & 10) \\
\hline 1051( & 5) & 1039 & 3) & 1010 & 2) & 1045( & 3) \\
\hline 1052( & 4) & 1044( & 5) & 1019 & 4) & 1058( & 3) \\
\hline 1085 & 1) & 1077( & 2) & 1052( & 2) & 1091( & 2) \\
\hline 1128 & 13) & 1119 & 10) & 1095 & 3) & 1129 & 5) \\
\hline 1144( & 11) & 1135 & 13) & 1108( & 14) & 1150 & 14) \\
\hline 1185 & 8) & 1173( & 7) & 1141( & 4) & 1183 & 7) \\
\hline 1211( & 1) & 1198( & 1) & 1162( & 0) & 1207 & 1) \\
\hline
\end{tabular}




\begin{tabular}{|c|c|c|c|c|c|c|c|}
\hline 1223( & 3) & 1210 & 4) & 1172( & 4) & 1225 & 3) \\
\hline 1232( & 1) & 1220 & 1) & 1183( & 1) & 1233 & 3) \\
\hline 1269 & 8) & 1256( & 8) & 1221( & 5) & 1255( & 8) \\
\hline 1278( & 10) & 1267( & 11) & 1233( & 12) & 1269 & 14) \\
\hline 1287( & 7) & 1273( & 7) & 1239 & 5) & 1277( & 5) \\
\hline 1322( & 0) & 1307( & 1) & 1264( & 0) & 1313( & 1) \\
\hline 1337( & 3) & 1321( & 3) & 1277( & 3) & 1330 & 1) \\
\hline 1338( & 1) & 1324( & 1) & 1280( & 2) & 1333( & 4) \\
\hline 1362( & 5) & 1347( & 4) & 1305( & 3) & 1356( & 4) \\
\hline 1408( & 2) & 1394( & 2) & 1352( & 1) & 1397( & 1) \\
\hline 1414( & 2) & 1401( & 2) & 1361( & 2) & 1408( & 3) \\
\hline 1427( & 1) & 1409 & 0) & 1366( & 1) & 1417( & 0) \\
\hline 1468( & 1) & 1458( & 3) & 1418( & 1) & 1461( & 4) \\
\hline 1474( & 3) & 1460 & 1) & 1421( & 3) & 1463( & 1) \\
\hline 1478( & 6) & 1465( & 13) & 1426( & 5) & 1469 & 8) \\
\hline 1480 & 11) & 1466( & 4) & 1431( & 9) & 1471( & 9) \\
\hline 1488( & 7) & 1475( & 5) & 1437( & 4) & 1475( & 3) \\
\hline 1490( & 12) & 1479 & 15) & 1439 & 15) & 1481( & 11) \\
\hline 1573 ( & 25) & 1553( & 27) & 1500 & 12) & 1564( & 33) \\
\hline 1580( & 42) & 1561( & 37) & 1505( & 11) & 1571( & 10) \\
\hline 1612( & 6) & 1577( & 7) & 1509 & 26) & 1585 & 36) \\
\hline 3006( & 33) & 2985( & 34) & 2925 & 43) & 3015 & 25) \\
\hline 3012( & 30) & 2992( & 24) & 2933 & 21) & 3018( & 84) \\
\hline 3023 ( & 64) & 3001( & 72) & 2941( & 87) & 3031( & 149) \\
\hline 3031( & 51) & 3010 & 50) & 2957( & 51) & 3040 & 76) \\
\hline 3068( & 19) & 3050 & 16) & 2998( & 5) & 3078( & 34) \\
\hline 3080( & 31) & 3061( & 30) & 3004 & 24) & 3082 & 12) \\
\hline 3090( & 18) & 3070( & 16) & 3014( & 17) & 3098( & 23) \\
\hline 3096( & 8) & 3080 & 7) & 3017( & 41) & 3101( & 39) \\
\hline 3100( & 5) & 3080 & 18) & 3034( & 2) & 3105( & 34) \\
\hline 3108( & 27) & 3092( & 15) & 3036( & 11) & 3112 & 47) \\
\hline 3121( & 8) & 3100 & 8) & 3040 & 9) & 3124 & 7) \\
\hline 3127( & 5) & 3103( & 3) & 3046( & 8) & 3135( & 15) \\
\hline 3128( & 2) & 3107( & 6) & 3054( & 6) & 3137( & 13) \\
\hline 3152( & 14) & 31260 & 15) & 3057( & 22) & 3145( & 38) \\
\hline 3169 & 11) & 3148( & 13) & 3094( & 16) & 3174( & 35) \\
\hline 3173( & 15) & 3155( & 15) & 3102 & 17) & 3193 & 29) \\
\hline 3178( & 19) & 3162( & 16) & 3119 & 11) & 3194( & 23) \\
\hline 3182( & 25) & 3172( & 18) & 31260 & 16) & 32010 & 33) \\
\hline
\end{tabular}


Table S80. Harmonic vibrational frequencies (in $\mathrm{cm}^{-1}$ ) and infrared intensities (in parentheses in $\mathrm{km} / \mathrm{mol})$ for the $\left(\mathbf{C}_{4} \mathbf{H}_{6}\right)_{3} \mathbf{F e}$ structure $\mathrm{Fe}-2 \mathrm{P}$.

\begin{tabular}{|c|c|c|c|c|c|c|c|}
\hline \multicolumn{2}{|r|}{ B3LYP } & \multicolumn{2}{|c|}{ B3LYP* } & \multicolumn{2}{|c|}{ BP86 } & \multicolumn{2}{|c|}{ M06L } \\
\hline 27( & 0) & 44( & 0) & 14( & 0) & -107( & 1) \\
\hline 46( & 0) & 63( & 0) & 50( & 0) & -27( & 0) \\
\hline 58( & 0) & 65( & 1) & 69 & 0) & 68( & 0) \\
\hline 77( & 0) & 67( & 0) & 77( & 0) & 104( & 0) \\
\hline 99 ( & 2) & 98( & 3) & 112( & 0) & 123( & 4) \\
\hline 117( & 4) & 103( & 2) & 122( & 2) & 136( & 1) \\
\hline 132( & 1) & 145( & 0) & 154( & 1) & 154( & 0) \\
\hline 195( & 1) & 193( & 1) & 185( & 0) & 208( & 0) \\
\hline 211( & 2) & 196( & 1) & 213 ( & 2) & 227 & 3) \\
\hline 248( & 0) & 250 & 0) & 246( & 0) & 272( & 0) \\
\hline 286( & 0) & 284( & 0) & 278( & 0) & 313( & 0) \\
\hline 302( & 1) & 295( & 1) & 303( & 0) & 346( & 0) \\
\hline 312( & 2) & 296( & 1) & 310( & 0) & 348( & 0) \\
\hline 343( & 0) & 336( & 0) & 342( & 1) & 363( & 1) \\
\hline 417( & 1) & 413 ( & 1) & 406( & 0) & 427( & 2) \\
\hline 4381 & 17) & 437 & 14) & 422( & 3) & 431( & 12) \\
\hline 477( & 23) & 476( & 30) & 4631 & 9) & 480( & 20) \\
\hline 523( & 18) & 519 & 19) & 510( & 10) & 517( & 19) \\
\hline 536( & 4) & 527( & 4) & 520( & 2) & 541( & 3) \\
\hline 5681 & 3) & 555( & 10) & 5531 & 4) & 565( & 3) \\
\hline 576( & 5) & 556( & 1) & 555( & 2) & 590( & 4) \\
\hline 622( & 0) & 613 ( & 0) & 599 & 0) & 611( & 1) \\
\hline 6581 & 7) & 644( & 2) & 6331 & 5) & 640( & 3) \\
\hline 6961 & 27) & 662( & 6) & 673 & 36) & 690 & 14) \\
\hline 7031 & 21) & 690 & 38) & 682( & 7) & 693( & 20) \\
\hline 730( & 75) & 723 & 21) & 6961 & 61) & 700( & 26) \\
\hline 7331 & 19) & 724( & 87) & 701( & 22) & 702( & 54) \\
\hline 817( & 6) & 814( & 5) & 794( & 8) & 824( & 4) \\
\hline 829 & 7) & 824( & 5) & 807( & 11) & 850( & 31) \\
\hline 847( & 9) & 834( & 0) & 814( & 1) & 853( & 7) \\
\hline 852( & 1) & 842( & 10) & 822( & 15) & 857( & 41) \\
\hline 8861 & 1) & 871( & 95) & 846( & 74) & 874( & 41) \\
\hline 886( & 125) & 880 & 2) & 857( & 2) & 898( & 1) \\
\hline 949 & 11) & 940( & 24) & 890( & 7) & 907( & 3) \\
\hline 951( & 0) & 944( & 0) & 897( & 1) & 919( & 1) \\
\hline 968( & 15) & 961( & 12) & 934( & 6) & 973( & 7) \\
\hline 969 & 8) & 962( & 7) & 941( & 5) & 980( & 3) \\
\hline 1000( & 1) & 991( & 1) & 957( & 0) & 991( & 0) \\
\hline 1001( & 6) & 994( & 4) & 970( & 4) & 1011( & 4) \\
\hline 1018( & 4) & 1009 & 10) & 984( & 10) & 1024( & 9) \\
\hline 1018( & 7) & 1010( & 3) & 988( & 2) & 1028( & 4) \\
\hline 1050( & 3) & 1040( & 3) & 1014( & 3) & 1055( & 5) \\
\hline 1059( & 7) & 1050( & 5) & 1025( & 5) & 1064( & 5) \\
\hline 1085( & 0) & 1077( & 0) & 1057 & 0) & 1092( & 1) \\
\hline 1120( & 16) & 1111( & 14) & 1086 & 11) & 1119 & 12) \\
\hline 1145( & 2) & 1136( & 2) & 1108( & 1) & 1152( & 1) \\
\hline 1186( & 7) & 1177( & 8) & 1139 & 6) & 1181( & 11) \\
\hline 1210( & 1) & 1202( & 1) & 1166( & 0) & 1203( & 1) \\
\hline
\end{tabular}




\begin{tabular}{|c|c|c|c|c|c|c|c|}
\hline 12300 & 5) & 12180 & 4) & 1182( & 4) & 1224( & 8) \\
\hline 12350 & 0) & 1223( & 1) & 1187( & 0) & 1225 & 0) \\
\hline 1262( & 0) & 1255( & 1) & 1218( & 0) & 1250 & 6) \\
\hline 1263( & 9) & 1255( & 6) & 1219 & 5) & 1252 & 0) \\
\hline 1290( & 5) & 1276( & 5) & 1246( & 6) & 1282( & 6) \\
\hline 1321( & 0) & 1308( & 0) & 1269 & 0) & 1316( & 0) \\
\hline 1339 & 3) & 1324( & 2) & 1282( & 4) & 1336( & 12) \\
\hline 1344( & 10) & 1330( & 7) & 1289 & 7) & 1336( & 4) \\
\hline 13460 & 2) & 1335( & 2) & 1293 & 2) & 1343( & 2) \\
\hline 1406( & 1) & 1392( & 1) & 1354( & 0) & 1396( & 0) \\
\hline 14100 & 4) & 13950 & 3) & 1357( & 3) & 1400 & 3) \\
\hline 1433( & 1) & 1418( & 1) & 1382( & 1) & 1433( & 1) \\
\hline 1469 & 2) & 1451( & 0) & 1412( & 2) & 1462( & 1) \\
\hline 1473( & 10) & 1452( & 14) & 1422( & 15) & 1463( & 13) \\
\hline 1473( & 3) & 1457( & 5) & 1424( & 0) & 1472( & 0) \\
\hline 14800 & 2) & 14660 & 9) & 1426( & 3) & 1473( & 4) \\
\hline 1486( & 11) & 1473( & 7) & 1430 & 6) & 1484( & 2) \\
\hline 1486( & 6) & 1474( & 6) & 1432( & 7) & 1485( & 9) \\
\hline 1565( & 14) & 1553( & 39) & 1502( & 4) & 1568( & 4) \\
\hline 1571( & 46) & 1560( & 29) & 1511( & 34) & 1579 & 54) \\
\hline 1720( & 1) & 1705( & 1) & 1665( & 1) & 1749 & 2) \\
\hline 3016( & 1) & 29960 & 1) & 2940( & 2) & 3002 & 1) \\
\hline 3018( & 42) & 2998( & 44) & 2942( & 45) & 3022 & 42) \\
\hline 3033( & 96) & 3013( & 95) & 2956( & 100) & 3035 & 135) \\
\hline 3034( & 24) & 3014( & 22) & 2957( & 16) & 3035 & 11) \\
\hline 30650 & 30) & 3049 & 24) & 2989 & 36) & 3066 & 89) \\
\hline 3072( & 10) & 3054( & 11) & 2994( & 13) & 3071( & 90) \\
\hline 3080( & 4) & 3063( & 3) & 3006 & 1) & 3092 & 21) \\
\hline 30860 & 9) & 3071( & 46) & 3010( & 68) & 3093 & 15) \\
\hline 30860 & 13) & 3072( & 4) & 3010 & 0) & 3094( & 1) \\
\hline 3089 & 64) & 3073 & 18) & 3012( & 19) & 3095 & 89) \\
\hline 3090( & 4) & 3081( & 1) & 3013( & 13) & 3101( & 4) \\
\hline 3091( & 25) & 3081( & 12) & 3014( & 11) & 3104 & 37) \\
\hline 31200 & 8) & 30960 & 8) & 3043( & 9) & 3140 & 14) \\
\hline 3147( & 43) & 3124( & 44) & 3069 & 47) & 3165( & 72) \\
\hline 3163( & 0) & 3152( & 0) & 3091( & 0) & 3178( & 0) \\
\hline 3163( & 29) & 3153( & 14) & 3092( & 26) & 3179 & 48) \\
\hline 3179 & 0) & 3158( & 0) & 3106( & 1) & 3192 & 38) \\
\hline 3179 & 27) & 3159 & 27) & 3107( & 24) & 31920 & 12) \\
\hline
\end{tabular}


Table S81. Harmonic vibrational frequencies (in $\mathrm{cm}^{-1}$ ) and infrared intensities (in parentheses in $\mathrm{km} / \mathrm{mol}$ ) for the $\left(\mathbf{C}_{4} \mathbf{H}_{6}\right)_{3}$ Co structure Co-1D.

\begin{tabular}{|c|c|c|c|c|c|c|c|}
\hline \multicolumn{2}{|c|}{ B3LYP } & \multicolumn{2}{|c|}{ B3LYP* } & \multicolumn{2}{|c|}{ BP86 } & \multicolumn{2}{|c|}{ M06L } \\
\hline 861 & 1) & 79( & 1) & 78( & 1) & 481 & 1) \\
\hline 96( & 0) & 97( & 0) & 96( & 0) & 85( & 0) \\
\hline 115( & 1) & 111( & 1) & 112( & 0) & 113( & 0) \\
\hline 146( & 1) & 141( & 1) & 141( & 1) & 149 & 1) \\
\hline 158( & 1) & 155( & 2) & 155 & 2) & 161( & 2) \\
\hline 204( & 0) & 204( & 0) & 199 & 1) & 211( & 0) \\
\hline 219 & 0) & 218( & 0) & 212 & 0) & 233 & 0) \\
\hline 230 & 0) & 230 & 0) & 221( & 0) & 241( & 0) \\
\hline 256( & 1) & 253( & 1) & 252( & 1) & 266( & 1) \\
\hline 288( & 0) & 285( & 1) & 282( & 0) & 304( & 2) \\
\hline 301( & 10) & 300( & 7) & 304( & 4) & 312( & 1) \\
\hline 321( & 2) & 323( & 1) & 322 & 1) & 339 & 1) \\
\hline 328( & 2) & 332( & 2) & 342( & 2) & 357 & 2) \\
\hline 369 & 12) & 367( & 12) & 372( & 9) & 383( & 11) \\
\hline 405( & 1) & 401( & 1) & 403( & 1) & 411( & 1) \\
\hline 449 & 0) & 446( & 0) & 439 & 1) & 454( & 0) \\
\hline 483( & 7) & 482( & 6) & 481( & 3) & 483( & 5) \\
\hline 494( & 5) & 492( & 4) & 490 & 3) & 503( & 1) \\
\hline 505( & 3) & 500 & 3) & 501( & 7) & 512( & 2) \\
\hline 576( & 3) & 571( & 3) & 556 & 3) & 576( & 2) \\
\hline 604( & 1) & 600( & 1) & 587( & 1) & 616( & 0) \\
\hline 652( & 2) & 647( & 2) & 632( & 1) & 659 & 2) \\
\hline 712( & 23) & 707( & 24) & 691( & 27) & 720 & 23) \\
\hline 735( & 16) & 728( & 15) & 707 & 13) & 7331 & 16) \\
\hline 751( & 12) & 7431 & 11) & 722( & 8) & 747( & 4) \\
\hline 772( & 6) & 767( & 7) & 750( & 7) & 7831 & 6) \\
\hline 812( & 9) & 807( & 12) & 790 & 7) & 810( & 1) \\
\hline 815( & 8) & 808( & 6) & 7931 & 12) & 826( & 3) \\
\hline 834( & 10) & 828( & 8) & 808( & 5) & 831( & 11) \\
\hline 860 & 5) & 852( & 5) & 832( & 3) & 857 & 2) \\
\hline 874( & 2) & 865 & 1) & 844( & 0) & 870( & 4) \\
\hline 877 & 12) & 871( & 10) & 849 & 6) & 876( & 5) \\
\hline 912( & 4) & 905 & 3) & 880 & 3) & 915( & 2) \\
\hline 945( & 2) & 937( & 2) & 914( & 3) & 9431 & 2) \\
\hline 951( & 6) & 943( & 6) & 916( & 6) & 951( & 2) \\
\hline 981( & 4) & 965 & 4) & 919 & 2) & 955( & 5) \\
\hline 1004( & 3) & 989 & 2) & 944( & 2) & 978( & 3) \\
\hline 1007( & 3) & 997( & 2) & 9631 & $0)$ & 1002( & 1) \\
\hline 1013( & 0) & 1002( & 1) & 972( & 1) & 1011( & 2) \\
\hline 1014( & 3) & 1006( & 4) & 981( & 5) & 1019 & 5) \\
\hline 1038( & 19) & 1030 & 19) & 1005( & 16) & 1043 & 19) \\
\hline 1044( & 4) & 1034( & 4) & 1008( & 5) & 1050 & 4) \\
\hline 1055( & 3) & 1046( & 3) & 1021( & 3) & 1061( & 3) \\
\hline
\end{tabular}




\begin{tabular}{|c|c|c|c|c|c|c|c|}
\hline 1109 & 1) & 1100( & 1) & 1075( & 0) & 1117( & 1) \\
\hline 1162( & 6) & 1152( & 6) & 1124( & 6) & 1158( & 7) \\
\hline 1173( & 3) & 1163( & 3) & 1133( & 4) & 1169 & 4) \\
\hline 1186( & 4) & 1176 & 3) & 1144( & 3) & 1188( & 4) \\
\hline 1221( & 1) & 1210( & 1) & 1178( & 1) & 1223( & 1) \\
\hline 1247( & 3) & 1235( & 3) & 1198( & 4) & 1242( & 6) \\
\hline 1250( & 5) & 1238( & 4) & 1201( & 2) & 1246( & 1) \\
\hline 1265( & 8) & 1254( & 8) & 1217( & 5) & 1260( & 0) \\
\hline 1269 & 2) & 1258( & 1) & 1221( & 0) & 1263( & 5) \\
\hline 1300( & 1) & 1290( & 1) & 1257( & 2) & 1300( & 3) \\
\hline 1329 & 1) & 1316 & 1) & 1276( & 1) & 1326( & 1) \\
\hline 1341( & 6) & 1329( & 5) & 1287( & 6) & 1347( & 6) \\
\hline 1351( & 4) & 1338( & 4) & 1298( & 2) & 1351( & 5) \\
\hline 1371( & 11) & 1357( & 10) & 1319x & 9) & 1374( & 9) \\
\hline 1401( & 1) & 1388( & 1) & 1346( & 1) & 1394( & 1) \\
\hline 1405( & 1) & 1391( & 1) & 1350( & 1) & 1396( & 1) \\
\hline 1431( & 1) & 1418( & 1) & 1380( & 2) & 1434( & 2) \\
\hline 1467( & 3) & 1454( & 3) & 1411( & 2) & 1458( & 0) \\
\hline 1473( & 2) & 1458( & 2) & 1417( & 3) & 1463( & 2) \\
\hline 1478( & 4) & 1464( & 2) & 1422( & 2) & 1466( & 5) \\
\hline 1480( & 3) & 1467( & 5) & 1425( & 7) & 1469 & 6) \\
\hline 1483( & 8) & 1469( & 6) & 1426( & 7) & 1475( & 5) \\
\hline 1497( & 11) & 1484( & 12) & 1441( & 10) & 1493( & 10) \\
\hline 1539( & 29) & 1525( & 31) & 1477( & 21) & 1525( & 17) \\
\hline 1542( & 13) & 1527( & 6) & 1481( & 5) & 1531( & 15) \\
\hline 1723( & 0) & 1709 & 0) & 1666( & 1) & 1748( & 1) \\
\hline 3010( & 35) & 2990( & 35) & 2935( & 37) & 3023( & 78) \\
\hline 3021( & 19) & 3001( & 19) & 2946( & 21) & 3032( & 32) \\
\hline 3030( & 64) & 3010( & 62) & 2955( & 63) & 3037( & 96) \\
\hline 3033( & 57) & 3013( & 54) & 2957( & 56) & 3050( & 86) \\
\hline 3063( & 17) & 3043( & 17) & 2991( & 16) & 3079) & 31) \\
\hline 3079 & 31) & 3060( & 29) & 3007( & 28) & 3097( & 18) \\
\hline 3097( & 10) & 3074( & 10) & 3012( & 11) & 3099 & 35) \\
\hline 3104( & 15) & 3081( & 15) & 3019x & 17) & 3104( & 38) \\
\hline 3107( & 1) & 3085( & 1) & 3028( & 1) & 3107( & 13) \\
\hline 3122( & 13) & 3100( & 13) & 3042( & 13) & 3119 & 19) \\
\hline 3133( & 4) & 3112( & 4) & 3052( & 5) & 31360 & 15) \\
\hline 3144( & 37) & 3122( & 35) & 3064( & 39) & 3143( & 33) \\
\hline 3152( & 33) & 3130( & 33) & 3071( & 32) & 3149( & 38) \\
\hline 3152( & 10) & 3131( & 10) & 3071( & 16) & 3153( & 31) \\
\hline 3158( & 13) & 3134( & 15) & 3073( & 14) & 3158( & 82) \\
\hline 3159 & 26) & 3138( & 23) & 3079) & 26) & 3159 & 41) \\
\hline 3237 ( & 12) & 3213( & 12) & 3151( & 13) & 3227( & 20) \\
\hline 3243( & 8) & 32210 & 7) & 3162( & 8) & 3243( & 16) \\
\hline
\end{tabular}


Table S82. Harmonic vibrational frequencies (in $\mathrm{cm}^{-1}$ ) and infrared intensities (in parentheses in $\mathrm{km} / \mathrm{mol}$ ) for the $\left(\mathbf{C}_{4} \mathbf{H}_{6}\right)_{3} \mathbf{C o}$ structure Co-2D.

\begin{tabular}{|c|c|c|c|c|c|c|c|}
\hline \multicolumn{2}{|c|}{ B3LYP } & \multicolumn{2}{|c|}{ B3LYP* } & \multicolumn{2}{|c|}{ BP86 } & \multicolumn{2}{|c|}{ M06L } \\
\hline 31( & 0) & 48( & 0) & 41( & 0) & 54( & 0) \\
\hline 47( & 0) & 62( & 0) & 61( & 0) & 75( & 0) \\
\hline 98( & 0) & 101( & 0) & 95( & 0) & 126( & 0) \\
\hline 166( & 0) & 170( & 0) & 161( & 0) & 188( & 0) \\
\hline 188( & 1) & 189 & 2) & 189( & 3) & 205( & 4) \\
\hline 193( & 5) & 197( & 3) & 196( & 0) & 219 & 0) \\
\hline 233( & 2) & 241( & 2) & 239 & 1) & 265( & 1) \\
\hline 250( & 3) & 257( & 4) & 259 & 1) & 274( & 5) \\
\hline 261( & 1) & 265( & 0) & 268( & 2) & 286( & 0) \\
\hline 274( & 5) & 278( & 1) & 275( & 1) & 307( & 1) \\
\hline 279 & 3) & 283( & 5) & 291( & 1) & 311( & 1) \\
\hline 284( & 1) & 287( & 3) & 299 & 3) & 320( & 4) \\
\hline 322( & 1) & 321( & 1) & 317( & 4) & 324( & 3) \\
\hline 386( & 5) & 388( & 6) & 389 & 5) & 413( & 4) \\
\hline 400( & 5) & 405( & 5) & 408( & 4) & 418( & 5) \\
\hline 438( & 1) & 439 & 1) & 438( & 2) & 481( & 2) \\
\hline 487( & 4) & 486( & 3) & 472( & 1) & 492( & 1) \\
\hline 499( & 2) & 498( & 2) & 493( & 3) & 518( & 5) \\
\hline 546( & 2) & 544( & 1) & 529 & 1) & 558( & 2) \\
\hline 5681 & 6) & 566( & 6) & 551( & 5) & 595( & 4) \\
\hline 600 & 1) & 599 & 1) & 586( & 0) & 624( & 8) \\
\hline 635( & 5) & 6331 & 5) & 618( & 6) & 652( & 7) \\
\hline 689 & 13) & 682( & 13) & 659 & 11) & 694( & 17) \\
\hline 732( & 6) & 727( & 6) & 716( & 5) & 769 & 3) \\
\hline 771( & 12) & 768( & 12) & 749 & 11) & 774( & 7) \\
\hline 8031 & 7) & 799 & 7) & 7831 & 5) & 813( & 6) \\
\hline 825( & 13) & 820 & 11) & 803( & 9) & 828( & 44) \\
\hline 858( & 59) & 849 & 64) & 813( & 56) & 832( & 18) \\
\hline 860( & 11) & 855 & 5) & 8381 & 5) & 862( & 9) \\
\hline 867 & 10) & 866( & 12) & 8481 & 11) & 876( & 5) \\
\hline 889 & 6) & 884( & 4) & 867( & 2) & 897( & 1) \\
\hline 906( & 1) & 900( & 1) & 880( & 0) & 915( & 1) \\
\hline 932( & 12) & 924( & 11) & 8931 & 10) & 931( & 17) \\
\hline 942( & 8) & 9331 & 7) & 908( & 5) & 941( & 8) \\
\hline 955( & 2) & 947( & 1) & 921( & 1) & 961( & 2) \\
\hline 980( & 3) & 971( & 4) & 934( & 3) & 965( & 1) \\
\hline 992( & 1) & 980( & 0) & 946( & 1) & 972( & 2) \\
\hline 1005( & 3) & 992( & 1) & 956( & 0) & 989 & 1) \\
\hline 1014( & 5) & 1004( & 6) & 976( & 5) & 1015 & 8) \\
\hline 1019 & 1) & 1011( & 1) & 982( & 2) & 1021( & 3) \\
\hline 1025 & 6) & 1016( & 7) & 986( & 9) & 1028( & 4) \\
\hline 1038( & 6) & 1030( & 6) & 1000( & 5) & 1047( & 3) \\
\hline 1047( & 4) & 1040( & 4) & 1011( & 3) & 1060 & 6) \\
\hline
\end{tabular}




\begin{tabular}{|c|c|c|c|c|c|c|c|}
\hline 1094( & 2) & 1087( & 2) & 1060 & 2) & 1097( & 0) \\
\hline 1124( & 12) & 1116( & 12) & 1086( & 10) & 1121( & 11) \\
\hline 1146( & 7) & 1138( & 7) & 1107( & 6) & 1147( & 5) \\
\hline 1185( & 2) & 1177( & 2) & 1144( & 3) & 1188( & 1) \\
\hline 1210 & 1) & 1202( & 2) & 1168( & 3) & 1219 & 4) \\
\hline 1228( & 2) & 1218( & 2) & 1187( & 2) & 1238( & 2) \\
\hline 1242( & 0) & 1235( & 0) & 1199 & 0) & 1244( & 0) \\
\hline 1259 & 4) & 1248( & 4) & 1214( & 3) & 1257( & 2) \\
\hline 1287( & 7) & 1273( & 7) & 1234( & 5) & 1274( & 10) \\
\hline 1296( & 2) & 1284( & 2) & 1244( & 3) & 1302( & 1) \\
\hline 1307( & 1) & 1296( & 1) & 1252( & 1) & 1309 & 1) \\
\hline 1319 & 1) & 1308( & 1) & 1266( & 3) & 1318( & 1) \\
\hline 1322( & 2) & 1310 & 2) & 1272( & 0) & 1320( & 1) \\
\hline 1337( & 3) & 1324( & 3) & 1288( & 3) & 1344( & 3) \\
\hline 1352( & 3) & 1340 & 3) & 1303( & 3) & 1359 & 8) \\
\hline 1414( & 1) & 1401( & 1) & 1358( & 4) & 1407( & 5) \\
\hline 1420 & 3) & 1405( & 3) & 1361( & 1) & 1410( & 2) \\
\hline 1444( & 4) & 1431( & 4) & 1393( & 2) & 1434( & 2) \\
\hline 1479 & 2) & 1469 & 3) & 1426( & 3) & 1462( & 1) \\
\hline 1486( & 5) & 1475( & 4) & 1431( & 3) & 1472( & 4) \\
\hline 1496( & 5) & 1482( & 6) & 1440 & 7) & 1480( & 7) \\
\hline 1497( & 2) & 1484( & 2) & 1442( & 2) & 1482( & 14) \\
\hline 1504( & 17) & 1492( & 18) & 1449 & 18) & 1489 & 2) \\
\hline 1556( & 8) & 1543( & 6) & 1500 & 4) & 1553( & 5) \\
\hline 1599 & 5) & 1576( & 5) & 1511( & 3) & 1575( & 8) \\
\hline 1649 & 71) & 1636 & 66) & 1590 & 42) & 1659 & 65) \\
\hline 3013( & 32) & 2994( & 35) & 2939 & 46) & 3010 & 102) \\
\hline 3025( & 74) & 3006( & 77) & 2953( & 82) & 3039 & 91) \\
\hline 3035( & 55) & 3017( & 47) & 2967( & 40) & 3042( & 45) \\
\hline 3044( & 21) & 3025( & 19) & 2968( & 20) & 3057( & 71) \\
\hline 3054( & 52) & 3033( & 52) & 2975( & 57) & 3067( & 24) \\
\hline 3060 & 28) & 3042( & 27) & 2991( & 25) & 3080 & 51) \\
\hline 3093( & 3) & 3073( & 2) & 3011( & 0) & 3090 & 42) \\
\hline 3098( & 5) & 3076( & 3) & 3020 & 2) & 3104( & 2) \\
\hline 3112( & 30) & 3090 & 34) & 3030 & 40) & 3118( & 57) \\
\hline 3117 & 12) & 3097( & 12) & 3039 & 11) & 3121( & 32) \\
\hline 3131( & 6) & 3109 & 5) & 3049 & 5) & 3129 & 13) \\
\hline 3136 & 5) & 3115( & 6) & 3057( & 10) & 3142( & 17) \\
\hline 3138( & 23) & 3119 & 21) & 3064( & 23) & 3144( & 41) \\
\hline 3153( & 6) & 3132( & 5) & 3067( & 15) & 3156( & 18) \\
\hline 3156( & 2) & 3135( & 5) & 3079 & 7) & 3161( & 27) \\
\hline 3165( & 38) & 3143( & 35) & 3084( & 35) & 3171( & 66) \\
\hline 3223( & 9) & 3202( & 8) & 3143( & 9) & 3225( & 23) \\
\hline 32370 & 21) & 3219 & 20) & 3164( & 20) & 32410 & 32) \\
\hline
\end{tabular}


Table S83. Harmonic vibrational frequencies (in $\mathrm{cm}^{-1}$ ) and infrared intensities (in parentheses in $\mathrm{km} / \mathrm{mol}$ ) for the $\left(\mathbf{C}_{4} \mathbf{H}_{6}\right)_{3}$ Co structure Co-3D.

\begin{tabular}{|c|c|c|c|c|c|c|c|}
\hline \multicolumn{2}{|c|}{ B3LYP } & \multicolumn{2}{|c|}{ B3LYP* } & \multicolumn{2}{|c|}{ BP86 } & \multicolumn{2}{|c|}{ M06L } \\
\hline 32( & 0) & 32( & 0) & $49 x$ & 0) & 51( & 0) \\
\hline 55( & 0) & 58( & 0) & 53( & 0) & 75( & 0) \\
\hline 83( & 0) & 87( & 0) & 84( & 0) & 103( & 0) \\
\hline 96( & 0) & 97( & 0) & 931 & 1) & 113( & 2) \\
\hline 113( & 4) & 113( & 2) & 112( & 0) & 122( & 1) \\
\hline 143( & 10) & 151( & 7) & 154( & 2) & 154( & 3) \\
\hline 153( & 0) & 154( & 0) & 166( & 1) & 160( & 0) \\
\hline 171( & 2) & 175( & 2) & 175( & 1) & 178( & 2) \\
\hline 184( & 2) & 192( & 2) & 194( & 0) & 196( & 2) \\
\hline 208( & 3) & 207( & 3) & 208( & 3) & 213( & 3) \\
\hline 227( & 1) & 231( & 0) & 241( & 0) & 241( & 0) \\
\hline 248( & 3) & 263( & 3) & 278( & 2) & 271( & 2) \\
\hline 268( & 4) & 278( & 4) & 289 & 1) & 299( & 8) \\
\hline 289 & 10) & 292( & 11) & 302( & 4) & 323( & 3) \\
\hline 297( & 0) & 298( & 0) & 311( & 5) & 331( & 4) \\
\hline 311( & 5) & 315( & 5) & 327( & 6) & 344( & 3) \\
\hline 365( & 7) & 377( & 10) & 385( & 5) & 406( & 10) \\
\hline 382( & 2) & 385( & 0) & 399 & 18) & 416( & 5) \\
\hline 401( & 14) & 402( & 14) & 408( & 6) & 419 & 14) \\
\hline 447( & 2) & 442( & 2) & 435( & 2) & 463( & 1) \\
\hline 461( & 3) & 461( & 3) & 470( & 3) & 495( & 3) \\
\hline 510( & 7) & 509 & 6) & 504( & 3) & 515( & 3) \\
\hline 555( & 4) & 553( & 4) & 540 & 4) & 560( & 4) \\
\hline 601( & 2) & 598( & 2) & 577( & 2) & 600( & 4) \\
\hline 631( & 9) & 634( & 10) & 634( & 14) & 650( & 11) \\
\hline 664( & 22) & 662( & 23) & 653( & 30) & 672( & 21) \\
\hline 695( & 14) & 689 & 12) & 675( & 3) & 699( & 4) \\
\hline 728( & 1) & 718( & 1) & 689 & 4) & 725( & 3) \\
\hline 750( & 14) & 7461 & 12) & 726( & 8) & 7466 & 7) \\
\hline 7731 & 2) & 767( & 2) & 745( & 1) & 767 ( & 4) \\
\hline 854( & 15) & 851( & 20) & 827( & 33) & 838( & 11) \\
\hline 871( & 6) & 862( & 9) & 836( & 7) & 851( & 2) \\
\hline 875( & 3) & 870 & 1) & 838( & 6) & 8606 & 30) \\
\hline 889 & 11) & 881( & 12) & 847( & 6) & 869) & 10) \\
\hline 893( & 10) & 8861 & 14) & 851( & 31) & 871( & 17) \\
\hline 899( & 29) & 891( & 23) & 859 & 53) & 878( & 26) \\
\hline 909 & 7) & 900( & 21) & 862( & 1) & 887 ( & 10) \\
\hline 916( & 22) & 905( & 9) & 875( & 1) & 896( & 3) \\
\hline 923( & 43) & 911( & 37) & 879 & 5) & 905( & 1) \\
\hline 928( & 3) & 922( & 5) & 8961 & 8) & 914( & 11) \\
\hline 938( & 1) & 929 & 1) & 902( & 0) & $919 x$ & 5) \\
\hline 944( & 3) & 9331 & 2) & 906( & 2) & 9331 & 0) \\
\hline 982( & 0) & 969 & 1) & 944( & 2) & 959 & 1) \\
\hline 1004( & 3) & 997( & 3) & 970( & 2) & 9966 & 2) \\
\hline 1025( & 12) & 1015( & 12) & 983( & 12) & 1015( & 9) \\
\hline 1030 & 14) & 1023( & 14) & 989 & 13) & 1017( & 9) \\
\hline
\end{tabular}




\begin{tabular}{|c|c|c|c|c|c|c|c|}
\hline 1061( & 2) & 1053( & 2) & 1027( & 3) & 1060( & 2) \\
\hline 1068( & 2) & 1061( & 2) & 1037( & 1) & 1065( & 3) \\
\hline 1085( & 4) & 1076( & 4) & 1048( & 2) & 1079( & 2) \\
\hline 1101( & 4) & 1091( & 4) & 1067( & 4) & 1098( & 3) \\
\hline 1224( & 21) & 1216( & 19) & 1189 & 7) & 1227( & 8) \\
\hline 1234( & 18) & 1224( & 11) & 1192( & 10) & 1230( & 28) \\
\hline 1239 & 39) & 1230( & 36) & 1194( & 25) & 1230( & 18) \\
\hline 1265( & 19) & 1250( & 18) & 1205( & 12) & 1251( & 24) \\
\hline 1284( & 22) & 1275( & 16) & 1245( & 9) & 1276( & 16) \\
\hline 1305( & 3) & 1294( & 4) & 1261( & 4) & 1298( & 5) \\
\hline 1324( & 5) & 1313( & 4) & 1278( & 1) & 1322( & 4) \\
\hline 1386( & 11) & 1374( & 10) & 1330( & 6) & 1375( & 6) \\
\hline 1413( & 16) & 1402( & 13) & 1363( & 7) & 1398( & 0) \\
\hline 1418( & 0) & 1406( & 0) & 1366( & 1) & 1399( & 7) \\
\hline 1451( & 29) & 1441( & 24) & 1405( & 13) & 1451( & 20) \\
\hline 1465( & 4) & 1454( & 2) & 1415( & 1) & 1452( & 5) \\
\hline 1467( & 10) & 1456( & 10) & 1420( & 8) & 1455( & 5) \\
\hline 1538( & 48) & 1525( & 40) & 1478( & 26) & 1526( & 28) \\
\hline 1544( & 24) & 1531( & 18) & 1486( & 4) & 1528( & 27) \\
\hline 1560( & 6) & 1546( & 4) & 1490( & 13) & 1541( & 16) \\
\hline 1570( & 23) & 1550( & 22) & 1502( & 6) & 1545( & 10) \\
\hline 1661( & 67) & 1648( & 63) & 1603( & 44) & 1674( & 69) \\
\hline 1673( & 63) & 1659 & 64) & 1611( & 45) & 1691( & 75) \\
\hline 3130( & 3) & 3105( & 3) & 3042( & 5) & 3111( & 12) \\
\hline 3137( & 4) & 3114( & 7) & 3051( & 6) & 3121( & 24) \\
\hline 3138( & 9) & 3115( & 11) & 30531 & 16) & 3124( & 8) \\
\hline 3139 & 17) & 3117( & 12) & 3055( & 14) & 3126( & 24) \\
\hline 3145( & 5) & 3121( & 5) & 3061( & 1) & 3127( & 30) \\
\hline 3151( & 2) & 3129 & 2) & 3064( & 16) & 3150( & 13) \\
\hline 3153( & 11) & 3133( & 11) & 3078( & 3) & 3152( & 23) \\
\hline 3153( & 1) & 3134( & 1) & 3080( & 7) & 3158( & 13) \\
\hline 3160( & 3) & 3140( & 2) & 3083( & 3) & 3159 & 9) \\
\hline 3167( & 8) & 3143( & 8) & 3085( & 12) & 3162( & 18) \\
\hline 3181( & 4) & 3157( & 4) & 3088( & 9) & 3172( & 23) \\
\hline 3197( & 4) & 3174( & 4) & 3108( & 7) & 3193( & 11) \\
\hline 3223( & 9) & 3201( & 8) & 3139 & 9) & 3217 ( & 20) \\
\hline 3230( & 6) & 3208( & 6) & 3144( & 12) & 3227( & 18) \\
\hline 3238( & 7) & 3213( & 7) & 3154( & 10) & 3233( & 23) \\
\hline 3244( & 4) & 3222( & 4) & 3156( & 2) & 3234( & 8) \\
\hline 3247( & 12) & 3228( & 12) & 3173( & 12) & 3251( & 19) \\
\hline 3249 & 9) & 32290 & 9) & 31730 & 11) & 32531 & 24) \\
\hline
\end{tabular}


Table S84. Harmonic vibrational frequencies (in $\mathrm{cm}^{-1}$ ) and infrared intensities (in parentheses in $\mathrm{km} / \mathrm{mol}$ ) for the $\left(\mathbf{C}_{4} \mathbf{H}_{6}\right)_{3}$ Co structure Co-4D.

\begin{tabular}{|c|c|c|c|c|c|c|c|}
\hline \multicolumn{2}{|c|}{ B3LYP } & \multicolumn{2}{|c|}{ B3LYP* } & \multicolumn{2}{|c|}{ BP86 } & \multicolumn{2}{|c|}{ M06L } \\
\hline 371 & 0) & 35( & 0) & 31( & 0) & 49 & 0) \\
\hline 56( & 0) & 55( & 0) & 60( & 0) & 72( & 0) \\
\hline 74( & 0) & 79 ( & 0) & 87( & 0) & 85( & 0) \\
\hline 84( & 0) & 90( & 0) & 98( & 0) & 101( & 0) \\
\hline 103( & 0) & 107( & 1) & 110( & 1) & 121( & 2) \\
\hline 115( & 3) & 137( & 0) & 148( & 0) & 156( & 1) \\
\hline 130( & 1) & 144( & 3) & 171( & 1) & 175( & 1) \\
\hline 162( & 1) & 167( & 0) & 183( & 1) & 180( & 1) \\
\hline 173( & 1) & 173( & 2) & 194( & 1) & 196( & 1) \\
\hline 186( & 4) & 187( & 2) & 223( & 1) & 200( & 1) \\
\hline 215( & 7) & 220( & 7) & 227( & 5) & 230( & 5) \\
\hline 265( & 2) & 265( & 1) & 261( & 2) & 270( & 4) \\
\hline 272( & 3) & 271( & 4) & 271( & 5) & 291( & 3) \\
\hline 291( & 3) & 288( & 1) & 284( & 2) & 299( & 2) \\
\hline 298( & 2) & 302( & 1) & 310( & 1) & 319 & 1) \\
\hline 307( & 22) & 312( & 20) & 345( & 11) & 336( & 16) \\
\hline 326( & 21) & 329 & 18) & 352( & 5) & 356( & 13) \\
\hline 376( & 4) & 3921 & 5) & 408( & 9) & 423( & 5) \\
\hline 422( & 1) & 425( & 1) & 428( & 2) & 455( & 1) \\
\hline 428( & 1) & 432( & 1) & 444( & 1) & 458( & 3) \\
\hline 453( & 5) & 452( & 5) & 452( & 6) & 477( & 7) \\
\hline 546( & 11) & 542( & 12) & 530( & 11) & 551( & 7) \\
\hline 5566 & 6) & 551( & 4) & 5371 & 2) & 566( & 6) \\
\hline 598( & 3) & 598( & 3) & 594( & 3) & 613( & 6) \\
\hline 646( & 40) & 643( & 40) & 625( & 35) & 644( & 29) \\
\hline 650( & 1) & 648( & 2) & 637( & 12) & 6521 & 11) \\
\hline 681( & 11) & 6861 & 12) & 6861 & 5) & 703( & 9) \\
\hline 719 & 8) & 711( & 4) & 707( & 2) & 7166 & 1) \\
\hline 745( & 12) & 743( & 12) & 727( & 8) & 744( & 8) \\
\hline 749 & 3) & 746( & 4) & 7331 & 4) & 751( & 2) \\
\hline 821( & 29) & 824( & 21) & 831( & 7) & 8066 & 12) \\
\hline 860( & 7) & 852( & 11) & 8399 & 24) & 842( & 7) \\
\hline 874( & 1) & 870( & 1) & 844( & 47) & 852( & 36) \\
\hline 881( & 6) & 876( & 6) & 854( & 3) & 869 & 2) \\
\hline 898( & 14) & 891( & 34) & 858( & 14) & 877( & 51) \\
\hline 906( & 32) & 898( & 15) & 864( & 3) & 884( & 2) \\
\hline 920( & 16) & 911( & 24) & 870( & 33) & 888( & 1) \\
\hline 921( & 4) & 914( & 14) & 874( & 13) & 902( & 1) \\
\hline 925( & 15) & 916( & 25) & 887( & 3) & 911( & 1) \\
\hline 926( & 28) & 922( & 0) & 895( & 2) & 922( & 1) \\
\hline 9431 & 1) & 9351 & 1) & 909x & 0) & 931( & 1) \\
\hline 956( & 1) & 945( & 0) & 915( & 2) & 944( & 1) \\
\hline 983( & 5) & 964( & 4) & 918( & 1) & 952( & 2) \\
\hline 1014( & 22) & 1002( & 15) & 9621 & 13) & 1003( & 10) \\
\hline 1015( & 2) & 1006( & 10) & 976( & 11) & 1016( & 9) \\
\hline 1061( & 1) & 1054( & 3) & 1027( & 1) & 1056( & 1) \\
\hline
\end{tabular}




\begin{tabular}{|c|c|c|c|c|c|c|c|}
\hline 1063( & 2) & 1056( & 0) & 1029 & 3) & 1057( & 2) \\
\hline 1066( & 5) & 1059 & 5) & 1034( & 2) & 1060( & 3) \\
\hline 1084( & 6) & 1073( & 5) & 1041( & 3) & 1071( & 3) \\
\hline 1096( & 1) & 1086( & 1) & 1054( & 5) & 1087( & 3) \\
\hline 1097( & 7) & 1087( & 7) & 1057( & 3) & 1090( & 4) \\
\hline 1227( & 16) & 1217( & 9) & 1178( & 4) & 1214( & 7) \\
\hline 1253( & 45) & 1242( & 36) & 1203( & 19) & 1242( & 32) \\
\hline 1257( & 31) & 1244( & 29) & 1206( & 18) & 1247( & 28) \\
\hline 1289 ( & 15) & 1272( & 12) & 12260 & 8) & 1266( & 10) \\
\hline 1321( & 3) & 1310 & 2) & 1275( & 0) & 1317( & 7) \\
\hline 1326( & 4) & 1314( & 3) & 1275( & 4) & 1318( & 2) \\
\hline 1412( & 3) & 1399 & 1) & 1357( & 0) & 1389 & 1) \\
\hline 1417( & 12) & 1405( & 10) & 1364( & 6) & 13980 & 5) \\
\hline 1423( & 10) & 1411( & 9) & 1371( & 5) & 1404( & 5) \\
\hline 1453( & 17) & 1441( & 15) & 1402( & 10) & 1439( & 7) \\
\hline 1459 ( & 2) & 1447( & 2) & 1410 & 4) & 1449 & 3) \\
\hline 1466( & 20) & 1457( & 13) & 1424( & 6) & 14560 & 5) \\
\hline 1539 ( & 49) & 1528( & 36) & 1486( & 19) & 1522( & 29) \\
\hline 1558( & 36) & 1546( & 27) & 1493( & 8) & 1541( & 35) \\
\hline 1569 ( & 26) & 1552( & 28) & 1496( & 7) & 1547( & 5) \\
\hline 1580( & 25) & 1557( & 17) & 1503( & 16) & 1550( & 25) \\
\hline 1659 & 24) & 1643( & 21) & 1578( & 15) & 1659( & 28) \\
\hline 1672( & 23) & 1658( & 25) & 1612( & 25) & 1683( & 32) \\
\hline 3139 & 8) & 3119 & 11) & 3059 & 8) & 3124( & 32) \\
\hline 3142( & 14) & 3119 & 8) & 3060 & 12) & 3132( & 17) \\
\hline 3151( & 3) & 3127( & 2) & 3060 & 8) & 31330 & 16) \\
\hline 3154( & 8) & 3128( & 10) & 3064( & 8) & 3142( & 11) \\
\hline 3155( & 3) & 3133( & 6) & 3070( & 5) & 3148( & 19) \\
\hline 3157( & 6) & 3134( & 8) & 3071( & 11) & 3149 & 17) \\
\hline 3158( & 7) & 31360 & 6) & 3074( & 12) & 3152( & 18) \\
\hline 3162( & 5) & 3140 & 4) & 3076( & 21) & 3156( & 18) \\
\hline 3170( & 2) & 3143( & 4) & 3082( & 5) & 3161( & 11) \\
\hline 3171( & 8) & 3144( & 5) & 3083( & 2) & 3165( & 10) \\
\hline 3172( & 8) & 3147( & 7) & 3085( & 5) & 3168( & 31) \\
\hline 3182( & 10) & 3158( & 11) & 3097( & 17) & 3179 & 35) \\
\hline 3226( & 9) & 3206( & 7) & 3149 & 7) & 3220 & 19) \\
\hline 3244( & 6) & 3219 & 5) & 3153( & 8) & 3227( & 15) \\
\hline 3245( & 3) & 3224( & 4) & 3159 & 7) & 3235( & 11) \\
\hline 3248( & 18) & 3228( & 14) & 3161( & 8) & 3244( & 16) \\
\hline 3250( & 6) & 3230 & 8) & 3172( & 14) & 3255( & 32) \\
\hline 32660 & 6) & 32380 & 6) & 3176( & 11) & 32550 & 17) \\
\hline
\end{tabular}


Table S85. Harmonic vibrational frequencies (in $\mathrm{cm}^{-1}$ ) and infrared intensities (in parentheses in $\mathrm{km} / \mathrm{mol}$ ) for the $\left(\mathbf{C}_{4} \mathbf{H}_{6}\right)_{3}$ Co structure Co-5D.

\begin{tabular}{|c|c|c|c|c|c|c|c|}
\hline \multicolumn{2}{|c|}{ B3LYP } & \multicolumn{2}{|c|}{ B3LYP* } & \multicolumn{2}{|c|}{ BP86 } & \multicolumn{2}{|c|}{ M06L } \\
\hline 36( & 4) & 46( & 3) & 66( & 0) & 53( & 1) \\
\hline 55( & 0) & 55( & 0) & 90( & 1) & 79 ( & 1) \\
\hline 74( & 0) & 83( & 0) & 103( & 0) & 91( & 1) \\
\hline 105( & 4) & 100( & 3) & 136( & 1) & 132( & 1) \\
\hline 132( & 1) & 137( & 0) & 151( & 3) & 143( & 3) \\
\hline 142( & 2) & 151( & 2) & 162( & 1) & 159( & 2) \\
\hline 161( & 2) & 181( & 0) & 193( & 1) & 181( & 1) \\
\hline 188( & 4) & 190( & 4) & 218( & 2) & 201( & 2) \\
\hline 205( & 14) & 221( & 2) & 266( & 0) & 263( & 0) \\
\hline 232( & 4) & 243( & 1) & 287( & 0) & 267 ( & 0) \\
\hline 249 & 1) & 261( & 0) & 299( & 0) & 303( & 0) \\
\hline 267( & 1) & 276( & 3) & 331( & 2) & 340( & 3) \\
\hline 270( & 15) & 315( & 2) & 342( & 3) & 343( & 3) \\
\hline 313( & 2) & 321( & 4) & 367( & 3) & 391( & 2) \\
\hline 316( & 0) & 340( & 5) & 394( & 2) & 407( & 3) \\
\hline 369 & 2) & 374( & 1) & 435( & 1) & 441( & 4) \\
\hline 430( & 1) & 428( & 1) & 452( & 3) & 459( & 11) \\
\hline 444( & 5) & 449( & 4) & 465( & 2) & 473( & 0) \\
\hline 477( & 17) & 480( & 18) & 506( & 4) & 511( & 6) \\
\hline 491( & 13) & 509( & 7) & 515( & 1) & 536( & 1) \\
\hline 581( & 2) & 579) & 2) & 584( & 3) & 587( & 2) \\
\hline 593( & 3) & 588( & 4) & 601( & 8) & 609 & 6) \\
\hline 609 & 8) & 612( & 2) & 625( & 4) & 641( & 3) \\
\hline 639 & 16) & 636( & 24) & 657( & 1) & 657( & 2) \\
\hline 653( & 24) & 658( & 11) & 680( & 8) & 699( & 10) \\
\hline 678( & 34) & 6891 & 13) & 7031 & 59) & 708( & 9) \\
\hline 693( & 7) & 697( & 22) & 719) & 18) & 716( & 27) \\
\hline 710 & 21) & 704( & 20) & 726( & 9) & 740( & 16) \\
\hline 746( & 24) & 742( & 30) & 748( & 10) & 755( & 26) \\
\hline 829 & 12) & 826( & 13) & 822( & 20) & 845( & 11) \\
\hline 837( & 92) & 834( & 8) & 827( & 1) & 856( & 13) \\
\hline 852( & 30) & 847( & 28) & 832( & 14) & 860( & 3) \\
\hline 855( & 14) & 877( & 16) & 867( & 2) & 865( & 3) \\
\hline 874( & 23) & 904( & 29) & 897( & 2) & 917( & 16) \\
\hline 922( & 8) & 918( & 34) & 908( & 5) & 9331 & 3) \\
\hline 928( & 1) & 927( & 11) & 912( & 6) & 940( & 4) \\
\hline 938( & 4) & 936( & 13) & 922( & 5) & 945( & 6) \\
\hline 969 & 2) & 960( & 3) & 924( & 6) & 957( & 3) \\
\hline 971( & 10) & 968( & 14) & 927( & 1) & 964( & 4) \\
\hline 976( & 1) & 970( & 9) & 945( & 0) & 973( & 6) \\
\hline 983( & 2) & 978( & 12) & 952( & 5) & 986( & 8) \\
\hline 1021( & 22) & 1015( & 10) & 988( & 1) & 1021( & 0) \\
\hline 1036( & 0) & 1031( & 2) & 992( & 2) & 1027( & 1) \\
\hline 1065( & 5) & 1057( & 9) & 1010( & 8) & 1046( & 10) \\
\hline 1066( & 7) & 1066 & 4) & 1026( & 2) & 1062( & 2) \\
\hline 1083( & 6) & 1073( & 3) & 1041( & 9) & 1078( & 13) \\
\hline
\end{tabular}




\begin{tabular}{|c|c|c|c|c|c|c|c|}
\hline 1101( & 3) & 1091( & 3) & 1050( & 0) & 1089 & 1) \\
\hline 1132( & 1) & 1118( & 4) & 1076( & 1) & 1113( & 2) \\
\hline 1155( & 17) & 1145( & 6) & 1107( & 1) & 1146( & 1) \\
\hline 1208( & 7) & 1198( & 4) & 1133( & 5) & 1177( & 6) \\
\hline 1222( & 8) & 1206( & 9) & 1137( & 2) & 1185( & 4) \\
\hline 1224( & 7) & 1215( & 5) & 1178( & 1) & 1224( & 7) \\
\hline 1264( & 22) & 1259 ( & 15) & 1182( & 6) & 1227( & 4) \\
\hline 1276( & 30) & 1261( & 12) & 1207( & 2) & 1251( & 2) \\
\hline 1288( & 1) & 12766 & 1) & 1215( & 1) & 1257( & 1) \\
\hline 1335( & 1) & 1323( & 0) & 1252( & 2) & 1307( & 1) \\
\hline 1352( & 1) & 1339x & 0) & 1281( & 1) & 1333( & 0) \\
\hline 1410( & 7) & 1396( & 5) & 1344( & 1) & 1383( & 0) \\
\hline 1415( & 1) & 1401( & 1) & 1349 & 7) & 1397( & 5) \\
\hline 1421( & 12) & 1407( & 10) & 1363( & 4) & 1411( & 8) \\
\hline 1428( & 4) & 1412( & 3) & 1383( & 3) & 1426( & 3) \\
\hline 1467( & 24) & 1455( & 16) & 1430( & 5) & 1467( & 3) \\
\hline 1479( & 2) & 1463( & 1) & 1435( & 8) & 1468( & 10) \\
\hline 1486( & 2) & 1466( & 2) & 1438( & 2) & 1470 & 4) \\
\hline 1489( & 5) & 1474( & 6) & 1442( & 10) & 1482( & 3) \\
\hline 1532( & 46) & 1510( & 25) & 1447( & 2) & 1491( & 4) \\
\hline 1554( & 27) & 1539( & 19) & 1463( & 1) & 1506( & 3) \\
\hline 1588( & 83) & 1623( & 35) & 1547( & 3) & 1645( & 3) \\
\hline 1686( & 20) & 1675( & 18) & 1635( & 25) & 1707( & 32) \\
\hline 2993( & 21) & 29666 & 21) & 27331 & 70) & 2923( & 58) \\
\hline 3017( & 28) & 2996( & 30) & 2929) & 18) & 3017( & 38) \\
\hline 3035( & 47) & 3011( & 43) & 2951( & 29) & 3036( & 75) \\
\hline 3040( & 8) & 3022( & 10) & 2964( & 70) & 3065( & 48) \\
\hline 3074( & 4) & 3041( & 12) & 2997( & 20) & 3068( & 63) \\
\hline 3088( & 41) & 3052 ( & 5) & 3002 ( & 14) & 3083( & 27) \\
\hline 3091( & 19) & 3068( & 57) & 3014( & 51) & 3106( & 72) \\
\hline 3097( & 27) & 3071( & 25) & 3019) & 21) & 3109 & 38) \\
\hline 3137( & 2) & 3102( & 2) & 3031( & 18) & 3111( & 36) \\
\hline 3140( & 5) & 3110( & 3) & 3063( & 12) & 3140 & 21) \\
\hline 3146( & 31) & 3122( & 4) & 3069 ( & 4) & 3154( & 7) \\
\hline 3146( & 28) & 3125( & 54) & 3076( & 39) & 3162( & 75) \\
\hline 3164( & 3) & 3132( & 12) & 3087( & 1) & 3164( & 12) \\
\hline 3175( & 23) & 3144( & 3) & 3092( & 20) & 3172( & 35) \\
\hline 3182( & 8) & 31531 & 34) & 3102( & 14) & 3191( & 23) \\
\hline 3189 & 18) & 3165( & 6) & 3115( & 14) & 3198( & 38) \\
\hline 3231( & 6) & 31999 & 6) & 3117( & 15) & 3209 & 24) \\
\hline 3243( & 3) & 32266 & 2) & 3149 & 6) & 3235( & 11) \\
\hline
\end{tabular}


Table S86. Harmonic vibrational frequencies (in $\mathrm{cm}^{-1}$ ) and infrared intensities (in parentheses in $\mathrm{km} / \mathrm{mol}$ ) for the $\left(\mathbf{C}_{4} \mathbf{H}_{6}\right)_{3}$ Co structure Co-1Q.

\begin{tabular}{|c|c|c|c|c|c|c|c|}
\hline \multicolumn{2}{|c|}{ B3LYP } & \multicolumn{2}{|c|}{ B3LYP* } & \multicolumn{2}{|c|}{ BP86 } & \multicolumn{2}{|c|}{ M06L } \\
\hline 35( & 0) & 44( & 0) & 52( & 0) & 80( & 0) \\
\hline 75( & 2) & 75( & 0) & 84( & 0) & 95( & 0) \\
\hline 86( & 0) & 92( & 0) & 105( & 0) & 132( & 0) \\
\hline 111( & 2) & 123( & 1) & 132( & 0) & 141( & 1) \\
\hline 121( & 2) & 138( & 0) & 154( & 0) & 173( & 1) \\
\hline 151( & 0) & 158( & 0) & 171( & 0) & 187( & 1) \\
\hline 160( & 0) & 173( & 0) & 190( & 0) & 213( & 1) \\
\hline 216( & 6) & 216( & 4) & 209( & 1) & 220( & 0) \\
\hline 227( & 0) & 227( & 1) & 230( & 1) & 238( & 0) \\
\hline 236( & 1) & 241( & 1) & 248( & 0) & 264( & 1) \\
\hline 255( & 1) & 268( & 2) & 278 ( & 0) & 295( & 0) \\
\hline 282( & 1) & 283( & 1) & 305( & 3) & 313( & 0) \\
\hline 347( & 1) & 3531 & 0) & 3531 & 1) & 334( & 1) \\
\hline 361( & 1) & 358( & 1) & 3666 & 1) & 370( & 2) \\
\hline 410( & 5) & 401( & 4) & 392( & 2) & 398( & 1) \\
\hline 433( & 20) & 420( & 11) & 3999 & 1) & 412( & 7) \\
\hline 460( & 9) & 457( & 4) & 438( & 2) & 445( & 1) \\
\hline 481( & 4) & 466( & 1) & 448( & 1) & 4766 & 1) \\
\hline 530( & 3) & 516( & 0) & 485( & 0) & 511( & 2) \\
\hline 577( & 7) & 576( & 4) & 558( & 2) & 570( & 2) \\
\hline 609 ( & 1) & 602( & 1) & 578( & 2) & 605( & 0) \\
\hline 655( & 3) & 647( & 7) & 614( & 30) & 663( & 1) \\
\hline 666( & 11) & 667( & 20) & 657( & 18) & 678( & 3) \\
\hline 681( & 18) & 675( & 4) & 662( & 12) & 693( & 11) \\
\hline 710( & 38) & 703( & 28) & 679) & 10) & 706( & 33) \\
\hline 728( & 38) & 720( & 51) & 692( & 41) & 730( & 16) \\
\hline 739( & 20) & 741( & 9) & $739 x$ & 10) & 757( & 9) \\
\hline 796( & 8) & 791( & 8) & 775( & 7) & 770( & 20) \\
\hline 819( & 1) & 805( & 6) & 792( & 5) & 802( & 14) \\
\hline 835( & 19) & 819( & 9) & 802( & 18) & 820( & 31) \\
\hline 861( & 2) & 856( & 2) & 822( & 13) & 848( & 23) \\
\hline 896( & 49) & 865( & 46) & 842( & 4) & 873( & 13) \\
\hline 912( & 6) & 901( & 5) & 868( & 2) & 908( & 1) \\
\hline 943( & 6) & 932( & 8) & 898( & 8) & 932( & 6) \\
\hline 947( & 3) & 938( & 2) & 908( & 2) & 950( & 1) \\
\hline 972( & 6) & 958( & 5) & 918( & 0) & 958( & 4) \\
\hline 981( & 4) & 966( & 3) & 920( & 3) & 967( & 14) \\
\hline 994( & 3) & 977( & 4) & 929( & 8) & 967( & 2) \\
\hline 1008( & 6) & 999( & 4) & 970( & 2) & 1004( & 3) \\
\hline 1011( & 7) & 1002( & 7) & 974( & 6) & 1009 & 4) \\
\hline 1020( & 4) & 1014( & 7) & 989( & 7) & 1026( & 12) \\
\hline 1050( & 6) & 1040( & 4) & 1011( & 2) & 1039 & 6) \\
\hline 1057( & 0) & 1047( & 2) & 1020( & 2) & 1057( & 2) \\
\hline 1084( & 3) & 1077( & 3) & 1054( & 2) & 1097( & 3) \\
\hline 1131( & 11) & 1125( & 7) & 1102( & 2) & 1139 & 4) \\
\hline 1144( & 10) & 1137( & 12) & 1112( & 11) & 1154( & 6) \\
\hline 1185( & 10) & 1175( & 8) & 1143( & 4) & 1186( & 3) \\
\hline 1212( & 1) & 1199( & 1) & 1162( & 0) & 1212( & 2) \\
\hline
\end{tabular}




\begin{tabular}{|c|c|c|c|c|c|c|c|}
\hline 1227( & 4) & 1212( & 5) & 1173( & 4) & 1227( & 3) \\
\hline 1233( & 2) & 1220 & 1) & 1182( & 1) & 1240 & 8) \\
\hline 1270( & 6) & 1258( & 9) & 1221( & 5) & 1264( & 10) \\
\hline 1272( & 8) & 1260 & 7) & 1226( & 10) & 1267( & 12) \\
\hline 1283( & 7) & 1272 & 9) & 1232 & 8) & 1279 & 9) \\
\hline 1320 & 0) & 1306( & 0) & 1262( & 0) & 1310 & 0) \\
\hline 1341( & 3) & 1324( & 5) & 1278( & 3) & 1329 & 6) \\
\hline 1342( & 3) & 1327( & 1) & 1284( & 2) & 1333( & 1) \\
\hline 1361( & 5) & 1345( & 4) & 1303( & 3) & 1349 & 8) \\
\hline 1411( & 2) & 1398( & 2) & 1355( & 2) & 1390 & 1) \\
\hline 1412( & 1) & 1401( & 1) & 1363( & 3) & 1410( & 2) \\
\hline 1427( & 2) & 1412( & 2) & 1367( & 1) & 1421( & 3) \\
\hline 1467( & 5) & 1455( & 6) & 1413( & 7) & 1454( & 9) \\
\hline 1472( & 1) & 1461( & 2) & 1420( & 3) & 1459 & 2) \\
\hline 1475( & 9) & 1468( & 5) & 1429 & 3) & 1463 & 3) \\
\hline 1483( & 4) & 1470 & 5) & 1437( & 3) & 1473 & 5) \\
\hline 1487( & 8) & 1475 & 7) & 1439 & 4) & 1481( & 4) \\
\hline 1493( & 11) & 1481( & 12) & 1440 & 15) & 1489 & 6) \\
\hline 1564( & 35) & 1543( & 34) & 1493( & 21) & 1542( & 54) \\
\hline 1608( & 22) & 1571( & 28) & 1506( & 23) & 1556( & 49) \\
\hline 1628( & 2) & 1598( & 4) & 1518( & 3) & 1608( & 8) \\
\hline 3006( & 28) & 2986( & 31) & 2925( & 42) & 3015 & 55) \\
\hline 3016( & 24) & 2995( & 20) & 2935( & 21) & 3023 & 84) \\
\hline 3025( & 67) & 3003( & 74) & 2943( & 84) & 3025 & 97) \\
\hline 3030 & 57) & 3009 & $56)$ & 2954( & 57) & 3036( & 155) \\
\hline 3071( & 20) & 3054 & 18) & 3003( & 14) & 3089 & 23) \\
\hline 3075( & 19) & 30620 & 33) & 3008( & 27) & 3095 & 43) \\
\hline 3081( & 33) & 3070 & 17) & 3017( & 13) & 3103 & 29) \\
\hline 3096( & 12) & 3076( & 10) & 3025 & 15) & 3107( & 21) \\
\hline 3108( & 9) & 3089 & 16) & 3032( & 13) & 3110 & 4) \\
\hline 3114( & 18) & 3096( & 9) & 3034 & 10) & 3113 & 20) \\
\hline 3116( & 12) & 3102( & 3) & 3043( & 8) & 3128( & 21) \\
\hline 3126( & 1) & 3103( & 9) & 3049 & 30) & 3131( & 13) \\
\hline 3129 & 5) & 3112( & 6) & 3054( & 9) & 3137( & 60) \\
\hline 3148( & 18) & 3125 & 16) & 3058( & 7) & 3162 & 45) \\
\hline 3151( & 15) & 3145( & 9) & 3099 & 15) & 3175 & 9) \\
\hline 3171( & 28) & 3153( & 28) & 3104( & 15) & 3186( & 28) \\
\hline 3175( & 16) & 3157( & 14) & 3117( & 14) & 3205( & 24) \\
\hline 3193( & 17) & 3186( & 13) & 3141( & 11) & 3273 & 17) \\
\hline
\end{tabular}


Table S87. Harmonic vibrational frequencies (in $\mathrm{cm}^{-1}$ ) and infrared intensities (in parentheses in $\mathrm{km} / \mathrm{mol})$ for the $\left(\mathbf{C}_{4} \mathbf{H}_{6}\right)_{3}$ Co structure Co-1Q.

\begin{tabular}{|c|c|c|c|c|c|c|c|}
\hline \multicolumn{2}{|c|}{ B3LYP } & \multicolumn{2}{|c|}{ B3LYP* } & \multicolumn{2}{|c|}{ BP86 } & \multicolumn{2}{|c|}{ M06L } \\
\hline 28( & 2) & 23( & 3) & 33( & 0) & 57( & 5) \\
\hline 56( & 0) & 59( & 0) & 59( & 0) & 61( & 0) \\
\hline 87( & 0) & 97( & 0) & 100( & 0) & 103( & 1) \\
\hline 105( & 0) & 107( & 0) & 127( & 1) & 107( & 0) \\
\hline 122( & 4) & 128( & 5) & 140( & 1) & 119( & 2) \\
\hline 126( & 1) & 130( & 1) & 157( & 1) & 126( & 4) \\
\hline 149 & 1) & 161( & 1) & 177( & 1) & 173( & 1) \\
\hline 180( & 4) & 186( & 3) & 225( & 2) & 202( & 0) \\
\hline 212( & 1) & 214( & 1) & 241( & 0) & 227( & 2) \\
\hline 261( & 0) & 269 & 0) & 285( & 1) & 285( & 1) \\
\hline 288( & 0) & 288( & 0) & 287( & 2) & 309( & 6) \\
\hline 306( & 2) & 307( & 2) & 295( & $0)$ & 340( & 1) \\
\hline 311( & 0) & 320( & 0) & 338( & 3) & 350( & 0) \\
\hline 357( & 1) & 363( & 0) & 373( & 15) & 373( & 0) \\
\hline 421( & 28) & 417( & 22) & 381( & 2) & 426( & 19) \\
\hline 436( & 14) & 432( & 11) & 417( & 5) & 436( & 10) \\
\hline 476( & 10) & 467( & 9) & 462( & 4) & 482( & 10) \\
\hline 526( & 0) & 5231 & 1) & 494( & 5) & 529 & 3) \\
\hline 537( & 1) & 531( & 0) & 506( & 9) & 554( & 3) \\
\hline 545( & 4) & 541( & 3) & 529 & 7) & 565( & 3) \\
\hline 570( & 4) & 570 & 4) & 5531 & 2) & 575( & 2) \\
\hline 602( & 0) & 595( & 0) & 570( & 1) & 5981 & 0) \\
\hline 632( & 14) & 6331 & 10) & 621( & 2) & 644( & 3) \\
\hline 657 & 8) & 651( & 7) & 649 & 7) & 669 & 19) \\
\hline 713( & 8) & 706( & 13) & 679 & 38) & 699 & 11) \\
\hline 719 ( & 71) & 711( & 68) & 6831 & 52) & 712( & 86) \\
\hline 762( & 44) & 757( & 35) & 744( & 22) & 747 ( & 17) \\
\hline 797( & 1) & 7861 & 0) & 768( & 2) & 783( & 10) \\
\hline 837( & 1) & 831( & 2) & 810( & 2) & 840( & 16) \\
\hline 852( & 3) & 850 & 3) & 828( & 15) & 866( & 4) \\
\hline 8731 & 0) & 870( & 1) & 839 & 7) & 879 & 38) \\
\hline 922( & 3) & 901( & 18) & 861( & 11) & 894( & 36) \\
\hline 951( & 4) & 941( & 7) & 884( & 14) & 930( & 1) \\
\hline 957( & 3) & 945( & 1) & 902( & 2) & 938( & 6) \\
\hline 967( & 1) & 956( & 0) & 914( & 7) & 955( & 12) \\
\hline 981( & 2) & 966( & 5) & 921( & 4) & 959 & 2) \\
\hline 991( & 2) & 971( & 0) & 926( & 11) & 971( & 9) \\
\hline 997( & 20) & 980( & 20) & 937( & 3) & 981( & 2) \\
\hline 1007( & 14) & 1002( & 6) & 977( & 0) & 1023( & 4) \\
\hline 1009( & 3) & 1004( & 1) & 987( & 2) & 1025( & 2) \\
\hline 1022( & 3) & 1013( & 2) & 991( & 1) & 1028( & 0) \\
\hline 1051( & 1) & 1044( & 1) & 1015( & 3) & 1062( & 4) \\
\hline 1055( & 2) & 1048( & 1) & 1023 & 1) & 1064( & 0) \\
\hline
\end{tabular}




\begin{tabular}{|c|c|c|c|c|c|c|c|}
\hline 1092( & 9) & 1083( & 7) & 1059 & 4) & 1098 & 3) \\
\hline 1122( & 4) & 1112( & 5) & 1085( & 9) & 1125 & 8) \\
\hline 1136( & 5) & 1125( & 4) & 1097( & 1) & 1142( & 1) \\
\hline 1195( & 3) & 1185( & 2) & 1151( & 2) & 1198( & 2) \\
\hline 1208( & 2) & 1197( & 1) & 1169 & 1) & 1216( & 0) \\
\hline 1224( & 3) & 1212( & 3) & 1177( & 2) & 1227( & 1) \\
\hline 1244( & 1) & 1231( & 2) & 1193 & 2) & 1244( & 2) \\
\hline 1257( & 3) & 1245( & 1) & 1206( & 2) & 1257( & 0) \\
\hline 1257( & 7) & 1246( & 7) & 1210 & 8) & 1258( & 7) \\
\hline 1287( & 8) & 1274( & 12) & 1231( & 16) & 1278( & 18) \\
\hline 1320( & 1) & 1307( & 1) & 1259 & 1) & 1320 & 0) \\
\hline 1321( & 1) & 1308( & 2) & 1266( & 1) & 1324( & 5) \\
\hline 1327( & 1) & 1314( & 2) & 1272( & 0) & 1324( & 4) \\
\hline 1348( & 2) & 1334( & 1) & 1289 & 1) & 1344( & 2) \\
\hline 1403( & 8) & 1390 & 5) & 1345( & 3) & 1400 & 4) \\
\hline 1409 & 0) & 1396( & 0) & 1355( & 1) & 1408( & 1) \\
\hline 1429 & 5) & 1414( & 7) & 1364( & 8) & 1426( & 5) \\
\hline 1462( & 2) & 1449 & 3) & 1400 & 5) & 1453 & 0) \\
\hline 1467( & 4) & 1454( & 4) & 1417( & 2) & 1463 & 2) \\
\hline 1471( & 1) & 1459 & 1) & 1425( & 1) & 1469 & 0) \\
\hline 1479 & 10) & 1467( & 8) & 1431( & 6) & 1472( & 3) \\
\hline 1488( & 2) & 1480( & 3) & 1436( & 11) & 1478( & 10) \\
\hline 1490 & 16) & 1481( & 17) & 1444( & 11) & 1481( & 17) \\
\hline 1624( & 0) & 1604( & 2) & 1499 & 9) & 1609 & 6) \\
\hline 1627( & 19) & 1607( & 6) & 1502( & 21) & 1620 & 17) \\
\hline 1641( & 3) & 1608( & 13) & 1566( & 3) & 1626( & 15) \\
\hline 3025( & 1) & 3004( & 1) & 2875 & 12) & 3040 & 13) \\
\hline 3027( & 41) & 30060 & 42) & 2944( & 25) & 3042 & 100) \\
\hline 3033( & 0) & 3016( & 0) & 2956( & 34) & 3046 & 17) \\
\hline 3036( & 34) & 3019 & 32) & 2962( & 32) & 3046( & 64) \\
\hline 3048( & 29) & 3029 & 28) & 2976( & 49) & 3052 & 15) \\
\hline 3048( & 60) & 3029 & 56) & 3005( & 19) & 3052 & 52) \\
\hline 3087( & 8) & 3065( & 8) & 3010 & 12) & 3104 & 48) \\
\hline 3091( & 41) & 3070 & 42) & 3015( & 12) & 3106 & 73) \\
\hline 3097( & 20) & 3077( & 20) & 3019 & 21) & 3111( & 10) \\
\hline 3105( & 28) & 3083( & 26) & 3020 & 41) & 3121( & 25) \\
\hline 3107( & 2) & 3091( & 4) & 3023( & 5) & 3121( & 14) \\
\hline 3110 & 6) & 3094( & 2) & 3030 & 23) & 3138 & 14) \\
\hline 3125( & 3) & 3101( & 3) & 3053( & 12) & 3139 & 6) \\
\hline 3128( & 15) & 3110 & 15) & 3056( & 12) & 3141( & 41) \\
\hline 3129 & 49) & 3112( & 49) & 3061( & 26) & 3154 & 1) \\
\hline 3149 & 17) & 3124( & 18) & 3096 & 8) & 3154 & 78) \\
\hline 3166( & 56) & 3148( & 51) & 3102 & 38) & 3177 & 116) \\
\hline 31660 & 21) & 3148( & 20) & 3103( & 24) & 3177( & 44) \\
\hline
\end{tabular}


Table S88. Harmonic vibrational frequencies (in $\mathrm{cm}^{-1}$ ) and infrared intensities (in parentheses in $\mathrm{km} / \mathrm{mol})$ for the $\left(\mathbf{C}_{4} \mathbf{H}_{6}\right)_{3} \mathbf{N i}$ structure Ni-1S.

\begin{tabular}{|c|c|c|c|c|c|c|c|}
\hline \multicolumn{2}{|c|}{ B3LYP } & \multicolumn{2}{|c|}{ B3LYP* } & \multicolumn{2}{|c|}{ BP86 } & \multicolumn{2}{|c|}{ M06L } \\
\hline 84( & 1) & 77( & 0) & 81( & 1) & 84( & 1) \\
\hline 97( & 0) & 97( & 0) & 88( & 0) & 110( & 0) \\
\hline 116( & 1) & 111( & 1) & 115( & 1) & 124( & 1) \\
\hline 144( & 1) & 140( & 1) & 141( & 1) & 151( & 1) \\
\hline 158( & 1) & 153( & 1) & 152( & 1) & 164( & 0) \\
\hline 205( & 0) & 204( & 0) & 198( & 0) & 207( & 0) \\
\hline 214( & 0) & 213( & 0) & 207( & 0) & 225( & 0) \\
\hline 234( & 0) & 2300 & 0) & 223( & 0) & 2360 & 0) \\
\hline 258( & 1) & 254( & 1) & 253( & 0) & 271( & 0) \\
\hline 291( & 0) & 288( & 1) & 282( & 0) & 304( & 0) \\
\hline 300( & 12) & 300( & 8) & 304( & 4) & 326( & 3) \\
\hline 325( & 1) & 325( & 1) & 322( & 1) & 341( & 1) \\
\hline 3351 & 3) & 338( & 3) & 345( & 3) & 368( & 5) \\
\hline 369 & 12) & 367( & 11) & 373( & 10) & 401( & 9) \\
\hline 412( & 1) & 409 & 1) & 410( & 1) & 433( & 1) \\
\hline 448( & 1) & 445( & 0) & 437( & 1) & 457( & 0) \\
\hline 479 & 7) & 478( & 6) & 476( & 4) & 482( & 8) \\
\hline 492( & 5) & 490( & 6) & 4861 & 2) & 502( & 2) \\
\hline 504( & 3) & 500( & 3) & 497( & 9) & 513( & 4) \\
\hline 576( & 5) & 571( & 5) & 556( & 5) & 581( & 6) \\
\hline 597( & 2) & 5931 & 2) & 579( & 1) & 604( & 0) \\
\hline 648( & 3) & 643( & 2) & 6261 & 2) & 651( & 3) \\
\hline 708( & 23) & 703( & 24) & 6861 & 27) & 714( & 21) \\
\hline 735( & 22) & 728( & 18) & 707( & 19) & 728( & 23) \\
\hline 748( & 11) & 741( & 13) & 717( & 8) & 742( & 5) \\
\hline 774( & 5) & 7681 & 6) & 751( & 6) & 781( & 5) \\
\hline 809 & 14) & 803( & 14) & 786( & 14) & 819 & 10) \\
\hline 823( & 5) & 816( & 5) & 798( & 6) & 825( & 5) \\
\hline 845( & 8) & 838( & 7) & 816( & 5) & 839 & 3) \\
\hline 865( & 6) & 858( & 5) & 834( & 3) & 857( & 2) \\
\hline 877( & 0) & 869 & 0) & 844( & 0) & 871( & 0) \\
\hline 8866 & 10) & 879 & 8) & 855( & 4) & 884( & 5) \\
\hline 915( & 4) & 909 & 4) & 884( & 3) & 923( & 2) \\
\hline 947( & 3) & 939 & 3) & 914( & 6) & 947( & 5) \\
\hline 951( & 5) & 943( & 4) & 916( & 1) & 951( & 5) \\
\hline 984( & 6) & 969 & 6) & 926( & 7) & 958( & 4) \\
\hline 1000 & 0) & 986( & 0) & 942( & 0) & 971( & 0) \\
\hline 1007( & 1) & 9961 & 1) & 960( & 0) & 999( & 0) \\
\hline 1011( & 2) & 1000( & 2) & 971( & 2) & 1008( & 2) \\
\hline 1016( & 5) & 1008( & 5) & 983( & 6) & 1022( & 7) \\
\hline 1040 & 19) & 1032( & 17) & 1006( & 17) & 1044( & 16) \\
\hline 1043( & 3) & 1034( & 4) & 1008( & 2) & 1049 & 4) \\
\hline 1054( & 3) & 1045( & 3) & 1020( & 3) & 1061( & 3) \\
\hline 1108( & 1) & 1099 & 1) & 1072( & 0) & 1114( & 1) \\
\hline 1165( & 5) & 1156( & 5) & 1127( & 4) & 1159 & 6) \\
\hline 1175( & 3) & 1165 & 3) & 11360 & 3) & 1171( & 4) \\
\hline
\end{tabular}




\begin{tabular}{|c|c|c|c|c|c|c|c|}
\hline 1189 & 3) & 1179 & 3) & 1148( & 3) & 1188( & 3) \\
\hline 1222( & 1) & 1212( & 1) & 1179 & 1) & 1223( & 1) \\
\hline 1248( & 2) & 1236( & 2) & 1201( & 2) & 1243( & 2) \\
\hline 1252( & 7) & 1240 & 6) & 1204( & 5) & 1248( & 3) \\
\hline 1263( & 5) & 1253( & 5) & 1217( & 3) & 1249 & 7) \\
\hline 1271( & 3) & 1259 & 3) & 1226( & 2) & 1263( & 2) \\
\hline 1298( & 1) & 1287( & 1) & 1254( & 2) & 1290( & 2) \\
\hline 1329( & 1) & 1316( & 1) & 1276( & 1) & 1325( & 1) \\
\hline 1341( & 5) & 1328( & 5) & 1286( & 5) & 1341( & 4) \\
\hline 1349( & 4) & 1337( & 3) & 1295( & 2) & 1346( & 7) \\
\hline 1372( & 10) & 1358( & 9) & 1320 & 7) & 1374( & 10) \\
\hline 1401( & 1) & 1388( & 1) & 1347( & 2) & 1390( & 1) \\
\hline 1408( & 1) & 1394( & 1) & 1354( & 1) & 1399 & 1) \\
\hline 1430( & 1) & 1417( & 1) & 1379 & 1) & 1431( & 2) \\
\hline 1468( & 3) & 1454( & 3) & 1412( & 2) & 1460( & 6) \\
\hline 1473( & 2) & 1458( & 3) & 1417( & 4) & 1460( & 0) \\
\hline 1477( & 2) & 1464( & 0) & 1420( & 2) & 1463( & 3) \\
\hline 1481( & 3) & 1467( & 5) & 1426( & 5) & 1472( & 5) \\
\hline 1485( & 8) & 1470( & 6) & 1429 & 8) & 1478( & 4) \\
\hline 1495( & 11) & 1483( & 13) & 1439 & 11) & 1484( & 11) \\
\hline 1538( & 33) & 1523( & 31) & 1477( & 22) & 1524( & 18) \\
\hline 1542( & 3) & 1528( & 1) & 1484( & 1) & 1533( & 8) \\
\hline 1724( & 1) & 1710 & 1) & 1667( & 1) & 1745( & 1) \\
\hline 3010 ( & 31) & 2990( & 30) & 2934( & 33) & 3013( & 60) \\
\hline 3022( & 23) & 3002( & 23) & 2947( & 25) & 3035( & 29) \\
\hline 3033( & 75) & 3013( & 72) & 2958( & 72) & 3036( & 111) \\
\hline 3034( & 47) & 3014( & 44) & 2959 & 48) & 3044( & 91) \\
\hline 3064( & 17) & 3044( & 16) & 2992( & 16) & 3081( & 30) \\
\hline 3079) & 29) & 3060 & 28) & 3007( & 27) & 3107( & 39) \\
\hline 3107( & 2) & 3086( & 2) & 3030 & 2) & 3115( & 4) \\
\hline 3122( & 7) & 3101( & 7) & 3041( & 9) & 3123( & 19) \\
\hline 3126( & 9) & 3104( & 10) & 3047( & 9) & 3126( & 27) \\
\hline 3130( & 7) & 3109 & 8) & 3049 & 10) & 3137( & 10) \\
\hline 3136( & 12) & 3115( & 10) & 3057( & 9) & 3139 & 23) \\
\hline 31460 & 29) & 3124( & 28) & 30660 & 37) & 3140( & 14) \\
\hline 3149 & 4) & 3128( & 25) & 3067( & 4) & 3147( & 26) \\
\hline 3150( & 37) & 3129 & 14) & 3070( & 44) & 3156( & 51) \\
\hline 3159r & 19) & 31360 & 23) & 3073( & 17) & 3159 & 74) \\
\hline 3161( & 30) & 3140( & 26) & 3082( & 28) & 31600 & 68) \\
\hline 3239 & 10) & 3216( & 10) & 3155( & 12) & 3231( & 19) \\
\hline 3241( & 7) & 3221( & 6) & 31600 & 6) & 32370 & 16) \\
\hline
\end{tabular}


Table S89. Harmonic vibrational frequencies (in $\mathrm{cm}^{-1}$ ) and infrared intensities (in parentheses in $\mathrm{km} / \mathrm{mol})$ for the $\left(\mathbf{C}_{4} \mathbf{H}_{6}\right)_{3} \mathbf{N i}$ structure Ni-2S.

\begin{tabular}{|c|c|c|c|c|c|c|c|}
\hline \multicolumn{2}{|c|}{ B3LYP } & \multicolumn{2}{|c|}{ B3LYP* } & \multicolumn{2}{|r|}{ BP86 } & \multicolumn{2}{|c|}{ M06L } \\
\hline 23( & 0) & 19) & 0) & 35( & 0) & 48( & 1) \\
\hline 631 & 0) & 56( & 0) & 53( & 1) & 54( & 1) \\
\hline 102( & 0) & 101( & 0) & 101( & 0) & 105( & 0) \\
\hline 135( & 0) & 128( & 0) & 123( & 0) & 116( & 0) \\
\hline 171( & 2) & 169 & 2) & 165( & 1) & 168( & 2) \\
\hline 188( & 1) & 187( & 1) & 187( & 1) & 190( & 1) \\
\hline 2331 & 4) & 235( & 3) & 237( & 3) & 240( & 4) \\
\hline 250( & 5) & 255( & 5) & 250( & 1) & 258( & 1) \\
\hline 262( & 1) & 260( & 0) & 262( & 5) & 270( & 3) \\
\hline 269 ( & 1) & 268( & 1) & 272( & 2) & 284( & 2) \\
\hline 282( & 6) & 284( & 1) & 287( & 1) & 301( & 1) \\
\hline 288( & 2) & 292( & 6) & 291( & 0) & 308( & 1) \\
\hline 298( & 1) & 297( & 3) & 320( & 7) & 321( & 8) \\
\hline 399( & 9) & 396( & 9) & 395( & 9) & 414( & 8) \\
\hline 414( & 3) & 414( & 3) & 411( & 3) & 428( & 5) \\
\hline 454( & 2) & 453( & 3) & 450( & 4) & 458( & 2) \\
\hline 488( & 6) & 487( & 5) & 482( & 2) & 507( & 3) \\
\hline 511( & 0) & 507( & 0) & 500( & 1) & 523( & 2) \\
\hline 550( & 1) & 545( & 1) & 530( & 3) & 550( & 4) \\
\hline 562( & 3) & 557( & 2) & 542( & 1) & 571( & 3) \\
\hline 573( & 15) & 567( & 16) & 554( & 15) & 574( & 12) \\
\hline 620( & 3) & 616( & 4) & 601( & 5) & 615( & 4) \\
\hline 677( & 11) & 669 & 12) & 647( & 11) & 673( & 8) \\
\hline 739 ( & 3) & 731( & 3) & 708( & 3) & 720( & 3) \\
\hline 772( & 9) & 767( & 9) & 750( & 9) & 770( & 9) \\
\hline 810( & 8) & 805( & 8) & 787( & 10) & 796( & 3) \\
\hline 834( & 8) & 828( & 8) & 8066 & 9) & 815( & 55) \\
\hline 8531 & 22) & 845( & 30) & 807( & 60) & 831( & 6) \\
\hline 861( & 47) & 853( & 38) & 828( & 2) & 854( & 6) \\
\hline 8699 & 10) & 864( & 9) & 842( & 6) & 862( & 3) \\
\hline 887( & 7) & 882( & 6) & 861( & 5) & 878( & 2) \\
\hline 913( & 2) & 906( & 2) & 879( & 2) & 899( & 4) \\
\hline 943( & 1) & 934( & 0) & 907( & 0) & 938( & 1) \\
\hline 954( & 3) & 947( & 2) & 923( & 2) & 946( & 6) \\
\hline 971( & 2) & 961( & 2) & 928( & 1) & 956( & 0) \\
\hline 988( & 4) & 977( & 3) & 939 & 2) & 966( & 1) \\
\hline 991( & 3) & 978( & 2) & 946( & 1) & 968( & 1) \\
\hline 1005( & 5) & 993( & 4) & 957( & 2) & 988( & 2) \\
\hline 1010( & 1) & 1000( & 1) & 970( & 3) & 1002( & 2) \\
\hline 1027( & 12) & 1015( & 12) & 981( & 12) & 1011( & 10) \\
\hline 1029( & 2) & 1020( & 3) & 993( & 4) & 1031( & 6) \\
\hline 1041( & 1) & 1032( & 1) & 1007( & 1) & 1039 & 2) \\
\hline 1051( & 4) & 1042( & 3) & 1015( & 4) & 1050 & 4) \\
\hline 1083( & 5) & 1075( & 4) & 1051( & 2) & 1090( & 1) \\
\hline 1134( & 6) & 1125( & 5) & 1098( & 3) & 1134( & 2) \\
\hline 1160( & 10) & 1151( & 10) & 1123( & 8) & 1159 & 9) \\
\hline
\end{tabular}




\begin{tabular}{|c|c|c|c|c|c|c|c|}
\hline 1189 & 2) & 1179 & 2) & 11466 & 1) & 1185( & 1) \\
\hline 1216( & 2) & 1204( & 2) & 1171( & 3) & 1216( & 2) \\
\hline 1227( & 1) & 1218( & 1) & 1185( & 2) & 1235 & 2) \\
\hline 1250( & 0) & 1239 & 1) & 1205( & 1) & 1249 & 1) \\
\hline 1262( & 2) & 1249 & 2) & 1216( & 1) & 1254( & 0) \\
\hline 1285( & 6) & 1273( & 6) & 1236( & 6) & 1274( & 8) \\
\hline 1303( & 0) & 1290( & 1) & 1251( & 1) & 1303 & 0) \\
\hline 1311( & 3) & 1299 & 3) & 1259 & 2) & 1307( & 3) \\
\hline 1321( & 1) & 1309 & 2) & 1269 & 3) & 1312( & 0) \\
\hline 1322( & 1) & 1310 & 1) & 1273( & 1) & 1322( & 2) \\
\hline 1332( & 3) & 1319 & 3) & 1280( & 2) & 1330( & 2) \\
\hline 1376( & 3) & 1365( & 3) & 1328( & 4) & 1380( & 4) \\
\hline 1414( & 1) & 1401( & 1) & 1360( & 1) & 1402( & 1) \\
\hline 1423( & 5) & 1409 & 5) & 1365( & 5) & 1417( & 7) \\
\hline 1443( & 4) & 1431( & 3) & 1392( & 3) & 1434( & 3) \\
\hline 1483( & 5) & 1468( & 4) & 1427( & 4) & 1461( & 3) \\
\hline 1487( & 1) & 1472( & 2) & 1432( & 1) & 1470 & 1) \\
\hline 1495( & 2) & 1482( & 2) & 1443( & 3) & 1479 & 3) \\
\hline 1499( & 2) & 1484( & 2) & 1444( & 2) & 1481( & 4) \\
\hline 1505( & 13) & 1491( & 14) & 1452( & 14) & 1489 & 10) \\
\hline 1554( & 8) & 1538( & 7) & 1493( & 5) & 1545( & 6) \\
\hline 1612( & 1) & 1590( & 1) & 1527( & 1) & 1597( & 2) \\
\hline 1648( & 76) & 1634( & 71) & 1588( & 58) & 1648( & 79) \\
\hline 3015( & 42) & 2995( & 44) & 2939 & 54) & 3031( & 72) \\
\hline 3038( & 76) & 3019 & 30) & 2963( & 30) & 3042( & 76) \\
\hline 3039) & 22) & 3020( & 64) & 2968( & 71) & 3053( & 111) \\
\hline 3051( & 67) & 3031( & 67) & 2973( & 80) & 3059 & 110) \\
\hline 3067( & 11) & 3049 & 7) & 3001( & 2) & 3094( & 11) \\
\hline 3082( & 13) & 3062( & 14) & 3015( & 12) & 3101( & 2) \\
\hline 3096( & 24) & 3077( & 24) & 3022( & 5) & 3105( & 19) \\
\hline 3100( & 13) & 3080 & 12) & 3026( & 31) & 3117( & 22) \\
\hline 3118( & 16) & 3098( & 16) & 3040( & 17) & 3119 & 41) \\
\hline 3125( & 22) & 3104( & 20) & 3046( & 9) & 3123 ( & 46) \\
\hline 3128( & 8) & 3105( & 8) & 3048( & 28) & 3130( & 49) \\
\hline 3131( & 13) & 3109 & 13) & 3049 & 10) & 3131( & 22) \\
\hline 3144( & 3) & 3123( & 3) & 3065( & 2) & 3151( & 10) \\
\hline 3153( & 5) & 3133( & 4) & 3072( & 22) & 3152( & 8) \\
\hline 3159 ( & 7) & 31360 & 11) & 3078( & 5) & 3160( & 45) \\
\hline 3165( & 31) & 3143( & 28) & 3084( & 27) & 3173( & 39) \\
\hline 3224( & 8) & 3201( & 8) & 3139 & 9) & 3217 ( & 18) \\
\hline 3238( & 22) & 3220 & 20) & 3166( & 19) & 3244 ( & 34) \\
\hline
\end{tabular}


Table S90. Harmonic vibrational frequencies (in $\mathrm{cm}^{-1}$ ) and infrared intensities (in parentheses in $\mathrm{km} / \mathrm{mol})$ for the $\left(\mathbf{C}_{4} \mathbf{H}_{6}\right)_{3} \mathbf{N i}$ structure Ni-3S.

\begin{tabular}{|c|c|c|c|c|c|c|c|}
\hline \multicolumn{2}{|c|}{ B3LYP } & \multicolumn{2}{|c|}{ B3LYP* } & \multicolumn{2}{|c|}{ BP86 } & \multicolumn{2}{|c|}{ M06L } \\
\hline 43( & 0) & 49 & 0) & 41( & 0) & 48( & 0) \\
\hline 68( & 0) & 75( & 0) & 74( & 0) & 76( & 0) \\
\hline 106( & 0) & 109 & 0) & 110( & 0) & 107( & 0) \\
\hline 172( & 0) & 171( & 0) & 169 & 0) & 184( & 0) \\
\hline 186( & 1) & 188( & 2) & 185( & 3) & 202( & 5) \\
\hline 196( & 5) & 198( & 4) & 195( & 2) & 204( & 1) \\
\hline 230( & 2) & 237( & 2) & 238( & 1) & 259 & 3) \\
\hline 240( & 3) & 2460 & 3) & 257( & 1) & 272 & 4) \\
\hline 2531 & 4) & 256( & 3) & 261( & 3) & 276( & 0) \\
\hline 262( & 3) & 264( & 3) & 272 & 2) & 286( & 2) \\
\hline 276( & 1) & 276( & 1) & 275( & 0) & 290( & 3) \\
\hline 279 & 2) & 281( & 2) & 292( & 7) & 315( & 3) \\
\hline 3310 & 1) & 326( & 1) & 321( & 1) & 3361 & 1) \\
\hline 394( & 5) & 395( & 6) & 397( & 7) & 414( & 5) \\
\hline 412( & 4) & 412( & 4) & 408( & 3) & 4300 & 5) \\
\hline 440 & 1) & 439 & 1) & 438( & 2) & 453( & 0) \\
\hline 487( & 3) & 484( & 2) & 476( & 1) & 494( & 1) \\
\hline 505( & 0) & 500( & 1) & 488( & 1) & 515( & 2) \\
\hline 543( & 1) & 540( & 1) & 526( & 1) & 547( & 2) \\
\hline 569 & 8) & 567( & 8) & 551( & 8) & 575( & 7) \\
\hline 604( & 1) & 600 & 1) & 587( & 0) & 608( & 0) \\
\hline 639 & 5) & 635( & 5) & 620( & 6) & 642( & 5) \\
\hline 692( & 14) & 684( & 14) & 665( & 15) & 683( & 12) \\
\hline 740 & 7) & 734( & 6) & 717( & 4) & 738( & 7) \\
\hline 772( & 11) & 768( & 11) & 752( & 9) & 771( & 7) \\
\hline 803( & 5) & 798( & 5) & 784( & 5) & 805( & 1) \\
\hline 825( & 14) & 819 & 12) & 801( & 8) & 823( & 51) \\
\hline 861( & 20) & 853( & 63) & 820 & 58) & 8331 & 8) \\
\hline 864( & 50) & 856( & 6) & 837( & 7) & 867( & 11) \\
\hline 870( & 7) & 867( & 8) & 848( & 7) & 878( & 3) \\
\hline 905( & 6) & 898( & 5) & 876( & 2) & 893( & 1) \\
\hline 915( & 0) & 907( & 0) & 885( & 1) & 914( & 2) \\
\hline 9361 & 7) & 927( & 7) & 899 & 7) & 928( & 11) \\
\hline 946( & 7) & 937( & 6) & 911( & 4) & 941( & 2) \\
\hline 960( & 1) & 951( & 1) & 924( & 1) & 959 & 1) \\
\hline 986( & 4) & 975( & 4) & 9361 & 4) & 974( & 2) \\
\hline 994( & 0) & 983( & 0) & 950( & 1) & 979 & 0) \\
\hline 1011( & 5) & 999 & 2) & 958( & 0) & 990( & 0) \\
\hline 1017( & 3) & 1006( & 5) & 978( & 5) & 1013( & 7) \\
\hline 1021( & 2) & 1013( & 2) & 985( & 2) & 1021( & 4) \\
\hline 1026( & 7) & 1016( & 8) & 988( & 10) & 1026( & 7) \\
\hline 1040( & 5) & 1030( & 4) & 1002( & 3) & 1037( & 3) \\
\hline 1051( & 3) & 1043( & 3) & 1015( & 2) & 1050 & 3) \\
\hline 1096( & 2) & 1088( & 2) & 1062( & 2) & 1097( & 1) \\
\hline 1127( & 15) & 1118( & 15) & 1088( & 13) & 1124( & 13) \\
\hline 1152( & 4) & 1143( & 4) & 1112( & 4) & 1151( & 4) \\
\hline
\end{tabular}




\begin{tabular}{|c|c|c|c|c|c|c|c|}
\hline 1188( & 2) & 1179 & 2) & 1147( & 3) & 1188( & 4) \\
\hline 1216( & 1) & 1206( & 1) & 1173( & 1) & 1222( & 2) \\
\hline 1229 ( & 1) & 1218( & 1) & 1188( & 1) & 1236( & 1) \\
\hline 1249 & 0) & 1239 & 0) & 1202( & 1) & 1252( & 1) \\
\hline 1262( & 3) & 1252( & 2) & 1218( & 2) & 1258( & 2) \\
\hline 1289 ( & 3) & 1277( & 3) & 1238( & 3) & 1281( & 5) \\
\hline 1303( & 3) & 1291( & 3) & 1253( & 4) & 1295( & 1) \\
\hline 1311( & 1) & 1298( & 1) & 1255( & 2) & 1307( & 1) \\
\hline 1321( & 1) & 1311( & 2) & 1268( & 3) & 1313 & 1) \\
\hline 1326( & 1) & 1312( & 1) & 1275( & 1) & 1320 & 1) \\
\hline 1339x & 3) & 1327( & 3) & 1290( & 3) & 1343( & 3) \\
\hline 1354( & 3) & 1341( & 3) & 1303( & 4) & 1359 & 5) \\
\hline 1415( & 1) & 1404( & 1) & 1362( & 1) & 1408( & 2) \\
\hline 1427( & 3) & 1412( & 3) & 1367( & 4) & 1419 & 5) \\
\hline 1445( & 4) & 1432( & 4) & 1393( & 3) & 1434( & 2) \\
\hline 1480( & 2) & 1468( & 3) & 1426( & 3) & 1463( & 1) \\
\hline 1486( & 5) & 1474( & 5) & 1431( & 4) & 1470 & 6) \\
\hline 1498( & 4) & 1483( & 5) & 1443( & 2) & 1479 & 5) \\
\hline 1499( & 2) & 1487( & 1) & 1445( & 7) & 1485( & 11) \\
\hline 1503( & 19) & 1490( & 19) & 1447( & 20) & 1490 & 6) \\
\hline 1561( & 5) & 1548( & 4) & 1502( & 2) & 1557( & 3) \\
\hline 1624( & 2) & 1601( & 2) & 1534( & 2) & 1602( & 4) \\
\hline 1652( & 68) & 1639 & 63) & 1596( & 52) & 1662( & 63) \\
\hline 3017( & 34) & 2997( & 36) & 2942( & 44) & 3020 & 104) \\
\hline 3028( & 74) & 3008( & 76) & 2954( & 81) & 3046( & 9) \\
\hline 3040( & 18) & 3020( & 19) & 2963( & 23) & 3047( & 129) \\
\hline 3041( & 44) & 3025( & 37) & 2972( & 79) & 3056( & 112) \\
\hline 3051( & 62) & 3031( & 61) & 2976( & 22) & 3075( & 29) \\
\hline 3064( & 26) & 3046( & 25) & 2996( & 22) & 3087( & 45) \\
\hline 3104( & 5) & 3083( & 5) & 3023( & 3) & 3106( & 4) \\
\hline 3108( & 4) & 3087( & 3) & 3030 & 2) & 3114( & 15) \\
\hline 3122( & 23) & 3100( & 24) & 3038( & 30) & 3124( & 8) \\
\hline 3125( & 8) & 3104( & 8) & 3047( & 7) & 3128( & 31) \\
\hline 3133( & 4) & 3113( & 4) & 3054( & 4) & 3129 ( & 39) \\
\hline 3140( & 20) & 3121( & 17) & 3064( & 23) & 3142( & 38) \\
\hline 3143( & 6) & 3122( & 8) & 3064( & 5) & 3150( & 15) \\
\hline 3155( & 6) & 3135( & 5) & 3072( & 14) & 3158( & 13) \\
\hline 3159 ( & 3) & 3137( & 4) & 3081( & 6) & 3170( & 6) \\
\hline 3167 ( & 37) & 3145( & 37) & 3084( & 37) & 3177 ( & 80) \\
\hline 3225( & 7) & 3205( & 7) & 3148( & 7) & 3221( & 16) \\
\hline 3238( & 21) & 3219 & 20) & 3166( & 20) & 3245( & 33) \\
\hline
\end{tabular}


Table S91. Harmonic vibrational frequencies (in $\mathrm{cm}^{-1}$ ) and infrared intensities (in parentheses in $\mathrm{km} / \mathrm{mol})$ for the $\left(\mathbf{C}_{4} \mathbf{H}_{6}\right)_{3} \mathbf{N i}$ structure Ni-4S.

\begin{tabular}{|c|c|c|c|c|c|c|c|}
\hline \multicolumn{2}{|c|}{ B3LYP } & \multicolumn{2}{|c|}{ B3LYP* } & \multicolumn{2}{|c|}{ BP86 } & \multicolumn{2}{|c|}{ M06L } \\
\hline 54( & 0) & 49( & 0) & 51( & 0) & 54( & 0) \\
\hline 79( & 0) & 71( & 0) & 76( & 0) & 78( & 0) \\
\hline 112( & 0) & 106( & 0) & 102( & 0) & 117( & 0) \\
\hline 148( & 0) & 147( & 0) & 144( & 0) & 158( & 1) \\
\hline 175( & 4) & 177( & 3) & 178( & 2) & 184( & 3) \\
\hline 195( & 2) & 204( & 3) & 210( & 4) & 217( & 4) \\
\hline 203( & 4) & 206( & 3) & 215( & 1) & 219 & 1) \\
\hline 233( & 3) & 235( & 3) & 246( & 2) & 254( & 4) \\
\hline 247( & 0) & 248( & 1) & 249 & 2) & 259 & 0) \\
\hline 263( & 2) & 264( & 2) & 273( & 2) & 284( & 4) \\
\hline 275( & 1) & 275( & 1) & 281( & 0) & 299 & 0) \\
\hline 312( & 2) & 311( & 3) & 337( & 5) & 335( & 4) \\
\hline 380( & 3) & 376( & 2) & 364( & 2) & 384( & 2) \\
\hline 403( & 3) & 401( & 4) & 396( & 6) & 416( & 5) \\
\hline 417( & 7) & 414( & 7) & 404( & 8) & 422( & 8) \\
\hline 423( & 3) & 425( & 2) & 428( & 3) & 438( & 1) \\
\hline 488( & 5) & 487( & 4) & 484( & 3) & 503( & 7) \\
\hline 507( & 7) & 501( & 6) & 485( & 4) & 508( & 3) \\
\hline 558( & 4) & 5531 & 5) & 535( & 4) & 566( & 3) \\
\hline 573( & 9) & 566( & 8) & 555( & 8) & 577( & 9) \\
\hline 597( & 3) & 594( & 3) & 584( & 3) & 602( & 2) \\
\hline 658( & 4) & 652( & 4) & 6381 & 4) & 660( & 3) \\
\hline 712( & 49) & 705( & 50) & 6831 & 54) & 706( & 46) \\
\hline 741( & 5) & 737( & 4) & 715 ( & 3) & 734( & 4) \\
\hline 751( & 5) & 747( & 4) & 7331 & 3) & 752( & 2) \\
\hline 789( & 10) & 783( & 10) & 7631 & 8) & 787( & 6) \\
\hline 793( & 6) & 789 & 6) & 775 ( & 7) & 794( & 2) \\
\hline 828( & 2) & 822( & 2) & 802( & 3) & 828( & 3) \\
\hline 850( & 4) & 845( & 4) & 830( & 5) & 861( & 10) \\
\hline 866( & 13) & 861( & 12) & 844( & 10) & 868( & 3) \\
\hline 894( & 2) & 888( & 2) & 870( & 3) & 893( & 1) \\
\hline 923( & 1) & 918( & 1) & 892( & 0) & 908( & 4) \\
\hline 953( & 2) & 944( & 1) & 918( & 1) & 952( & 0) \\
\hline 960( & 2) & 952( & 1) & 919 & 0) & 954( & 0) \\
\hline 969 & 0) & 958( & 0) & 930( & 0) & 963( & 1) \\
\hline 989( & 6) & 977( & 5) & 9431 & 3) & 971( & 1) \\
\hline 996( & 1) & 984( & 1) & 947( & 1) & 977( & 3) \\
\hline 1005( & 2) & 996( & 2) & 967( & 4) & 1004( & 5) \\
\hline 1011( & 2) & 1002( & 2) & 978( & 1) & 1016( & 1) \\
\hline 1028( & 1) & 1018 & 2) & 991( & 3) & 1026( & 3) \\
\hline 1038( & 1) & 1029 & 0) & 1002( & 1) & 1035( & 1) \\
\hline 1056( & 2) & 1046( & 1) & 1015( & 1) & 1049 & 1) \\
\hline 1088( & 9) & 1080 & 10) & 1053( & 10) & 1089 & 14) \\
\hline 1102( & 12) & 1095 & 13) & 1073( & 10) & 1109 & 5) \\
\hline 1148( & 1) & 1138( & 1) & 1108( & 1) & 1146( & 1) \\
\hline 1157( & 9) & 1150 & 7) & 1117( & 5) & 1156( & 8) \\
\hline
\end{tabular}




\begin{tabular}{|c|c|c|c|c|c|c|c|}
\hline 1202( & 7) & 1190( & 7) & 1159 & 9) & 1199( & 9) \\
\hline 1217( & 1) & 1205( & 1) & 1175( & 0) & 1219 & 1) \\
\hline 1230( & 1) & 1218( & 1) & 1183( & 1) & 1235( & 1) \\
\hline 1239 & 1) & 1228( & 1) & 1194( & 1) & 1242( & 1) \\
\hline 1265( & 1) & 1253( & 0) & 1220 & 0) & 1253( & 0) \\
\hline 1272( & 4) & 1260( & 3) & 1225( & 2) & 1264( & 7) \\
\hline 1279 & 7) & 1267( & 8) & 1230 & 7) & 1266( & 5) \\
\hline 1308( & 0) & 1294( & 0) & 1255( & 1) & 1304( & 1) \\
\hline 1313( & 2) & 1299 & 2) & 1259 & 1) & 1311( & 1) \\
\hline 1332( & 3) & 1317( & 3) & 1274( & 2) & 1325( & 3) \\
\hline 1368( & 3) & 1354( & 2) & 1313( & 2) & 1366( & 2) \\
\hline 1409( & 9) & 1396( & 9) & 1352( & 9) & 1400( & 11) \\
\hline 1418( & 1) & 1405( & 1) & 1365( & 0) & 1409 & 1) \\
\hline 1423( & 1) & 1407( & 0) & 13660 & 1) & 1416( & 1) \\
\hline 1470( & 2) & 1455( & 2) & 1414( & 1) & 1458( & 1) \\
\hline 1480( & 6) & 1466( & 6) & 1425( & 6) & 1466( & 3) \\
\hline 1483( & 1) & 1469 & 1) & 1428( & 1) & 1471( & 6) \\
\hline 1497( & 6) & 1480( & 8) & 1442( & 13) & 1483( & 7) \\
\hline 1501( & 15) & 1487( & 14) & 1445( & 10) & 1487( & 6) \\
\hline 1505( & 1) & 1490( & 1) & 1449 & 1) & 1493( & 3) \\
\hline 1561( & 8) & 1547( & 7) & 1502( & 6) & 1555( & 6) \\
\hline 1607( & 2) & 1582( & 2) & 1513( & 0) & 1579( & 3) \\
\hline 1675( & 16) & 1661( & 12) & 1617( & 6) & 1695( & 13) \\
\hline 3015( & 42) & 2995( & 41) & 2941( & 40) & 3023( & 68) \\
\hline 3030( & 43) & 3010( & 42) & 2954( & 45) & 3038( & 83) \\
\hline 3032( & 40) & 3012( & 40) & 2956( & 49) & 3040( & 75) \\
\hline 3039( & 22) & 3022( & 20) & 2972( & 21) & 3041( & 26) \\
\hline 3050( & 52) & 3030( & 51) & 2975( & 56) & 3056( & 93) \\
\hline 3056( & 25) & 3037( & 22) & 2987( & 17) & 3079) & 5) \\
\hline 3097( & 7) & 3076( & 16) & 3016( & 26) & 3098( & 64) \\
\hline 3103( & 19) & 3079) & 10) & 3022( & 3) & 3103( & 16) \\
\hline 3108( & 2) & 3091( & 4) & 3039 & 13) & 3126( & 7) \\
\hline 3115( & 16) & 3096 & 14) & 3042( & 4) & 3127( & 38) \\
\hline 3128( & 32) & 3108( & 10) & 3050( & 12) & 3128( & 5) \\
\hline 3129 & 15) & 3110( & 35) & 3059 & 2) & 3146( & 60) \\
\hline 3141( & 4) & 3120( & 3) & 3061( & 34) & 3147( & 8) \\
\hline 3153( & 1) & 3129 & 3) & 3065( & 5) & 3151( & 9) \\
\hline 3161( & 51) & 3140( & 20) & 3080( & 6) & 3166r & 46) \\
\hline 3161( & 21) & 3141( & 49) & 3083( & 36) & 3172( & 32) \\
\hline 3176( & 14) & 3151( & 16) & 3088( & 53) & 3180( & 81) \\
\hline 3225( & 9) & 3205( & 9) & 3145( & 11) & 3226( & 21) \\
\hline
\end{tabular}


Table S92. Harmonic vibrational frequencies (in $\mathrm{cm}^{-1}$ ) and infrared intensities (in parentheses in $\mathrm{km} / \mathrm{mol})$ for the $\left(\mathbf{C}_{4} \mathbf{H}_{6}\right)_{3} \mathbf{N i}$ structure Ni-5S.

\begin{tabular}{|c|c|c|c|c|c|c|c|}
\hline \multicolumn{2}{|c|}{ B3LYP } & \multicolumn{2}{|c|}{ B3LYP* } & \multicolumn{2}{|c|}{ BP86 } & \multicolumn{2}{|c|}{ M06L } \\
\hline 42( & 1) & 32( & 1) & 34( & 1) & 57( & 1) \\
\hline 57( & 1) & 54( & 1) & 59 ( & 1) & 69( & 2) \\
\hline 108( & 2) & 110( & 2) & 113( & 1) & 111( & 2) \\
\hline 113( & 1) & 112( & 1) & 122( & 0) & 129( & 0) \\
\hline 120( & 1) & 122( & 1) & 129( & 1) & 138( & 1) \\
\hline 139 & 2) & 139( & 1) & 141( & 1) & 149( & 1) \\
\hline 191( & 3) & 196( & 3) & 210( & 2) & 218( & 3) \\
\hline 212( & 3) & 209 & 3) & 215( & 1) & 230( & 0) \\
\hline 220 & 2) & 223( & 3) & 228( & 6) & 241( & 7) \\
\hline 232( & 0) & 2331 & 0) & 2531 & 0) & 272( & 1) \\
\hline 276( & 1) & 272( & 1) & 279) & 2) & 292( & 1) \\
\hline 302( & 4) & 302( & 3) & 312( & 2) & 327( & 3) \\
\hline 341( & 1) & 3360 & 2) & 3331 & 2) & 345( & 3) \\
\hline 353( & 1) & 353( & 4) & 351( & 3) & 375( & 3) \\
\hline 358( & 4) & 357( & 1) & 378( & 0) & 393( & 1) \\
\hline 382( & 1) & 383( & 1) & 380( & 0) & 401( & 0) \\
\hline 402( & 1) & 400( & 2) & 402( & 2) & 434( & 2) \\
\hline 449 & 2) & 443( & 2) & 432( & 1) & 445( & 3) \\
\hline 531( & 8) & 528( & 8) & 513( & 5) & 521( & 6) \\
\hline 560( & 1) & 555( & 1) & 541( & 1) & 549 & 1) \\
\hline 571( & 2) & 566( & 2) & 552( & 2) & 570( & 2) \\
\hline 587( & 1) & 584( & 1) & 573( & 2) & 599( & 1) \\
\hline 655( & 24) & 653( & 7) & 638( & 7) & 666( & 10) \\
\hline 659 & 4) & 654( & 20) & 652( & 22) & 676( & 16) \\
\hline 713( & 43) & 707( & 28) & 678( & 11) & 709( & 28) \\
\hline 722( & 10) & 711( & 26) & 691( & 45) & 714( & 26) \\
\hline 735( & 30) & 724( & 34) & 708( & 38) & 729 ( & 22) \\
\hline 782( & 22) & 777( & 18) & 759) & 14) & 762( & 18) \\
\hline 786( & 5) & 785( & 5) & 773( & 3) & 779) & 3) \\
\hline 820( & 15) & 815( & 13) & 802( & 12) & 842( & 11) \\
\hline 853( & 5) & 847( & 5) & 827( & 7) & 848( & 12) \\
\hline 889 & 30) & 876( & 33) & 843( & 28) & 861( & 8) \\
\hline 8966 & 3) & 880( & 3) & 847( & 4) & 864( & 7) \\
\hline 928( & 4) & 919( & 2) & 894( & 2) & 930( & 1) \\
\hline 939 & 3) & 930( & 3) & 903( & 0) & 934( & 4) \\
\hline 965( & 5) & 949( & 3) & 903( & 2) & 938( & 2) \\
\hline 972( & 15) & 964( & 4) & 916( & 1) & 946( & 2) \\
\hline 978( & 0) & 965( & 9) & 929( & 2) & 961( & 11) \\
\hline 982( & 1) & 968( & 1) & 935( & 3) & 966( & 2) \\
\hline 985( & 3) & 976( & 5) & 952( & 8) & 975( & 2) \\
\hline 1007( & 5) & 999( & 7) & 958( & 4) & 994( & 6) \\
\hline 1021( & 9) & 1009( & 6) & 986( & 8) & 1013( & 12) \\
\hline 1038( & 3) & 1028( & 2) & 996( & 9) & 1022( & 11) \\
\hline 1048( & 19) & 1036( & 18) & 1005( & 6) & 1039x & 1) \\
\hline 1067( & 1) & 1058( & 1) & 1035( & 3) & 1066( & 4) \\
\hline 1079( & 5) & 1071( & 5) & 1048( & 3) & 1080( & 1) \\
\hline
\end{tabular}




\begin{tabular}{|c|c|c|c|c|c|c|c|}
\hline 1092( & 2) & 1080 & 2) & 1048( & 2) & 1081( & 1) \\
\hline 1123( & 5) & 1113( & 7) & 1088( & 5) & 1125( & 10) \\
\hline 1150( & 8) & 1138( & 8) & 1110( & 10) & 1149 & 11) \\
\hline 1216( & 7) & 1204( & 6) & 1173( & 4) & 1216( & 7) \\
\hline 1233( & 1) & 1221( & 1) & 1184( & 1) & 1232( & 1) \\
\hline 1253( & 1) & 1238( & 1) & 1193( & 1) & 1235( & 1) \\
\hline 1277( & 1) & 1264( & 1) & 1234( & 1) & 1272 & 2) \\
\hline 1303( & 10) & 1288( & 11) & 1244( & 11) & 1281( & 14) \\
\hline 1307( & 1) & 1297( & 0) & 1264( & 0) & 1301( & 0) \\
\hline 1335( & 1) & 1322( & 1) & 1282( & 1) & 1331( & 1) \\
\hline 1382( & 3) & 1370( & 3) & 1330( & 2) & 1386( & 4) \\
\hline 1418( & 4) & 1404( & 3) & 1364( & 1) & 1399 & 1) \\
\hline 1422( & 2) & 1407( & 2) & 1367( & 2) & 1414( & 4) \\
\hline 1426( & 1) & 1412( & 1) & 1375( & 1) & 1425( & 1) \\
\hline 1469 ( & 15) & 1455( & 13) & 1418( & 6) & 1459 ( & 6) \\
\hline 1473( & 4) & 1459 & 5) & 1420( & 9) & 1460 & 3) \\
\hline 1478( & 2) & 1464( & 3) & 1426( & 3) & 1467( & 3) \\
\hline 1488( & 2) & 1475( & 2) & 1435( & 2) & 1476( & 5) \\
\hline 1507( & 14) & 1492( & 14) & 1454( & 15) & 1500 & 10) \\
\hline 1578( & 57) & 1558( & 53) & 1504( & 38) & 1549 & 50) \\
\hline 1601( & 3) & 1580( & 3) & 1520( & 5) & 1566( & 6) \\
\hline 1664( & 1) & 1648( & 1) & 1599( & 3) & 1671( & 4) \\
\hline 1666( & 5) & 1651( & 5) & 1604( & 5) & 1682( & 5) \\
\hline 2996( & 45) & 2977( & 44) & 2922( & 46) & 3014( & 66) \\
\hline 3021( & 81) & 3002( & 78) & 2949( & 77) & 3032( & 139) \\
\hline 3046( & 27) & 3026( & 25) & 2974( & 22) & 3065( & 32) \\
\hline 3073( & 22) & 3057( & 21) & 3015 ( & 24) & 3096( & 36) \\
\hline 3082( & 26) & 3069 & 24) & 3024( & 27) & 3109 & 42) \\
\hline 3104( & 8) & 3085( & 7) & 3034( & 6) & 3119 & 13) \\
\hline 3114( & 7) & 3093( & 7) & 3043( & 7) & 3126( & 14) \\
\hline 3132( & 7) & 3108( & 8) & 3051( & 9) & 3150 & 6) \\
\hline 3142( & 42) & 3122( & 39) & 3068( & 38) & 3154( & 17) \\
\hline 3149 & 34) & 3129 & 33) & 3077( & 32) & 3157( & 53) \\
\hline 3164( & 23) & 3143( & 0) & 3080( & 3) & 3161( & 57) \\
\hline 3167( & 2) & 3146( & 20) & 3085( & 3) & 3162( & 24) \\
\hline 3170( & 3) & 3147( & 2) & 3096 & 18) & 3184( & 4) \\
\hline 3183( & 2) & 3159 & 2) & 3103( & 2) & 3190 & 20) \\
\hline 3186( & 36) & 3164( & 35) & 3106( & 28) & 3197( & 31) \\
\hline 3197( & 3) & 3174( & 3) & 3117 ( & 5) & 3198( & 19) \\
\hline 3257 ( & 4) & 3234( & 4) & 3168 & 5) & 3249 & 9) \\
\hline 3262( & 1) & 3239 & 1) & 3174( & 4) & 3257 & 12) \\
\hline
\end{tabular}


Table S93. Harmonic vibrational frequencies (in $\mathrm{cm}^{-1}$ ) and infrared intensities (in parentheses in $\mathrm{km} / \mathrm{mol})$ for the $\left(\mathbf{C}_{4} \mathbf{H}_{6}\right)_{3} \mathbf{N i}$ structure Ni-1T.

\begin{tabular}{|c|c|c|c|c|c|c|c|}
\hline \multicolumn{2}{|c|}{ B3LYP } & \multicolumn{2}{|c|}{ B3LYP* } & \multicolumn{2}{|c|}{ BP86 } & \multicolumn{2}{|c|}{ M06L } \\
\hline 54( & 0) & 56( & 0) & 631 & 0) & 70( & 0) \\
\hline 83( & 0) & 89( & 0) & 97( & 0) & 104( & 1) \\
\hline 99( & 1) & 93( & 1) & 113( & 0) & 124( & 0) \\
\hline 105( & 2) & 110( & 1) & 120( & 1) & 137( & 0) \\
\hline 118( & 1) & 126( & 1) & 133( & 1) & 152( & 3) \\
\hline 127( & 0) & 142( & 0) & 154( & 0) & 163( & 1) \\
\hline 134( & 1) & 151( & 3) & 194( & 2) & 167( & 2) \\
\hline 206( & 2) & 209 & 2) & 210( & 2) & 220( & 1) \\
\hline 223( & 0) & 225( & 0) & 226( & 0) & 227( & 0) \\
\hline 233( & 1) & 229 & 1) & 237( & 1) & 238( & 1) \\
\hline 240( & 2) & 255( & 3) & 270( & 0) & 263( & 2) \\
\hline 282( & 0) & 281( & 1) & 295( & 3) & 288( & 1) \\
\hline 320( & 2) & 321( & 1) & 318( & 1) & 324( & 2) \\
\hline 347( & 0) & 340( & 1) & 341( & 6) & 348( & 1) \\
\hline 393( & 2) & 389 & 1) & 382( & 1) & 408( & 2) \\
\hline 410( & 1) & 403( & 0) & 402( & 1) & 421( & 0) \\
\hline 452( & 2) & 450( & 2) & 440( & 5) & 468( & 2) \\
\hline 479( & 2) & 470( & 2) & 454( & 2) & 478( & 0) \\
\hline 503( & 1) & 501( & 0) & 485( & 0) & 514( & 1) \\
\hline 572( & 3) & 570 & 2) & 552( & 1) & 573( & 2) \\
\hline 595( & 0) & 590( & 1) & 570 & 5) & 595( & 1) \\
\hline 637( & 7) & 629 & 18) & 596( & 30) & 624( & 27) \\
\hline 671( & 33) & 661( & 31) & 646( & 15) & 670 & 29) \\
\hline 689( & 19) & 681( & 9) & 659 & 9) & 691( & 5) \\
\hline 703( & 15) & 699 & 13) & 685( & 7) & 702( & 0) \\
\hline 739) & 29) & 731( & 20) & 716( & 21) & 730 & 11) \\
\hline 744( & 18) & 734( & 26) & 728( & 23) & 736( & 26) \\
\hline 784( & 45) & 784( & 35) & 773 ( & 1) & 779 & 30) \\
\hline 797( & 5) & 792( & 5) & 779 ( & 29) & 800( & 7) \\
\hline 811( & 5) & 807( & 8) & 798( & 3) & 811( & 9) \\
\hline 843( & 12) & 835( & 5) & 824( & 1) & 835( & 1) \\
\hline 859 & 4) & 854( & 2) & 837( & 1) & 874 ( & 3) \\
\hline 906( & 3) & 896( & 2) & 8681 & 1) & 908( & 2) \\
\hline 941( & 2) & 927( & 3) & 897( & 7) & 929( & 5) \\
\hline 948( & 2) & 939( & 3) & 907( & 0) & 9431 & 0) \\
\hline 966( & 6) & 954( & 5) & 915( & 6) & 957( & 9) \\
\hline 986( & 5) & 968( & 5) & 921( & 2) & 958( & 0) \\
\hline 993( & 1) & 975( & 0) & 927( & 1) & 966( & 0) \\
\hline 1002( & 3) & 990( & 4) & 962( & 3) & 1000( & 2) \\
\hline 1011( & 4) & 1002( & 2) & 974( & 2) & 1009 & 2) \\
\hline 1021( & 8) & 1011( & 6) & 986( & 5) & 1026( & 4) \\
\hline 1041( & 1) & 1033( & 1) & 1002( & 2) & 1041( & 3) \\
\hline 1058( & 5) & 1048( & 4) & 1019 & 3) & 1057( & 3) \\
\hline 1090( & 3) & 1082( & 3) & 1056( & 2) & 1093( & 2) \\
\hline 1138( & 4) & 1132( & 3) & 1105( & 1) & 1138( & 2) \\
\hline 1157( & 6) & 1148( & 5) & 1122( & 4) & 1160( & 4) \\
\hline
\end{tabular}




\begin{tabular}{|c|c|c|c|c|c|c|c|}
\hline 1192( & 4) & 1181( & 3) & 1149 & 1) & 1191( & 2) \\
\hline 1204( & 1) & 1193( & 1) & 1157( & 2) & 1205( & 0) \\
\hline 1222( & 3) & 1210( & 3) & 1172( & 3) & 1223 & 3) \\
\hline 1227( & 1) & 1217( & 2) & 1180( & 1) & 1230 & 2) \\
\hline 1260( & 18) & 1251( & 14) & 1215( & 9) & 1250 & 11) \\
\hline 1262( & 12) & 1253( & 11) & 1219 ( & 5) & 1253( & 9) \\
\hline 1291( & 5) & 1275( & 8) & 1234( & 12) & 1276( & 9) \\
\hline 1317( & 1) & 1305( & 1) & 1260( & 0) & 1309 & 0) \\
\hline 1333( & 5) & 1317( & 4) & 1275( & 3) & 1326( & 3) \\
\hline 1338( & 2) & 1324( & 2) & 1282( & 2) & 1338( & 3) \\
\hline 1348( & 2) & 1333( & 1) & 1293( & 1) & 1342( & 1) \\
\hline 1410( & 1) & 1398( & 1) & 1355( & 2) & 1399 & 1) \\
\hline 1411( & 1) & 1399 & 1) & 1358( & 1) & 1402( & 1) \\
\hline 1427( & 3) & 1411( & 4) & 1365( & 3) & 1420 & 3) \\
\hline 1472( & 5) & 1460 & 3) & 1421( & 4) & 1460( & 13) \\
\hline 1476( & 13) & 1463( & 13) & 1423( & 9) & 1461( & 3) \\
\hline 1484( & 2) & 1472( & 1) & 1431( & 1) & 1467( & 1) \\
\hline 1487( & 3) & 1474( & 4) & 1433( & 2) & 1477( & 5) \\
\hline 1491( & 12) & 1479 & 12) & 1439 & 2) & 1478( & 6) \\
\hline 1491( & 1) & 1480( & 2) & 1441( & 10) & 1480 & 3) \\
\hline 1537( & 55) & 1521( & 41) & 1476( & 23) & 1534( & 50) \\
\hline 1541( & 38) & 1525( & 37) & 1480( & 23) & 1538( & 26) \\
\hline 1649( & 1) & 1612( & 3) & 1526( & 4) & 1627( & 4) \\
\hline 3013( & 37) & 2989 & 42) & 2929 ( & 52) & 3021( & 20) \\
\hline 3021( & 16) & 2998( & 17) & 2935( & 29) & 3024( & 49) \\
\hline 3030( & 78) & 3008( & 72) & 2947( & 68) & 3029 & 142) \\
\hline 3032( & 68) & 3011( & 68) & 2956( & 73) & 3035( & 102) \\
\hline 3070( & 0) & 3051( & 1) & 2994( & 2) & 3062( & 32) \\
\hline 3073( & 22) & 3053( & 21) & 3000( & 19) & 3089 & 61) \\
\hline 3090( & 33) & 3072( & 31) & 3019x & 18) & 3099 & 28) \\
\hline 3096( & 1) & 3077( & 19) & 3021( & 35) & 3101( & 25) \\
\hline 3098( & 34) & 3081( & 14) & 30331 & 9) & 3114( & 49) \\
\hline 3131( & 2) & 3111( & 4) & 3044( & 12) & 3125( & 25) \\
\hline 3136( & 13) & 3116( & 7) & 3065( & 7) & 3131( & 18) \\
\hline 3136( & 7) & 3119 & 10) & 3067( & 7) & 3146( & 11) \\
\hline 3146( & 4) & 3126( & 4) & 3068 & 10) & 3146( & 14) \\
\hline 3166r & 8) & 3143( & 9) & 3073( & 15) & 3165( & 24) \\
\hline 3169 & 16) & 3148( & 16) & 3090( & 19) & 3165( & 39) \\
\hline 3179 ( & 18) & 3160 & 17) & 3107 ( & 17) & 3186( & 35) \\
\hline 3192( & 18) & 3179 & 14) & 3132( & 13) & 3207 ( & 26) \\
\hline 3226( & 14) & 3208( & 13) & $3157 x$ & 12) & 3222( & 26) \\
\hline
\end{tabular}


Table S94. Harmonic vibrational frequencies (in $\mathrm{cm}^{-1}$ ) and infrared intensities (in parentheses in $\mathrm{km} / \mathrm{mol})$ for the $\left(\mathbf{C}_{4} \mathbf{H}_{6}\right)_{3} \mathbf{N i}$ structure Ni-2T.

\begin{tabular}{|c|c|c|c|c|c|c|c|}
\hline \multicolumn{2}{|c|}{ B3LYP } & \multicolumn{2}{|c|}{ B3LYP* } & \multicolumn{2}{|c|}{ BP86 } & \multicolumn{2}{|c|}{ M06L } \\
\hline 48( & 1) & 40( & 0) & 38( & 0) & 42( & 0) \\
\hline 66( & 0) & 64( & 0) & 62( & 0) & 69 & 0) \\
\hline 93( & 0) & 94( & 0) & 83( & 0) & 98( & 0) \\
\hline 118( & 2) & 122( & 2) & 120( & 1) & 116( & 1) \\
\hline 122( & 1) & 128( & 1) & 131( & 1) & 138( & 1) \\
\hline 142( & 0) & 144( & 0) & 148( & 0) & 172( & 1) \\
\hline 179 & 1) & 178( & 1) & 181( & 1) & 187( & 2) \\
\hline 195( & 0) & 200( & 0) & 212( & 1) & 215( & 0) \\
\hline 230 & 2) & 234( & 1) & 239 & 0) & 246( & 3) \\
\hline 239 & 1) & 239 & 1) & 2461 & 2) & 271( & 0) \\
\hline 267( & 1) & 272( & 1) & 282( & 1) & 277( & 1) \\
\hline 298( & 2) & 295( & 2) & 290( & 1) & 292( & 2) \\
\hline 306( & 0) & 307( & 0) & 318( & 1) & 337( & 1) \\
\hline 319 & 1) & 327( & 1) & 344( & 2) & 374( & 2) \\
\hline 383( & 8) & 381( & 5) & 3761 & 0) & 390( & 1) \\
\hline 425( & 7) & 421( & 7) & 412( & 5) & 4331 & 6) \\
\hline 475( & 5) & 466( & 3) & 441( & 3) & 471( & 3) \\
\hline 494( & 3) & 490( & 4) & 474( & 6) & 489 & 5) \\
\hline 539 & 6) & 5331 & 8) & 519 & 9) & 545( & 6) \\
\hline 567( & 2) & 563( & 2) & 546( & 3) & 566( & 2) \\
\hline 582( & 2) & 580( & 2) & 561( & 1) & 585( & 1) \\
\hline 623( & 8) & 620( & 7) & 597( & 6) & 631( & 5) \\
\hline 660 & 1) & 6531 & 1) & 617( & 8) & 657( & 7) \\
\hline 673( & 10) & 664( & 9) & 641( & 10) & 665( & 9) \\
\hline 718( & 26) & 711( & 27) & 6931 & 35) & 706( & 31) \\
\hline 759 & 29) & 749 & 20) & 724( & 7) & 727( & 8) \\
\hline 796( & 5) & 788( & 8) & 765( & 10) & 799 & 12) \\
\hline 816( & 26) & 805( & 38) & 775( & 41) & 808( & 47) \\
\hline 856( & 72) & 844( & 73) & 800( & 58) & 818( & 44) \\
\hline 861( & 2) & 854( & 5) & 807( & 1) & 859 & 18) \\
\hline 877( & 9) & 863( & 28) & 840 & 9) & 871( & 17) \\
\hline 882( & 42) & 875( & 17) & 860( & 7) & 893( & 4) \\
\hline 919 & 36) & 902( & 33) & 864( & 11) & 901( & 28) \\
\hline 939 & 0) & 931( & 0) & 902( & 2) & 934( & 0) \\
\hline 959 & 1) & 949 & 1) & 912( & 4) & 936( & 1) \\
\hline 977( & 10) & 963( & 7) & 913( & 5) & 9531 & 5) \\
\hline 988( & 0) & 973( & 1) & 9261 & 0) & 967( & 1) \\
\hline 1005( & 3) & 983( & 2) & 951( & 1) & 993( & 1) \\
\hline 1012( & 6) & 1005( & 11) & 970( & 17) & 1012( & 8) \\
\hline 1015( & 10) & 1010( & 7) & 988( & 3) & 1029 & 6) \\
\hline 1031( & 10) & 1021( & 8) & 995( & 2) & 1034( & 3) \\
\hline 1036( & 3) & 1027( & 4) & 999 & 7) & 1036( & 9) \\
\hline 1052( & 1) & 1044( & 1) & 1020( & 1) & 1067( & 1) \\
\hline 1095( & 2) & 1087( & 2) & 1062( & 2) & 1102( & 2) \\
\hline 1125( & 4) & 1116( & 3) & 1089 & 2) & 1124( & 3) \\
\hline 1138( & 18) & 1128( & 16) & 1099 & 7) & 1139 & 12) \\
\hline
\end{tabular}




\begin{tabular}{|c|c|c|c|c|c|c|c|}
\hline 11866 & 5) & 1175( & 5) & 1138( & 3) & 1183( & 6) \\
\hline 1214( & 2) & 1203( & 3) & 1170( & 2) & 1220 & 2) \\
\hline 1219 ( & 7) & 1207( & 5) & 1176( & 1) & 1224( & 4) \\
\hline 1240( & 2) & 1230 & 2) & 1194( & 2) & 1242( & 2) \\
\hline 1253( & 4) & 1239 & 4) & 1202( & 5) & 1251( & 4) \\
\hline 1288( & 3) & 1274( & 5) & 1229 & 9) & 1269 & 8) \\
\hline 1315( & 1) & 1303( & 0) & 1257( & 2) & 1309 & 0) \\
\hline 1318( & 0) & 1303( & 0) & 1258( & 1) & 1310 & 1) \\
\hline 1322( & 1) & 1305( & 2) & 1264( & 0) & 1313 & 1) \\
\hline 1339x & 4) & 1327( & 2) & 1284( & 2) & 1334( & 1) \\
\hline 1342( & 4) & 1328( & 7) & 1289 & 3) & 1345( & 6) \\
\hline 1357( & 2) & 1345( & 2) & 1316( & 2) & 1368( & 1) \\
\hline 1406( & 1) & 1391( & 1) & 1348( & 1) & 1398( & 2) \\
\hline 1426( & 4) & 1410( & 6) & 1362( & 7) & 1417( & 7) \\
\hline 1444( & 4) & 1432( & 3) & 1393( & 2) & 1438( & 2) \\
\hline 1471( & 3) & 1458( & 3) & 1418( & 2) & 1460 & 5) \\
\hline 1476( & 3) & 1463( & 4) & 1422( & 6) & 1464( & 5) \\
\hline 1482( & 9) & 1469 & 8) & 1428( & 3) & 1470 & 5) \\
\hline 1490( & 5) & 1475( & 5) & 1437( & 4) & 1473( & 2) \\
\hline 1495( & 17) & 1485( & 19) & 1447( & 20) & 1500 & 15) \\
\hline 1602( & 24) & 1582( & 27) & 1518( & 3) & 1607( & 23) \\
\hline 1640( & 37) & 1615( & 2) & 1524( & 24) & 1610 & 4) \\
\hline 1646( & 3) & 1621( & 31) & 1556( & 14) & 1634( & 31) \\
\hline 3011( & 60) & 2992( & 60) & 2946( & 25) & 3007( & 112) \\
\hline 3030( & 13) & 3008( & 17) & 2950 & 24) & 3034( & 39) \\
\hline 3032( & 39) & 3013( & 40) & 2955( & 85) & 3043( & 95) \\
\hline 3036( & 44) & 3016( & 35) & 2961( & 20) & 3050 & 18) \\
\hline 3055( & 41) & 3035( & 34) & 2977( & 26) & 3061( & 23) \\
\hline 3058( & 14) & 3038( & 16) & 2984( & 17) & 3069 & 49) \\
\hline 3064( & 24) & 3047( & 22) & 3001( & 15) & 3079 & 40) \\
\hline 3092( & 5) & 3069 & 4) & 3005( & 1) & 3095( & 6) \\
\hline 3098( & 11) & 3076( & 15) & 3017 ( & 19) & 3108 & 15) \\
\hline 3110( & 14) & 3088( & 12) & 3030 & 25) & 3127( & 34) \\
\hline 3121( & 5) & 3100( & 21) & 3031( & 24) & 3130( & 35) \\
\hline 3123( & 21) & 3103( & 7) & 3056( & 19) & 3132( & 27) \\
\hline 3126( & 21) & 3106( & 19) & 3058( & 8) & 3140( & 10) \\
\hline 3137( & 22) & 3119 & 19) & 3061( & 10) & 3150( & 13) \\
\hline 3155( & 5) & 31360 & 3) & 3074( & 14) & 3155 ( & 34) \\
\hline 3162( & 6) & 3138( & 7) & 3081( & 5) & 3166( & 17) \\
\hline 3169 & 41) & 3151( & 36) & 3104( & 32) & 3189 ( & 64) \\
\hline 3242( & 18) & 3224 ( & 16) & 3173 & 15) & 3242( & 27) \\
\hline
\end{tabular}


Table S95. Titanium-carbon distances (in $\AA$ ) for the $\left(\mathbf{C}_{4} \mathbf{H}_{6}\right)_{3} \mathbf{T i}$ structures. The bold face indicates the existence of Ti-C bonds.

\begin{tabular}{|c|c|c|c|c|c|c|c|c|}
\hline & \multicolumn{4}{|c|}{ Ti-1S } & \multicolumn{4}{|c|}{ Ti-2S } \\
\hline & B3LYP & B3LYP* & BP86 & M06L & B3LYP & B3LYP* & BP86 & M06L \\
\hline $\mathrm{TiC} 1$ & 2.282 & 2.282 & 2.282 & 2.264 & 2.488 & 2.476 & 2.423 & 2.453 \\
\hline $\mathrm{TiC} 2$ & 2.357 & 2.353 & 2.339 & 2.331 & 2.344 & 2.340 & 2.332 & 2.340 \\
\hline $\mathrm{TiC} 3$ & 2.496 & 2.488 & 2.459 & 2.440 & 2.402 & 2.394 & 2.371 & 2.393 \\
\hline $\mathrm{TiC} 4$ & 3.230 & 3.225 & 3.211 & 3.151 & 2.514 & 2.494 & 2.425 & 2.458 \\
\hline TiC5 & 2.963 & 2.957 & 2.943 & 2.856 & 2.346 & 2.348 & 2.373 & 2.305 \\
\hline TiC6 & 2.379 & 2.374 & 2.361 & 2.333 & 2.309 & 2.311 & 2.320 & 2.281 \\
\hline $\mathrm{TiC} 7$ & 2.347 & 2.344 & 2.339 & 2.329 & 2.314 & 2.317 & 2.330 & 2.301 \\
\hline TiC8 & 2.304 & 2.303 & 2.301 & 2.286 & 2.313 & 2.321 & 2.359 & 2.293 \\
\hline TiC9 & 2.249 & 2.251 & 2.259 & 2.227 & 2.432 & 2.426 & 2.404 & 2.372 \\
\hline $\mathrm{TiC} 10$ & 2.321 & 2.322 & 2.323 & 2.293 & 2.347 & 2.347 & 2.357 & 2.335 \\
\hline $\mathrm{TiC} 11$ & 2.312 & 2.312 & 2.311 & 2.286 & 2.341 & 2.340 & 2.342 & 2.319 \\
\hline \multirow[t]{3}{*}{$\mathrm{TiC} 12$} & 2.211 & 2.209 & 2.207 & 2.190 & 2.419 & 2.414 & 2.388 & 2.342 \\
\hline & \multicolumn{4}{|c|}{ Ti-1T } & & & & \\
\hline & B3LYP & B3LYP* & BP86 & M06L & & & & \\
\hline $\mathrm{TiC} 1$ & 2.315 & 2.320 & 2.331 & 2.299 & & & & \\
\hline $\mathrm{TiC} 2$ & 2.369 & 2.353 & 2.291 & 2.303 & & & & \\
\hline $\mathrm{TiC} 3$ & 2.488 & 2.456 & 2.392 & 2.388 & & & & \\
\hline $\mathrm{TiC} 4$ & 3.148 & 3.122 & 3.179 & 3.057 & & & & \\
\hline TiC5 & 2.839 & 2.817 & 2.956 & 2.752 & & & & \\
\hline TiC6 & 2.323 & 2.287 & 2.307 & 2.233 & & & & \\
\hline $\mathrm{TiC} 7$ & 2.362 & 2.340 & 2.308 & 2.284 & & & & \\
\hline TiC8 & 2.350 & 2.347 & 2.331 & 2.302 & & & & \\
\hline TiC9 & 2.516 & 2.522 & 2.468 & 2.512 & & & & \\
\hline $\mathrm{TiC} 10$ & 2.404 & 2.394 & 2.328 & 2.363 & & & & \\
\hline $\mathrm{TiC} 11$ & 2.405 & 2.397 & 2.382 & 2.358 & & & & \\
\hline $\mathrm{TiC} 12$ & 2.538 & 2.534 & 2.511 & 2.489 & & & & \\
\hline
\end{tabular}


Table S96. Vanadium-carbon distances (in $\AA$ ) for the $\left(\mathbf{C}_{4} \mathbf{H}_{6}\right)_{3} \mathbf{V}$ structures. The bold face indicates the existence of $\mathrm{V}-\mathrm{C}$ bonds.

\begin{tabular}{|c|c|c|c|c|c|c|c|c|}
\hline & \multicolumn{4}{|c|}{ V-1D } & \multicolumn{4}{|c|}{$V-2 D$} \\
\hline & B3LYP & B3LYP* & BP86 & M06L & B3LYP & B3LYP* & BP86 & M06L \\
\hline VC1 & 2.187 & 2.183 & 2.174 & 2.164 & 2.401 & 2.394 & 2.369 & 2.332 \\
\hline $\mathrm{VC} 2$ & 2.187 & 2.177 & 2.151 & 2.143 & 2.295 & 2.29 & 2.276 & 2.269 \\
\hline VC3 & 2.452 & 2.441 & 2.401 & 2.382 & 2.292 & 2.289 & 2.277 & 2.271 \\
\hline $\mathrm{VC} 4$ & 3.257 & 3.258 & 3.258 & 3.209 & 2.399 & 2.395 & 2.374 & 2.35 \\
\hline VC5 & 2.977 & 2.980 & 2.996 & 2.932 & 2.41 & 2.399 & 2.369 & 2.358 \\
\hline VC6 & 2.172 & 2.172 & 2.178 & 2.146 & 2.275 & 2.27 & 2.259 & 2.26 \\
\hline VC7 & 2.152 & 2.148 & 2.140 & 2.120 & 2.357 & 2.348 & 2.324 & 2.337 \\
\hline VC8 & 3.030 & 3.028 & 3.034 & 2.980 & 2.487 & 2.466 & 2.406 & 2.43 \\
\hline VC9 & 3.233 & 3.220 & 3.189 & 3.164 & 2.349 & 2.343 & 2.329 & 2.299 \\
\hline VC10 & 2.537 & 2.513 & 2.441 & 2.458 & 2.278 & 2.276 & 2.274 & 2.253 \\
\hline VC11 & 2.267 & 2.256 & 2.231 & 2.232 & 2.271 & 2.270 & 2.269 & 2.254 \\
\hline VC12 & 2.182 & 2.181 & 2.175 & 2.166 & 2.295 & 2.296 & 2.298 & 2.259 \\
\hline & \multicolumn{4}{|c|}{ V-3D } & \multicolumn{4}{|c|}{ V-4D } \\
\hline & B3LYP & B3LYP* & BP86 & M06L & B3LYP & B3LYP* & BP86 & M06L \\
\hline $\mathrm{VC} 1$ & 2.058 & 2.057 & 2.056 & 2.077 & 2.132 & 2.142 & 2.141 & 2.128 \\
\hline $\mathrm{VC} 2$ & 3.191 & 3.183 & 3.173 & 3.176 & 2.166 & 2.161 & 2.153 & 2.144 \\
\hline VC3 & 3.651 & 3.640 & 3.637 & 3.646 & 2.329 & 2.326 & 2.319 & 2.268 \\
\hline VC4 & 3.481 & 3.454 & 3.421 & 3.436 & 3.070 & 3.077 & 3.093 & 2.985 \\
\hline VC5 & 2.371 & 2.342 & 2.315 & 2.332 & 3.236 & 3.224 & 3.216 & 3.184 \\
\hline VC6 & 2.107 & 2.104 & 2.109 & 2.096 & 2.281 & 2.260 & 2.223 & 2.232 \\
\hline VC7 & 2.194 & 2.175 & 2.170 & 2.163 & 2.136 & 2.149 & 2.136 & 2.123 \\
\hline VC8 & 2.889 & 2.856 & 2.873 & 2.841 & 2.910 & 2.944 & 2.959 & 2.904 \\
\hline VC9 & 3.131 & 3.105 & 3.105 & 3.085 & 3.036 & 3.069 & 3.082 & 3.054 \\
\hline VC10 & 2.336 & 2.330 & 2.286 & 2.271 & 2.142 & 2.103 & 2.093 & 2.117 \\
\hline VC11 & 2.155 & 2.162 & 2.144 & 2.120 & 3.138 & 3.129 & 3.125 & 3.120 \\
\hline VC12 & 2.182 & 2.185 & 2.172 & 2.148 & 4.239 & 4.304 & 4.325 & 4.264 \\
\hline & \multicolumn{4}{|c|}{$\mathrm{V}-1 \mathrm{Q}$} & \multicolumn{4}{|c|}{$\mathrm{V}-2 \mathrm{Q}$} \\
\hline & B3LYP & B3LYP* & BP86 & M06L & B3LYP & B3LYP* & BP86 & M06L \\
\hline VC1 & 2.278 & 2.269 & 2.246 & 2.242 & 2.313 & 2.298 & 2.317 & 2.265 \\
\hline $\mathrm{VC} 2$ & 2.279 & 2.271 & 2.249 & 2.250 & 2.249 & 2.243 & 2.24 & 2.22 \\
\hline VC3 & 2.362 & 2.355 & 2.324 & 2.325 & 2.405 & 2.403 & 2.361 & 2.351 \\
\hline VC4 & 2.944 & 2.935 & 2.912 & 2.881 & 3.267 & 3.267 & 3.202 & 3.207 \\
\hline VC5 & 2.607 & 2.581 & 2.532 & 2.485 & 3.152 & 3.149 & 2.992 & 3.093 \\
\hline VC6 & 2.366 & 2.333 & 2.267 & 2.278 & 2.556 & 2.524 & 2.427 & 2.478 \\
\hline VC7 & 2.577 & 2.541 & 2.454 & 2.467 & 2.499 & 2.461 & 2.405 & 2.418 \\
\hline VC8 & 3.129 & 3.103 & 3.027 & 3.006 & 3.014 & 2.983 & 2.912 & 2.906 \\
\hline VC9 & 3.128 & 3.115 & 3.077 & 3.046 & 2.831 & 2.843 & 3.076 & 2.784 \\
\hline VC10 & 2.347 & 2.342 & 2.324 & 2.310 & 2.365 & 2.357 & 2.336 & 2.332 \\
\hline VC11 & 2.240 & 2.235 & 2.224 & 2.217 & 2.316 & 2.304 & 2.249 & 2.28 \\
\hline VC12 & 2.269 & 2.260 & 2.231 & 2.231 & 2.31 & 2.306 & 2.316 & 2.271 \\
\hline & \multicolumn{4}{|c|}{$\mathrm{V}-3 \mathrm{Q}$} & \multicolumn{4}{|c|}{$\mathrm{V}-4 \mathrm{Q}$} \\
\hline & B3LYP & B3LYP* & BP86 & M06L & B3LYP & B3LYP* & BP86 & M06L \\
\hline $\mathrm{VC} 1$ & 2.31 & 2.313 & 2.309 & 2.28 & 2.312 & 2.313 & 2.311 & 2.279 \\
\hline VC2 & 2.283 & 2.283 & 2.279 & 2.261 & 2.290 & 2.280 & 2.256 & 2.247 \\
\hline VC3 & 2.277 & 2.279 & 2.275 & 2.259 & 2.295 & 2.281 & 2.250 & 2.248 \\
\hline VC4 & 2.294 & 2.296 & 2.284 & 2.258 & 2.788 & 2.787 & 2.783 & 2.747 \\
\hline VC5 & 2.402 & 2.386 & 2.36 & 2.349 & 3.111 & 3.085 & 3.038 & 2.957 \\
\hline VC6 & 2.473 & 2.454 & 2.427 & 2.399 & 2.452 & 2.409 & 2.330 & 2.332 \\
\hline VC7 & 3.306 & 3.285 & 3.255 & 3.163 & 2.517 & 2.486 & 2.419 & 2.438 \\
\hline VC8 & 4.209 & 4.18 & 4.142 & 3.985 & 3.181 & 3.165 & 3.116 & 3.143 \\
\hline VC9 & 2.347 & 2.332 & 2.305 & 2.29 & 3.078 & 3.054 & 2.999 & 2.984 \\
\hline VC10 & 2.297 & 2.29 & 2.277 & 2.266 & 2.181 & 2.171 & 2.147 & 2.158 \\
\hline VC11 & 2.303 & 2.295 & 2.282 & 2.27 & 3.074 & 3.090 & 3.127 & 3.058 \\
\hline VC12 & 2.357 & 2.339 & 2.311 & 2.303 & 4.077 & 4.106 & 4.173 & 4.094 \\
\hline \multicolumn{9}{|c|}{ V-5Q } \\
\hline & B3LYP & B3LYP* & BP86 & M06L & & & & \\
\hline VC1 & 2.38 & 2.372 & 2.348 & 2.34 & & & & \\
\hline $\mathrm{VC} 2$ & 2.276 & 2.271 & 2.257 & 2.241 & & & & \\
\hline VC3 & 2.261 & 2.253 & 2.233 & 2.225 & & & & \\
\hline VC4 & 2.37 & 2.355 & 2.318 & 2.306 & & & & \\
\hline VC5 & 2.354 & 2.348 & 2.334 & 2.333 & & & & \\
\hline VC6 & 2.45 & 2.447 & 2.439 & 2.428 & & & & \\
\hline
\end{tabular}


$\begin{array}{lllll}\text { VC7 } & 3.294 & 3.293 & 3.286 & 3.222\end{array}$

$\begin{array}{lllll}\text { VC8 } & 4.132 & 4.131 & 4.13 & 4.02\end{array}$

$\begin{array}{lllll}\text { VC9 } & \mathbf{2 . 3 9 9} & \mathbf{2 . 3 9 4} & \mathbf{2 . 3 7 4} & \mathbf{2 . 3 5 7}\end{array}$

$\begin{array}{llllr}V C 10 & \mathbf{2 . 2 8 9} & \mathbf{2 . 2 8 6} & \mathbf{2 . 2 7 5} & \mathbf{2 . 2 6}\end{array}$

$\begin{array}{lllll}V C 11 & \mathbf{2 . 2 6 7} & \mathbf{2 . 2 6} & \mathbf{2 . 2 4 2} & \mathbf{2 . 2 3 6}\end{array}$

$\begin{array}{lllll}\text { VC12 } & \mathbf{2 . 3 7 6} & \mathbf{2 . 3 6 1} & \mathbf{2 . 3 2 5} & \mathbf{2 . 3 0 9}\end{array}$ 
Table S97. Chromium-carbon distances (in $\AA$ ) for the $\left(\mathbf{C}_{4} \mathbf{H}_{6}\right)_{3} \mathbf{C r}$ structures. The bold face indicates the existence of $\mathrm{Cr}-\mathrm{C}$ bonds.

\begin{tabular}{|c|c|c|c|c|c|c|c|c|}
\hline & \multicolumn{4}{|c|}{$\mathrm{Cr}-1 \mathrm{~S}$} & \multicolumn{4}{|c|}{$\mathrm{Cr}-2 \mathrm{~S}$} \\
\hline & B3LYP & B3LYP* & BP86 & M06L & B3LYP & B3LYP* & BP86 & M06L \\
\hline $\mathrm{CrCl}$ & 2.186 & 2.180 & 2.165 & 2.137 & 2.343 & 2.326 & 2.274 & 2.247 \\
\hline $\mathrm{CrC} 2$ & 2.211 & 2.209 & 2.206 & 2.173 & 2.238 & 2.232 & 2.220 & 2.210 \\
\hline $\mathrm{CrC} 3$ & 2.218 & 2.219 & 2.220 & 2.217 & 2.232 & 2.226 & 2.211 & 2.189 \\
\hline $\mathrm{CrC} 4$ & 2.192 & 2.189 & 2.178 & 2.170 & 2.348 & 2.336 & 2.298 & 2.270 \\
\hline $\mathrm{CrC} 5$ & 2.181 & 2.176 & 2.161 & 2.152 & 2.439 & 2.420 & 2.358 & 2.370 \\
\hline CrC6 & 2.215 & 2.216 & 2.215 & 2.207 & 2.305 & 2.300 & 2.291 & 2.287 \\
\hline $\mathrm{CrC} 7$ & 2.226 & 2.225 & 2.227 & 2.191 & 2.192 & 2.189 & 2.195 & 2.174 \\
\hline $\mathrm{CrC} 8$ & 2.195 & 2.189 & 2.176 & 2.148 & 2.281 & 2.266 & 2.226 & 2.206 \\
\hline $\mathrm{CrC} 9$ & 2.190 & 2.180 & 2.160 & 2.137 & 2.374 & 2.360 & 2.315 & 2.303 \\
\hline $\mathrm{CrC} 10$ & 2.252 & 2.244 & 2.227 & 2.205 & 2.256 & 2.252 & 2.244 & 2.236 \\
\hline $\mathrm{CrC} 11$ & 3.276 & 3.273 & 3.271 & 3.220 & 2.243 & 2.244 & 2.255 & 2.240 \\
\hline \multirow[t]{2}{*}{$\mathrm{CrC} 12$} & 4.209 & 4.198 & 4.187 & 4.075 & 2.305 & 2.303 & 2.296 & 2.268 \\
\hline & \multicolumn{4}{|c|}{ Cr-1T } & \multicolumn{4}{|c|}{$\mathrm{Cr}-2 \mathrm{~T}$} \\
\hline & B3LYP & B3LYP* & BP86 & M06L & B3LYP & B3LYP* & BP86 & M06L \\
\hline $\mathrm{CrCl}$ & 2.198 & 2.192 & 2.186 & 2.168 & 2.136 & 2.131 & 2.120 & 2.111 \\
\hline $\mathrm{CrC} 2$ & 2.228 & 2.223 & 2.213 & 2.202 & 2.118 & 2.116 & 2.112 & 2.096 \\
\hline $\mathrm{CrC} 3$ & 2.236 & 2.230 & 2.215 & 2.203 & 2.293 & 2.290 & 2.275 & 2.246 \\
\hline $\mathrm{CrC} 4$ & 2.212 & 2.207 & 2.199 & 2.174 & 3.233 & 3.235 & 3.240 & 3.192 \\
\hline $\mathrm{CrC} 5$ & 2.285 & 2.276 & 2.254 & 2.246 & 3.102 & 3.101 & 3.102 & 3.059 \\
\hline CrC6 & 2.277 & 2.266 & 2.232 & 2.237 & 2.316 & 2.303 & 2.265 & 2.275 \\
\hline $\mathrm{CrC} 7$ & 2.303 & 2.292 & 2.261 & 2.259 & 2.212 & 2.202 & 2.183 & 2.190 \\
\hline $\mathrm{CrC} 8$ & 2.953 & 2.946 & 2.936 & 2.891 & 2.821 & 2.818 & 2.819 & 2.763 \\
\hline $\mathrm{CrC9}$ & 3.129 & 3.119 & 3.105 & 3.072 & 2.742 & 2.740 & 2.738 & 2.680 \\
\hline $\mathrm{CrC} 10$ & 2.351 & 2.333 & 2.293 & 2.294 & 2.280 & 2.272 & 2.250 & 2.245 \\
\hline $\mathrm{CrC} 11$ & 2.219 & 2.208 & 2.184 & 2.184 & 2.253 & 2.244 & 2.216 & 2.221 \\
\hline \multirow[t]{3}{*}{$\mathrm{CrC} 12$} & 2.268 & 2.262 & 2.246 & 2.238 & 2.232 & 2.227 & 2.211 & 2.201 \\
\hline & \multicolumn{4}{|c|}{ Cr-3T } & \multicolumn{4}{|c|}{ Cr-1P } \\
\hline & B3LYP & B3LYP* & BP86 & M06L & B3LYP & B3LYP* & BP86 & M06L \\
\hline $\mathrm{CrC} 1$ & 2.248 & 2.245 & 2.235 & 2.222 & 2.280 & 2.272 & 2.257 & 2.249 \\
\hline $\mathrm{CrC} 2$ & 2.197 & 2.192 & 2.181 & 2.173 & 2.218 & 2.215 & 2.200 & 2.186 \\
\hline $\mathrm{CrC} 3$ & 2.238 & 2.231 & 2.211 & 2.199 & 2.360 & 2.363 & 2.333 & 2.313 \\
\hline $\mathrm{CrC} 4$ & 2.258 & 2.250 & 2.231 & 2.211 & 3.194 & 3.186 & 3.134 & 3.130 \\
\hline $\mathrm{CrC} 5$ & 2.268 & 2.260 & 2.240 & 2.221 & 3.138 & 3.104 & 2.998 & 3.044 \\
\hline $\mathrm{CrC} 6$ & 2.240 & 2.232 & 2.211 & 2.197 & 2.789 & 2.698 & 2.467 & 2.666 \\
\hline $\mathrm{CrC} 7$ & 2.191 & 2.185 & 2.173 & 2.165 & 2.777 & 2.687 & 2.481 & 2.649 \\
\hline $\mathrm{CrC} 8$ & 2.244 & 2.239 & 2.228 & 2.212 & 3.096 & 3.063 & 2.982 & 2.988 \\
\hline CrC9 & 2.295 & 2.278 & 2.247 & 2.270 & 3.082 & 3.062 & 3.014 & 3.012 \\
\hline $\mathrm{CrC} 10$ & 2.354 & 2.334 & 2.296 & 2.321 & 2.267 & 2.257 & 2.231 & 2.243 \\
\hline $\mathrm{CrC} 11$ & 3.240 & 3.225 & 3.198 & 3.140 & 2.221 & 2.217 & 2.205 & 2.194 \\
\hline \multirow[t]{3}{*}{$\mathrm{CrC} 12$} & 4.064 & 4.048 & 4.029 & 3.859 & 2.334 & 2.333 & 2.319 & 2.278 \\
\hline & & $\mathrm{Cr}-2 \mathrm{P}$ & & & & & & \\
\hline & B3LYP & B3LYP* & BP86 & M06L & & & & \\
\hline $\mathrm{CrC} 1$ & 2.240 & 2.234 & 2.216 & 2.215 & & & & \\
\hline $\mathrm{CrC} 2$ & 2.205 & 2.196 & 2.170 & 2.174 & & & & \\
\hline $\mathrm{CrC} 3$ & 2.376 & 2.365 & 2.330 & 2.330 & & & & \\
\hline $\mathrm{CrC} 4$ & 3.248 & 3.241 & 3.220 & 3.145 & & & & \\
\hline $\mathrm{CrC} 5$ & 3.378 & 3.373 & 3.363 & 3.152 & & & & \\
\hline CrC6 & 3.714 & 3.710 & 3.680 & 3.481 & & & & \\
\hline $\mathrm{CrC} 7$ & 3.714 & 3.710 & 3.680 & 3.481 & & & & \\
\hline $\mathrm{CrC} 8$ & 3.378 & 3.373 & 3.363 & 3.152 & & & & \\
\hline $\mathrm{CrC9}$ & 3.248 & 3.241 & 3.220 & 3.145 & & & & \\
\hline $\mathrm{CrC} 10$ & 2.376 & 2.365 & 2.330 & 2.330 & & & & \\
\hline $\mathrm{CrC} 11$ & 2.205 & 2.196 & 2.170 & 2.174 & & & & \\
\hline $\mathrm{CrC} 12$ & 2.240 & 2.234 & 2.216 & 2.215 & & & & \\
\hline
\end{tabular}


Table S98. Manganese-carbon distances (in $\AA$ ) for the $\left(\mathbf{C}_{4} \mathbf{H}_{6}\right)_{3} \mathbf{M n}$ structures. The bold face indicates the existence of $\mathrm{Mn}-\mathrm{C}$ bonds.

\begin{tabular}{|c|c|c|c|c|c|c|c|c|}
\hline & \multicolumn{4}{|c|}{ Mn-1D } & \multicolumn{4}{|c|}{ Mn-2D } \\
\hline & B3LYP & B3LYP* & BP86 & M06L & B3LYP & B3LYP* & BP86 & M06L \\
\hline $\mathrm{MnC1}$ & 2.201 & 2.196 & 2.186 & 2.161 & 2.159 & 2.149 & 2.128 & 2.106 \\
\hline $\mathrm{MnC} 2$ & 2.130 & 2.125 & 2.119 & 2.099 & 2.119 & 2.106 & 2.077 & 2.075 \\
\hline $\mathrm{MnC} 3$ & 2.124 & 2.119 & 2.110 & 2.091 & 2.223 & 2.218 & 2.208 & 2.197 \\
\hline $\mathrm{MnC4}$ & 2.174 & 2.166 & 2.150 & 2.127 & 2.842 & 2.849 & 2.874 & 2.822 \\
\hline $\mathrm{MnC5}$ & 2.173 & 2.166 & 2.151 & 2.123 & 2.918 & 2.920 & 2.932 & 2.892 \\
\hline MnC6 & 2.126 & 2.121 & 2.113 & 2.093 & 2.268 & 2.253 & 2.230 & 2.244 \\
\hline $\mathrm{MnC7}$ & 2.128 & 2.123 & 2.115 & 2.098 & 2.342 & 2.321 & 2.268 & 2.291 \\
\hline $\mathrm{MnC} 8$ & 2.204 & 2.197 & 2.184 & 2.161 & 3.131 & 3.130 & 3.122 & 3.094 \\
\hline $\mathrm{MnC9}$ & 2.209 & 2.196 & 2.170 & 2.176 & 3.223 & 3.225 & 3.218 & 3.179 \\
\hline $\mathrm{MnC} 10$ & 2.256 & 2.242 & 2.218 & 2.231 & 2.270 & 2.266 & 2.232 & 2.210 \\
\hline $\mathrm{MnC} 11$ & 3.214 & 3.209 & 3.210 & 3.171 & 2.089 & 2.085 & 2.072 & 2.057 \\
\hline \multirow[t]{2}{*}{$\mathrm{MnC} 12$} & 4.171 & 4.163 & 4.162 & 4.065 & 2.098 & 2.089 & 2.070 & 2.058 \\
\hline & \multicolumn{4}{|c|}{ Mn-3D } & \multicolumn{4}{|c|}{ Mn-1Q } \\
\hline & B3LYP & B3LYP* & BP86 & M06L & B3LYP & B3LYP* & BP86 & M06L \\
\hline $\mathrm{MnC1}$ & 2.236 & 2.228 & 2.208 & 2.201 & 2.188 & 2.176 & 2.140 & 2.151 \\
\hline $\mathrm{MnC} 2$ & 2.181 & 2.176 & 2.167 & 2.153 & 2.235 & 2.220 & 2.204 & 2.198 \\
\hline $\mathrm{MnC} 3$ & 2.135 & 2.127 & 2.115 & 2.095 & 3.016 & 2.999 & 2.927 & 2.966 \\
\hline $\mathrm{MnC} 4$ & 2.192 & 2.179 & 2.154 & 2.135 & 3.739 & 3.730 & 3.671 & 3.694 \\
\hline $\mathrm{MnC5}$ & 2.198 & 2.187 & 2.165 & 2.139 & 2.170 & 2.161 & 2.158 & 2.211 \\
\hline $\mathrm{MnC6}$ & 2.139 & 2.132 & 2.119 & 2.100 & 2.188 & 2.173 & 2.155 & 2.170 \\
\hline $\mathrm{MnC7}$ & 2.177 & 2.171 & 2.159 & 2.152 & 2.829 & 2.817 & 3.000 & 2.472 \\
\hline $\mathrm{MnC} 8$ & 2.231 & 2.222 & 2.202 & 2.203 & 3.395 & 3.396 & 3.877 & 2.838 \\
\hline $\mathrm{MnC9}$ & 2.151 & 2.148 & 2.138 & 2.121 & 2.186 & 2.178 & 2.217 & 2.195 \\
\hline $\mathrm{MnC} 10$ & 2.219 & 2.217 & 2.212 & 2.195 & 2.196 & 2.181 & 2.183 & 2.176 \\
\hline $\mathrm{MnC} 11$ & 3.223 & 3.228 & 3.242 & 3.195 & 2.757 & 2.728 & 2.174 & 2.611 \\
\hline \multirow[t]{3}{*}{$\mathrm{MnC} 12$} & 4.145 & 4.148 & 4.161 & 4.082 & 3.248 & 3.218 & 2.292 & 3.045 \\
\hline & \multicolumn{4}{|c|}{$\mathrm{Mn}-2 \mathrm{Q}$} & \multicolumn{4}{|c|}{$\mathrm{Mn}-1 \mathrm{H}$} \\
\hline & B3LYP & B3LYP* & BP86 & M06L & B3LYP & B3LYP* & BP86 & M06L \\
\hline $\mathrm{MnCl}$ & 2.270 & 2.263 & 2.241 & 2.233 & 2.119 & 2.111 & 2.089 & 2.110 \\
\hline $\mathrm{MnC} 2$ & 2.255 & 2.244 & 2.203 & 2.152 & 2.843 & 2.780 & 2.743 & 2.580 \\
\hline $\mathrm{MnC} 3$ & 2.346 & 2.331 & 2.266 & 2.204 & 3.585 & 3.530 & 3.433 & 3.277 \\
\hline $\mathrm{MnC} 4$ & 3.036 & 3.031 & 3.023 & 3.074 & 3.998 & 3.942 & 3.775 & 3.745 \\
\hline $\mathrm{MnC5}$ & 3.868 & 3.852 & 3.784 & 3.714 & 3.332 & 3.280 & 3.076 & 3.151 \\
\hline MnC6 & 3.676 & 3.654 & 3.551 & 3.338 & 2.650 & 2.597 & 2.327 & 2.570 \\
\hline $\mathrm{MnC} 7$ & 3.003 & 2.981 & 2.886 & 2.688 & 2.661 & 2.611 & 2.365 & 2.540 \\
\hline $\mathrm{MnC} 8$ & 2.059 & 2.043 & 2.039 & 2.118 & 3.387 & 3.342 & 3.179 & 3.158 \\
\hline $\mathrm{MnC} 9$ & 2.047 & 2.037 & 2.046 & 2.212 & 3.501 & 3.462 & 3.334 & 3.283 \\
\hline $\mathrm{MnC} 10$ & 2.833 & 2.823 & 2.758 & 2.172 & 2.919 & 2.883 & 2.810 & 2.676 \\
\hline $\mathrm{MnC} 11$ & 2.839 & 2.830 & 2.762 & 2.124 & 2.392 & 2.369 & 2.319 & 2.279 \\
\hline $\mathrm{MnC} 12$ & 2.063 & 2.059 & 2.060 & 2.133 & 2.144 & 2.142 & 2.115 & 2.162 \\
\hline \multicolumn{9}{|c|}{$\mathrm{Mn}-2 \mathrm{H}$} \\
\hline & B3LYP & B3LYP* & BP86 & M06L & & & & \\
\hline $\mathrm{MnC1}$ & 2.116 & 2.107 & 2.074 & 2.101 & & & & \\
\hline $\mathrm{MnC} 2$ & 3.046 & 3.018 & 2.906 & 3.015 & & & & \\
\hline $\mathrm{MnC} 3$ & 3.662 & 3.628 & 3.481 & 3.585 & & & & \\
\hline $\mathrm{MnC} 4$ & 3.665 & 3.626 & 3.452 & 3.511 & & & & \\
\hline $\mathrm{MnC5}$ & 3.647 & 3.580 & 3.284 & 3.479 & & & & \\
\hline MnC6 & 2.706 & 2.633 & 2.327 & 2.544 & & & & \\
\hline $\mathrm{MnC7}$ & 2.706 & 2.633 & 2.327 & 2.544 & & & & \\
\hline $\mathrm{MnC} 8$ & 3.647 & 3.580 & 3.284 & 3.479 & & & & \\
\hline $\mathrm{MnC} 9$ & 3.665 & 3.626 & 3.452 & 3.511 & & & & \\
\hline $\mathrm{MnC} 10$ & 3.662 & 3.628 & 3.481 & 3.585 & & & & \\
\hline $\mathrm{MnC} 11$ & 3.046 & 3.018 & 2.906 & 3.015 & & & & \\
\hline $\mathrm{MnC} 12$ & 2.116 & 2.107 & 2.074 & 2.101 & & & & \\
\hline
\end{tabular}


Table S99. Iron-carbon distances (in $\AA$ ) for the $\left(\mathbf{C}_{4} \mathbf{H}_{6}\right)_{3} \mathbf{F e}$ structures. The bold face indicates the existence of $\mathrm{Fe}-\mathrm{C}$ bonds.

\begin{tabular}{|c|c|c|c|c|c|c|c|c|}
\hline & \multicolumn{4}{|c|}{ Fe-1S } & \multicolumn{4}{|c|}{$\mathrm{Fe}-2 \mathrm{~S}$} \\
\hline & B3LYP & B3LYP* & BP86 & M06L & B3LYP & B3LYP* & BP86 & M06L \\
\hline $\mathrm{FeC} 1$ & 2.088 & 2.085 & 2.082 & 2.063 & 2.205 & 2.205 & 2.194 & 2.159 \\
\hline $\mathrm{FeC} 2$ & 2.091 & 2.085 & 2.070 & 2.056 & 2.103 & 2.101 & 2.092 & 2.068 \\
\hline $\mathrm{FeC} 3$ & 2.202 & 2.197 & 2.179 & 2.148 & 2.114 & 2.101 & 2.075 & 2.06 \\
\hline $\mathrm{FeC} 4$ & 2.822 & 2.814 & 2.792 & 2.749 & 2.218 & 2.196 & 2.15 & 2.136 \\
\hline $\mathrm{FeC} 5$ & 2.395 & 2.364 & 2.290 & 2.278 & 2.21 & 2.191 & 2.149 & 2.128 \\
\hline FeC6 & 2.120 & 2.091 & 2.044 & 2.051 & 2.11 & 2.098 & 2.072 & 2.052 \\
\hline $\mathrm{FeC} 7$ & 2.294 & 2.252 & 2.178 & 2.185 & 2.094 & 2.09 & 2.079 & 2.052 \\
\hline $\mathrm{FeC} 8$ & 3.010 & 2.991 & 2.959 & 2.926 & 2.189 & 2.186 & 2.175 & 2.137 \\
\hline FeC9 & 3.052 & 3.050 & 3.043 & 3.003 & 2.127 & 2.119 & 2.102 & 2.096 \\
\hline $\mathrm{FeC} 10$ & 2.144 & 2.144 & 2.134 & 2.110 & 2.202 & 2.198 & 2.192 & 2.18 \\
\hline $\mathrm{FeC} 11$ & 2.034 & 2.029 & 2.019 & 2.007 & 3.201 & 3.205 & 3.221 & 3.17 \\
\hline \multirow[t]{2}{*}{$\mathrm{FeC} 12$} & 2.076 & 2.069 & 2.052 & 2.044 & 4.112 & 4.111 & 4.125 & 4.037 \\
\hline & \multicolumn{4}{|c|}{ Fe-1T } & \multicolumn{4}{|c|}{$\mathrm{Fe}-2 \mathrm{~T}$} \\
\hline & B3LYP & B3LYP* & BP86 & M06L & B3LYP & B3LYP* & BP86 & M06L \\
\hline $\mathrm{FeC1}$ & 2.146 & 2.147 & 2.145 & 2.114 & 2.11 & 2.103 & 2.083 & 2.072 \\
\hline $\mathrm{FeC} 2$ & 2.061 & 2.065 & 2.059 & 2.041 & 2.02 & 2.012 & 1.992 & 1.987 \\
\hline $\mathrm{FeC} 3$ & 2.268 & 2.264 & 2.214 & 2.207 & 2.159 & 2.15 & 2.117 & 2.116 \\
\hline $\mathrm{FeC} 4$ & 3.146 & 3.129 & 3.091 & 3.073 & 3.174 & 3.171 & 3.182 & 3.114 \\
\hline $\mathrm{FeC} 5$ & 3.038 & 2.989 & 2.932 & 2.931 & 3.524 & 3.531 & 3.654 & 3.393 \\
\hline FeC6 & 2.425 & 2.314 & 2.179 & 2.259 & 3.901 & 3.91 & 3.986 & 3.779 \\
\hline $\mathrm{FeC} 7$ & 2.406 & 2.307 & 2.184 & 2.255 & 3.901 & 3.91 & 3.986 & 3.779 \\
\hline $\mathrm{FeC} 8$ & 2.965 & 2.938 & 2.921 & 2.888 & 3.524 & 3.531 & 3.654 & 3.393 \\
\hline FeC9 & 2.995 & 2.989 & 2.992 & 2.950 & 3.174 & 3.171 & 3.182 & 3.114 \\
\hline $\mathrm{FeC} 10$ & 2.128 & 2.127 & 2.135 & 2.107 & 2.159 & 2.15 & 2.117 & 2.116 \\
\hline $\mathrm{FeC} 11$ & 2.058 & 2.057 & 2.056 & 2.036 & 2.02 & 2.012 & 1.992 & 1.987 \\
\hline \multirow[t]{3}{*}{$\mathrm{FeC} 12$} & 2.206 & 2.194 & 2.147 & 2.144 & 2.11 & 2.103 & 2.083 & 2.072 \\
\hline & \multicolumn{4}{|c|}{$\mathrm{Fe}-3 \mathrm{~T}$} & \multicolumn{4}{|c|}{$\mathrm{Fe}-1 \mathrm{P}$} \\
\hline & B3LYP & B3LYP* & BP86 & M06L & B3LYP & B3LYP* & BP86 & M06L \\
\hline $\mathrm{FeCl}$ & 2.224 & 2.191 & 2.18 & 2.128 & 2.092 & 2.096 & 2.086 & 2.104 \\
\hline $\mathrm{FeC} 2$ & 2.163 & 2.146 & 2.129 & 2.112 & 2.378 & 2.334 & 2.297 & 2.253 \\
\hline $\mathrm{FeC} 3$ & 2.171 & 2.162 & 2.106 & 2.15 & 2.969 & 2.909 & 2.904 & 2.771 \\
\hline $\mathrm{FeC} 4$ & 2.272 & 2.255 & 2.158 & 2.24 & 3.533 & 3.484 & 3.465 & 3.370 \\
\hline $\mathrm{FeC} 5$ & 2.168 & 2.155 & 2.124 & 2.111 & 3.059 & 3.018 & 2.972 & 2.931 \\
\hline FeC6 & 2.221 & 2.209 & 2.151 & 2.187 & 2.299 & 2.237 & 2.142 & 2.159 \\
\hline $\mathrm{FeC} 7$ & 3.038 & 3.012 & 3.102 & 2.964 & 2.275 & 2.212 & 2.120 & 2.137 \\
\hline $\mathrm{FeC} 8$ & 3.8 & 3.761 & 3.932 & 3.649 & 3.064 & 3.028 & 2.984 & 2.946 \\
\hline FeC9 & 2.106 & 2.093 & 2.069 & 2.066 & 3.341 & 3.327 & 3.303 & 3.256 \\
\hline $\mathrm{FeC} 10$ & 2.158 & 2.146 & 2.178 & 2.129 & 2.822 & 2.824 & 2.800 & 2.758 \\
\hline $\mathrm{FeC} 11$ & 3.091 & 3.087 & 3.143 & 3.033 & 2.326 & 2.321 & 2.277 & 2.289 \\
\hline \multirow[t]{3}{*}{$\mathrm{FeC} 12$} & 3.891 & 3.881 & 3.949 & 3.756 & 2.099 & 2.096 & 2.089 & 2.103 \\
\hline & \multicolumn{4}{|c|}{ Fe-2P } & & & & \\
\hline & B3LYP & B3LYP* & BP86 & M06L & & & & \\
\hline $\mathrm{FeC1}$ & 2.061 & 2.047 & 2.040 & 2.056 & & & & \\
\hline $\mathrm{FeC} 2$ & 2.293 & 2.320 & 2.224 & 2.215 & & & & \\
\hline $\mathrm{FeC} 3$ & 3.037 & 3.090 & 2.993 & 2.917 & & & & \\
\hline $\mathrm{FeC} 4$ & 3.784 & 3.819 & 3.764 & 3.619 & & & & \\
\hline $\mathrm{FeC} 5$ & 3.753 & 3.786 & 3.769 & 3.465 & & & & \\
\hline FeC6 & 4.227 & 4.299 & 4.299 & 3.973 & & & & \\
\hline $\mathrm{FeC} 7$ & 4.227 & 4.299 & 4.299 & 3.973 & & & & \\
\hline $\mathrm{FeC} 8$ & 3.753 & 3.786 & 3.769 & 3.465 & & & & \\
\hline $\mathrm{FeC} 9$ & 3.784 & 3.819 & 3.764 & 3.619 & & & & \\
\hline $\mathrm{FeC} 10$ & 3.037 & 3.090 & 2.993 & 2.917 & & & & \\
\hline $\mathrm{FeC} 11$ & 2.293 & 2.320 & 2.224 & 2.215 & & & & \\
\hline $\mathrm{FeC} 12$ & 2.061 & 2.047 & 2.040 & 2.056 & & & & \\
\hline
\end{tabular}


Table S100. Cobalt-carbon distances (in $\AA$ ) for the $\left(\mathbf{C}_{4} \mathbf{H}_{6}\right)_{3}$ Co structures. The bold face indicates the existence of $\mathrm{Co}-\mathrm{C}$ bonds.

\begin{tabular}{|c|c|c|c|c|c|c|c|c|}
\hline & \multicolumn{4}{|c|}{ Co-1D } & \multicolumn{4}{|c|}{ Co-2D } \\
\hline & B3LYP & B3LYP* & BP86 & M06L & B3LYP & B3LYP* & BP86 & M06L \\
\hline $\mathrm{CoC} 1$ & 2.094 & 2.088 & 2.075 & 2.060 & 2.058 & 2.056 & 2.053 & 2.043 \\
\hline $\mathrm{CoC} 2$ & 1.992 & 1.985 & 1.969 & 1.961 & 2.087 & 2.079 & 2.060 & 2.059 \\
\hline $\mathrm{CoC} 3$ & 2.125 & 2.118 & 2.102 & 2.102 & 2.185 & 2.179 & 2.160 & 2.137 \\
\hline $\mathrm{CoC} 4$ & 3.119 & 3.116 & 3.113 & 3.089 & 2.738 & 2.743 & 2.738 & 2.602 \\
\hline CoC5 & 3.351 & 3.353 & 3.351 & 3.264 & 2.816 & 2.795 & 2.767 & 2.765 \\
\hline CoC6 & 3.375 & 3.379 & 3.366 & 3.191 & 2.144 & 2.121 & 2.077 & 2.081 \\
\hline $\mathrm{CoC} 7$ & 3.346 & 3.350 & 3.339 & 3.160 & 2.177 & 2.154 & 2.098 & 2.092 \\
\hline $\mathrm{CoC} 8$ & 3.259 & 3.262 & 3.267 & 3.162 & 2.944 & 2.940 & 2.932 & 2.902 \\
\hline CoC9 & 3.075 & 3.075 & 3.074 & 3.025 & 2.980 & 2.980 & 2.986 & 2.979 \\
\hline $\mathrm{CoC} 10$ & 2.107 & 2.101 & 2.086 & 2.072 & 2.066 & 2.067 & 2.070 & 2.077 \\
\hline $\mathrm{CoC} 11$ & 1.994 & 1.987 & 1.970 & 1.965 & 2.896 & 2.893 & 2.875 & 2.825 \\
\hline \multirow[t]{2}{*}{$\mathrm{CoC} 12$} & 2.104 & 2.098 & 2.082 & 2.075 & 3.859 & 3.857 & 3.840 & 3.748 \\
\hline & \multicolumn{4}{|c|}{ Co-3D } & \multicolumn{4}{|c|}{ Co-4D } \\
\hline & B3LYP & B3LYP* & BP86 & M06L & B3LYP & B3LYP* & BP86 & M06L \\
\hline $\mathrm{CoC} 1$ & 2.168 & 2.155 & 2.134 & 2.11 & 2.152 & 2.149 & 2.136 & 2.103 \\
\hline $\mathrm{CoC} 2$ & 2.132 & 2.126 & 2.114 & 2.096 & 2.059 & 2.056 & 2.059 & 2.034 \\
\hline $\mathrm{CoC} 3$ & 2.095 & 2.09 & 2.082 & 2.063 & 2.187 & 2.14 & 2.08 & 2.094 \\
\hline $\mathrm{CoC} 4$ & 2.171 & 2.161 & 2.121 & 2.109 & 2.446 & 2.351 & 2.193 & 2.253 \\
\hline CoC5 & 3.784 & 3.779 & 3.746 & 3.621 & 2.099 & 2.09 & 2.076 & 2.067 \\
\hline CoC6 & 3.009 & 3.009 & 2.985 & 2.93 & 2.196 & 2.182 & 2.154 & 2.146 \\
\hline $\mathrm{CoC} 7$ & 2.104 & 2.102 & 2.091 & 2.078 & 3.132 & 3.129 & 3.125 & 3.067 \\
\hline $\mathrm{CoC} 8$ & 2.05 & 2.046 & 2.032 & 2.026 & 4.038 & 4.041 & 4.05 & 3.926 \\
\hline CoC9 & 2.158 & 2.127 & 2.071 & 2.087 & 2.125 & 2.119 & 2.086 & 2.087 \\
\hline $\mathrm{CoC} 10$ & 2.347 & 2.304 & 2.191 & 2.224 & 2.119 & 2.116 & 2.086 & 2.084 \\
\hline $\mathrm{CoC} 11$ & 3.255 & 3.22 & 3.126 & 3.117 & 2.843 & 2.847 & 2.8 & 2.737 \\
\hline \multirow[t]{3}{*}{$\mathrm{CoC} 12$} & 4.28 & 4.254 & 4.182 & 4.149 & 3.575 & 3.609 & 3.597 & 3.415 \\
\hline & \multicolumn{4}{|c|}{ Co-5D } & \multicolumn{4}{|c|}{ Co-1Q } \\
\hline & B3LYP & B3LYP* & BP86 & M06L & B3LYP & B3LYP* & BP86 & M06L \\
\hline $\mathrm{CoC} 1$ & 2.148 & 2.075 & 1.977 & 1.971 & 2.069 & 2.084 & 2.085 & 2.478 \\
\hline $\mathrm{CoC} 2$ & 2.167 & 2.143 & 2.052 & 2.038 & 2.343 & 2.273 & 2.234 & 2.108 \\
\hline $\mathrm{CoC} 3$ & 2.206 & 2.205 & 2.118 & 2.105 & 2.954 & 2.852 & 2.857 & 2.167 \\
\hline $\mathrm{CoC} 4$ & 2.196 & 2.18 & 2.086 & 2.08 & 3.532 & 3.454 & 3.448 & 3.050 \\
\hline CoC5 & 1.989 & 1.976 & 1.994 & 1.999 & 3.064 & 3.012 & 2.976 & 2.926 \\
\hline CoC6 & 3.087 & 3.075 & 2.978 & 2.976 & 2.304 & 2.231 & 2.121 & 2.142 \\
\hline $\mathrm{CoC} 7$ & 3.594 & 3.571 & 3.285 & 3.292 & 2.316 & 2.239 & 2.120 & 2.187 \\
\hline $\mathrm{CoC} 8$ & 3.389 & 3.353 & 2.867 & 2.896 & 3.156 & 3.087 & 3.013 & 3.020 \\
\hline CoC9 & 4.077 & 4.032 & 3.476 & 3.507 & 3.492 & 3.428 & 3.343 & 3.325 \\
\hline $\mathrm{CoC} 10$ & 3.82 & 3.778 & 3.208 & 3.274 & 3.062 & 2.969 & 2.838 & 2.804 \\
\hline CoC11 & 3.053 & 3.001 & 2.563 & 2.646 & 2.462 & 2.388 & 2.271 & 2.255 \\
\hline \multirow[t]{3}{*}{$\mathrm{CoC} 12$} & 2.148 & 2.061 & 2.028 & 2.031 & 2.035 & 2.046 & 2.060 & 2.083 \\
\hline & \multicolumn{4}{|c|}{$\mathrm{Co}-2 \mathrm{Q}$} & & & & \\
\hline & B3LYP & B3LYP* & BP86 & M06L & & & & \\
\hline $\mathrm{CoC} 1$ & 2.027 & 2.023 & 2.021 & 2.027 & & & & \\
\hline $\mathrm{CoC} 2$ & 2.861 & 2.845 & 2.484 & 2.812 & & & & \\
\hline $\mathrm{CoC} 3$ & 3.49 & 3.472 & 3.15 & 3.419 & & & & \\
\hline $\mathrm{CoC} 4$ & 3.546 & 3.521 & 3.407 & 3.438 & & & & \\
\hline CoC5 & 3.411 & 3.334 & 3.167 & 3.235 & & & & \\
\hline CoC6 & 2.421 & 2.332 & 2.124 & 2.234 & & & & \\
\hline $\mathrm{CoC} 7$ & 2.421 & 2.332 & 2.111 & 2.234 & & & & \\
\hline $\mathrm{CoC} 8$ & 3.411 & 3.334 & 3.123 & 3.235 & & & & \\
\hline CoC9 & 3.546 & 3.521 & 3.521 & 3.438 & & & & \\
\hline $\mathrm{CoC} 10$ & 3.49 & 3.472 & 3.645 & 3.419 & & & & \\
\hline $\mathrm{CoC} 11$ & 2.861 & 2.845 & 3.049 & 2.812 & & & & \\
\hline $\mathrm{CoC} 12$ & 2.027 & 2.023 & 2.017 & 2.027 & & & & \\
\hline
\end{tabular}


Table S101. Nickel-carbon distances (in $\AA$ ) for the $\left(\mathbf{C}_{4} \mathbf{H}_{6}\right)_{3} \mathbf{N i}$ structures. The bold face indicates the existence of $\mathrm{Ni}-\mathrm{C}$ bonds.

\begin{tabular}{|c|c|c|c|c|c|c|c|c|}
\hline & \multicolumn{4}{|c|}{$\mathrm{Ni}-1 \mathrm{~S}$} & \multicolumn{4}{|c|}{$\mathrm{Ni}-2 \mathrm{~S}$} \\
\hline & B3LYP & B3LYP* & BP86 & M06L & B3LYP & B3LYP* & BP86 & M06L \\
\hline $\mathrm{NiC} 1$ & 2.066 & 2.063 & 2.055 & 2.039 & 2.01 & 2.011 & 2.016 & 1.997 \\
\hline $\mathrm{NiC} 2$ & 1.979 & 1.973 & 1.96 & 1.953 & 2.043 & 2.038 & 2.024 & 2.012 \\
\hline $\mathrm{NiC} 3$ & 2.071 & 2.069 & 2.063 & 2.042 & 2.158 & 2.154 & 2.14 & 2.108 \\
\hline $\mathrm{NiC} 4$ & 3.026 & 3.029 & 3.035 & 2.971 & 2.792 & 2.795 & 2.804 & 2.716 \\
\hline $\mathrm{NiC} 5$ & 3.227 & 3.232 & 3.241 & 3.138 & 2.873 & 2.862 & 2.843 & 2.816 \\
\hline NiC6 & 3.367 & 3.374 & 3.382 & 3.264 & 2.151 & 2.129 & 2.082 & 2.082 \\
\hline $\mathrm{NiC} 7$ & 3.396 & 3.403 & 3.409 & 3.305 & 2.147 & 2.126 & 2.085 & 2.085 \\
\hline $\mathrm{NiC} 8$ & 3.316 & 3.319 & 3.322 & 3.254 & 2.868 & 2.863 & 2.856 & 2.822 \\
\hline NiC9 & 3.065 & 3.065 & 3.067 & 3.035 & 2.961 & 2.964 & 2.976 & 2.955 \\
\hline $\mathrm{NiC} 10$ & 2.084 & 2.081 & 2.071 & 2.056 & 2.016 & 2.02 & 2.029 & 2.022 \\
\hline $\mathrm{NiC} 11$ & 1.977 & 1.971 & 1.957 & 1.948 & 2.736 & 2.723 & 2.653 & 2.588 \\
\hline \multirow[t]{3}{*}{$\mathrm{NiC} 12$} & 2.055 & 2.052 & 2.044 & 2.026 & 3.669 & 3.652 & 3.56 & 3.45 \\
\hline & \multicolumn{4}{|c|}{$\mathrm{Ni}-3 \mathrm{~S}$} & \multicolumn{4}{|c|}{$\mathrm{Ni}-4 \mathrm{~S}$} \\
\hline & B3LYP & B3LYP* & BP86 & M06L & B3LYP & B3LYP* & BP86 & M06L \\
\hline $\mathrm{NiCl}$ & 2.005 & 2.007 & 2.016 & 1.999 & 2.003 & 2.005 & 2.013 & 1.997 \\
\hline $\mathrm{NiC} 2$ & 2.063 & 2.057 & 2.038 & 2.031 & 2.057 & 2.051 & 2.038 & 2.03 \\
\hline $\mathrm{NiC} 3$ & 2.186 & 2.182 & 2.162 & 2.131 & 2.181 & 2.175 & 2.155 & 2.123 \\
\hline $\mathrm{NiC} 4$ & 2.757 & 2.758 & 2.757 & 2.662 & 2.792 & 2.793 & 2.793 & 2.709 \\
\hline $\mathrm{NiC} 5$ & 2.803 & 2.791 & 2.767 & 2.711 & 2.937 & 2.922 & 2.897 & 2.872 \\
\hline $\mathrm{NiC} 6$ & 2.16 & 2.135 & 2.083 & 2.079 & 2.169 & 2.14 & 2.084 & 2.087 \\
\hline $\mathrm{NiC} 7$ & 2.195 & 2.169 & 2.109 & 2.11 & 2.212 & 2.18 & 2.119 & 2.116 \\
\hline NiC8 & 2.927 & 2.921 & 2.913 & 2.897 & 3.105 & 3.09 & 3.067 & 3.018 \\
\hline $\mathrm{NiC9}$ & 2.93 & 2.934 & 2.95 & 2.935 & 3.984 & 3.979 & 3.971 & 3.928 \\
\hline $\mathrm{NiC} 10$ & 2.024 & 2.03 & 2.048 & 2.041 & 3.778 & 3.78 & 3.787 & 3.771 \\
\hline $\mathrm{NiC} 11$ & 2.892 & 2.895 & 2.904 & 2.829 & 3.002 & 3.005 & 3.01 & 3.011 \\
\hline \multirow[t]{3}{*}{$\mathrm{NiC} 12$} & 3.884 & 3.889 & 3.906 & 3.803 & 2.006 & 2.012 & 2.027 & 2.029 \\
\hline & \multicolumn{4}{|c|}{$\mathrm{Ni}-5 \mathrm{~S}$} & \multicolumn{4}{|c|}{$\mathrm{Ni}-1 \mathrm{~T}$} \\
\hline & B3LYP & B3LYP* & BP86 & M06L & B3LYP & B3LYP* & BP86 & M06L \\
\hline $\mathrm{NiCl}$ & 2.274 & 2.253 & 2.199 & 2.179 & 2.141 & 2.136 & 2.142 & 2.135 \\
\hline $\mathrm{NiC} 2$ & 2.128 & 2.117 & 2.094 & 2.068 & 2.087 & 2.088 & 2.068 & 2.06 \\
\hline $\mathrm{NiC} 3$ & 2.114 & 2.104 & 2.086 & 2.057 & 2.325 & 2.347 & 2.304 & 2.25 \\
\hline $\mathrm{NiC} 4$ & 2.238 & 2.217 & 2.163 & 2.133 & 3.062 & 3.059 & 3.014 & 2.981 \\
\hline $\mathrm{NiC} 5$ & 1.967 & 1.97 & 1.98 & 1.979 & 3.026 & 2.973 & 2.893 & 2.902 \\
\hline NiC6 & 2.637 & 2.62 & 2.562 & 2.496 & 2.373 & 2.26 & 2.116 & 2.222 \\
\hline $\mathrm{NiC} 7$ & 3.478 & 3.462 & 3.406 & 3.301 & 2.331 & 2.231 & 2.116 & 2.189 \\
\hline $\mathrm{NiC} 8$ & 3.992 & 3.982 & 3.946 & 3.849 & 2.908 & 2.87 & 2.846 & 2.761 \\
\hline $\mathrm{NiC} 9$ & 4.359 & 4.348 & 4.31 & 4.243 & 3.427 & 3.401 & 3.408 & 3.289 \\
\hline $\mathrm{NiC} 10$ & 3.646 & 3.635 & 3.595 & 3.548 & 2.963 & 2.944 & 2.959 & 2.838 \\
\hline NiC11 & 2.702 & 2.693 & 2.657 & 2.63 & 2.354 & 2.333 & 2.297 & 2.258 \\
\hline \multirow[t]{3}{*}{$\mathrm{NiC} 12$} & 1.974 & 1.978 & 1.989 & 1.989 & 2.036 & 2.045 & 2.056 & 2.053 \\
\hline & \multicolumn{4}{|c|}{$\mathrm{Ni}-2 \mathrm{~T}$} & & & & \\
\hline & B3LYP & B3LYP* & BP86 & M06L & & & & \\
\hline $\mathrm{NiC} 1$ & 2.004 & 2.002 & 2.006 & 2.008 & & & & \\
\hline $\mathrm{NiC} 2$ & 2.819 & 2.827 & 2.815 & 2.827 & & & & \\
\hline $\mathrm{NiC} 3$ & 3.455 & 3.474 & 3.499 & 3.472 & & & & \\
\hline $\mathrm{NiC} 4$ & 3.512 & 3.526 & 3.565 & 3.499 & & & & \\
\hline $\mathrm{NiC} 5$ & 3.352 & 3.291 & 3.18 & 3.188 & & & & \\
\hline NiC6 & 2.429 & 2.34 & 2.154 & 2.213 & & & & \\
\hline $\mathrm{NiC} 7$ & 2.26 & 2.193 & 2.058 & 2.109 & & & & \\
\hline $\mathrm{NiC} 8$ & 2.976 & 2.941 & 2.873 & 2.907 & & & & \\
\hline NiC9 & 2.903 & 2.896 & 2.918 & 2.896 & & & & \\
\hline $\mathrm{NiC} 10$ & 2.036 & 2.033 & 2.033 & 2.032 & & & & \\
\hline $\mathrm{NiC} 11$ & 2.821 & 2.804 & 2.747 & 2.72 & & & & \\
\hline $\mathrm{NiC} 12$ & 3.809 & 3.787 & 3.72 & 3.673 & & & & \\
\hline
\end{tabular}


Table S102. Total energies ( $E$ in hartree), relative energies $(\Delta E$ in $\mathrm{kcal} / \mathrm{mol})$, and the spin expectation values $\left\langle\mathrm{S}^{2}\right\rangle$ for the $\left(\mathrm{C}_{4} \mathrm{H}_{6}\right)_{3}$ Ti structure by all four methods. Neither structure has any imaginary vibrational frequencies.

\begin{tabular}{|c|c|c|c|c|}
\hline \multicolumn{5}{|c|}{ Ti-1S } \\
\hline & $\operatorname{B3LYP}\left(\boldsymbol{C}_{1}\right)$ & $\operatorname{B3LYP}^{*}\left(\boldsymbol{C}_{1}\right)$ & $\operatorname{BP} 86\left(\boldsymbol{C}_{1}\right)$ & $\operatorname{M06L}\left(\boldsymbol{C}_{1}\right)$ \\
\hline$E+1317$ & -0.61403 & -0.14284 & -0.72126 & -0.53230 \\
\hline$\Delta \mathrm{E}$ & 0.0 & 0.0 & 0.0 & 0.0 \\
\hline \multicolumn{5}{|c|}{ Ti-2S } \\
\hline & $\operatorname{B3LYP}\left(\boldsymbol{C}_{1}\right)$ & $\operatorname{B3LYP}^{*}\left(\boldsymbol{C}_{1}\right)$ & $\operatorname{BP86}\left(\boldsymbol{C}_{1}\right)$ & $\operatorname{M06L}\left(\boldsymbol{C}_{1}\right)$ \\
\hline$E+1317$ & -0.58775 & -0.11816 & -0.70125 & -0.51437 \\
\hline$\Delta \mathrm{E}$ & 16.5 & 15.5 & 12.6 & 11.3 \\
\hline \multicolumn{5}{|c|}{ Ti-1T } \\
\hline & $\operatorname{B3LYP}\left(\boldsymbol{C}_{1}\right)$ & $\operatorname{B3LYP}^{*}\left(\boldsymbol{C}_{1}\right)$ & $\operatorname{BP86}\left(\boldsymbol{C}_{1}\right)$ & $\operatorname{M06L}\left(\boldsymbol{C}_{1}\right)$ \\
\hline$E+1317$ & -0.58065 & -0.10658 & -0.68330 & -0.49311 \\
\hline$\Delta \mathrm{E}$ & 20.9 & 22.8 & 23.8 & 24.6 \\
\hline$\left\langle\mathrm{S}^{2}\right\rangle$ & 2.01 & 2.01 & 2.01 & 2.02 \\
\hline
\end{tabular}


Table S103. Total energies ( $E$ in hartree), relative energies ( $\Delta E$ in $\mathrm{kcal} / \mathrm{mol})$, and the spin expectation values $\left\langle\mathrm{S}^{2}\right\rangle$ for the $\left(\mathrm{C}_{4} \mathrm{H}_{6}\right)_{3} \mathrm{~V}$ structure by all four methods. Neither structure has any imaginary vibrational frequencies.

\begin{tabular}{|c|c|c|c|c|}
\hline \multicolumn{5}{|c|}{$\overline{V-1 Q}$} \\
\hline & $\operatorname{B3LYP}\left(\boldsymbol{C}_{\mathbf{1}}\right)$ & $\operatorname{B3LYP}^{*}\left(\boldsymbol{C}_{\mathbf{1}}\right)$ & $\operatorname{BP86}\left(\boldsymbol{C}_{\mathbf{1}}\right)$ & $\operatorname{M06L}\left(\boldsymbol{C}_{1}\right)$ \\
\hline$E+1411$ & -1.13001 & -0.64924 & -1.24973 & -1.03503 \\
\hline$\Delta \mathrm{E}$ & 0.0 & 0.0 & 0.0 & 0.0 \\
\hline$\left\langle\mathrm{S}^{2}\right\rangle$ & 3.78 & 3.78 & 3.78 & 3.80 \\
\hline \multicolumn{5}{|c|}{$\mathrm{V}-2 \mathrm{Q}$} \\
\hline & $\operatorname{B3LYP}\left(\boldsymbol{C}_{1}\right)$ & \left.${\mathrm{B} 3 L Y P^{*}}^{*} \boldsymbol{C}_{\mathbf{1}}\right)$ & $\operatorname{BP86}\left(\boldsymbol{C}_{\mathbf{1}}\right)$ & $\operatorname{M06L}\left(C_{1}\right)$ \\
\hline$E+1411$ & -1.11332 & -0.63249 & -1.2332 & -1.01658 \\
\hline$\Delta \mathrm{E}$ & 10.5 & 10.5 & 10.4 & 11.6 \\
\hline$\left\langle\mathrm{S}^{2}\right\rangle$ & 3.79 & 3.79 & 3.79 & 3.8 \\
\hline \multicolumn{5}{|c|}{$V-3 Q$} \\
\hline & $\operatorname{B3LYP}\left(\boldsymbol{C}_{\mathbf{1}}\right)$ & \left.${\mathrm{B} 3 L Y P^{*}}^{*} \boldsymbol{C}_{\boldsymbol{1}}\right)$ & $\operatorname{BP86}\left(\boldsymbol{C}_{1}\right)$ & $\operatorname{M06L}\left(\boldsymbol{C}_{1}\right)$ \\
\hline $\mathrm{E}+1411$ & -1.09315 & -0.61623 & -1.21963 & -1.00245 \\
\hline$\Delta \mathrm{E}$ & 23.1 & 20.7 & 18.9 & 20.4 \\
\hline$\left\langle\mathrm{S}^{2}\right\rangle$ & 3.78 & 3.79 & 3.77 & 3.8 \\
\hline \multicolumn{5}{|c|}{$\mathrm{V}-4 \mathrm{Q}$} \\
\hline & $\operatorname{B3LYP}\left(\boldsymbol{C}_{\mathbf{1}}\right)$ & $\operatorname{B3LYP}^{*}\left(\boldsymbol{C}_{\mathbf{1}}\right)$ & $\mathrm{BP} 86\left(\boldsymbol{C}_{\mathbf{1}}\right)$ & $\operatorname{M06L}\left(\boldsymbol{C}_{1}\right)$ \\
\hline$E+1411$ & -1.09834 & -0.61541 & -1.21194 & -0.99279 \\
\hline$\Delta \mathrm{E}$ & 19.9 & 21.2 & 23.7 & 26.5 \\
\hline$\left\langle\mathrm{S}^{2}\right\rangle$ & 3.79 & 3.78 & 3.81 & 3.81 \\
\hline \multicolumn{5}{|c|}{$\mathrm{V}-5 \mathrm{Q}$} \\
\hline & $\operatorname{B3LYP}\left(\boldsymbol{C}_{\mathbf{1}}\right)$ & B3LYP* $^{*}\left(\boldsymbol{C}_{1}\right)$ & $\operatorname{BP86}\left(\boldsymbol{C}_{1}\right)$ & $\operatorname{M06L}\left(C_{1}\right)$ \\
\hline$E+1411$ & -1.09295 & -0.61485 & -1.21905 & -1.00058 \\
\hline$\Delta \mathrm{E}$ & 23.3 & 21.6 & 19.2 & 21.6 \\
\hline$\left\langle\mathrm{S}^{2}\right\rangle$ & 3.79 & 3.79 & 3.78 & 3.81 \\
\hline \multicolumn{5}{|c|}{ V-1D } \\
\hline & $\operatorname{B3LYP}\left(\boldsymbol{C}_{\mathbf{1}}\right)$ & B3LYP* $^{*}\left(\boldsymbol{C}_{1}\right)$ & $\operatorname{BP86}\left(\boldsymbol{C}_{1}\right)$ & $\operatorname{M06L}\left(\boldsymbol{C}_{1}\right)$ \\
\hline$E+1411$ & -1.11884 & -0.64227 & -1.25130 & -1.02444 \\
\hline$\Delta \mathrm{E}$ & 7.0 & 4.4 & -1.0 & 6.6 \\
\hline$\left\langle\mathrm{S}^{2}\right\rangle$ & 0.77 & 0.77 & 0.76 & 0.78 \\
\hline \multicolumn{5}{|c|}{ V-2D } \\
\hline & $\operatorname{B3LYP}\left(\boldsymbol{C}_{\mathbf{1}}\right)$ & $\mathrm{B} 3 L Y P^{*}\left(\boldsymbol{C}_{\mathbf{1}}\right)$ & $\operatorname{BP86}\left(\boldsymbol{C}_{\mathbf{1}}\right)$ & $\operatorname{M06L}\left(\boldsymbol{C}_{1}\right)$ \\
\hline$E+1411$ & -1.10769 & -0.63367 & -1.24778 & -1.02849 \\
\hline$\Delta \mathrm{E}$ & 14.0 & 9.8 & 1.2 & 4.1 \\
\hline$\left\langle\mathrm{S}^{2}\right\rangle$ & 0.81 & 0.79 & 0.77 & 0.81 \\
\hline \multicolumn{5}{|c|}{ V-3D } \\
\hline & $\operatorname{B3LYP}\left(\boldsymbol{C}_{1}\right)$ & B3LYP $^{*}\left(\boldsymbol{C}_{\mathbf{1}}\right)$ & $\mathrm{BP} 86\left(\boldsymbol{C}_{1}\right)$ & $\operatorname{M06L}\left(C_{1}\right)$ \\
\hline$E+1411$ & -1.10305 & -0.62571 & -1.23404 & -1.00329 \\
\hline$\Delta \mathrm{E}$ & 16.9 & 14.8 & 9.8 & 19.9 \\
\hline$\left\langle\mathrm{S}^{2}\right\rangle$ & 0.77 & 0.77 & 0.76 & 0.78 \\
\hline \multicolumn{5}{|c|}{ V-4D } \\
\hline & $\operatorname{B3LYP}\left(\boldsymbol{C}_{1}\right)$ & $\mathrm{B} 3 \mathrm{LYP}^{*}\left(\boldsymbol{C}_{\mathbf{1}}\right)$ & $\mathrm{BP86}\left(\boldsymbol{C}_{\mathbf{1}}\right)$ & $\operatorname{M06L}\left(\boldsymbol{C}_{1}\right)$ \\
\hline$E+1411$ & -1.08083 & -0.60354 & -1.21064 & -0.97959 \\
\hline$\Delta \mathrm{E}$ & 30.9 & 28.7 & 24.5 & 34.8 \\
\hline$\left\langle\mathrm{S}^{2}\right\rangle$ & 0.81 & 0.79 & 0.78 & 0.85 \\
\hline
\end{tabular}


Table S104. Total energies ( $\mathrm{E}$ in hartree), relative energies $(\Delta \mathrm{E}$ in $\mathrm{kcal} / \mathrm{mol})$, and the spin expectation values $\left\langle\mathrm{S}^{2}\right\rangle$ for the $\left(\mathrm{C}_{4} \mathrm{H}_{6}\right)_{3} \mathrm{Cr}$ structure by all four methods. Neither structure has any imaginary vibrational frequencies.

\begin{tabular}{|c|c|c|c|c|}
\hline \multicolumn{5}{|c|}{ Cr-1S } \\
\hline & B3LYP $\left(\boldsymbol{C}_{\mathbf{1}}\right)$ & B3LYP $^{*}\left(\boldsymbol{C}_{\mathbf{1}}\right)$ & $\mathrm{BP} 86\left(\boldsymbol{C}_{1}\right)$ & $\operatorname{M06L}\left(\boldsymbol{C}_{1}\right)$ \\
\hline$E+1512$ & -0.54969 & -0.07332 & -0.72136 & -0.46333 \\
\hline$\Delta \mathrm{E}$ & 36.7 & 28.5 & 9.5 & 26.0 \\
\hline \multicolumn{5}{|c|}{ Cr-2S } \\
\hline & $\operatorname{B3LYP}\left(\boldsymbol{c}_{1}\right)$ & $\operatorname{B3LYP}^{*}\left(\boldsymbol{C}_{1}\right)$ & $\operatorname{BP86}\left(\boldsymbol{C}_{1}\right)$ & $\operatorname{M06L}\left(\boldsymbol{C}_{1}\right)$ \\
\hline$E+1512$ & -0.53231 & -0.05589 & -0.70671 & -0.45431 \\
\hline$\Delta \mathrm{E}$ & 47.6 & 39.5 & 18.7 & 31.6 \\
\hline \multicolumn{5}{|c|}{$\mathrm{Cr}-1 \mathrm{~T}$} \\
\hline & $\operatorname{B3LYP}\left(\boldsymbol{c}_{1}\right)$ & $\operatorname{B3LYP}^{*}\left(\boldsymbol{C}_{1}\right)$ & $\operatorname{BP} 86\left(\boldsymbol{C}_{1}\right)$ & $\operatorname{M06L}\left(\boldsymbol{C}_{1}\right)$ \\
\hline $\mathrm{E}+1512$ & -0.59605 & -0.11503 & -0.75365 & -0.50623 \\
\hline$\Delta \mathrm{E}$ & 7.6 & 2.3 & -10.7 & -0.9 \\
\hline$\left\langle\mathrm{S}^{2}\right\rangle$ & 2.25 & 2.18 & 2.07 & 2.22 \\
\hline \multicolumn{5}{|c|}{$\mathrm{Cr}-2 \mathrm{~T}$} \\
\hline & $\operatorname{B3LYP}\left(\boldsymbol{c}_{1}\right)$ & B3LYP* $^{*}\left(\boldsymbol{C}_{1}\right)$ & $\operatorname{BP86}\left(\boldsymbol{C}_{1}\right)$ & $\operatorname{M06L}\left(\boldsymbol{C}_{1}\right)$ \\
\hline$E+1512$ & -0.57662 & -0.09443 & -0.72989 & -0.48070 \\
\hline$\Delta \mathrm{E}$ & 19.8 & 15.3 & 4.2 & 15.1 \\
\hline$\left\langle\mathrm{S}^{2}\right\rangle$ & 2.15 & 2.13 & 2.08 & 2.18 \\
\hline \multicolumn{5}{|c|}{$\mathrm{Cr}-3 \mathrm{~T}$} \\
\hline & $\operatorname{B3LYP}\left(\boldsymbol{C}_{1}\right)$ & $\mathrm{B}^{2} \mathrm{LYP}^{*}\left(\boldsymbol{C}_{1}\right)$ & $\operatorname{BP86}\left(\boldsymbol{C}_{1}\right)$ & $\operatorname{M06L}\left(\boldsymbol{C}_{1}\right)$ \\
\hline$E+1512$ & -0.57256 & -0.09109 & -0.72676 & -0.47946 \\
\hline$\Delta \mathrm{E}$ & 22.3 & 17.4 & 6.2 & 15.9 \\
\hline$\left\langle\mathrm{S}^{2}\right\rangle$ & 2.19 & 2.14 & 2.06 & 2.19 \\
\hline \multicolumn{5}{|c|}{ Cr-1P } \\
\hline & $\operatorname{B3LYP}\left(\boldsymbol{C}_{1}\right)$ & $\operatorname{B3LYP}^{*}\left(\boldsymbol{C}_{\mathbf{1}}\right)$ & $\operatorname{BP} 86\left(\boldsymbol{C}_{1}\right)$ & $\operatorname{M06L}\left(\boldsymbol{C}_{1}\right)$ \\
\hline$E+1512$ & -0.60807 & -0.11877 & -0.73653 & -0.50478 \\
\hline$\Delta \mathrm{E}$ & 0.0 & 0.0 & 0.0 & 0.0 \\
\hline$\left\langle\mathrm{S}^{2}\right\rangle$ & 6.07 & 6.07 & 6.06 & 6.10 \\
\hline \multicolumn{5}{|c|}{$\mathrm{Cr}-2 \mathrm{P}$} \\
\hline & $\operatorname{B3LYP}\left(\boldsymbol{c}_{\mathbf{s}}\right)$ & $\operatorname{B3LYP}^{*}\left(\boldsymbol{C}_{\mathbf{s}}\right)$ & $\operatorname{BP} 86\left(\boldsymbol{C}_{\mathrm{s}}\right)$ & $\operatorname{M06L}\left(\boldsymbol{C}_{\mathbf{s}}\right)$ \\
\hline$E+1512$ & -0.60718 & -0.11742 & -0.73178 & -0.50138 \\
\hline$\Delta \mathrm{E}$ & 0.6 & 0.8 & 3.0 & 2.1 \\
\hline$\left\langle\mathrm{S}^{2}\right\rangle$ & 6.07 & 6.07 & 6.05 & 6.09 \\
\hline
\end{tabular}


Table S105. Total energies ( $\mathrm{E}$ in hartree), relative energies ( $\Delta \mathrm{E}$ in $\mathrm{kcal} / \mathrm{mol})$, and the spin expectation values $\left\langle\mathrm{S}^{2}\right\rangle$ for the $\left(\mathrm{C}_{4} \mathrm{H}_{6}\right)_{3} \mathrm{Mn}$ structure by all four methods. Neither structure has any imaginary vibrational frequencies.

\begin{tabular}{|c|c|c|c|c|}
\hline \multicolumn{5}{|c|}{ Mn-1D } \\
\hline & $\operatorname{B3LYP}\left(\boldsymbol{C}_{\mathbf{1}}\right)$ & $\operatorname{B3LYP}^{*}\left(\boldsymbol{C}_{1}\right)$ & $\operatorname{BP86}\left(\boldsymbol{C}_{1}\right)$ & $\operatorname{M06L}\left(\boldsymbol{C}_{1}\right)$ \\
\hline$E+1618$ & -1.08177 & -0.59910 & -1.27298 & -0.99038 \\
\hline$\Delta \mathrm{E}$ & 34.8 & 23.9 & -3.9 & 18.8 \\
\hline$\left\langle\mathrm{S}^{2}\right\rangle$ & 0.97 & 0.89 & 0.80 & 0.94 \\
\hline \multicolumn{5}{|c|}{ Mn-2D } \\
\hline & $\operatorname{B3LYP}\left(\boldsymbol{C}_{1}\right)$ & $\operatorname{B3LYP}^{*}\left(\boldsymbol{C}_{1}\right)$ & $\operatorname{BP86}\left(\boldsymbol{C}_{1}\right)$ & $\operatorname{M06L}\left(\boldsymbol{C}_{1}\right)$ \\
\hline$E+1618$ & -1.08200 & -0.59587 & -1.26269 & -0.97849 \\
\hline$\Delta \mathrm{E}$ & 34.7 & 25.9 & 2.6 & 26.2 \\
\hline$\left\langle\mathrm{S}^{2}\right\rangle$ & 0.83 & 0.82 & 0.79 & 0.86 \\
\hline \multicolumn{5}{|c|}{ Mn-3D } \\
\hline & $\operatorname{B3LYP}\left(\boldsymbol{c}_{1}\right)$ & B3LYP* $^{*}\left(\boldsymbol{C}_{1}\right)$ & $\operatorname{BP86}\left(\boldsymbol{C}_{1}\right)$ & $\operatorname{M06L}\left(\boldsymbol{C}_{1}\right)$ \\
\hline$E+1618$ & -1.07579 & -0.59334 & -1.26744 & -0.98259 \\
\hline$\Delta \mathrm{E}$ & 38.6 & 27.5 & -0.4 & 23.7 \\
\hline$\left\langle\mathrm{S}^{2}\right\rangle$ & 0.86 & 0.83 & 0.79 & 0.89 \\
\hline \multicolumn{5}{|c|}{ Mn-1Q } \\
\hline & $\operatorname{B3LYP}\left(\boldsymbol{c}_{1}\right)$ & $\operatorname{B3LYP}^{*}\left(\boldsymbol{C}_{1}\right)$ & $\operatorname{BP} 86\left(C_{1}\right)$ & $\operatorname{M06L}\left(\boldsymbol{C}_{1}\right)$ \\
\hline$E+1618$ & -1.09764 & -0.60454 & -1.25239 & -0.98511 \\
\hline$\Delta \mathrm{E}$ & 24.9 & 20.5 & 9.02 & 22.1 \\
\hline$\left\langle\mathrm{S}^{2}\right\rangle$ & 4.16 & 4.06 & 3.86 & 4.17 \\
\hline \multicolumn{5}{|c|}{ Mn-2Q } \\
\hline & $\operatorname{B3LYP}\left(\boldsymbol{C}_{1}\right)$ & $\mathrm{B} \mathrm{LYP}^{*}\left(\boldsymbol{C}_{1}\right)$ & $\operatorname{BP86}\left(\boldsymbol{C}_{1}\right)$ & $\operatorname{M06L}\left(\boldsymbol{C}_{1}\right)$ \\
\hline$E+1618$ & -1.08923 & -0.59736 & -1.24642 & -1.00571 \\
\hline$\Delta \mathrm{E}$ & 30.1 & 25.0 & 12.8 & 9.2 \\
\hline$\left\langle\mathrm{S}^{2}\right\rangle$ & 4.10 & 4.01 & 3.87 & 4.06 \\
\hline \multicolumn{5}{|c|}{ Mn-1H } \\
\hline & $\operatorname{B3LYP}\left(\boldsymbol{C}_{1}\right)$ & $\operatorname{B3LYP}^{*}\left(\boldsymbol{C}_{1}\right)$ & $\operatorname{BP86}\left(\boldsymbol{C}_{1}\right)$ & $\operatorname{M06L}\left(\boldsymbol{C}_{1}\right)$ \\
\hline$E+1618$ & -1.13728 & -0.63714 & -1.26684 & -1.02028 \\
\hline$\Delta \mathrm{E}$ & 0.0 & 0.0 & 0.0 & 0.0 \\
\hline$\left\langle\mathrm{S}^{2}\right\rangle$ & 8.78 & 8.78 & 8.79 & 8.79 \\
\hline \multicolumn{5}{|c|}{ Mn-2H } \\
\hline & $\operatorname{B3LYP}\left(\boldsymbol{C}_{\mathbf{s}}\right)$ & B3LYP* $^{*}\left(\boldsymbol{C}_{\mathbf{s}}\right)$ & $\operatorname{BP86}\left(\boldsymbol{C}_{\mathrm{s}}\right)$ & $\operatorname{M06L}\left(\boldsymbol{C}_{\mathbf{s}}\right)$ \\
\hline$E+1618$ & -1.13295 & -0.63217 & -1.26039 & -1.01012 \\
\hline$\Delta \mathrm{E}$ & 2.7 & 3.1 & 4.0 & 6.4 \\
\hline$\left\langle\mathrm{S}^{2}\right\rangle$ & 8.78 & 8.78 & 8.79 & 8.79 \\
\hline
\end{tabular}


Table S106. Total energies ( $E$ in hartree), relative energies ( $\Delta E$ in $\mathrm{kcal} / \mathrm{mol})$, and the spin expectation values $\left\langle\mathrm{S}^{2}\right\rangle$ for the $\left(\mathrm{C}_{4} \mathrm{H}_{6}\right)_{3} \mathrm{Fe}$ structure by all four methods. Neither structure has any imaginary vibrational frequencies.

\begin{tabular}{|c|c|c|c|c|}
\hline \multicolumn{5}{|c|}{ Fe-1T } \\
\hline & $\operatorname{B3LYP}\left(\boldsymbol{C}_{\mathbf{s}}\right)$ & $\operatorname{B3LYP}^{*}\left(\boldsymbol{C}_{\mathbf{s}}\right)$ & $\operatorname{BP86}\left(\boldsymbol{C}_{\mathrm{s}}\right)$ & M06L $\left(\boldsymbol{C}_{\mathbf{s}}\right)$ \\
\hline$E+1731$ & -0.85253 & -0.35682 & -1.04125 & -0.73650 \\
\hline$\Delta \mathrm{E}$ & 0.0 & 0.0 & 0.0 & 0.0 \\
\hline$\left\langle\mathrm{S}^{2}\right\rangle$ & 2.14 & 2.12 & 2.06 & 2.16 \\
\hline \multicolumn{5}{|c|}{ Fe-2T } \\
\hline & $\operatorname{B3LYP}\left(\boldsymbol{C}_{\mathbf{s}}\right)$ & B3LYP* $\left.^{*} \boldsymbol{C}_{\mathbf{s}}\right)$ & BP86( $\left.\boldsymbol{C}_{\mathrm{s}}\right)$ & M06L( $\left.\boldsymbol{C}_{\mathbf{s}}\right)$ \\
\hline$E+1731$ & -0.85307 & -0.35545 & -1.03022 & -0.73223 \\
\hline$\Delta \mathrm{E}$ & -0.3 & 0.9 & 6.9 & 2.7 \\
\hline$\left\langle\mathrm{S}^{2}\right\rangle$ & 2.1 & 2.09 & 2.05 & 2.13 \\
\hline \multicolumn{5}{|c|}{$\mathrm{Fe}-3 \mathrm{~T}$} \\
\hline & $\operatorname{B3LYP}\left(\boldsymbol{c}_{1}\right)$ & $\operatorname{B3LYP}^{*}\left(\boldsymbol{C}_{1}\right)$ & $\operatorname{BP86}\left(\boldsymbol{C}_{1}\right)$ & $\operatorname{M06L}\left(\boldsymbol{C}_{1}\right)$ \\
\hline$E+1731$ & -0.81548 & -0.32066 & -1.00077 & -0.69876 \\
\hline$\Delta \mathrm{E}$ & 23.3 & 22.7 & 25.4 & 23.7 \\
\hline$\left\langle\mathrm{S}^{2}\right\rangle$ & 2.38 & 2.25 & 2.08 & 2.31 \\
\hline \multicolumn{5}{|c|}{ Fe-1S } \\
\hline & $\operatorname{B3LYP}\left(\boldsymbol{c}_{\mathbf{1}}\right)$ & $\operatorname{B3LYP}^{*}\left(\boldsymbol{C}_{1}\right)$ & $\operatorname{BP86}\left(\boldsymbol{C}_{1}\right)$ & $\operatorname{M06L}\left(\boldsymbol{C}_{1}\right)$ \\
\hline$E+1731$ & -0.83072 & -0.34125 & -1.04168 & -0.72857 \\
\hline$\Delta \mathrm{E}$ & 13.7 & 9.8 & -0.3 & 5.0 \\
\hline \multicolumn{5}{|c|}{ Fe-2S } \\
\hline & $\operatorname{B3LYP}\left(\boldsymbol{C}_{1}\right)$ & $\operatorname{B3LYP}^{*}\left(\boldsymbol{C}_{1}\right)$ & $\operatorname{BP86}\left(\boldsymbol{C}_{1}\right)$ & $\operatorname{M06L}\left(\boldsymbol{C}_{1}\right)$ \\
\hline $\mathrm{E}+1731$ & -0.79411 & -0.30745 & -1.01189 & -0.69835 \\
\hline$\Delta \mathrm{E}$ & 36.7 & 31.0 & 18.4 & 23.9 \\
\hline \multicolumn{5}{|c|}{ Fe-1P } \\
\hline & $\operatorname{B3LYP}\left(\boldsymbol{C}_{1}\right)$ & B3LYP* $\left.^{*} \boldsymbol{C}_{1}\right)$ & $\operatorname{BP} 86\left(\boldsymbol{C}_{1}\right)$ & $\operatorname{M06L}\left(\boldsymbol{C}_{1}\right)$ \\
\hline$E+1731$ & -0.84494 & -0.34389 & -1.01269 & -0.72419 \\
\hline$\Delta \mathrm{E}$ & 4.8 & 8.1 & 17.9 & 7.7 \\
\hline$\left\langle\mathrm{S}^{2}\right\rangle$ & 6.07 & 6.08 & 6.06 & 6.13 \\
\hline \multicolumn{5}{|c|}{$\mathrm{Fe}-2 \mathrm{P}$} \\
\hline & $\operatorname{B3LYP}\left(\boldsymbol{C}_{\mathbf{1}}\right)$ & $\operatorname{B3LYP}^{*}\left(\boldsymbol{C}_{1}\right)$ & $\operatorname{BP} 86\left(\boldsymbol{C}_{1}\right)$ & $\operatorname{M06L}\left(\boldsymbol{C}_{1}\right)$ \\
\hline$E+1731$ & -0.82268 & -0.33003 & -0.97597 & -0.69291 \\
\hline$\Delta \mathrm{E}$ & 18.7 & 16.8 & 41.0 & 27.4 \\
\hline$\left\langle\mathrm{S}^{2}\right\rangle$ & 6.06 & 6.05 & 6.03 & 6.05 \\
\hline
\end{tabular}


Table S107. Total energies ( $\mathrm{E}$ in hartree), relative energies ( $\Delta \mathrm{E}$ in $\mathrm{kcal} / \mathrm{mol})$, and the spin expectation values $\left\langle\mathrm{S}^{2}\right\rangle$ for the $\left(\mathrm{C}_{4} \mathrm{H}_{6}\right)_{3} \mathrm{Co}$ structure by all four methods. Neither structure has any imaginary vibrational frequencies.

\begin{tabular}{|c|c|c|c|c|}
\hline \multicolumn{5}{|c|}{ Co-1D } \\
\hline & $\operatorname{B3LYP}\left(\boldsymbol{C}_{1}\right)$ & $\operatorname{B3LYP}^{*}\left(\boldsymbol{C}_{1}\right)$ & $\operatorname{BP86}\left(\boldsymbol{C}_{1}\right)$ & $\operatorname{M06L}\left(\boldsymbol{C}_{1}\right)$ \\
\hline$E+1850$ & -0.9208 & -0.41808 & -1.11934 & -0.79587 \\
\hline $\mathrm{DE}$ & 0.0 & 0.0 & 0.0 & 0.0 \\
\hline$\left\langle\mathrm{S}^{2}\right\rangle$ & 0.80 & 0.78 & 0.77 & 0.80 \\
\hline \multicolumn{5}{|c|}{ Co-2D } \\
\hline & $\operatorname{B3LYP}\left(\boldsymbol{C}_{1}\right)$ & B3LYP $^{*}\left(\boldsymbol{C}_{1}\right)$ & $\operatorname{BP86}\left(\boldsymbol{C}_{1}\right)$ & $\operatorname{M06L}\left(\boldsymbol{C}_{1}\right)$ \\
\hline$E+1850$ & -0.90697 & -0.40453 & -1.10771 & -0.7794 \\
\hline$\Delta \mathrm{E}$ & 8.7 & 8.5 & 7.3 & 10.3 \\
\hline$\left\langle\mathrm{S}^{2}\right\rangle$ & 0.80 & 0.79 & 0.77 & 0.81 \\
\hline \multicolumn{5}{|c|}{ Co-3D } \\
\hline & $\operatorname{B3LYP}\left(\boldsymbol{C}_{1}\right)$ & B3LYP* $^{*}\left(\boldsymbol{C}_{1}\right)$ & $\operatorname{BP86}\left(\boldsymbol{C}_{1}\right)$ & $\operatorname{M06L}\left(\boldsymbol{C}_{1}\right)$ \\
\hline$E+1850$ & -0.88429 & -0.38479 & -1.09459 & -0.75788 \\
\hline$\Delta \mathrm{E}$ & 22.9 & 20.9 & 15.5 & 23.8 \\
\hline$\left\langle\mathrm{S}^{2}\right\rangle$ & 0.87 & 0.82 & 0.77 & 0.82 \\
\hline \multicolumn{5}{|c|}{ Co-4D } \\
\hline & B3LYP( $\left.\boldsymbol{C}_{1}\right)$ & $\operatorname{B3LYP}^{*}\left(\boldsymbol{C}_{1}\right)$ & $\operatorname{BP86}\left(\boldsymbol{C}_{1}\right)$ & $\operatorname{M06L}\left(\boldsymbol{C}_{1}\right)$ \\
\hline$E+1850$ & -0.87605 & -0.37673 & -1.08834 & -0.75288 \\
\hline$\Delta \mathrm{E}$ & 28.1 & 25.9 & 19.5 & 27.0 \\
\hline$\left\langle\mathrm{S}^{2}\right\rangle$ & 0.85 & 0.82 & 0.77 & 0.82 \\
\hline \multicolumn{5}{|c|}{ Co-5D } \\
\hline & B3LYP( $\left.\boldsymbol{C}_{1}\right)$ & B3LYP $^{*}\left(\boldsymbol{C}_{1}\right)$ & $\operatorname{BP86}\left(\boldsymbol{C}_{1}\right)$ & $\operatorname{M06L}\left(\boldsymbol{C}_{1}\right)$ \\
\hline$E+1850$ & -0.86601 & -0.36291 & -1.0722 & -0.73553 \\
\hline$\Delta \mathrm{E}$ & 34.4 & 34.6 & 29.6 & 37.9 \\
\hline$\left\langle\mathrm{S}^{2}\right\rangle$ & 1.48 & 1.11 & 0.76 & 0.82 \\
\hline \multicolumn{5}{|c|}{ Co-1Q } \\
\hline & $\operatorname{B3LYP}\left(\boldsymbol{C}_{1}\right)$ & $\mathrm{B} 3 \mathrm{LYP}^{*}\left(\boldsymbol{C}_{1}\right)$ & $\operatorname{BP86}\left(\boldsymbol{C}_{1}\right)$ & $\operatorname{M06L}\left(\boldsymbol{C}_{1}\right)$ \\
\hline$E+1850$ & -0.90061 & -0.39465 & -1.0928 & -0.77236 \\
\hline$\Delta \mathrm{E}$ & 12.7 & 14.7 & 16.7 & 14.8 \\
\hline$\left\langle\mathrm{S}^{2}\right\rangle$ & 3.80 & 3.80 & 3.78 & 3.81 \\
\hline \multicolumn{5}{|c|}{ Co-2Q } \\
\hline & B3LYP( $\left.\boldsymbol{C}_{1}\right)$ & B3LYP $^{*}\left(\boldsymbol{C}_{1}\right)$ & $\operatorname{BP} 86\left(\boldsymbol{C}_{1}\right)$ & $\operatorname{M06L}\left(\boldsymbol{C}_{1}\right)$ \\
\hline$E+1850$ & -0.89728 & -0.38857 & -1.07819 & -0.75374 \\
\hline$\Delta \mathrm{E}$ & 14.8 & 18.5 & 25.8 & 26.4 \\
\hline$\left\langle\mathrm{S}^{2}\right\rangle$ & 3.78 & 3.78 & 3.77 & 3.78 \\
\hline
\end{tabular}


Table S108. Total energies ( $\mathrm{E}$ in hartree), relative energies $(\Delta \mathrm{E}$ in $\mathrm{kcal} / \mathrm{mol})$ for the $\left(\mathrm{C}_{4} \mathrm{H}_{6}\right)_{3} \mathrm{Ni}$ structure by all four methods. Neither structure has any imaginary vibrational frequencies.

\begin{tabular}{|c|c|c|c|c|}
\hline \multicolumn{5}{|c|}{ Ni-1S } \\
\hline & $\operatorname{B3LYP}\left(\boldsymbol{C}_{1}\right)$ & $\operatorname{B3LYP}^{*}\left(\boldsymbol{C}_{1}\right)$ & $\overline{B P 86}\left(\boldsymbol{C}_{\mathbf{1}}\right)$ & $\overline{\operatorname{M06L}\left(\boldsymbol{C}_{1}\right)}$ \\
\hline$E+1975$ & -1.48422 & -0.97691 & -1.70525 & -1.35156 \\
\hline$\Delta \mathrm{E}$ & 0 & 0 & 0 & 0 \\
\hline \multicolumn{5}{|c|}{ Ni-2S } \\
\hline & $\operatorname{B3LYP}\left(\boldsymbol{C}_{1}\right)$ & $\operatorname{B3LYP}^{*}\left(\boldsymbol{C}_{1}\right)$ & $\operatorname{BP86}\left(\boldsymbol{C}_{\mathbf{1}}\right)$ & $\operatorname{M06L}\left(\boldsymbol{C}_{1}\right)$ \\
\hline$E+1975$ & -1.47325 & -0.96623 & -1.69684 & -1.33632 \\
\hline$\Delta \mathrm{E}$ & 6.9 & 6.7 & 5.3 & 9.6 \\
\hline \multicolumn{5}{|c|}{ Ni-3S } \\
\hline & $\operatorname{B3LYP}\left(\boldsymbol{C}_{\mathbf{1}}\right)$ & $\mathrm{B}^{2} \operatorname{LYP}^{*}\left(\boldsymbol{C}_{1}\right)$ & $\operatorname{BP86}\left(\boldsymbol{C}_{1}\right)$ & $\operatorname{M06L}\left(\boldsymbol{C}_{1}\right)$ \\
\hline$E+1975$ & -1.46999 & -0.96275 & -1.69221 & -1.33384 \\
\hline$\Delta \mathrm{E}$ & 8.9 & 8.9 & 8.2 & 11.1 \\
\hline \multicolumn{5}{|c|}{ Ni-4S } \\
\hline & $\operatorname{B3LYP}\left(\boldsymbol{C}_{1}\right)$ & $\mathrm{B3LYP}^{*}\left(\boldsymbol{C}_{1}\right)$ & $\operatorname{BP86}\left(\boldsymbol{C}_{1}\right)$ & $\operatorname{M06L}\left(\boldsymbol{C}_{1}\right)$ \\
\hline$E+1975$ & -1.46877 & -0.96171 & -1.69198 & -1.33243 \\
\hline$\Delta \mathrm{E}$ & 9.7 & 9.5 & 8.3 & 12 \\
\hline \multicolumn{5}{|c|}{ Ni-5S } \\
\hline & $\operatorname{B3LYP}\left(\boldsymbol{c}_{1}\right)$ & $\operatorname{B3LYP}^{*}\left(\boldsymbol{C}_{1}\right)$ & $\operatorname{BP86}\left(\boldsymbol{C}_{1}\right)$ & $\operatorname{M06L}\left(\boldsymbol{C}_{1}\right)$ \\
\hline$E+1975$ & -1.43891 & -0.93287 & -1.66383 & -1.30504 \\
\hline$\Delta \mathrm{E}$ & 28.4 & 27.6 & 26 & 29.2 \\
\hline \multicolumn{5}{|c|}{ Ni-1T } \\
\hline & $\operatorname{B3LYP}\left(\boldsymbol{C}_{\mathbf{1}}\right)$ & 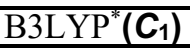 & $\operatorname{BP86}\left(\boldsymbol{C}_{\mathbf{1}}\right)$ & $\operatorname{M06L}\left(\boldsymbol{C}_{1}\right)$ \\
\hline$E+1975$ & -1.45098 & -0.94098 & -1.66833 & -1.31329 \\
\hline$\Delta \mathrm{E}$ & 20.9 & 22.5 & 23.2 & 24 \\
\hline$\left\langle\mathrm{S}^{2}\right\rangle$ & 2.04 & 2.03 & 2.01 & 2.03 \\
\hline \multicolumn{5}{|c|}{ Ni-2T } \\
\hline & $\operatorname{B3LYP}\left(\boldsymbol{C}_{\mathbf{1}}\right)$ & $\mathrm{B}^{2} \mathrm{LYP}^{*}\left(\boldsymbol{C}_{1}\right)$ & $\operatorname{BP86}\left(\boldsymbol{C}_{\mathbf{1}}\right)$ & $\overline{\mathrm{M} 06 \mathrm{~L}\left(\boldsymbol{C}_{1}\right)}$ \\
\hline$E+1975$ & -1.44388 & -0.93126 & -1.6516 & -1.29562 \\
\hline$\Delta \mathrm{E}$ & 25.3 & 28.6 & 33.7 & 35.1 \\
\hline$\left\langle\mathrm{S}^{2}\right\rangle$ & 2.02 & 2.02 & 2.01 & 2.02 \\
\hline
\end{tabular}


Table S109. The theoretical (B3LYP*) Ni-C and C-C distances (in $\AA$ ) of the $\mathrm{Ni}\left(\mathrm{C}_{12} \mathrm{H}_{19}\right)^{+}$ cation. The experimental results of those in $\left[\mathrm{Ni}\left(\mathrm{C}_{12} \mathrm{H}_{19}\right)\right]^{+} \mathrm{X}^{-}\left(\mathrm{X}=\mathrm{BF}_{4}, \mathrm{~F}_{3} \mathrm{CSO}_{3}\right)$ are listed for comparison.

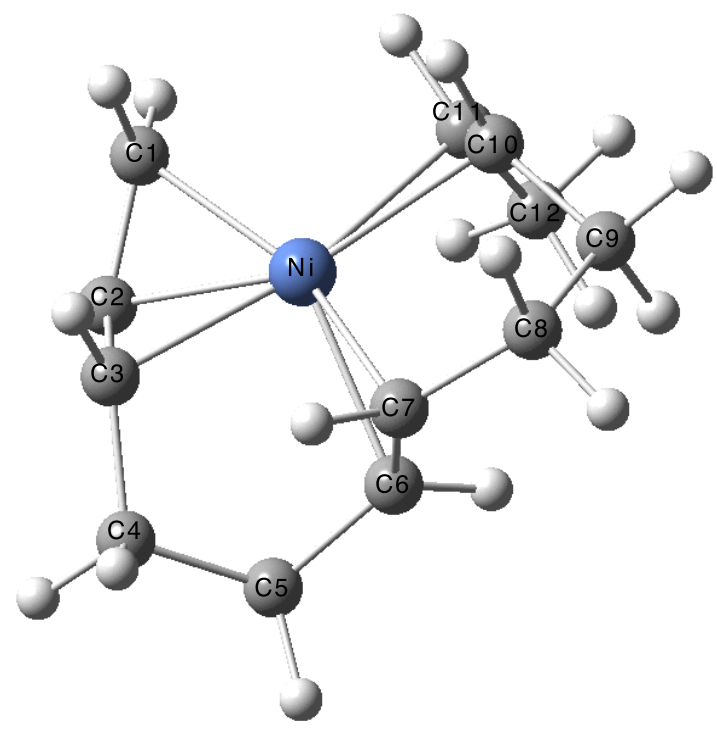

\begin{tabular}{lccc}
\hline & This work & $\mathrm{X}=\mathrm{BF}_{4}(\exp )$. & $\mathrm{X}=\mathrm{F}_{3} \mathrm{CSO}_{3}(\mathrm{exp})$. \\
\hline $\mathrm{Ni}-\mathrm{C} 1$ & 2.077 & $2.079(6)$ & $2.13(1)$ \\
$\mathrm{Ni}-\mathrm{C} 2$ & 2.055 & $2.021(5)$ & $2.02(1)$ \\
$\mathrm{Ni}-\mathrm{C} 3$ & 2.051 & $2.031(5)$ & $2.01(1)$ \\
$\mathrm{Ni}-\mathrm{C} 4$ & 3.001 & $2.956(3)$ & $2.967(8)$ \\
$\mathrm{Ni}-\mathrm{C} 5$ & 2.914 & $2.907(3)$ & $2.953(8)$ \\
$\mathrm{Ni}-\mathrm{C6}$ & 2.157 & $2.111(6)$ & $2.12(1)$ \\
$\mathrm{Ni}-\mathrm{C} 7$ & 2.173 & $2.119(4)$ & $2.11(1)$ \\
$\mathrm{Ni}-\mathrm{C} 8$ & 3.001 & $2.964(3)$ & $2.952(8)$ \\
$\mathrm{Ni}-\mathrm{C} 9$ & $2.934(3)$ & $3.009(8)$ \\
$\mathrm{Ni}-\mathrm{C} 10$ & 2.975 & $2.145(4)$ & $2.21(1)$ \\
$\mathrm{Ni}-\mathrm{C} 11$ & 2.149 & $2.227(5)$ & $2.25(1)$ \\
$\mathrm{C} 1-\mathrm{C} 2$ & 2.266 & $1.367(7)$ & $1.38(2)$ \\
$\mathrm{C} 2-\mathrm{C} 3$ & 1.409 & $1.395(7)$ & $1.41(2)$ \\
$\mathrm{C} 3-\mathrm{C} 4$ & 1.416 & $1.488(8)$ & $1.53(2)$ \\
$\mathrm{C} 4-\mathrm{C} 5$ & 1.520 & $1.528(8)$ & $1.55(2)$ \\
$\mathrm{C} 5-\mathrm{C} 6$ & 1.542 & $1.505(6)$ & $1.49(2)$ \\
$\mathrm{C} 6-\mathrm{C} 7$ & 1.522 & $1.343(6)$ & $1.39(2)$ \\
C7-C8 & 1.383 & $1.517(6)$ & $1.53(2)$ \\
C8-C9 & 1.520 & $1.506(8)$ & $1.52(2)$ \\
C9-C10 & 1.540 & $1.500(8)$ & $1.50(2)$ \\
C10-C11 & 1.515 & $1.334(7)$ & $1.38(2)$ \\
C11-C12 & 1.380 & $1.497(7)$ & $1.48(2)$ \\
\hline
\end{tabular}


Table S110. The Mulliken spin density on the metal center. It shows that the spin states are mainly metal-centered, and the contribution from the ligand-based spin density are less than \pm 1.0 , except for the high-lying Ni-1T.

\begin{tabular}{|c|c|c|c|c|c|c|c|c|}
\hline $\mathrm{Ni}-1 \mathrm{~S}$ & $\mathrm{Ni}-2 \mathrm{~S}$ & Ni-3S & $\mathrm{Ni}-4 \mathrm{~S}$ & $\mathrm{Ni}-5 \mathrm{~S}$ & $\mathrm{Ni}-1 \mathrm{~T}$ & $\mathrm{Ni}-2 \mathrm{~T}$ & & \\
\hline 0.00 & 0.00 & 0.00 & 0.00 & 0.00 & 0.96 & 1.21 & & \\
\hline Co-1D & Co-2D & Co-3D & Co-4D & Co-1Q & Co-2Q & & & \\
\hline 1.20 & 1.22 & 1.24 & 1.12 & 2.22 & 2.36 & & & \\
\hline Fe-1T & Fe-2T & Fe-3T & Fe-1P & Fe-2P & $\mathrm{Fe}-1 \mathrm{~S}$ & Fe-2S & & \\
\hline 2.33 & 2.34 & 2.53 & 3.40 & 3.45 & 0.00 & 0.00 & & \\
\hline$M n-1 H$ & $\mathrm{Mn}-2 \mathrm{H}$ & $M n-1 Q$ & $M n-2 Q$ & $M n-1 D$ & Mn-2D & Mn-3D & & \\
\hline 4.75 & 4.85 & 3.65 & 3.61 & 1.63 & 1.37 & 1.43 & & \\
\hline Cr-1P & $\mathrm{Cr}-2 \mathrm{P}$ & $\mathrm{Cr}-1 \mathrm{~T}$ & $\mathrm{Cr}-2 \mathrm{~T}$ & Cr-3T & Cr-1S & Cr-2S & & \\
\hline 4.11 & 4.16 & 2.59 & 2.50 & 2.51 & 0.00 & 0.00 & & \\
\hline V-1Q & $V-2 Q$ & V-3Q & $V-4 Q$ & $V-5 Q$ & V-1D & $V-2 D$ & V-3D & V-4D \\
\hline 2.92 & 3.00 & 2.50 & 3.02 & 2.61 & 1.15 & 1.34 & 1.18 & 1.24 \\
\hline Ti-1S & Ti-2S & Ti-1T & & & & & & \\
\hline 0.00 & 0.00 & 1.65 & & & & & & \\
\hline
\end{tabular}

Note: The italics denotes the Mulliken spin density on the metal center less than the unpaired electrons of this molecule.

\section{Complete Gaussian 09 reference (Reference 39)}

Gaussian 09, Revision B.01, Frisch, M. J.; Trucks, G. W.; Schlegel, H. B.; Scuseria, G. E.; Robb, M. A.; Cheeseman, J. R.; Scalmani, G.; Barone, V.; Mennucci, B.; Petersson, G. A.; Nakatsuji, H.; Caricato, M.; Li, X.; Hratchian, H. P.; Izmaylov, A. F.; Bloino, J.; Zheng, G.; Sonnenberg, J. L.; Hada, M.; Ehara, M.; Toyota, K.; Fukuda, R.; Hasegawa, J.; Ishida, M.; Nakajima, T.; Honda, Y.; Kitao, O.; Nakai, H.; Vreven, T.; Montgomery, Jr., J. A.; Peralta, J. E.; Ogliaro, F.; Bearpark, M.; Heyd, J. J.; Brothers, E.; Kudin, K. N.; Staroverov, V. N.; Keith, T.; Kobayashi, R.; Normand, J.; Raghavachari, K.; Rendell, A.; Burant, J. C.; Iyengar, S. S.; Tomasi, J.; Cossi, M.; Rega, N.; Millam, N. J.; Klene, M.; Knox, J. E.; Cross, J. B.; Bakken, V.; Adamo, C.; Jaramillo, J.; Gomperts, R.; Stratmann, R. E.; Yazyev, O.; Austin, A. J.; Cammi, R.; Pomelli, C.; Ochterski, J. W.; Martin, R. L.; Morokuma, K.; Zakrzewski, V. G.; Voth, G. A.; Salvador, P.; Dannenberg, J. J.; Dapprich, S.; Daniels, A. D.; Farkas, Ö.;

Foresman, J. B.; Ortiz, J. V.; Cioslowski, J.; Fox, D. J. Gaussian, Inc., Wallingford CT, 2010. 